\title{
BROOKHSWEN
}

NATIONAL LABORATORY

BNL-77290-2006-IR

\section{Recent References: \\ October 1, 2005 to December 31, 2005}

\author{
David F. Winchell \\ National Nuclear Data Center, Brookhaven National Laboratory
}

November 2006

\author{
Energy Sciences \& Technology Department \\ National Nuclear Data Center \\ Brookhaven National Laboratory \\ P.O. Box 5000 \\ Upton, NY 11973-5000 \\ www.bnl.gov
}

\begin{abstract}
Notice: This manuscript has been authored by employees of Brookhaven Science Associates, LLC under Contract No. DE-AC02-98CH10886 with the U.S. Department of Energy. The publisher by accepting the manuscript for publication acknowledges that the United States Government retains a non-exclusive, paid-up, irrevocable, world-wide license to publish or reproduce the published form of this manuscript, or allow others to do so, for United States Government purposes.
\end{abstract}




\section{DISCLAIMER}

This report was prepared as an account of work sponsored by an agency of the United States Government. Neither the United States Government nor any agency thereof, nor any of their employees, nor any of their contractors, subcontractors, or their employees, makes any warranty, express or implied, or assumes any legal liability or responsibility for the accuracy, completeness, or any third party's use or the results of such use of any information, apparatus, product, or process disclosed, or represents that its use would not infringe privately owned rights. Reference herein to any specific commercial product, process, or service by trade name, trademark, manufacturer, or otherwise, does not necessarily constitute or imply its endorsement, recommendation, or favoring by the United States Government or any agency thereof or its contractors or subcontractors. The views and opinions of authors expressed herein do not necessarily state or reflect those of the United States Government or any agency thereof. 


\title{
Recent References: \\ October 1, 2005 to December 31, 2005
}

\author{
David F. Winchell \\ National Nuclear Data Center, Brookhaven National Laboratory
}

This document lists experimental references added to Nuclear Science References (NSR) during the period October 1, 2005 to December 31, 2005. The first section lists keynumbers and keywords sorted by mass and nuclide. The second section lists all references, ordered by keynumber.

For more information, and access to the most recent NSR updates, please visit the NSR web site at http://www.nndc.bnl.gov/nsr/.

\section{Contents}

Keynumbers and Keywords $\quad 2$

$\begin{array}{ll}\text { References } & 120\end{array}$ 


\title{
Keynumbers and Keywords
}

\author{
$\mathrm{A}=1$ \\ ${ }^{1} \mathrm{n} \quad 2005$ AHO7 \\ 2005GR28 \\ 2005JA17 \\ 2005J021 \\ 2005KHZX \\ 2005KI19 \\ 2005KR14 \\ 2005R037 \\ ${ }^{1} \mathrm{H}$ \\ 2005AH07 \\ 2005AN30 \\ 2005BA93 \\ 2005DEZT \\ 2005GA45 \\ 2005GR26 \\ NUCLEAR REACTIONS ${ }^{1} \mathrm{H}\left(\right.$ polarized $\left.\gamma, \pi^{0}\right),\left(\right.$ polarized $\left.\gamma, \pi^{+}\right)$, e $\approx$ \\ $340 \mathrm{MeV}$; measured $\sigma(\theta), \mathrm{G}$ asymmetries, related polarization \\ observables. Polarized target. JOUR ZAANE 26135 \\ NUCLEAR REACTIONS ${ }^{1} \mathrm{H}\left(\pi^{-}, \pi^{+} \pi^{-}\right),\left(\pi^{+}, 2 \pi^{+}\right), \mathrm{E}=243,264,284$, \\ $305 \mathrm{MeV} ;{ }^{2} \mathrm{H},{ }^{12} \mathrm{C},{ }^{40} \mathrm{Ca},{ }^{208} \mathrm{~Pb}\left(\pi^{+}, 2 \pi^{+}\right),\left(\pi^{+}, \pi^{+} \pi^{-}\right), \mathrm{E}=283 \mathrm{MeV}$; \\ $\mathrm{Sc}\left(\pi^{+}, 2 \pi^{+} \mathrm{X}\right),\left(\pi^{+}, \pi^{+} \pi^{-} \mathrm{X}\right), \mathrm{E}=243,264,284,305 \mathrm{MeV}$; measured \\ invariant mass distributions, $\sigma(\theta)$, correlations; deduced partial chiral \\ symmetry restoration. JOUR NUPAB 76380 \\ NUCLEAR REACTIONS ${ }^{1} \mathrm{H}(\mathrm{n}, \mathrm{p}), \mathrm{E}=11 \mathrm{MeV}$; measured recoil \\ proton spectra in scintillator. JOUR NIMAE 551245 \\ NUCLEAR REACTIONS ${ }^{1} \mathrm{H}\left(\right.$ polarized e, e' $\pi^{+}$), (polarized e, e' $\pi^{0}$ ), \\ $\mathrm{E}=1.515 \mathrm{GeV}$; measured $\sigma(\mathrm{E}, \theta)$, polarized longitudinal-transverse \\ structure function; deduced sensitivity to Roper resonance. JOUR \\ PRVCA 72058202 \\ RADIOACTIVITY ${ }^{1} \mathrm{n}\left(\beta^{-}\right)$; measured $\beta \mathrm{p}-, \beta \mathrm{p} \gamma$-coin; deduced \\ branching ratio for radiative decay. Comparison with model \\ predictions. PREPRINT nucl-ex/0512001,12/1/2005 \\ NUCLEAR REACTIONS ${ }^{2} \mathrm{H}(\mathrm{p}, 2 \mathrm{p}), \mathrm{E}=130 \mathrm{MeV}$; measured Ep, \\ pp-coin, $\sigma\left(\theta_{1}, \theta_{2}\right)$; deduced three-nucleon force effects. JOUR PRVCA \\ 72044006 \\ NUCLEAR REACTIONS ${ }^{3} \mathrm{He}$ (polarized e, e'), E=3.465-5.727 GeV; \\ measured parallel and perpendicular cross section differences. ${ }^{1} \mathrm{n},{ }^{3} \mathrm{He}$ \\ deduced momentum transfer dependence of spin structure function. \\ JOUR PRLTA 95142002 \\ NUCLEAR REACTIONS ${ }^{1} \mathrm{H}\left({ }^{8} \mathrm{He},{ }^{8} \mathrm{He}\right)$, E not given; measured recoil \\ proton spectrum; deduced excitation function. ${ }^{1} \mathrm{H}\left({ }^{6} \mathrm{He},{ }^{6} \mathrm{Li}\right)$, E not \\ given; measured neutron spectrum, n $\gamma$-coin; deduced excitation \\ function. ${ }^{7,9} \mathrm{Li}$ deduced resonance parameters. ${ }^{7,9} \mathrm{He}$ deduced analog \\ states features. JOUR NIMBE 241977 \\ NUCLEAR REACTIONS ${ }^{1} \mathrm{H}\left(\right.$ polarized $\left.\gamma, \pi^{0}\right),\left(\right.$ polarized $\left.\gamma, \pi^{+}\right), \mathrm{e} \approx$ \\ $340 \mathrm{MeV}$; measured $\sigma(\theta), \mathrm{G}$ asymmetries, related polarization \\ observables. Polarized target. JOUR ZAANE 26135 \\ NUCLEAR REACTIONS ${ }^{2} \mathrm{H}\left({ }^{7} \mathrm{Be}, 2 \alpha\right)$, E=1.71, $5.55 \mathrm{MeV}$; measured \\ particle spectra, $\sigma .{ }^{7} \mathrm{Be}(\mathrm{d}, \mathrm{p}), \mathrm{E}(\mathrm{cm}) \approx 0.38,1.2 \mathrm{MeV}$; deduced \\ astrophysical S-factors. Implications for primordial ${ }^{7} \mathrm{Li}$ abundance \\ discussed. JOUR ASJOA 630 L105 \\ NUCLEAR REACTIONS ${ }^{1} \mathrm{H}\left(\gamma, \pi^{0}\right), \mathrm{E}=0.3-3.0 \mathrm{GeV}$; measured pion \\ production $\sigma(\theta), \sigma$. Tagged photons. JOUR PRLTA 94012003 \\ NUCLEAR REACTIONS ${ }^{1} \mathrm{H}\left(\pi^{+}, \pi^{+}\right),\left(\pi^{-}, \pi^{-}\right), \mathrm{E}=19,26,32,37,43$ \\ $\mathrm{MeV}$; measured $\sigma(\theta)$; deduced real part of isospin forward scattering \\ amplitude. PREPRINT nucl-ex/0512006,12/3/2005 \\ NUCLEAR REACTIONS ${ }^{2} \mathrm{H}\left({ }^{44} \mathrm{Ar},{ }^{45} \mathrm{Ar}\right),\left({ }^{40} \mathrm{Ar},{ }^{41} \mathrm{Ar}\right), \mathrm{E}=10 \mathrm{MeV} /$ \\ nucleon; measured particle spectra, $\sigma(\mathrm{E}, \theta) .{ }^{45} \mathrm{Ar}$ deduced levels, \\ spectroscopic factors. JOUR JPGPE 31 S1623 \\ NUCLEAR REACTIONS ${ }^{1} \mathrm{H}(\mathrm{e}, \mathrm{e} \cdot \gamma), \mathrm{E}=$ high; measured $\sigma\left(\mathrm{Q}^{2}, \mathrm{~W}\right)$ for \\ deeply virtual Compton scattering. JOUR ZCCNE 44 S1
}




\section{$\mathrm{A}=1$ (continued)}

2005GU29 NUCLEAR REACTIONS ${ }^{2} \mathrm{H}\left({ }^{8} \mathrm{Li},{ }^{9} \mathrm{Li}\right), \mathrm{E}(\mathrm{cm})=7.8 \mathrm{MeV}$; measured $\sigma(\theta)$; deduced asymptotic normalization coefficient. ${ }^{8} \mathrm{~B}(\mathrm{p}, \gamma), \mathrm{E}=\mathrm{low}$; calculated astrophysical S-factor. DWBA analysis, inverse kinematics, comparison with data. JOUR NUPAB 761162

2005HA64 NUCLEAR REACTIONS ${ }^{1} \mathrm{H}\left({ }^{6} \mathrm{He},{ }^{6} \mathrm{He}\right), \mathrm{E}=71 \mathrm{MeV} /$ nucleon; measured $\sigma(\theta)$, analyzing powers; deduced optical model parameters. ${ }^{6} \mathrm{He}$ deduced rms radius. Polarized target. Comparison with model predictions. JOUR ZAANE 25 s01 255

2005J021 NUCLEAR REACTIONS ${ }^{1} \mathrm{H}$ (polarized e, e' $\left.\pi^{+}\right)$, (polarized e, e' $\left.\pi^{0}\right)$, $\mathrm{E}=1.515 \mathrm{GeV}$; measured $\sigma(\mathrm{E}, \theta)$, polarized longitudinal-transverse structure function; deduced sensitivity to Roper resonance. JOUR PRVCA 72058202

2005KHZX RADIOACTIVITY ${ }^{1} \mathrm{n}\left(\beta^{-}\right)$; measured $\beta \mathrm{p}-, \beta \mathrm{p} \gamma$-coin; deduced branching ratio for radiative decay. Comparison with model predictions. PREPRINT nucl-ex/0512001,12/1/2005

2005MAZM NUCLEAR REACTIONS ${ }^{2} \mathrm{H}\left({ }^{48} \mathrm{Ca},{ }^{49} \mathrm{Ca}\right), \mathrm{E}=105 \mathrm{MeV}$; measured $\mathrm{E} \gamma$, I $\gamma$, (particle) $\gamma$-coin. ${ }^{48} \mathrm{Ca}$ (polarized d, p), $\mathrm{E}=14 \mathrm{MeV}$; measured proton spectra, $\sigma(\theta) .{ }^{49} \mathrm{Ca}$ deduced levels, J, $\pi$. REPT MLL 2004

Annual,P8,Maierbeck

2005NA36 NUCLEAR REACTIONS ${ }^{2} \mathrm{H}, \mathrm{C}\left({ }^{7} \mathrm{Li},{ }^{7} \mathrm{Be}\right), \mathrm{E}=65 \mathrm{MeV} /$ nucleon; measured spin-flip and spin-nonflip particle spectra; deduced charge-exchange spin-flip $\sigma .{ }^{2} \mathrm{H}(\gamma, \mathrm{n}), \mathrm{E} \approx 1.5-10 \mathrm{MeV}$; deduced magnetic dipole $\sigma$. Comparison with previous results, model predictions. JOUR PRVCA 72041001

20050N04 NUCLEAR REACTIONS ${ }^{1} \mathrm{H}\left({ }^{16} \mathrm{C},{ }^{16} \mathrm{C}\right), \mathrm{E}=33 \mathrm{MeV} /$ nucleon; measured $\mathrm{E} \gamma, \mathrm{I} \gamma$, (particle) $\gamma$-coin; deduced $\sigma .{ }^{16} \mathrm{C}$ deduced deformation parameter. JOUR ZAANE 25 s01 347

$2005 R 037$ NUCLEAR REACTIONS ${ }^{1} \mathrm{H}\left({ }^{8} \mathrm{He},{ }^{8} \mathrm{He}\right)$, E not given; measured recoil proton spectrum; deduced excitation function. ${ }^{1} \mathrm{H}\left({ }^{6} \mathrm{He},{ }^{6} \mathrm{Li}\right)$, E not given; measured neutron spectrum, $\mathrm{n} \gamma$-coin; deduced excitation function. ${ }^{7,9} \mathrm{Li}$ deduced resonance parameters. ${ }^{7,9} \mathrm{He}$ deduced analog states features. JOUR NIMBE 241977

2005SE22 NUCLEAR REACTIONS ${ }^{2} \mathrm{H}(\mathrm{p}, \mathrm{p}), \mathrm{E}=135 \mathrm{MeV} ;{ }^{1} \mathrm{H}(\mathrm{d}, \mathrm{d}), \mathrm{E}=135$ $\mathrm{MeV}$ / nucleon; measured $\sigma(\theta)$; deduced relativistic effects, three-nucleon force effects. Comparison with previous results. JOUR PRLTA 95162301

2005SEZV NUCLEAR REACTIONS ${ }^{2} \mathrm{H}(\mathrm{p}, \mathrm{p}), \mathrm{E}=135 \mathrm{MeV} ;{ }^{1} \mathrm{H}(\mathrm{d}, \mathrm{d}), \mathrm{E}=270$ $\mathrm{MeV}$; measured $\sigma(\theta)$. Comparison with model predictions and previous data. PREPRINT nucl-ex/0510005,10/3/2005

2005TU09 NUCLEAR REACTIONS ${ }^{2} \mathrm{H}\left({ }^{6} \mathrm{Li}, \mathrm{t} \alpha\right), \mathrm{E}=14 \mathrm{MeV}$; measured triton and $\alpha$ spectra. ${ }^{6} \mathrm{Li}(\mathrm{n}, \alpha), \mathrm{E} \approx 0-1 \mathrm{MeV}$; deduced $\sigma(\theta)$. JOUR ZAANE $25 \mathrm{~s} 01649$

$$
\mathrm{A}=\mathbf{2}
$$

${ }^{2} \mathrm{H} \quad 2005 \mathrm{AIO6}$

NUCLEAR REACTIONS ${ }^{1} \mathrm{H}\left(\mathrm{e}^{+}, \mathrm{e}^{+} \mathrm{X}\right), \mathrm{E}$ at $27.7 \mathrm{GeV} / \mathrm{c}$; measured tensor asymmetry. ${ }^{2} \mathrm{H}$ deduced tensor structure function. Polarized target. JOUR PRLTA 95242001 


\section{$\mathrm{A}=2$ (continued)}

2005CA42 NUCLEAR MOMENTS ${ }^{2} \mathrm{H},{ }^{15} \mathrm{~N}$; measured hfs; deduced parameters. JOUR APJSA 159181

2005CU06 NUCLEAR REACTIONS ${ }^{7} \mathrm{Li}\left({ }^{7} \mathrm{Li},{ }^{11} \mathrm{~B}\right),\left({ }^{7} \mathrm{Li},{ }^{12} \mathrm{~B}\right), \mathrm{E}=58 \mathrm{MeV} ;{ }^{12} \mathrm{C}$, ${ }^{16} \mathrm{O}\left({ }^{7} \mathrm{Li},{ }^{10} \mathrm{~B}\right), \mathrm{E}=58 \mathrm{MeV}$; measured particle spectra. ${ }^{10,11,12} \mathrm{~B}$ deduced relative yields for $\alpha+\mathrm{Li}$ and $\mathrm{H}+\mathrm{Be}$ decay channels from excited states. JOUR PRVCA 72044320

2005LA30 NUCLEAR REACTIONS H, C(polarized d, pX), E at $9 \mathrm{GeV} / \mathrm{c}$; measured tensor analyzing power vs proton transverse momentum. ${ }^{2} \mathrm{H}$ deduced wave function features. JOUR PYLBB 62960

2005SE22 NUCLEAR REACTIONS ${ }^{2} \mathrm{H}(\mathrm{p}, \mathrm{p}), \mathrm{E}=135 \mathrm{MeV} ;{ }^{1} \mathrm{H}(\mathrm{d}, \mathrm{d}), \mathrm{E}=135$ $\mathrm{MeV}$ / nucleon; measured $\sigma(\theta)$; deduced relativistic effects, three-nucleon force effects. Comparison with previous results. JOUR PRLTA 95162301

2005SEZV NUCLEAR REACTIONS ${ }^{2} \mathrm{H}(\mathrm{p}, \mathrm{p}), \mathrm{E}=135 \mathrm{MeV} ;{ }^{1} \mathrm{H}(\mathrm{d}, \mathrm{d}), \mathrm{E}=270$ $\mathrm{MeV}$; measured $\sigma(\theta)$. Comparison with model predictions and previous data. PREPRINT nucl-ex/0510005,10/3/2005

2005SH51 NUCLEAR REACTIONS ${ }^{4} \mathrm{He}(\gamma, \mathrm{p}),(\gamma, \mathrm{n}),(\gamma, \mathrm{np}), \mathrm{E}=21.8-29.8 \mathrm{MeV}$; ${ }^{12} \mathrm{C}(\gamma, \mathrm{p}),(\gamma, \mathrm{n}), \mathrm{E}=22.3-32 \mathrm{MeV}$; measured charged particle spectra, photodisintegration $\sigma, \sigma(\theta)$. Monoenergetic pulsed photons, comparison with previous results and model predictions. JOUR PRVCA 72044004

$$
\mathrm{A}=3
$$

${ }^{3} \mathrm{H} \quad 2005 \mathrm{CU} 06$

NUCLEAR REACTIONS ${ }^{7} \mathrm{Li}\left({ }^{7} \mathrm{Li},{ }^{11} \mathrm{~B}\right),\left({ }^{7} \mathrm{Li},{ }^{12} \mathrm{~B}\right), \mathrm{E}=58 \mathrm{MeV} ;{ }^{12} \mathrm{C}$, ${ }^{16} \mathrm{O}\left({ }^{7} \mathrm{Li},{ }^{10} \mathrm{~B}\right), \mathrm{E}=58 \mathrm{MeV}$; measured particle spectra. ${ }^{10,11,12} \mathrm{~B}$ deduced relative yields for $\alpha+\mathrm{Li}$ and $\mathrm{H}+\mathrm{Be}$ decay channels from excited states. JOUR PRVCA 72044320

2005GI18 NUCLEAR REACTIONS ${ }^{1} \mathrm{H}\left({ }^{6} \mathrm{He}, \alpha\right), \mathrm{E}=25 \mathrm{MeV} /$ nucleon; measured $\sigma(\theta)$; deduced particle transfer contributions, entrance potential dependence. ${ }^{6} \mathrm{He}$ deduced spectroscopic factors for $\mathrm{t}+\mathrm{t}$ and $\mathrm{a}+2 \mathrm{n}$ cluster configurations. SPEG spectrometer and MUST array at GANIL. DWBA and coupled-channels calculations. JOUR ZAANE 25 s01 267

2005KI17 NUCLEAR REACTIONS ${ }^{4} \mathrm{He}(\gamma, \mathrm{p}),(\gamma, \mathrm{n}), \mathrm{E} \approx 27.6 \mathrm{MeV}$; measured particle spectra, tp-, $\left({ }^{3} \mathrm{He}\right) \mathrm{n}$-coin. Time projection chamber. JOUR NIMAE 552329

2005MI32 NUCLEAR REACTIONS ${ }^{4} \mathrm{He}\left({ }^{22} \mathrm{O},{ }^{23} \mathrm{~F}\right), \mathrm{E}=35 \mathrm{MeV} /$ nucleon; ${ }^{4} \mathrm{He}\left({ }^{23} \mathrm{~F},{ }^{23} \mathrm{~F}\right.$ '), $\mathrm{E}=41.5 \mathrm{MeV} /$ nucleon; ${ }^{4} \mathrm{He}\left({ }^{24} \mathrm{~F},{ }^{23} \mathrm{~F}\right), \mathrm{E}=36 \mathrm{MeV} /$ nucleon; measured $\mathrm{E} \gamma$, I $\gamma$, (particle) $\gamma-, \gamma \gamma$-coin; deduced $\sigma(\mathrm{E}) .{ }^{23} \mathrm{~F}$ deduced levels, J, $\pi$. DWBA analysis. JOUR ZAANE 25 s01 367 2005MIZT NUCLEAR REACTIONS ${ }^{4} \mathrm{He}\left({ }^{22} \mathrm{O},{ }^{23} \mathrm{~F}\right),\left({ }^{23} \mathrm{~F},{ }^{23} \mathrm{~F}^{\prime}\right),\left({ }^{24} \mathrm{~F},{ }^{23} \mathrm{~F}\right)$, $\left({ }^{25} \mathrm{Ne},{ }^{23} \mathrm{~F}\right), \mathrm{E} \approx 35-43 \mathrm{MeV} /$ nucleon; measured $\mathrm{E} \gamma, \mathrm{I} \gamma$, (particle) $\gamma-$, $\gamma \gamma$-coin. ${ }^{4} \mathrm{He}\left({ }^{22} \mathrm{O},{ }^{23} \mathrm{~F}\right), \mathrm{E}=35 \mathrm{MeV} /$ nucleon; measured $\sigma(\theta) .{ }^{23} \mathrm{~F}$ deduced levels, J, $\pi$, configurations. REPT CNS-REP-67,Michimasa

2005RA27 NUCLEAR REACTIONS ${ }^{2} \mathrm{H}(\mathrm{d}, \mathrm{p}), \mathrm{E} \approx 4-23 \mathrm{keV}$; measured S-factors, electron screening effects for reactions in deuterated metals, temperature dependence. JOUR JPGPE 311141 


\section{$\mathrm{A}=3$ (continued)}

$2005 \mathrm{SH} 46$ NUCLEAR REACTIONS ${ }^{4} \mathrm{He}\left({ }^{22} \mathrm{O},{ }^{23} \mathrm{~F}\right), \mathrm{E}=35 \mathrm{MeV} /$ nucleon; measured $\mathrm{E} \gamma, \mathrm{I} \gamma$, (particle) $\gamma$-coin, $\sigma(\theta) .{ }^{23} \mathrm{~F}$ deduced levels, J, $\pi$. JOUR JPGPE 31 S1759

2005SH51 NUCLEAR REACTIONS ${ }^{4} \mathrm{He}(\gamma, \mathrm{p}),(\gamma, \mathrm{n}),(\gamma, \mathrm{np}), \mathrm{E}=21.8-29.8 \mathrm{MeV}$; ${ }^{12} \mathrm{C}(\gamma, \mathrm{p}),(\gamma, \mathrm{n}), \mathrm{E}=22.3-32 \mathrm{MeV}$; measured charged particle spectra, photodisintegration $\sigma, \sigma(\theta)$. Monoenergetic pulsed photons, comparison with previous results and model predictions. JOUR PRVCA 72044004

2005ST30 NUCLEAR REACTIONS ${ }^{4} \mathrm{He}\left(\mathrm{e}, \mathrm{e}\right.$ 'p $\left.\pi^{-}\right),\left(\mathrm{e}, \mathrm{e} \mathrm{p} \pi^{0}\right), \mathrm{E}=672 \mathrm{MeV}$; measured Ep, recoil spectra, $\sigma(\theta)$. Comparison with model predictions. JOUR PRLTA 95172501

2005TU09 NUCLEAR REACTIONS ${ }^{2} \mathrm{H}\left({ }^{6} \mathrm{Li}, \mathrm{t} \alpha\right), \mathrm{E}=14 \mathrm{MeV}$; measured triton and $\alpha$ spectra. ${ }^{6} \mathrm{Li}(\mathrm{n}, \alpha), \mathrm{E} \approx 0-1 \mathrm{MeV}$; deduced $\sigma(\theta)$. JOUR ZAANE $25 \mathrm{~s} 01649$

2005VE08 NUCLEAR REACTIONS ${ }^{6} \operatorname{Li}(\mathrm{n}, \alpha), \mathrm{E}=$ reactor; measured triton spectra, angular distribution; deduced P-odd asymmetry coefficient. JOUR PZETA 82519

${ }^{3} \mathrm{He} \quad 2005 \mathrm{KI} 17 \quad$ NUCLEAR REACTIONS ${ }^{4} \mathrm{He}(\gamma, \mathrm{p}),(\gamma, \mathrm{n}), \mathrm{E} \approx 27.6 \mathrm{MeV}$; measured particle spectra, tp-, $\left({ }^{3} \mathrm{He}\right)$ n-coin. Time projection chamber. JOUR NIMAE 552329

2005KR14 NUCLEAR REACTIONS ${ }^{3} \mathrm{He}$ (polarized e, e'), E=3.465-5.727 GeV; measured parallel and perpendicular cross section differences. ${ }^{1} \mathrm{n},{ }^{3} \mathrm{He}$ deduced momentum transfer dependence of spin structure function. JOUR PRLTA 95142002

2005NI20 NUCLEAR REACTIONS ${ }^{4} \mathrm{He}(\gamma, \mathrm{n}), \mathrm{E}=23-42 \mathrm{MeV}$; measured $\sigma(\theta)$; deduced angle-integrated $\sigma$. Comparison with previous data and various model calculations. Liquid target, tagged photons. JOUR PYLBB 62665

2005SH51 NUCLEAR REACTIONS ${ }^{4} \mathrm{He}(\gamma, \mathrm{p}),(\gamma, \mathrm{n}),(\gamma, \mathrm{np}), \mathrm{E}=21.8-29.8 \mathrm{MeV}$; ${ }^{12} \mathrm{C}(\gamma, \mathrm{p}),(\gamma, \mathrm{n}), \mathrm{E}=22.3-32 \mathrm{MeV}$; measured charged particle spectra, photodisintegration $\sigma, \sigma(\theta)$. Monoenergetic pulsed photons, comparison with previous results and model predictions. JOUR PRVCA 72044004

2005ST30 NUCLEAR REACTIONS ${ }^{4} \mathrm{He}\left(\mathrm{e}, \mathrm{e} \mathrm{p} \pi^{-}\right),\left(\mathrm{e}, \mathrm{e} \mathrm{p} \pi^{0}\right), \mathrm{E}=672 \mathrm{MeV}$; measured Ep, recoil spectra, $\sigma(\theta)$. Comparison with model predictions. JOUR PRLTA 95172501

$$
\mathrm{A}=4
$$

${ }^{4} \mathrm{n} \quad 2005 \mathrm{KI} 20$

NUCLEAR REACTIONS ${ }^{4} \mathrm{He}\left(\pi^{+}, \pi^{-}\right), \mathrm{E}=120,150,180,240,270$ $\mathrm{MeV} ;{ }^{4} \mathrm{He}\left(\pi^{-}, \pi^{+}\right), \mathrm{E}=180,240 \mathrm{MeV}$; measured $\sigma(\mathrm{E}, \theta)$; deduced multiple scattering effects, total $\sigma$. JOUR PRVCA 72044608

${ }^{4} \mathrm{He} \quad 2005$ MI32 NUCLEAR REACTIONS ${ }^{4} \mathrm{He}\left({ }^{22} \mathrm{O},{ }^{23} \mathrm{~F}\right), \mathrm{E}=35 \mathrm{MeV} /$ nucleon; ${ }^{4} \mathrm{He}\left({ }^{23} \mathrm{~F},{ }^{23} \mathrm{~F}\right.$ '), $\mathrm{E}=41.5 \mathrm{MeV} /$ nucleon; ${ }^{4} \mathrm{He}\left({ }^{24} \mathrm{~F},{ }^{23} \mathrm{~F}\right), \mathrm{E}=36 \mathrm{MeV} /$ nucleon; measured $\mathrm{E} \gamma, \mathrm{I} \gamma$, (particle) $\gamma$-, $\gamma \gamma$-coin; deduced $\sigma(\mathrm{E}) .{ }^{23} \mathrm{~F}$ deduced levels, J, $\pi$. DWBA analysis. JOUR ZAANE 25 s01 367 


\section{$\mathrm{A}=4$ (continued)}

2005MIZT NUCLEAR REACTIONS ${ }^{4} \mathrm{He}\left({ }^{22} \mathrm{O},{ }^{23} \mathrm{~F}\right),\left({ }^{23} \mathrm{~F},{ }^{23} \mathrm{~F}\right),\left({ }^{24} \mathrm{~F},{ }^{23} \mathrm{~F}\right)$, $\left({ }^{25} \mathrm{Ne},{ }^{23} \mathrm{~F}\right), \mathrm{E} \approx 35-43 \mathrm{MeV} /$ nucleon; measured $\mathrm{E} \gamma, \mathrm{I} \gamma$, (particle) $\gamma-$, $\gamma \gamma$-coin. ${ }^{4} \mathrm{He}\left({ }^{22} \mathrm{O},{ }^{23} \mathrm{~F}\right), \mathrm{E}=35 \mathrm{MeV} /$ nucleon; measured $\sigma(\theta) \cdot{ }^{23} \mathrm{~F}$ deduced levels, J, $\pi$, configurations. REPT CNS-REP-67,Michimasa

2005SU25 RADIOACTIVITY ${ }^{8} \mathrm{~B}(\mathrm{EC} \alpha)\left[\right.$ from $\left.{ }^{6} \mathrm{Li}\left({ }^{3} \mathrm{He}, \mathrm{n}\right)\right] ;{ }^{8} \mathrm{Li}\left(\beta^{-} \alpha\right)\left[\right.$ from ${ }^{7} \mathrm{Li}(\mathrm{d}$, p)]; measured $\beta$-NMR spectra; angular correlations; deduced limit on G-parity term. JOUR ZAANE 25 s01 709

2005WRZZ NUCLEAR REACTIONS ${ }^{2} \mathrm{H}(\mathrm{d}, \mathrm{X})^{4} \mathrm{He}, \mathrm{E} \approx$ threshold; measured $\eta$ production $\sigma, \sigma(\theta)$. PREPRINT nucl-ex/0510056,10/20/2005

${ }^{4} \mathrm{Be} \quad 2005 \mathrm{KI} 20$ NUCLEAR REACTIONS ${ }^{4} \mathrm{He}\left(\pi^{+}, \pi^{-}\right), \mathrm{E}=120,150,180,240,270$ $\mathrm{MeV} ;{ }^{4} \mathrm{He}\left(\pi^{-}, \pi^{+}\right), \mathrm{E}=180,240 \mathrm{MeV}$; measured $\sigma(\mathrm{E}, \theta)$; deduced multiple scattering effects, total $\sigma$. JOUR PRVCA 72044608

\section{$\mathrm{A}=5$}

${ }^{5} \mathrm{H} \quad 2005 \mathrm{TE} 05$

NUCLEAR REACTIONS ${ }^{3} \mathrm{H}(\mathrm{t}, \mathrm{p}), \mathrm{E}=58 \mathrm{MeV} ;{ }^{2} \mathrm{H}\left({ }^{6} \mathrm{He}, \mathrm{t}\right),\left({ }^{6} \mathrm{He},{ }^{3} \mathrm{He}\right)$, $\mathrm{E}=132 \mathrm{MeV}$; measured particle spectra, angular correlations following residual nucleus decay. ${ }^{5} \mathrm{He}$ deduced resonances J, $\pi$, IAS features. ${ }^{5} \mathrm{H}$ deduced ground-state resonance energy. JOUR ZAANE 25 s01 315

${ }^{5} \mathrm{He} \quad$ 2005MI32 NUCLEAR REACTIONS ${ }^{4} \mathrm{He}\left({ }^{22} \mathrm{O},{ }^{23} \mathrm{~F}\right), \mathrm{E}=35 \mathrm{MeV} /$ nucleon; ${ }^{4} \mathrm{He}\left({ }^{23} \mathrm{~F},{ }^{23} \mathrm{~F}\right.$ '), $\mathrm{E}=41.5 \mathrm{MeV} /$ nucleon; ${ }^{4} \mathrm{He}\left({ }^{24} \mathrm{~F},{ }^{23} \mathrm{~F}\right), \mathrm{E}=36 \mathrm{MeV} /$ nucleon; measured $\mathrm{E} \gamma, \mathrm{I} \gamma$, (particle) $\gamma$-, $\gamma \gamma$-coin; deduced $\sigma(\mathrm{E}) \cdot{ }^{23} \mathrm{~F}$ deduced levels, J, $\pi$. DWBA analysis. JOUR ZAANE 25 s01 367 2005MIZT NUCLEAR REACTIONS ${ }^{4} \mathrm{He}\left({ }^{22} \mathrm{O},{ }^{23} \mathrm{~F}\right),\left({ }^{23} \mathrm{~F},{ }^{23} \mathrm{~F}\right),\left({ }^{24} \mathrm{~F},{ }^{23} \mathrm{~F}\right)$, $\left({ }^{25} \mathrm{Ne},{ }^{23} \mathrm{~F}\right), \mathrm{E} \approx 35-43 \mathrm{MeV} /$ nucleon; measured $\mathrm{E} \gamma, \mathrm{I} \gamma$, (particle) $\gamma-$, $\gamma \gamma$-coin. ${ }^{4} \mathrm{He}\left({ }^{22} \mathrm{O},{ }^{23} \mathrm{~F}\right), \mathrm{E}=35 \mathrm{MeV} /$ nucleon; measured $\sigma(\theta) \cdot{ }^{23} \mathrm{~F}$ deduced levels, J, $\pi$, configurations. REPT CNS-REP-67,Michimasa $2005 \mathrm{~S} 013$ NUCLEAR REACTIONS ${ }^{16} \mathrm{O}\left({ }^{9} \mathrm{Be}, \alpha^{7} \mathrm{Be}\right),{ }^{7} \mathrm{Li}\left({ }^{9} \mathrm{Be}, \alpha^{7} \mathrm{Li}\right),\left({ }^{9} \mathrm{Be}, \mathrm{t} 2 \alpha\right)$, $\mathrm{E}=55,70 \mathrm{MeV}$; measured excitation energy spectra. ${ }^{11} \mathrm{~B},{ }^{11} \mathrm{C}$ deduced excited states energies, configurations. JOUR JPGPE 31 S1701

2005TE05 NUCLEAR REACTIONS ${ }^{3} \mathrm{H}(\mathrm{t}, \mathrm{p}), \mathrm{E}=58 \mathrm{MeV} ;{ }^{2} \mathrm{H}\left({ }^{6} \mathrm{He}, \mathrm{t}\right),\left({ }^{6} \mathrm{He},{ }^{3} \mathrm{He}\right)$, $\mathrm{E}=132 \mathrm{MeV}$; measured particle spectra, angular correlations following residual nucleus decay. ${ }^{5} \mathrm{He}$ deduced resonances J, $\pi$, IAS features. ${ }^{5} \mathrm{H}$ deduced ground-state resonance energy. JOUR ZAANE 25 s01 315

$$
\mathrm{A}=6
$$

${ }^{6} \mathrm{He} \quad$ 2005GI18

NUCLEAR REACTIONS ${ }^{1} \mathrm{H}\left({ }^{6} \mathrm{He}, \alpha\right), \mathrm{E}=25 \mathrm{MeV} /$ nucleon; measured $\sigma(\theta)$; deduced particle transfer contributions, entrance potential dependence. ${ }^{6} \mathrm{He}$ deduced spectroscopic factors for $\mathrm{t}+\mathrm{t}$ and $\mathrm{a}+2 \mathrm{n}$ cluster configurations. SPEG spectrometer and MUST array at GANIL. DWBA and coupled-channels calculations. JOUR ZAANE 25 s01 267

2005HA64 NUCLEAR REACTIONS ${ }^{1} \mathrm{H}\left({ }^{6} \mathrm{He},{ }^{6} \mathrm{He}\right), \mathrm{E}=71 \mathrm{MeV} /$ nucleon; measured $\sigma(\theta)$, analyzing powers; deduced optical model parameters. ${ }^{6}$ He deduced rms radius. Polarized target. Comparison with model predictions. JOUR ZAANE 25 s01 255 


\section{$\mathrm{A}=6$ (continued)}

2005KI21 NUCLEAR REACTIONS ${ }^{1} \mathrm{H}\left({ }^{6} \mathrm{He}, \mathrm{p}\right),\left({ }^{8} \mathrm{He}, \mathrm{p}\right), \mathrm{E} \approx 700 \mathrm{MeV} /$ nucleon; measured recoil proton spectra, $\sigma(\mathrm{E}, \theta) .{ }^{6,8} \mathrm{He}$ deduced nuclear matter density distributions, charge radii, cluster configurations, spectroscopic factors. JOUR ZAANE 25 s01 215 2005Ye05 NUCLEAR REACTIONS ${ }^{9} \mathrm{Be}\left({ }^{6} \mathrm{He},{ }^{6} \mathrm{He}\right),\left({ }^{6} \mathrm{He},{ }^{5} \mathrm{He}\right),\left({ }^{6} \mathrm{He}, \alpha\right),\left({ }^{6} \mathrm{He}\right.$, $\alpha \mathrm{X}),\left({ }^{6} \mathrm{He}, \mathrm{tX}\right), \mathrm{E}=25 \mathrm{MeV} /$ nucleon; measured quasielastic, breakup, and transfer $\sigma(\theta)$. ${ }^{6} \mathrm{He}$ deduced two-triton configuration. JOUR JPGPE 31 S1647

${ }^{6} \mathrm{Li} \quad 2004 \mathrm{KU} 36$ NUCLEAR REACTIONS ${ }^{3} \mathrm{He}\left({ }^{7} \mathrm{Li}, \alpha\right), \mathrm{E}=31.2 \mathrm{MeV}$; measured $\mathrm{E} \alpha$. ${ }^{6} \mathrm{Li}$ deduced resonance energies, widths. JOUR BJPHE 34933

2005B049 NUCLEAR REACTIONS ${ }^{1} \mathrm{H}\left({ }^{6} \mathrm{He}, \mathrm{n}\right)$, E not given; measured Doppler-shifted $\mathrm{E} \gamma, \mathrm{I} \gamma .{ }^{7} \mathrm{Li}$ deduced resonance features, IAS. JOUR ZAANE 25 s01 259

2005MIZT NUCLEAR REACTIONS ${ }^{4} \mathrm{He}\left({ }^{22} \mathrm{O},{ }^{23} \mathrm{~F}\right),\left({ }^{23} \mathrm{~F},{ }^{23} \mathrm{~F}\right),\left({ }^{24} \mathrm{~F},{ }^{23} \mathrm{~F}\right)$, $\left({ }^{25} \mathrm{Ne},{ }^{23} \mathrm{~F}\right), \mathrm{E} \approx 35-43 \mathrm{MeV} /$ nucleon; measured $\mathrm{E} \gamma, \mathrm{I} \gamma$, (particle) $\gamma-$, $\gamma \gamma$-coin. ${ }^{4} \mathrm{He}\left({ }^{22} \mathrm{O},{ }^{23} \mathrm{~F}\right), \mathrm{E}=35 \mathrm{MeV} /$ nucleon; measured $\sigma(\theta) \cdot{ }^{23} \mathrm{~F}$ deduced levels, J, $\pi$, configurations. REPT CNS-REP-67,Michimasa 2005N015 NUCLEAR MOMENTS ${ }^{6,7,8,9} \mathrm{Li}$; measured hfs, isotope shifts; deduced charge radii. Resonance ionization mass spectroscopy, comparison with model predictions. JOUR ZAANE 25 s01 199

${ }^{6} \mathrm{Be} \quad 2004 \mathrm{GU} 21$ NUCLEAR REACTIONS ${ }^{9} \mathrm{Be}\left({ }^{14} \mathrm{~B},{ }^{13} \mathrm{BX}\right), \mathrm{E}=60 \mathrm{MeV} /$ nucleon; measured $\mathrm{E} \gamma, \mathrm{I} \gamma$, particle momentum distribution, $\sigma(\mathrm{E}) .{ }^{13} \mathrm{~B}$ deduced levels, $\mathrm{J}, \pi$, asymptotic normalization coefficients. ${ }^{2} \mathrm{H}\left({ }^{8} \mathrm{~B}, \alpha\right), \mathrm{E}=28.5$ $\mathrm{MeV}$; measured E $\alpha$. JOUR BJPHE 341012

$$
\mathrm{A}=7
$$

${ }^{7} \mathrm{He} \quad$ 2005R037

NUCLEAR REACTIONS ${ }^{1} \mathrm{H}\left({ }^{8} \mathrm{He},{ }^{8} \mathrm{He}\right)$, E not given; measured recoil proton spectrum; deduced excitation function. ${ }^{1} \mathrm{H}\left({ }^{6} \mathrm{He},{ }^{6} \mathrm{Li}\right)$, E not given; measured neutron spectrum, $\mathrm{n} \gamma$-coin; deduced excitation function. ${ }^{7,9} \mathrm{Li}$ deduced resonance parameters. ${ }^{7,9} \mathrm{He}$ deduced analog states features. JOUR NIMBE 241977

${ }^{7} \mathrm{Li} \quad 2005 \mathrm{BA96}$ NUCLEAR REACTIONS ${ }^{7} \mathrm{Li}\left({ }^{7} \mathrm{Be},{ }^{7} \mathrm{Be}\right), \mathrm{E}(\mathrm{cm})=8.87,9.87 \mathrm{MeV}$; measured $\sigma(\theta)$; deduced optical model parameters. JOUR PRVCA 72 044602

2005B049 NUCLEAR REACTIONS ${ }^{1} \mathrm{H}\left({ }^{6} \mathrm{He}, \mathrm{n}\right)$, E not given; measured Doppler-shifted E $\gamma, \mathrm{I} \gamma .{ }^{7} \mathrm{Li}$ deduced resonance features, IAS. JOUR ZAANE 25 s01 259

2005N015 NUCLEAR MOMENTS ${ }^{6,7,8,9} \mathrm{Li}$; measured hfs, isotope shifts; deduced charge radii. Resonance ionization mass spectroscopy, comparison with model predictions. JOUR ZAANE 25 s01 199

2005R037 NUCLEAR REACTIONS ${ }^{1} \mathrm{H}\left({ }^{8} \mathrm{He},{ }^{8} \mathrm{He}\right)$, E not given; measured recoil proton spectrum; deduced excitation function. ${ }^{1} \mathrm{H}\left({ }^{6} \mathrm{He},{ }^{6} \mathrm{Li}\right)$, E not given; measured neutron spectrum, n $\gamma$-coin; deduced excitation function. ${ }^{7,9} \mathrm{Li}$ deduced resonance parameters. ${ }^{7,9} \mathrm{He}$ deduced analog states features. JOUR NIMBE 241977 


\section{$\mathrm{A}=7$ (continued)}

2005RU18 NUCLEAR REACTIONS ${ }^{7} \mathrm{Li}\left({ }^{11} \mathrm{~B}, \mathrm{X}\right), \mathrm{E}=44 \mathrm{MeV}$; measured particle spectra, charge distributions. ${ }^{7} \mathrm{Li}\left({ }^{11} \mathrm{~B},{ }^{11} \mathrm{~B}\right),\left({ }^{11} \mathrm{~B},{ }^{11} \mathrm{~B}\right), \mathrm{E}=44 \mathrm{MeV}$; measured $\sigma(\mathrm{E}, \theta) ;{ }^{11} \mathrm{~B}\left({ }^{7} \mathrm{Li},{ }^{7} \mathrm{Li}\right),\left({ }^{7} \mathrm{Li},{ }^{7} \mathrm{Li}\right), \mathrm{E}=34 \mathrm{MeV}$; analyzed $\sigma(\mathrm{E}$, $\theta)$; deduced optical model parameters, transfer channel contributions, reorientation effects. ${ }^{7} \mathrm{Li},{ }^{11} \mathrm{~B}$ deduced deformation parameters. Optical model and coupled-reaction-channels analysis. JOUR PRVCA 72034608

${ }^{7} \mathrm{Be} \quad$ 2005DA41 NUCLEAR REACTIONS H, C $\left({ }^{7} \mathrm{Li}, \mathrm{X}\right){ }^{7} \mathrm{Be}, \mathrm{E} \approx 25-30 \mathrm{MeV}$; measured yields. JOUR NIMBE 241953

2005SE23 NUCLEAR REACTIONS ${ }^{197} \mathrm{Au}(\mathrm{n}, \gamma), \mathrm{E}=$ spectrum; measured $\mathrm{E} \gamma$, I $\gamma$; deduced neutron flux. ${ }^{7} \mathrm{Li}(\mathrm{p}, \mathrm{n}), \mathrm{E}$ not given; deduced neutron spectrum. ${ }^{62} \mathrm{Ni}(\mathrm{n}, \gamma), \mathrm{E} \approx 5.5-20 \mathrm{keV}$; measured $\sigma$; deduced Maxwellian-averaged $\sigma$. JOUR JUPSA 742981

\section{$\mathrm{A}=8$}

${ }^{8} \mathrm{He} \quad 2005 \mathrm{KI} 21 \quad$ NUCLEAR REACTIONS ${ }^{1} \mathrm{H}\left({ }^{6} \mathrm{He}, \mathrm{p}\right),\left({ }^{8} \mathrm{He}, \mathrm{p}\right), \mathrm{E} \approx 700 \mathrm{MeV} /$ nucleon; measured recoil proton spectra, $\sigma(\mathrm{E}, \theta) .{ }^{6,8} \mathrm{He}$ deduced nuclear matter density distributions, charge radii, cluster configurations, spectroscopic factors. JOUR ZAANE 25 s01 215

${ }^{8} \mathrm{Li} \quad 2005 \mathrm{~B} 045$ RADIOACTIVITY ${ }^{8,9} \operatorname{Li}\left(\beta^{-}\right)$[from Ta $(\mathrm{p}, \mathrm{X})$ ]; measured $\beta$-asymmetries, $\beta$-NMR spectra from polarized sources. ${ }^{8,9} \mathrm{Li}$ deduced quadrupole moments. ${ }^{9} \mathrm{Li}$ deduced $\mu$. Comparisons with previous results and model predictions. JOUR PRVCA 72044309

2005N015 NUCLEAR MOMENTS ${ }^{6,7,8,9} \mathrm{Li}$; measured hfs, isotope shifts; deduced charge radii. Resonance ionization mass spectroscopy, comparison with model predictions. JOUR ZAANE 25 s01 199

2005SU25 RADIOACTIVITY ${ }^{8} \mathrm{~B}(\mathrm{EC} \alpha)\left[\right.$ from $\left.{ }^{6} \mathrm{Li}\left({ }^{3} \mathrm{He}, \mathrm{n}\right)\right] ;{ }^{8} \mathrm{Li}\left(\beta^{-} \alpha\right)\left[\right.$ from ${ }^{7} \mathrm{Li}(\mathrm{d}$, p)]; measured $\beta$-NMR spectra; angular correlations; deduced limit on G-parity term. JOUR ZAANE 25 s01 709

${ }^{8} \mathrm{Be} \quad 2005 \mathrm{AN} 30$ NUCLEAR REACTIONS ${ }^{2} \mathrm{H}\left({ }^{7} \mathrm{Be}, 2 \alpha\right), \mathrm{E}=1.71,5.55 \mathrm{MeV}$; measured particle spectra, $\sigma .{ }^{7} \mathrm{Be}(\mathrm{d}, \mathrm{p}), \mathrm{E}(\mathrm{cm}) \approx 0.38,1.2 \mathrm{MeV}$; deduced astrophysical S-factors. Implications for primordial ${ }^{7} \mathrm{Li}$ abundance discussed. JOUR ASJOA 630 L105

2005B045 RADIOACTIVITY ${ }^{8,9} \operatorname{Li}\left(\beta^{-}\right)$[from Ta(p, X)]; measured $\beta$-asymmetries, $\beta$-NMR spectra from polarized sources. ${ }^{8,9} \mathrm{Li}$ deduced quadrupole moments. ${ }^{9} \mathrm{Li}$ deduced $\mu$. Comparisons with previous results and model predictions. JOUR PRVCA 72044309

2005SCZV NUCLEAR REACTIONS ${ }^{9} \mathrm{Be}\left({ }^{26} \mathrm{Mg},{ }^{27} \mathrm{Mg}\right), \mathrm{E}=57 \mathrm{MeV}$; measured $\mathrm{E} \gamma$, $\mathrm{I} \gamma, \alpha \alpha$-coin, $\sigma(\theta) .{ }^{27} \mathrm{Mg}$ deduced transitions. REPT MLL 2004 Annual,P4,Schwerdtfeger

2005SPZY NUCLEAR REACTIONS ${ }^{12} \mathrm{C}\left({ }^{32} \mathrm{~S},{ }^{36} \mathrm{Ar}\right), \mathrm{E}=65 \mathrm{MeV} ;{ }^{12} \mathrm{C}\left({ }^{34} \mathrm{~S},{ }^{38} \mathrm{Ar}\right)$, $\mathrm{E}=67 \mathrm{MeV}$; measured $\mathrm{E} \gamma, \mathrm{I} \gamma(\theta, \mathrm{H}, \mathrm{t}), \alpha \gamma$-coin. ${ }^{36,38}$ Ar levels deduced $\mathrm{g}$ factors. Transient field technique. Comparison with shell model predictions. REPT MLL 2004 Annual,P5,Speidel

${ }^{8} \mathrm{~B} 2005 \mathrm{Su} 25 \quad$ RADIOACTIVITY ${ }^{8} \mathrm{~B}(\mathrm{EC} \alpha)\left[\right.$ from $\left.{ }^{6} \mathrm{Li}\left({ }^{3} \mathrm{He}, \mathrm{n}\right)\right] ;{ }^{8} \mathrm{Li}\left(\beta^{-} \alpha\right)\left[\right.$ from ${ }^{7} \mathrm{Li}(\mathrm{d}$, p)]; measured $\beta$-NMR spectra; angular correlations; deduced limit on G-parity term. JOUR ZAANE 25 s01 709 


\section{$\mathrm{A}=8$ (continued)}

2005TA32 NUCLEAR REACTIONS Be, C, Al $\left({ }^{12} \mathrm{C}, \mathrm{X}\right), \mathrm{E}=30-200 \mathrm{MeV} /$ nucleon; $\mathrm{Be}\left({ }^{9} \mathrm{Be}, \mathrm{X}\right), \mathrm{E}=70-100 \mathrm{MeV} /$ nucleon; measured reaction $\sigma(\mathrm{E})$; deduced nucleon-nucleon interaction range. ${ }^{8} \mathrm{~B}$ deduced nuclear matter density distribution. Comparison with Glauber calculations.

JOUR ZAANE 25 s01 217

$$
\mathrm{A}=\mathbf{9}
$$

${ }^{9} \mathrm{He} \quad 2005 \mathrm{R} 037$

NUCLEAR REACTIONS ${ }^{1} \mathrm{H}\left({ }^{8} \mathrm{He},{ }^{8} \mathrm{He}\right)$, E not given; measured recoil proton spectrum; deduced excitation function. ${ }^{1} \mathrm{H}\left({ }^{6} \mathrm{He},{ }^{6} \mathrm{Li}\right)$, E not given; measured neutron spectrum, $\mathrm{n} \gamma$-coin; deduced excitation function. ${ }^{7,9} \mathrm{Li}$ deduced resonance parameters. ${ }^{7,9} \mathrm{He}$ deduced analog states features. JOUR NIMBE 241977

${ }^{9} \mathrm{Li} \quad$ 2005B045 RADIOACTIVITY ${ }^{8,9} \mathrm{Li}\left(\beta^{-}\right)$[from Ta $\left.(\mathrm{p}, \mathrm{X})\right]$; measured $\beta$-asymmetries, $\beta$-NMR spectra from polarized sources. ${ }^{8,9} \mathrm{Li}$ deduced quadrupole moments. ${ }^{9} \mathrm{Li}$ deduced $\mu$. Comparisons with previous results and model predictions. JOUR PRVCA 72044309

2005N015 NUCLEAR MOMENTS ${ }^{6,7,8,9} \mathrm{Li}$; measured hfs, isotope shifts; deduced charge radii. Resonance ionization mass spectroscopy, comparison with model predictions. JOUR ZAANE 25 s01 199

2005R037 NUCLEAR REACTIONS ${ }^{1} \mathrm{H}\left({ }^{8} \mathrm{He},{ }^{8} \mathrm{He}\right)$, E not given; measured recoil proton spectrum; deduced excitation function. ${ }^{1} \mathrm{H}\left({ }^{6} \mathrm{He},{ }^{6} \mathrm{Li}\right)$, E not given; measured neutron spectrum, $\mathrm{n} \gamma$-coin; deduced excitation function. ${ }^{7,9} \mathrm{Li}$ deduced resonance parameters. ${ }^{7,9} \mathrm{He}$ deduced analog states features. JOUR NIMBE 241977

${ }^{9} \mathrm{Be} \quad 2005 \mathrm{AD} 35$ NCLEAR REACTIONS ${ }^{6} \mathrm{Li}\left({ }^{6} \mathrm{Li}, \alpha \mathrm{X}\right),\left({ }^{7} \mathrm{Li}, \alpha \mathrm{X}\right), \mathrm{E}=14-20 \mathrm{MeV}$; measured $\alpha$-spectra. ${ }^{12} \mathrm{C}(\mathrm{n}, \alpha), \mathrm{E}=72.8 \mathrm{MeV} ;{ }^{28} \mathrm{Si}\left({ }^{6} \mathrm{Li}, \alpha\right), \mathrm{E}=36 \mathrm{MeV}$; analyzed $\alpha$-spectra. Statistical model calculations. Target-projectile symmetry discussed. JOUR ZAANE 25 s01 299

2005B045 RADIOACTIVITY ${ }^{8,9} \operatorname{Li}\left(\beta^{-}\right)$[from Ta(p, X)]; measured $\beta$-asymmetries, $\beta$-NMR spectra from polarized sources. ${ }^{8,9} \mathrm{Li}$ deduced quadrupole moments. ${ }^{9} \mathrm{Li}$ deduced $\mu$. Comparisons with previous results and model predictions. JOUR PRVCA 72044309 2005 YE05 NUCLEAR REACTIONS ${ }^{9} \mathrm{Be}\left({ }^{6} \mathrm{He},{ }^{6} \mathrm{He}\right),\left({ }^{6} \mathrm{He},{ }^{5} \mathrm{He}\right),\left({ }^{6} \mathrm{He}, \alpha\right),\left({ }^{6} \mathrm{He}\right.$, $\alpha \mathrm{X}),\left({ }^{6} \mathrm{He}, \mathrm{tX}\right), \mathrm{E}=25 \mathrm{MeV} /$ nucleon; measured quasielastic, breakup, and transfer $\sigma(\theta) .{ }^{6} \mathrm{He}$ deduced two-triton configuration. JOUR JPGPE 31 S1647

${ }^{9} \mathrm{C} \quad 2005 \mathrm{GU} 29 \quad$ NUCLEAR REACTIONS ${ }^{2} \mathrm{H}\left({ }^{8} \mathrm{Li},{ }^{9} \mathrm{Li}\right), \mathrm{E}(\mathrm{cm})=7.8 \mathrm{MeV}$; measured $\sigma(\theta)$; deduced asymptotic normalization coefficient. ${ }^{8} \mathrm{~B}(\mathrm{p}, \gamma), \mathrm{E}=\mathrm{low}$; calculated astrophysical S-factor. DWBA analysis, inverse kinematics, comparison with data. JOUR NUPAB 761162 


\section{$\mathrm{A}=\mathbf{1 0}$}

\begin{tabular}{|c|c|c|}
\hline${ }^{10} \mathrm{Be}$ & 2005YE05 & $\begin{array}{l}\text { NUCLEAR REACTIONS }{ }^{9} \mathrm{Be}\left({ }^{6} \mathrm{He},{ }^{6} \mathrm{He}\right),\left({ }^{6} \mathrm{He},{ }^{5} \mathrm{He}\right),\left({ }^{6} \mathrm{He}, \alpha\right),\left({ }^{6} \mathrm{He},\right. \\
\alpha \mathrm{X}),\left({ }^{6} \mathrm{He}, \mathrm{tX}\right), \mathrm{E}=25 \mathrm{MeV} / \text { nucleon; measured quasielastic, breakup, } \\
\text { and transfer } \sigma(\theta) .{ }^{6} \mathrm{He} \text { deduced two-triton configuration. JOUR } \\
\text { JPGPE } 31 \mathrm{~S} 1647\end{array}$ \\
\hline${ }^{10} \mathrm{~B}$ & 2005CU06 & $\begin{array}{l}\text { NUCLEAR REACTIONS }{ }^{7} \mathrm{Li}\left({ }^{7} \mathrm{Li},{ }^{11} \mathrm{~B}\right),\left({ }^{7} \mathrm{Li},{ }^{12} \mathrm{~B}\right), \mathrm{E}=58 \mathrm{MeV} ;{ }^{12} \mathrm{C} \text {, } \\
{ }^{16} \mathrm{O}\left({ }^{7} \mathrm{Li},{ }^{10} \mathrm{~B}\right), \mathrm{E}=58 \mathrm{MeV} \text {; measured particle spectra. }{ }^{10,11,12} \mathrm{~B} \\
\text { deduced relative yields for } \alpha+\mathrm{Li} \text { and } \mathrm{H}+\text { Be decay channels from } \\
\text { excited states. JOUR PRVCA } 72044320\end{array}$ \\
\hline
\end{tabular}

\section{$\mathrm{A}=11$}

${ }^{11} \mathrm{Li} \quad$ 2005BB01 ATOMIC MASSES ${ }^{11} \mathrm{Li}$; measured mass; deduced two-neutron separation energy. ${ }^{11} \mathrm{Be}$; measured mass. Transmission mass spectrometer. JOUR ZAANE 25 s01 31

${ }^{11} \mathrm{Be} \quad 2005 \mathrm{BB} 01$

ATOMIC MASSES ${ }^{11} \mathrm{Li}$; measured mass; deduced two-neutron separation energy. ${ }^{11} \mathrm{Be}$; measured mass. Transmission mass spectrometer. JOUR ZAANE 25 s01 31

2005PA68 NUCLEAR REACTIONS C $\left({ }^{12} \mathrm{Be}, \mathrm{n}^{11} \mathrm{Be}\right), \mathrm{E}=39.3 \mathrm{MeV} /$ nucleon; measured En, E $\gamma$, projectile-like fragments spectra, relative energy spectra; deduced $\sigma(\mathrm{E}) .{ }^{11} \mathrm{Be}$ deduced excited states. ${ }^{12} \mathrm{Be}$ deduced ground state configuration. Kinematically complete measurement. JOUR ZAANE 25 s01 349

2005PAZV NUCLEAR REACTIONS C $\left({ }^{12} \mathrm{Be},{ }^{11} \mathrm{BeX}\right), \mathrm{E}(\mathrm{cm}) \approx 39.3 \mathrm{MeV}$; measured E $\gamma$, En, (particle) $\gamma-$, (particle)n-coin; deduced one-neutron removal $\sigma(\mathrm{E}) .{ }^{11}$ Be levels deduced spectroscopic factors. ${ }^{12} \mathrm{Be}$ deduced ground-state configuration. PREPRINT nucl-ex/0510048,10/16/2005

2005YE05 NUCLEAR REACTIONS ${ }^{9} \mathrm{Be}\left({ }^{6} \mathrm{He},{ }^{6} \mathrm{He}\right),\left({ }^{6} \mathrm{He},{ }^{5} \mathrm{He}\right),\left({ }^{6} \mathrm{He}, \alpha\right),\left({ }^{6} \mathrm{He}\right.$, $\alpha \mathrm{X}),\left({ }^{6} \mathrm{He}, \mathrm{tX}\right), \mathrm{E}=25 \mathrm{MeV} /$ nucleon; measured quasielastic, breakup, and transfer $\sigma(\theta) .{ }^{6} \mathrm{He}$ deduced two-triton configuration. JOUR JPGPE 31 S1647

${ }^{11} \mathrm{~B} \quad 2005 \mathrm{CU} 06$ NUCLEAR REACTIONS ${ }^{7} \mathrm{Li}\left({ }^{7} \mathrm{Li},{ }^{11} \mathrm{~B}\right),\left({ }^{7} \mathrm{Li},{ }^{12} \mathrm{~B}\right), \mathrm{E}=58 \mathrm{MeV} ;{ }^{12} \mathrm{C}$, ${ }^{16} \mathrm{O}\left({ }^{7} \mathrm{Li},{ }^{10} \mathrm{~B}\right), \mathrm{E}=58 \mathrm{MeV}$; measured particle spectra. ${ }^{10,11,12} \mathrm{~B}$ deduced relative yields for $\alpha+\mathrm{Li}$ and $\mathrm{H}+\mathrm{Be}$ decay channels from excited states. JOUR PRVCA 72044320

2005KAZU NUCLEAR REACTIONS ${ }^{11} \mathrm{~B}(\mathrm{~d}$, d'), $\mathrm{E}=200 \mathrm{MeV}$; measured $\sigma(\mathrm{E}, \theta)$. ${ }^{11} \mathrm{~B}$ levels deduced isoscalar monopole and quadrupole strengths, cluster structure. Comparison with antisymmetrized molecular dynamics model predictions. PREPRINT nucl-ex/0512040,12/25/2005 2005RU18 NUCLEAR REACTIONS ${ }^{7} \operatorname{Li}\left({ }^{11} \mathrm{~B}, \mathrm{X}\right), \mathrm{E}=44 \mathrm{MeV}$; measured particle spectra, charge distributions. ${ }^{7} \mathrm{Li}\left({ }^{11} \mathrm{~B},{ }^{11} \mathrm{~B}\right),\left({ }^{11} \mathrm{~B},{ }^{11} \mathrm{~B}\right), \mathrm{E}=44 \mathrm{MeV}$; measured $\sigma(\mathrm{E}, \theta) ;{ }^{11} \mathrm{~B}\left({ }^{7} \mathrm{Li},{ }^{7} \mathrm{Li}\right),\left({ }^{7} \mathrm{Li},{ }^{7} \mathrm{Li} '\right), \mathrm{E}=34 \mathrm{MeV}$; analyzed $\sigma(\mathrm{E}$, $\theta)$; deduced optical model parameters, transfer channel contributions, reorientation effects. ${ }^{7} \mathrm{Li},{ }^{11} \mathrm{~B}$ deduced deformation parameters. Optical model and coupled-reaction-channels analysis. JOUR PRVCA 72034608 


\section{$\mathrm{A}=11$ (continued)}

2005SH51 NUCLEAR REACTIONS ${ }^{4} \mathrm{He}(\gamma, \mathrm{p}),(\gamma, \mathrm{n}),(\gamma, \mathrm{np}), \mathrm{E}=21.8-29.8 \mathrm{MeV}$; ${ }^{12} \mathrm{C}(\gamma, \mathrm{p}),(\gamma, \mathrm{n}), \mathrm{E}=22.3-32 \mathrm{MeV}$; measured charged particle spectra, photodisintegration $\sigma, \sigma(\theta)$. Monoenergetic pulsed photons, comparison with previous results and model predictions. JOUR PRVCA 72044004

$2005 \mathrm{~S} 013 \quad$ NUCLEAR REACTIONS ${ }^{16} \mathrm{O}\left({ }^{9} \mathrm{Be}, \alpha^{7} \mathrm{Be}\right),{ }^{7} \mathrm{Li}\left({ }^{9} \mathrm{Be}, \alpha^{7} \mathrm{Li}\right),\left({ }^{9} \mathrm{Be}, \mathrm{t} 2 \alpha\right)$, $\mathrm{E}=55,70 \mathrm{MeV}$; measured excitation energy spectra. ${ }^{11} \mathrm{~B},{ }^{11} \mathrm{C}$ deduced excited states energies, configurations. JOUR JPGPE 31 S1701

${ }^{11} \mathrm{C} \quad 2005 \mathrm{SH} 51$ NUCLEAR REACTIONS ${ }^{4} \mathrm{He}(\gamma, \mathrm{p}),(\gamma, \mathrm{n}),(\gamma, \mathrm{np}), \mathrm{E}=21.8-29.8 \mathrm{MeV}$; ${ }^{12} \mathrm{C}(\gamma, \mathrm{p}),(\gamma, \mathrm{n}), \mathrm{E}=22.3-32 \mathrm{MeV}$; measured charged particle spectra, photodisintegration $\sigma, \sigma(\theta)$. Monoenergetic pulsed photons, comparison with previous results and model predictions. JOUR PRVCA 72044004

2005S013 NUCLEAR REACTIONS ${ }^{16} \mathrm{O}\left({ }^{9} \mathrm{Be}, \alpha^{7} \mathrm{Be}\right),{ }^{7} \mathrm{Li}\left({ }^{9} \mathrm{Be}, \alpha^{7} \mathrm{Li}\right),\left({ }^{9} \mathrm{Be}, \mathrm{t} 2 \alpha\right)$, $\mathrm{E}=55,70 \mathrm{MeV}$; measured excitation energy spectra. ${ }^{11} \mathrm{~B},{ }^{11} \mathrm{C}$ deduced excited states energies, configurations. JOUR JPGPE 31 S1701

\section{$\mathrm{A}=12$}

${ }^{12} \mathrm{Be} \quad$ 2005PA68

NUCLEAR REACTIONS C $\left({ }^{12} \mathrm{Be}, \mathrm{n}^{11} \mathrm{Be}\right), \mathrm{E}=39.3 \mathrm{MeV} /$ nucleon; measured En, E $\gamma$, projectile-like fragments spectra, relative energy spectra; deduced $\sigma(\mathrm{E}) .{ }^{11} \mathrm{Be}$ deduced excited states. ${ }^{12} \mathrm{Be}$ deduced ground state configuration. Kinematically complete measurement. JOUR ZAANE 25 s01 349

2005PAZV NUCLEAR REACTIONS C $\left({ }^{12} \mathrm{Be},{ }^{11} \mathrm{BeX}\right), \mathrm{E}(\mathrm{cm}) \approx 39.3 \mathrm{MeV}$; measured E $\gamma$, En, (particle) $\gamma$-, (particle)n-coin; deduced one-neutron removal $\sigma(\mathrm{E}) .{ }^{11}$ Be levels deduced spectroscopic factors. ${ }^{12}$ Be deduced ground-state configuration. PREPRINT nucl-ex/0510048,10/16/2005

\section{${ }^{12} \mathrm{~B} \quad 2005 \mathrm{CU} 06$} NUCLEAR REACTIONS ${ }^{7} \mathrm{Li}\left({ }^{7} \mathrm{Li},{ }^{11} \mathrm{~B}\right),\left({ }^{7} \mathrm{Li},{ }^{12} \mathrm{~B}\right), \mathrm{E}=58 \mathrm{MeV} ;{ }^{12} \mathrm{C}$, ${ }^{16} \mathrm{O}\left({ }^{7} \mathrm{Li},{ }^{10} \mathrm{~B}\right), \mathrm{E}=58 \mathrm{MeV}$; measured particle spectra. ${ }^{10,11,12} \mathrm{~B}$ deduced relative yields for $\alpha+\mathrm{Li}$ and $\mathrm{H}+\mathrm{Be}$ decay channels from excited states. JOUR PRVCA 72044320

2005DI16 RADIOACTIVITY ${ }^{12} \mathrm{~B}\left(\beta^{-}\right),\left(\beta^{-} 3 \alpha\right)$ [from Ta(p, X)]; measured $\beta$-delayed $\mathrm{E} \alpha, \alpha \alpha$-coin. ${ }^{12} \mathrm{C}$ deduced excited states, J, $\pi$. R-matrix analysis. JOUR NUPAB 7603

${ }^{12} \mathrm{C} \quad 2005$ AL37 NUCLEAR REACTIONS ${ }^{12} \mathrm{C}\left({ }^{3} \mathrm{He}, \mathrm{t} \pi^{+}\right), \mathrm{E}=2 \mathrm{GeV}$; measured excitation energy spectra. ${ }^{1} \mathrm{H}\left(\mathrm{d}, \mathrm{d}^{\prime} \mathrm{X}\right),\left(\alpha, \alpha^{\prime} \mathrm{X}\right), \mathrm{E} \approx 1 \mathrm{GeV} /$ nucleon; measured missing mass spectra. JOUR NIMAE 551290

2005DA42 NUCLEAR REACTIONS ${ }^{12} \mathrm{C}\left({ }^{132} \mathrm{Te},{ }^{132} \mathrm{Te}\right),\left({ }^{130} \mathrm{Te},{ }^{130} \mathrm{Te}\right),\left({ }^{126} \mathrm{Te}\right.$, $\left.{ }^{126} \mathrm{Te}^{\prime}\right),\left({ }^{122} \mathrm{Te},{ }^{122} \mathrm{Te}\right.$ '), $\mathrm{E}=3 \mathrm{MeV} /$ nucleon; measured $\mathrm{E} \gamma, \mathrm{I} \gamma(\theta)$, (particle) $\gamma$-coin following projectile Coulomb excitation. ${ }^{132} \mathrm{Te}$ level deduced g-factor. Recoil-in-vacuum technique. JOUR NIMBE 241971

2005DI16 RADIOACTIVITY ${ }^{12} \mathrm{~B}\left(\beta^{-}\right),\left(\beta^{-} 3 \alpha\right)$ [from Ta(p, X)]; measured $\beta$-delayed $\mathrm{E} \alpha, \alpha \alpha$-coin. ${ }^{12} \mathrm{C}$ deduced excited states, J, $\pi$. R-matrix analysis. JOUR NUPAB 7603

2005G036 ATOMIC MASSES ${ }^{12} \mathrm{C},{ }^{16} \mathrm{O},{ }^{20} \mathrm{Ne},{ }^{32} \mathrm{~S},{ }^{36,40} \mathrm{Ar}$; measured masses. Cyclotron-based mass spectrometry. JOUR JPGPE 31 S1869 


\section{$\mathrm{A}=12$ (continued)}

2005GR25 NUCLEAR REACTIONS ${ }^{64} \mathrm{Ni}\left({ }^{132} \mathrm{Sn}, \mathrm{X}\right),\left({ }^{134} \mathrm{Sn}, \mathrm{X}\right), \mathrm{E}=450-620 \mathrm{MeV}$; measured fusion $\sigma . \mathrm{C}\left({ }^{130} \mathrm{Te},{ }^{130} \mathrm{Te}\right),\left({ }^{132} \mathrm{Te},{ }^{132} \mathrm{Te}\right.$ '), $\mathrm{E}=3 \mathrm{MeV} /$ nucleon; measured $\mathrm{E} \gamma, \mathrm{I} \gamma$, (particle) $\gamma$-coin following projectile Coulomb excitation. ${ }^{132} \mathrm{Te}$ level deduced g factor. ${ }^{13} \mathrm{C}\left({ }^{134} \mathrm{Te},{ }^{135} \mathrm{Te}\right), \mathrm{E}=550$ $\mathrm{MeV} ;$ measured $\mathrm{E} \gamma, \mathrm{I} \gamma$. ${ }^{135} \mathrm{Te}$ level deduced J, $\pi$. JOUR JPGPE 31 S1639

2005KN02 RADIOACTIVITY ${ }^{13} \mathrm{O}\left(\beta^{+} \mathrm{p}\right)$ [from ${ }^{14} \mathrm{~N}(\mathrm{p}, 2 \mathrm{n})$ ]; measured $\beta$-delayed $\mathrm{Ep}$, Ip; deduced log ft. ${ }^{13} \mathrm{~N}$ deduced branching ratios for proton decay from excited states. JOUR PRVCA 72044312

$2005 K U 36$ NUCLEAR REACTIONS ${ }^{15} \mathrm{~N}(\mathrm{p}, \alpha \gamma), \mathrm{E} \approx 429,897 \mathrm{keV}$; measured $\gamma$-ray yields for nitrogen in various materials; deduced depth profiles. JOUR NIMBE 240704

2005S014 NUCLEAR REACTIONS ${ }^{12} \mathrm{C}\left({ }^{6} \mathrm{Li}, \mathrm{d} \alpha\right), \mathrm{E}=26 \mathrm{MeV} ;{ }^{59} \mathrm{Co}\left({ }^{6} \mathrm{Li}, \mathrm{d} \alpha\right)$, $\mathrm{E}=30 \mathrm{MeV}$; measured particle spectra, $\sigma(\theta(\alpha), \theta(\mathrm{d}))$, three-body final state correlations; deduced reaction mechanism features. JOUR BJPHE 35888

\section{$\mathrm{A}=13$}

${ }^{13} \mathrm{~B} \quad 2004 \mathrm{GU} 21$

NUCLEAR REACTIONS ${ }^{9} \mathrm{Be}\left({ }^{14} \mathrm{~B},{ }^{13} \mathrm{BX}\right), \mathrm{E}=60 \mathrm{MeV} /$ nucleon; measured $\mathrm{E} \gamma, \mathrm{I} \gamma$, particle momentum distribution, $\sigma(\mathrm{E}) .{ }^{13} \mathrm{~B}$ deduced levels, J, $\pi$, asymptotic normalization coefficients. ${ }^{2} \mathrm{H}\left({ }^{8} \mathrm{~B}, \alpha\right), \mathrm{E}=28.5$ $\mathrm{MeV}$; measured E $\alpha$. JOUR BJPHE 341012

${ }^{13} \mathrm{C} \quad 2005 \mathrm{CU} 06$
NUCLEAR REACTIONS ${ }^{7} \mathrm{Li}\left({ }^{7} \mathrm{Li},{ }^{11} \mathrm{~B}\right),\left({ }^{7} \mathrm{Li},{ }^{12} \mathrm{~B}\right), \mathrm{E}=58 \mathrm{MeV} ;{ }^{12} \mathrm{C}$, ${ }^{16} \mathrm{O}\left({ }^{7} \mathrm{Li},{ }^{10} \mathrm{~B}\right), \mathrm{E}=58 \mathrm{MeV}$; measured particle spectra. ${ }^{10,11,12} \mathrm{~B}$ deduced relative yields for $\alpha+\mathrm{Li}$ and $\mathrm{H}+\mathrm{Be}$ decay channels from excited states. JOUR PRVCA 72044320

${ }^{13} \mathrm{~N} \quad 2005 \mathrm{FE} 11$
NUCLEAR REACTIONS ${ }^{1} \mathrm{H}\left({ }^{12} \mathrm{C}, \gamma\right), \mathrm{E}(\mathrm{cm})=206.8,229.5 \mathrm{keV}$; measured yields. Accelerator mass spectrometry. JOUR NIMBE 240 495

2005KN02 RADIOACTIVITY ${ }^{13} \mathrm{O}\left(\beta^{+} \mathrm{p}\right)$ [from $\left.{ }^{14} \mathrm{~N}(\mathrm{p}, 2 \mathrm{n})\right]$; measured $\beta$-delayed $\mathrm{Ep}$, Ip; deduced log ft. ${ }^{13} \mathrm{~N}$ deduced branching ratios for proton decay from excited states. JOUR PRVCA 72044312

2006LE01 NUCLEAR REACTIONS ${ }^{13} \mathrm{C}(\mathrm{p}, \mathrm{n}), \mathrm{E}=5-30 \mathrm{MeV}$; measured neutron yield. Comparison with previous results. JOUR NIMAE 556397

${ }^{13} \mathrm{O} \quad 2005 \mathrm{KNO} 2$ RADIOACTIVITY ${ }^{13} \mathrm{O}\left(\beta^{+} \mathrm{p}\right)$ [from ${ }^{14} \mathrm{~N}(\mathrm{p}, 2 \mathrm{n})$ ]; measured $\beta$-delayed $\mathrm{Ep}$, Ip; deduced log ft. ${ }^{13} \mathrm{~N}$ deduced branching ratios for proton decay from excited states. JOUR PRVCA 72044312

\section{$\mathrm{A}=14$}

${ }^{14} \mathrm{C} \quad 2005 \mathrm{MC12}$

NUCLEAR REACTIONS ${ }^{12} \mathrm{C}\left({ }^{16} \mathrm{O},{ }^{14} \mathrm{O}\right)$, E not given; measured excitation energy spectra. ${ }^{14} \mathrm{C}$ deduced decay branch widths. JOUR JPGPE 31 S1921

2005NE14 NUCLEAR REACTIONS ${ }^{14} \mathrm{~N}(\mathrm{~d}, 2 \mathrm{p}), \mathrm{E}=170 \mathrm{MeV} ;{ }^{14} \mathrm{~N}\left({ }^{3} \mathrm{He}, \mathrm{t}\right)$, $\mathrm{E}=420 \mathrm{MeV}$; measured excitation energy spectra; deduced isospin symmetry features. JOUR JPGPE 31 S1931 


\section{$\mathrm{A}=14$ (continued)}

2005S013 NUCLEAR REACTIONS ${ }^{16} \mathrm{O}\left({ }^{9} \mathrm{Be}, \alpha^{7} \mathrm{Be}\right),{ }^{7} \mathrm{Li}\left({ }^{9} \mathrm{Be}, \alpha{ }^{7} \mathrm{Li}\right),\left({ }^{9} \mathrm{Be}, \mathrm{t} 2 \alpha\right)$, $\mathrm{E}=55,70 \mathrm{MeV}$; measured excitation energy spectra. ${ }^{11} \mathrm{~B},{ }^{11} \mathrm{C}$ deduced excited states energies, configurations. JOUR JPGPE 31 S1701

${ }^{14} \mathrm{~N} \quad 2005 \mathrm{BL} 23$ NUCLEAR REACTIONS ${ }^{12} \mathrm{C},{ }^{14} \mathrm{~N}\left({ }^{17} \mathrm{~F},{ }^{17} \mathrm{~F}\right), \mathrm{E}=10 \mathrm{MeV} /$ nucleon; measured $\sigma(\theta)$; deduced parameters, reaction mechanism features. Double-folding procedure. JOUR PRVCA 72034606

2005MA92 NUCLEAR REACTIONS ${ }^{13} \mathrm{C}(\mathrm{p}, \gamma), \mathrm{E} \approx 450-680 \mathrm{MeV}$; measured $\mathrm{E} \gamma$, $\mathrm{I} \gamma .{ }^{14} \mathrm{~N}$ deduced resonance width. Monolayer target. JOUR NIMAE 55531

2005RA26 NUCLEAR MOMENTS ${ }^{14} \mathrm{~N}$; measured hfs; deduced parameters. JOUR CHPLB 415161

${ }^{14} \mathrm{O} \quad$ 2005GU25 NUCLEAR REACTIONS ${ }^{1} \mathrm{H}\left({ }^{14} \mathrm{O}, \mathrm{p}\right), \mathrm{E}=120 \mathrm{MeV}$; measured recoil proton spectra, $\sigma(\theta) .{ }^{15} \mathrm{~F}$ deduced resonance energies, J, $\pi$. JOUR PRVCA 72034312

2005NE14 NUCLEAR REACTIONS ${ }^{14} \mathrm{~N}(\mathrm{~d}, 2 \mathrm{p}), \mathrm{E}=170 \mathrm{MeV} ;{ }^{14} \mathrm{~N}\left({ }^{3} \mathrm{He}, \mathrm{t}\right)$, $\mathrm{E}=420 \mathrm{MeV}$; measured excitation energy spectra; deduced isospin symmetry features. JOUR JPGPE 31 S1931

$$
\mathrm{A}=15
$$

${ }^{15} \mathrm{C}$ 2005DA38 NUCLEAR REACTIONS $\mathrm{Pb}\left({ }^{17} \mathrm{C}, \mathrm{n}^{16} \mathrm{C}\right),\left({ }^{23} \mathrm{O}, \mathrm{n}^{22} \mathrm{O}\right), \mathrm{E} \approx 400-600$ $\mathrm{MeV} /$ nucleon; measured $\mathrm{E} \gamma, \mathrm{I} \gamma$, Coulomb dissociation $\sigma \cdot{ }^{14} \mathrm{C}(\mathrm{n}, \gamma)$, $\mathrm{E}(\mathrm{cm})=23 \mathrm{keV}$; deduced capture $\sigma$. JOUR JPGPE $31 \mathrm{~S} 1583$

2005DA43 NUCLEAR REACTIONS $\mathrm{Pb}\left({ }^{17} \mathrm{C}, \mathrm{n}^{16} \mathrm{C}\right),\left({ }^{23} \mathrm{O}, \mathrm{n}^{22} \mathrm{O}\right), \mathrm{E} \approx 400-600$ $\mathrm{MeV} /$ nucleon; measured $\mathrm{E} \gamma, \mathrm{I} \gamma$, Coulomb dissociation $\sigma .{ }^{14} \mathrm{C}(\mathrm{n}, \gamma)$, $\mathrm{E}(\mathrm{cm})=23 \mathrm{keV}$; deduced capture $\sigma$. JOUR ZAANE $25 \mathrm{~s} 01339$

${ }^{15} \mathrm{~N} \quad$ 2005CA42 NUCLEAR MOMENTS ${ }^{2} \mathrm{H},{ }^{15} \mathrm{~N}$; measured hfs; deduced parameters. JOUR APJSA 159181

2005LA28 NUCLEAR REACTIONS ${ }^{2} \mathrm{H}\left({ }^{14} \mathrm{~N}, \mathrm{p}\right), \mathrm{E}=10.6 \mathrm{MeV} /$ nucleon; measured Ep, E $\gamma, \sigma(\theta)$. Comparison with previous results. JOUR JPGPE 31 S1691

${ }^{15} \mathrm{O}$ 2005BB05 NUCLEAR REACTIONS ${ }^{1} \mathrm{H}\left({ }^{18} \mathrm{~F}, \mathrm{p}\right), \mathrm{E}(\mathrm{cm}) \approx 0.3-1.3 \mathrm{MeV}$; measured Ep, $\sigma(\theta)$; deduced excitation functions. ${ }^{19} \mathrm{Ne}$ deduced resonance energies, J, $\pi$, analog states. ${ }^{18} \mathrm{~F}(\mathrm{p}, \alpha),(\mathrm{p}, \gamma), \mathrm{E}=$ low; calculated astrophysical reaction rates. JOUR ZAANE 25 s01 643

2005 Im02 NUCLEAR REACTIONS ${ }^{14} \mathrm{~N}(\mathrm{p}, \gamma)$, E $(\mathrm{cm})=119-367 \mathrm{keV}$; measured $\mathrm{E} \gamma, \mathrm{I} \gamma$, excitation functions; deduced astrophysical S-factors. R-matrix analysis. JOUR ZAANE 25455

2005PR20 NUCLEAR REACTIONS ${ }^{14} \mathrm{~N}(\mathrm{p}, \gamma), \mathrm{E}=$ low; measured $\mathrm{E} \gamma$, I $\gamma$; deduced astrophysical S-factor. Solid and gas targets. JOUR JPGPE 31 S1537

${ }^{15} \mathrm{~F} \quad 2005 \mathrm{GU} 25$
NUCLEAR REACTIONS ${ }^{1} \mathrm{H}\left({ }^{14} \mathrm{O}, \mathrm{p}\right), \mathrm{E}=120 \mathrm{MeV}$; measured recoil proton spectra, $\sigma(\theta) .{ }^{15} \mathrm{~F}$ deduced resonance energies, J, $\pi$. JOUR PRVCA 72034312 


\section{$\mathrm{A}=16$}

\begin{tabular}{|c|c|c|}
\hline${ }^{16} \mathrm{Be}$ & 2004 TH15 & $\begin{array}{l}\text { NUCLEAR REACTIONS Be }\left({ }^{40} \mathrm{Ar}, \mathrm{X}\right), \mathrm{E}=140 \mathrm{MeV} / \text { nucleon; } \\
\text { measured fragment isotopic yields; deduced no evidence for }{ }^{16} \mathrm{Be} \text {. } \\
{ }^{12} \mathrm{C}\left({ }^{24} \mathrm{~F}, \mathrm{X}\right),\left({ }^{25} \mathrm{~F}, \mathrm{X}\right),\left({ }^{26} \mathrm{~F}, \mathrm{X}\right){ }^{20} \mathrm{O} /{ }^{21} \mathrm{O} /{ }^{22} \mathrm{O} /{ }^{23} \mathrm{O} /{ }^{24} \mathrm{O}, \mathrm{E} \approx 50 \\
\mathrm{MeV} / \text { nucleon; measured yields; deduced no evidence for }{ }^{25} \mathrm{O} \text {. JOUR } \\
\text { APHPF } 21379\end{array}$ \\
\hline \multirow[t]{2}{*}{${ }^{16} \mathrm{C}$} & 2005B039 & $\begin{array}{l}\text { NUCLEAR REACTIONS }{ }^{13,14} \mathrm{C}\left({ }^{12} \mathrm{C},{ }^{9} \mathrm{C}\right), \mathrm{E}=231 \mathrm{MeV} \text {; measured } \\
\text { excitation energy spectra. }{ }^{16,17} \mathrm{C} \text { deduced levels, J, } \pi \text {, configurations. } \\
\text { JOUR JPGPE } 31 \text { S1461 }\end{array}$ \\
\hline & 20050N04 & $\begin{array}{l}\text { NUCLEAR REACTIONS }{ }^{1} \mathrm{H}\left({ }^{16} \mathrm{C},{ }^{16} \mathrm{C}^{\prime}\right), \mathrm{E}=33 \mathrm{MeV} / \text { nucleon; } \\
\text { measured } \mathrm{E} \gamma, \mathrm{I} \gamma \text {, (particle) } \gamma \text {-coin; deduced } \sigma .{ }^{16} \mathrm{C} \text { deduced } \\
\text { deformation parameter. JOUR ZAANE } 25 \mathrm{~s} 01347\end{array}$ \\
\hline \multirow[t]{3}{*}{${ }^{16} \mathrm{O}$} & $2005 \mathrm{G0} 36$ & $\begin{array}{l}\text { ATOMIC MASSES }{ }^{12} \mathrm{C},{ }^{16} \mathrm{O},{ }^{20} \mathrm{Ne},{ }^{32} \mathrm{~S},{ }^{36,40} \mathrm{Ar} \text {; measured masses. } \\
\text { Cyclotron-based mass spectrometry. JOUR JPGPE } 31 \mathrm{~S} 1869\end{array}$ \\
\hline & $2005 \mathrm{SC} 29$ & $\begin{array}{l}\text { NUCLEAR REACTIONS }{ }^{4} \mathrm{He}\left({ }^{12} \mathrm{C}, \gamma\right), \mathrm{E}=0.7-5.0 \mathrm{MeV} \text {; measured total } \\
\text { recoil spectra, } \sigma .{ }^{12} \mathrm{C}(\alpha, \gamma), \mathrm{E}=1.9-4.9 \mathrm{MeV} \text {; deduced astrophysical } \\
\text { S-factors. JOUR ZAANE } 26301\end{array}$ \\
\hline & $2005 \mathrm{SCZT}$ & $\begin{array}{l}\text { NUCLEAR REACTIONS }{ }^{4} \mathrm{He}\left({ }^{12} \mathrm{C}, \gamma\right), \mathrm{E} \text { not given; measured recoil } \\
\text { particle spectra. }{ }^{12} \mathrm{C}(\alpha, \gamma), \mathrm{E}=1.9-4.9 \mathrm{MeV} \text {; deduced astrophysical } \\
\text { S-factors. resonance features. PREPRINT nucl-ex } / 0511050.11 / 29 / 2005\end{array}$ \\
\hline
\end{tabular}

\section{$\mathrm{A}=17$}

$\begin{array}{ll}{ }^{17} \mathrm{C} & \text { 2005B039 } \\ { }^{17} \mathrm{O} & \text { 2005DE54 } \\ { }^{17} \mathrm{~F} & \text { 2005AN24 } \\ { }^{17} \mathrm{Ne} & \text { 2005KA51 }\end{array}$

2005TA33

NUCLEAR REACTIONS ${ }^{13,14} \mathrm{C}\left({ }^{12} \mathrm{C},{ }^{9} \mathrm{C}\right), \mathrm{E}=231 \mathrm{MeV}$; measured excitation energy spectra. ${ }^{16,17} \mathrm{C}$ deduced levels, $\mathrm{J}, \pi$, configurations. JOUR JPGPE 31 S1461 NUCLEAR REACTIONS ${ }^{14} \mathrm{~N}(\alpha, \mathrm{p}), \mathrm{E}=4893-6047 \mathrm{keV}$; measured $\sigma\left(\theta=172^{\circ}\right)$. Application to nitrogen depth profiling discussed. JOUR NIMBE 240803

NUCLEAR REACTIONS ${ }^{14} \mathrm{~N},{ }^{12} \mathrm{C},{ }^{16} \mathrm{O}(\mathrm{d}, \mathrm{n})$, E not given; measured activation yields in plasma focus device. JOUR ARISE 63545

NUCLEAR REACTIONS Be $\left({ }^{17} \mathrm{Ne},{ }^{15} \mathrm{OX}\right), \mathrm{E}=64 \mathrm{MeV} /$ nucleon; measured fragments longitudinal momentum distributions, interaction $\sigma .{ }^{17} \mathrm{Ne}$ deduced two-proton halo features. Few-body Glauber model analysis. JOUR ZAANE 25 s01 327

NUCLEAR REACTIONS ${ }^{9} \mathrm{Be},{ }^{12} \mathrm{C},{ }^{27} \mathrm{Al}\left({ }^{17} \mathrm{Ne}, \mathrm{X}\right), \mathrm{E}=42,62 \mathrm{MeV} /$ nucleon; measured interaction and reaction $\sigma .{ }^{17} \mathrm{Ne}$ deduced matter density distribution. JOUR ZAANE 25 s01 221

\section{$\mathrm{A}=18$}

${ }^{18} \mathrm{O}$ 2005N013 NUCLEAR REACTIONS ${ }^{2} \mathrm{H},{ }^{3,4} \mathrm{He},{ }^{6,7} \mathrm{Li},{ }^{9} \mathrm{Be},{ }^{10,11} \mathrm{~B},{ }^{16} \mathrm{O}$, ${ }^{19} \mathrm{~F}$ (polarized p, 2p), E=392 MeV; measured analyzing powers. Comparison with model predictions. JOUR PRVCA 72041602

${ }^{18} \mathrm{~F} \quad 2005 \mathrm{BB} 05 \quad$ NUCLEAR REACTIONS ${ }^{1} \mathrm{H}\left({ }^{18} \mathrm{~F}, \mathrm{p}\right), \mathrm{E}(\mathrm{cm}) \approx 0.3-1.3 \mathrm{MeV}$; measured Ep, $\sigma(\theta)$; deduced excitation functions. ${ }^{19} \mathrm{Ne}$ deduced resonance energies, $\mathrm{J}, \pi$, analog states. ${ }^{18} \mathrm{~F}(\mathrm{p}, \alpha),(\mathrm{p}, \gamma), \mathrm{E}=$ low; calculated astrophysical reaction rates. JOUR ZAANE 25 s01 643 


\section{$\mathrm{A}=19$}

${ }^{19} \mathrm{O}$ 2005KO43 NUCLEAR REACTIONS U(p, X) ${ }^{19} \mathrm{O} /{ }^{20} \mathrm{O} /{ }^{21} \mathrm{O} /{ }^{22} \mathrm{O}, \mathrm{E}=1.4 \mathrm{GeV}$; measured yields. JOUR ZAANE 25 s01 729

${ }^{19} \mathrm{Ne} \quad 2005 \mathrm{BB} 05 \quad$ NUCLEAR REACTIONS ${ }^{1} \mathrm{H}\left({ }^{18} \mathrm{~F}, \mathrm{p}\right), \mathrm{E}(\mathrm{cm}) \approx 0.3-1.3 \mathrm{MeV}$; measured Ep, $\sigma(\theta)$; deduced excitation functions. ${ }^{19} \mathrm{Ne}$ deduced resonance energies, J, $\pi$, analog states. ${ }^{18} \mathrm{~F}(\mathrm{p}, \alpha),(\mathrm{p}, \gamma), \mathrm{E}=$ low; calculated astrophysical reaction rates. JOUR ZAANE 25 s01 643

2005TA28 NUCLEAR REACTIONS ${ }^{17} \mathrm{O}\left({ }^{3} \mathrm{He}, \mathrm{n}\right), \mathrm{E}=3.0 \mathrm{MeV}$; measured $\mathrm{E} \gamma$, I $\gamma$, $\mathrm{n} \gamma$-coin, DSA. ${ }^{19} \mathrm{Ne}$ levels deduced energies, $\mathrm{T}_{1 / 2}$. Astrophysical implications discussed. Comparison with model predictions. JOUR PRVCA 72041302

\section{$\mathrm{A}=\mathbf{2 0}$}

${ }^{20} \mathrm{O} \quad 2004 \mathrm{TH} 15$

NUCLEAR REACTIONS Be $\left({ }^{40} \mathrm{Ar}, \mathrm{X}\right), \mathrm{E}=140 \mathrm{MeV} /$ nucleon; measured fragment isotopic yields; deduced no evidence for ${ }^{16} \mathrm{Be}$. ${ }^{12} \mathrm{C}\left({ }^{24} \mathrm{~F}, \mathrm{X}\right),\left({ }^{25} \mathrm{~F}, \mathrm{X}\right),\left({ }^{26} \mathrm{~F}, \mathrm{X}\right){ }^{20} \mathrm{O} /{ }^{21} \mathrm{O} /{ }^{22} \mathrm{O} /{ }^{23} \mathrm{O} /{ }^{24} \mathrm{O}, \mathrm{E} \approx 50$ $\mathrm{MeV} /$ nucleon; measured yields; deduced no evidence for ${ }^{25} \mathrm{O}$. JOUR APHPF 21379

2005K043 NUCLEAR REACTIONS U(p, X) ${ }^{19} \mathrm{O} /{ }^{20} \mathrm{O} /{ }^{21} \mathrm{O} /{ }^{22} \mathrm{O}, \mathrm{E}=1.4 \mathrm{GeV}$; measured yields. JOUR ZAANE 25 s01 729

2005BB06 NUCLEAR REACTIONS ${ }^{12} \mathrm{C}\left({ }^{12} \mathrm{C}, \mathrm{X}\right), \mathrm{E}=5.3-7 \mathrm{MeV}$; measured $\mathrm{E} \gamma$, I $\gamma$, thick-target yields. ${ }^{12} \mathrm{C}\left({ }^{12} \mathrm{C}, \mathrm{p}\right),\left({ }^{12} \mathrm{C}, \alpha\right), \mathrm{E}=5.3-7 \mathrm{MeV}$; deduced $\sigma$. Astrophysical implications discussed. JOUR ZAANE 25 s01 645 2005G036 ATOMIC MASSES ${ }^{12} \mathrm{C},{ }^{16} \mathrm{O},{ }^{20} \mathrm{Ne},{ }^{32} \mathrm{~S},{ }^{36,40} \mathrm{Ar}$; measured masses. Cyclotron-based mass spectrometry. JOUR JPGPE 31 S1869

2005 IL03 NUCLEAR REACTIONS ${ }^{23} \mathrm{Na}(\mathrm{p}, \gamma),(\mathrm{p}, \alpha), \mathrm{E}=130-155 \mathrm{keV}$; measured $\mathrm{E} \gamma, \mathrm{I} \gamma$; deduced resonance strength upper limits, astrophysical reaction rates. JOUR JPGPE 31 S1785

\section{$\mathrm{A}=\mathbf{2 1}$}

${ }^{21} \mathrm{O} \quad 2004 \mathrm{TH} 15$

NUCLEAR REACTIONS Be $\left({ }^{40} \mathrm{Ar}, \mathrm{X}\right), \mathrm{E}=140 \mathrm{MeV} /$ nucleon; measured fragment isotopic yields; deduced no evidence for ${ }^{16} \mathrm{Be}$. ${ }^{12} \mathrm{C}\left({ }^{24} \mathrm{~F}, \mathrm{X}\right),\left({ }^{25} \mathrm{~F}, \mathrm{X}\right),\left({ }^{26} \mathrm{~F}, \mathrm{X}\right){ }^{20} \mathrm{O} /{ }^{21} \mathrm{O} /{ }^{22} \mathrm{O} /{ }^{23} \mathrm{O} /{ }^{24} \mathrm{O}, \mathrm{E} \approx 50$ $\mathrm{MeV} /$ nucleon; measured yields; deduced no evidence for ${ }^{25} \mathrm{O}$. JOUR APHPF 21379

2005K043 NUCLEAR REACTIONS U(p, X) ${ }^{19} \mathrm{O} /{ }^{20} \mathrm{O} /{ }^{21} \mathrm{O} /{ }^{22} \mathrm{O}, \mathrm{E}=1.4 \mathrm{GeV}$; measured yields. JOUR ZAANE 25 s01 729

\section{$\mathrm{A}=22$}

${ }^{22} \mathrm{O} \quad 2004 \mathrm{TH} 15$

NUCLEAR REACTIONS Be $\left({ }^{40} \mathrm{Ar}, \mathrm{X}\right), \mathrm{E}=140 \mathrm{MeV} /$ nucleon; measured fragment isotopic yields; deduced no evidence for ${ }^{16} \mathrm{Be}$. ${ }^{12} \mathrm{C}\left({ }^{24} \mathrm{~F}, \mathrm{X}\right),\left({ }^{25} \mathrm{~F}, \mathrm{X}\right),\left({ }^{26} \mathrm{~F}, \mathrm{X}\right){ }^{20} \mathrm{O} /{ }^{21} \mathrm{O} /{ }^{22} \mathrm{O} /{ }^{23} \mathrm{O} /{ }^{24} \mathrm{O}, \mathrm{E} \approx 50$ $\mathrm{MeV} /$ nucleon; measured yields; deduced no evidence for ${ }^{25} \mathrm{O}$. JOUR APHPF 21379 


\section{$\mathrm{A}=22$ (continued)}

2005KO43 NUCLEAR REACTIONS U(p, X) ${ }^{19} \mathrm{O} /{ }^{20} \mathrm{O} /{ }^{21} \mathrm{O} /{ }^{22} \mathrm{O}, \mathrm{E}=1.4 \mathrm{GeV}$; measured yields. JOUR ZAANE 25 s01 729

${ }^{22} \mathrm{Ne} \quad$ 2005KE08

2005KE11

NUCLEAR REACTIONS ${ }^{150} \mathrm{Nd}\left({ }^{26} \mathrm{Ne}, \mathrm{X}\right){ }^{22} \mathrm{Ne} /{ }^{23} \mathrm{Na} /{ }^{28} \mathrm{Mg}, \mathrm{E}=160$ $\mathrm{MeV}$; measured $\mathrm{E} \gamma, \mathrm{I} \gamma, \gamma \gamma-$, (particle) $\gamma$-coin. ${ }^{22} \mathrm{Ne},{ }^{23} \mathrm{Na},{ }^{28} \mathrm{Mg}$ deduced levels, J, $\pi$. Euroball IV array, fragment separator. JOUR JPGPE 31 S1903

NUCLEAR REACTIONS ${ }^{150} \mathrm{Nd}\left({ }^{26} \mathrm{Mg}, \mathrm{X}\right), \mathrm{E}=160 \mathrm{MeV}$; measured $\mathrm{E} \gamma$, $\mathrm{I} \gamma, \gamma \gamma$-, (particle) $\gamma$-coin. ${ }^{22} \mathrm{Ne},{ }^{23} \mathrm{Na}$ deduced levels, J, $\pi$. Euroball IV array, binary reaction spectrometer. JOUR ZAANE 25 s01 431

$$
\mathrm{A}=23
$$

${ }^{23} \mathrm{O} \quad 2004 \mathrm{TH} 15$

$2005 C 024$

${ }^{23} \mathrm{~F}$

2005 MI32

2005MIZT

$2005 \mathrm{SH} 46$

${ }^{23} \mathrm{Na} \quad 2005 \mathrm{BB} 06$

2005KE08

2005KE11

${ }^{23} \mathrm{Al} \quad 2005 \mathrm{GO34}$
NUCLEAR REACTIONS Be $\left({ }^{40} \mathrm{Ar}, \mathrm{X}\right), \mathrm{E}=140 \mathrm{MeV} /$ nucleon; measured fragment isotopic yields; deduced no evidence for ${ }^{16} \mathrm{Be}$. ${ }^{12} \mathrm{C}\left({ }^{24} \mathrm{~F}, \mathrm{X}\right),\left({ }^{25} \mathrm{~F}, \mathrm{X}\right),\left({ }^{26} \mathrm{~F}, \mathrm{X}\right){ }^{20} \mathrm{O} /{ }^{21} \mathrm{O} /{ }^{22} \mathrm{O} /{ }^{23} \mathrm{O} /{ }^{24} \mathrm{O}, \mathrm{E} \approx 50$ $\mathrm{MeV} /$ nucleon; measured yields; deduced no evidence for ${ }^{25} \mathrm{O}$. JOUR APHPF 21379 NUCLEAR REACTIONS C $\left({ }^{23} \mathrm{O},{ }^{22} \mathrm{OX}\right), \mathrm{E}=938 \mathrm{MeV} /$ nucleon; measured longitudinal momentum distributions, one-neutron removal $\sigma .{ }^{23} \mathrm{O}$ deduced ground-state $\mathrm{J}, \pi$, configuration. JOUR ZAANE 25 s01 343 NUCLEAR REACTIONS ${ }^{4} \mathrm{He}\left({ }^{22} \mathrm{O},{ }^{23} \mathrm{~F}\right), \mathrm{E}=35 \mathrm{MeV} /$ nucleon; nucleon; measured $\mathrm{E} \gamma, \mathrm{I} \gamma$, (particle) $\gamma-, \gamma \gamma$-coin; deduced $\sigma(\mathrm{E}) .{ }^{23} \mathrm{~F}$ deduced levels, J, $\pi$. DWBA analysis. JOUR ZAANE 25 s01 367 NUCLEAR REACTIONS ${ }^{4} \mathrm{He}\left({ }^{22} \mathrm{O},{ }^{23} \mathrm{~F}\right),\left({ }^{23} \mathrm{~F},{ }^{23} \mathrm{~F}^{\prime}\right),\left({ }^{24} \mathrm{~F},{ }^{23} \mathrm{~F}\right)$, $\left({ }^{25} \mathrm{Ne},{ }^{23} \mathrm{~F}\right), \mathrm{E} \approx 35-43 \mathrm{MeV} /$ nucleon; measured $\mathrm{E} \gamma, \mathrm{I} \gamma$, (particle) $\gamma-$, $\gamma \gamma$-coin. ${ }^{4} \mathrm{He}\left({ }^{22} \mathrm{O},{ }^{23} \mathrm{~F}\right), \mathrm{E}=35 \mathrm{MeV} /$ nucleon; measured $\sigma(\theta) \cdot{ }^{23} \mathrm{~F}$ NUCLEAR REACTIONS ${ }^{4} \mathrm{He}\left({ }^{22} \mathrm{O},{ }^{23} \mathrm{~F}\right), \mathrm{E}=35 \mathrm{MeV} /$ nucleon; measured $\mathrm{E} \gamma, \mathrm{I} \gamma$, (particle) $\gamma$-coin, $\sigma(\theta) .{ }^{23} \mathrm{~F}$ deduced levels, $\mathrm{J}, \pi$. JOUR JPGPE 31 S1759

NUCLEAR REACTIONS ${ }^{12} \mathrm{C}\left({ }^{12} \mathrm{C}, \mathrm{X}\right), \mathrm{E}=5.3-7 \mathrm{MeV}$; measured $\mathrm{E} \gamma$, $\mathrm{I} \gamma$, thick-target yields. ${ }^{12} \mathrm{C}\left({ }^{12} \mathrm{C}, \mathrm{p}\right),\left({ }^{12} \mathrm{C}, \alpha\right), \mathrm{E}=5.3-7 \mathrm{MeV}$; deduced $\sigma$. Astrophysical implications discussed. JOUR ZAANE 25 s01 645 NUCLEAR REACTIONS ${ }^{150} \mathrm{Nd}\left({ }^{26} \mathrm{Ne}, \mathrm{X}\right){ }^{22} \mathrm{Ne} /{ }^{23} \mathrm{Na} /{ }^{28} \mathrm{Mg}, \mathrm{E}=160$ $\mathrm{MeV}$; measured $\mathrm{E} \gamma, \mathrm{I} \gamma, \gamma \gamma$-, (particle) $\gamma$-coin. ${ }^{22} \mathrm{Ne},{ }^{23} \mathrm{Na},{ }^{28} \mathrm{Mg}$ deduced levels, J, $\pi$. Euroball IV array, fragment separator. JOUR JPGPE 31 S1903

NUCLEAR REACTIONS ${ }^{150} \mathrm{Nd}\left({ }^{26} \mathrm{Mg}, \mathrm{X}\right), \mathrm{E}=160 \mathrm{MeV}$; measured $\mathrm{E} \gamma$, $\mathrm{I} \gamma, \gamma \gamma$-, (particle) $\gamma$-coin. ${ }^{22} \mathrm{Ne},{ }^{23} \mathrm{Na}$ deduced levels, J, $\pi$. Euroball IV array, binary reaction spectrometer. JOUR ZAANE 25 s01 431 NUCLEAR REACTIONS ${ }^{208} \mathrm{~Pb}\left({ }^{23} \mathrm{Al}, \mathrm{p}^{22} \mathrm{Mg}\right), \mathrm{E}=50 \mathrm{MeV} /$ nucleon; measured relative energy spectrum, $\sigma(\theta) .{ }^{23} \mathrm{Al}$ deduced excited state radiative width. Astrophysical implications discussed. JOUR JPGPE 31 S1517 ${ }^{4} \mathrm{He}\left({ }^{23} \mathrm{~F},{ }^{23} \mathrm{~F}^{\prime}\right), \mathrm{E}=41.5 \mathrm{MeV} /$ nucleon; ${ }^{4} \mathrm{He}\left({ }^{24} \mathrm{~F},{ }^{23} \mathrm{~F}\right), \mathrm{E}=36 \mathrm{MeV} /$ deduced levels, J, $\pi$, configurations. REPT CNS-REP-67,Michimasa 


\section{$\mathrm{A}=24$}

$\begin{array}{lll}{ }^{24} \mathrm{O} & \text { 2004TH15 } & \text { NUCLEAR REACTIONS } \mathrm{Be}\left({ }^{40} \mathrm{Ar}, \mathrm{X}\right), \mathrm{E}=140 \mathrm{MeV} / \text { nucleon; } \\ & \text { measured fragment isotopic yields; deduced no evidence for }{ }^{16} \mathrm{Be} . \\ & & { }^{12} \mathrm{C}\left({ }^{24} \mathrm{~F}, \mathrm{X}\right),\left({ }^{25} \mathrm{~F}, \mathrm{X}\right),\left({ }^{26} \mathrm{~F}, \mathrm{X}\right)^{20} \mathrm{O} /{ }^{21} \mathrm{O} /{ }^{22} \mathrm{O} /{ }^{23} \mathrm{O} /{ }^{24} \mathrm{O}, \mathrm{E} \approx 50 \\ & \mathrm{MeV} / \text { nucleon; measured yields; deduced no evidence for }{ }^{25} \mathrm{O} . \mathrm{JOUR} \\ & \text { APHPF } 21379 \\ & \\ & \text { NUCLEAR REACTIONS }{ }^{23} \mathrm{Na}(\mathrm{p}, \gamma),(\mathrm{p}, \alpha), \mathrm{E}=130-155 \mathrm{keV} ; \\ & \text { measured } \mathrm{E} \gamma, \mathrm{I} \gamma ; \text { deduced resonance strength upper limits, } \\ & \text { astrophysical reaction rates. JOUR JPGPE } 31 \text { S1785 }\end{array}$

$$
\mathrm{A}=25
$$

${ }^{25} \mathrm{O}$ 2004TH15 NUCLEAR REACTIONS Be $\left({ }^{40} \mathrm{Ar}, \mathrm{X}\right), \mathrm{E}=140 \mathrm{MeV} /$ nucleon; measured fragment isotopic yields; deduced no evidence for ${ }^{16} \mathrm{Be}$. ${ }^{12} \mathrm{C}\left({ }^{24} \mathrm{~F}, \mathrm{X}\right),\left({ }^{25} \mathrm{~F}, \mathrm{X}\right),\left({ }^{26} \mathrm{~F}, \mathrm{X}\right){ }^{20} \mathrm{O} /{ }^{21} \mathrm{O} /{ }^{22} \mathrm{O} /{ }^{23} \mathrm{O} /{ }^{24} \mathrm{O}, \mathrm{E} \approx 50$ $\mathrm{MeV} /$ nucleon; measured yields; deduced no evidence for ${ }^{25} \mathrm{O}$. JOUR APHPF 21379

${ }^{25} \mathrm{Ne} \quad 2005 \mathrm{BE} 60$ NUCLEAR REACTIONS ${ }^{9} \mathrm{Be}, \mathrm{C}\left({ }^{36} \mathrm{~S}, \mathrm{X}\right){ }^{25} \mathrm{Ne} /{ }^{26} \mathrm{Ne} /{ }^{27} \mathrm{Ne} /{ }^{28} \mathrm{Ne}$, $\mathrm{E}=77.5 \mathrm{MeV} /$ nucleon; measured $\mathrm{E} \gamma, \mathrm{I} \gamma, \gamma \gamma$-coin. ${ }^{26,28} \mathrm{Ne}$ deduced levels, J, $\pi .{ }^{27,29} \mathrm{Ne}$ deduced excited states. Comparison with shell model predictions. JOUR PRVCA 72054316

2005CA44 NUCLEAR REACTIONS ${ }^{2} \mathrm{H}\left({ }^{24} \mathrm{Ne}, \mathrm{p}\right), \mathrm{E}=10 \mathrm{MeV} /$ nucleon; measured Ep, E $\gamma$, p $\gamma$-coin, $\sigma(\theta) .{ }^{25}$ Ne deduced levels, J, $\pi$. JOUR JPGPE 31 S1655

2005CA50 NUCLEAR REACTIONS ${ }^{2} \mathrm{H}\left({ }^{24} \mathrm{Ne}, \mathrm{p}\right), \mathrm{E}=10 \mathrm{MeV} /$ nucleon; measured Ep, E $\gamma$, p $\gamma$-coin, $\sigma(\theta) .{ }^{25}$ Ne deduced levels, J, $\pi$. JOUR ZAANE 25 s01 245

\section{$\mathrm{A}=\mathbf{2 6}$}

\begin{tabular}{|c|c|c|}
\hline${ }^{26} \mathrm{O}$ & $2005 \mathrm{SC} 20$ & $\begin{array}{l}\text { NUCLEAR REACTIONS } \mathrm{C}\left({ }^{27} \mathrm{~F}, \mathrm{X}\right),\left({ }^{29} \mathrm{Ne}, \mathrm{X}\right), \mathrm{E} \approx 90 \mathrm{MeV} / \\
\text { nucleon; measured fragment yields, production } \sigma \text { upper limits; deduced } \\
\text { no evidence for }{ }^{26} \mathrm{O},{ }^{28} \mathrm{~F} \text {. JOUR PRVCA } 72037601\end{array}$ \\
\hline${ }^{26} \mathrm{Ne}$ & 2005BE60 & $\begin{array}{l}\text { NUCLEAR REACTIONS }{ }^{9} \mathrm{Be}, \mathrm{C}\left({ }^{36} \mathrm{~S}, \mathrm{X}\right){ }^{25} \mathrm{Ne} /{ }^{26} \mathrm{Ne} /{ }^{27} \mathrm{Ne} /{ }^{28} \mathrm{Ne} \text {, } \\
\mathrm{E}=77.5 \mathrm{MeV} / \text { nucleon; measured } \mathrm{E} \gamma, \mathrm{I} \gamma, \gamma \gamma \text {-coin. }{ }^{26,28} \mathrm{Ne} \text { deduced } \\
\text { levels, J, } \pi .{ }^{27,29} \mathrm{Ne} \text { deduced excited states. Comparison with shell } \\
\text { model predictions. JOUR PRVCA } 72054316\end{array}$ \\
\hline & 2005GAZT & $\begin{array}{l}\text { ATOMIC MASSES }{ }^{26} \mathrm{Ne},{ }^{26,27,28,29,30} \mathrm{Na},{ }^{29,30,31,32,33} \mathrm{Mg} ; \text { measured } \\
\text { masses. Reanalysis of data using new calibration. PREPRINT } \\
\text { nucl-ex/0511007,11/2/2005 }\end{array}$ \\
\hline${ }^{26} \mathrm{Na}$ & 2005GAZT & $\begin{array}{l}\text { ATOMIC MASSES }{ }^{26} \mathrm{Ne},{ }^{26,27,28,29,30} \mathrm{Na},{ }^{29,30,31,32,33} \mathrm{Mg} ; \text { measured } \\
\text { masses. Reanalysis of data using new calibration. PREPRINT } \\
\text { nucl-ex/0511007,11/2/2005 }\end{array}$ \\
\hline & 2005 WI20 & $\begin{array}{l}\text { RADIOACTIVITY }{ }^{26} \mathrm{Na}\left(\beta^{-}\right) ;{ }^{152} \mathrm{Eu}\left(\beta^{-}\right) \text {, (EC); measured } \mathrm{E} \gamma, \mathrm{I} \gamma, \gamma \gamma-\text {, } \\
\beta \gamma \text {-coin. }{ }^{152} \mathrm{Sm} \text { level deduced } \mathrm{T}_{1 / 2} \text {. JOUR JPGPE } 31 \mathrm{~S} 1979\end{array}$ \\
\hline
\end{tabular}




\section{$\mathrm{A}=\mathbf{2 6}$ (continued)}

2005ZEZZ NUCLEAR REACTIONS ${ }^{26} \mathrm{Mg}\left({ }^{3} \mathrm{He}, \mathrm{t}\right), \mathrm{E}=140 \mathrm{MeV} /$ nucleon; ${ }^{26} \mathrm{Mg}\left(\mathrm{t},{ }^{3} \mathrm{He}\right), \mathrm{E}=115 \mathrm{MeV}$ / nucleon; measured excitation energy spectra, $\sigma(\theta)$; deduced Gamow-Teller strengths. PREPRINT nucl-ex/0512025,12/20/2005

${ }^{26} \mathrm{Mg}$ 2005BE61 NUCLEAR REACTIONS ${ }^{26} \mathrm{Mg}\left({ }^{76} \mathrm{Kr},{ }^{76} \mathrm{Kr}\right.$ '), E=230 MeV; measured $\mathrm{E} \gamma, \mathrm{I} \gamma(\theta, \mathrm{H}, \mathrm{t})$, (particle) $\gamma$-coin following projectile Coulomb excitation. ${ }^{76} \mathrm{Kr}$ level deduced $\mathrm{g}$ factor. Transient field technique. JOUR ZAANE 25 s01 203

2005CH66 NUCLEAR REACTIONS ${ }^{209} \mathrm{Bi}\left({ }^{26} \mathrm{Mg},{ }^{26} \mathrm{Mg}\right.$ '), $\mathrm{E}=78.6 \mathrm{MeV}$ / nucleon; ${ }^{197} \mathrm{Au}\left({ }^{32} \mathrm{Mg},{ }^{32} \mathrm{Mg}\right.$ '), E=81.1 MeV / nucleon; ${ }^{209} \mathrm{Bi}\left({ }^{34} \mathrm{Mg},{ }^{34} \mathrm{Mg}{ }^{\prime}\right)$, $\mathrm{E}=76.4 \mathrm{MeV} /$ nucleon; measured $\mathrm{E} \gamma, \mathrm{I} \gamma$, (particle) $\gamma$-coin following projectile Coulomb excitation. ${ }^{26,32,34} \mathrm{Mg}$ deduced transitions $\mathrm{B}(\mathrm{E} 2)$, deformation parameters. Comparison with previous work, model predictions. JOUR PRVCA 72054320

2005WI20 RADIOACTIVITY ${ }^{26} \mathrm{Na}\left(\beta^{-}\right) ;{ }^{152} \mathrm{Eu}\left(\beta^{-}\right)$, (EC); measured $\mathrm{E} \gamma, \mathrm{I} \gamma, \gamma \gamma-$, $\beta \gamma$-coin. ${ }^{152} \mathrm{Sm}$ level deduced $\mathrm{T}_{1 / 2}$. JOUR JPGPE $31 \mathrm{~S} 1979$

${ }^{26} \mathrm{Al}$ 2005HE24 NUCLEAR REACTIONS ${ }^{14} \mathrm{~N}\left({ }^{16} \mathrm{O}, \alpha\right), \mathrm{E}(\mathrm{cm})=6.6,7.9,9.5 \mathrm{MeV}$; measured $\sigma$. Accelerator mass spectrometry. JOUR NIMBE 240612

2005ZEZZ NUCLEAR REACTIONS ${ }^{26} \mathrm{Mg}\left({ }^{3} \mathrm{He}, \mathrm{t}\right), \mathrm{E}=140 \mathrm{MeV} /$ nucleon; ${ }^{26} \mathrm{Mg}\left(\mathrm{t},{ }^{3} \mathrm{He}\right), \mathrm{E}=115 \mathrm{MeV} /$ nucleon; measured excitation energy spectra, $\sigma(\theta)$; deduced Gamow-Teller strengths. PREPRINT nucl-ex/0512025,12/20/2005

\section{$\mathrm{A}=\mathbf{2 7}$}

${ }^{27} \mathrm{Ne} \quad 2005 \mathrm{BE} 60$

NUCLEAR REACTIONS ${ }^{9} \mathrm{Be}, \mathrm{C}\left({ }^{36} \mathrm{~S}, \mathrm{X}\right){ }^{25} \mathrm{Ne} /{ }^{26} \mathrm{Ne} /{ }^{27} \mathrm{Ne} /{ }^{28} \mathrm{Ne}$, $\mathrm{E}=77.5 \mathrm{MeV} /$ nucleon; measured $\mathrm{E} \gamma, \mathrm{I} \gamma, \gamma \gamma$-coin. ${ }^{26,28} \mathrm{Ne}$ deduced levels, J, $\pi .{ }^{27,29} \mathrm{Ne}$ deduced excited states. Comparison with shell model predictions. JOUR PRVCA 72054316

${ }^{27} \mathrm{Na} \quad$ 2005GAZT ATOMIC MASSES ${ }^{26} \mathrm{Ne},{ }^{26,27,28,29,30} \mathrm{Na},{ }^{29,30,31,32,33} \mathrm{Mg}$; measured masses. Reanalysis of data using new calibration. PREPRINT nucl-ex/0511007,11/2/2005

${ }^{27} \mathrm{Mg} \quad 2005 \mathrm{SCZV}$

NUCLEAR REACTIONS ${ }^{9} \mathrm{Be}\left({ }^{26} \mathrm{Mg},{ }^{27} \mathrm{Mg}\right), \mathrm{E}=57 \mathrm{MeV}$; measured $\mathrm{E} \gamma$, $\mathrm{I} \gamma, \alpha \alpha$-coin, $\sigma(\theta) .{ }^{27} \mathrm{Mg}$ deduced transitions. REPT MLL 2004 Annual,P4,Schwerdtfeger

\section{$\mathrm{A}=\mathbf{2 8}$}

$\begin{array}{ll}{ }^{28} \mathrm{~F} & 2005 \mathrm{SC} 20 \\ { }^{28} \mathrm{Ne} & 2005 \mathrm{BE} 60\end{array}$

NUCLEAR REACTIONS C $\left({ }^{27} \mathrm{~F}, \mathrm{X}\right),\left({ }^{29} \mathrm{Ne}, \mathrm{X}\right), \mathrm{E} \approx 90 \mathrm{MeV} /$ nucleon; measured fragment yields, production $\sigma$ upper limits; deduced no evidence for ${ }^{26} \mathrm{O},{ }^{28} \mathrm{~F}$. JOUR PRVCA 72037601

NUCLEAR REACTIONS ${ }^{9} \mathrm{Be}, \mathrm{C}\left({ }^{36} \mathrm{~S}, \mathrm{X}\right){ }^{25} \mathrm{Ne} /{ }^{26} \mathrm{Ne} /{ }^{27} \mathrm{Ne} /{ }^{28} \mathrm{Ne}$, $\mathrm{E}=77.5 \mathrm{MeV} /$ nucleon; measured $\mathrm{E} \gamma, \mathrm{I} \gamma, \gamma \gamma$-coin. ${ }^{26,28} \mathrm{Ne}$ deduced levels, J, $\pi .{ }^{27,29} \mathrm{Ne}$ deduced excited states. Comparison with shell model predictions. JOUR PRVCA 72054316 


\section{$\mathrm{A}=\mathbf{2 8}$ (continued)}

\begin{tabular}{|c|c|c|}
\hline${ }^{28} \mathrm{Na}$ & 2005GAZT & $\begin{array}{l}\text { ATOMIC MASSES }{ }^{26} \mathrm{Ne},{ }^{26,27,28,29,30} \mathrm{Na},{ }^{29,30,31,32,33} \mathrm{Mg} \text {; measured } \\
\text { masses. Reanalysis of data using new calibration. PREPRINT }\end{array}$ \\
\hline${ }^{28} \mathrm{Mg}$ & 2005KE08 & $\begin{array}{l}\text { NUCLEAR REACTIONS }{ }^{150} \mathrm{Nd}\left({ }^{26} \mathrm{Ne}, \mathrm{X}\right){ }^{22} \mathrm{Ne} /{ }^{23} \mathrm{Na} /{ }^{28} \mathrm{Mg}, \mathrm{E}=160 \\
\mathrm{MeV} ; \text { measured } \mathrm{E} \gamma, \mathrm{I} \gamma, \gamma \gamma-,(\text { particle }) \gamma \text {-coin. }{ }^{22} \mathrm{Ne},{ }^{23} \mathrm{Na},{ }^{28} \mathrm{Mg} \\
\text { deduced levels, J, } \pi \text {. Euroball IV array, fragment separator. JOUR } \\
\text { JPGPE } 31 \mathrm{~S} 1903\end{array}$ \\
\hline${ }^{28} \mathrm{Si}$ & 2004MB08 & $\begin{array}{l}\text { NUCLEAR REACTIONS }{ }^{28} \mathrm{Si}\left({ }^{16} \mathrm{O},{ }^{16} \mathrm{O}^{\prime}\right), \mathrm{E}=40-46,71,73,75 \mathrm{MeV} \text {; } \\
\text { measured } \mathrm{E} \gamma, \mathrm{I} \gamma \text {, (particle) } \gamma \text {-coin, } \sigma(\theta) \text {. Gammasphere, Chico arrays. } \\
\text { JOUR BJPHE } 34885\end{array}$ \\
\hline
\end{tabular}

\section{$A=29$}

${ }^{29} \mathrm{Ne} \quad 2005 \mathrm{BE} 60 \quad$ NUCLEAR REACTIONS ${ }^{9} \mathrm{Be}, \mathrm{C}\left({ }^{36} \mathrm{~S}, \mathrm{X}\right){ }^{25} \mathrm{Ne} /{ }^{26} \mathrm{Ne} /{ }^{27} \mathrm{Ne} /{ }^{28} \mathrm{Ne}$, $\mathrm{E}=77.5 \mathrm{MeV} /$ nucleon; measured $\mathrm{E} \gamma, \mathrm{I} \gamma, \gamma \gamma$-coin. ${ }^{26,28} \mathrm{Ne}$ deduced levels, J, $\pi .{ }^{27,29} \mathrm{Ne}$ deduced excited states. Comparison with shell model predictions. JOUR PRVCA 72054316

2005TR13 RADIOACTIVITY ${ }^{29} \mathrm{Ne}\left(\beta^{-}\right)$[from $\mathrm{Be}\left({ }^{48} \mathrm{Ca}, \mathrm{X}\right)$ ]; measured $\mathrm{E} \gamma, \mathrm{E} \beta$, $\gamma \gamma-, \beta \gamma$-coin; deduced log ft. ${ }^{29} \mathrm{Na}$ deduced levels, $\beta$-feeding intensities. Comparison with shell model calculations. JOUR ZAANE 25 s01 101

${ }^{29} \mathrm{Na} \quad$ 2005GAZT ATOMIC MASSES ${ }^{26} \mathrm{Ne},{ }^{26,27,28,29,30} \mathrm{Na},{ }^{29,30,31,32,33} \mathrm{Mg}$; measured masses. Reanalysis of data using new calibration. PREPRINT nucl-ex/0511007,11/2/2005

2005TR13 RADIOACTIVITY ${ }^{29} \mathrm{Ne}\left(\beta^{-}\right)\left[\right.$from $\left.\mathrm{Be}\left({ }^{48} \mathrm{Ca}, \mathrm{X}\right)\right]$; measured $\mathrm{E} \gamma, \mathrm{E} \beta$, $\gamma \gamma-, \beta \gamma$-coin; deduced log ft. ${ }^{29} \mathrm{Na}$ deduced levels, $\beta$-feeding intensities. Comparison with shell model calculations. JOUR ZAANE 25 s01 101

${ }^{29} \mathrm{Mg} \quad$ 2005GAZT ATOMIC MASSES ${ }^{26} \mathrm{Ne},{ }^{26,27,28,29,30} \mathrm{Na},{ }^{29,30,31,32,33} \mathrm{Mg}$; measured masses. Reanalysis of data using new calibration. PREPRINT nucl-ex/0511007,11/2/2005

\section{$\mathrm{A}=\mathbf{3 0}$}

\begin{tabular}{|c|c|c|}
\hline \multirow[t]{2}{*}{${ }^{30} \mathrm{Na}$} & 2005GAZT & $\begin{array}{l}\text { ATOMIC MASSES }{ }^{26} \mathrm{Ne},{ }^{26,27,28,29,30} \mathrm{Na},{ }^{29,30,31,32,33} \mathrm{Mg} \text {; measured } \\
\text { masses. Reanalysis of data using new calibration. PREPRINT } \\
\text { nucl-ex/0511007,11/2/2005 }\end{array}$ \\
\hline & 2005MA96 & $\begin{array}{l}\text { RADIOACTIVITY }{ }^{30,31,32} \mathrm{Na}\left(\beta^{-}\right) ;{ }^{31,32} \mathrm{Na}\left(\beta^{-} \mathrm{n}\right) ; \text { measured } \mathrm{E} \gamma, \mathrm{I} \gamma \\
\gamma \gamma-, \beta \gamma \text {-coin. } \\
\text { techniques. JOUR ZAM } \mathrm{Mg} \text { deduced levels } \mathrm{T}_{1 / 2} \text {. Ultra-fast timing } 25 \text { s01 } 105\end{array}$ \\
\hline${ }^{30} \mathrm{Mg}$ & $2005 \mathrm{GAZT}$ & $\begin{array}{l}\text { ATOMIC MASSES }{ }^{26} \mathrm{Ne},{ }^{26,27,28,29,30} \mathrm{Na},{ }^{29,30,31,32,33} \mathrm{Mg} \text {; measured } \\
\text { masses. Reanalysis of data using new calibration. PREPRINT } \\
\text { nucl-ex/0511007,11/2/2005 }\end{array}$ \\
\hline & 2005MA96 & $\begin{array}{l}\text { RADIOACTIVITY }{ }^{30,31,32} \mathrm{Na}\left(\beta^{-}\right) ;{ }^{31,32} \mathrm{Na}\left(\beta^{-} \mathrm{n}\right) ; \text { measured } \mathrm{E} \gamma, \mathrm{I} \gamma \\
\gamma \gamma-, \beta \gamma \text {-coin. } \\
\text { techniques. JOUR ZAA } \mathrm{Mg} \text { deduced levels } \mathrm{T}_{1 / 2} \text {. Ultra-fast timing } 25 \mathrm{~s} 01105\end{array}$ \\
\hline
\end{tabular}




\section{$\mathrm{A}=30$ (continued)}

2005SC27 NUCLEAR REACTIONS Ni $\left({ }^{30} \mathrm{Mg},{ }^{30} \mathrm{Mg}\right.$ '), $\mathrm{E}=2.2 \mathrm{MeV} /$ nucleon; measured $\mathrm{E} \gamma, \mathrm{I} \gamma$, (particle) $\gamma$-coin following projectile Coulomb excitation. ${ }^{2}$ Hmeasured $\mathrm{E} \gamma, \mathrm{I} \gamma, \gamma \gamma$-, (particle) $\gamma$-coin. ${ }^{30} \mathrm{Mg}$ deduced transitions $\mathrm{B}(\mathrm{E} 2) .{ }^{31} \mathrm{Mg}$ deduced transitions. Miniball array. JOUR ZAANE 25 s01 397

${ }^{30} \mathrm{P} \quad 2005$ AD35 $\quad$ NUCLEAR REACTIONS ${ }^{6} \mathrm{Li}\left({ }^{6} \mathrm{Li}, \alpha \mathrm{X}\right),\left({ }^{7} \mathrm{Li}, \alpha \mathrm{X}\right), \mathrm{E}=14-20 \mathrm{MeV}$; measured $\alpha$-spectra. ${ }^{12} \mathrm{C}(\mathrm{n}, \alpha), \mathrm{E}=72.8 \mathrm{MeV} ;{ }^{28} \mathrm{Si}\left({ }^{6} \mathrm{Li}, \alpha\right), \mathrm{E}=36 \mathrm{MeV}$; analyzed $\alpha$-spectra. Statistical model calculations. Target-projectile symmetry discussed. JOUR ZAANE 25 s01 299

2005KA46 RADIOACTIVITY ${ }^{31} \mathrm{Cl}\left(\beta^{+} \mathrm{p}\right)$ [from $\mathrm{S}(\mathrm{p}, \mathrm{X}), \mathrm{E}=40 \mathrm{MeV}$; ; measured $\beta$-delayed $\mathrm{E} \gamma, \mathrm{Ep} .{ }^{58} \mathrm{Zn}\left(\beta^{+}\right)$[from $\mathrm{Nb}(\mathrm{p}, \mathrm{X}), \mathrm{E}=1.4 \mathrm{GeV}$ ]; measured $\mathrm{E} \gamma, \mathrm{I} \gamma, \beta \gamma$-coin, $\mathrm{T}_{1 / 2} .{ }^{58} \mathrm{Cu}$ deduced levels, $\beta$-feeding intensities. ${ }^{81 m} \mathrm{Kr}(\mathrm{EC}),(\mathrm{IT}) ;{ }^{81} \mathrm{Y},{ }^{81} \mathrm{Sr},{ }^{85} \mathrm{Nb},{ }^{85} \mathrm{Zr},{ }^{86} \mathrm{Mo},{ }^{86} \mathrm{Nb}(\mathrm{EC})$ [from Ni, $\left.{ }^{54} \mathrm{Fe}\left({ }^{32} \mathrm{~S}, \mathrm{X}\right)\right]$; measured $\mathrm{E} \gamma, \mathrm{I} \gamma, \mathrm{E}(\mathrm{ce}), \mathrm{I}(\mathrm{ce}), \mathrm{T}_{1 / 2} \cdot{ }^{81} \mathrm{Kr},{ }^{85} \mathrm{Zr},{ }^{85} \mathrm{Nb}$ deduced isomeric transitions $\mathrm{T}_{1 / 2}$, ICC. ${ }^{85} \mathrm{Zr},{ }^{86} \mathrm{Nb}$ deduced levels, J, $\pi$, ICC. ${ }^{81} \mathrm{Br}$ deduced neutrino capture rate. Mass-separated sources. JOUR ZAANE 25 s01 129

\section{$\mathrm{A}=31$}

$\begin{array}{ll}{ }^{31} \mathrm{Na} & \text { 2005MA96 } \\ { }^{31} \mathrm{Mg} & \text { 2005GAZT }\end{array}$

2005K041

2005MA86

2005MA96

2005SC27

${ }^{31} \mathrm{Al} \quad 2005 \mathrm{KO} 41$

2005MA86
RADIOACTIVITY ${ }^{30,31,32} \mathrm{Na}\left(\beta^{-}\right) ;{ }^{31,32} \mathrm{Na}\left(\beta^{-}\right.$n); measured $\mathrm{E} \gamma, \mathrm{I} \gamma$, $\gamma \gamma-, \beta \gamma$-coin. ${ }^{30,31,32} \mathrm{Mg}$ deduced levels $\mathrm{T}_{1 / 2}$. Ultra-fast timing techniques. JOUR ZAANE 25 s01 105 ATOMIC MASSES ${ }^{26} \mathrm{Ne},{ }^{26,27,28,29,30} \mathrm{Na},{ }^{29,30,31,32,33} \mathrm{Mg}$; measured masses. Reanalysis of data using new calibration. PREPRINT nucl-ex/0511007,11/2/2005

RADIOACTIVITY ${ }^{31} \mathrm{Mg}\left(\beta^{-}\right)$[from $\mathrm{U}(\mathrm{p}, \mathrm{X})$ ]; measured $\beta$-asymmetry and hfs, $\beta$-NMR spectra from polarized source. ${ }^{31} \mathrm{Mg}$ deduced ground-state $\mathrm{J}, \pi, \mu$. JOUR ZAANE 25 s01 193

RADIOACTIVITY ${ }^{31} \mathrm{Mg}\left(\beta^{-}\right)$[from $\mathrm{Be}\left({ }^{36} \mathrm{~S}, \mathrm{X}\right)$ ]; measured $\mathrm{E} \gamma, \mathrm{I} \gamma$, $\beta \gamma$-coin, $\mathrm{T}_{1 / 2}$; deduced log ft. ${ }^{31} \mathrm{Al}$ deduced levels, feeding intensities. ${ }^{31} \mathrm{Mg}$ deduced ground-state intruder configuration. JOUR PRVCA 72 044314

RADIOACTIVITY ${ }^{30,31,32} \mathrm{Na}\left(\beta^{-}\right) ;{ }^{31,32} \mathrm{Na}\left(\beta^{-} \mathrm{n}\right)$; measured $\mathrm{E} \gamma, \mathrm{I} \gamma$, $\gamma \gamma$-, $\beta \gamma$-coin. ${ }^{30,31,32} \mathrm{Mg}$ deduced levels $\mathrm{T}_{1 / 2}$. Ultra-fast timing techniques. JOUR ZAANE 25 s01 105 NUCLEAR REACTIONS Ni $\left({ }^{30} \mathrm{Mg},{ }^{30} \mathrm{Mg}\right.$ '), $\mathrm{E}=2.2 \mathrm{MeV} /$ nucleon; measured $\mathrm{E} \gamma, \mathrm{I} \gamma$, (particle) $\gamma$-coin following projectile Coulomb excitation. ${ }^{2}$ Hmeasured $\mathrm{E} \gamma, \mathrm{I} \gamma, \gamma \gamma-$, (particle) $\gamma$-coin. ${ }^{30} \mathrm{Mg}$ deduced transitions $\mathrm{B}(\mathrm{E} 2) .{ }^{31} \mathrm{Mg}$ deduced transitions. Miniball array. JOUR ZAANE 25 s01 397

RADIOACTIVITY ${ }^{31} \mathrm{Mg}\left(\beta^{-}\right)$[from $\mathrm{U}(\mathrm{p}, \mathrm{X})$ ]; measured $\beta$-asymmetry and hfs, $\beta$-NMR spectra from polarized source. ${ }^{31} \mathrm{Mg}$ deduced ground-state $\mathrm{J}, \pi, \mu$. JOUR ZAANE 25 s01 193

RADIOACTIVITY ${ }^{31} \mathrm{Mg}\left(\beta^{-}\right)$[from $\mathrm{Be}\left({ }^{36} \mathrm{~S}, \mathrm{X}\right)$ ]; measured $\mathrm{E} \gamma, \mathrm{I} \gamma$, $\beta \gamma$-coin, $\mathrm{T}_{1 / 2}$; deduced $\log \mathrm{ft} .{ }^{31} \mathrm{Al}$ deduced levels, feeding intensities.

${ }^{31} \mathrm{Mg}$ deduced ground-state intruder configuration. JOUR PRVCA 72 044314 


\section{$\mathrm{A}=31$ (continued)}

\begin{tabular}{|c|c|c|}
\hline${ }^{1} \mathrm{P}$ & 2005JE07 & $\begin{array}{l}\text { NUCLEAR REACTIONS }{ }^{12} \mathrm{C}\left({ }^{20} \mathrm{Ne}, \mathrm{p}\right),\left({ }^{20} \mathrm{Ne}, \mathrm{n}\right), \mathrm{E}=32 \mathrm{MeV} \text {; } \\
\text { measured } \mathrm{E} \gamma, \mathrm{I} \gamma, \gamma \gamma-,(\text { recoil }) \gamma \text {-coin. }{ }^{31} \mathrm{~S},{ }^{31} \mathrm{P} \text { deduced high-spin levels, } \\
\text { J, } \pi \text {. Gammasphere array, fragment mass analyzer. JOUR PRVCA } 72 \\
031303\end{array}$ \\
\hline${ }^{31} \mathrm{~S}$ & 2005 GA54 & $\begin{array}{l}\text { NUCLEAR REACTIONS }{ }^{9} \mathrm{Be}\left({ }^{32} \mathrm{~S},{ }^{31} \mathrm{SX}\right),\left({ }^{33} \mathrm{Cl},{ }^{32} \mathrm{ClX}\right),\left({ }^{32} \mathrm{Ar} \text {, }\right. \\
\left.{ }^{31} \mathrm{ArX}\right),\left({ }^{34} \mathrm{Ar},{ }^{33} \mathrm{ArX}\right), \mathrm{E} \approx 65 \mathrm{MeV} / \text { nucleon; measured } \mathrm{E} \gamma, \mathrm{I} \gamma, \\
\text { (particle) } \gamma \text {-coin, particle momentum distributions; deduced one-neutron } \\
\text { removal } \sigma .{ }^{31} \mathrm{~S},{ }^{32} \mathrm{Cl},{ }^{31,33} \mathrm{Ar} \text { levels deduced spectroscopic factors. } \\
\text { Comparison with shell model predictions. JOUR ZAANE } 25 \text { s01 } 251\end{array}$ \\
\hline & 2005JE07 & $\begin{array}{l}\text { NUCLEAR REACTIONS }{ }^{12} \mathrm{C}\left({ }^{20} \mathrm{Ne}, \mathrm{p}\right),\left({ }^{20} \mathrm{Ne}, \mathrm{n}\right), \mathrm{E}=32 \mathrm{MeV} ; \\
\text { measured } \mathrm{E} \gamma, \mathrm{I} \gamma, \gamma \gamma-,(\text { recoil }) \gamma \text {-coin. }{ }^{31} \mathrm{~S},{ }^{11} \mathrm{P} \text { deduced high-spin levels, } \\
\mathrm{J}, \pi \text {. Gammasphere array, fragment mass analyzer. JOUR PRVCA } 72 \\
031303\end{array}$ \\
\hline $1 \mathrm{Cl}$ & $2005 \mathrm{KA} 46$ & $\begin{array}{l}\left.\text { RADIOACTIVITY }{ }^{31} \mathrm{Cl}\left(\beta^{+} \mathrm{p}\right) \text { [from } \mathrm{S}(\mathrm{p}, \mathrm{X}), \mathrm{E}=40 \mathrm{MeV}\right] ; \text { measured } \\
\left.\beta \text {-delayed E } \gamma, \mathrm{Ep} .{ }^{58} \mathrm{Zn}\left(\beta^{+}\right) \text {[from } \mathrm{Nb}(\mathrm{p}, \mathrm{X}), \mathrm{E}=1.4 \mathrm{GeV}\right] ; \text { measured } \\
\mathrm{E} \gamma, \mathrm{I} \gamma, \beta \gamma \text {-coin, } \mathrm{T}_{1 / 2} \cdot{ }^{58} \mathrm{Cu} \text { deduced levels, } \beta \text {-feeding intensities. } \\
{ }^{81 m} \mathrm{Kr}(\mathrm{EC}),(\mathrm{IT}) ;{ }^{81} \mathrm{Y},{ }^{81} \mathrm{Sr},{ }^{85} \mathrm{Nb},{ }^{85} \mathrm{Zr},{ }^{86} \mathrm{Mo},{ }^{86} \mathrm{Nb}(\mathrm{EC})[\text { from Ni, } \\
\left.{ }^{54} \mathrm{Fe}\left({ }^{32} \mathrm{~S}, \mathrm{X}\right)\right] ; \text { measured } \mathrm{E} \gamma, \mathrm{I} \gamma, \mathrm{E}(\mathrm{ce}), \mathrm{I}(\mathrm{ce}), \mathrm{T}_{1 / 2} \cdot{ }^{81} \mathrm{Kr},{ }^{85} \mathrm{Zr},{ }^{85} \mathrm{Nb} \\
\text { deduced isomeric transitions } \mathrm{T}_{1 / 2}, \mathrm{ICC} .{ }^{85} \mathrm{Zr},{ }^{86} \mathrm{Nb} \text { deduced levels, J, } \\
\pi, \text { ICC. }{ }^{81} \mathrm{Br} \text { deduced neutrino capture rate. Mass-separated sources. } \\
\text { JOUR ZAANE } 25 \text { s01 } 129\end{array}$ \\
\hline 1 & $2005 \mathrm{GA} 54$ & $\begin{array}{l}\text { NUCLEAR REACTIONS }{ }^{9} \mathrm{Be}\left({ }^{32} \mathrm{~S},{ }^{31} \mathrm{SX}\right),\left({ }^{33} \mathrm{Cl},{ }^{32} \mathrm{ClX}\right),\left({ }^{32} \mathrm{Ar} \text {, }\right. \\
\left.{ }^{31} \mathrm{ArX}\right),\left({ }^{34} \mathrm{Ar},{ }^{33} \mathrm{ArX}\right), \mathrm{E} \approx 65 \mathrm{MeV} / \text { nucleon; measured } \mathrm{E} \gamma, \mathrm{I} \gamma \text {, } \\
\text { (particle) } \gamma \text {-coin, particle momentum distributions; deduced one-neutron } \\
\text { removal } \sigma .{ }^{31} \mathrm{~S},{ }^{32} \mathrm{Cl},{ }^{31,33} \mathrm{Ar} \text { levels deduced spectroscopic factors. } \\
\text { Comparison with shell model predictions. JOUR ZAANE } 25 \text { s01 } 251\end{array}$ \\
\hline
\end{tabular}

\section{$\mathrm{A}=32$}

${ }^{32} \mathrm{Na} \quad$ 2005MA81

2005MA96

${ }^{32} \mathrm{Mg} \quad 2004 \mathrm{CO} 29$

2005 CH66
RADIOACTIVITY ${ }^{32} \mathrm{Na},{ }^{80} \mathrm{Ga}\left(\beta^{-}\right)$; measured $\mathrm{E} \gamma, \mathrm{I} \gamma, \gamma \gamma-, \beta \gamma$-coin. ${ }^{32} \mathrm{Mg},{ }^{80} \mathrm{Ge}$ levels deduced $\mathrm{T}_{1 / 2}$. Ultra-fast timing techniques. JOUR JPGPE 31 S1421

RADIOACTIVITY ${ }^{30,31,32} \mathrm{Na}\left(\beta^{-}\right) ;{ }^{31,32} \mathrm{Na}\left(\beta^{-} \mathrm{n}\right)$; measured $\mathrm{E} \gamma, \mathrm{I} \gamma$, $\gamma \gamma$-, $\beta \gamma$-coin. ${ }^{30,31,32} \mathrm{Mg}$ deduced levels $\mathrm{T}_{1 / 2}$. Ultra-fast timing techniques. JOUR ZAANE $25 \mathrm{~s} 01105$ RADIOACTIVITY ${ }^{74} \mathrm{Kr}(\mathrm{EC}),\left(\beta^{+}\right)$[from $\mathrm{Nb}(\mathrm{p}, \mathrm{X})$ ]; measured $\beta \gamma$-coin; deduced Gamow-Teller strength distribution. ${ }^{33} \mathrm{Na}\left(\beta^{-}\right)$, $\left(\beta^{-} \mathrm{n}\right)$ [from $\mathrm{U}(\mathrm{p}, \mathrm{X})$ ]; measured $\beta \gamma-, \mathrm{n} \beta-, \gamma \gamma$-coin, $\mathrm{T}_{1 / 2} .{ }^{33} \mathrm{Mg}$ deduced ground-state $\mathrm{J}, \pi$. Total absorption spectrometer. JOUR BJPHE 34 850

NUCLEAR REACTIONS ${ }^{209} \mathrm{Bi}\left({ }^{26} \mathrm{Mg},{ }^{26} \mathrm{Mg}\right.$ '), E=78.6 MeV / nucleon; ${ }^{197} \mathrm{Au}\left({ }^{32} \mathrm{Mg},{ }^{32} \mathrm{Mg}\right.$ '), E=81.1 MeV / nucleon; ${ }^{209} \mathrm{Bi}\left({ }^{34} \mathrm{Mg},{ }^{34} \mathrm{Mg}\right)$, $\mathrm{E}=76.4 \mathrm{MeV} /$ nucleon; measured $\mathrm{E} \gamma, \mathrm{I} \gamma$, (particle) $\gamma$-coin following projectile Coulomb excitation. ${ }^{26,32,34} \mathrm{Mg}$ deduced transitions $\mathrm{B}(\mathrm{E} 2)$, deformation parameters. Comparison with previous work, model predictions. JOUR PRVCA 72054320 


\section{$\mathrm{A}=32$ (continued)}

2005GAZT ATOMIC MASSES ${ }^{26} \mathrm{Ne},{ }^{26,27,28,29,30} \mathrm{Na},{ }^{29,30,31,32,33} \mathrm{Mg}$; measured masses. Reanalysis of data using new calibration. PREPRINT nucl-ex/0511007,11/2/2005

2005MA81 RADIOACTIVITY ${ }^{32} \mathrm{Na},{ }^{80} \mathrm{Ga}\left(\beta^{-}\right)$; measured $\mathrm{E} \gamma, \mathrm{I} \gamma, \gamma \gamma-, \beta \gamma$-coin. ${ }^{32} \mathrm{Mg},{ }^{80} \mathrm{Ge}$ levels deduced $\mathrm{T}_{1 / 2}$. Ultra-fast timing techniques. JOUR JPGPE 31 S1421

2005MA96 RADIOACTIVITY ${ }^{30,31,32} \mathrm{Na}\left(\beta^{-}\right) ;{ }^{31,32} \mathrm{Na}\left(\beta^{-} \mathrm{n}\right)$; measured $\mathrm{E} \gamma$, I $\gamma$, $\gamma \gamma-, \beta \gamma$-coin. ${ }^{30,31,32} \mathrm{Mg}$ deduced levels $\mathrm{T}_{1 / 2}$. Ultra-fast timing techniques. JOUR ZAANE 25 s01 105

${ }^{32} \mathrm{~S} \quad 2005 \mathrm{GO} 36 \quad$ ATOMIC MASSES ${ }^{12} \mathrm{C},{ }^{16} \mathrm{O},{ }^{20} \mathrm{Ne},{ }^{32} \mathrm{~S},{ }^{36,40} \mathrm{Ar}$; measured masses. Cyclotron-based mass spectrometry. JOUR JPGPE 31 S1869

2005GA54 NUCLEAR REACTIONS ${ }^{9} \mathrm{Be}\left({ }^{32} \mathrm{Sl},{ }^{31} \mathrm{SX}\right),\left({ }^{33} \mathrm{Cl},{ }^{32} \mathrm{ClX}\right),\left({ }^{32} \mathrm{Ar}\right.$, $\left.{ }^{31} \mathrm{ArX}\right),\left({ }^{34} \mathrm{Ar},{ }^{33} \mathrm{ArX}\right), \mathrm{E} \approx 65 \mathrm{MeV} /$ nucleon; measured $\mathrm{E} \gamma, \mathrm{I} \gamma$, (particle) $\gamma$-coin, particle momentum distributions; deduced one-neutron removal $\sigma \cdot{ }^{31} \mathrm{~S},{ }^{32} \mathrm{Cl},{ }^{31,33} \mathrm{Ar}$ levels deduced spectroscopic factors. Comparison with shell model predictions. JOUR ZAANE 25 s01 251

\section{$\mathrm{A}=33$}

${ }^{33} \mathrm{Na} \quad 2004 \mathrm{CO} 29$

RADIOACTIVITY ${ }^{74} \mathrm{Kr}(\mathrm{EC}),\left(\beta^{+}\right)$[from $\mathrm{Nb}(\mathrm{p}, \mathrm{X})$ ]; measured $\beta \gamma$-coin; deduced Gamow-Teller strength distribution. ${ }^{33} \mathrm{Na}\left(\beta^{-}\right)$, $\left(\beta^{-} \mathrm{n}\right)$ [from $\mathrm{U}(\mathrm{p}, \mathrm{X})$ ]; measured $\beta \gamma-, \mathrm{n} \beta-, \gamma \gamma$-coin, $\mathrm{T}_{1 / 2} .{ }^{33} \mathrm{Mg}$ deduced ground-state $\mathrm{J}, \pi$. Total absorption spectrometer. JOUR BJPHE 34 850

${ }^{33} \mathrm{Mg} \quad$ 2004CO29 RADIOACTIVITY ${ }^{74} \mathrm{Kr}(\mathrm{EC}),\left(\beta^{+}\right)$[from $\left.\mathrm{Nb}(\mathrm{p}, \mathrm{X})\right]$; measured $\beta \gamma$-coin; deduced Gamow-Teller strength distribution. ${ }^{33} \mathrm{Na}\left(\beta^{-}\right)$, $\left(\beta^{-} \mathrm{n}\right)$ [from $\left.\mathrm{U}(\mathrm{p}, \mathrm{X})\right]$; measured $\beta \gamma-, \mathrm{n} \beta-, \gamma \gamma-$ coin, $\mathrm{T}_{1 / 2} .{ }^{33} \mathrm{Mg}$ deduced ground-state $\mathrm{J}, \pi$. Total absorption spectrometer. JOUR BJPHE 34 850

2005GAZT ATOMIC MASSES ${ }^{26} \mathrm{Ne},{ }^{26,27,28,29,30} \mathrm{Na},{ }^{29,30,31,32,33} \mathrm{Mg}$; measured masses. Reanalysis of data using new calibration. PREPRINT nucl-ex/0511007,11/2/2005

${ }^{33} \mathrm{Ar}$ 2005GA54 NUCLEAR REACTIONS ${ }^{9} \mathrm{Be}\left({ }^{32} \mathrm{~S},{ }^{31} \mathrm{SX}\right),\left({ }^{33} \mathrm{Cl},{ }^{32} \mathrm{ClX}\right),\left({ }^{32} \mathrm{Ar}\right.$, $\left.{ }^{31} \mathrm{ArX}\right),\left({ }^{34} \mathrm{Ar},{ }^{33} \mathrm{ArX}\right), \mathrm{E} \approx 65 \mathrm{MeV} /$ nucleon; measured $\mathrm{E} \gamma, \mathrm{I} \gamma$, (particle) $\gamma$-coin, particle momentum distributions; deduced one-neutron removal $\sigma \cdot{ }^{31} \mathrm{~S},{ }^{32} \mathrm{Cl},{ }^{31,33} \mathrm{Ar}$ levels deduced spectroscopic factors. Comparison with shell model predictions. JOUR ZAANE 25 s01 251

\section{$\mathrm{A}=34$}

${ }^{34} \mathrm{Mg} \quad 2005 \mathrm{CH} 66$

NUCLEAR REACTIONS ${ }^{209} \mathrm{Bi}\left({ }^{26} \mathrm{Mg},{ }^{26} \mathrm{Mg}\right.$ '), $\mathrm{E}=78.6 \mathrm{MeV} /$ nucleon; ${ }^{197} \mathrm{Au}\left({ }^{32} \mathrm{Mg},{ }^{32} \mathrm{Mg}\right.$ '), E=81.1 MeV / nucleon; ${ }^{209} \mathrm{Bi}\left({ }^{34} \mathrm{Mg},{ }^{34} \mathrm{Mg}\right.$ '), $\mathrm{E}=76.4 \mathrm{MeV} /$ nucleon; measured $\mathrm{E} \gamma, \mathrm{I} \gamma$, (particle) $\gamma$-coin following projectile Coulomb excitation. ${ }^{26,32,34} \mathrm{Mg}$ deduced transitions $\mathrm{B}(\mathrm{E} 2)$, deformation parameters. Comparison with previous work, model predictions. JOUR PRVCA 72054320 


\section{$\mathrm{A}=34$ (continued)}

\begin{tabular}{|c|c|c|}
\hline${ }^{34} \mathrm{Al}$ & 20050B04 & $\begin{array}{l}\text { NUCLEAR REACTIONS }{ }^{251} \mathrm{Cf}(\mathrm{n}, \mathrm{F}), \mathrm{E}=\text { thermal; measured light } \\
\text { charged particle yields, energy distributions following ternary fission; } \\
\text { deduced evidence for }{ }^{34} \mathrm{Al},{ }^{36} \mathrm{Si} \text {. JOUR NUPAB } 761173\end{array}$ \\
\hline${ }^{34} \mathrm{Si}$ & 2005 TI11 & $\begin{array}{l}\text { RADIOACTIVITY }{ }^{35} \mathrm{Al}\left(\beta^{-}\right),\left(\beta^{-} \mathrm{n}\right)\left[\text { from }{ }^{36} \mathrm{~S} \text { fragmentation]; }\right. \\
\text { measured } \beta \text {-delayed } \mathrm{E} \gamma, \mathrm{En}, \mathrm{T}_{1 / 2}, \text { neutron emission probability; } \\
\text { deduced log ft. }{ }^{34,35} \mathrm{Si} \text { deduced levels, J, } \pi \text {, feeding intensities. JOUR } \\
\text { JPGPE } 31 \mathrm{~S} 1965\end{array}$ \\
\hline 2 & 20050L04 & $\begin{array}{l}\text { NUCLEAR REACTIONS }{ }^{176} \mathrm{Yb}\left({ }^{36} \mathrm{~S}, \mathrm{X}\right){ }^{34} \mathrm{P}, \mathrm{E}=230 \mathrm{MeV} ;{ }^{208} \mathrm{~Pb}\left({ }^{36} \mathrm{~S} \text {, }\right. \\
\mathrm{X}){ }^{36} \mathrm{~S} /{ }^{38} \mathrm{~S} /{ }^{34} \mathrm{P} /{ }^{36} \mathrm{P}, \mathrm{E}=215 \mathrm{MeV} \text {; measured } \mathrm{E} \gamma, \mathrm{I} \gamma, \gamma \gamma- \\
\text { (particle) } \gamma \text {-coin. } \\
\text { JPGPE } 31 \text { S1935 }\end{array}$ \\
\hline
\end{tabular}

\section{$\mathrm{A}=35$}

${ }^{35} \mathrm{Al} 2005 \mathrm{TI} 11 \quad \mathrm{RADIOACTIVITY}{ }^{35} \mathrm{Al}\left(\beta^{-}\right),\left(\beta^{-} \mathrm{n}\right)$ [from ${ }^{36} \mathrm{~S}$ fragmentation]; measured $\beta$-delayed $\mathrm{E} \gamma, \mathrm{En}, \mathrm{T}_{1 / 2}$, neutron emission probability; deduced $\log \mathrm{ft} .{ }^{34,35} \mathrm{Si}$ deduced levels, J, $\pi$, feeding intensities. JOUR JPGPE 31 S1965

${ }^{35} \mathrm{Si} \quad 2005$ TI11

RADIOACTIVITY ${ }^{35} \mathrm{Al}\left(\beta^{-}\right),\left(\beta^{-} \mathrm{n}\right)$ [from ${ }^{36} \mathrm{~S}$ fragmentation]; measured $\beta$-delayed $\mathrm{E} \gamma, \mathrm{En}, \mathrm{T}_{1 / 2}$, neutron emission probability; deduced $\log \mathrm{ft} .{ }^{34,35} \mathrm{Si}$ deduced levels, J, $\pi$, feeding intensities. JOUR JPGPE 31 S1965

${ }^{35} \mathrm{Cl}$ 2005EK01 NUCLEAR REACTIONS ${ }^{16} \mathrm{O}\left({ }^{24} \mathrm{Mg}, \mathrm{n} \alpha\right),\left({ }^{24} \mathrm{Mg}, \mathrm{p} \alpha\right), \mathrm{E}=60 \mathrm{MeV}$; ${ }^{28} \mathrm{Si}\left({ }^{32} \mathrm{~S}, \mathrm{n} 2 \alpha\right),\left({ }^{32} \mathrm{~S}, \mathrm{p} 2 \alpha\right), \mathrm{E}=130 \mathrm{MeV} ;{ }^{24} \mathrm{Mg}\left({ }^{40} \mathrm{Ca}, 2 \mathrm{np}\right),\left({ }^{40} \mathrm{Ca}, \mathrm{n} 2 \mathrm{p}\right)$, $\mathrm{E}=104 \mathrm{MeV}$; measured $\mathrm{E} \gamma, \mathrm{I} \gamma, \gamma \gamma-$, (charged particle) $\gamma_{-}$, (neutron) $\gamma$-coin. ${ }^{35} \mathrm{Ar},{ }^{35} \mathrm{Cl},{ }^{51} \mathrm{Fe},{ }^{51} \mathrm{Mn},{ }^{61} \mathrm{Ga},{ }^{61} \mathrm{Zn}$ deduced levels, J, $\pi$, mirror energy difference. Discussed electromagnetic spin-orbit effect. Large-scale shell model calculations. JOUR ZAANE 25 s01 363

${ }^{35} \mathrm{Ar} \quad$ 2005EK01 NUCLEAR REACTIONS ${ }^{16} \mathrm{O}\left({ }^{24} \mathrm{Mg}, \mathrm{n} \alpha\right),\left({ }^{24} \mathrm{Mg}, \mathrm{p} \alpha\right), \mathrm{E}=60 \mathrm{MeV}$; ${ }^{28} \mathrm{Si}\left({ }^{32} \mathrm{~S}, \mathrm{n} 2 \alpha\right),\left({ }^{32} \mathrm{~S}, \mathrm{p} 2 \alpha\right), \mathrm{E}=130 \mathrm{MeV} ;{ }^{24} \mathrm{Mg}\left({ }^{40} \mathrm{Ca}, 2 \mathrm{np}\right),\left({ }^{40} \mathrm{Ca}, \mathrm{n} 2 \mathrm{p}\right)$, $\mathrm{E}=104 \mathrm{MeV}$; measured $\mathrm{E} \gamma, \mathrm{I} \gamma, \gamma \gamma-$, (charged particle) $\gamma-$, (neutron) $\gamma$-coin. ${ }^{35} \mathrm{Ar},{ }^{35} \mathrm{Cl},{ }^{51} \mathrm{Fe},{ }^{51} \mathrm{Mn},{ }^{61} \mathrm{Ga},{ }^{61} \mathrm{Zn}$ deduced levels, J, $\pi$, mirror energy difference. Discussed electromagnetic spin-orbit effect. Large-scale shell model calculations. JOUR ZAANE 25 s01 363

\section{$\mathrm{A}=36$}

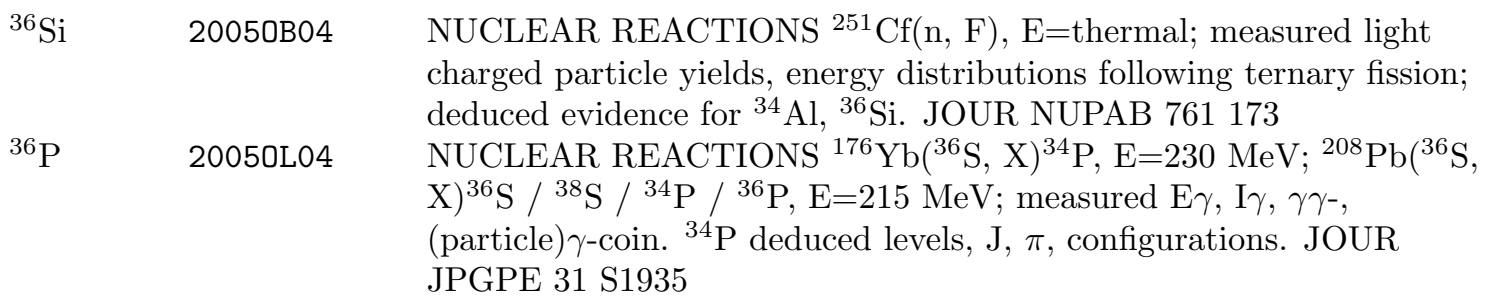




\section{$\mathrm{A}=36$ (continued)}

\begin{tabular}{|c|c|c|}
\hline${ }^{36} \mathrm{~S}$ & 20050L04 & $\begin{array}{l}\text { NUCLEAR REACTIONS }{ }^{176} \mathrm{Yb}\left({ }^{36} \mathrm{~S}, \mathrm{X}\right){ }^{34} \mathrm{P}, \mathrm{E}=230 \mathrm{MeV} ;{ }^{208} \mathrm{~Pb}\left({ }^{36} \mathrm{~S} \text {, }\right. \\
\mathrm{X})^{36} \mathrm{~S} /{ }^{38} \mathrm{~S} /{ }^{34} \mathrm{P} /{ }^{36} \mathrm{P}, \mathrm{E}=215 \mathrm{MeV} ; \text { measured } \mathrm{E} \gamma, \mathrm{I} \gamma, \gamma \gamma- \\
\text { (particle) } \gamma \text {-coin. }{ }^{34} \mathrm{P} \text { deduced levels, J, } \pi \text {, configurations. JOUR } \\
\text { JPGPE } 31 \text { S1935 }\end{array}$ \\
\hline${ }^{36} \mathrm{Ar}$ & $2005 \mathrm{G0} 36$ & $\begin{array}{l}\text { ATOMIC MASSES }{ }^{12} \mathrm{C},{ }^{16} \mathrm{O},{ }^{20} \mathrm{Ne},{ }^{32} \mathrm{~S},{ }^{36,40} \mathrm{Ar} \text {; measured masses. } \\
\text { Cyclotron-based mass spectrometry. JOUR JPGPE } 31 \mathrm{~S} 1869\end{array}$ \\
\hline & 2005SPZY & $\begin{array}{l}\text { NUCLEAR REACTIONS }{ }^{12} \mathrm{C}\left({ }^{32} \mathrm{~S},{ }^{36} \mathrm{Ar}\right), \mathrm{E}=65 \mathrm{MeV} ;{ }^{12} \mathrm{C}\left({ }^{34} \mathrm{~S},{ }^{38} \mathrm{Ar}\right) \text {, } \\
\mathrm{E}=67 \mathrm{MeV} ; \text { measured } \mathrm{E} \gamma, \mathrm{I} \gamma(\theta, \mathrm{H}, \mathrm{t}), \alpha \gamma \text {-coin. }{ }^{36,38} \mathrm{Ar} \text { levels deduced } \mathrm{g} \\
\text { factors. Transient field technique. Comparison with shell model } \\
\text { predictions. REPT MLL } 2004 \text { Annual,P5,Speidel }\end{array}$ \\
\hline
\end{tabular}

$$
\mathrm{A}=37
$$

No references found

\section{$\mathrm{A}=38$}
${ }^{38} \mathrm{~S}$ 20050L04 NUCLEAR REACTIONS ${ }^{176} \mathrm{Yb}\left({ }^{36} \mathrm{~S}, \mathrm{X}\right){ }^{34} \mathrm{P}, \mathrm{E}=230 \mathrm{MeV} ;{ }^{208} \mathrm{~Pb}\left({ }^{36} \mathrm{~S}\right.$, $\mathrm{X})^{36} \mathrm{~S} /{ }^{38} \mathrm{~S} /{ }^{34} \mathrm{P} /{ }^{36} \mathrm{P}, \mathrm{E}=215 \mathrm{MeV}$; measured $\mathrm{E} \gamma, \mathrm{I} \gamma, \gamma \gamma-$ (particle) $\gamma$-coin. ${ }^{34} \mathrm{P}$ deduced levels, J, $\pi$, configurations. JOUR JPGPE 31 S1935
${ }^{38} \mathrm{Ar}$ 2005SPzY NUCLEAR REACTIONS ${ }^{12} \mathrm{C}\left({ }^{32} \mathrm{~S},{ }^{36} \mathrm{Ar}\right), \mathrm{E}=65 \mathrm{MeV} ;{ }^{12} \mathrm{C}\left({ }^{34} \mathrm{~S},{ }^{38} \mathrm{Ar}\right)$, $\mathrm{E}=67 \mathrm{MeV}$; measured $\mathrm{E} \gamma, \mathrm{I} \gamma(\theta, \mathrm{H}, \mathrm{t}), \alpha \gamma$-coin. ${ }^{36,38} \mathrm{Ar}$ levels deduced $\mathrm{g}$ factors. Transient field technique. Comparison with shell model predictions. REPT MLL 2004 Annual,P5,Speidel

$$
\mathrm{A}=39
$$

No references found

$$
\mathrm{A}=\mathbf{4 0}
$$

2005 G036 ATOMIC MASSES ${ }^{40} \mathrm{Ar},{ }^{16} \mathrm{O},{ }^{20} \mathrm{Ne},{ }^{32} \mathrm{~S},{ }^{36,40} \mathrm{Ar}$; measured masses. Cyclotron-based mass spectrometry. JOUR JPGPE 31 S1869

$$
\mathrm{A}=41
$$

${ }^{41} \mathrm{~K}$ 2005GUZX NUCLEAR REACTIONS ${ }^{44} \mathrm{Ca}$ (polarized $\mathrm{p}, \alpha$ ), $\mathrm{E}=24.6 \mathrm{MeV}$; measured $\sigma(\theta), \operatorname{Ay}(\theta)$. DWBA analysis. REPT MLL 2004 Annual,P6,Guazzoni 


\section{$\mathrm{A}=42$}

${ }^{42} \mathrm{Ca} \quad 2005 \mathrm{CO} 25 \quad$ NUCLEAR REACTIONS ${ }^{208} \mathrm{~Pb}\left({ }^{40} \mathrm{Ca},{ }^{42} \mathrm{Ca}\right), \mathrm{E}=225 \mathrm{MeV}$; measured $\sigma(\mathrm{E}, \theta) .{ }^{42} \mathrm{Ca}$ deduced excited states configurations. ${ }^{208} \mathrm{~Pb}\left({ }^{90} \mathrm{Zr}, \mathrm{X}\right)$, $\mathrm{E}=560 \mathrm{MeV}$; measured $\mathrm{E} \gamma, \mathrm{I} \gamma$, (fragment) $\gamma$-coin, isotopic yields for projectile-like fragments. ${ }^{90} \mathrm{Zr}$ deduced transitions. JOUR ZAANE 25 s01 427

2005GUZW NUCLEAR REACTIONS ${ }^{45} \mathrm{Sc}$ (polarized $\mathrm{p}, \alpha$ ), E=24.6 MeV; measured $\sigma(\theta), \operatorname{Ay}(\theta) .{ }^{42} \mathrm{Ca}$ levels deduced configurations. REPT MLL 2004 Annual,P7,Guazzoni

\section{$\mathrm{A}=43$}

${ }^{43} \mathrm{Cr} \quad$ 2005BL31

RADIOACTIVITY ${ }^{45} \mathrm{Fe},{ }^{48} \mathrm{Ni},{ }^{54} \mathrm{Zn}(2 \mathrm{p})$ [from Ni( $\left.\left.{ }^{58} \mathrm{Ni}, \mathrm{X}\right)\right]$; measured proton spectra, $\mathrm{T}_{1 / 2}$. Comparison with theory. JOUR ZAANE $25 \mathrm{~s} 01$ 169

2005D020 RADIOACTIVITY ${ }^{45} \mathrm{Fe}(2 \mathrm{p})$ [from Ni $\left.\left({ }^{58} \mathrm{Ni}, \mathrm{X}\right)\right]$; measured Ep, $\mathrm{T}_{1 / 2}$, branching ratio. ${ }^{48} \mathrm{Ni}$; measured decay energy, $\mathrm{T}_{1 / 2}$; deduced probable two-proton decay. Comparisons with model predictions. JOUR PRVCA 72054315

2005GI15 RADIOACTIVITY ${ }^{45} \mathrm{Fe},{ }^{54} \mathrm{Zn}(\mathrm{p}),(2 \mathrm{p})$ [from Ni $\left.\left({ }^{58} \mathrm{Ni}, \mathrm{X}\right)\right]$; measured proton spectra, $\mathrm{T}_{1 / 2}$. JOUR JPGPE $31 \mathrm{~S} 1509$

\section{$\mathrm{A}=44$}

${ }^{44} \mathrm{~S}$

${ }^{44} \mathrm{Mn}$

2005GI15

2005GR30

${ }^{45} \mathrm{Ar}$

2005GA45

${ }^{45} \mathrm{Fe}$

2005BL31

2005D020

2005D020

2005GI15
$\mathrm{A}=45$

NUCLEAR REACTIONS ${ }^{2} \mathrm{H}\left({ }^{44} \mathrm{Ar},{ }^{45} \mathrm{Ar}\right),\left({ }^{40} \mathrm{Ar},{ }^{41} \mathrm{Ar}\right), \mathrm{E}=10 \mathrm{MeV} /$ nucleon; measured particle spectra, $\sigma(\mathrm{E}, \theta) .{ }^{45} \mathrm{Ar}$ deduced levels, spectroscopic factors. JOUR JPGPE 31 S1623

RADIOACTIVITY ${ }^{45} \mathrm{Fe},{ }^{48} \mathrm{Ni},{ }^{54} \mathrm{Zn}(2 \mathrm{p})$ [from Ni $\left.\left({ }^{58} \mathrm{Ni}, \mathrm{X}\right)\right]$; measured proton spectra, $\mathrm{T}_{1 / 2}$. Comparison with theory. JOUR ZAANE 25 s01 169

RADIOACTIVITY ${ }^{44} \mathrm{~S}(\mathrm{IT})$ [from Be $\left({ }^{48} \mathrm{Ca}, \mathrm{X}\right)$ ]; measured $\mathrm{E}(\mathrm{ce}), \mathrm{T}_{1 / 2}$. ${ }^{44} \mathrm{~S}$ deduced levels, $\mathrm{J}, \pi$. Comparison with shell model calculations. JOUR ZAANE 25 s01 111 RADIOACTIVITY ${ }^{45} \mathrm{Fe},{ }^{54} \mathrm{Zn}(\mathrm{p}),(2 \mathrm{p})$ [from $\left.\mathrm{Ni}\left({ }^{58} \mathrm{Ni}, \mathrm{X}\right)\right]$; measured proton spectra, $\mathrm{T}_{1 / 2}$. JOUR JPGPE $31 \mathrm{~S} 1509$

NUCLEAR REACTIONS Ni( $\left.{ }^{58} \mathrm{Ni}, \mathrm{X}\right), \mathrm{E}=74.5 \mathrm{MeV} /$ nucleon; measured fragments isotopic yields; deduced evidence for ${ }^{48} \mathrm{Ni},{ }^{45} \mathrm{Fe}$. JOUR PRVCA 72054315 RADIOACTIVITY ${ }^{45} \mathrm{Fe}(2 \mathrm{p})$ [from $\mathrm{Ni}\left({ }^{58} \mathrm{Ni}, \mathrm{X}\right)$ ]; measured Ep, $\mathrm{T}_{1 / 2}$, branching ratio. ${ }^{48} \mathrm{Ni}$; measured decay energy, $\mathrm{T}_{1 / 2}$; deduced probable two-proton decay. Comparisons with model predictions. JOUR PRVCA 72054315 RADIOACTIVITY ${ }^{45} \mathrm{Fe},{ }^{54} \mathrm{Zn}(\mathrm{p}),(2 \mathrm{p})$ [from $\left.\mathrm{Ni}\left({ }^{58} \mathrm{Ni}, \mathrm{X}\right)\right]$; measured proton spectra, $\mathrm{T}_{1 / 2}$. JOUR JPGPE $31 \mathrm{~S} 1509$ 


\section{$\mathrm{A}=46$}

${ }^{46} \mathrm{Cr}$ 2005YA26 NUCLEAR REACTIONS $\mathrm{Pb}\left({ }^{46} \mathrm{Cr},{ }^{46} \mathrm{Cr}\right),\left({ }^{50} \mathrm{Fe},{ }^{50} \mathrm{Fe}\right),\left({ }^{54} \mathrm{Ni},{ }^{54} \mathrm{Ni}{ }^{\prime}\right)$, $\mathrm{E}=41-44 \mathrm{MeV} /$ nucleon; measured $\sigma(\theta), \mathrm{E} \gamma, \mathrm{I} \gamma$, (particle) $\gamma$-coin following projectile Coulomb excitation. ${ }^{46} \mathrm{Cr},{ }^{50} \mathrm{Fe},{ }^{54} \mathrm{Ni}$ deduced excitation B(E2). DWBA analysis. JOUR ZAANE 25 s01 409

${ }^{46} \mathrm{Fe} \quad 2005 \mathrm{BL} 31$ RADIOACTIVITY ${ }^{45} \mathrm{Fe},{ }^{48} \mathrm{Ni},{ }^{54} \mathrm{Zn}(2 \mathrm{p})$ [from Ni( $\left.\left.{ }^{58} \mathrm{Ni}, \mathrm{X}\right)\right]$; measured proton spectra, $\mathrm{T}_{1 / 2}$. Comparison with theory. JOUR ZAANE 25 s01 169

$$
\mathrm{A}=47
$$

No references found

$$
\mathrm{A}=48
$$

${ }^{48} \mathrm{Ti}$ 2005VA31 NUCLEAR REACTIONS ${ }^{48} \mathrm{Ti}\left({ }^{132} \mathrm{Sn},{ }^{132} \mathrm{Sn}\right), \mathrm{E}=470-495 \mathrm{MeV}$; ${ }^{90} \mathrm{Zr}\left({ }^{134} \mathrm{Sn},{ }^{134} \mathrm{Sn}\right.$ '), $\mathrm{E}=400 \mathrm{MeV}$; measured $\mathrm{E} \gamma, \mathrm{I} \gamma$, (particle) $\gamma$-coin following projectile Coulomb excitation. ${ }^{132,134} \mathrm{Sn}$ deduced transitions B(E2). JOUR ZAANE 25 s01 391

${ }^{48} \mathrm{Ni}$ 2005BL31 RADIOACTIVITY ${ }^{45} \mathrm{Fe},{ }^{48} \mathrm{Ni},{ }^{54} \mathrm{Zn}(2 \mathrm{p})\left[\right.$ from $\left.\mathrm{Ni}\left({ }^{58} \mathrm{Ni}, \mathrm{X}\right)\right]$; measured proton spectra, $\mathrm{T}_{1 / 2}$. Comparison with theory. JOUR ZAANE $25 \mathrm{~s} 01$ 169

2005D020 NUCLEAR REACTIONS Ni( $\left.{ }^{58} \mathrm{Ni}, \mathrm{X}\right), \mathrm{E}=74.5 \mathrm{MeV} /$ nucleon; measured fragments isotopic yields; deduced evidence for ${ }^{48} \mathrm{Ni},{ }^{45} \mathrm{Fe}$. JOUR PRVCA 72054315

2005D020 RADIOACTIVITY ${ }^{45} \mathrm{Fe}(2 \mathrm{p})$ [from $\left.\mathrm{Ni}\left({ }^{58} \mathrm{Ni}, \mathrm{X}\right)\right]$; measured $\mathrm{Ep}, \mathrm{T}_{1 / 2}$, branching ratio. ${ }^{48} \mathrm{Ni}$; measured decay energy, $\mathrm{T}_{1 / 2}$; deduced probable two-proton decay. Comparisons with model predictions. JOUR PRVCA 72054315

2005GI15 NUCLEAR REACTIONS Ni $\left({ }^{58} \mathrm{Ni}, \mathrm{X}\right), \mathrm{E}=75 \mathrm{MeV} /$ nucleon; measured fragments isotopic yields; deduced evidence for ${ }^{48} \mathrm{Ni}$. JOUR JPGPE 31 S1509

$$
\mathrm{A}=\mathbf{4 9}
$$

${ }^{49} \mathrm{Ca}$ 2005MAzM NUCLEAR REACTIONS ${ }^{2} \mathrm{H}\left({ }^{48} \mathrm{Ca},{ }^{49} \mathrm{Ca}\right), \mathrm{E}=105 \mathrm{MeV}$; measured $\mathrm{E} \gamma$, $\mathrm{I} \gamma$, (particle) $\gamma$-coin. ${ }^{48} \mathrm{Ca}$ (polarized d, p), $\mathrm{E}=14 \mathrm{MeV}$; measured proton spectra, $\sigma(\theta) .{ }^{49} \mathrm{Ca}$ deduced levels, J, $\pi$. REPT MLL 2004

Annual,P8,Maierbeck

${ }^{49} \mathrm{Ti} \quad 2005 \mathrm{ID} 03 \quad$ NUCLEAR REACTIONS ${ }^{9} \mathrm{Be}\left({ }^{46} \mathrm{Ar}, 3 \mathrm{n}\right),\left({ }^{46} \mathrm{Ar}, 4 \mathrm{n}\right),\left({ }^{46} \mathrm{Ar}, 5 \mathrm{n}\right),\left({ }^{46} \mathrm{Ar}\right.$, $6 \mathrm{n}), \mathrm{E} \approx 2-6 \mathrm{MeV} /$ nucleon; measured $\mathrm{E} \gamma, \mathrm{I} \gamma$, (particle) $\gamma$-coin; deduced excitation functions. ${ }^{49,50,51} \mathrm{Ti}$ deduced high-spin levels, J, $\pi$. JOUR ZAANE 25 s01 429 


\section{$\mathrm{A}=50$}

\begin{tabular}{|c|c|c|}
\hline${ }^{50} \mathrm{Ti}$ & 2005ID03 & $\begin{array}{l}\text { NUCLEAR REACTIONS }{ }^{9} \mathrm{Be}\left({ }^{46} \mathrm{Ar}, 3 \mathrm{n}\right),\left({ }^{46} \mathrm{Ar}, 4 \mathrm{n}\right),\left({ }^{46} \mathrm{Ar}, 5 \mathrm{n}\right),\left({ }^{46} \mathrm{Ar} \text {, }\right. \\
6 \mathrm{n}), \mathrm{E} \approx 2-6 \mathrm{MeV} / \text { nucleon; measured } \mathrm{E} \gamma, \mathrm{I} \gamma,(\text { particle }) \gamma \text {-coin; } \\
\text { deduced excitation functions. }{ }^{49,50,51} \mathrm{Ti} \text { deduced high-spin levels, J, } \pi \text {. }\end{array}$ \\
\hline & 2005YU07 & NUCLEAR REACTIONS ${ }^{50} \mathrm{Ti}\left({ }^{129} \mathrm{Sb},{ }^{129} \mathrm{Sb}\right),\left({ }^{129} \mathrm{Te},{ }^{129} \mathrm{Te}\right), \mathrm{E}=400$ \\
\hline & & $\begin{array}{l}\mathrm{MeV} \text {; measured } \mathrm{E} \gamma, \mathrm{I} \gamma, \text { (particle) } \gamma \text {-coin following projectile Coulomb } \\
\text { excitation. }{ }^{129} \mathrm{Te},{ }^{129} \mathrm{Sb} \text { deduced transitions B(E2). Clarion, Hyball } \\
\text { arrays. JOUR ZAANE } 25 \text { s01 } 395\end{array}$ \\
\hline${ }^{50} \mathrm{~V}$ & 2005 SUZU & $\begin{array}{l}\text { NUCLEAR REACTIONS }{ }^{51} \mathrm{~V}\left({ }^{3} \mathrm{He},{ }^{3} \mathrm{He} e^{\prime}\right),\left({ }^{3} \mathrm{He}, \alpha\right), \mathrm{E}=30 \mathrm{MeV} \text {; } \\
\text { measured E } \gamma, \mathrm{I} \gamma, \text { (particle) } \gamma \text {-coin. }{ }^{50,51} \mathrm{~V} \text { deduced level densities, } \\
\text { radiative strength functions, microcanonical entropies. PREPRINT } \\
\text { nucl-ex/0511054,11/30/2005 }\end{array}$ \\
\hline${ }^{50} \mathrm{Mn}$ & 2005FU16 & $\begin{array}{l}\text { NUCLEAR REACTIONS }{ }^{50} \mathrm{Cr}\left({ }^{3} \mathrm{He}, \mathrm{t}\right), \mathrm{E}=140 \mathrm{MeV} / \text { nucleon; } \\
\text { measured triton spectra; deduced Gamow-Teller transition strengths. } \\
{ }^{50} \mathrm{Mn} \text { deduced level energies. }{ }^{50} \mathrm{Fe} \text { deduced } \beta \text {-decay intensities. }\end{array}$ \\
\hline & & Astrophysical implications discussed. JOUR PRLTA 95212501 \\
\hline${ }^{50} \mathrm{Fe}$ & $2005 \mathrm{FU} 16$ & $\begin{array}{l}\text { NUCLEAR REACTIONS }{ }^{50} \mathrm{Cr}\left({ }^{3} \mathrm{He}, \mathrm{t}\right), \mathrm{E}=140 \mathrm{MeV} / \text { nucleon; } \\
\text { measured triton spectra; deduced Gamow-Teller transition strengths. } \\
{ }^{50} \mathrm{Mn} \text { deduced level energies. }{ }^{50} \mathrm{Fe} \text { deduced } \beta \text {-decay intensities. }\end{array}$ \\
\hline & 2005YA26 & $\begin{array}{l}\text { Astrophysical implications discussed. JOUR PRLTA } 95212501 \\
\text { NUCLEAR REACTIONS } \mathrm{Pb}\left({ }^{46} \mathrm{Cr},{ }^{46} \mathrm{Cr}^{\prime}\right),\left({ }^{50} \mathrm{Fe},{ }^{50} \mathrm{Fe}\right),\left({ }^{54} \mathrm{Ni},{ }^{54} \mathrm{Ni}^{\prime}\right) \text {, } \\
\mathrm{E}=41-44 \mathrm{MeV} / \text { nucleon; measured } \sigma(\theta), \mathrm{E} \gamma, \mathrm{I} \gamma,(\text { particle }) \gamma \text {-coin } \\
\text { following projectile Coulomb excitation. }{ }^{46} \mathrm{Cr},{ }^{50} \mathrm{Fe},{ }^{54} \mathrm{Ni} \text { deduced } \\
\text { excitation B(E2). DWBA analysis. JOUR ZAANE } 25 \text { s01 } 409\end{array}$ \\
\hline
\end{tabular}

\section{$\mathrm{A}=51$}

${ }^{51} \mathrm{Ti}$ 2005ID03 NUCLEAR REACTIONS ${ }^{9} \mathrm{Be}\left({ }^{46} \mathrm{Ar}, 3 \mathrm{n}\right),\left({ }^{46} \mathrm{Ar}, 4 \mathrm{n}\right),\left({ }^{46} \mathrm{Ar}, 5 \mathrm{n}\right),\left({ }^{46} \mathrm{Ar}\right.$, $6 \mathrm{n}), \mathrm{E} \approx 2-6 \mathrm{MeV} /$ nucleon; measured $\mathrm{E} \gamma, \mathrm{I} \gamma$, (particle) $\gamma$-coin; deduced excitation functions. ${ }^{49,50,51} \mathrm{Ti}$ deduced high-spin levels, $\mathrm{J}, \pi$. JOUR ZAANE 25 s01 429

${ }^{51} \mathrm{~V}$ 2005SUZU NUCLEAR REACTIONS ${ }^{51} \mathrm{~V}\left({ }^{3} \mathrm{He},{ }^{3} \mathrm{He}\right),\left({ }^{3} \mathrm{He}, \alpha\right), \mathrm{E}=30 \mathrm{MeV}$; measured $\mathrm{E} \gamma, \mathrm{I} \gamma$, (particle) $\gamma$-coin. ${ }^{50,51} \mathrm{~V}$ deduced level densities, radiative strength functions, microcanonical entropies. PREPRINT nucl-ex/0511054,11/30/2005

${ }^{51} \mathrm{Mn} \quad$ 2005EKO1 NUCLEAR REACTIONS ${ }^{16} \mathrm{O}\left({ }^{24} \mathrm{Mg}, \mathrm{n} \alpha\right),\left({ }^{24} \mathrm{Mg}, \mathrm{p} \alpha\right), \mathrm{E}=60 \mathrm{MeV}$; ${ }^{28} \mathrm{Si}\left({ }^{32} \mathrm{~S}, \mathrm{n} 2 \alpha\right),\left({ }^{32} \mathrm{~S}, \mathrm{p} 2 \alpha\right), \mathrm{E}=130 \mathrm{MeV} ;{ }^{24} \mathrm{Mg}\left({ }^{40} \mathrm{Ca}, 2 \mathrm{np}\right),\left({ }^{40} \mathrm{Ca}, \mathrm{n} 2 \mathrm{p}\right)$, $\mathrm{E}=104 \mathrm{MeV}$; measured $\mathrm{E} \gamma, \mathrm{I} \gamma, \gamma \gamma-$, (charged particle) $\gamma-$, (neutron) $\gamma$-coin. ${ }^{35} \mathrm{Ar},{ }^{35} \mathrm{Cl},{ }^{51} \mathrm{Fe},{ }^{51} \mathrm{Mn},{ }^{61} \mathrm{Ga},{ }^{61} \mathrm{Zn}$ deduced levels, J, $\pi$, mirror energy difference. Discussed electromagnetic spin-orbit effect. Large-scale shell model calculations. JOUR ZAANE 25 s01 363

2005MA81 NUCLEAR REACTIONS ${ }^{40} \mathrm{Ca}\left({ }^{14} \mathrm{~N}, \mathrm{n} 2 \mathrm{p}\right)$, E not given; measured $\mathrm{E} \gamma$, $\mathrm{I} \gamma, \gamma \gamma$-coin. ${ }^{51} \mathrm{Mn}$ levels deduced $\mathrm{T}_{1 / 2}$. Ultra-fast timing techniques. JOUR JPGPE 31 S1421 


\section{$\mathrm{A}=51$ (continued)}

${ }^{51} \mathrm{Fe} \quad$ 2005EK01 NUCLEAR REACTIONS ${ }^{16} \mathrm{O}\left({ }^{24} \mathrm{Mg}, \mathrm{n} \alpha\right),\left({ }^{24} \mathrm{Mg}, \mathrm{p} \alpha\right), \mathrm{E}=60 \mathrm{MeV}$ ${ }^{28} \mathrm{Si}\left({ }^{32} \mathrm{~S}, \mathrm{n} 2 \alpha\right),\left({ }^{32} \mathrm{~S}, \mathrm{p} 2 \alpha\right), \mathrm{E}=130 \mathrm{MeV} ;{ }^{24} \mathrm{Mg}\left({ }^{40} \mathrm{Ca}, 2 \mathrm{np}\right),\left({ }^{40} \mathrm{Ca}, \mathrm{n} 2 \mathrm{p}\right)$, $\mathrm{E}=104 \mathrm{MeV}$; measured $\mathrm{E} \gamma, \mathrm{I} \gamma, \gamma \gamma-$, (charged particle) $\gamma-$, (neutron) $\gamma$-coin. ${ }^{35} \mathrm{Ar},{ }^{35} \mathrm{Cl},{ }^{51} \mathrm{Fe},{ }^{51} \mathrm{Mn},{ }^{61} \mathrm{Ga},{ }^{61} \mathrm{Zn}$ deduced levels, J, $\pi$, mirror energy difference. Discussed electromagnetic spin-orbit effect. Large-scale shell model calculations. JOUR ZAANE 25 s01 363

\section{$\mathrm{A}=52$}
2005 ID03 NUCLEAR REACTIONS ${ }^{9} \mathrm{Be}\left({ }^{46} \mathrm{Ar}, 3 \mathrm{n}\right),\left({ }^{46} \mathrm{Ar}, 4 \mathrm{n}\right),\left({ }^{46} \mathrm{Ar}, 5 \mathrm{n}\right),\left({ }^{46} \mathrm{Ar}\right.$, $6 \mathrm{n}$ ), $\mathrm{E} \approx 2-6 \mathrm{MeV} /$ nucleon; measured $\mathrm{E} \gamma, \mathrm{I} \gamma$, (particle) $\gamma$-coin; deduced excitation functions. ${ }^{49,50,51} \mathrm{Ti}$ deduced high-spin levels, J, $\pi$. JOUR ZAANE 25 s01 429
${ }^{52} \mathrm{Ni}$ 2005BL31 RADIOACTIVITY ${ }^{45} \mathrm{Fe},{ }^{48} \mathrm{Ni},{ }^{54} \mathrm{Zn}(2 \mathrm{p})$ [from Ni( $\left.\left.{ }^{58} \mathrm{Ni}, \mathrm{X}\right)\right]$; measured proton spectra, $\mathrm{T}_{1 / 2}$. Comparison with theory. JOUR ZAANE 25 s01 169
2005GI15 RADIOACTIVITY ${ }^{45} \mathrm{Fe},{ }^{54} \mathrm{Zn}(\mathrm{p}),(2 \mathrm{p})$ [from Ni $\left.\left({ }^{58} \mathrm{Ni}, \mathrm{X}\right)\right]$; measured proton spectra, $\mathrm{T}_{1 / 2}$. JOUR JPGPE $31 \mathrm{~S} 1509$

\section{$\mathrm{A}=53$}

${ }^{53} \mathrm{Ti}$ 2005F014 NUCLEAR REACTIONS ${ }^{208} \mathrm{~Pb}\left({ }^{48} \mathrm{Ca}, \mathrm{X}\right), \mathrm{E}=305 \mathrm{MeV} ;{ }^{238} \mathrm{U}\left({ }^{48} \mathrm{Ca}\right.$, $\mathrm{X}), \mathrm{E}=330 \mathrm{MeV}$; measured $\mathrm{E} \gamma, \mathrm{I} \gamma, \gamma \gamma$-coin. ${ }^{53} \mathrm{Ti}$ deduced levels, $\mathrm{J}, \pi$. Gammasphere array, cross-coincidence with reaction partners. Comparison with model predictions. JOUR PRVCA 72044315

${ }^{53} \mathrm{Cu} \quad 2005 \mathrm{GI} 15$ RADIOACTIVITY ${ }^{45} \mathrm{Fe},{ }^{54} \mathrm{Zn}(\mathrm{p}),(2 \mathrm{p})$ [from $\left.\mathrm{Ni}\left({ }^{58} \mathrm{Ni}, \mathrm{X}\right)\right]$; measured proton spectra, $\mathrm{T}_{1 / 2}$. JOUR JPGPE $31 \mathrm{~S} 1509$

\section{$\mathrm{A}=54$}

$\begin{array}{ll}{ }^{54} \mathrm{Cr} & \text { 2006B001 } \\ { }^{54} \mathrm{Mn} & 2005 \mathrm{SI} 22 \\ & \text { 2006B001 } \\ & \text { 2005TA27 } \\ { }^{54} \mathrm{Fe} & \\ & \\ { }^{54} \mathrm{Ni} & \text { 2005TA27 }\end{array}$

RADIOACTIVITY ${ }^{54} \mathrm{Mn},{ }^{65} \mathrm{Zn}(\mathrm{EC})$; measured $\beta \gamma$-coin. Triple to double coincidence ratio method. JOUR ARISE 64124

NUCLEAR REACTIONS Cu(n, X $)^{54} \mathrm{Mn} /{ }^{59} \mathrm{Fe} /{ }^{56} \mathrm{Co} /{ }^{57} \mathrm{Co} /{ }^{58} \mathrm{Co}$ $/{ }^{60} \mathrm{Co}, \mathrm{E} \approx 70.7,110.8 \mathrm{MeV}$; measured $\sigma$. Comparison with previous results, model predictions. JOUR NIMBE 240617

RADIOACTIVITY ${ }^{54} \mathrm{Mn},{ }^{65} \mathrm{Zn}(\mathrm{EC})$; measured $\beta \gamma$-coin. Triple to double coincidence ratio method. JOUR ARISE 64124 NUCLEAR REACTIONS ${ }^{9} \mathrm{Be}\left({ }^{55} \mathrm{Ni}, \mathrm{X}\right),\left({ }^{55} \mathrm{Co}, \mathrm{X}\right), \mathrm{E} \approx 170 \mathrm{MeV} /$ nucleon; measured $\mathrm{E} \gamma, \mathrm{I} \gamma$, (fragment) $\gamma$-coin. ${ }^{54} \mathrm{Ni},{ }^{54} \mathrm{Fe}$ deduced transitions. JOUR JPGPE 31 S1527 NUCLEAR REACTIONS ${ }^{9} \mathrm{Be}\left({ }^{55} \mathrm{Ni}, \mathrm{X}\right),\left({ }^{55} \mathrm{Co}, \mathrm{X}\right), \mathrm{E} \approx 170 \mathrm{MeV} /$ nucleon; measured $\mathrm{E} \gamma$, I $\gamma$, (fragment) $\gamma$-coin. ${ }^{54} \mathrm{Ni},{ }^{54} \mathrm{Fe}$ deduced transitions. JOUR JPGPE 31 S1527 


\section{$\mathrm{A}=54$ (continued)}

2005 YA26 NUCLEAR REACTIONS $\mathrm{Pb}\left({ }^{46} \mathrm{Cr},{ }^{46} \mathrm{Cr}{ }^{\prime}\right),\left({ }^{50} \mathrm{Fe},{ }^{50} \mathrm{Fe} '\right),\left({ }^{54} \mathrm{Ni},{ }^{54} \mathrm{Ni}^{\prime}\right)$, $\mathrm{E}=41-44 \mathrm{MeV} /$ nucleon; measured $\sigma(\theta), \mathrm{E} \gamma, \mathrm{I} \gamma$, (particle) $\gamma$-coin following projectile Coulomb excitation. ${ }^{46} \mathrm{Cr},{ }^{50} \mathrm{Fe},{ }^{54} \mathrm{Ni}$ deduced excitation B(E2). DWBA analysis. JOUR ZAANE 25 s01 409

${ }^{54} \mathrm{Zn} \quad 2005 \mathrm{BL} 31$

RADIOACTIVITY ${ }^{45} \mathrm{Fe},{ }^{48} \mathrm{Ni},{ }^{54} \mathrm{Zn}(2 \mathrm{p})$ [from Ni( $\left.\left.{ }^{58} \mathrm{Ni}, \mathrm{X}\right)\right]$; measured proton spectra, $\mathrm{T}_{1 / 2}$. Comparison with theory. JOUR ZAANE 25 s01 169

2005GI15 RADIOACTIVITY ${ }^{45} \mathrm{Fe},{ }^{54} \mathrm{Zn}(\mathrm{p}),(2 \mathrm{p})$ [from Ni $\left.\left({ }^{58} \mathrm{Ni}, \mathrm{X}\right)\right]$; measured proton spectra, $\mathrm{T}_{1 / 2}$. JOUR JPGPE $31 \mathrm{~S} 1509$

\section{$\mathrm{A}=55$}

${ }^{55} \mathrm{Fe}$

2005MAZL

NUCLEAR REACTIONS ${ }^{58} \mathrm{Ni}$ (polarized p, d), E=24.6 MeV; measured $\sigma(\theta), \operatorname{Ay}(\theta) .{ }^{2} \mathrm{H}\left({ }^{54} \mathrm{Fe}, \mathrm{p}\right), \mathrm{E}=4.8 \mathrm{MeV} /$ nucleon; measured $\sigma(\theta)$. Other reactions discussed. REPT MLL 2004 Annual,P9,Mahgoub

\section{$\mathrm{A}=56$}

$\begin{array}{ll}{ }^{56} \mathrm{Sc} & \\ { }^{56} \mathrm{Ti} & \text { 2005MA93 } \\ & \\ { }^{56} \mathrm{Cr} & \text { 2005MA93 } \\ { }^{56} \mathrm{Mn} & \text { 2004AG07 }\end{array}$

RADIOACTIVITY ${ }^{56} \mathrm{Sc}\left(\beta^{-}\right)$[from $\left.\mathrm{Be}\left({ }^{78} \mathrm{Kr}, \mathrm{X}\right)\right]$; measured $\mathrm{E} \gamma, \mathrm{I} \gamma$, $\beta \gamma$-coin. ${ }^{56} \mathrm{Ti}$ deduced levels. Mass-separated source. JOUR NIMBE 241195

RADIOACTIVITY ${ }^{56} \mathrm{Sc}\left(\beta^{-}\right)$[from $\left.\operatorname{Be}\left({ }^{78} \mathrm{Kr}, \mathrm{X}\right)\right]$; measured $\mathrm{E} \gamma, \mathrm{I} \gamma$, $\beta \gamma$-coin. ${ }^{56} \mathrm{Ti}$ deduced levels. Mass-separated source. JOUR NIMBE 241195

ATOMIC MASSES ${ }^{56,57} \mathrm{Cr}$; measured masses. Penning trap mass spectrometer. JOUR JPGPE 31 S1765

NUCLEAR REACTIONS ${ }^{103} \mathrm{Rh}\left(\mathrm{n}, \mathrm{n}^{\prime}\right){ }^{103 m} \mathrm{Rh}, \mathrm{E} \approx 4.8 \mathrm{MeV} ;{ }^{115} \mathrm{In}(\mathrm{n}$, $\left.\mathrm{n}^{\prime}\right){ }^{115 m} \mathrm{In}, \mathrm{E} \approx 5 \mathrm{MeV} ;{ }^{232} \mathrm{Th},{ }^{238} \mathrm{U}(\mathrm{n}, \mathrm{F}), \mathrm{E} \approx 5 \mathrm{MeV} ;{ }^{24} \mathrm{Mg},{ }^{27} \mathrm{Al}$, ${ }^{46,47,48} \mathrm{Ti},{ }^{54,56} \mathrm{Fe},{ }^{58} \mathrm{Ni},{ }^{64} \mathrm{Zn}(\mathrm{n}, \mathrm{p}), \mathrm{E} \approx 2-8 \mathrm{MeV} ;{ }^{27} \mathrm{Al},{ }^{59} \mathrm{Co}(\mathrm{n}, \alpha), \mathrm{E}$ $\approx 8.3 \mathrm{MeV}$; measured activation $\sigma$. Spectrum average technique, comparison with previous results. JOUR RAACA 9263

2005GU37 ATOMIC MASSES ${ }^{56,57} \mathrm{Mn},{ }^{82 m} \mathrm{Rb},{ }^{92} \mathrm{Sr},{ }^{124,127} \mathrm{Cs},{ }^{130} \mathrm{Ba}$; measured masses. Penning trap mass spectrometer. JOUR ZAANE 25 s01 35

${ }^{56} \mathrm{Co} \quad 2005 \mathrm{Siz} 2 \quad$ NUCLEAR REACTIONS $\mathrm{Cu}(\mathrm{n}, \mathrm{X}){ }^{54} \mathrm{Mn} /{ }^{59} \mathrm{Fe} /{ }^{56} \mathrm{Co} /{ }^{57} \mathrm{Co} /{ }^{58} \mathrm{Co}$ $/{ }^{60} \mathrm{Co}, \mathrm{E} \approx 70.7,110.8 \mathrm{MeV}$; measured $\sigma$. Comparison with previous results, model predictions. JOUR NIMBE 240617

\section{$\mathrm{A}=57$}

${ }^{57} \mathrm{Ti} \quad 2005 \mathrm{LI53}$

RADIOACTIVITY ${ }^{57} \mathrm{Ti},{ }^{59} \mathrm{~V},{ }^{59} \mathrm{Cr}\left(\beta^{-}\right)$[from $\left.\mathrm{Be}\left({ }^{86} \mathrm{Kr}, \mathrm{X}\right)\right]$; measured $\beta$-delayed $\mathrm{E} \gamma, \mathrm{I} \gamma, \gamma \gamma$-coin, $\mathrm{T}_{1 / 2}$; deduced $\log \mathrm{ft} .{ }^{57} \mathrm{~V},{ }^{59} \mathrm{Cr},{ }^{59} \mathrm{Mn}$ deduced levels, $\beta$-feeding intensities, deformation. Comparison with shell-model predictions. JOUR PRVCA 72054321 


\section{$\mathrm{A}=57$ (continued)}

\begin{tabular}{|c|c|c|}
\hline${ }^{57} \mathrm{~V}$ & 2005LI53 & $\begin{array}{l}\text { RADIOACTIVITY }{ }^{57} \mathrm{Ti},{ }^{59} \mathrm{~V},{ }^{59} \mathrm{Cr}\left(\beta^{-}\right)\left[\text {from } \mathrm{Be}\left({ }^{86} \mathrm{Kr}, \mathrm{X}\right)\right] ; \text { measured } \\
\beta \text {-delayed } \mathrm{E} \gamma, \mathrm{I} \gamma, \gamma \gamma \text {-coin, } \mathrm{T}_{1 / 2} ; \text { deduced } \log \mathrm{ft} .{ }^{57} \mathrm{~V},{ }^{59} \mathrm{Cr} \text {, }{ }^{59} \mathrm{Mn} \\
\text { deduced levels, } \beta \text {-feeding intensities, deformation. Comparison with } \\
\text { shell-model predictions. JOUR PRVCA } 72054321\end{array}$ \\
\hline${ }^{57} \mathrm{Cr}$ & $2005 \mathrm{GU} 27$ & $\begin{array}{l}\text { ATOMIC MASSES }{ }^{56,57} \mathrm{Cr} \text {; measured masses. Penning trap mass } \\
\text { spectrometer. JOUR JPGPE } 31 \text { S1765 }\end{array}$ \\
\hline${ }^{57} \mathrm{Mn}$ & 2005GU37 & $\begin{array}{l}\text { ATOMIC MASSES }{ }^{56,57} \mathrm{Mn},{ }^{82 m} \mathrm{Rb},{ }^{92} \mathrm{Sr},{ }^{124,127} \mathrm{Cs},{ }^{130} \mathrm{Ba} \text {; measured } \\
\text { masses. Penning trap mass spectrometer. JOUR ZAANE } 25 \text { s01 } 35\end{array}$ \\
\hline${ }^{57} \mathrm{C}$ & 2005SI32 & $\begin{array}{l}\text { NUCLEAR REACTIONS Cu }(\mathrm{n}, \mathrm{X}){ }^{54} \mathrm{Mn} /{ }^{59} \mathrm{Fe} /{ }^{56} \mathrm{Co} /{ }^{57} \mathrm{Co} /{ }^{58} \mathrm{Co} \\
/{ }^{60} \mathrm{Co}, \mathrm{E} \approx 70.7,110.8 \mathrm{MeV} ; \text { measured } \sigma . \text { Comparison with previous } \\
\text { results, model predictions. JOUR NIMBE } 240617\end{array}$ \\
\hline${ }^{57} \mathrm{Ni}$ & 2005MAZL & $\begin{array}{l}\left.\text { NUCLEAR REACTIONS }{ }^{58} \mathrm{Ni} \text { (polarized } \mathrm{p}, \mathrm{d}\right), \mathrm{E}=24.6 \mathrm{MeV} \text {; measured } \\
\sigma(\theta), \mathrm{Ay}(\theta) .{ }^{2} \mathrm{H}\left({ }^{54} \mathrm{Fe}, \mathrm{p}\right), \mathrm{E}=4.8 \mathrm{MeV} / \text { nucleon; measured } \sigma(\theta) \text {. Other } \\
\text { reactions discussed. REPT MLL } 2004 \text { Annual,P9,Mahgoub }\end{array}$ \\
\hline
\end{tabular}

\section{$\mathrm{A}=58$}

${ }^{58} \mathrm{Cr} \quad 2005 \mathrm{GA} 44$

2005GA56

${ }^{58} \mathrm{Co}$

2005SI28

$2005 \mathrm{SI} 32$

2005SI37

${ }^{58} \mathrm{Ni}$

2005AL45

$2005 \mathrm{C} 022$

${ }^{58} \mathrm{Cu} \quad 2005 \mathrm{CO} 22$
NUCLEAR REACTIONS ${ }^{208} \mathrm{~Pb}\left({ }^{90} \mathrm{Zr}, \mathrm{X}\right){ }^{90} \mathrm{Zr} /{ }^{92} \mathrm{Zr} /{ }^{88} \mathrm{Sr}, \mathrm{E}=560$ $\mathrm{MeV} ;{ }^{238} \mathrm{U}\left({ }^{64} \mathrm{Ni}, \mathrm{X}\right){ }^{58} \mathrm{Cr}, \mathrm{E}=400 \mathrm{MeV}$; measured $\mathrm{E} \gamma, \mathrm{I} \gamma, \gamma \gamma-$, (recoil) $\gamma$-coin. ${ }^{58} \mathrm{Cr},{ }^{90,92} \mathrm{Zr},{ }^{88} \mathrm{Sr}$ deduced transitions. Clara array, mass separator. JOUR JPGPE 31 S1443 NUCLEAR REACTIONS ${ }^{238} \mathrm{U}\left({ }^{82} \mathrm{Se}, \mathrm{X}\right), \mathrm{E}=505 \mathrm{MeV} ;{ }^{238} \mathrm{U}\left({ }^{64} \mathrm{Ni}, \mathrm{X}\right)$, $\mathrm{E}=400 \mathrm{MeV}$; measured $\mathrm{E} \gamma, \mathrm{I} \gamma, \gamma \gamma-$, (fragment) $\gamma$-coin, projectile-like fragments isotopic yields. ${ }^{58} \mathrm{Cr},{ }^{80} \mathrm{As},{ }^{82} \mathrm{Ge},{ }^{84} \mathrm{Se}$ deduced levels, $\mathrm{J}, \pi$. Clara array, Prisma spectrometer. JOUR ZAANE 25 s01 421 NUCLEAR REACTIONS ${ }^{51} \mathrm{~V}\left({ }^{10} \mathrm{~B}, 2 \mathrm{np}\right), \mathrm{E}=33,36 \mathrm{MeV}$; measured $\mathrm{E} \gamma, \mathrm{I} \gamma, \gamma \gamma$-coin, DSA. ${ }^{58} \mathrm{Co}$ deduced levels, J, $\pi, \mathrm{T}_{1 / 2}, \mathrm{~B}(\mathrm{M} 1)$. Comparison with shell model predictions. JOUR JPGPE 31 S1577 NUCLEAR REACTIONS Cu(n, X) ${ }^{54} \mathrm{Mn} /{ }^{59} \mathrm{Fe} /{ }^{56} \mathrm{Co} /{ }^{57} \mathrm{Co} /{ }^{58} \mathrm{Co}$ $/{ }^{60} \mathrm{Co}, \mathrm{E} \approx 70.7,110.8 \mathrm{MeV}$; measured $\sigma$. Comparison with previous results, model predictions. JOUR NIMBE 240617

NUCLEAR REACTIONS ${ }^{51} \mathrm{~V}\left({ }^{10} \mathrm{~B}, 2 \mathrm{np}\right), \mathrm{E}=33 \mathrm{MeV}$; measured $\mathrm{E} \gamma, \mathrm{I} \gamma$, $\gamma \gamma-$, (charged particle) $\gamma$-coin. ${ }^{58} \mathrm{Co}$ deduced levels, $\mathrm{J}, \pi$, configurations. Comparison with shell model predictions. JOUR BJPHE 35821 NUCLEAR REACTIONS ${ }^{58} \mathrm{Ni}\left({ }^{16} \mathrm{O},{ }^{16} \mathrm{O}\right),\left({ }^{16} \mathrm{O},{ }^{16} \mathrm{O}\right),\left({ }^{16} \mathrm{O}, \mathrm{X}\right),\left({ }^{18} \mathrm{O}\right.$, $\left.{ }^{18} \mathrm{O}\right),\left({ }^{18} \mathrm{O},{ }^{18} \mathrm{O}\right),\left({ }^{18} \mathrm{O}, \mathrm{X}\right), \mathrm{E}=46 \mathrm{MeV}$; measured elastic, inelastic, and transfer $\sigma(\theta)$. Comparison with model predictions. JOUR BJPHE 35909

NUCLEAR REACTIONS ${ }^{58} \mathrm{Ni}(\mathrm{p}, \mathrm{p} \gamma),(\mathrm{p}, \mathrm{n} \gamma), \mathrm{E}=14 \mathrm{MeV}$; measured prompt and delayed $\mathrm{E} \gamma, \mathrm{I} \gamma .{ }^{58} \mathrm{Cu}$ level deduced $\mathrm{T}_{1 / 2}, \mathrm{~B}(\mathrm{E} 2)$, collective features. Comparison with model predictions. JOUR PRVCA 72 054305

NUCLEAR REACTIONS ${ }^{58} \mathrm{Ni}(\mathrm{p}, \mathrm{p} \gamma),(\mathrm{p}, \mathrm{n} \gamma), \mathrm{E}=14 \mathrm{MeV}$; measured prompt and delayed $\mathrm{E} \gamma, \mathrm{I} \gamma .{ }^{58} \mathrm{Cu}$ level deduced $\mathrm{T}_{1 / 2}, \mathrm{~B}(\mathrm{E} 2)$, collective features. Comparison with model predictions. JOUR PRVCA 72 054305 


\section{$\mathrm{A}=58$ (continued)}

2005KA46 RADIOACTIVITY ${ }^{31} \mathrm{Cl}\left(\beta^{+} \mathrm{p}\right)$ [from $\mathrm{S}(\mathrm{p}, \mathrm{X}), \mathrm{E}=40 \mathrm{MeV}$ ]; measured $\beta$-delayed $\mathrm{E} \gamma, \mathrm{Ep} .{ }^{58} \mathrm{Zn}\left(\beta^{+}\right)$[from $\mathrm{Nb}(\mathrm{p}, \mathrm{X}), \mathrm{E}=1.4 \mathrm{GeV}$ ]; measured $\mathrm{E} \gamma, \mathrm{I} \gamma, \beta \gamma$-coin, $\mathrm{T}_{1 / 2} .{ }^{58} \mathrm{Cu}$ deduced levels, $\beta$-feeding intensities. ${ }^{81 m} \mathrm{Kr}(\mathrm{EC}),(\mathrm{IT}) ;{ }^{81} \mathrm{Y},{ }^{81} \mathrm{Sr},{ }^{85} \mathrm{Nb},{ }^{85} \mathrm{Zr},{ }^{86} \mathrm{Mo},{ }^{86} \mathrm{Nb}(\mathrm{EC})$ [from Ni, $\left.{ }^{54} \mathrm{Fe}\left({ }^{32} \mathrm{~S}, \mathrm{X}\right)\right]$; measured $\mathrm{E} \gamma, \mathrm{I} \gamma, \mathrm{E}(\mathrm{ce}), \mathrm{I}(\mathrm{ce}), \mathrm{T}_{1 / 2} \cdot{ }^{81} \mathrm{Kr},{ }^{85} \mathrm{Zr},{ }^{85} \mathrm{Nb}$ deduced isomeric transitions $\mathrm{T}_{1 / 2}$, ICC. ${ }^{85} \mathrm{Zr},{ }^{86} \mathrm{Nb}$ deduced levels, J, $\pi$, ICC. ${ }^{81} \mathrm{Br}$ deduced neutrino capture rate. Mass-separated sources. JOUR ZAANE 25 s01 129

${ }^{58} \mathrm{Zn} \quad$ 2005KA46 RADIOACTIVITY ${ }^{31} \mathrm{Cl}\left(\beta^{+} \mathrm{p}\right)$ [from $\mathrm{S}(\mathrm{p}, \mathrm{X}), \mathrm{E}=40 \mathrm{MeV}$ ]; measured $\beta$-delayed $\mathrm{E} \gamma, \mathrm{Ep} .{ }^{58} \mathrm{Zn}\left(\beta^{+}\right)$[from $\mathrm{Nb}(\mathrm{p}, \mathrm{X}), \mathrm{E}=1.4 \mathrm{GeV}$ ]; measured $\mathrm{E} \gamma, \mathrm{I} \gamma, \beta \gamma$-coin, $\mathrm{T}_{1 / 2} .{ }^{58} \mathrm{Cu}$ deduced levels, $\beta$-feeding intensities. ${ }^{81 m} \mathrm{Kr}(\mathrm{EC}),(\mathrm{IT}) ;{ }^{81} \mathrm{Y},{ }^{81} \mathrm{Sr},{ }^{85} \mathrm{Nb},{ }^{85} \mathrm{Zr},{ }^{86} \mathrm{Mo},{ }^{86} \mathrm{Nb}(\mathrm{EC})$ [from Ni, $\left.{ }^{54} \mathrm{Fe}\left({ }^{32} \mathrm{~S}, \mathrm{X}\right)\right]$; measured $\mathrm{E} \gamma, \mathrm{I} \gamma, \mathrm{E}(\mathrm{ce}), \mathrm{I}(\mathrm{ce}), \mathrm{T}_{1 / 2} \cdot{ }^{81} \mathrm{Kr},{ }^{85} \mathrm{Zr},{ }^{85} \mathrm{Nb}$ deduced isomeric transitions $\mathrm{T}_{1 / 2}$, ICC. ${ }^{85} \mathrm{Zr},{ }^{86} \mathrm{Nb}$ deduced levels, $\mathrm{J}$, $\pi$, ICC. ${ }^{81} \mathrm{Br}$ deduced neutrino capture rate. Mass-separated sources. JOUR ZAANE 25 s01 129

\section{$\mathrm{A}=59$}

\begin{tabular}{|c|c|c|}
\hline${ }^{59} \mathrm{~V}$ & 2005LI53 & $\begin{array}{l}\text { RADIOACTIVITY }{ }^{57} \mathrm{Ti},{ }^{59} \mathrm{~V},{ }^{59} \mathrm{Cr}\left(\beta^{-}\right)\left[\text {from } \mathrm{Be}\left({ }^{86} \mathrm{Kr}, \mathrm{X}\right)\right] ; \text { measured } \\
\beta \text {-delayed } \mathrm{E} \gamma, \mathrm{I} \gamma, \gamma \gamma \text {-coin, } \mathrm{T}_{1 / 2} ; \text { deduced } \log \mathrm{ft} .{ }^{57} \mathrm{~V},{ }^{59} \mathrm{Cr},{ }^{59} \mathrm{Mn} \\
\text { deduced levels, } \beta \text {-feeding intensities, deformation. Comparison with } \\
\text { shell-model predictions. JOUR PRVCA } 72054321\end{array}$ \\
\hline${ }^{59} \mathrm{Cr}$ & 2005FR29 & $\begin{array}{l}\text { NUCLEAR REACTIONS }{ }^{13,14} \mathrm{C}\left({ }^{48} \mathrm{Ca}, 2 \mathrm{p}\right), \mathrm{E}=130 \mathrm{MeV} \text {; measured } \\
\mathrm{E} \gamma, \mathrm{I} \gamma, \gamma \gamma-\text {, (recoil }) \gamma \text {-coin. }{ }^{59,60} \mathrm{Cr} \text { deduced levels, J, } \pi \text {. Gammasphere } \\
\text { array, comparison with model predictions. JOUR JPGPE } 31 \text { S1465 }\end{array}$ \\
\hline & 2005LI53 & $\begin{array}{l}\text { RADIOACTIVITY }{ }^{57} \mathrm{Ti},{ }^{59} \mathrm{~V},{ }^{59} \mathrm{Cr}\left(\beta^{-}\right)\left[\text {from } \mathrm{Be}\left({ }^{86} \mathrm{Kr}, \mathrm{X}\right)\right] \text {; measured } \\
\beta \text {-delayed } \mathrm{E} \gamma, \mathrm{I} \gamma, \gamma \gamma \text {-coin, } \mathrm{T}_{1 / 2} ; \text { deduced } \log \mathrm{ft} .{ }^{57} \mathrm{~V},{ }^{59} \mathrm{Cr},{ }^{59} \mathrm{Mn} \\
\text { deduced levels, } \beta \text {-feeding intensities, deformation. Comparison with } \\
\text { shell-model predictions. JOUR PRVCA } 72054321\end{array}$ \\
\hline M & 2005LI53 & $\begin{array}{l}\text { RADIOACTIVITY }{ }^{57} \mathrm{Ti},{ }^{59} \mathrm{~V},{ }^{59} \mathrm{Cr}\left(\beta^{-}\right)\left[\text {from } \mathrm{Be}\left({ }^{86} \mathrm{Kr}, \mathrm{X}\right)\right] ; \text { measured } \\
\beta \text {-delayed } \mathrm{E} \gamma, \mathrm{I} \gamma, \gamma \gamma \text {-coin, } \mathrm{T}_{1 / 2} ; \text { deduced } \log \mathrm{ft} .{ }^{57} \mathrm{~V},{ }^{59} \mathrm{Cr},{ }^{59} \mathrm{Mn} \\
\text { deduced levels, } \beta \text {-feeding intensities, deformation. Comparison with } \\
\text { shell-model predictions. JOUR PRVCA } 72054321\end{array}$ \\
\hline $\mathrm{Fe}$ & 2005SI32 & $\begin{array}{l}\text { NUCLEAR REACTIONS } \mathrm{Cu}(\mathrm{n}, \mathrm{X}){ }^{54} \mathrm{Mn} /{ }^{59} \mathrm{Fe} /{ }^{56} \mathrm{Co} /{ }^{57} \mathrm{Co} /{ }^{58} \mathrm{Co} \\
/{ }^{60} \mathrm{Co}, \mathrm{E} \approx 70.7,110.8 \mathrm{MeV} \text {; measured } \sigma \text {. Comparison with previous } \\
\text { results, model predictions. JOUR NIMBE } 240617\end{array}$ \\
\hline$C_{0}$ & $2004 \mathrm{~S} 036$ & $\begin{array}{l}\text { NUCLEAR REACTIONS }{ }^{59} \mathrm{Co}\left({ }^{6} \mathrm{Li}, \mathrm{X}\right),\left({ }^{7} \mathrm{Li}, \mathrm{X}\right), \mathrm{E}=12-26 \mathrm{MeV} \text {; } \\
\text { measured fusion } \sigma \text {; deduced breakup effects. }{ }^{59} \mathrm{Co}\left({ }^{6} \mathrm{Li}, \mathrm{d} \alpha\right), \mathrm{E}=26 \\
\mathrm{MeV} \text {; measured } \mathrm{E} \alpha \text {, Ed, d } \alpha \text {-coin. JOUR BJPHE } 34907\end{array}$ \\
\hline & $2005 \mathrm{~S} 014$ & $\begin{array}{l}\text { NUCLEAR REACTIONS }{ }^{12} \mathrm{C}\left({ }^{6} \mathrm{Li}, \mathrm{d} \alpha\right), \mathrm{E}=26 \mathrm{MeV} ;{ }^{59} \mathrm{Co}\left({ }^{6} \mathrm{Li}, \mathrm{d} \alpha\right) \text {, } \\
\mathrm{E}=30 \mathrm{MeV} ; \text { measured particle spectra, } \sigma(\theta(\alpha), \theta(\mathrm{d})) \text {, three-body final } \\
\text { state correlations; deduced reaction mechanism features. JOUR } \\
\text { BJPHE } 35888\end{array}$ \\
\hline
\end{tabular}
BJPHE 35888 


\section{$\mathrm{A}=59$ (continued)}

${ }^{59} \mathrm{Ga} \quad$ 2005St29 NUCLEAR REACTIONS ${ }^{9} \mathrm{Be}\left({ }^{78} \mathrm{Kr}, \mathrm{X}\right){ }^{60} \mathrm{Ge} /{ }^{61} \mathrm{Ge} /{ }^{62} \mathrm{Ge} /{ }^{63} \mathrm{Ge} /$ ${ }^{64} \mathrm{Ge} /{ }^{64} \mathrm{Se} /{ }^{65} \mathrm{Se} /{ }^{66} \mathrm{Se} /{ }^{67} \mathrm{Se} /{ }^{68} \mathrm{Se}, \mathrm{E}=140 \mathrm{MeV} /$ nucleon; measured production $\sigma$, isotopic yields; deduced no evidence for ${ }^{59} \mathrm{Ga}$, ${ }^{63}$ As. ${ }^{60} \mathrm{Ge},{ }^{64}$ Se deduced $\mathrm{T}_{1 / 2}$ lower limits. ${ }^{59} \mathrm{Ga},{ }^{63}$ As deduced $\mathrm{T}_{1 / 2}$ upper limits. JOUR PYLBB 62732

2005ST34 NUCLEAR REACTIONS ${ }^{9} \mathrm{Be}\left({ }^{78} \mathrm{Kr}, \mathrm{X}\right){ }^{60} \mathrm{Ge} /{ }^{61} \mathrm{Ge} /{ }^{62} \mathrm{Ge} /{ }^{63} \mathrm{Ge} /$ ${ }^{64} \mathrm{Ge} /{ }^{64} \mathrm{Se} /{ }^{65} \mathrm{Se} /{ }^{66} \mathrm{Se} /{ }^{67} \mathrm{Se} /{ }^{68} \mathrm{Se}, \mathrm{E}=140 \mathrm{MeV} /$ nucleon; measured production $\sigma$, isotopic yields; deduced no evidence for ${ }^{59} \mathrm{Ga}$, ${ }^{63}$ As. JOUR ZAANE 25 s01 335

\section{$\mathrm{A}=60$}

${ }^{60} \mathrm{Cr}$ 2005FR29 NUCLEAR REACTIONS ${ }^{13,14} \mathrm{C}\left({ }^{48} \mathrm{Ca}, 2 \mathrm{p}\right), \mathrm{E}=130 \mathrm{MeV}$; measured $\mathrm{E} \gamma, \mathrm{I} \gamma, \gamma \gamma$-, (recoil) $\gamma$-coin. ${ }^{59,60} \mathrm{Cr}$ deduced levels, J, $\pi$. Gammasphere array, comparison with model predictions. JOUR JPGPE 31 S1465

${ }^{60} \mathrm{Co} \quad 2004 \mathrm{GE} 20$ RADIOACTIVITY ${ }^{155} \mathrm{Sm}\left(\beta^{-}\right)$[from ${ }^{154} \mathrm{Sm}(\mathrm{n}, \gamma)$ ]; ${ }^{60} \mathrm{Co},{ }^{133} \mathrm{Ba},{ }^{152} \mathrm{Eu}$; measured $\gamma$-ray angular correlations. ${ }^{155} \mathrm{Eu},{ }^{60} \mathrm{Ni},{ }^{133} \mathrm{Cs},{ }^{152} \mathrm{Gd}$ transitions deduced $\delta$. Comparison with previous results. JOUR BJPHE 34722

2005SI32 NUCLEAR REACTIONS Cu(n, X $){ }^{54} \mathrm{Mn} /{ }^{59} \mathrm{Fe} /{ }^{56} \mathrm{Co} /{ }^{57} \mathrm{Co} /{ }^{58} \mathrm{Co}$ $/{ }^{60} \mathrm{Co}, \mathrm{E} \approx 70.7,110.8 \mathrm{MeV}$; measured $\sigma$. Comparison with previous results, model predictions. JOUR NIMBE 240617

${ }^{60} \mathrm{Ni} \quad$ 2004GE20 RADIOACTIVITY ${ }^{155} \mathrm{Sm}\left(\beta^{-}\right)\left[\right.$from $\left.{ }^{154} \mathrm{Sm}(\mathrm{n}, \gamma)\right] ;{ }^{60} \mathrm{Co},{ }^{133} \mathrm{Ba},{ }^{152} \mathrm{Eu}$ measured $\gamma$-ray angular correlations. ${ }^{155} \mathrm{Eu},{ }^{60} \mathrm{Ni},{ }^{133} \mathrm{Cs},{ }^{152} \mathrm{Gd}$ transitions deduced $\delta$. Comparison with previous results. JOUR BJPHE 34722

2005WI23 NUCLEAR REACTIONS ${ }^{100} \mathrm{Mo}\left({ }^{11} \mathrm{~B}\right.$, xnypz $\left.\alpha\right){ }^{104} \mathrm{Rh} /{ }^{105} \mathrm{Rh} /{ }^{107} \mathrm{Pd} /$ ${ }^{108} \mathrm{Pd}, \mathrm{E}=43 \mathrm{MeV} ;{ }^{51} \mathrm{~V}\left({ }^{16} \mathrm{O}\right.$, xnypz $\left.\alpha\right){ }^{60} \mathrm{Ni} /{ }^{61} \mathrm{Ni} /{ }^{61} \mathrm{Cu} /{ }^{62} \mathrm{Cu}, \mathrm{E}=70$ $\mathrm{MeV}$; measured $\mathrm{E} \gamma, \mathrm{I} \gamma, \gamma \gamma-$, (charged particle) $\gamma$-coin; deduced $\gamma$-ray yield ratios. Application to exit channel determination discussed. JOUR BJPHE 35898

${ }^{60} \mathrm{Ge} \quad$ 2005ST29 NUCLEAR REACTIONS ${ }^{9} \mathrm{Be}\left({ }^{78} \mathrm{Kr}, \mathrm{X}\right){ }^{60} \mathrm{Ge} /{ }^{61} \mathrm{Ge} /{ }^{62} \mathrm{Ge} /{ }^{63} \mathrm{Ge} /$ ${ }^{64} \mathrm{Ge} /{ }^{64} \mathrm{Se} /{ }^{65} \mathrm{Se} /{ }^{66} \mathrm{Se} /{ }^{67} \mathrm{Se} /{ }^{68} \mathrm{Se}, \mathrm{E}=140 \mathrm{MeV} /$ nucleon; measured production $\sigma$, isotopic yields; deduced no evidence for ${ }^{59} \mathrm{Ga}$, ${ }^{63}$ As. ${ }^{60} \mathrm{Ge},{ }^{64}$ Se deduced $\mathrm{T}_{1 / 2}$ lower limits. ${ }^{59} \mathrm{Ga},{ }^{63}$ As deduced $\mathrm{T}_{1 / 2}$ upper limits. JOUR PYLBB 62732

2005ST34 NUCLEAR REACTIONS ${ }^{9} \mathrm{Be}\left({ }^{78} \mathrm{Kr}, \mathrm{X}\right){ }^{60} \mathrm{Ge} /{ }^{61} \mathrm{Ge} /{ }^{62} \mathrm{Ge} /{ }^{63} \mathrm{Ge} /$ ${ }^{64} \mathrm{Ge} /{ }^{64} \mathrm{Se} /{ }^{65} \mathrm{Se} /{ }^{66} \mathrm{Se} /{ }^{67} \mathrm{Se} /{ }^{68} \mathrm{Se}, \mathrm{E}=140 \mathrm{MeV} /$ nucleon; measured production $\sigma$, isotopic yields; deduced no evidence for ${ }^{59} \mathrm{Ga}$, ${ }^{63}$ As. JOUR ZAANE 25 s01 335 


\section{$\mathrm{A}=61$}

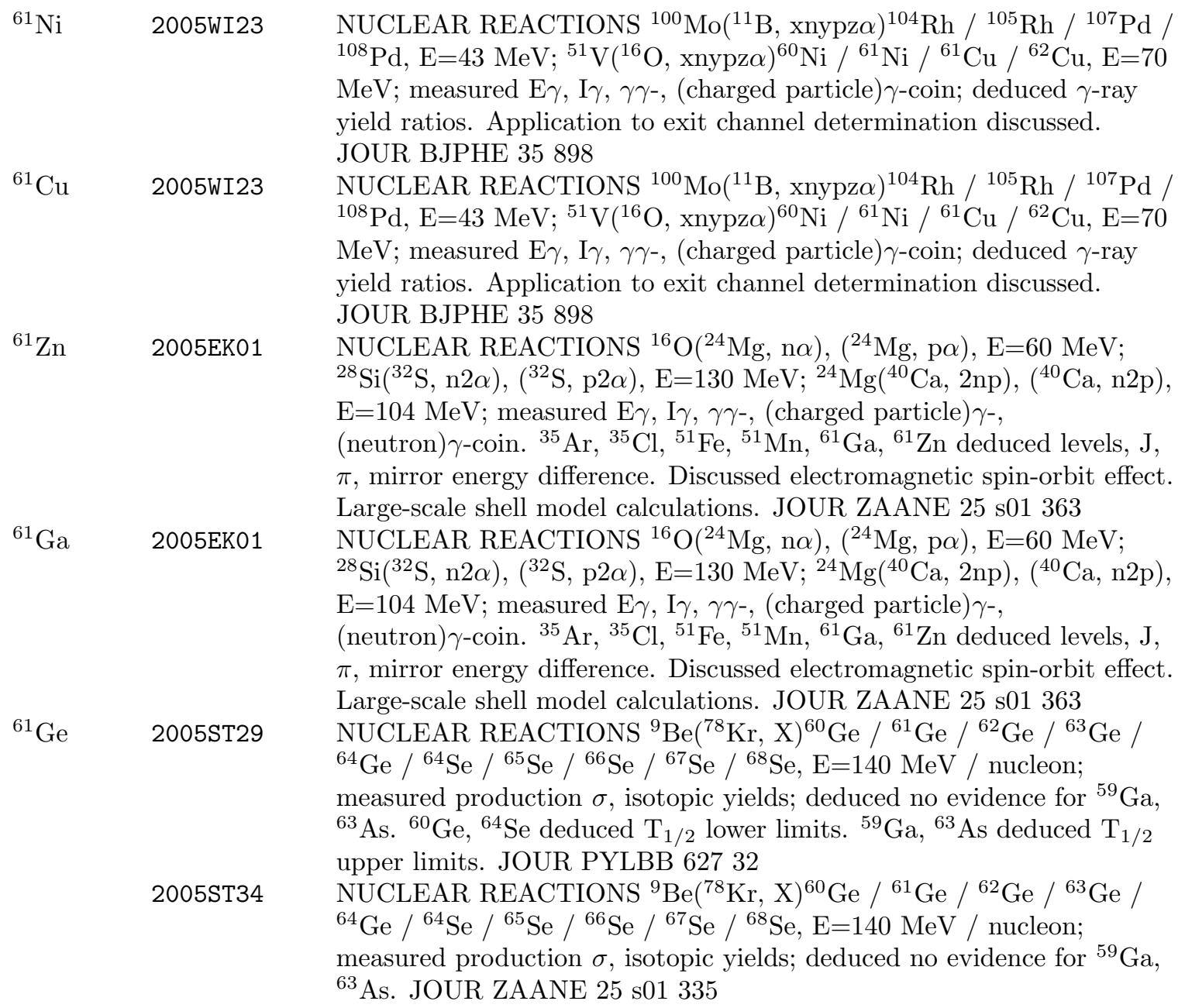

\section{$\mathrm{A}=62$}

\begin{tabular}{|c|c|c|}
\hline${ }^{62} \mathrm{Ni}$ & $2005 \mathrm{~T} 014$ & $\begin{array}{l}\text { NUCLEAR REACTIONS }{ }^{62} \mathrm{Ni}(\nu, \gamma), \mathrm{E}=5.5-90 \mathrm{keV} \text {; measured } \mathrm{E} \gamma \text {, } \\
\text { capture } \sigma \text {; deduced Maxwellian-averaged } \sigma . \text { JOUR ASJOA } 623 \mathrm{~L} 153\end{array}$ \\
\hline \multirow[t]{3}{*}{${ }^{62} \mathrm{Cu}$} & 2005ERZZ & $\begin{array}{l}\text { ATOMIC MASSES }{ }^{62} \mathrm{Ga},{ }^{62} \mathrm{Zn},{ }^{62} \mathrm{Cu} \text {; measured masses. }{ }^{62} \mathrm{Ga} \text { deduced } \\
\text { Q(EC) for superallowed } \beta \text {-decay. Penning trap. PREPRINT } \\
\text { nucl-ex/0512010,12/12/2005 }\end{array}$ \\
\hline & 2005PE23 & $\begin{array}{l}\text { NUCLEAR REACTIONS }{ }^{197} \mathrm{Au}\left({ }^{65} \mathrm{Cu}, \mathrm{X}\right){ }^{62} \mathrm{Cu} /{ }^{63} \mathrm{Cu}, \mathrm{E}=443 \mathrm{MeV} \text {; } \\
\text { measured yields. JOUR ZAANE } 25 \mathrm{~s} 01749\end{array}$ \\
\hline & $2005 W I 23$ & $\begin{array}{l}\text { NUCLEAR REACTIONS }{ }^{100} \mathrm{Mo}\left({ }^{11} \mathrm{~B} \text {, xnypz } \alpha\right){ }^{104} \mathrm{Rh} /{ }^{105} \mathrm{Rh} /{ }^{107} \mathrm{Pd} / \\
{ }^{108} \mathrm{Pd}, \mathrm{E}=43 \mathrm{MeV} ;{ }^{51} \mathrm{~V}\left({ }^{16} \mathrm{O} \text {, xnypz } \alpha\right)^{60} \mathrm{Ni} /{ }^{61} \mathrm{Ni} /{ }^{61} \mathrm{Cu} /{ }^{62} \mathrm{Cu}, \mathrm{E}=70 \\
\mathrm{MeV} \text {; measured } \mathrm{E} \gamma, \mathrm{I} \gamma, \gamma \gamma-\text { (charged particle }) \gamma \text {-coin; deduced } \gamma \text {-ray } \\
\text { yield ratios. Application to exit channel determination discussed. } \\
\text { JOUR BJPHE } 35898\end{array}$ \\
\hline
\end{tabular}
JOUR BJPHE 35898 


\section{$\mathrm{A}=62$ (continued)}

\begin{tabular}{|c|c|c|}
\hline \multirow[t]{2}{*}{${ }^{62} \mathrm{Zn}$} & 2005ERZZ & $\begin{array}{l}\text { ATOMIC MASSES }{ }^{62} \mathrm{Ga},{ }^{62} \mathrm{Zn},{ }^{62} \mathrm{Cu} \text {; measured masses. }{ }^{62} \mathrm{Ga} \text { deduced } \\
\text { Q(EC) for superallowed } \beta \text {-decay. Penning trap. PREPRINT } \\
\text { nucl-ex/0512010,12/12/2005 }\end{array}$ \\
\hline & 2005 HYO4 & $\begin{array}{l}\text { RADIOACTIVITY }{ }^{62} \mathrm{Ga}\left(\beta^{+}\right) ; \text {measured } \mathrm{T}_{1 / 2} . \text { Comparison with } \\
\text { previous results. JOUR JPGPE } 31 \mathrm{~S} 1885\end{array}$ \\
\hline \multirow[t]{2}{*}{${ }^{62} \mathrm{Ga}$} & 2005ERZZ & $\begin{array}{l}\text { ATOMIC MASSES }{ }^{62} \mathrm{Ga},{ }^{62} \mathrm{Zn},{ }^{62} \mathrm{Cu} \text {; measured masses. }{ }^{62} \mathrm{Ga} \text { deduced } \\
\text { Q(EC) for superallowed } \beta \text {-decay. Penning trap. PREPRINT } \\
\text { nucl-ex/0512010,12/12/2005 }\end{array}$ \\
\hline & 2005HYO4 & $\begin{array}{l}\text { RADIOACTIVITY }{ }^{62} \mathrm{Ga}\left(\beta^{+}\right) \text {; measured } \mathrm{T}_{1 / 2} \text {. Comparison with } \\
\text { previous results. JOUR JPGPE } 31 \mathrm{~S} 1885\end{array}$ \\
\hline \multirow[t]{2}{*}{${ }^{62} \mathrm{Ge}$} & 2005ST29 & $\begin{array}{l}\text { NUCLEAR REACTIONS }{ }^{9} \mathrm{Be}\left({ }^{78} \mathrm{Kr}, \mathrm{X}\right){ }^{60} \mathrm{Ge} /{ }^{61} \mathrm{Ge} /{ }^{62} \mathrm{Ge} /{ }^{63} \mathrm{Ge} / \\
{ }^{64} \mathrm{Ge} /{ }^{64} \mathrm{Se} /{ }^{65} \mathrm{Se} /{ }^{66} \mathrm{Se} /{ }^{67} \mathrm{Se} /{ }^{68} \mathrm{Se}, \mathrm{E}=140 \mathrm{MeV} / \text { nucleon; } \\
\text { measured production } \sigma \text {, isotopic yields; deduced no evidence for }{ }^{59} \mathrm{Ga} \text {, } \\
{ }^{63} \text { As. }{ }^{60} \mathrm{Ge},{ }^{64} \mathrm{Se} \text { deduced } \mathrm{T}_{1 / 2} \text { lower limits. }{ }^{59} \mathrm{Ga},{ }^{63} \text { As deduced } \mathrm{T}_{1 / 2} \\
\text { upper limits. JOUR PYLBB } 62732\end{array}$ \\
\hline & 2005ST34 & $\begin{array}{l}\text { NUCLEAR REACTIONS }{ }^{9} \mathrm{Be}\left({ }^{78} \mathrm{Kr}, \mathrm{X}\right){ }^{60} \mathrm{Ge} /{ }^{61} \mathrm{Ge} /{ }^{62} \mathrm{Ge} /{ }^{63} \mathrm{Ge} / \\
{ }^{64} \mathrm{Ge} /{ }^{64} \mathrm{Se} /{ }^{65} \mathrm{Se} /{ }^{66} \mathrm{Se} /{ }^{67} \mathrm{Se} /{ }^{68} \mathrm{Se}, \mathrm{E}=140 \mathrm{MeV} / \text { nucleon; } \\
\text { measured production } \sigma \text {, isotopic yields; deduced no evidence for }{ }^{59} \mathrm{Ga} \text {, } \\
{ }^{63} \text { As. JOUR ZAANE } 25 \text { s01 } 335\end{array}$ \\
\hline
\end{tabular}

\section{$\mathrm{A}=63$}

${ }^{63} \mathrm{Ni} \quad$ 2005GE09 NUCLEAR REACTIONS ${ }^{62,64} \mathrm{Ni}(\mathrm{d}, \mathrm{p})$, E not given; measured $\mathrm{E} \gamma$, $\mathrm{I} \gamma(\theta, \mathrm{H}, \mathrm{t}) .{ }^{65} \mathrm{Ni}$ deduced isomeric state $\mathrm{g}$ factor. Time dependent perturbed angular correlation technique, comparison with model predictions. JOUR JPGPE 31 S1439

2005PE23 RADIOACTIVITY ${ }^{63} \mathrm{Cu}(\mathrm{EC})\left[\right.$ from $\left.{ }^{197} \mathrm{Au}\left({ }^{65} \mathrm{Cu}, \mathrm{X}\right)\right]$; measured $\beta$-delayed E $\gamma$, I $\gamma$. JOUR ZAANE 25 s01 749

2005SE23 NUCLEAR REACTIONS ${ }^{197} \mathrm{Au}(\mathrm{n}, \gamma), \mathrm{E}=$ spectrum; measured $\mathrm{E} \gamma, \mathrm{I} \gamma$; deduced neutron flux. ${ }^{7} \operatorname{Li}(\mathrm{p}, \mathrm{n})$, E not given; deduced neutron spectrum. ${ }^{62} \mathrm{Ni}(\mathrm{n}, \gamma), \mathrm{E} \approx 5.5-20 \mathrm{keV}$; measured $\sigma$; deduced Maxwellian-averaged $\sigma$. JOUR JUPSA 742981

${ }^{63} \mathrm{Cu}$ 2005PE23 RADIOACTIVITY ${ }^{63} \mathrm{Cu}(\mathrm{EC})\left[\right.$ from $\left.{ }^{197} \mathrm{Au}\left({ }^{65} \mathrm{Cu}, \mathrm{X}\right)\right] ;$ measured $\beta$-delayed $\mathrm{E} \gamma, \mathrm{I} \gamma$. JOUR ZAANE 25 s01 749

2005PE23 NUCLEAR REACTIONS ${ }^{197} \mathrm{Au}\left({ }^{65} \mathrm{Cu}, \mathrm{X}\right){ }^{62} \mathrm{Cu} /{ }^{63} \mathrm{Cu}, \mathrm{E}=443 \mathrm{MeV}$; measured yields. JOUR ZAANE 25 s01 749

${ }^{63} \mathrm{Ge} \quad$ 2005St29 NUCLEAR REACTIONS ${ }^{9} \mathrm{Be}\left({ }^{78} \mathrm{Kr}, \mathrm{X}\right){ }^{60} \mathrm{Ge} /{ }^{61} \mathrm{Ge} /{ }^{62} \mathrm{Ge} /{ }^{63} \mathrm{Ge} /$ ${ }^{64} \mathrm{Ge} /{ }^{64} \mathrm{Se} /{ }^{65} \mathrm{Se} /{ }^{66} \mathrm{Se} /{ }^{67} \mathrm{Se} /{ }^{68} \mathrm{Se}, \mathrm{E}=140 \mathrm{MeV} /$ nucleon; measured production $\sigma$, isotopic yields; deduced no evidence for ${ }^{59} \mathrm{Ga}$, ${ }^{63}$ As. ${ }^{60} \mathrm{Ge},{ }^{64}$ Se deduced $\mathrm{T}_{1 / 2}$ lower limits. ${ }^{59} \mathrm{Ga},{ }^{63}$ As deduced $\mathrm{T}_{1 / 2}$ upper limits. JOUR PYLBB 62732

2005ST34 NUCLEAR REACTIONS ${ }^{9} \mathrm{Be}\left({ }^{78} \mathrm{Kr}, \mathrm{X}\right){ }^{60} \mathrm{Ge} /{ }^{61} \mathrm{Ge} /{ }^{62} \mathrm{Ge} /{ }^{63} \mathrm{Ge} /$ ${ }^{64} \mathrm{Ge} /{ }^{64} \mathrm{Se} /{ }^{65} \mathrm{Se} /{ }^{66} \mathrm{Se} /{ }^{67} \mathrm{Se} /{ }^{68} \mathrm{Se}, \mathrm{E}=140 \mathrm{MeV} /$ nucleon; measured production $\sigma$, isotopic yields; deduced no evidence for ${ }^{59} \mathrm{Ga}$, ${ }^{63}$ As. JOUR ZAANE 25 s01 335 


\section{$\mathrm{A}=63$ (continued)}

${ }^{63} \mathrm{As}$

2005ST29

2005ST34

2005P017

2005SHZS

2005SZ04

${ }^{64} \mathrm{Zn}$

2005 CH60

2005LE38

${ }^{64} \mathrm{Ga}$

$2005 \mathrm{CH} 60$

${ }^{64} \mathrm{Ge} \quad$ 2005CL08

2005ST29
NUCLEAR REACTIONS ${ }^{9} \mathrm{Be}\left({ }^{78} \mathrm{Kr}, \mathrm{X}\right){ }^{60} \mathrm{Ge} /{ }^{61} \mathrm{Ge} /{ }^{62} \mathrm{Ge} /{ }^{63} \mathrm{Ge} /$ ${ }^{64} \mathrm{Ge} /{ }^{64} \mathrm{Se} /{ }^{65} \mathrm{Se} /{ }^{66} \mathrm{Se} /{ }^{67} \mathrm{Se} /{ }^{68} \mathrm{Se}, \mathrm{E}=140 \mathrm{MeV} /$ nucleon; measured production $\sigma$, isotopic yields; deduced no evidence for ${ }^{59} \mathrm{Ga}$, ${ }^{63}$ As. ${ }^{60} \mathrm{Ge},{ }^{64}$ Se deduced $\mathrm{T}_{1 / 2}$ lower limits. ${ }^{59} \mathrm{Ga},{ }^{63}$ As deduced $\mathrm{T}_{1 / 2}$ upper limits. JOUR PYLBB 62732

NUCLEAR REACTIONS ${ }^{9} \mathrm{Be}\left({ }^{78} \mathrm{Kr}, \mathrm{X}\right){ }^{60} \mathrm{Ge} /{ }^{61} \mathrm{Ge} /{ }^{62} \mathrm{Ge} /{ }^{63} \mathrm{Ge} /$ ${ }^{64} \mathrm{Ge} /{ }^{64} \mathrm{Se} /{ }^{65} \mathrm{Se} /{ }^{66} \mathrm{Se} /{ }^{67} \mathrm{Se} /{ }^{68} \mathrm{Se}, \mathrm{E}=140 \mathrm{MeV} /$ nucleon; measured production $\sigma$, isotopic yields; deduced no evidence for ${ }^{59} \mathrm{Ga}$, ${ }^{63}$ As. JOUR ZAANE 25 s01 335

\section{$\mathrm{A}=64$}

NUCLEAR REACTIONS ${ }^{103} \mathrm{Rh}\left(\mathrm{n}, \mathrm{n}^{\prime}\right){ }^{103 m} \mathrm{Rh}, \mathrm{E} \approx 4.8 \mathrm{MeV} ;{ }^{115} \mathrm{In}(\mathrm{n}$, $\left.\mathrm{n}^{\prime}\right)^{115 m} \mathrm{In}, \mathrm{E} \approx 5 \mathrm{MeV} ;{ }^{232} \mathrm{Th},{ }^{238} \mathrm{U}(\mathrm{n}, \mathrm{F}), \mathrm{E} \approx 5 \mathrm{MeV} ;{ }^{24} \mathrm{Mg},{ }^{27} \mathrm{Al}$, ${ }^{46,47,48} \mathrm{Ti},{ }^{54,56} \mathrm{Fe},{ }^{58} \mathrm{Ni},{ }^{64} \mathrm{Zn}(\mathrm{n}, \mathrm{p}), \mathrm{E} \approx 2-8 \mathrm{MeV} ;{ }^{27} \mathrm{Al},{ }^{59} \mathrm{Co}(\mathrm{n}, \alpha), \mathrm{E}$ $\approx 8.3 \mathrm{MeV}$; measured activation $\sigma$. Spectrum average technique, comparison with previous results. JOUR RAACA 9263 NUCLEAR REACTIONS ${ }^{64} \mathrm{Ni}\left({ }^{3} \mathrm{He}, \mathrm{t}\right), \mathrm{E}=140 \mathrm{MeV} /$ nucleon; measured triton spectra, $\sigma(\theta) .{ }^{64} \mathrm{Cu}$ deduced levels, J, $\pi$, Gamow-Teller strength distribution. JOUR JPGPE 31 S1945 NUCLEAR REACTIONS ${ }^{65} \mathrm{Cu}\left({ }^{6} \mathrm{Li}, \mathrm{d} \alpha\right),\left({ }^{6} \mathrm{Li},{ }^{7} \mathrm{Li}\right),\left({ }^{6} \mathrm{Li},{ }^{3} \mathrm{He}\right),\left({ }^{6} \mathrm{Li}\right.$, $\alpha),\left({ }^{6} \mathrm{Li}, \alpha \mathrm{X}\right),\left({ }^{7} \mathrm{Li}, \mathrm{t} \alpha\right),\left({ }^{7} \mathrm{Li}, \mathrm{d} \alpha\right),\left({ }^{7} \mathrm{Li},{ }^{6} \mathrm{Li}\right),\left({ }^{7} \mathrm{Li},{ }^{6} \mathrm{He}\right),\left({ }^{7} \mathrm{Li}, \alpha\right),\left({ }^{7} \mathrm{Li}\right.$, $\alpha \mathrm{X}), \mathrm{E}=25 \mathrm{MeV}$; measured particle spectra, $\sigma, \sigma(\theta)$; deduced reaction mechanism features. Comparison with coupled channels predictions. PREPRINT nucl-ex/0512032,12/21/2005 NUCLEAR REACTIONS Zn, ${ }^{68} \mathrm{Zn}(\mathrm{p}, \mathrm{X}){ }^{64} \mathrm{Cu}, \mathrm{E} \approx 18-100 \mathrm{MeV}$; ${ }^{66} \mathrm{Zn}(\mathrm{p}, \mathrm{n} 2 \mathrm{p}), \mathrm{E} \approx 35-100 \mathrm{MeV}$; measured production $\sigma$. Stacked-foil activation technique. JOUR NIMBE 240625 ATOMIC MASSES ${ }^{64} \mathrm{Zn},{ }^{64} \mathrm{Ga},{ }^{68} \mathrm{Ge},{ }^{68} \mathrm{As},{ }^{68,72} \mathrm{Se},{ }^{76} \mathrm{Kr},{ }^{76} \mathrm{Rb},{ }^{80} \mathrm{Sr}$, ${ }^{80} \mathrm{Y}$; measured masses. Direct time-of-flight technique, comparison with previous results. JOUR JPGPE 31 S1771

NUCLEAR REACTIONS C( ${ }^{68} \mathrm{Zn},{ }^{68} \mathrm{Zn}$ '), E=180 MeV; measured E $\gamma$, $\mathrm{I} \gamma(\theta, \mathrm{H}, \mathrm{t})$ (particle) $\gamma$-coin following projectile Coulomb excitation.

${ }^{68} \mathrm{Zn}$ levels deduced $\mathrm{g}$ factors, $\mathrm{T}_{1 / 2}, \mathrm{~B}(\mathrm{E} 2)$, configurations. ${ }^{64} \mathrm{Zn}$ levels analyzed $\mathrm{g}$ factors, B(E2). Large-scale shell-model calculations. JOUR PRVCA 72044301

ATOMIC MASSES ${ }^{64} \mathrm{Zn},{ }^{64} \mathrm{Ga},{ }^{68} \mathrm{Ge},{ }^{68} \mathrm{As},{ }^{68,72} \mathrm{Se},{ }^{76} \mathrm{Kr},{ }^{76} \mathrm{Rb},{ }^{80} \mathrm{Sr}$, ${ }^{80} \mathrm{Y}$; measured masses. Direct time-of-flight technique, comparison with previous results. JOUR JPGPE 31 S1771

ATOMIC MASSES ${ }^{64} \mathrm{Ge},{ }^{68} \mathrm{Se}$; analyzed masses; deduced effective $\mathrm{T}_{1 / 2} \cdot{ }^{90,91} \mathrm{Mo},{ }^{90,91,92,93} \mathrm{Tc},{ }^{93,94} \mathrm{Ru},{ }^{94,95} \mathrm{Rh},{ }^{104,105,106,107} \mathrm{In}$, $104,105,107,108 \mathrm{Sn},{ }^{107,108} \mathrm{Sb}$; measured masses. Penning trap, astrophysical implications discussed. JOUR ZAANE 25 s01 629 NUCLEAR REACTIONS ${ }^{9} \mathrm{Be}\left({ }^{78} \mathrm{Kr}, \mathrm{X}\right){ }^{60} \mathrm{Ge} /{ }^{61} \mathrm{Ge} /{ }^{62} \mathrm{Ge} /{ }^{63} \mathrm{Ge} /$ ${ }^{64} \mathrm{Ge} /{ }^{64} \mathrm{Se} /{ }^{65} \mathrm{Se} /{ }^{66} \mathrm{Se} /{ }^{67} \mathrm{Se} /{ }^{68} \mathrm{Se}, \mathrm{E}=140 \mathrm{MeV} /$ nucleon; measured production $\sigma$, isotopic yields; deduced no evidence for ${ }^{59} \mathrm{Ga}$, ${ }^{63}$ As. ${ }^{60} \mathrm{Ge},{ }^{64}$ Se deduced $\mathrm{T}_{1 / 2}$ lower limits. ${ }^{59} \mathrm{Ga},{ }^{63}$ As deduced $\mathrm{T}_{1 / 2}$ upper limits. JOUR PYLBB 62732 


\section{$\mathrm{A}=64$ (continued)}

2005ST34 NUCLEAR REACTIONS ${ }^{9} \mathrm{Be}\left({ }^{78} \mathrm{Kr}, \mathrm{X}\right){ }^{60} \mathrm{Ge} /{ }^{61} \mathrm{Ge} /{ }^{62} \mathrm{Ge} /{ }^{63} \mathrm{Ge} /$ ${ }^{64} \mathrm{Ge} /{ }^{64} \mathrm{Se} /{ }^{65} \mathrm{Se} /{ }^{66} \mathrm{Se} /{ }^{67} \mathrm{Se} /{ }^{68} \mathrm{Se}, \mathrm{E}=140 \mathrm{MeV} /$ nucleon; measured production $\sigma$, isotopic yields; deduced no evidence for ${ }^{59} \mathrm{Ga}$, ${ }^{63}$ As. JOUR ZAANE 25 s01 335

${ }^{64} \mathrm{Se} \quad 2005 \mathrm{ST} 29$ NUCLEAR REACTIONS ${ }^{9} \mathrm{Be}\left({ }^{78} \mathrm{Kr}, \mathrm{X}\right){ }^{60} \mathrm{Ge} /{ }^{61} \mathrm{Ge} /{ }^{62} \mathrm{Ge} /{ }^{63} \mathrm{Ge} /$ ${ }^{64} \mathrm{Ge} /{ }^{64} \mathrm{Se} /{ }^{65} \mathrm{Se} /{ }^{66} \mathrm{Se} /{ }^{67} \mathrm{Se} /{ }^{68} \mathrm{Se}, \mathrm{E}=140 \mathrm{MeV} /$ nucleon; measured production $\sigma$, isotopic yields; deduced no evidence for ${ }^{59} \mathrm{Ga}$, ${ }^{63}$ As. ${ }^{60} \mathrm{Ge},{ }^{64}$ Se deduced $\mathrm{T}_{1 / 2}$ lower limits. ${ }^{59} \mathrm{Ga},{ }^{63}$ As deduced $\mathrm{T}_{1 / 2}$ upper limits. JOUR PYLBB 62732

2005ST34 NUCLEAR REACTIONS ${ }^{9} \mathrm{Be}\left({ }^{78} \mathrm{Kr}, \mathrm{X}\right){ }^{60} \mathrm{Ge} /{ }^{61} \mathrm{Ge} /{ }^{62} \mathrm{Ge} /{ }^{63} \mathrm{Ge} /$ ${ }^{64} \mathrm{Ge} /{ }^{64} \mathrm{Se} /{ }^{65} \mathrm{Se} /{ }^{66} \mathrm{Se} /{ }^{67} \mathrm{Se} /{ }^{68} \mathrm{Se}, \mathrm{E}=140 \mathrm{MeV} /$ nucleon; measured production $\sigma$, isotopic yields; deduced no evidence for ${ }^{59} \mathrm{Ga}$, ${ }^{63}$ As. JOUR ZAANE 25 s01 335

\section{$\mathrm{A}=65$}

${ }^{65} \mathrm{Ni} \quad$ 2005GE09

2005SHZS

2006B001

${ }^{65} \mathrm{Zn}$

2006B001

${ }^{65} \mathrm{Se}$

2005ST29

2005ST34

\section{$\mathrm{A}=66$}

${ }^{66} \mathrm{Cu} \quad$ 2005SHZS
NUCLEAR REACTIONS ${ }^{62,64} \mathrm{Ni}(\mathrm{d}, \mathrm{p})$, E not given; measured $\mathrm{E} \gamma$, $\mathrm{I} \gamma(\theta, \mathrm{H}, \mathrm{t}) .{ }^{65} \mathrm{Ni}$ deduced isomeric state $\mathrm{g}$ factor. Time dependent perturbed angular correlation technique, comparison with model predictions. JOUR JPGPE 31 S1439

NUCLEAR REACTIONS ${ }^{65} \mathrm{Cu}\left({ }^{6} \mathrm{Li}, \mathrm{d} \alpha\right),\left({ }^{6} \mathrm{Li},{ }^{7} \mathrm{Li}\right),\left({ }^{6} \mathrm{Li},{ }^{3} \mathrm{He}\right),\left({ }^{6} \mathrm{Li}\right.$, $\alpha),\left({ }^{6} \mathrm{Li}, \alpha \mathrm{X}\right),\left({ }^{7} \mathrm{Li}, \mathrm{t} \alpha\right),\left({ }^{7} \mathrm{Li}, \mathrm{d} \alpha\right),\left({ }^{7} \mathrm{Li},{ }^{6} \mathrm{Li}\right),\left({ }^{7} \mathrm{Li},{ }^{6} \mathrm{He}\right),\left({ }^{7} \mathrm{Li}, \alpha\right),\left({ }^{7} \mathrm{Li}\right.$, $\alpha \mathrm{X}), \mathrm{E}=25 \mathrm{MeV}$; measured particle spectra, $\sigma, \sigma(\theta)$; deduced reaction mechanism features. Comparison with coupled channels predictions. PREPRINT nucl-ex/0512032,12/21/2005 RADIOACTIVITY ${ }^{54} \mathrm{Mn},{ }^{65} \mathrm{Zn}(\mathrm{EC})$; measured $\beta \gamma$-coin. Triple to double coincidence ratio method. JOUR ARISE 64124

RADIOACTIVITY ${ }^{54} \mathrm{Mn},{ }^{65} \mathrm{Zn}(\mathrm{EC})$; measured $\beta \gamma$-coin. Triple to double coincidence ratio method. JOUR ARISE 64124 NUCLEAR REACTIONS ${ }^{9} \mathrm{Be}\left({ }^{78} \mathrm{Kr}, \mathrm{X}\right){ }^{60} \mathrm{Ge} /{ }^{61} \mathrm{Ge} /{ }^{62} \mathrm{Ge} /{ }^{63} \mathrm{Ge} /$ ${ }^{64} \mathrm{Ge} /{ }^{64} \mathrm{Se} /{ }^{65} \mathrm{Se} /{ }^{66} \mathrm{Se} /{ }^{67} \mathrm{Se} /{ }^{68} \mathrm{Se}, \mathrm{E}=140 \mathrm{MeV} /$ nucleon; measured production $\sigma$, isotopic yields; deduced no evidence for ${ }^{59} \mathrm{Ga}$, ${ }^{63}$ As. ${ }^{60} \mathrm{Ge},{ }^{64}$ Se deduced $\mathrm{T}_{1 / 2}$ lower limits. ${ }^{59} \mathrm{Ga},{ }^{63}$ As deduced $\mathrm{T}_{1 / 2}$ upper limits. JOUR PYLBB 62732

NUCLEAR REACTIONS ${ }^{9} \mathrm{Be}\left({ }^{78} \mathrm{Kr}, \mathrm{X}\right){ }^{60} \mathrm{Ge} /{ }^{61} \mathrm{Ge} /{ }^{62} \mathrm{Ge} /{ }^{63} \mathrm{Ge} /$ ${ }^{64} \mathrm{Ge} /{ }^{64} \mathrm{Se} /{ }^{65} \mathrm{Se} /{ }^{66} \mathrm{Se} /{ }^{67} \mathrm{Se} /{ }^{68} \mathrm{Se}, \mathrm{E}=140 \mathrm{MeV} / \mathrm{nucleon} ;$ measured production $\sigma$, isotopic yields; deduced no evidence for ${ }^{59} \mathrm{Ga}$, ${ }^{63}$ As. JOUR ZAANE 25 s01 335

NUCLEAR REACTIONS ${ }^{65} \mathrm{Cu}\left({ }^{6} \mathrm{Li}, \mathrm{d} \alpha\right),\left({ }^{6} \mathrm{Li},{ }^{7} \mathrm{Li}\right),\left({ }^{6} \mathrm{Li},{ }^{3} \mathrm{He}\right),\left({ }^{6} \mathrm{Li}\right.$, $\alpha),\left({ }^{6} \mathrm{Li}, \alpha \mathrm{X}\right),\left({ }^{7} \mathrm{Li}, \mathrm{t} \alpha\right),\left({ }^{7} \mathrm{Li}, \mathrm{d} \alpha\right),\left({ }^{7} \mathrm{Li},{ }^{6} \mathrm{Li}\right),\left({ }^{7} \mathrm{Li},{ }^{6} \mathrm{He}\right),\left({ }^{7} \mathrm{Li}, \alpha\right),\left({ }^{7} \mathrm{Li}\right.$, $\alpha \mathrm{X}), \mathrm{E}=25 \mathrm{MeV}$; measured particle spectra, $\sigma, \sigma(\theta)$; deduced reaction mechanism features. Comparison with coupled channels predictions. PREPRINT nucl-ex/0512032,12/21/2005 


\section{$\mathrm{A}=66$ (continued)}

\begin{tabular}{|c|c|c|}
\hline${ }^{66} \mathrm{Zn}$ & 2005SHZS & $\begin{array}{l}\text { NUCLEAR REACTIONS }{ }^{65} \mathrm{Cu}\left({ }^{6} \mathrm{Li}, \mathrm{d} \alpha\right),\left({ }^{6} \mathrm{Li},{ }^{7} \mathrm{Li}\right),\left({ }^{6} \mathrm{Li},{ }^{3} \mathrm{He}\right),\left({ }^{6} \mathrm{Li} \text {, }\right. \\
\alpha),\left({ }^{6} \mathrm{Li}, \alpha \mathrm{X}\right),\left({ }^{7} \mathrm{Li}, \mathrm{t} \alpha\right),\left({ }^{7} \mathrm{Li}, \mathrm{d} \alpha\right),\left({ }^{7} \mathrm{Li},{ }^{6} \mathrm{Li}\right),\left({ }^{7} \mathrm{Li},{ }^{6} \mathrm{He}\right),\left({ }^{7} \mathrm{Li}, \alpha\right),\left({ }^{7} \mathrm{Li} \text {, }\right. \\
\alpha \mathrm{X}), \mathrm{E}=25 \mathrm{MeV} \text {; measured particle spectra, } \sigma, \sigma(\theta) ; \text { deduced reaction } \\
\text { mechanism features. Comparison with coupled channels predictions. } \\
\text { PREPRINT nucl-ex/0512032,12/21/2005 }\end{array}$ \\
\hline${ }^{66} \mathrm{Se}$ & 2005ST29 & $\begin{array}{l}\text { NUCLEAR REACTIONS }{ }^{9} \mathrm{Be}\left({ }^{78} \mathrm{Kr}, \mathrm{X}\right){ }^{60} \mathrm{Ge} /{ }^{61} \mathrm{Ge} /{ }^{62} \mathrm{Ge} /{ }^{63} \mathrm{Ge} / \\
{ }^{64} \mathrm{Ge} /{ }^{64} \mathrm{Se} /{ }^{65} \mathrm{Se} /{ }^{66} \mathrm{Se} /{ }^{67} \mathrm{Se} /{ }^{68} \mathrm{Se}, \mathrm{E}=140 \mathrm{MeV} / \text { nucleon; } \\
\text { measured production } \sigma \text {, isotopic yields; deduced no evidence for }{ }^{59} \mathrm{Ga} \text {, } \\
{ }^{63} \text { As. }{ }^{60} \mathrm{Ge},{ }^{64} \mathrm{Se} \text { deduced } \mathrm{T}_{1 / 2} \text { lower limits. }{ }^{59} \mathrm{Ga},{ }^{63} \mathrm{As} \text { deduced } \mathrm{T}_{1 / 2} \\
\text { upper limits. JOUR PYLBB } 62732\end{array}$ \\
\hline & 2005ST34 & $\begin{array}{l}\text { NUCLEAR REACTIONS }{ }^{9} \mathrm{Be}\left({ }^{78} \mathrm{Kr}, \mathrm{X}\right){ }^{60} \mathrm{Ge} /{ }^{61} \mathrm{Ge} /{ }^{62} \mathrm{Ge} /{ }^{63} \mathrm{Ge} / \\
{ }^{64} \mathrm{Ge} /{ }^{64} \mathrm{Se} /{ }^{65} \mathrm{Se} /{ }^{66} \mathrm{Se} /{ }^{67} \mathrm{Se} /{ }^{68} \mathrm{Se}, \mathrm{E}=140 \mathrm{MeV} / \text { nucleon; } \\
\text { measured production } \sigma \text {, isotopic yields; deduced no evidence for }{ }^{59} \mathrm{Ga} \text {, } \\
{ }^{63} \text { As. JOUR ZAANE } 25 \text { s01 } 335\end{array}$ \\
\hline
\end{tabular}

\section{$\mathrm{A}=67$}

${ }^{67} \mathrm{Zn}$ 2005SHZS NUCLEAR REACTIONS ${ }^{65} \mathrm{Cu}\left({ }^{6} \mathrm{Li}, \mathrm{d} \alpha\right),\left({ }^{6} \mathrm{Li},{ }^{7} \mathrm{Li}\right),\left({ }^{6} \mathrm{Li},{ }^{3} \mathrm{He}\right),\left({ }^{6} \mathrm{Li}\right.$, $\alpha),\left({ }^{6} \mathrm{Li}, \alpha \mathrm{X}\right),\left({ }^{7} \mathrm{Li}, \mathrm{t} \alpha\right),\left({ }^{7} \mathrm{Li}, \mathrm{d} \alpha\right),\left({ }^{7} \mathrm{Li},{ }^{6} \mathrm{Li}\right),\left({ }^{7} \mathrm{Li},{ }^{6} \mathrm{He}\right),\left({ }^{7} \mathrm{Li}, \alpha\right),\left({ }^{7} \mathrm{Li}\right.$, $\alpha \mathrm{X}), \mathrm{E}=25 \mathrm{MeV}$; measured particle spectra, $\sigma, \sigma(\theta)$; deduced reaction mechanism features. Comparison with coupled channels predictions. PREPRINT nucl-ex/0512032,12/21/2005

${ }^{67} \mathrm{Se} \quad$ 2005St29 NUCLEAR REACTIONS ${ }^{9} \mathrm{Be}\left({ }^{78} \mathrm{Kr}, \mathrm{X}\right){ }^{60} \mathrm{Ge} /{ }^{61} \mathrm{Ge} /{ }^{62} \mathrm{Ge} /{ }^{63} \mathrm{Ge} /$ ${ }^{64} \mathrm{Ge} /{ }^{64} \mathrm{Se} /{ }^{65} \mathrm{Se} /{ }^{66} \mathrm{Se} /{ }^{67} \mathrm{Se} /{ }^{68} \mathrm{Se}, \mathrm{E}=140 \mathrm{MeV} /$ nucleon; measured production $\sigma$, isotopic yields; deduced no evidence for ${ }^{59} \mathrm{Ga}$, ${ }^{63}$ As. ${ }^{60} \mathrm{Ge},{ }^{64}$ Se deduced $\mathrm{T}_{1 / 2}$ lower limits. ${ }^{59} \mathrm{Ga},{ }^{63}$ As deduced $\mathrm{T}_{1 / 2}$ upper limits. JOUR PYLBB 62732

2005ST34 NUCLEAR REACTIONS ${ }^{9} \mathrm{Be}\left({ }^{78} \mathrm{Kr}, \mathrm{X}\right){ }^{60} \mathrm{Ge} /{ }^{61} \mathrm{Ge} /{ }^{62} \mathrm{Ge} /{ }^{63} \mathrm{Ge} /$ ${ }^{64} \mathrm{Ge} /{ }^{64} \mathrm{Se} /{ }^{65} \mathrm{Se} /{ }^{66} \mathrm{Se} /{ }^{67} \mathrm{Se} /{ }^{68} \mathrm{Se}, \mathrm{E}=140 \mathrm{MeV} /$ nucleon; measured production $\sigma$, isotopic yields; deduced no evidence for ${ }^{59} \mathrm{Ga}$, ${ }^{63}$ As. JOUR ZAANE 25 s01 335

\section{$\mathrm{A}=68$}

${ }^{68} \mathrm{Zn}$ 2005LE38 NUCLEAR REACTIONS C( $\left.{ }^{68} \mathrm{Zn},{ }^{68} \mathrm{Zn}\right), \mathrm{E}=180 \mathrm{MeV}$; measured E $\gamma$, $\mathrm{I} \gamma(\theta, \mathrm{H}, \mathrm{t})$ (particle) $\gamma$-coin following projectile Coulomb excitation. ${ }^{68} \mathrm{Zn}$ levels deduced $\mathrm{g}$ factors, $\mathrm{T}_{1 / 2}, \mathrm{~B}(\mathrm{E} 2)$, configurations. ${ }^{64} \mathrm{Zn}$ levels analyzed $\mathrm{g}$ factors, $\mathrm{B}(\mathrm{E} 2)$. Large-scale shell-model calculations. JOUR PRVCA 72044301

2005SHZS NUCLEAR REACTIONS ${ }^{65} \mathrm{Cu}\left({ }^{6} \mathrm{Li}, \mathrm{d} \alpha\right),\left({ }^{6} \mathrm{Li},{ }^{7} \mathrm{Li}\right),\left({ }^{6} \mathrm{Li},{ }^{3} \mathrm{He}\right),\left({ }^{6} \mathrm{Li}\right.$, $\alpha),\left({ }^{6} \mathrm{Li}, \alpha \mathrm{X}\right),\left({ }^{7} \mathrm{Li}, \mathrm{t} \alpha\right),\left({ }^{7} \mathrm{Li}, \mathrm{d} \alpha\right),\left({ }^{7} \mathrm{Li},{ }^{6} \mathrm{Li}\right),\left({ }^{7} \mathrm{Li},{ }^{6} \mathrm{He}\right),\left({ }^{7} \mathrm{Li}, \alpha\right),\left({ }^{7} \mathrm{Li}\right.$, $\alpha \mathrm{X}), \mathrm{E}=25 \mathrm{MeV}$; measured particle spectra, $\sigma, \sigma(\theta)$; deduced reaction mechanism features. Comparison with coupled channels predictions. PREPRINT nucl-ex/0512032,12/21/2005

${ }^{68} \mathrm{Ge} \quad 2005 \mathrm{CH} 60 \quad$ ATOMIC MASSES ${ }^{64} \mathrm{Zn},{ }^{64} \mathrm{Ga},{ }^{68} \mathrm{Ge},{ }^{68} \mathrm{As},{ }^{68,72} \mathrm{Se},{ }^{76} \mathrm{Kr},{ }^{76} \mathrm{Rb},{ }^{80} \mathrm{Sr}$, ${ }^{80} \mathrm{Y}$; measured masses. Direct time-of-flight technique, comparison with previous results. JOUR JPGPE 31 S1771 


\section{$\mathrm{A}=68$ (continued)}

\begin{tabular}{|c|c|c|}
\hline${ }^{68} \mathrm{As}$ & $2005 \mathrm{CH} 60$ & $\begin{array}{l}\text { ATOMIC MASSES }{ }^{64} \mathrm{Zn},{ }^{64} \mathrm{Ga},{ }^{68} \mathrm{Ge},{ }^{68} \mathrm{As},{ }^{68,72} \mathrm{Se},{ }^{76} \mathrm{Kr},{ }^{76} \mathrm{Rb},{ }^{80} \mathrm{Sr} \text {, } \\
{ }^{80} \mathrm{Y} \text {; measured masses. Direct time-of-flight technique, comparison with } \\
\text { previous results. JOUR JPGPE } 31 \text { S1771 }\end{array}$ \\
\hline \multirow[t]{4}{*}{${ }^{68} \mathrm{Se}$} & $2005 \mathrm{CH} 60$ & $\begin{array}{l}\text { ATOMIC MASSES }{ }^{64} \mathrm{Zn},{ }^{64} \mathrm{Ga},{ }^{68} \mathrm{Ge},{ }^{68} \mathrm{As},{ }^{68,72} \mathrm{Se},{ }^{76} \mathrm{Kr},{ }^{76} \mathrm{Rb},{ }^{80} \mathrm{Sr} \text {, } \\
{ }^{80} \mathrm{Y} \text {; measured masses. Direct time-of-flight technique, comparison with } \\
\text { previous results. JOUR JPGPE } 31 \mathrm{~S} 1771\end{array}$ \\
\hline & 2005CL08 & $\begin{array}{l}\text { ATOMIC MASSES }{ }^{64} \mathrm{Ge},{ }^{68} \mathrm{Se} \text {; analyzed masses; deduced effective } \\
\mathrm{T}_{1 / 2 \cdot} \cdot{ }^{90,91} \mathrm{Mo},{ }^{90,91,92,93} \mathrm{Tc},{ }^{93,94} \mathrm{Ru},{ }^{94,95} \mathrm{Rh},{ }^{104,105,106,107} \mathrm{In}, \\
104,105,107,108 \mathrm{Sn},{ }^{107,108} \mathrm{Sb} \text {; measured masses. Penning trap, }\end{array}$ \\
\hline & 2005ST29 & $\begin{array}{l}\text { astrophysical implications discussed. JOUR ZAANE } 25 \text { s01 } 629 \\
\text { NUCLEAR REACTIONS }{ }^{9} \mathrm{Be}\left({ }^{78} \mathrm{Kr}, \mathrm{X}\right){ }^{60} \mathrm{Ge} /{ }^{61} \mathrm{Ge} /{ }^{62} \mathrm{Ge} /{ }^{63} \mathrm{Ge} / \\
{ }^{64} \mathrm{Ge} /{ }^{64} \mathrm{Se} /{ }^{65} \mathrm{Se} /{ }^{66} \mathrm{Se} /{ }^{67} \mathrm{Se} /{ }^{68} \mathrm{Se}, \mathrm{E}=140 \mathrm{MeV} / \text { nucleon; } \\
\text { measured production } \sigma \text {, isotopic yields; deduced no evidence for }{ }^{59} \mathrm{Ga} \text {, } \\
\text { As. }{ }^{60} \mathrm{Ge},{ }^{64} \mathrm{Se} \text { deduced T } 1 / 2 \text { lower limits. }{ }^{59} \mathrm{Ga},{ }^{63} \mathrm{As} \text { deduced } \mathrm{T}_{1 / 2} \\
\text { upper limits. JOUR PYLBB } 62732\end{array}$ \\
\hline & 2005ST34 & $\begin{array}{l}\text { NUCLEAR REACTIONS }{ }^{9} \mathrm{Be}\left({ }^{78} \mathrm{Kr}, \mathrm{X}\right){ }^{60} \mathrm{Ge} /{ }^{61} \mathrm{Ge} /{ }^{62} \mathrm{Ge} /{ }^{63} \mathrm{Ge} / \\
{ }^{64} \mathrm{Ge} /{ }^{64} \mathrm{Se} /{ }^{65} \mathrm{Se} /{ }^{66} \mathrm{Se} /{ }^{67} \mathrm{Se} /{ }^{68} \mathrm{Se}, \mathrm{E}=140 \mathrm{MeV} / \text { nucleon; } \\
\text { measured production } \sigma \text {, isotopic yields; deduced no evidence for }{ }^{59} \mathrm{Ga} \text {, } \\
{ }^{63} \mathrm{As} \text {. JOUR ZAANE } 25 \text { s01 } 335\end{array}$ \\
\hline
\end{tabular}

\section{$\mathrm{A}=69$}

No references found

\section{$\mathrm{A}=70$}

$\begin{array}{ll}{ }^{70} \mathrm{Ni} & \text { 2005MA95 } \\ { }^{70} \mathrm{Ga} & \text { 2005WA29 }\end{array}$

RADIOACTIVITY ${ }^{71,72,73,74} \mathrm{Co}\left(\beta^{-}\right),\left(\beta^{-} \mathrm{n}\right)$ [from ${ }^{9} \mathrm{Be}\left({ }^{86} \mathrm{Kr}, \mathrm{X}\right)$ ]; measured $\mathrm{E} \gamma, \mathrm{E} \beta, \beta \gamma$-coin; deduced $\beta \mathrm{n}$ branching fraction. 70,71,72,73,74 $\mathrm{Ni}$ deduced levels, J, $\pi$. JOUR ZAANE 25 s01 93 NUCLEAR REACTIONS ${ }^{71} \mathrm{Ga}(\mathrm{n}, 2 \mathrm{n}), \mathrm{E}=13.5,14.1,14.7 \mathrm{MeV}$; measured $\sigma$. Activation technique, comparison with previous results. JOUR PRVCA 72037604

\section{$\mathrm{A}=71$}

$\begin{array}{ll}{ }^{71} \mathrm{Co} & 2005 \text { MA95 } \\ { }^{71} \mathrm{Ni} & 2005 \text { MA95 } \\ { }^{71} \mathrm{Ge} & 2004 \mathrm{HO} 25\end{array}$

RADIOACTIVITY ${ }^{71,72,73,74} \mathrm{Co}\left(\beta^{-}\right),\left(\beta^{-} \mathrm{n}\right)$ [from ${ }^{9} \mathrm{Be}\left({ }^{86} \mathrm{Kr}, \mathrm{X}\right)$; measured $\mathrm{E} \gamma, \mathrm{E} \beta, \beta \gamma$-coin; deduced $\beta \mathrm{n}$ branching fraction. 70,71,72,73,74 $\mathrm{Ni}$ deduced levels, J, $\pi$. JOUR ZAANE 25 s01 93 RADIOACTIVITY ${ }^{71,72,73,74} \mathrm{Co}\left(\beta^{-}\right),\left(\beta^{-} \mathrm{n}\right)$ [from ${ }^{9} \mathrm{Be}\left({ }^{86} \mathrm{Kr}, \mathrm{X}\right)$ ]; measured $\mathrm{E} \gamma, \mathrm{E} \beta, \beta \gamma$-coin; deduced $\beta \mathrm{n}$ branching fraction. 70,71,72,73,74 Ni deduced levels, J, $\pi$. JOUR ZAANE 25 s01 93 NUCLEAR REACTIONS ${ }^{70,73} \mathrm{Ge}(\mathrm{n}, \gamma), \mathrm{E}=$ thermal; measured $\mathrm{E} \gamma, \mathrm{I} \gamma$, $\gamma \gamma$-coin. ${ }^{71,74} \mathrm{Ge}$ deduced transitions, two-quantum cascade intensities. JOUR BRSPE 681324 


\begin{tabular}{|c|c|c|}
\hline & & $\mathrm{A}=72$ \\
\hline${ }^{72} \mathrm{Co}$ & 2005MA95 & $\begin{array}{l}\text { RADIOACTIVITY }{ }^{71,72,73,74} \mathrm{Co}\left(\beta^{-}\right),\left(\beta^{-} \mathrm{n}\right)\left[\text { from }{ }^{9} \mathrm{Be}\left({ }^{86} \mathrm{Kr}, \mathrm{X}\right)\right] \\
\text { measured E } \gamma, \mathrm{E} \beta, \beta \gamma \text {-coin; deduced } \beta \text { branching fraction. } \\
70,71,72,73,74 \mathrm{Ni} \text { deduced levels, J, } \pi \text {. JOUR ZAANE } 25 \text { s01 } 93\end{array}$ \\
\hline${ }^{72} \mathrm{Ni}$ & 2005MA95 & $\begin{array}{l}\text { RADIOACTIVITY }{ }^{71,72,73,74} \mathrm{Co}\left(\beta^{-}\right),\left(\beta^{-} \mathrm{n}\right)\left[\text { from }{ }^{9} \mathrm{Be}\left({ }^{86} \mathrm{Kr}, \mathrm{X}\right)\right] \\
\text { measured } \mathrm{E} \gamma, \mathrm{E} \beta, \beta \gamma \text {-coin; deduced } \beta \mathrm{n} \text { branching fraction. } \\
{ }_{70,71,72,73,74} \mathrm{Ni} \text { deduced levels, J, } \pi \text {. JOUR ZAANE } 25 \mathrm{~s} 0193\end{array}$ \\
\hline${ }^{72} \mathrm{Se}$ & 2005 CH 60 & $\begin{array}{l}\text { ATOMIC MASSES }{ }^{64} \mathrm{Zn},{ }^{64} \mathrm{Ga},{ }^{68} \mathrm{Ge},{ }^{68} \mathrm{As},{ }^{68,72} \mathrm{Se},{ }^{76} \mathrm{Kr},{ }^{76} \mathrm{Rb},{ }^{80} \mathrm{Sr} \text {, } \\
{ }^{80} \mathrm{Y} \text {; measured masses. Direct time-of-flight technique, comparison with } \\
\text { previous results. JOUR JPGPE } 31 \mathrm{~S} 1771\end{array}$ \\
\hline${ }^{72} \mathrm{Kr}$ & 2005R039 & $\begin{array}{l}\text { ATOMIC MASSES }{ }^{72,73,74} \mathrm{Kr},{ }^{73} \mathrm{Rb},{ }^{74} \mathrm{Sr} \text {; measured masses. Penning } \\
\text { trap mass spectrometer. JOUR ZAANE } 25 \text { s01 } 41\end{array}$ \\
\hline
\end{tabular}

\section{$\mathrm{A}=73$}

${ }^{73} \mathrm{Co} \quad$ 2005MA95

${ }^{73} \mathrm{Ni} \quad$ 2005MA95

${ }^{73} \mathrm{Ge} \quad$ 2004VA37

${ }^{73}$ As $\quad 2004$ VA37

${ }^{73} \mathrm{Kr} \quad$ 2005R039

${ }^{73} \mathrm{Rb} \quad 2005 \mathrm{R} 039$

RADIOACTIVITY ${ }^{71,72,73,74} \mathrm{Co}\left(\beta^{-}\right),\left(\beta^{-} \mathrm{n}\right)$ [from ${ }^{9} \mathrm{Be}\left({ }^{86} \mathrm{Kr}, \mathrm{X}\right)$ ]; measured $\mathrm{E} \gamma, \mathrm{E} \beta, \beta \gamma$-coin; deduced $\beta \mathrm{n}$ branching fraction. 70,71,72,73,74 Ni deduced levels, J, $\pi$. JOUR ZAANE 25 s01 93 RADIOACTIVITY ${ }^{71,72,73,74} \mathrm{Co}\left(\beta^{-}\right),\left(\beta^{-} \mathrm{n}\right)$ [from $\left.{ }^{9} \mathrm{Be}\left({ }^{86} \mathrm{Kr}, \mathrm{X}\right)\right]$; measured $\mathrm{E} \gamma, \mathrm{E} \beta, \beta \gamma$-coin; deduced $\beta \mathrm{n}$ branching fraction. 70,71,72,73,74 Ni deduced levels, J, $\pi$. JOUR ZAANE 25 s01 93 RADIOACTIVITY ${ }^{73} \mathrm{Ge}\left(\beta^{-}\right)$; measured $\mathrm{T}_{1 / 2}$ lower limit for charge-nonconserving $\beta$-decay. JOUR BRSPE 681255 RADIOACTIVITY ${ }^{73} \mathrm{Ge}\left(\beta^{-}\right)$; measured $\mathrm{T}_{1 / 2}$ lower limit for charge-nonconserving $\beta$-decay. JOUR BRSPE 681255 ATOMIC MASSES ${ }^{72,73,74} \mathrm{Kr},{ }^{73} \mathrm{Rb},{ }^{74} \mathrm{Sr}$; measured masses. Penning trap mass spectrometer. JOUR ZAANE 25 s01 41 ATOMIC MASSES ${ }^{72,73,74} \mathrm{Kr},{ }^{73} \mathrm{Rb},{ }^{74} \mathrm{Sr}$; measured masses. Penning trap mass spectrometer. JOUR ZAANE 25 s01 41

\section{$\mathrm{A}=74$}

$\begin{array}{ll}{ }^{74} \mathrm{Co} & \text { 2005MA95 } \\ { }^{74} \mathrm{Ni} & 2005 \text { MA95 } \\ { }^{74} \mathrm{Ge} & 2004 \mathrm{HO} 25 \\ & \\ { }^{74} \mathrm{Br} & 2004 \mathrm{CO} 29\end{array}$

RADIOACTIVITY ${ }^{71,72,73,74} \mathrm{Co}\left(\beta^{-}\right),\left(\beta^{-} \mathrm{n}\right)$ [from ${ }^{9} \mathrm{Be}\left({ }^{86} \mathrm{Kr}, \mathrm{X}\right)$; measured $\mathrm{E} \gamma, \mathrm{E} \beta, \beta \gamma$-coin; deduced $\beta \mathrm{n}$ branching fraction. 70,71,72,73,74 Ni deduced levels, J, $\pi$. JOUR ZAANE 25 s01 93 RADIOACTIVITY ${ }^{71,72,73,74} \mathrm{Co}\left(\beta^{-}\right),\left(\beta^{-} \mathrm{n}\right)$ [from ${ }^{9} \mathrm{Be}\left({ }^{86} \mathrm{Kr}, \mathrm{X}\right)$ ]; measured $\mathrm{E} \gamma, \mathrm{E} \beta, \beta \gamma$-coin; deduced $\beta \mathrm{n}$ branching fraction. 70,71,72,73,74 Ni deduced levels, J, $\pi$. JOUR ZAANE 25 s01 93 NUCLEAR REACTIONS ${ }^{70,73} \mathrm{Ge}(\mathrm{n}, \gamma), \mathrm{E}=$ thermal; measured $\mathrm{E} \gamma, \mathrm{I} \gamma$, $\gamma \gamma$-coin. ${ }^{71,74} \mathrm{Ge}$ deduced transitions, two-quantum cascade intensities. JOUR BRSPE 681324 RADIOACTIVITY ${ }^{74} \mathrm{Kr}(\mathrm{EC}),\left(\beta^{+}\right)$[from $\mathrm{Nb}(\mathrm{p}, \mathrm{X})$ ]; measured $\beta \gamma$-coin; deduced Gamow-Teller strength distribution. ${ }^{33} \mathrm{Na}\left(\beta^{-}\right)$, $\left(\beta^{-} \mathrm{n}\right)$ [from $\left.\mathrm{U}(\mathrm{p}, \mathrm{X})\right]$; measured $\beta \gamma-, \mathrm{n} \beta-, \gamma \gamma$-coin, $\mathrm{T}_{1 / 2} \cdot{ }^{33} \mathrm{Mg}$ deduced ground-state $\mathrm{J}, \pi$. Total absorption spectrometer. JOUR BJPHE 34 850 


\section{$\mathrm{A}=74$ (continued)}

${ }^{74} \mathrm{Kr} \quad$ 2004C029 RADIOACTIVITY ${ }^{74} \mathrm{Kr}(\mathrm{EC}),\left(\beta^{+}\right)[$from $\mathrm{Nb}(\mathrm{p}, \mathrm{X})]$; measured $\beta \gamma$-coin; deduced Gamow-Teller strength distribution. ${ }^{33} \mathrm{Na}\left(\beta^{-}\right)$, $\left(\beta^{-} \mathrm{n}\right)$ [from $\left.\mathrm{U}(\mathrm{p}, \mathrm{X})\right]$; measured $\beta \gamma-, \mathrm{n} \beta-, \gamma \gamma$-coin, $\mathrm{T}_{1 / 2} .{ }^{33} \mathrm{Mg}$ deduced ground-state $\mathrm{J}, \pi$. Total absorption spectrometer. JOUR BJPHE 34 850

2005 G043 NUCLEAR REACTIONS ${ }^{40} \mathrm{Ca}\left({ }^{40} \mathrm{Ca}, 2 \mathrm{p} \alpha\right),\left({ }^{40} \mathrm{Ca}, 4 \mathrm{p}\right), \mathrm{E}=147 \mathrm{MeV}$; measured Doppler-shifted $\mathrm{E} \gamma, \mathrm{I} \gamma, \gamma \gamma$-coin. ${ }^{74,76} \mathrm{Kr}$ levels deduced $\mathrm{T}_{1 / 2}$, B(E2). GASP array, recoil-distance technique. JOUR ZAANE 26153 2005R039 ATOMIC MASSES ${ }^{72,73,74} \mathrm{Kr},{ }^{73} \mathrm{Rb},{ }^{74} \mathrm{Sr}$; measured masses. Penning trap mass spectrometer. JOUR ZAANE 25 s01 41 2005VA30 NUCLEAR REACTIONS ${ }^{40} \mathrm{Ca}\left({ }^{40} \mathrm{Ca}, 2 \mathrm{p} \alpha\right), \mathrm{E}=165,185 \mathrm{MeV}$; measured E $\gamma, \mathrm{I} \gamma, \gamma \gamma-$, (charged particle) $\gamma-$, (neutron) $\gamma$-coin, DSA. ${ }^{74} \mathrm{Kr}$ deduced high-spin levels, $\mathrm{J}, \pi, \mathrm{T}_{1 / 2}$, transition quadrupole moments, configurations, nontermination of rotational bands. Euroball III, ISIS, Gammasphere, and Microball arrays. JOUR PRLTA 95232501

${ }^{74} \mathrm{Sr} \quad 2005 \mathrm{R} 039$ ATOMIC MASSES ${ }^{72,73,74} \mathrm{Kr},{ }^{73} \mathrm{Rb},{ }^{74} \mathrm{Sr}$; measured masses. Penning trap mass spectrometer. JOUR ZAANE 25 s01 41

\section{$\mathrm{A}=75$}

${ }^{75} \mathrm{As}$

2005RA29

${ }^{75} \mathrm{Se}$

2005RA29

${ }^{75} \mathrm{Br}$

$2004 \mathrm{SC} 48$

${ }^{76} \mathrm{Ge} \quad 2005$ IW03

${ }^{76} \mathrm{Br}$

$2004 \mathrm{SC} 48$

${ }^{76} \mathrm{Kr}$

2005BE61

2005 CH60

\section{$\mathrm{A}=76$}

RADIOACTIVITY ${ }^{75} \mathrm{Se}(\mathrm{EC})$; measured $\mathrm{E} \gamma, \mathrm{I} \gamma, \mathrm{E}(\mathrm{ce}), \mathrm{I}(\mathrm{ce})$; deduced $\log \mathrm{ft} .{ }^{75}$ As deduced levels, J, $\pi$, ICC, B(E2), $\delta$. Mini-orange spectrometer. JOUR ZAANE 2641

RADIOACTIVITY ${ }^{75} \mathrm{Se}(\mathrm{EC})$; measured $\mathrm{E} \gamma, \mathrm{I} \gamma, \mathrm{E}(\mathrm{ce}), \mathrm{I}(\mathrm{ce})$; deduced $\log \mathrm{ft} .{ }^{75}$ As deduced levels, J, $\pi$, ICC, B(E2), $\delta$. Mini-orange spectrometer. JOUR ZAANE 2641

NUCLEAR REACTIONS ${ }^{78} \operatorname{Kr}(\mathrm{d}, \mathrm{n}),(\mathrm{d}, \mathrm{p}),(\mathrm{d}, \alpha),(\mathrm{d}, \mathrm{n} \alpha), \mathrm{E} \approx 4-13$ $\mathrm{MeV}$; measured excitation functions. Stacked gas cell activation technique. JOUR RAACA 92203

NUCLEAR REACTIONS Pb $\left({ }^{76} \mathrm{Ge},{ }^{76} \mathrm{Ge}\right),\left({ }^{78} \mathrm{Ge},{ }^{78} \mathrm{Ge}\right),\left({ }^{80} \mathrm{Ge}\right.$, $\left.{ }^{80} \mathrm{Ge}^{\prime}\right),\left({ }^{82} \mathrm{Ge},{ }^{82} \mathrm{Ge}\right), \mathrm{E} \approx 40 \mathrm{MeV} /$ nucleon; measured $\mathrm{E} \gamma, \mathrm{I} \gamma$, (particle) $\gamma$-coin following projectile Coulomb excitation. ${ }^{76,78,80,82} \mathrm{Ge}$ deduced transitions B(E2). JOUR ZAANE 25 s01 415

NUCLEAR REACTIONS ${ }^{78} \operatorname{Kr}(\mathrm{d}, \mathrm{n}),(\mathrm{d}, \mathrm{p}),(\mathrm{d}, \alpha),(\mathrm{d}, \mathrm{n} \alpha), \mathrm{E} \approx 4-13$ $\mathrm{MeV}$; measured excitation functions. Stacked gas cell activation technique. JOUR RAACA 92203

NUCLEAR REACTIONS ${ }^{26} \mathrm{Mg}\left({ }^{76} \mathrm{Kr},{ }^{76} \mathrm{Kr}\right), \mathrm{E}=230 \mathrm{MeV}$; measured $\mathrm{E} \gamma, \mathrm{I} \gamma(\theta, \mathrm{H}, \mathrm{t}),($ particle$) \gamma$-coin following projectile Coulomb excitation. ${ }^{76} \mathrm{Kr}$ level deduced $\mathrm{g}$ factor. Transient field technique. JOUR ZAANE 25 s01 203 ATOMIC MASSES ${ }^{64} \mathrm{Zn},{ }^{64} \mathrm{Ga},{ }^{68} \mathrm{Ge},{ }^{68} \mathrm{As},{ }^{68,72} \mathrm{Se},{ }^{76} \mathrm{Kr},{ }^{76} \mathrm{Rb},{ }^{80} \mathrm{Sr}$, ${ }^{80} \mathrm{Y}$; measured masses. Direct time-of-flight technique, comparison with previous results. JOUR JPGPE 31 S1771 


\section{$\mathrm{A}=76$ (continued)}

2005GI17 RADIOACTIVITY ${ }^{76} \mathrm{Rb}\left(\beta^{+}\right)$, (EC) [from $\left.\mathrm{Nb}(\mathrm{p}, \mathrm{X})\right]$; measured $\mathrm{E} \gamma, \mathrm{I} \gamma$, $\mathrm{E}(\mathrm{ce}), \mathrm{I}(\mathrm{ce}), \gamma \gamma-, \beta \gamma$-coin; deduced log ft. ${ }^{76} \mathrm{Kr}$ deduced levels $\mathrm{J}, \pi$, $\mathrm{T}_{1 / 2}$, ICC. ${ }^{76} \mathrm{Rb}$ deduced ground state $\mathrm{J}, \pi$. JOUR PRVCA 72044308

$2005 \mathrm{G043}$ NUCLEAR REACTIONS ${ }^{40} \mathrm{Ca}\left({ }^{40} \mathrm{Ca}, 2 \mathrm{p} \alpha\right),\left({ }^{40} \mathrm{Ca}, 4 \mathrm{p}\right), \mathrm{E}=147 \mathrm{MeV}$; measured Doppler-shifted $\mathrm{E} \gamma, \mathrm{I} \gamma, \gamma \gamma$-coin. ${ }^{74,76} \mathrm{Kr}$ levels deduced $\mathrm{T}_{1 / 2}$, B(E2). GASP array, recoil-distance technique. JOUR ZAANE 26153

${ }^{76} \mathrm{Rb} \quad 2005 \mathrm{CH} 60 \quad$ ATOMIC MASSES ${ }^{64} \mathrm{Zn},{ }^{64} \mathrm{Ga},{ }^{68} \mathrm{Ge},{ }^{68} \mathrm{As},{ }^{68,72} \mathrm{Se},{ }^{76} \mathrm{Kr},{ }^{76} \mathrm{Rb},{ }^{80} \mathrm{Sr}$,
${ }^{80} \mathrm{Y}$; measured masses. Direct time-of-flight technique, comparison with previous results. JOUR JPGPE 31 S1771

2005GI17 RADIOACTIVITY ${ }^{76} \mathrm{Rb}\left(\beta^{+}\right)$, (EC) [from $\mathrm{Nb}(\mathrm{p}, \mathrm{X})$ ]; measured $\mathrm{E} \gamma, \mathrm{I} \gamma$, $\mathrm{E}(\mathrm{ce}), \mathrm{I}(\mathrm{ce}), \gamma \gamma-, \beta \gamma$-coin; deduced log ft. ${ }^{76} \mathrm{Kr}$ deduced levels $\mathrm{J}, \pi$, $\mathrm{T}_{1 / 2}$, ICC. ${ }^{76} \mathrm{Rb}$ deduced ground state $\mathrm{J}, \pi$. JOUR PRVCA 72044308

${ }^{76} \mathrm{Sr} \quad 2005 \mathrm{SI} 34$ ATOMIC MASSES $76,77,80,81,86,88 \mathrm{Sr},{ }^{124,129,130,131,132} \mathrm{Sn}$; measured masses. Penning trap mass spectrometer, comparison with previous results. JOUR NUPAB 76345

\section{$\mathrm{A}=77$}

${ }^{77} \mathrm{Sr} \quad 2005 \mathrm{SI} 34$

ATOMIC MASSES $76,77,80,81,86,88 \mathrm{Sr},{ }^{124,129,130,131,132} \mathrm{Sn}$; measured masses. Penning trap mass spectrometer, comparison with previous results. JOUR NUPAB 76345

\section{$\mathrm{A}=78$}

${ }^{78} \mathrm{Ni}$

$2005 \mathrm{SC} 28$

RADIOACTIVITY ${ }^{78} \mathrm{Ni}\left(\beta^{-}\right)$[from ${ }^{86} \mathrm{Kr}$ fragmentation]; measured $\mathrm{T}_{1 / 2}$. Comparison with model predictions, astrophysical implications discussed. JOUR ZAANE 25 s01 639

${ }^{78} \mathrm{Cu} \quad 2005 \mathrm{SC} 28$ RADIOACTIVITY ${ }^{78} \mathrm{Ni}\left(\beta^{-}\right)$[from ${ }^{86} \mathrm{Kr}$ fragmentation]; measured $\mathrm{T}_{1 / 2}$. Comparison with model predictions, astrophysical implications discussed. JOUR ZAANE 25 s01 639

${ }^{78} \mathrm{Ge} \quad 2005 \mathrm{IW} 03$ NUCLEAR REACTIONS Pb( $\left({ }^{76} \mathrm{Ge},{ }^{76} \mathrm{Ge}\right),\left({ }^{78} \mathrm{Ge},{ }^{78} \mathrm{Ge}\right),\left({ }^{80} \mathrm{Ge}\right.$, $\left.{ }^{80} \mathrm{Ge} '\right),\left({ }^{82} \mathrm{Ge},{ }^{82} \mathrm{Ge}^{\prime}\right), \mathrm{E} \approx 40 \mathrm{MeV} /$ nucleon; measured $\mathrm{E} \gamma, \mathrm{I} \gamma$, (particle) $\gamma$-coin following projectile Coulomb excitation. ${ }^{76,78,80,82} \mathrm{Ge}$ deduced transitions B(E2). JOUR ZAANE 25 s01 415

${ }^{78} \mathrm{Se} \quad 2005 \mathrm{GAZV} \quad$ RADIOACTIVITY ${ }^{78} \mathrm{Kr}(2 \mathrm{EC})$; measured $2 \mathrm{~K}(2 \nu)$-capture $\mathrm{T}_{1 / 2}$ lower limit. PREPRINT nucl-ex/0510070,10/26/2005

${ }^{78} \mathrm{Kr} \quad 2005 \mathrm{GAZV}$ RADIOACTIVITY ${ }^{78} \mathrm{Kr}(2 \mathrm{EC})$; measured $2 \mathrm{~K}(2 \nu)$-capture $\mathrm{T}_{1 / 2}$ lower limit. PREPRINT nucl-ex/0510070,10/26/2005

2005 SC26 ATOMIC MASSES ${ }^{78,80,82,83,84,86} \mathrm{Kr}$; measured masses. Penning trap mass spectrometer. JOUR ZAANE 25 s01 51

$$
\mathrm{A}=79
$$

${ }^{79} \mathrm{Kr} \quad 2004 \mathrm{SC} 48 \quad$ NUCLEAR REACTIONS ${ }^{78} \mathrm{Kr}(\mathrm{d}, \mathrm{n}),(\mathrm{d}, \mathrm{p}),(\mathrm{d}, \alpha),(\mathrm{d}, \mathrm{n} \alpha), \mathrm{E} \approx 4-13$ $\mathrm{MeV}$; measured excitation functions. Stacked gas cell activation technique. JOUR RAACA 92203 


\section{$\mathrm{A}=79$ (continued)}

${ }^{79} \mathrm{Rb} \quad 2004 \mathrm{SC} 48 \quad$ NUCLEAR REACTIONS ${ }^{78} \mathrm{Kr}(\mathrm{d}, \mathrm{n}),(\mathrm{d}, \mathrm{p}),(\mathrm{d}, \alpha),(\mathrm{d}, \mathrm{n} \alpha), \mathrm{E} \approx 4-13$ $\mathrm{MeV}$; measured excitation functions. Stacked gas cell activation technique. JOUR RAACA 92203

\section{$\mathrm{A}=80$}

$\begin{array}{ll}{ }^{80} \mathrm{Ga} & \text { 2005MA81 } \\ { }^{80} \mathrm{Ge} & 2005 \text { IW03 }\end{array}$

RADIOACTIVITY ${ }^{32} \mathrm{Na},{ }^{80} \mathrm{Ga}\left(\beta^{-}\right)$; measured $\mathrm{E} \gamma, \mathrm{I} \gamma, \gamma \gamma-, \beta \gamma$-coin. ${ }^{32} \mathrm{Mg},{ }^{80} \mathrm{Ge}$ levels deduced $\mathrm{T}_{1 / 2}$. Ultra-fast timing techniques. JOUR JPGPE 31 S1421 NUCLEAR REACTIONS Pb $\left({ }^{76} \mathrm{Ge},{ }^{76} \mathrm{Ge}\right),\left({ }^{78} \mathrm{Ge},{ }^{78} \mathrm{Ge}\right),\left({ }^{80} \mathrm{Ge}\right.$, $\left.{ }^{80} \mathrm{Ge}^{\prime}\right),\left({ }^{82} \mathrm{Ge},{ }^{82} \mathrm{Ge}\right), \mathrm{E} \approx 40 \mathrm{MeV} /$ nucleon; measured $\mathrm{E} \gamma, \mathrm{I} \gamma$, (particle) $\gamma$-coin following projectile Coulomb excitation. ${ }^{76,78,80,82} \mathrm{Ge}$ deduced transitions B(E2). JOUR ZAANE 25 s01 415

2005MA81 RADIOACTIVITY ${ }^{32} \mathrm{Na},{ }^{80} \mathrm{Ga}\left(\beta^{-}\right)$; measured $\mathrm{E} \gamma, \mathrm{I} \gamma, \gamma \gamma-, \beta \gamma$-coin. ${ }^{32} \mathrm{Mg},{ }^{80} \mathrm{Ge}$ levels deduced $\mathrm{T}_{1 / 2}$. Ultra-fast timing techniques. JOUR JPGPE 31 S1421

${ }^{80} \mathrm{As} \quad 2005 \mathrm{GA} 56$ NUCLEAR REACTIONS ${ }^{238} \mathrm{U}\left({ }^{82} \mathrm{Se}, \mathrm{X}\right), \mathrm{E}=505 \mathrm{MeV} ;{ }^{238} \mathrm{U}\left({ }^{64} \mathrm{Ni}, \mathrm{X}\right)$, $\mathrm{E}=400 \mathrm{MeV}$; measured $\mathrm{E} \gamma, \mathrm{I} \gamma, \gamma \gamma-$, (fragment) $\gamma$-coin, projectile-like fragments isotopic yields. ${ }^{58} \mathrm{Cr},{ }^{80} \mathrm{As},{ }^{82} \mathrm{Ge},{ }^{84}$ Se deduced levels, $\mathrm{J}, \pi$. Clara array, Prisma spectrometer. JOUR ZAANE 25 s01 421

${ }^{80} \mathrm{Kr} \quad 2005 \mathrm{SC} 26 \quad$ ATOMIC MASSES ${ }^{78,80,82,83,84,86} \mathrm{Kr}$; measured masses. Penning trap mass spectrometer. JOUR ZAANE 25 s01 51

${ }^{80} \mathrm{Sr} \quad 2005 \mathrm{CH} 60$ ATOMIC MASSES ${ }^{64} \mathrm{Zn},{ }^{64} \mathrm{Ga},{ }^{68} \mathrm{Ge},{ }^{68} \mathrm{As},{ }^{68,72} \mathrm{Se},{ }^{76} \mathrm{Kr},{ }^{76} \mathrm{Rb},{ }^{80} \mathrm{Sr}$, ${ }^{80} \mathrm{Y}$; measured masses. Direct time-of-flight technique, comparison with previous results. JOUR JPGPE 31 S1771

2005SI34 ATOMIC MASSES ${ }^{76,77,80,81,86,88} \mathrm{Sr},{ }^{124,129,130,131,132} \mathrm{Sn}$; measured masses. Penning trap mass spectrometer, comparison with previous results. JOUR NUPAB 76345

${ }^{80} \mathrm{Y} \quad 2005 \mathrm{CH} 60$ ATOMIC MASSES ${ }^{64} \mathrm{Zn},{ }^{64} \mathrm{Ga},{ }^{68} \mathrm{Ge},{ }^{68} \mathrm{As},{ }^{68,72} \mathrm{Se},{ }^{76} \mathrm{Kr},{ }^{76} \mathrm{Rb},{ }^{80} \mathrm{Sr}$, ${ }^{80} \mathrm{Y}$; measured masses. Direct time-of-flight technique, comparison with previous results. JOUR JPGPE 31 S1771

\section{$\mathrm{A}=81$}

$\begin{array}{ll}{ }^{81} \mathrm{Zn} & \text { 2004VE14 } \\ { }^{81} \mathrm{Ga} & 2004 \mathrm{VE} 14 \\ { }^{81} \mathrm{Br} & 2005 \mathrm{KA} 39\end{array}$

RADIOACTIVITY ${ }^{83} \mathrm{Ga},{ }^{81} \mathrm{Zn}\left(\beta^{-}\right)$[from $\mathrm{U}(\mathrm{d}, \mathrm{X})$ ]; measured $\mathrm{E} \gamma, \mathrm{I} \gamma$, $\gamma \gamma$-, $\beta \gamma$-coin, $\mathrm{T}_{1 / 2} \cdot{ }^{81} \mathrm{Ga},{ }^{83} \mathrm{As}$ deduced levels. JOUR BJPHE 34979 RADIOACTIVITY ${ }^{83} \mathrm{Ga},{ }^{81} \mathrm{Zn}\left(\beta^{-}\right)$[from $\mathrm{U}(\mathrm{d}, \mathrm{X})$ ]; measured $\mathrm{E} \gamma, \mathrm{I} \gamma$, $\gamma \gamma-, \beta \gamma$-coin, $\mathrm{T}_{1 / 2} .{ }^{81} \mathrm{Ga},{ }^{83} \mathrm{As}$ deduced levels. JOUR BJPHE 34979 RADIOACTIVITY ${ }^{81 m} \mathrm{Kr}(\mathrm{EC}),(\mathrm{IT}) ;{ }^{81} \mathrm{Y},{ }^{81} \mathrm{Sr},{ }^{85} \mathrm{Nb},{ }^{85} \mathrm{Zr},{ }^{86} \mathrm{Mo}$, ${ }^{86} \mathrm{Nb}(\mathrm{EC})$ [from $\mathrm{Ni},{ }^{54} \mathrm{Fe}\left({ }^{32} \mathrm{~S}, \mathrm{X}\right)$ ]; measured $\mathrm{E} \gamma, \mathrm{I} \gamma, \mathrm{E}(\mathrm{ce}), \mathrm{I}(\mathrm{ce}), \mathrm{T}_{1 / 2}$. ${ }^{81} \mathrm{Kr},{ }^{85} \mathrm{Zr},{ }^{85} \mathrm{Nb}$ deduced isomeric transitions $\mathrm{T}_{1 / 2}$, ICC. ${ }^{85} \mathrm{Zr},{ }^{86} \mathrm{Nb}$ deduced levels, J, $\pi$, ICC. ${ }^{81} \mathrm{Br}$ deduced neutrino capture rate. Astrophysical implications discussed. JOUR ZAANE 25355 


\section{$\mathrm{A}=81$ (continued)}

2005KA46 RADIOACTIVITY ${ }^{31} \mathrm{Cl}\left(\beta^{+} \mathrm{p}\right)$ [from $\mathrm{S}(\mathrm{p}, \mathrm{X}), \mathrm{E}=40 \mathrm{MeV}$ ]; measured $\beta$-delayed $\mathrm{E} \gamma, \mathrm{Ep} .{ }^{58} \mathrm{Zn}\left(\beta^{+}\right)$[from $\mathrm{Nb}(\mathrm{p}, \mathrm{X}), \mathrm{E}=1.4 \mathrm{GeV}$ ]; measured $\mathrm{E} \gamma, \mathrm{I} \gamma, \beta \gamma$-coin, $\mathrm{T}_{1 / 2} .{ }^{58} \mathrm{Cu}$ deduced levels, $\beta$-feeding intensities. ${ }^{81 m} \mathrm{Kr}(\mathrm{EC}),(\mathrm{IT}) ;{ }^{81} \mathrm{Y},{ }^{81} \mathrm{Sr},{ }^{85} \mathrm{Nb},{ }^{85} \mathrm{Zr},{ }^{86} \mathrm{Mo},{ }^{86} \mathrm{Nb}(\mathrm{EC})$ [from Ni, $\left.{ }^{54} \mathrm{Fe}\left({ }^{32} \mathrm{~S}, \mathrm{X}\right)\right]$; measured $\mathrm{E} \gamma, \mathrm{I} \gamma, \mathrm{E}(\mathrm{ce}), \mathrm{I}(\mathrm{ce}), \mathrm{T}_{1 / 2} \cdot{ }^{81} \mathrm{Kr},{ }^{85} \mathrm{Zr},{ }^{85} \mathrm{Nb}$ deduced isomeric transitions $\mathrm{T}_{1 / 2}$, ICC. ${ }^{85} \mathrm{Zr},{ }^{86} \mathrm{Nb}$ deduced levels, J, $\pi$, ICC. ${ }^{81} \mathrm{Br}$ deduced neutrino capture rate. Mass-separated sources. JOUR ZAANE 25 s01 129

${ }^{81} \mathrm{Kr} \quad 2005 \mathrm{KA39}$ RADIOACTIVITY ${ }^{81 m} \mathrm{Kr}$ (EC), (IT); ${ }^{81} \mathrm{Y},{ }^{81} \mathrm{Sr},{ }^{85} \mathrm{Nb},{ }^{85} \mathrm{Zr},{ }^{86} \mathrm{Mo}$, ${ }^{86} \mathrm{Nb}(\mathrm{EC})\left[\right.$ from $\left.\mathrm{Ni},{ }^{54} \mathrm{Fe}\left({ }^{32} \mathrm{~S}, \mathrm{X}\right)\right]$; measured $\mathrm{E} \gamma, \mathrm{I} \gamma, \mathrm{E}(\mathrm{ce}), \mathrm{I}(\mathrm{ce}), \mathrm{T}_{1 / 2}$. ${ }^{81} \mathrm{Kr},{ }^{85} \mathrm{Zr},{ }^{85} \mathrm{Nb}$ deduced isomeric transitions $\mathrm{T}_{1 / 2}$, ICC. ${ }^{85} \mathrm{Zr},{ }^{86} \mathrm{Nb}$ deduced levels, J, $\pi$, ICC. ${ }^{81} \mathrm{Br}$ deduced neutrino capture rate. Astrophysical implications discussed. JOUR ZAANE 25355 2005KA39 NUCLEAR REACTIONS ${ }^{54} \mathrm{Fe}\left({ }^{32} \mathrm{~S}, \mathrm{X}\right){ }^{81} \mathrm{Zr} /{ }^{81} \mathrm{Y} /{ }^{81} \mathrm{Sr} /{ }^{81 m} \mathrm{Kr}$, $\mathrm{E}=150-170 \mathrm{MeV} ; \mathrm{Ni}\left({ }^{32} \mathrm{~S}, \mathrm{X}\right){ }^{85} \mathrm{Nb} /{ }^{85 m} \mathrm{Nb} /{ }^{85 Z r} /{ }^{85 m} \mathrm{Zr} /{ }^{86} \mathrm{Mo} /$ ${ }^{86} \mathrm{Nb}, \mathrm{E}=150-170 \mathrm{MeV}$; measured yields. JOUR ZAANE 25355

2005KA46 RADIOACTIVITY ${ }^{31} \mathrm{Cl}\left(\beta^{+} \mathrm{p}\right)$ [from $\mathrm{S}(\mathrm{p}, \mathrm{X}), \mathrm{E}=40 \mathrm{MeV}$ ]; measured $\beta$-delayed $\mathrm{E} \gamma, \mathrm{Ep} .{ }^{58} \mathrm{Zn}\left(\beta^{+}\right)$[from $\mathrm{Nb}(\mathrm{p}, \mathrm{X}), \mathrm{E}=1.4 \mathrm{GeV}$ ]; measured $\mathrm{E} \gamma, \mathrm{I} \gamma, \beta \gamma$-coin, $\mathrm{T}_{1 / 2} .{ }^{58} \mathrm{Cu}$ deduced levels, $\beta$-feeding intensities. ${ }^{81 m} \mathrm{Kr}(\mathrm{EC}),(\mathrm{IT}) ;{ }^{81} \mathrm{Y},{ }^{81} \mathrm{Sr},{ }^{85} \mathrm{Nb},{ }^{85} \mathrm{Zr},{ }^{86} \mathrm{Mo},{ }^{86} \mathrm{Nb}(\mathrm{EC})$ [from Ni, $\left.{ }^{54} \mathrm{Fe}\left({ }^{32} \mathrm{~S}, \mathrm{X}\right)\right]$; measured $\mathrm{E} \gamma, \mathrm{I} \gamma, \mathrm{E}(\mathrm{ce}), \mathrm{I}(\mathrm{ce}), \mathrm{T}_{1 / 2} \cdot{ }^{81} \mathrm{Kr},{ }^{85} \mathrm{Zr},{ }^{85} \mathrm{Nb}$ deduced isomeric transitions $\mathrm{T}_{1 / 2}$, ICC. ${ }^{85} \mathrm{Zr},{ }^{86} \mathrm{Nb}$ deduced levels, $\mathrm{J}$, $\pi$, ICC. ${ }^{81} \mathrm{Br}$ deduced neutrino capture rate. Mass-separated sources. JOUR ZAANE 25 s01 129

${ }^{81} \mathrm{Rb}$ 2004KA68 NUCLEAR REACTIONS ${ }^{85} \mathrm{Rb}(\mathrm{p}, \mathrm{np}),(\mathrm{p}, 2 \mathrm{np}),(\mathrm{p}, 3 \mathrm{np}),(\mathrm{p}, 4 \mathrm{np}), \mathrm{E}$ $\approx 17-100 \mathrm{MeV}$; measured excitation functions. Activation technique, comparison with model predictions. JOUR RAACA 92449

2005KA39 RADIOACTIVITY ${ }^{81 m} \mathrm{Kr}$ (EC), (IT); ${ }^{81} \mathrm{Y},{ }^{81} \mathrm{Sr},{ }^{85} \mathrm{Nb},{ }^{85} \mathrm{Zr},{ }^{86} \mathrm{Mo}$, ${ }^{86} \mathrm{Nb}(\mathrm{EC})\left[\right.$ from $\left.\mathrm{Ni},{ }^{54} \mathrm{Fe}\left({ }^{32} \mathrm{~S}, \mathrm{X}\right)\right]$; measured $\mathrm{E} \gamma, \mathrm{I} \gamma, \mathrm{E}(\mathrm{ce}), \mathrm{I}(\mathrm{ce}), \mathrm{T}_{1 / 2}$. ${ }^{81} \mathrm{Kr},{ }^{85} \mathrm{Zr},{ }^{85} \mathrm{Nb}$ deduced isomeric transitions $\mathrm{T}_{1 / 2}$, ICC. ${ }^{85} \mathrm{Zr},{ }^{86} \mathrm{Nb}$ deduced levels, J, $\pi$, ICC. ${ }^{81} \mathrm{Br}$ deduced neutrino capture rate. Astrophysical implications discussed. JOUR ZAANE 25355

2005KA46 RADIOACTIVITY ${ }^{31} \mathrm{Cl}\left(\beta^{+} \mathrm{p}\right)$ [from $\mathrm{S}(\mathrm{p}, \mathrm{X}), \mathrm{E}=40 \mathrm{MeV}$; ; measured $\beta$-delayed $\mathrm{E} \gamma, \mathrm{Ep} .{ }^{58} \mathrm{Zn}\left(\beta^{+}\right)$[from $\mathrm{Nb}(\mathrm{p}, \mathrm{X}), \mathrm{E}=1.4 \mathrm{GeV}$ ]; measured $\mathrm{E} \gamma, \mathrm{I} \gamma, \beta \gamma$-coin, $\mathrm{T}_{1 / 2} .{ }^{58} \mathrm{Cu}$ deduced levels, $\beta$-feeding intensities. ${ }^{81 m} \mathrm{Kr}(\mathrm{EC}),(\mathrm{IT}) ;{ }^{81} \mathrm{Y},{ }^{81} \mathrm{Sr},{ }^{85} \mathrm{Nb},{ }^{85} \mathrm{Zr},{ }^{86} \mathrm{Mo},{ }^{86} \mathrm{Nb}(\mathrm{EC})$ [from Ni, $\left.{ }^{54} \mathrm{Fe}\left({ }^{32} \mathrm{~S}, \mathrm{X}\right)\right]$; measured $\mathrm{E} \gamma, \mathrm{I} \gamma, \mathrm{E}(\mathrm{ce}), \mathrm{I}(\mathrm{ce}), \mathrm{T}_{1 / 2} \cdot{ }^{81} \mathrm{Kr},{ }^{85} \mathrm{Zr},{ }^{85} \mathrm{Nb}$ deduced isomeric transitions $\mathrm{T}_{1 / 2}$, ICC. ${ }^{85} \mathrm{Zr},{ }^{86} \mathrm{Nb}$ deduced levels, $\mathrm{J}$, $\pi$, ICC. ${ }^{81} \mathrm{Br}$ deduced neutrino capture rate. Mass-separated sources. JOUR ZAANE 25 s01 129

${ }^{81} \mathrm{Sr} \quad$ 2005KA39 RADIOACTIVITY ${ }^{81 m} \mathrm{Kr}(\mathrm{EC}),(\mathrm{IT}) ;{ }^{81} \mathrm{Y},{ }^{81} \mathrm{Sr},{ }^{85} \mathrm{Nb},{ }^{85} \mathrm{Zr},{ }^{86} \mathrm{Mo}$, ${ }^{86} \mathrm{Nb}(\mathrm{EC})\left[\right.$ from $\left.\mathrm{Ni},{ }^{54} \mathrm{Fe}\left({ }^{32} \mathrm{~S}, \mathrm{X}\right)\right]$; measured $\mathrm{E} \gamma, \mathrm{I} \gamma, \mathrm{E}(\mathrm{ce}), \mathrm{I}(\mathrm{ce}), \mathrm{T}_{1 / 2}$. ${ }^{81} \mathrm{Kr},{ }^{85} \mathrm{Zr},{ }^{85} \mathrm{Nb}$ deduced isomeric transitions $\mathrm{T}_{1 / 2}$, ICC. ${ }^{85} \mathrm{Zr},{ }^{86} \mathrm{Nb}$ deduced levels, J, $\pi$, ICC. ${ }^{81} \mathrm{Br}$ deduced neutrino capture rate. Astrophysical implications discussed. JOUR ZAANE 25355 2005KA39 NUCLEAR REACTIONS ${ }^{54} \mathrm{Fe}\left({ }^{32} \mathrm{~S}, \mathrm{X}\right){ }^{81} \mathrm{Zr} /{ }^{81} \mathrm{Y} /{ }^{81} \mathrm{Sr} /{ }^{81 m} \mathrm{Kr}$, $\mathrm{E}=150-170 \mathrm{MeV} ; \mathrm{Ni}\left({ }^{32} \mathrm{~S}, \mathrm{X}\right){ }^{85} \mathrm{Nb} /{ }^{85 m} \mathrm{Nb} /{ }^{85 Z r} /{ }^{85 m} \mathrm{Zr} /{ }^{86} \mathrm{Mo} /$ ${ }^{86} \mathrm{Nb}, \mathrm{E}=150-170 \mathrm{MeV}$; measured yields. JOUR ZAANE 25355 


\section{$\mathrm{A}=81$ (continued)}

2005KA46 RADIOACTIVITY ${ }^{31} \mathrm{Cl}\left(\beta^{+} \mathrm{p}\right)$ [from $\mathrm{S}(\mathrm{p}, \mathrm{X}), \mathrm{E}=40 \mathrm{MeV}$ ]; measured $\beta$-delayed $\mathrm{E} \gamma, \mathrm{Ep} .{ }^{58} \mathrm{Zn}\left(\beta^{+}\right)$[from $\mathrm{Nb}(\mathrm{p}, \mathrm{X}), \mathrm{E}=1.4 \mathrm{GeV}$ ]; measured $\mathrm{E} \gamma, \mathrm{I} \gamma, \beta \gamma$-coin, $\mathrm{T}_{1 / 2} .{ }^{58} \mathrm{Cu}$ deduced levels, $\beta$-feeding intensities. ${ }^{81 m} \mathrm{Kr}(\mathrm{EC}),(\mathrm{IT}) ;{ }^{81} \mathrm{Y},{ }^{81} \mathrm{Sr},{ }^{85} \mathrm{Nb},{ }^{85} \mathrm{Zr},{ }^{86} \mathrm{Mo},{ }^{86} \mathrm{Nb}(\mathrm{EC})$ [from Ni, $\left.{ }^{54} \mathrm{Fe}\left({ }^{32} \mathrm{~S}, \mathrm{X}\right)\right]$; measured $\mathrm{E} \gamma, \mathrm{I} \gamma, \mathrm{E}(\mathrm{ce}), \mathrm{I}(\mathrm{ce}), \mathrm{T}_{1 / 2} \cdot{ }^{81} \mathrm{Kr},{ }^{85} \mathrm{Zr},{ }^{85} \mathrm{Nb}$ deduced isomeric transitions $\mathrm{T}_{1 / 2}$, ICC. ${ }^{85} \mathrm{Zr},{ }^{86} \mathrm{Nb}$ deduced levels, J, $\pi$, ICC. ${ }^{81} \mathrm{Br}$ deduced neutrino capture rate. Mass-separated sources. JOUR ZAANE 25 s01 129

2005SI34 ATOMIC MASSES ${ }^{76,77,80,81,86,88} \mathrm{Sr},{ }^{124,129,130,131,132} \mathrm{Sn}$; measured masses. Penning trap mass spectrometer, comparison with previous results. JOUR NUPAB 76345

${ }^{81} \mathrm{Y}$ 2005KA39 RADIOACTIVITY ${ }^{81 m} \mathrm{Kr}(\mathrm{EC}),(\mathrm{IT}) ;{ }^{81} \mathrm{Y},{ }^{81} \mathrm{Sr},{ }^{85} \mathrm{Nb},{ }^{85} \mathrm{Zr},{ }^{86} \mathrm{Mo}$, ${ }^{86} \mathrm{Nb}(\mathrm{EC})\left[\right.$ from $\left.\mathrm{Ni},{ }^{54} \mathrm{Fe}\left({ }^{32} \mathrm{~S}, \mathrm{X}\right)\right]$; measured $\mathrm{E} \gamma, \mathrm{I} \gamma, \mathrm{E}(\mathrm{ce}), \mathrm{I}(\mathrm{ce}), \mathrm{T}_{1 / 2}$.

${ }^{81} \mathrm{Kr},{ }^{85} \mathrm{Zr},{ }^{85} \mathrm{Nb}$ deduced isomeric transitions $\mathrm{T}_{1 / 2}$, ICC. ${ }^{85} \mathrm{Zr},{ }^{86} \mathrm{Nb}$ deduced levels, J, $\pi$, ICC. ${ }^{81} \mathrm{Br}$ deduced neutrino capture rate. Astrophysical implications discussed. JOUR ZAANE 25355

2005KA39 NUCLEAR REACTIONS ${ }^{54} \mathrm{Fe}\left({ }^{32} \mathrm{~S}, \mathrm{X}\right){ }^{81} \mathrm{Zr} /{ }^{81} \mathrm{Y} /{ }^{81} \mathrm{Sr} /{ }^{81 m} \mathrm{Kr}$, $\mathrm{E}=150-170 \mathrm{MeV} ; \mathrm{Ni}\left({ }^{32} \mathrm{~S}, \mathrm{X}\right){ }^{85} \mathrm{Nb} /{ }^{85 m} \mathrm{Nb} /{ }^{85 Z r} /{ }^{85 m} \mathrm{Zr} /{ }^{86} \mathrm{Mo} /$ ${ }^{86} \mathrm{Nb}, \mathrm{E}=150-170 \mathrm{MeV}$; measured yields. JOUR ZAANE 25355

2005KA46 RADIOACTIVITY ${ }^{31} \mathrm{Cl}\left(\beta^{+} \mathrm{p}\right)$ [from $\mathrm{S}(\mathrm{p}, \mathrm{X}), \mathrm{E}=40 \mathrm{MeV}$ ]; measured $\beta$-delayed $\mathrm{E} \gamma, \mathrm{Ep} .{ }^{58} \mathrm{Zn}\left(\beta^{+}\right)$[from $\mathrm{Nb}(\mathrm{p}, \mathrm{X}), \mathrm{E}=1.4 \mathrm{GeV}$ ]; measured $\mathrm{E} \gamma, \mathrm{I} \gamma, \beta \gamma$-coin, $\mathrm{T}_{1 / 2} .{ }^{58} \mathrm{Cu}$ deduced levels, $\beta$-feeding intensities. ${ }^{81 m} \mathrm{Kr}(\mathrm{EC}),(\mathrm{IT}) ;{ }^{81} \mathrm{Y},{ }^{81} \mathrm{Sr},{ }^{85} \mathrm{Nb},{ }^{85} \mathrm{Zr},{ }^{86} \mathrm{Mo},{ }^{86} \mathrm{Nb}(\mathrm{EC})$ [from Ni, $\left.{ }^{54} \mathrm{Fe}\left({ }^{32} \mathrm{~S}, \mathrm{X}\right)\right]$; measured $\mathrm{E} \gamma, \mathrm{I} \gamma, \mathrm{E}(\mathrm{ce}), \mathrm{I}(\mathrm{ce}), \mathrm{T}_{1 / 2} \cdot{ }^{81} \mathrm{Kr},{ }^{85} \mathrm{Zr},{ }^{85} \mathrm{Nb}$ deduced isomeric transitions $\mathrm{T}_{1 / 2}$, ICC. ${ }^{85} \mathrm{Zr},{ }^{86} \mathrm{Nb}$ deduced levels, $\mathrm{J}$, $\pi$, ICC. ${ }^{81} \mathrm{Br}$ deduced neutrino capture rate. Mass-separated sources. JOUR ZAANE 25 s01 129

${ }^{81} \mathrm{Zr} \quad$ 2005KA39 NUCLEAR REACTIONS ${ }^{54} \mathrm{Fe}\left({ }^{32} \mathrm{~S}, \mathrm{X}\right){ }^{81} \mathrm{Zr} /{ }^{81} \mathrm{Y} /{ }^{81} \mathrm{Sr} /{ }^{81 m} \mathrm{Kr}$, $\mathrm{E}=150-170 \mathrm{MeV} ; \mathrm{Ni}\left({ }^{32} \mathrm{~S}, \mathrm{X}\right){ }^{85} \mathrm{Nb} /{ }^{85 m} \mathrm{Nb} /{ }^{85 Z r} /{ }^{85 m} \mathrm{Zr} /{ }^{86} \mathrm{Mo} /$ ${ }^{86} \mathrm{Nb}, \mathrm{E}=150-170 \mathrm{MeV}$; measured yields. JOUR ZAANE 25355

\section{$\mathrm{A}=82$}

$\quad{ }^{82} \mathrm{Ge} \quad$ 2005GA56

NUCLEAR REACTIONS ${ }^{238} \mathrm{U}\left({ }^{82} \mathrm{Se}, \mathrm{X}\right), \mathrm{E}=505 \mathrm{MeV} ;{ }^{238} \mathrm{U}\left({ }^{64} \mathrm{Ni}, \mathrm{X}\right)$, $\mathrm{E}=400 \mathrm{MeV}$; measured $\mathrm{E} \gamma, \mathrm{I} \gamma, \gamma \gamma-$, (fragment) $\gamma$-coin, projectile-like fragments isotopic yields. ${ }^{58} \mathrm{Cr},{ }^{80} \mathrm{As},{ }^{82} \mathrm{Ge},{ }^{84}$ Se deduced levels, $\mathrm{J}, \pi$. Clara array, Prisma spectrometer. JOUR ZAANE 25 s01 421

2005IW03 NUCLEAR REACTIONS Pb $\left({ }^{76} \mathrm{Ge},{ }^{76} \mathrm{Ge}\right),\left({ }^{78} \mathrm{Ge},{ }^{78} \mathrm{Ge}\right),\left({ }^{80} \mathrm{Ge}\right.$, $\left.{ }^{80} \mathrm{Ge}^{\prime}\right),\left({ }^{82} \mathrm{Ge},{ }^{82} \mathrm{Ge}\right), \mathrm{E} \approx 40 \mathrm{MeV} /$ nucleon; measured $\mathrm{E} \gamma, \mathrm{I} \gamma$, (particle) $\gamma$-coin following projectile Coulomb excitation. ${ }^{76,78,80,82} \mathrm{Ge}$ deduced transitions B(E2). JOUR ZAANE 25 s01 415

${ }^{82} \mathrm{Se} \quad 2005$ AR27 RADIOACTIVITY ${ }^{82} \mathrm{Se},{ }^{100} \mathrm{Mo}\left(2 \beta^{-}\right)$; measured $2 \nu \beta \beta$-decay $\mathrm{T}_{1 / 2}$, $0 \nu \beta \beta$-decay $\mathrm{T}_{1 / 2}$ lower limits; deduced neutrino mass limits. JOUR PRLTA 95182302

${ }^{82} \mathrm{Kr} \quad$ 2005AR27 RADIOACTIVITY ${ }^{82} \mathrm{Se},{ }^{100} \mathrm{Mo}\left(2 \beta^{-}\right)$; measured $2 \nu \beta \beta$-decay $\mathrm{T}_{1 / 2}$, $0 \nu \beta \beta$-decay $\mathrm{T}_{1 / 2}$ lower limits; deduced neutrino mass limits. JOUR PRLTA 95182302 


\section{$\mathrm{A}=82$ (continued)}

2005SC26 ATOMIC MASSES $78,80,82,83,84,86 \mathrm{Kr}$; measured masses. Penning trap mass spectrometer. JOUR ZAANE 25 s01 51

${ }^{82} \mathrm{Rb} \quad$ 2004KA68

2005GU37

${ }^{83} \mathrm{Ga} \quad$ 2004VE14

${ }^{83} \mathrm{Ge} \quad$ 2004VE14

2005CI07

2005 TH12

${ }^{83}$ As $\quad 2004$ VE14

${ }^{83} \mathrm{Kr} \quad 2005 \mathrm{SC} 26$

${ }^{83} \mathrm{Rb} \quad 2004 \mathrm{KA} 68$

${ }^{83} \mathrm{Sr} \quad 2005 \mathrm{DU} 23$

${ }^{83} \mathrm{Y} \quad 2005 \mathrm{DU} 23$
NUCLEAR REACTIONS ${ }^{85} \mathrm{Rb}(\mathrm{p}, \mathrm{np}),(\mathrm{p}, 2 \mathrm{np}),(\mathrm{p}, 3 \mathrm{np}),(\mathrm{p}, 4 \mathrm{np}), \mathrm{E}$ $\approx 17-100 \mathrm{MeV}$; measured excitation functions. Activation technique, comparison with model predictions. JOUR RAACA 92449 ATOMIC MASSES ${ }^{56,57} \mathrm{Mn},{ }^{82 m} \mathrm{Rb},{ }^{92} \mathrm{Sr},{ }^{124,127} \mathrm{Cs},{ }^{130} \mathrm{Ba}$; measured masses. Penning trap mass spectrometer. JOUR ZAANE 25 s01 35

\section{$\mathrm{A}=83$}

RADIOACTIVITY ${ }^{83} \mathrm{Ga},{ }^{81} \mathrm{Zn}\left(\beta^{-}\right)$[from $\mathrm{U}(\mathrm{d}, \mathrm{X})$ ]; measured $\mathrm{E} \gamma, \mathrm{I} \gamma$, $\gamma \gamma$-, $\beta \gamma$-coin, $\mathrm{T}_{1 / 2} \cdot{ }^{81} \mathrm{Ga},{ }^{83}$ As deduced levels. JOUR BJPHE 34979 RADIOACTIVITY ${ }^{83} \mathrm{Ga},{ }^{81} \mathrm{Zn}\left(\beta^{-}\right)$[from $\mathrm{U}(\mathrm{d}, \mathrm{X})$ ]; measured $\mathrm{E} \gamma, \mathrm{I} \gamma$, $\gamma \gamma-, \beta \gamma$-coin, $\mathrm{T}_{1 / 2} .{ }^{81} \mathrm{Ga},{ }^{83} \mathrm{As}$ deduced levels. JOUR BJPHE 34979 NUCLEAR REACTIONS ${ }^{2} \mathrm{H}\left({ }^{82} \mathrm{Ge}, \mathrm{p}\right),\left({ }^{84} \mathrm{Se}, \mathrm{p}\right), \mathrm{E}=4 \mathrm{MeV} /$ nucleon; measured Ep, $\sigma(\theta) .{ }^{83} \mathrm{Ge},{ }^{85} \mathrm{Se}$ deduced ground and excited states energies, J, $\pi$. JOUR NIMBE 241200

NUCLEAR REACTIONS ${ }^{2} \mathrm{H}\left({ }^{82} \mathrm{Ge}, \mathrm{p}\right), \mathrm{E}=4 \mathrm{MeV} /$ nucleon; ${ }^{2} \mathrm{H}\left({ }^{84} \mathrm{Se}\right.$, p), $\mathrm{E}=4.5 \mathrm{MeV}$ / nucleon; measured Ep, recoil particle spectrum, proton angular distribution. ${ }^{83} \mathrm{Ge},{ }^{85}$ Se deduced levels J, $\pi$, spectroscopic factors. DWBA analysis. JOUR ZAANE 25 s01 371 RADIOACTIVITY ${ }^{83} \mathrm{Ga},{ }^{81} \mathrm{Zn}\left(\beta^{-}\right)$[from U(d, X)]; measured $\mathrm{E} \gamma, \mathrm{I} \gamma$, $\gamma \gamma-, \beta \gamma$-coin, $\mathrm{T}_{1 / 2} \cdot{ }^{81} \mathrm{Ga},{ }^{83}$ As deduced levels. JOUR BJPHE 34979 ATOMIC MASSES $78,80,82,83,84,86 \mathrm{Kr}$; measured masses. Penning trap mass spectrometer. JOUR ZAANE 25 s01 51

NUCLEAR REACTIONS ${ }^{85} \mathrm{Rb}(\mathrm{p}, \mathrm{np}),(\mathrm{p}, 2 \mathrm{np}),(\mathrm{p}, 3 \mathrm{np}),(\mathrm{p}, 4 \mathrm{np}), \mathrm{E}$ $\approx 17-100 \mathrm{MeV}$; measured excitation functions. Activation technique, comparison with model predictions. JOUR RAACA 92449

NUCLEAR REACTIONS Ge $\left({ }^{18} \mathrm{O}, \mathrm{X}\right)^{83 m} \mathrm{Sr} /{ }^{83} \mathrm{Y} /{ }^{84 m} \mathrm{Y} /{ }^{88 m} \mathrm{Y} /$ ${ }^{85} \mathrm{Zr} /{ }^{87} \mathrm{Zr}, \mathrm{E}=82.8 \mathrm{GeV} ;{ }^{84} \mathrm{Se}\left({ }^{18} \mathrm{O}, \mathrm{X}\right){ }^{86 m} \mathrm{Y} /{ }^{85} \mathrm{Zr} /{ }^{87} \mathrm{Nb} /{ }^{87 m} \mathrm{Nb} /$ ${ }^{88} \mathrm{Nb} /{ }^{88} \mathrm{Mo}, \mathrm{E}=82.7 \mathrm{MeV} ;{ }^{124} \mathrm{Sn}\left({ }^{50} \mathrm{Ti}, \mathrm{X}\right){ }^{168 m} \mathrm{Lu} /{ }^{167} \mathrm{Hf} /{ }^{168} \mathrm{Hf}$, $\mathrm{E}=223.7 \mathrm{MeV} ;{ }^{116} \mathrm{Sn}\left({ }^{50} \mathrm{Ti}, \mathrm{X}\right){ }^{162} \mathrm{Tm} /{ }^{161} \mathrm{Yb} /{ }^{162} \mathrm{Yb} /{ }^{163} \mathrm{Yb} /{ }^{162} \mathrm{Lu} /$ ${ }^{162} \mathrm{Hf}, \mathrm{E}=224.4 \mathrm{MeV}$; measured delayed $\mathrm{E} \gamma$, I $\gamma$ following residual nucleus decay. Physical preseparation technique. JOUR NIMAE 551 528 NUCLEAR REACTIONS Ge $\left({ }^{18} \mathrm{O}, \mathrm{X}\right)^{83 m} \mathrm{Sr} /{ }^{83} \mathrm{Y} /{ }^{84 m} \mathrm{Y} /{ }^{88 m} \mathrm{Y} /$ ${ }^{85} \mathrm{Zr} /{ }^{87} \mathrm{Zr}, \mathrm{E}=82.8 \mathrm{GeV} ;{ }^{84} \mathrm{Se}\left({ }^{18} \mathrm{O}, \mathrm{X}\right){ }^{86 m} \mathrm{Y} /{ }^{85} \mathrm{Zr} /{ }^{87} \mathrm{Nb} /{ }^{87 m} \mathrm{Nb} /$ ${ }^{88} \mathrm{Nb} /{ }^{88} \mathrm{Mo}, \mathrm{E}=82.7 \mathrm{MeV} ;{ }^{124} \mathrm{Sn}\left({ }^{50} \mathrm{Ti}, \mathrm{X}\right){ }^{168 m} \mathrm{Lu} /{ }^{167} \mathrm{Hf} /{ }^{168} \mathrm{Hf}$, $\mathrm{E}=223.7 \mathrm{MeV} ;{ }^{116} \mathrm{Sn}\left({ }^{50} \mathrm{Ti}, \mathrm{X}\right){ }^{162} \mathrm{Tm} /{ }^{161} \mathrm{Yb} /{ }^{162} \mathrm{Yb} /{ }^{163} \mathrm{Yb} /{ }^{162} \mathrm{Lu} /$ ${ }^{162} \mathrm{Hf}, \mathrm{E}=224.4 \mathrm{MeV}$; measured delayed $\mathrm{E} \gamma, \mathrm{I} \gamma$ following residual nucleus decay. Physical preseparation technique. JOUR NIMAE 551 528 


\section{$\mathrm{A}=84$}

\begin{tabular}{|c|c|c|}
\hline${ }^{84} \mathrm{Se}$ & 2005GA56 & $\begin{array}{l}\text { NUCLEAR REACTIONS }{ }^{238} \mathrm{U}\left({ }^{82} \mathrm{Se}, \mathrm{X}\right), \mathrm{E}=505 \mathrm{MeV} ;{ }^{238} \mathrm{U}\left({ }^{64} \mathrm{Ni}, \mathrm{X}\right) \text {, } \\
\mathrm{E}=400 \mathrm{MeV} ; \text { measured } \mathrm{E} \gamma, \mathrm{I} \gamma, \gamma \gamma-\text {, (fragment) } \gamma \text {-coin, projectile-like } \\
\text { fragments isotopic yields. }{ }^{58} \mathrm{Cr},{ }^{80} \mathrm{As},{ }^{82} \mathrm{Ge},{ }^{84} \mathrm{Se} \text { deduced levels, J, } \pi \text {. } \\
\text { Clara array, Prisma spectrometer. JOUR ZAANE } 25 \mathrm{~s} 01421\end{array}$ \\
\hline${ }^{84} \mathrm{Kr}$ & 2005 SC26 & $\begin{array}{l}\text { ATOMIC MASSES }{ }^{78,80,82,83,84,86} \mathrm{Kr} \text {; measured masses. Penning trap } \\
\text { mass spectrometer. JOUR ZAANE } 25 \text { s01 } 51\end{array}$ \\
\hline${ }^{84} \mathrm{Rb}$ & 2004KA68 & $\begin{array}{l}\text { NUCLEAR REACTIONS }{ }^{85} \mathrm{Rb}(\mathrm{p}, \mathrm{np}),(\mathrm{p}, 2 \mathrm{np}),(\mathrm{p}, 3 \mathrm{np}),(\mathrm{p}, 4 \mathrm{np}), \mathrm{E} \\
\approx 17-100 \mathrm{MeV} \text {; measured excitation functions. Activation technique, } \\
\text { comparison with model predictions. JOUR RAACA } 92449\end{array}$ \\
\hline${ }^{84} \mathrm{Y}$ & 2005DU23 & $\begin{array}{l}\text { NUCLEAR REACTIONS Ge }\left({ }^{18} \mathrm{O}, \mathrm{X}\right){ }^{83 m} \mathrm{Sr} /{ }^{83} \mathrm{Y} /{ }^{84 m} \mathrm{Y} /{ }^{88 m} \mathrm{Y} / \\
{ }^{85} \mathrm{Zr} /{ }^{87} \mathrm{Zr}, \mathrm{E}=82.8 \mathrm{GeV} ;{ }^{84} \mathrm{Se}\left({ }^{18} \mathrm{O}, \mathrm{X}\right){ }^{86 m} \mathrm{Y} /{ }^{85} \mathrm{Zr} /{ }^{87} \mathrm{Nb} /{ }^{87 m} \mathrm{Nb} / \\
{ }^{88} \mathrm{Nb} /{ }^{88} \mathrm{Mo}, \mathrm{E}=82.7 \mathrm{MeV} ;{ }^{124} \mathrm{Sn}\left({ }^{50} \mathrm{Ti}, \mathrm{X}\right){ }^{168 m} \mathrm{Lu} /{ }^{167} \mathrm{Hf} /{ }^{168} \mathrm{Hf}, \\
\mathrm{E}=223.7 \mathrm{MeV} ;{ }^{116} \mathrm{Sn}\left({ }^{50} \mathrm{Ti}, \mathrm{X}\right){ }^{162} \mathrm{Tm} /{ }^{161} \mathrm{Yb} /{ }^{162} \mathrm{Yb} /{ }^{163} \mathrm{Yb} /{ }^{162} \mathrm{Lu} / \\
{ }^{162} \mathrm{Hf}, \mathrm{E}=224.4 \mathrm{MeV} \text {; measured delayed } \mathrm{E} \gamma, \mathrm{I} \gamma \text { following residual } \\
\text { nucleus decay. Physical preseparation technique. JOUR NIMAE } 551 \\
528\end{array}$ \\
\hline
\end{tabular}

20051002 NUCLEAR REACTIONS ${ }^{84} \mathrm{Sr}(\mathrm{p}, \mathrm{n}), \mathrm{E}=13.5 \mathrm{MeV}$; measured $\mathrm{E} \gamma, \mathrm{I} \gamma(\theta$, $\mathrm{H}, \mathrm{t}) .{ }^{84} \mathrm{Y}$ deduced levels, $\mathrm{J}, \pi$, configurations, $\mathrm{g}$ factors, isomeric states $\mathrm{T}_{1 / 2}$. Time-differential perturbed angular distribution method. JOUR PRVCA 72044313

\section{$\mathrm{A}=85$}

${ }^{85} \mathrm{Se} \quad 2005 \mathrm{CIO}$

NUCLEAR REACTIONS ${ }^{2} \mathrm{H}\left({ }^{82} \mathrm{Ge}, \mathrm{p}\right),\left({ }^{84} \mathrm{Se}, \mathrm{p}\right), \mathrm{E}=4 \mathrm{MeV} /$ nucleon; measured Ep, $\sigma(\theta) .{ }^{83} \mathrm{Ge},{ }^{85} \mathrm{Se}$ deduced ground and excited states energies, J, $\pi$. JOUR NIMBE 241200

2005TH12 NUCLEAR REACTIONS ${ }^{2} \mathrm{H}\left({ }^{82} \mathrm{Ge}, \mathrm{p}\right), \mathrm{E}=4 \mathrm{MeV} /$ nucleon; ${ }^{2} \mathrm{H}\left({ }^{84} \mathrm{Se}\right.$, p), $\mathrm{E}=4.5 \mathrm{MeV}$ / nucleon; measured Ep, recoil particle spectrum, proton angular distribution. ${ }^{83} \mathrm{Ge},{ }^{85} \mathrm{Se}$ deduced levels $\mathrm{J}, \pi$, spectroscopic factors. DWBA analysis. JOUR ZAANE 25 s01 371 ${ }^{85} \mathrm{Rb} \quad 2005 \mathrm{KR} 15$ NUCLEAR MOMENTS ${ }^{85,87} \mathrm{Rb}$; measured excited-state hfs. Electromagnetically induced transparency. JOUR EULEE 72221 ${ }^{85} \mathrm{Y} \quad$ 2005KA39 RADIOACTIVITY ${ }^{81 m} \mathrm{Kr}(\mathrm{EC}),(\mathrm{IT}) ;{ }^{81} \mathrm{Y},{ }^{81} \mathrm{Sr},{ }^{85} \mathrm{Nb},{ }^{85} \mathrm{Zr},{ }^{86} \mathrm{Mo}$, ${ }^{86} \mathrm{Nb}(\mathrm{EC})$ [from $\mathrm{Ni},{ }^{54} \mathrm{Fe}\left({ }^{32} \mathrm{~S}, \mathrm{X}\right)$ ]; measured $\mathrm{E} \gamma, \mathrm{I} \gamma, \mathrm{E}(\mathrm{ce}), \mathrm{I}(\mathrm{ce}), \mathrm{T}_{1 / 2}$. ${ }^{81} \mathrm{Kr},{ }^{85} \mathrm{Zr},{ }^{85} \mathrm{Nb}$ deduced isomeric transitions $\mathrm{T}_{1 / 2}$, ICC. ${ }^{85} \mathrm{Zr},{ }^{86} \mathrm{Nb}$ deduced levels, J, $\pi$, ICC. ${ }^{81} \mathrm{Br}$ deduced neutrino capture rate. Astrophysical implications discussed. JOUR ZAANE 25355

2005KA46 RADIOACTIVITY ${ }^{31} \mathrm{Cl}\left(\beta^{+} \mathrm{p}\right)$ [from $\mathrm{S}(\mathrm{p}, \mathrm{X}), \mathrm{E}=40 \mathrm{MeV}$ ]; measured $\beta$-delayed $\mathrm{E} \gamma, \mathrm{Ep} .{ }^{58} \mathrm{Zn}\left(\beta^{+}\right)$[from $\mathrm{Nb}(\mathrm{p}, \mathrm{X}), \mathrm{E}=1.4 \mathrm{GeV}$ ]; measured $\mathrm{E} \gamma, \mathrm{I} \gamma, \beta \gamma$-coin, $\mathrm{T}_{1 / 2} .{ }^{58} \mathrm{Cu}$ deduced levels, $\beta$-feeding intensities. ${ }^{81 m} \mathrm{Kr}(\mathrm{EC}),(\mathrm{IT}) ;{ }^{81} \mathrm{Y},{ }^{81} \mathrm{Sr},{ }^{85} \mathrm{Nb},{ }^{85} \mathrm{Zr},{ }^{86} \mathrm{Mo},{ }^{86} \mathrm{Nb}(\mathrm{EC})$ [from $\mathrm{Ni}$, $\left.{ }^{54} \mathrm{Fe}\left({ }^{32} \mathrm{~S}, \mathrm{X}\right)\right]$; measured $\mathrm{E} \gamma, \mathrm{I} \gamma, \mathrm{E}(\mathrm{ce}), \mathrm{I}(\mathrm{ce}), \mathrm{T}_{1 / 2} \cdot{ }^{81} \mathrm{Kr},{ }^{85} \mathrm{Zr},{ }^{85} \mathrm{Nb}$ deduced isomeric transitions $\mathrm{T}_{1 / 2}$, ICC. ${ }^{85} \mathrm{Zr},{ }^{86} \mathrm{Nb}$ deduced levels, $\mathrm{J}$, $\pi$, ICC. ${ }^{81} \mathrm{Br}$ deduced neutrino capture rate. Mass-separated sources. JOUR ZAANE 25 s01 129 


\section{$\mathrm{A}=85$ (continued)}

${ }^{85} \mathrm{Zr}$

2005DU23

2005KA46

${ }^{85} \mathrm{Nb}$

2005KA39

2005KA39

2005KA46

$2005 \mathrm{SC} 26$

2005SI34

$\begin{array}{ll}{ }^{86} \mathrm{Kr} & 2005 \mathrm{SC} 26 \\ { }^{86} \mathrm{Sr} & 2005 \mathrm{SI} 34\end{array}$

NUCLEAR REACTIONS Ge $\left({ }^{18} \mathrm{O}, \mathrm{X}\right)^{83 m} \mathrm{Sr} /{ }^{83} \mathrm{Y} /{ }^{84 m} \mathrm{Y} /{ }^{88 m} \mathrm{Y} /$ ${ }^{85} \mathrm{Zr} /{ }^{87} \mathrm{Zr}, \mathrm{E}=82.8 \mathrm{GeV} ;{ }^{84} \mathrm{Se}\left({ }^{18} \mathrm{O}, \mathrm{X}\right){ }^{86 m} \mathrm{Y} /{ }^{85} \mathrm{Zr} /{ }^{87} \mathrm{Nb} /{ }^{87 m} \mathrm{Nb} /$ ${ }^{88} \mathrm{Nb} /{ }^{88} \mathrm{Mo}, \mathrm{E}=82.7 \mathrm{MeV} ;{ }^{124} \mathrm{Sn}\left({ }^{50} \mathrm{Ti}, \mathrm{X}\right){ }^{168 m} \mathrm{Lu} /{ }^{167} \mathrm{Hf} /{ }^{168} \mathrm{Hf}$, $\mathrm{E}=223.7 \mathrm{MeV} ;{ }^{116} \mathrm{Sn}\left({ }^{50} \mathrm{Ti}, \mathrm{X}\right){ }^{162} \mathrm{Tm} /{ }^{161} \mathrm{Yb} /{ }^{162} \mathrm{Yb} /{ }^{163} \mathrm{Yb} /{ }^{162} \mathrm{Lu} /$ ${ }^{162} \mathrm{Hf}, \mathrm{E}=224.4 \mathrm{MeV}$; measured delayed $\mathrm{E} \gamma$, I $\gamma$ following residual nucleus decay. Physical preseparation technique. JOUR NIMAE 551 528

RADIOACTIVITY ${ }^{81 m} \mathrm{Kr}(\mathrm{EC}),(\mathrm{IT}) ;{ }^{81} \mathrm{Y},{ }^{81} \mathrm{Sr},{ }^{85} \mathrm{Nb},{ }^{85} \mathrm{Zr},{ }^{86} \mathrm{Mo}$, ${ }^{86} \mathrm{Nb}(\mathrm{EC})$ [from $\mathrm{Ni},{ }^{54} \mathrm{Fe}\left({ }^{32} \mathrm{~S}, \mathrm{X}\right)$ ]; measured $\mathrm{E} \gamma, \mathrm{I} \gamma, \mathrm{E}(\mathrm{ce}), \mathrm{I}(\mathrm{ce}), \mathrm{T}_{1 / 2}$. ${ }^{81} \mathrm{Kr},{ }^{85} \mathrm{Zr}$, ${ }^{85} \mathrm{Nb}$ deduced isomeric transitions $\mathrm{T}_{1 / 2}$, ICC. ${ }^{85} \mathrm{Zr},{ }^{86} \mathrm{Nb}$ deduced levels, J, $\pi$, ICC. ${ }^{81} \mathrm{Br}$ deduced neutrino capture rate. Astrophysical implications discussed. JOUR ZAANE 25355 RADIOACTIVITY ${ }^{31} \mathrm{Cl}\left(\beta^{+} \mathrm{p}\right)$ [from $\mathrm{S}(\mathrm{p}, \mathrm{X}), \mathrm{E}=40 \mathrm{MeV}$ ]; measured $\beta$-delayed $\mathrm{E} \gamma, \mathrm{Ep} .{ }^{58} \mathrm{Zn}\left(\beta^{+}\right)$[from $\mathrm{Nb}(\mathrm{p}, \mathrm{X}), \mathrm{E}=1.4 \mathrm{GeV}$ ]; measured $\mathrm{E} \gamma, \mathrm{I} \gamma, \beta \gamma$-coin, $\mathrm{T}_{1 / 2} .{ }^{58} \mathrm{Cu}$ deduced levels, $\beta$-feeding intensities. ${ }^{81 m} \mathrm{Kr}$ (EC), (IT); ${ }^{81} \mathrm{Y},{ }^{81} \mathrm{Sr},{ }^{85} \mathrm{Nb},{ }^{85} \mathrm{Zr},{ }^{86} \mathrm{Mo},{ }^{86} \mathrm{Nb}(\mathrm{EC})$ [from Ni, $\left.{ }^{54} \mathrm{Fe}\left({ }^{32} \mathrm{~S}, \mathrm{X}\right)\right]$; measured $\mathrm{E} \gamma, \mathrm{I} \gamma, \mathrm{E}(\mathrm{ce}), \mathrm{I}(\mathrm{ce}), \mathrm{T}_{1 / 2} \cdot{ }^{81} \mathrm{Kr},{ }^{85} \mathrm{Zr},{ }^{85} \mathrm{Nb}$ deduced isomeric transitions $\mathrm{T}_{1 / 2}$, ICC. ${ }^{85} \mathrm{Zr},{ }^{86} \mathrm{Nb}$ deduced levels, J, $\pi$, ICC. ${ }^{81} \mathrm{Br}$ deduced neutrino capture rate. Mass-separated sources. JOUR ZAANE 25 s01 129 RADIOACTIVITY ${ }^{81 m} \mathrm{Kr}(\mathrm{EC}),(\mathrm{IT}) ;{ }^{81} \mathrm{Y},{ }^{81} \mathrm{Sr},{ }^{85} \mathrm{Nb},{ }^{85} \mathrm{Zr},{ }^{86} \mathrm{Mo}$, ${ }^{86} \mathrm{Nb}(\mathrm{EC})$ [from $\mathrm{Ni},{ }^{54} \mathrm{Fe}\left({ }^{32} \mathrm{~S}, \mathrm{X}\right)$ ]; measured $\mathrm{E} \gamma, \mathrm{I} \gamma, \mathrm{E}(\mathrm{ce}), \mathrm{I}(\mathrm{ce}), \mathrm{T}_{1 / 2}$. ${ }^{81} \mathrm{Kr},{ }^{85} \mathrm{Zr},{ }^{85} \mathrm{Nb}$ deduced isomeric transitions $\mathrm{T}_{1 / 2}$, ICC. ${ }^{85} \mathrm{Zr},{ }^{86} \mathrm{Nb}$ deduced levels, J, $\pi$, ICC. ${ }^{81} \mathrm{Br}$ deduced neutrino capture rate. Astrophysical implications discussed. JOUR ZAANE 25355 NUCLEAR REACTIONS ${ }^{54} \mathrm{Fe}\left({ }^{32} \mathrm{~S}, \mathrm{X}\right){ }^{81} \mathrm{Zr} /{ }^{81} \mathrm{Y} /{ }^{81} \mathrm{Sr} /{ }^{81 m} \mathrm{Kr}$, $\mathrm{E}=150-170 \mathrm{MeV} ; \mathrm{Ni}\left({ }^{32} \mathrm{~S}, \mathrm{X}\right){ }^{85} \mathrm{Nb} /{ }^{85 m} \mathrm{Nb} /{ }^{85 Z r} /{ }^{85 m} \mathrm{Zr} /{ }^{86} \mathrm{Mo} /$ ${ }^{86} \mathrm{Nb}, \mathrm{E}=150-170 \mathrm{MeV}$; measured yields. JOUR ZAANE 25355 RADIOACTIVITY ${ }^{31} \mathrm{Cl}\left(\beta^{+} \mathrm{p}\right)$ [from $\mathrm{S}(\mathrm{p}, \mathrm{X}), \mathrm{E}=40 \mathrm{MeV}$ ]; measured $\beta$-delayed $\mathrm{E} \gamma, \mathrm{Ep} .{ }^{58} \mathrm{Zn}\left(\beta^{+}\right)$[from $\mathrm{Nb}(\mathrm{p}, \mathrm{X}), \mathrm{E}=1.4 \mathrm{GeV}$ ]; measured $\mathrm{E} \gamma, \mathrm{I} \gamma, \beta \gamma$-coin, $\mathrm{T}_{1 / 2} .{ }^{58} \mathrm{Cu}$ deduced levels, $\beta$-feeding intensities. ${ }^{81 m} \mathrm{Kr}(\mathrm{EC}),(\mathrm{IT}) ;{ }^{81} \mathrm{Y},{ }^{81} \mathrm{Sr},{ }^{85} \mathrm{Nb},{ }^{85} \mathrm{Zr},{ }^{86} \mathrm{Mo},{ }^{86} \mathrm{Nb}(\mathrm{EC})$ [from $\mathrm{Ni}$, $\left.{ }^{54} \mathrm{Fe}\left({ }^{32} \mathrm{~S}, \mathrm{X}\right)\right]$; measured $\mathrm{E} \gamma, \mathrm{I} \gamma, \mathrm{E}(\mathrm{ce}), \mathrm{I}(\mathrm{ce}), \mathrm{T}_{1 / 2} \cdot{ }^{81} \mathrm{Kr},{ }^{85} \mathrm{Zr},{ }^{85} \mathrm{Nb}$ deduced isomeric transitions $\mathrm{T}_{1 / 2}$, ICC. ${ }^{85} \mathrm{Zr},{ }^{86} \mathrm{Nb}$ deduced levels, J, $\pi$, ICC. ${ }^{81} \mathrm{Br}$ deduced neutrino capture rate. Mass-separated sources. JOUR ZAANE 25 s01 129

\section{$\mathrm{A}=86$}

ATOMIC MASSES $78,80,82,83,84,86 \mathrm{Kr}$; measured masses. Penning trap mass spectrometer. JOUR ZAANE 25 s01 51

ATOMIC MASSES $76,77,80,81,86,88 \mathrm{Sr},{ }^{124,129,130,131,132} \mathrm{Sn}$; measured masses. Penning trap mass spectrometer, comparison with previous results. JOUR NUPAB 76345 


\section{$A=86$ (continued)}

\begin{tabular}{|c|c|c|}
\hline${ }^{86} \mathrm{Y}$ & 2005DU23 & $\begin{array}{l}\text { NUCLEAR REACTIONS Ge }\left({ }^{18} \mathrm{O}, \mathrm{X}\right){ }^{83 m} \mathrm{Sr} /{ }^{83} \mathrm{Y} /{ }^{84 m} \mathrm{Y} /{ }^{88 m} \mathrm{Y} / \\
{ }^{85} \mathrm{Zr} /{ }^{87} \mathrm{Zr}, \mathrm{E}=82.8 \mathrm{GeV} ;{ }^{84} \mathrm{Se}\left({ }^{18} \mathrm{O}, \mathrm{X}\right){ }^{86 m} \mathrm{Y} /{ }^{85} \mathrm{Zr} /{ }^{87} \mathrm{Nb} /{ }^{87 m} \mathrm{Nb} / \\
{ }^{88} \mathrm{Nb} /{ }^{88} \mathrm{Mo}, \mathrm{E}=82.7 \mathrm{MeV} ;{ }^{124} \mathrm{Sn}\left({ }^{50} \mathrm{Ti}, \mathrm{X}\right){ }^{168 m} \mathrm{Lu} /{ }^{167} \mathrm{Hf} /{ }^{168} \mathrm{Hf}, \\
\mathrm{E}=223.7 \mathrm{MeV} ;{ }^{116} \mathrm{Sn}\left({ }^{50} \mathrm{Ti}, \mathrm{X}\right){ }^{162} \mathrm{Tm} /{ }^{161} \mathrm{Yb} /{ }^{162} \mathrm{Yb} /{ }^{163} \mathrm{Yb} /{ }^{162} \mathrm{Lu} / \\
{ }^{162} \mathrm{Hf}, \mathrm{E}=224.4 \mathrm{MeV} \text {; measured delayed } \mathrm{E} \gamma, \mathrm{I} \gamma \text { following residual } \\
\text { nucleus decay. Physical preseparation technique. JOUR NIMAE } 551 \\
528\end{array}$ \\
\hline \multirow[t]{3}{*}{${ }^{86} \mathrm{Zr}$} & 2005 BI25 & $\begin{array}{l}\text { NUCLEAR MOMENTS } 86,87,88,89,90,91,92,93,94,95,96,97,98,99,100,101,102 \mathrm{Zr} \text {; } \\
\text { measured charge radii. }{ }^{176} \mathrm{Yb} \text {; measured isomer shift. Ion-beam cooler, } \\
\text { laser spectroscopy. JOUR ZAANE } 25 \text { s01 } 187\end{array}$ \\
\hline & 2005KA39 & $\begin{array}{l}\text { RADIOACTIVITY }{ }^{81 m} \mathrm{Kr}(\mathrm{EC}),(\mathrm{IT}) ;{ }^{81} \mathrm{Y},{ }^{81} \mathrm{Sr},{ }^{85} \mathrm{Nb},{ }^{85} \mathrm{Zr},{ }^{86} \mathrm{Mo} \text {, } \\
\left.{ }^{86} \mathrm{Nb}(\mathrm{EC}) \text { [from Ni, }{ }^{54} \mathrm{Fe}\left({ }^{32} \mathrm{~S}, \mathrm{X}\right)\right] ; \text { measured } \mathrm{E} \gamma, \mathrm{I} \gamma, \mathrm{E}(\mathrm{ce}), \mathrm{I}(\mathrm{ce}), \mathrm{T}_{1 / 2} \text {. } \\
{ }^{81} \mathrm{Kr},{ }^{85} \mathrm{Zr},{ }^{85} \mathrm{Nb} \text { deduced isomeric transitions } \mathrm{T}_{1 / 2}, \mathrm{ICC} .{ }^{85} \mathrm{Zr},{ }^{86} \mathrm{Nb} \\
\text { deduced levels, J, } \pi \text {, ICC. }{ }^{81} \mathrm{Br} \text { deduced neutrino capture rate. } \\
\text { Astrophysical implications discussed. JOUR ZAANE } 25355\end{array}$ \\
\hline & $2005 K A 46$ & $\begin{array}{l}\text { RADIOACTIVITY }{ }^{31} \mathrm{Cl}\left(\beta^{+} \mathrm{p}\right)[\text { from } \mathrm{S}(\mathrm{p}, \mathrm{X}), \mathrm{E}=40 \mathrm{MeV}] ; \text { measured } \\
\left.\beta \text {-delayed } \mathrm{E} \gamma, \mathrm{Ep} .{ }^{58} \mathrm{Zn}\left(\beta^{+}\right) \text {[from } \mathrm{Nb}(\mathrm{p}, \mathrm{X}), \mathrm{E}=1.4 \mathrm{GeV}\right] ; \text { measured } \\
\mathrm{E} \gamma, \mathrm{I} \gamma, \beta \gamma \text {-coin, } \mathrm{T}_{1 / 2} \cdot{ }^{58} \mathrm{Cu} \text { deduced levels, } \beta \text {-feeding intensities. } \\
{ }^{81 m} \mathrm{Kr}(\mathrm{EC}),(\mathrm{IT}) ;{ }^{81} \mathrm{Y},{ }^{81} \mathrm{Sr},{ }^{85} \mathrm{Nb},{ }^{85} \mathrm{Zr},{ }^{86} \mathrm{Mo},{ }^{86} \mathrm{Nb}(\mathrm{EC})[\text { from } \mathrm{Ni} \text {, } \\
\left.{ }^{54} \mathrm{Fe}\left({ }^{32} \mathrm{~S}, \mathrm{X}\right)\right] ; \text { measured } \mathrm{E} \gamma, \mathrm{I} \gamma, \mathrm{E}(\mathrm{ce}), \mathrm{I}(\mathrm{ce}), \mathrm{T}_{1 / 2} \cdot{ }^{81} \mathrm{Kr},{ }^{85} \mathrm{Zr},{ }^{85} \mathrm{Nb} \\
\text { deduced isomeric transitions } \mathrm{T}_{1 / 2}, \mathrm{ICC} .{ }^{85} \mathrm{Zr},{ }^{86} \mathrm{Nb} \text { deduced levels, J, } \\
\pi, \text { ICC. }{ }^{81} \mathrm{Br} \text { deduced neutrino capture rate. Mass-separated sources. } \\
\text { JOUR ZAANE } 25 \text { s01 } 129\end{array}$ \\
\hline \multirow[t]{2}{*}{${ }^{86} \mathrm{Nb}$} & 2005KA39 & $\begin{array}{l}\text { RADIOACTIVITY }{ }^{81 m} \mathrm{Kr}(\mathrm{EC}),(\mathrm{IT}) ;{ }^{81} \mathrm{Y},{ }^{81} \mathrm{Sr},{ }^{85} \mathrm{Nb},{ }^{85} \mathrm{Zr},{ }^{86} \mathrm{Mo} \text {, } \\
{ }^{86} \mathrm{Nb}(\mathrm{EC})\left[\text { from Ni, }{ }^{54} \mathrm{Fe}\left({ }^{32} \mathrm{~S}, \mathrm{X}\right)\right] ; \text { measured } \mathrm{E} \gamma, \mathrm{I} \gamma, \mathrm{E}(\mathrm{ce}), \mathrm{I}(\mathrm{ce}), \mathrm{T}_{1 / 2} \text {. } \\
{ }^{81} \mathrm{Kr},{ }^{85} \mathrm{Zr},{ }^{85} \mathrm{Nb} \text { deduced isomeric transitions } \mathrm{T}_{1 / 2}, \mathrm{ICC} .{ }^{85} \mathrm{Zr},{ }^{86} \mathrm{Nb} \\
\text { deduced levels, J, } \pi \text {, ICC. }{ }^{81} \mathrm{Br} \text { deduced neutrino capture rate. } \\
\text { Astrophysical implications discussed. JOUR ZAANE } 25355\end{array}$ \\
\hline & $2005 K A 46$ & $\begin{array}{l}\left.\text { RADIOACTIVITY }{ }^{31} \mathrm{Cl}\left(\beta^{+} \mathrm{p}\right) \text { [from } \mathrm{S}(\mathrm{p}, \mathrm{X}), \mathrm{E}=40 \mathrm{MeV}\right] ; \text { measured } \\
\left.\beta \text {-delayed E } \gamma, \mathrm{Ep} .{ }^{58} \mathrm{Zn}\left(\beta^{+}\right) \text {[from } \mathrm{Nb}(\mathrm{p}, \mathrm{X}), \mathrm{E}=1.4 \mathrm{GeV}\right] ; \text { measured } \\
\mathrm{E} \gamma, \mathrm{I} \gamma, \beta \gamma \text {-coin, } \mathrm{T}_{1 / 2} \cdot{ }^{58} \mathrm{Cu} \text { deduced levels, } \beta \text {-feeding intensities. } \\
{ }^{81 m} \mathrm{Kr}(\mathrm{EC}),(\mathrm{IT}) ;{ }^{81} \mathrm{Y},{ }^{81} \mathrm{Sr},{ }^{85} \mathrm{Nb},{ }^{85} \mathrm{Zr},{ }^{86} \mathrm{Mo},{ }^{86} \mathrm{Nb}(\mathrm{EC})[\text { from } \mathrm{Ni} \text {, } \\
\left.{ }^{54} \mathrm{Fe}\left({ }^{32} \mathrm{~S}, \mathrm{X}\right)\right] ; \text { measured } \mathrm{E} \gamma, \mathrm{I} \gamma, \mathrm{E}(\mathrm{ce}), \mathrm{I}(\mathrm{ce}), \mathrm{T}_{1 / 2} \cdot{ }^{81} \mathrm{Kr},{ }^{85} \mathrm{Zr},{ }^{85} \mathrm{Nb} \\
\text { deduced isomeric transitions } \mathrm{T}_{1 / 2}, \mathrm{ICC} .{ }^{85} \mathrm{Zr},{ }^{86} \mathrm{Nb} \text { deduced levels, J, } \\
\pi, \text { ICC. }{ }^{81} \mathrm{Br} \text { deduced neutrino capture rate. Mass-separated sources. } \\
\text { JOUR ZAANE } 25 \text { s01 } 129\end{array}$ \\
\hline \multirow[t]{2}{*}{${ }^{86} \mathrm{Mo}$} & 2005KA39 & $\begin{array}{l}\text { RADIOACTIVITY }{ }^{81 m} \mathrm{Kr}(\mathrm{EC}),(\mathrm{IT}) ;{ }^{81} \mathrm{Y},{ }^{81} \mathrm{Sr},{ }^{85} \mathrm{Nb},{ }^{85} \mathrm{Zr},{ }^{86} \mathrm{Mo} \text {, } \\
{ }^{86} \mathrm{Nb}(\mathrm{EC})\left[\text { from Ni},{ }^{54} \mathrm{Fe}\left({ }^{32} \mathrm{~S}, \mathrm{X}\right)\right] ; \text { measured } \mathrm{E} \gamma, \mathrm{I} \gamma, \mathrm{E}(\mathrm{ce}), \mathrm{I}(\mathrm{ce}), \mathrm{T}_{1 / 2} \text {. } \\
\text { de } \mathrm{Kr},{ }^{85} \mathrm{Zr},{ }^{85} \mathrm{Nb} \text { deduced isomeric transitions } \mathrm{T}_{1 / 2}, \mathrm{ICC} .{ }^{85} \mathrm{Zr},{ }^{86} \mathrm{Nb} \\
\text { deduced levels, J, } \pi \text {, ICC. }{ }^{81} \mathrm{Br} \text { deduced neutrino capture rate. } \\
\text { Astrophysical implications discussed. JOUR ZAANE } 25355\end{array}$ \\
\hline & $2005 \mathrm{KA} 46$ & $\begin{array}{l}\left.\text { RADIOACTIVITY }{ }^{31} \mathrm{Cl}\left(\beta^{+} \mathrm{p}\right) \text { [from } \mathrm{S}(\mathrm{p}, \mathrm{X}), \mathrm{E}=40 \mathrm{MeV}\right] ; \text { measured } \\
\left.\beta \text {-delayed } \mathrm{E} \gamma, \mathrm{Ep} .{ }^{58} \mathrm{Zn}\left(\beta^{+}\right) \text {[from } \mathrm{Nb}(\mathrm{p}, \mathrm{X}), \mathrm{E}=1.4 \mathrm{GeV}\right] ; \text { measured } \\
\mathrm{E} \gamma, \mathrm{I} \gamma, \beta \gamma \text {-coin, } \mathrm{T}_{1 / 2} \cdot{ }^{58} \mathrm{Cu} \text { deduced levels, } \beta \text {-feeding intensities. } \\
{ }^{81 m} \mathrm{Kr}(\mathrm{EC}),(\mathrm{IT}) ;{ }^{81} \mathrm{Y},{ }^{81} \mathrm{Sr},{ }^{85} \mathrm{Nb},{ }^{85} \mathrm{Zr},{ }^{86} \mathrm{Mo},{ }^{86} \mathrm{Nb}(\mathrm{EC})[\text { from } \mathrm{Ni} \text {, } \\
\left.{ }^{54} \mathrm{Fe}\left({ }^{32} \mathrm{~S}, \mathrm{X}\right)\right] ; \text { measured } \mathrm{E} \gamma, \mathrm{I} \gamma, \mathrm{E}(\mathrm{ce}), \mathrm{I}(\mathrm{ce}), \mathrm{T}_{1 / 2} \cdot{ }^{81} \mathrm{Kr},{ }^{85} \mathrm{Zr},{ }^{85} \mathrm{Nb} \\
\text { deduced isomeric transitions } \mathrm{T}_{1 / 2}, \mathrm{ICC} .{ }^{85} \mathrm{Zr},{ }^{86} \mathrm{Nb} \text { deduced levels, J, } \\
\pi, \text { ICC. }{ }^{81} \mathrm{Br} \text { deduced neutrino capture rate. Mass-separated sources. } \\
\text { JOUR ZAANE } 25 \text { s01 } 129\end{array}$ \\
\hline
\end{tabular}




\section{$\mathrm{A}=87$}

\begin{tabular}{|c|c|c|}
\hline${ }^{87} \mathrm{Rb}$ & 2005KR15 & $\begin{array}{l}\text { NUCLEAR MOMENTS }{ }^{85,87} \mathrm{Rb} \text {; measured excited-state hfs. } \\
\text { Electromagnetically induced transparency. JOUR EULEE } 72221\end{array}$ \\
\hline${ }^{87} \mathrm{Zr}$ & 2005BI25 & $\begin{array}{l}\text { NUCLEAR MOMENTS }{ }^{86,87,88,89,90,91,92,93,94,95,96,97,98,99,100,101,102} \mathrm{Zr} \text {; } \\
\text { measured charge radii. }{ }^{176} \mathrm{Yb} \text {; measured isomer shift. Ion-beam cooler, } \\
\text { laser spectroscopy. JOUR ZAANE } 25 \text { s01 } 187\end{array}$ \\
\hline & 2005DU23 & $\begin{array}{l}\text { NUCLEAR REACTIONS Ge }\left({ }^{18} \mathrm{O}, \mathrm{X}\right){ }^{83 m} \mathrm{Sr} /{ }^{83} \mathrm{Y} /{ }^{84 m} \mathrm{Y} /{ }^{88 m} \mathrm{Y} / \\
{ }^{85} \mathrm{Zr} /{ }^{87} \mathrm{Zr}, \mathrm{E}=82.8 \mathrm{GeV} ;{ }^{84} \mathrm{Se}\left({ }^{18} \mathrm{O}, \mathrm{X}\right){ }^{86 m} \mathrm{Y} /{ }^{85} \mathrm{Zr} /{ }^{87} \mathrm{Nb} /{ }^{87 m} \mathrm{Nb} / \\
{ }^{88} \mathrm{Nb} /{ }^{88} \mathrm{Mo}, \mathrm{E}=82.7 \mathrm{MeV} ;{ }^{124} \mathrm{Sn}\left({ }^{50} \mathrm{Ti}, \mathrm{X}\right){ }^{168 m} \mathrm{Lu} /{ }^{167} \mathrm{Hf} /{ }^{168} \mathrm{Hf} \\
\mathrm{E}=223.7 \mathrm{MeV} ;{ }^{116} \mathrm{Sn}\left({ }^{50} \mathrm{Ti}, \mathrm{X}\right){ }^{162} \mathrm{Tm} /{ }^{161} \mathrm{Yb} /{ }^{162} \mathrm{Yb} /{ }^{163} \mathrm{Yb} /{ }^{162} \mathrm{Lu} / \\
{ }^{162} \mathrm{Hf}, \mathrm{E}=224.4 \mathrm{MeV} ; \text { measured delayed } \mathrm{E} \gamma, \mathrm{I} \gamma \text { following residual } \\
\text { nucleus decay. Physical preseparation technique. JOUR NIMAE } 551 \\
528\end{array}$ \\
\hline${ }^{87} \mathrm{Nb}$ & 2005DU23 & $\begin{array}{l}\text { NUCLEAR REACTIONS Ge }\left({ }^{18} \mathrm{O}, \mathrm{X}\right){ }^{83 m} \mathrm{Sr} /{ }^{83} \mathrm{Y} /{ }^{84 m} \mathrm{Y} /{ }^{88 m} \mathrm{Y} / \\
{ }^{85} \mathrm{Zr} /{ }^{87} \mathrm{Zr}, \mathrm{E}=82.8 \mathrm{GeV} ;{ }^{84} \mathrm{Se}\left({ }^{18} \mathrm{O}, \mathrm{X}\right){ }^{86 m} \mathrm{Y} /{ }^{85} \mathrm{Zr} /{ }^{87} \mathrm{Nb} /{ }^{87 m} \mathrm{Nb} / \\
{ }^{88} \mathrm{Nb} /{ }^{88} \mathrm{Mo}, \mathrm{E}=82.7 \mathrm{MeV} ;{ }^{124} \mathrm{Sn}\left({ }^{50} \mathrm{Ti}, \mathrm{X}\right){ }^{168 m} \mathrm{Lu} /{ }^{167} \mathrm{Hf} /{ }^{168} \mathrm{Hf} \\
\mathrm{E}=223.7 \mathrm{MeV} ;{ }^{116} \mathrm{Sn}\left({ }^{50} \mathrm{Ti}, \mathrm{X}\right){ }^{162} \mathrm{Tm} /{ }^{161} \mathrm{Yb} /{ }^{162} \mathrm{Yb} /{ }^{163} \mathrm{Yb} /{ }^{162} \mathrm{Lu} / \\
{ }^{162} \mathrm{Hf}, \mathrm{E}=224.4 \mathrm{MeV} \text {; measured delayed } \mathrm{E} \gamma, \mathrm{I} \gamma \text { following residual } \\
\text { nucleus decay. Physical preseparation technique. JOUR NIMAE } 551 \\
528\end{array}$ \\
\hline
\end{tabular}

\section{$\mathrm{A}=88$}

${ }^{88} \mathrm{Sr} \quad$ 2005GA44 NUCLEAR REACTIONS ${ }^{208} \mathrm{~Pb}\left({ }^{90} \mathrm{Zr}, \mathrm{X}\right){ }^{90} \mathrm{Zr} /{ }^{92} \mathrm{Zr} /{ }^{88} \mathrm{Sr}, \mathrm{E}=560$ $\mathrm{MeV} ;{ }^{238} \mathrm{U}\left({ }^{64} \mathrm{Ni}, \mathrm{X}\right){ }^{58} \mathrm{Cr}, \mathrm{E}=400 \mathrm{MeV}$; measured $\mathrm{E} \gamma, \mathrm{I} \gamma, \gamma \gamma-$ (recoil) $\gamma$-coin. ${ }^{58} \mathrm{Cr},{ }^{90,92} \mathrm{Zr},{ }^{88} \mathrm{Sr}$ deduced transitions. Clara array, mass separator. JOUR JPGPE 31 S1443

2005SI34 ATOMIC MASSES ${ }^{76,77,80,81,86,88} \mathrm{Sr},{ }^{124,129,130,131,132} \mathrm{Sn}$; measured masses. Penning trap mass spectrometer, comparison with previous results. JOUR NUPAB 76345

${ }^{88} \mathrm{Y} 2005 \mathrm{DU} 23 \quad$ NUCLEAR REACTIONS Ge$\left({ }^{18} \mathrm{O}, \mathrm{X}\right)^{83 m} \mathrm{Sr} /{ }^{83} \mathrm{Y} /{ }^{84 m} \mathrm{Y} /{ }^{88 m} \mathrm{Y} /$ ${ }^{85} \mathrm{Zr} /{ }^{87} \mathrm{Zr}, \mathrm{E}=82.8 \mathrm{GeV} ;{ }^{84} \mathrm{Se}\left({ }^{18} \mathrm{O}, \mathrm{X}\right){ }^{86 m} \mathrm{Y} /{ }^{85} \mathrm{Zr} /{ }^{87} \mathrm{Nb} /{ }^{87 m} \mathrm{Nb} /$ ${ }^{88} \mathrm{Nb} /{ }^{88} \mathrm{Mo}, \mathrm{E}=82.7 \mathrm{MeV} ;{ }^{124} \mathrm{Sn}\left({ }^{50} \mathrm{Ti}, \mathrm{X}\right){ }^{168 m} \mathrm{Lu} /{ }^{167} \mathrm{Hf} /{ }^{168} \mathrm{Hf}$, $\mathrm{E}=223.7 \mathrm{MeV} ;{ }^{116} \mathrm{Sn}\left({ }^{50} \mathrm{Ti}, \mathrm{X}\right){ }^{162} \mathrm{Tm} /{ }^{161} \mathrm{Yb} /{ }^{162} \mathrm{Yb} /{ }^{163} \mathrm{Yb} /{ }^{162} \mathrm{Lu} /$ ${ }^{162} \mathrm{Hf}, \mathrm{E}=224.4 \mathrm{MeV}$; measured delayed $\mathrm{E} \gamma$, I $\gamma$ following residual nucleus decay. Physical preseparation technique. JOUR NIMAE 551 528

${ }^{88} \mathrm{Zr} \quad 2005 B I 25 \quad$ NUCLEAR MOMENTS $86,87,88,89,90,91,92,93,94,95,96,97,98,99,100,101,102 \mathrm{Zr}$; measured charge radii. ${ }^{176} \mathrm{Yb}$; measured isomer shift. Ion-beam cooler, laser spectroscopy. JOUR ZAANE 25 s01 187

2005WA31 NUCLEAR REACTIONS ${ }^{92,98,100} \mathrm{Mo}\left(\gamma, \gamma^{\prime}\right), \mathrm{E}=13.2 \mathrm{MeV}$ bremsstrahlung; measured $\mathrm{E} \gamma, \mathrm{I} \gamma .{ }^{92,100} \mathrm{Mo},{ }^{197} \mathrm{Au}(\gamma, \mathrm{n}),{ }^{92} \mathrm{Mo}(\gamma, \mathrm{p})$, $(\gamma, \alpha), \mathrm{E} \approx 11.8-16.5 \mathrm{MeV}$ bremsstrahlung; measured integrated $\sigma$. JOUR JPGPE 31 S1969 


\section{$\mathrm{A}=88$ (continued)}

\begin{tabular}{|c|c|c|}
\hline${ }^{88} \mathrm{Nb}$ & 2005DU23 & $\begin{array}{l}\left.\text { NUCLEAR REACTIONS Ge( }{ }^{18} \mathrm{O}, \mathrm{X}\right){ }^{83 m} \mathrm{Sr} /{ }^{83} \mathrm{Y} /{ }^{84 m} \mathrm{Y} /{ }^{88 m} \mathrm{Y} / \\
\\
{ }^{85} \mathrm{Zr} /{ }^{87} \mathrm{Zr}, \mathrm{E}=82.8 \mathrm{GeV} ;{ }^{84} \mathrm{Se}\left({ }^{18} \mathrm{O}, \mathrm{X}\right){ }^{86 m} \mathrm{Y} /{ }^{85} \mathrm{Zr} /{ }^{87} \mathrm{Nb} /{ }^{87 m} \mathrm{Nb} / \\
{ }^{88} \mathrm{Nb} /{ }^{88} \mathrm{Mo}, \mathrm{E}=82.7 \mathrm{MeV} ;{ }^{124} \mathrm{Sn}\left({ }^{50} \mathrm{Ti}, \mathrm{X}\right)^{168 m} \mathrm{Lu} /{ }^{167} \mathrm{Hf} /{ }^{168} \mathrm{Hf} \\
\mathrm{E}=223.7 \mathrm{MeV} ;{ }^{116} \mathrm{Sn}\left({ }^{50} \mathrm{Ti}, \mathrm{X}\right){ }^{162} \mathrm{Tm} /{ }^{161} \mathrm{Yb} /{ }^{162} \mathrm{Yb} /{ }^{163} \mathrm{Yb} /{ }^{162} \mathrm{Lu} / \\
{ }^{162} \mathrm{Hf}, \mathrm{E}=224.4 \mathrm{MeV} \text {; measured delayed } \mathrm{E} \gamma, \mathrm{I} \gamma \text { following residual } \\
\text { nucleus decay. Physical preseparation technique. JOUR NIMAE } 551 \\
528\end{array}$ \\
\hline${ }^{88} \mathrm{Mo}$ & 2005DU23 & $\begin{array}{l}\text { NUCLEAR REACTIONS Ge }\left({ }^{18} \mathrm{O}, \mathrm{X}\right){ }^{83 m} \mathrm{Sr} /{ }^{83} \mathrm{Y} /{ }^{84 m} \mathrm{Y} /{ }^{88 m} \mathrm{Y} / \\
{ }^{85} \mathrm{Zr} /{ }^{87} \mathrm{Zr}, \mathrm{E}=82.8 \mathrm{GeV} ;{ }^{84} \mathrm{Se}\left({ }^{18} \mathrm{O}, \mathrm{X}\right){ }^{86 m} \mathrm{Y} /{ }^{85} \mathrm{Zr} /{ }^{87} \mathrm{Nb} /{ }^{87 m} \mathrm{Nb} / \\
{ }^{88} \mathrm{Nb} /{ }^{88} \mathrm{Mo}, \mathrm{E}=82.7 \mathrm{MeV} ;{ }^{124} \mathrm{Sn}\left({ }^{50} \mathrm{Ti}, \mathrm{X}\right){ }^{168 m} \mathrm{Lu} /{ }^{167} \mathrm{Hf} /{ }^{168} \mathrm{Hf}, \\
\mathrm{E}=223.7 \mathrm{MeV} ;{ }^{116} \mathrm{Sn}\left({ }^{50} \mathrm{Ti}, \mathrm{X}\right){ }^{162} \mathrm{Tm} /{ }^{161} \mathrm{Yb} /{ }^{162} \mathrm{Yb} /{ }^{163} \mathrm{Yb} /{ }^{162} \mathrm{Lu} / \\
{ }^{162} \mathrm{Hf}, \mathrm{E}=224.4 \mathrm{MeV} \text {; measured delayed } \mathrm{E} \gamma, \mathrm{I} \gamma \text { following residual } \\
\text { nucleus decay. Physical preseparation technique. JOUR NIMAE } 551 \\
528\end{array}$ \\
\hline
\end{tabular}

\section{$\mathrm{A}=89$}

${ }^{89} \mathrm{Kr} \quad$ 2004GA60

NUCLEAR REACTIONS ${ }^{237} \mathrm{~Np}(\gamma, \mathrm{F}){ }^{135} \mathrm{Xe} /{ }^{137} \mathrm{Xe} /{ }^{138} \mathrm{Xe} /{ }^{139} \mathrm{Xe} /$ ${ }^{140} \mathrm{Xe} /{ }^{141} \mathrm{Xe} /{ }^{142} \mathrm{Xe} /{ }^{89} \mathrm{Kr} /{ }^{91} \mathrm{Kr} /{ }^{92} \mathrm{Kr} /{ }^{93} \mathrm{Kr}, \mathrm{E}=25 \mathrm{MeV}$ bremsstrahlung; measured fission yields, isotopic distribution parameters. Comparison with results from other targets. JOUR BRSPE 681298 2005GA50 NUCLEAR REACTIONS ${ }^{237} \mathrm{~Np},{ }^{243} \mathrm{Am}(\gamma, \mathrm{F}){ }^{135} \mathrm{Xe} /{ }^{137} \mathrm{Xe} /{ }^{138} \mathrm{Xe} /$ ${ }^{139} \mathrm{Xe} /{ }^{140} \mathrm{Xe} /{ }^{141} \mathrm{Xe} /{ }^{142} \mathrm{Xe} /{ }^{89} \mathrm{Kr} /{ }^{91} \mathrm{Kr} /{ }^{92} \mathrm{Kr} /{ }^{93} \mathrm{Kr}, \mathrm{E}=25 \mathrm{MeV}$ bremsstrahlung; measured fission yields, isotopic distribution parameters. JOUR YAFIA 681475

${ }^{89} \mathrm{Sr} \quad 2004 \mathrm{SP} 06$ NUCLEAR REACTIONS ${ }^{64,67} \mathrm{Zn},{ }^{89} \mathrm{Y}(\mathrm{n}, \mathrm{p}), \mathrm{E}=14 \mathrm{MeV}$; measured $\sigma$. Comparison with results using fission neutrons. JOUR RAACA 92183 NUCLEAR MOMENTS 86,87,88,89,90,91,92,93,94,95,96,97,98,99,100,101,102 Zr; measured charge radii. ${ }^{176} \mathrm{Yb}$; measured isomer shift. Ion-beam cooler, laser spectroscopy. JOUR ZAANE 25 s01 187

\section{$\mathrm{A}=\mathbf{9 0}$}

${ }^{90} \mathrm{Zr} \quad 2005 \mathrm{BI} 25$

NUCLEAR MOMENTS 86,87,88,89,90,91,92,93,94,95,96,97,98,99,100,101,102 Zr; measured charge radii. ${ }^{176} \mathrm{Yb}$; measured isomer shift. Ion-beam cooler, laser spectroscopy. JOUR ZAANE 25 s01 187

$2005 \mathrm{CO} 25$ NUCLEAR REACTIONS ${ }^{208} \mathrm{~Pb}\left({ }^{40} \mathrm{Ca},{ }^{42} \mathrm{Ca}\right), \mathrm{E}=225 \mathrm{MeV}$; measured $\sigma(\mathrm{E}, \theta) .{ }^{42} \mathrm{Ca}$ deduced excited states configurations. ${ }^{208} \mathrm{~Pb}\left({ }^{90} \mathrm{Zr}, \mathrm{X}\right)$, $\mathrm{E}=560 \mathrm{MeV}$; measured $\mathrm{E} \gamma, \mathrm{I} \gamma$, (fragment) $\gamma$-coin, isotopic yields for projectile-like fragments. ${ }^{90} \mathrm{Zr}$ deduced transitions. JOUR ZAANE 25 s01 427

2005GA44 NUCLEAR REACTIONS ${ }^{208} \mathrm{~Pb}\left({ }^{90} \mathrm{Zr}, \mathrm{X}\right){ }^{90} \mathrm{Zr} /{ }^{92} \mathrm{Zr} /{ }^{88} \mathrm{Sr}, \mathrm{E}=560$ $\mathrm{MeV} ;{ }^{238} \mathrm{U}\left({ }^{64} \mathrm{Ni}, \mathrm{X}\right){ }^{58} \mathrm{Cr}, \mathrm{E}=400 \mathrm{MeV}$; measured $\mathrm{E} \gamma, \mathrm{I} \gamma, \gamma \gamma-$ (recoil) $\gamma$-coin. ${ }^{58} \mathrm{Cr},{ }^{90,92} \mathrm{Zr},{ }^{88} \mathrm{Sr}$ deduced transitions. Clara array, mass separator. JOUR JPGPE 31 S1443 


\section{$\mathrm{A}=90$ (continued)}

2005VA31 NUCLEAR REACTIONS ${ }^{48} \mathrm{Ti}\left({ }^{132} \mathrm{Sn},{ }^{132} \mathrm{Sn}\right), \mathrm{E}=470-495 \mathrm{MeV}$; ${ }^{90} \mathrm{Zr}\left({ }^{134} \mathrm{Sn},{ }^{134} \mathrm{Sn}\right.$ '), $\mathrm{E}=400 \mathrm{MeV}$; measured $\mathrm{E} \gamma, \mathrm{I} \gamma$, (particle) $\gamma$-coin following projectile Coulomb excitation. ${ }^{132,134} \mathrm{Sn}$ deduced transitions B(E2). JOUR ZAANE 25 s01 391

${ }^{90} \mathrm{Nb} \quad 2004 \mathrm{ZH} 45 \quad$ NUCLEAR REACTIONS ${ }^{90,94} \mathrm{Zr}(\mathrm{p}, \mathrm{n}), \mathrm{E}=7-11 \mathrm{MeV}$; measured En, $\sigma(\mathrm{E}, \theta)$, excitation functions. ${ }^{90,94} \mathrm{Nb}$ deduced level densities, shell effects. Optical-statistical analysis. JOUR BRSPE 681319

2005CH65 NUCLEAR REACTIONS ${ }^{63} \mathrm{Cu}\left({ }^{31} \mathrm{P}, \mathrm{n} 3 \mathrm{p}\right), \mathrm{E}=120,125 \mathrm{MeV}$; measured prompt and delayed $\mathrm{E} \gamma, \mathrm{I} \gamma, \gamma \gamma-$, (recoil) $\gamma$-coin. ${ }^{90} \mathrm{Nb}$ deduced levels, J, $\pi$, configurations, isomeric states $\mathrm{T}_{1 / 2}, \mathrm{~B}(\mathrm{E} 2)$. Large-basis shell model calculations. JOUR PRVCA 72054309

2005CU07 NUCLEAR REACTIONS ${ }^{76} \mathrm{Ge}\left({ }^{19} \mathrm{~F}, 5 \mathrm{n}\right), \mathrm{E}=80 \mathrm{MeV}$; measured $\mathrm{E} \gamma, \mathrm{I} \gamma$, $\gamma \gamma$-coin. ${ }^{90} \mathrm{Nb}$ deduced high-spin levels, $\mathrm{J}, \pi$, configurations. Semi-empirical shell model calculations. JOUR PRVCA 72044322

${ }^{90} \mathrm{Mo} \quad 2005 \mathrm{CL} 08$ ATOMIC MASSES ${ }^{64} \mathrm{Ge},{ }^{68} \mathrm{Se}$; analyzed masses; deduced effective $\mathrm{T}_{1 / 2} \cdot{ }^{90,91} \mathrm{Mo},{ }^{90,91,92,93} \mathrm{Tc},{ }^{93,94} \mathrm{Ru},{ }^{94,95} \mathrm{Rh},{ }^{104,105,106,107} \mathrm{In}$,

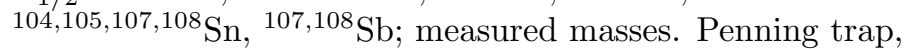
astrophysical implications discussed. JOUR ZAANE 25 s01 629

${ }^{90} \mathrm{Tc} \quad 2005 \mathrm{CL} 08$ ATOMIC MASSES ${ }^{64} \mathrm{Ge},{ }^{68} \mathrm{Se}$; analyzed masses; deduced effective $\mathrm{T}_{1 / 2} .{ }^{90,91} \mathrm{Mo},{ }^{90,91,92,93} \mathrm{Tc},{ }^{93,94} \mathrm{Ru},{ }^{94,95} \mathrm{Rh},{ }^{104,105,106,107} \mathrm{In}$, $104,105,107,108 \mathrm{Sn},{ }^{107,108} \mathrm{Sb}$; measured masses. Penning trap, astrophysical implications discussed. JOUR ZAANE 25 s01 629

\section{$\mathrm{A}=\mathbf{9 1}$}

${ }^{91} \mathrm{Kr} \quad 2004 \mathrm{GA} 60$

NUCLEAR REACTIONS $\left.{ }^{237} \mathrm{~Np}(\gamma, \mathrm{F})\right)^{135} \mathrm{Xe} /{ }^{137} \mathrm{Xe} /{ }^{138} \mathrm{Xe} /{ }^{139} \mathrm{Xe} /$ ${ }^{140} \mathrm{Xe} /{ }^{141} \mathrm{Xe} /{ }^{142} \mathrm{Xe} /{ }^{89} \mathrm{Kr} /{ }^{91} \mathrm{Kr} /{ }^{92} \mathrm{Kr} /{ }^{93} \mathrm{Kr}, \mathrm{E}=25 \mathrm{MeV}$ bremsstrahlung; measured fission yields, isotopic distribution parameters. Comparison with results from other targets. JOUR BRSPE 681298

2005GA50 NUCLEAR REACTIONS ${ }^{237} \mathrm{~Np},{ }^{243} \mathrm{Am}(\gamma, \mathrm{F}){ }^{135} \mathrm{Xe} /{ }^{137} \mathrm{Xe} /{ }^{138} \mathrm{Xe} /$ ${ }^{139} \mathrm{Xe} /{ }^{140} \mathrm{Xe} /{ }^{141} \mathrm{Xe} /{ }^{142} \mathrm{Xe} /{ }^{89} \mathrm{Kr} /{ }^{91} \mathrm{Kr} /{ }^{92} \mathrm{Kr} /{ }^{93} \mathrm{Kr}, \mathrm{E}=25 \mathrm{MeV}$ bremsstrahlung; measured fission yields, isotopic distribution parameters. JOUR YAFIA 681475

${ }^{91} \mathrm{Zr} \quad$ 2005BI25 NUCLEAR MOMENTS 86,87,88,89,90,91,92,93,94,95,96,97,98,99,100,101,102 Zr; measured charge radii. ${ }^{176} \mathrm{Yb}$; measured isomer shift. Ion-beam cooler, laser spectroscopy. JOUR ZAANE 25 s01 187

${ }^{91} \mathrm{Nb}$ 2005WA31 NUCLEAR REACTIONS ${ }^{92,98,100} \mathrm{Mo}\left(\gamma, \gamma^{\prime}\right), \mathrm{E}=13.2 \mathrm{MeV}$ bremsstrahlung; measured $\mathrm{E} \gamma, \mathrm{I} \gamma .{ }^{92,100} \mathrm{Mo},{ }^{197} \mathrm{Au}(\gamma, \mathrm{n}),{ }^{92} \mathrm{Mo}(\gamma, \mathrm{p})$, $(\gamma, \alpha), \mathrm{E} \approx 11.8-16.5 \mathrm{MeV}$ bremsstrahlung; measured integrated $\sigma$. JOUR JPGPE 31 S1969

${ }^{91} \mathrm{Mo} \quad$ 2005CL08 ATOMIC MASSES ${ }^{64} \mathrm{Ge},{ }^{68} \mathrm{Se}$; analyzed masses; deduced effective $\mathrm{T}_{1 / 2} .{ }^{90,91} \mathrm{Mo},{ }^{90,91,92,93} \mathrm{Tc},{ }^{93,94} \mathrm{Ru},{ }^{94,95} \mathrm{Rh},{ }^{104,105,106,107} \mathrm{In}$, $104,105,107,108 \mathrm{Sn},{ }^{107,108} \mathrm{Sb}$; measured masses. Penning trap, astrophysical implications discussed. JOUR ZAANE 25 s01 629 


\section{$\mathrm{A}=91$ (continued)}

${ }^{91} \mathrm{Tc} \quad$ 2005CL08

ATOMIC MASSES ${ }^{64} \mathrm{Ge},{ }^{68} \mathrm{Se}$; analyzed masses; deduced effective $\mathrm{T}_{1 / 2} \cdot{ }^{90,91} \mathrm{Mo},{ }^{90,91,92,93} \mathrm{Tc},{ }^{93,94} \mathrm{Ru},{ }^{94,95} \mathrm{Rh},{ }^{104,105,106,107} \mathrm{In}$, $104,105,107,108$ Sn, ${ }^{107,108}$ Sb; measured masses. Penning trap, astrophysical implications discussed. JOUR ZAANE 25 s01 629

\section{$\mathrm{A}=92$}

${ }^{92} \mathrm{Kr} \quad$ 2004GA60

NUCLEAR REACTIONS ${ }^{237} \mathrm{~Np}(\gamma, \mathrm{F}){ }^{135} \mathrm{Xe} /{ }^{137} \mathrm{Xe} /{ }^{138} \mathrm{Xe} /{ }^{139} \mathrm{Xe} /$ ${ }^{140} \mathrm{Xe} /{ }^{141} \mathrm{Xe} /{ }^{142} \mathrm{Xe} /{ }^{89} \mathrm{Kr} /{ }^{91} \mathrm{Kr} /{ }^{92} \mathrm{Kr} /{ }^{93} \mathrm{Kr}, \mathrm{E}=25 \mathrm{MeV}$ bremsstrahlung; measured fission yields, isotopic distribution parameters. Comparison with results from other targets. JOUR BRSPE 681298 2005GA50 NUCLEAR REACTIONS ${ }^{237} \mathrm{~Np},{ }^{243} \mathrm{Am}(\gamma, \mathrm{F}){ }^{135} \mathrm{Xe} /{ }^{137} \mathrm{Xe} /{ }^{138} \mathrm{Xe} /$ ${ }^{139} \mathrm{Xe} /{ }^{140} \mathrm{Xe} /{ }^{141} \mathrm{Xe} /{ }^{142} \mathrm{Xe} /{ }^{89} \mathrm{Kr} /{ }^{91} \mathrm{Kr} /{ }^{92} \mathrm{Kr} /{ }^{93} \mathrm{Kr}, \mathrm{E}=25 \mathrm{MeV}$ bremsstrahlung; measured fission yields, isotopic distribution parameters. JOUR YAFIA 681475

${ }^{92} \mathrm{Sr} \quad 2005$ GU37
ATOMIC MASSES ${ }^{56,57} \mathrm{Mn},{ }^{82 m} \mathrm{Rb},{ }^{92} \mathrm{Sr},{ }^{124,127} \mathrm{Cs},{ }^{130} \mathrm{Ba}$; measured masses. Penning trap mass spectrometer. JOUR ZAANE 25 s01 35 NUCLEAR MOMENTS 86,87,88,89,90,91,92,93,94,95,96,97,98,99,100,101,102 Zr; measured charge radii. ${ }^{176} \mathrm{Yb}$; measured isomer shift. Ion-beam cooler, laser spectroscopy. JOUR ZAANE 25 s01 187

2005GA44 NUCLEAR REACTIONS ${ }^{208} \mathrm{~Pb}\left({ }^{90} \mathrm{Zr}, \mathrm{X}\right){ }^{90} \mathrm{Zr} /{ }^{92} \mathrm{Zr} /{ }^{88} \mathrm{Sr}, \mathrm{E}=560$ $\mathrm{MeV} ;{ }^{238} \mathrm{U}\left({ }^{64} \mathrm{Ni}, \mathrm{X}\right){ }^{58} \mathrm{Cr}, \mathrm{E}=400 \mathrm{MeV}$; measured $\mathrm{E} \gamma, \mathrm{I} \gamma, \gamma \gamma-$, (recoil) $\gamma$-coin. ${ }^{58} \mathrm{Cr},{ }^{90,92} \mathrm{Zr},{ }^{88} \mathrm{Sr}$ deduced transitions. Clara array, mass separator. JOUR JPGPE 31 S1443

${ }^{92} \mathrm{Mo} \quad$ 2005RUZZ

NUCLEAR REACTIONS ${ }^{92,98,100} \mathrm{Mo}\left(\gamma, \gamma^{\prime}\right), \mathrm{E}=14 \mathrm{MeV}$

bremsstrahlung; measured $\mathrm{E} \gamma, \mathrm{I} \gamma .{ }^{92,98,100}$ Mo deduced dipole strength functions, resonance features. PREPRINT nucl-ex/0512027,12/20/2005

2005WA31 NUCLEAR REACTIONS ${ }^{92,98,100} \mathrm{Mo}\left(\gamma, \gamma^{\prime}\right), \mathrm{E}=13.2 \mathrm{MeV}$ bremsstrahlung; measured $\mathrm{E} \gamma, \mathrm{I} \gamma .{ }^{92,100} \mathrm{Mo},{ }^{197} \mathrm{Au}(\gamma, \mathrm{n}),{ }^{92} \mathrm{Mo}(\gamma, \mathrm{p})$, $(\gamma, \alpha), \mathrm{E} \approx 11.8-16.5 \mathrm{MeV}$ bremsstrahlung; measured integrated $\sigma$. JOUR JPGPE 31 S1969

${ }^{92} \mathrm{Tc} \quad$ 2005CL08 ATOMIC MASSES ${ }^{64} \mathrm{Ge},{ }^{68} \mathrm{Se}$; analyzed masses; deduced effective $\mathrm{T}_{1 / 2} \cdot{ }^{90,91} \mathrm{Mo},{ }^{90,91,92,93} \mathrm{Tc},{ }^{93,94} \mathrm{Ru},{ }^{94,95} \mathrm{Rh},{ }^{104,105,106,107} \mathrm{In}$, ${ }_{104,105,107,108} \mathrm{Sn},{ }^{107,108} \mathrm{Sb}$; measured masses. Penning trap, astrophysical implications discussed. JOUR ZAANE 25 s01 629

\section{$\mathrm{A}=93$}

${ }^{93} \mathrm{Kr} \quad$ 2004GA60

NUCLEAR REACTIONS ${ }^{237} \mathrm{~Np}(\gamma, \mathrm{F}){ }^{135} \mathrm{Xe} /{ }^{137} \mathrm{Xe} /{ }^{138} \mathrm{Xe} /{ }^{139} \mathrm{Xe} /$ ${ }^{140} \mathrm{Xe} /{ }^{141} \mathrm{Xe} /{ }^{142} \mathrm{Xe} /{ }^{89} \mathrm{Kr} /{ }^{91} \mathrm{Kr} /{ }^{92} \mathrm{Kr} /{ }^{93} \mathrm{Kr}, \mathrm{E}=25 \mathrm{MeV}$ bremsstrahlung; measured fission yields, isotopic distribution parameters. Comparison with results from other targets. JOUR BRSPE 681298 


\section{$\mathrm{A}=93$ (continued)}

2005GA50 NUCLEAR REACTIONS ${ }^{237} \mathrm{~Np},{ }^{243} \mathrm{Am}(\gamma, \mathrm{F}){ }^{135} \mathrm{Xe} /{ }^{137} \mathrm{Xe} /{ }^{138} \mathrm{Xe} /$ ${ }^{139} \mathrm{Xe} /{ }^{140} \mathrm{Xe} /{ }^{141} \mathrm{Xe} /{ }^{142} \mathrm{Xe} /{ }^{89} \mathrm{Kr} /{ }^{91} \mathrm{Kr} /{ }^{92} \mathrm{Kr} /{ }^{93} \mathrm{Kr}, \mathrm{E}=25 \mathrm{MeV}$ bremsstrahlung; measured fission yields, isotopic distribution parameters. JOUR YAFIA 681475

${ }^{93} \mathrm{Zr} \quad 2005 \mathrm{BI} 25$ NUCLEAR MOMENTS 86,87,88,89,90,91,92,93,94,95,96,97,98,99,100,101,102 Zr; measured charge radii. ${ }^{176} \mathrm{Yb}$; measured isomer shift. Ion-beam cooler, laser spectroscopy. JOUR ZAANE 25 s01 187

${ }^{93} \mathrm{Nb} \quad 2005 \mathrm{MC} 13$
NUCLEAR REACTIONS ${ }^{93} \mathrm{Nb}\left(\mathrm{n}, \mathrm{n}\right.$ '), $\mathrm{E}=1.5-2.6 \mathrm{MeV} ;{ }^{94} \mathrm{Zr}(\mathrm{p}, 2 \mathrm{n})$, $\mathrm{E}=11.5-19 \mathrm{MeV}$; measured $\mathrm{E} \gamma, \mathrm{I} \gamma$, DSA, branching ratios, excitation functions. ${ }^{93} \mathrm{Nb}$ deduced levels $\mathrm{J}, \pi, \mathrm{T}_{1 / 2}$, mixed-symmetry states. JOUR ZAANE 25 s01 377
${ }^{93} \mathrm{Tc} \quad$ 2005CL08 ATOMIC MASSES ${ }^{64} \mathrm{Ge},{ }^{68} \mathrm{Se}$; analyzed masses; deduced effective $\mathrm{T}_{1 / 2} \cdot{ }^{90,91} \mathrm{Mo},{ }^{90,91,92,93} \mathrm{Tc},{ }^{93,94} \mathrm{Ru},{ }^{94,95} \mathrm{Rh},{ }^{104,105,106,107} \mathrm{In}$, ${ }_{104,105,107,108} \mathrm{Sn},{ }^{107,108} \mathrm{Sb}$; measured masses. Penning trap, astrophysical implications discussed. JOUR ZAANE 25 s01 629

2005NA43 RADIOACTIVITY ${ }^{93 m} \mathrm{Tc}$ (IT) [from ${ }^{45} \mathrm{Sc}\left({ }^{52} \mathrm{Cr}, 2 \mathrm{n} 2 \mathrm{p}\right)$ ]; measured $\gamma$-ray anisotropies from oriented source; deduced parity-nonconserving matrix element. JOUR ZAANE 25 s01 703

${ }^{93} \mathrm{Ru} \quad$ 2005CL08 ATOMIC MASSES ${ }^{64} \mathrm{Ge},{ }^{68} \mathrm{Se}$; analyzed masses; deduced effective $\mathrm{T}_{1 / 2} \cdot{ }^{90,91} \mathrm{Mo},{ }^{90,91,92,93} \mathrm{Tc},{ }^{93,94} \mathrm{Ru},{ }^{94,95} \mathrm{Rh},{ }^{104,105,106,107} \mathrm{In}$, ${ }_{104,105,107,108} \mathrm{Sn},{ }^{107,108} \mathrm{Sb}$; measured masses. Penning trap, astrophysical implications discussed. JOUR ZAANE 25 s01 629

\section{$\mathrm{A}=94$}

${ }^{94} \mathrm{Zr} \quad 2005 \mathrm{BI} 25$

NUCLEAR MOMENTS 86,87,88,89,90,91,92,93,94,95,96,97,98,99,100,101,102Zr; measured charge radii. ${ }^{176} \mathrm{Yb}$; measured isomer shift. Ion-beam cooler, laser spectroscopy. JOUR ZAANE 25 s01 187

${ }^{94} \mathrm{Nb} 2004 \mathrm{ZH} 45 \quad$ NUCLEAR REACTIONS ${ }^{90,94} \mathrm{Zr}(\mathrm{p}, \mathrm{n}), \mathrm{E}=7-11 \mathrm{MeV}$; measured En, $\sigma(\mathrm{E}, \theta)$, excitation functions. ${ }^{90,94} \mathrm{Nb}$ deduced level densities, shell effects. Optical-statistical analysis. JOUR BRSPE 681319

${ }^{94} \mathrm{Ru} \quad$ 2005CL08 ATOMIC MASSES ${ }^{64} \mathrm{Ge},{ }^{68} \mathrm{Se}$; analyzed masses; deduced effective $\mathrm{T}_{1 / 2} \cdot{ }^{90,91} \mathrm{Mo},{ }^{90,91,92,93} \mathrm{Tc},{ }^{93,94} \mathrm{Ru},{ }^{94,95} \mathrm{Rh},{ }^{104,105,106,107} \mathrm{In}$, $104,105,107,108 \mathrm{Sn},{ }^{107,108} \mathrm{Sb}$; measured masses. Penning trap, astrophysical implications discussed. JOUR ZAANE 25 s01 629

${ }^{94} \mathrm{Rh} \quad$ 2005CL08 ATOMIC MASSES ${ }^{64} \mathrm{Ge},{ }^{68} \mathrm{Se}$; analyzed masses; deduced effective $\mathrm{T}_{1 / 2} .{ }^{90,91} \mathrm{Mo},{ }^{90,91,92,93} \mathrm{Tc},{ }^{93,94} \mathrm{Ru},{ }^{94,95} \mathrm{Rh},{ }^{104,105,106,107} \mathrm{In}$, $104,105,107,108$ Sn, ${ }^{107,108} \mathrm{Sb}$; measured masses. Penning trap, astrophysical implications discussed. JOUR ZAANE 25 s01 629

\section{$\mathrm{A}=95$}

${ }^{95} \mathrm{Sr} \quad 2005 \mathrm{HW} 06 \quad$ RADIOACTIVITY ${ }^{252} \mathrm{Cf}(\mathrm{SF})$; measured $\mathrm{E} \gamma, \mathrm{I} \gamma, \gamma \gamma$-coin. ${ }^{95,97} \mathrm{Sr},{ }^{99} \mathrm{Zr}$, ${ }^{108} \mathrm{Tc},{ }^{133,134} \mathrm{Te},{ }^{137} \mathrm{Xe}$ levels deduced $\mathrm{T}_{1 / 2}$. Gammasphere array, time-gated triple-coincidence method. JOUR ZAANE 25 s01 463 


\section{$\mathrm{A}=95$ (continued)}

$\begin{array}{ll}{ }^{95} \mathrm{Zr} & \text { 2005BI25 } \\ { }^{95} \mathrm{Nb} & \text { 2005RA30 } \\ { }^{95} \mathrm{Rh} & \text { 2005CL08 }\end{array}$

NUCLEAR MOMENTS 86,87,88,89,90,91,92,93,94,95,96,97,98,99,100,101,102 Zr; measured charge radii. ${ }^{176} \mathrm{Yb}$; measured isomer shift. Ion-beam cooler, laser spectroscopy. JOUR ZAANE 25 s01 187

NUCLEAR REACTIONS ${ }^{93} \mathrm{Nb}(\mathrm{t}, \mathrm{p}), \mathrm{E}=12 \mathrm{MeV}$; measured Ep, $\sigma(\mathrm{E}$, $\theta) .{ }^{95} \mathrm{Nb}$ deduced levels, $\mathrm{J}, \pi$. Comparison with previous results and model predictions. JOUR PRVCA 72054303

ATOMIC MASSES ${ }^{64} \mathrm{Ge},{ }^{68} \mathrm{Se}$; analyzed masses; deduced effective $\mathrm{T}_{1 / 2} \cdot{ }^{90,91} \mathrm{Mo},{ }^{90,91,92,93} \mathrm{Tc},{ }^{93,94} \mathrm{Ru},{ }^{94,95} \mathrm{Rh},{ }^{104,105,106,107} \mathrm{In}$, ${ }_{104,105,107,108} \mathrm{Sn},{ }^{107,108} \mathrm{Sb}$; measured masses. Penning trap, astrophysical implications discussed. JOUR ZAANE 25 s01 629

\section{$\mathrm{A}=96$}

${ }^{96} \mathrm{Zr} \quad 2005 \mathrm{BI} 25$

NUCLEAR MOMENTS 86,87,88,89,90,91,92,93,94,95,96,97,98,99,100,101,102 Zr; measured charge radii. ${ }^{176} \mathrm{Yb}$; measured isomer shift. Ion-beam cooler, laser spectroscopy. JOUR ZAANE 25 s01 187

2005SM08 RADIOACTIVITY ${ }^{252} \mathrm{Cf}(\mathrm{SF})$; measured $\mathrm{E} \gamma, \mathrm{I} \gamma(\theta, \mathrm{H}, \mathrm{t}), \gamma \gamma$-coin. 96,100,102 Zr, ${ }^{102,104,106,108} \mathrm{Mo},{ }^{106,108,110,112} \mathrm{Ru},{ }^{110,114,116} \mathrm{Pd}$ levels

deduced $\mathrm{g}$ factors, $\mathrm{B}(\mathrm{E} 2)$. Gammasphere array, time-integral perturbed angular correlation technique. Comparison with interacting boson model predictions. JOUR JPGPE 31 S1433

\section{$\mathrm{A}=\mathbf{9 7}$}

$\begin{array}{ll}{ }^{97} \mathrm{Sr} & 2005 \mathrm{HW} 06 \\ { }^{97} \mathrm{Zr} & 2005 \mathrm{BI} 25 \\ { }^{97} \mathrm{Rh} & 2005 \mathrm{~T} 015\end{array}$

RADIOACTIVITY ${ }^{252} \mathrm{Cf}(\mathrm{SF}) ;$ measured $\mathrm{E} \gamma, \mathrm{I} \gamma, \gamma \gamma$-coin. ${ }^{95,97} \mathrm{Sr},{ }^{99} \mathrm{Zr}$, ${ }^{108} \mathrm{Tc},{ }^{133,134} \mathrm{Te},{ }^{137} \mathrm{Xe}$ levels deduced $\mathrm{T}_{1 / 2}$. Gammasphere array, time-gated triple-coincidence method. JOUR ZAANE 25 s01 463 NUCLEAR MOMENTS 86,87,88,89,90,91,92,93,94,95,96,97,98,99,100,101,102 Zr; measured charge radii. ${ }^{176} \mathrm{Yb}$; measured isomer shift. Ion-beam cooler, laser spectroscopy. JOUR ZAANE 25 s01 187

NUCLEAR REACTIONS ${ }^{93} \mathrm{Nb}\left({ }^{12} \mathrm{C}, \mathrm{X}\right){ }^{97} \mathrm{Rh} /{ }^{99} \mathrm{Rh}, \mathrm{E}=55.7-77.5$ $\mathrm{MeV} ;{ }^{89} \mathrm{Y}\left({ }^{16} \mathrm{O}, \mathrm{X}\right){ }^{99} \mathrm{Rh}, \mathrm{E}=68-81 \mathrm{MeV}$; measured isomeric $\sigma$ ratios following complete and incomplete fusion; deduced angular momentum transfer. Recoil catcher technique. JOUR PRAMC 641

\section{$\mathrm{A}=98$}

$\begin{array}{ll}{ }^{98} \mathrm{Sr} & 2005 \mathrm{~F} 017 \\ { }^{98} \mathrm{Zr} & 2005 \mathrm{BI} 25\end{array}$

RADIOACTIVITY ${ }^{252} \mathrm{Cf}(\mathrm{SF})$; measured $\mathrm{E} \gamma, \mathrm{I} \gamma, \gamma \gamma$-coin. ${ }^{98} \mathrm{Sr}$, ${ }^{102,104} \mathrm{Zr},{ }^{137} \mathrm{Xe},{ }^{143} \mathrm{Ba},{ }^{152} \mathrm{Ce}$ levels deduced $\mathrm{T}_{1 / 2}$. Gammasphere array, time-gated triple-coincidence method. JOUR ZAANE 25 s01 465 NUCLEAR MOMENTS $86,87,88,89,90,91,92,93,94,95,96,97,98,99,100,101,102 \mathrm{Zr}$; measured charge radii. ${ }^{176} \mathrm{Yb}$; measured isomer shift. Ion-beam cooler, laser spectroscopy. JOUR ZAANE 25 s01 187

2005J022 ATOMIC MASSES ${ }^{98,99,100,101,102,103,104,105} \mathrm{Zr}$; measured masses. Penning trap mass spectrometer. JOUR ZAANE 25 s01 27 


\section{$\mathrm{A}=98$ (continued)}

$\begin{array}{cl}{ }^{98} \mathrm{Mo} & \text { NUCLEAR REACTIONS }{ }^{92,98,100} \mathrm{Mo}\left(\gamma, \gamma^{\prime}\right), \mathrm{E}=14 \mathrm{MeV} \\ & \text { bremsstrahlung; measured } \mathrm{E} \gamma, \mathrm{I} \gamma \cdot{ }^{92,98,100} \mathrm{Mo} \text { deduced dipole strength } \\ & \text { functions, resonance features. PREPRINT nucl-ex } / 0512027,12 / 20 / 2005 \\ & \text { NUCLEAR REACTIONS }{ }^{92,98,100} \mathrm{Mo}\left(\gamma, \gamma \gamma^{\prime}\right), \mathrm{E}=13.2 \mathrm{MeV} \\ & \text { bremsstrahlung; measured } \mathrm{E} \gamma, \mathrm{I} \gamma \cdot{ }^{92,100} \mathrm{Mo},{ }^{197} \mathrm{Au}(\gamma, \mathrm{n}),{ }^{92} \mathrm{Mo}(\gamma, \mathrm{p}), \\ & (\gamma, \alpha), \mathrm{E} \approx 11.8-16.5 \mathrm{MeV} \text { bremsstrahlung; measured integrated } \sigma . \\ & \text { JOUR JPGPE } 31 \mathrm{~S} 1969\end{array}$

\section{$\mathrm{A}=99$}

RADIOACTIVITY ${ }^{99} \mathrm{Y}\left(\beta^{-}\right)$; measured $\beta$-delayed $\mathrm{E} \gamma, \mathrm{I} \gamma, \gamma \gamma$-coin; deduced logft. ${ }^{99} \mathrm{Zr}$ deduced levels, $\mathrm{J}, \pi$, configurations. Interacting boson-fermion model calculations. JOUR PRVCA 72034308

2005LU21 RADIOACTIVITY ${ }^{252} \mathrm{Cf}(\mathrm{SF})$; measured $\mathrm{E} \gamma, \mathrm{I} \gamma, \gamma \gamma$-coin. ${ }^{99,101} \mathrm{Y}$, ${ }^{101,105} \mathrm{Nb}$ deduced levels, J, $\pi$, configurations, rotational bands, shape transition features. Gammasphere array, triaxial-rotor-plus-quasiparticle calculations. JOUR JPGPE 311303 2005LU24 RADIOACTIVITY ${ }^{252} \mathrm{Cf}(\mathrm{SF})$; measured E $\gamma$, I $\gamma, \gamma \gamma$-coin. ${ }^{99,101} \mathrm{Y}$, ${ }^{101,105} \mathrm{Nb}$ deduced levels, J, $\pi$, configurations, deformation.

Gammasphere array, triaxial-rotor-plus-particle calculations. JOUR ZAANE 25 s01 469

${ }^{99} \mathrm{Zr} \quad 2005 \mathrm{BI} 25$ NUCLEAR MOMENTS 86,87,88,89,90,91,92,93,94,95,96,97,98,99,100,101,102Zr; measured charge radii. ${ }^{176} \mathrm{Yb}$; measured isomer shift. Ion-beam cooler, laser spectroscopy. JOUR ZAANE 25 s01 187

2005HW06 RADIOACTIVITY ${ }^{252} \mathrm{Cf}(\mathrm{SF})$; measured $\mathrm{E} \gamma, \mathrm{I} \gamma, \gamma \gamma$-coin. ${ }^{95,97} \mathrm{Sr},{ }^{99} \mathrm{Zr}$, ${ }^{108} \mathrm{Tc},{ }^{133,134} \mathrm{Te},{ }^{137} \mathrm{Xe}$ levels deduced $\mathrm{T}_{1 / 2}$. Gammasphere array, time-gated triple-coincidence method. JOUR ZAANE 25 s01 463 2005J022 ATOMIC MASSES ${ }^{98,99,100,101,102,103,104,105} \mathrm{Zr}$; measured masses. Penning trap mass spectrometer. JOUR ZAANE 25 s01 27

2005LH01 RADIOACTIVITY ${ }^{99} \mathrm{Y}\left(\beta^{-}\right)$; measured $\beta$-delayed $\mathrm{E} \gamma, \mathrm{I} \gamma, \gamma \gamma$-coin; deduced logft. ${ }^{99} \mathrm{Zr}$ deduced levels, $\mathrm{J}, \pi$, configurations. Interacting boson-fermion model calculations. JOUR PRVCA 72034308

${ }^{99} \mathrm{Ru}$ 2004R047 NUCLEAR REACTIONS ${ }^{99,101} \mathrm{Ru}(\mathrm{d}, \mathrm{d}$ '), $\mathrm{E}=13 \mathrm{MeV}$; measured $\sigma(\mathrm{E}$, $\theta$ ). ${ }^{99,101} \mathrm{Ru}$ levels deduced deformation lengths, Coulomb-nuclear interference parameters. DWBA-deformed optical model analysis. JOUR BJPHE 34760

${ }^{99} \mathrm{Rh} \quad 2005 \mathrm{T015} \quad$ NUCLEAR REACTIONS ${ }^{93} \mathrm{Nb}\left({ }^{12} \mathrm{C}, \mathrm{X}\right){ }^{97} \mathrm{Rh} /{ }^{99} \mathrm{Rh}, \mathrm{E}=55.7-77.5$ $\mathrm{MeV} ;{ }^{89} \mathrm{Y}\left({ }^{16} \mathrm{O}, \mathrm{X}\right){ }^{99} \mathrm{Rh}, \mathrm{E}=68-81 \mathrm{MeV}$; measured isomeric $\sigma$ ratios following complete and incomplete fusion; deduced angular momentum transfer. Recoil catcher technique. JOUR PRAMC 641

\section{$\mathrm{A}=\mathbf{1 0 0}$}

${ }^{100} \mathrm{Zr} \quad 2005 \mathrm{BI} 25$

NUCLEAR MOMENTS 86,87,88,89,90,91,92,93,94,95,96,97,98,99,100,101,102 Zr; measured charge radii. ${ }^{176} \mathrm{Yb}$; measured isomer shift. Ion-beam cooler, laser spectroscopy. JOUR ZAANE 25 s01 187 


\section{$\mathrm{A}=100$ (continued)}

2005J022 ATOMIC MASSES ${ }^{98,99,100,101,102,103,104,105} \mathrm{Zr}$; measured masses. Penning trap mass spectrometer. JOUR ZAANE 25 s01 27

2005SM08 RADIOACTIVITY ${ }^{252} \mathrm{Cf}(\mathrm{SF})$; measured $\mathrm{E} \gamma, \mathrm{I} \gamma(\theta, \mathrm{H}, \mathrm{t}), \gamma \gamma$-coin. ${ }^{96,100,102} \mathrm{Zr},{ }^{102,104,106,108} \mathrm{Mo},{ }^{106,108,110,112} \mathrm{Ru},{ }^{110,114,116} \mathrm{Pd}$ levels

deduced $\mathrm{g}$ factors, $\mathrm{B}(\mathrm{E} 2)$. Gammasphere array, time-integral perturbed angular correlation technique. Comparison with interacting boson model predictions. JOUR JPGPE 31 S1433

${ }^{100} \mathrm{Mo} \quad$ 2005AR27 RADIOACTIVITY ${ }^{82} \mathrm{Se},{ }^{100} \mathrm{Mo}\left(2 \beta^{-}\right)$; measured $2 \nu \beta \beta$-decay $\mathrm{T}_{1 / 2}$, $0 \nu \beta \beta$-decay $\mathrm{T}_{1 / 2}$ lower limits; deduced neutrino mass limits. JOUR PRLTA 95182302

2005HOZW RADIOACTIVITY ${ }^{100} \mathrm{Mo}\left(2 \beta^{-}\right)$; measured $\mathrm{E} \gamma, \mathrm{I} \gamma, \gamma \gamma$-coin, $\mathrm{T}_{1 / 2}$ for decay to excited states. ${ }^{100} \mathrm{Ru}$ levels deduced feeding intensities. PREPRINT nucl-ex/0512030,12/20/2005

2005RUZZ NUCLEAR REACTIONS ${ }^{92,98,100} \operatorname{Mo}\left(\gamma, \gamma^{\prime}\right), \mathrm{E}=14 \mathrm{MeV}$ bremsstrahlung; measured $\mathrm{E} \gamma, \mathrm{I} \gamma .{ }^{92,98,100}$ Mo deduced dipole strength functions, resonance features. PREPRINT nucl-ex/0512027,12/20/2005

2005WA31 NUCLEAR REACTIONS ${ }^{92,98,100} \mathrm{Mo}\left(\gamma, \gamma^{\prime}\right), \mathrm{E}=13.2 \mathrm{MeV}$ bremsstrahlung; measured $\mathrm{E} \gamma, \mathrm{I} \gamma .{ }^{92,100} \mathrm{Mo},{ }^{197} \mathrm{Au}(\gamma, \mathrm{n}),{ }^{92} \mathrm{Mo}(\gamma, \mathrm{p})$, $(\gamma, \alpha), \mathrm{E} \approx 11.8-16.5 \mathrm{MeV}$ bremsstrahlung; measured integrated $\sigma$. JOUR JPGPE 31 S1969

${ }^{100} \mathrm{Ru} \quad$ 2005AR27 RADIOACTIVITY ${ }^{82} \mathrm{Se},{ }^{100} \mathrm{Mo}\left(2 \beta^{-}\right)$; measured $2 \nu \beta \beta$-decay $\mathrm{T}_{1 / 2}$, $0 \nu \beta \beta$-decay $\mathrm{T}_{1 / 2}$ lower limits; deduced neutrino mass limits. JOUR PRLTA 95182302

2005HOZW RADIOACTIVITY ${ }^{100} \mathrm{Mo}\left(2 \beta^{-}\right)$; measured $\mathrm{E} \gamma, \mathrm{I} \gamma, \gamma \gamma$-coin, $\mathrm{T}_{1 / 2}$ for decay to excited states. ${ }^{100} \mathrm{Ru}$ levels deduced feeding intensities. PREPRINT nucl-ex/0512030,12/20/2005

${ }^{100} \mathrm{In}$ 2005KA47 RADIOACTIVITY ${ }^{102} \mathrm{Sn}\left(\beta^{+}\right)\left[\right.$from $\left.{ }^{58} \mathrm{Ni}\left({ }^{50} \mathrm{Cr}, \mathrm{X}\right)\right] ;$ measured $\mathrm{E} \gamma, \mathrm{I} \gamma$, $\gamma \gamma$-coin, $\mathrm{E} \beta, \mathrm{B}(\mathrm{GT}) .{ }^{102} \mathrm{In}$ levels deduced $\beta$-feeding intensities, log ft, hindrance factor. ${ }^{100} \mathrm{Sn}\left(\beta^{+}\right)$; analyzed data; deduced $\mathrm{B}(\mathrm{GT})$, hindrance factor. JOUR ZAANE 25 s01 135

${ }^{100} \mathrm{Sn} \quad$ 2005KA47 RADIOACTIVITY ${ }^{102} \mathrm{Sn}\left(\beta^{+}\right)\left[\right.$from $\left.{ }^{58} \mathrm{Ni}\left({ }^{50} \mathrm{Cr}, \mathrm{X}\right)\right] ;$ measured $\mathrm{E} \gamma, \mathrm{I} \gamma$, $\gamma \gamma$-coin, $\mathrm{E} \beta, \mathrm{B}(\mathrm{GT}) .{ }^{102}$ In levels deduced $\beta$-feeding intensities, $\log \mathrm{ft}$, hindrance factor. ${ }^{100} \mathrm{Sn}\left(\beta^{+}\right)$; analyzed data; deduced $\mathrm{B}(\mathrm{GT})$, hindrance factor. JOUR ZAANE 25 s01 135

2005KA47 NUCLEAR REACTIONS ${ }^{58} \mathrm{Ni}\left({ }^{50} \mathrm{Cr}, \mathrm{X}\right){ }^{101} \mathrm{Sn} /{ }^{102} \mathrm{Sn} /{ }^{103} \mathrm{Sn} /{ }^{104} \mathrm{Sn} /$ ${ }^{105} \mathrm{Sn}, \mathrm{E} \approx 5 \mathrm{MeV} /$ nucleon; measured production $\sigma \cdot{ }^{58} \mathrm{Ni}\left({ }^{50} \mathrm{Cr}\right.$, $\mathrm{X})^{100} \mathrm{Sn}, \mathrm{E}=5.8 \mathrm{MeV} /$ nucleon; deduced approximate production $\sigma$. JOUR ZAANE 25 s01 135

$$
\mathrm{A}=\mathbf{1 0 1}
$$

${ }^{101} \mathrm{Y}$ 2005LU21 RADIOACTIVITY ${ }^{252} \mathrm{Cf}(\mathrm{SF})$; measured $\mathrm{E} \gamma, \mathrm{I} \gamma, \gamma \gamma$-coin. ${ }^{99,101} \mathrm{Y}$, ${ }^{101,105} \mathrm{Nb}$ deduced levels, J, $\pi$, configurations, rotational bands, shape transition features. Gammasphere array, triaxial-rotor-plus-quasiparticle calculations. JOUR JPGPE 311303 


\section{$\mathrm{A}=101$ (continued)}

2005LU24 RADIOACTIVITY ${ }^{252} \mathrm{Cf}(\mathrm{SF})$; measured $\mathrm{E} \gamma, \mathrm{I} \gamma, \gamma \gamma$-coin. ${ }^{99,101} \mathrm{Y}$, ${ }^{101,105} \mathrm{Nb}$ deduced levels, $\mathrm{J}, \pi$, configurations, deformation.

Gammasphere array, triaxial-rotor-plus-particle calculations. JOUR ZAANE 25 s01 469

${ }^{101} \mathrm{Zr} \quad 2005 \mathrm{BI} 25$ NUCLEAR MOMENTS 86,87,88,89,90,91,92,93,94,95,96,97,98,99,100,101,102 Zr; measured charge radii. ${ }^{176} \mathrm{Yb}$; measured isomer shift. Ion-beam cooler, laser spectroscopy. JOUR ZAANE 25 s01 187

2005J022 ATOMIC MASSES ${ }^{98,99,100,101,102,103,104,105} \mathrm{Zr}$; measured masses. Penning trap mass spectrometer. JOUR ZAANE 25 s01 27

${ }^{101} \mathrm{Nb} \quad$ 2005LU21 RADIOACTIVITY ${ }^{252} \mathrm{Cf}(\mathrm{SF})$; measured $\mathrm{E} \gamma, \mathrm{I} \gamma, \gamma \gamma$-coin. ${ }^{99,101} \mathrm{Y}$, ${ }^{101,105} \mathrm{Nb}$ deduced levels, $\mathrm{J}, \pi$, configurations, rotational bands, shape transition features. Gammasphere array, triaxial-rotor-plus-quasiparticle calculations. JOUR JPGPE 311303 2005LU24 RADIOACTIVITY ${ }^{252} \mathrm{Cf}(\mathrm{SF})$; measured $\mathrm{E} \gamma, \mathrm{I} \gamma, \gamma \gamma$-coin. ${ }^{99,101} \mathrm{Y}$, $101,105 \mathrm{Nb}$ deduced levels, J, $\pi$, configurations, deformation.

Gammasphere array, triaxial-rotor-plus-particle calculations. JOUR ZAANE 25 s01 469

${ }^{101} \mathrm{Ru} \quad 2004 \mathrm{R} 047$ NUCLEAR REACTIONS ${ }^{99,101} \mathrm{Ru}(\mathrm{d}, \mathrm{d}$ '), $\mathrm{E}=13 \mathrm{MeV}$; measured $\sigma(\mathrm{E}$, $\theta$ ). ${ }^{99,101} \mathrm{Ru}$ levels deduced deformation lengths, Coulomb-nuclear interference parameters. DWBA-deformed optical model analysis. JOUR BJPHE 34760

${ }^{101} \mathrm{Sn}$ 2005KA47 NUCLEAR REACTIONS ${ }^{58} \mathrm{Ni}\left({ }^{50} \mathrm{Cr}, \mathrm{X}\right){ }^{101} \mathrm{Sn} /{ }^{102} \mathrm{Sn} /{ }^{103} \mathrm{Sn} /{ }^{104} \mathrm{Sn} /$ ${ }^{105} \mathrm{Sn}, \mathrm{E} \approx 5 \mathrm{MeV} /$ nucleon; measured production $\sigma \cdot{ }^{58} \mathrm{Ni}\left({ }^{50} \mathrm{Cr}\right.$, $\mathrm{X})^{100} \mathrm{Sn}, \mathrm{E}=5.8 \mathrm{MeV} /$ nucleon; deduced approximate production $\sigma$. JOUR ZAANE 25 s01 135

\section{$\mathrm{A}=102$}

${ }^{102} \mathrm{Zr} \quad 2005 \mathrm{BI} 25$

2005F017

NUCLEAR MOMENTS 86,87,88,89,90,91,92,93,94,95,96,97,98,99,100,101,102 Zr; measured charge radii. ${ }^{176} \mathrm{Yb}$; measured isomer shift. Ion-beam cooler, laser spectroscopy. JOUR ZAANE 25 s01 187

RADIOACTIVITY ${ }^{252} \mathrm{Cf}(\mathrm{SF})$; measured $\mathrm{E} \gamma, \mathrm{I} \gamma, \gamma \gamma$-coin. ${ }^{98} \mathrm{Sr}$, ${ }^{102,104} \mathrm{Zr},{ }^{137} \mathrm{Xe},{ }^{143} \mathrm{Ba},{ }^{152} \mathrm{Ce}$ levels deduced $\mathrm{T}_{1 / 2}$. Gammasphere array, time-gated triple-coincidence method. JOUR ZAANE 25 s01 465

2005J022 ATOMIC MASSES ${ }^{98,99,100,101,102,103,104,105} \mathrm{Zr}$; measured masses. Penning trap mass spectrometer. JOUR ZAANE 25 s01 27

2005SM08 RADIOACTIVITY ${ }^{252} \mathrm{Cf}(\mathrm{SF}) ;$ measured $\mathrm{E} \gamma, \mathrm{I} \gamma(\theta, \mathrm{H}, \mathrm{t}), \gamma \gamma$-coin.

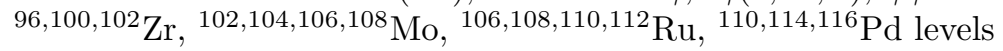
deduced $\mathrm{g}$ factors, $\mathrm{B}(\mathrm{E} 2)$. Gammasphere array, time-integral perturbed angular correlation technique. Comparison with interacting boson model predictions. JOUR JPGPE 31 S1433

${ }^{102} \mathrm{Mo} \quad$ 2005SM08 RADIOACTIVITY ${ }^{252} \mathrm{Cf}(\mathrm{SF}) ;$ measured $\mathrm{E} \gamma, \mathrm{I} \gamma(\theta, \mathrm{H}, \mathrm{t}), \gamma \gamma$-coin. ${ }^{96,100,102} \mathrm{Zr},{ }^{102,104,106,108} \mathrm{Mo},{ }^{106,108,110,112} \mathrm{Ru},{ }^{110,114,116} \mathrm{Pd}$ levels deduced $\mathrm{g}$ factors, $\mathrm{B}(\mathrm{E} 2)$. Gammasphere array, time-integral perturbed angular correlation technique. Comparison with interacting boson model predictions. JOUR JPGPE 31 S1433 


\section{$\mathrm{A}=102$ (continued)}

\begin{tabular}{|c|c|c|}
\hline${ }^{102} \mathrm{In}$ & $2005 \mathrm{KA} 47$ & $\begin{array}{l}\text { RADIOACTIVITY }{ }^{102} \mathrm{Sn}\left(\beta^{+}\right)\left[\text {from }{ }^{58} \mathrm{Ni}\left({ }^{50} \mathrm{Cr}, \mathrm{X}\right)\right] \text {; measured } \mathrm{E} \gamma, \mathrm{I} \gamma \text {, } \\
\gamma \gamma \text {-coin, } \mathrm{E} \beta, \mathrm{B}(\mathrm{GT}) .{ }^{102} \mathrm{In} \text { levels deduced } \beta \text {-feeding intensities, log ft, } \\
\text { hindrance factor. }{ }^{100} \mathrm{Sn}\left(\beta^{+}\right) \text {; analyzed data; deduced } \mathrm{B}(\mathrm{GT}) \text {, hindrance } \\
\text { factor. JOUR ZAANE } 25 \mathrm{~s} 01135\end{array}$ \\
\hline \multirow[t]{3}{*}{${ }^{102} \mathrm{Sn}$} & 2005HA57 & $\begin{array}{l}\text { RADIOACTIVITY }{ }^{106} \mathrm{Te}(\alpha)\left[\text { from }{ }^{54} \mathrm{Fe}\left({ }^{54} \mathrm{Fe}, 2 \mathrm{n}\right)\right] ; \text { measured } \mathrm{E} \alpha, \mathrm{T}_{1 / 2} \text {. } \\
\text { JOUR PRVCA } 72041303\end{array}$ \\
\hline & $2005 \mathrm{KA} 47$ & $\begin{array}{l}\left.\text { RADIOACTIVITY }{ }^{102} \mathrm{Sn}\left(\beta^{+}\right) \text {[from }{ }^{58} \mathrm{Ni}\left({ }^{50} \mathrm{Cr}, \mathrm{X}\right)\right] \text {; measured } \mathrm{E} \gamma, \mathrm{I} \gamma \text {, } \\
\gamma \gamma \text {-coin, } \mathrm{E} \beta, \mathrm{B}(\mathrm{GT}) \cdot{ }^{102} \mathrm{In} \text { levels deduced } \beta \text {-feeding intensities, log ft, } \\
\text { hindrance factor. }{ }^{100} \mathrm{Sn}\left(\beta^{+}\right) \text {; analyzed data; deduced } \mathrm{B}(\mathrm{GT}) \text {, hindrance } \\
\text { factor. JOUR ZAANE } 25 \mathrm{~s} 01135\end{array}$ \\
\hline & $2005 \mathrm{KA} 47$ & $\begin{array}{l}\text { NUCLEAR REACTIONS }{ }^{58} \mathrm{Ni}\left({ }^{50} \mathrm{Cr}, \mathrm{X}\right){ }^{101} \mathrm{Sn} /{ }^{102} \mathrm{Sn} /{ }^{103} \mathrm{Sn} /{ }^{104} \mathrm{Sn} / \\
{ }^{105} \mathrm{Sn}, \mathrm{E} \approx 5 \mathrm{MeV} / \text { nucleon; measured production } \sigma .{ }^{58} \mathrm{Ni}\left({ }^{50} \mathrm{Cr} \text {, }\right. \\
\mathrm{X})^{100} \mathrm{Sn}, \mathrm{E}=5.8 \mathrm{MeV} / \text { nucleon; deduced approximate production } \sigma . \\
\text { JOUR ZAANE } 25 \mathrm{~s} 01135\end{array}$ \\
\hline
\end{tabular}

\section{$A=103$}

$\begin{array}{ll}{ }^{103} \mathrm{Zr} & \text { 2005J022 } \\ { }^{103} \mathrm{Rh} & \text { 2004AG09 }\end{array}$

ATOMIC MASSES 98,99,100,101,102,103,104,105 Zr; measured masses. Penning trap mass spectrometer. JOUR ZAANE $25 \mathrm{~s} 0127$ NUCLEAR REACTIONS ${ }^{103} \mathrm{Rh}\left(\mathrm{n}, \mathrm{n}\right.$ ') ${ }^{103 m} \mathrm{Rh}, \mathrm{E} \approx 4.8 \mathrm{MeV} ;{ }^{115} \mathrm{In}(\mathrm{n}$, n') ${ }^{115 m} \mathrm{In}, \mathrm{E} \approx 5 \mathrm{MeV} ;{ }^{232} \mathrm{Th},{ }^{238} \mathrm{U}(\mathrm{n}, \mathrm{F}), \mathrm{E} \approx 5 \mathrm{MeV} ;{ }^{24} \mathrm{Mg},{ }^{27} \mathrm{Al}$, ${ }^{46,47,48} \mathrm{Ti},{ }^{54,56} \mathrm{Fe},{ }^{58} \mathrm{Ni},{ }^{64} \mathrm{Zn}(\mathrm{n}, \mathrm{p}), \mathrm{E} \approx 2-8 \mathrm{MeV} ;{ }^{27} \mathrm{Al},{ }^{59} \mathrm{Co}(\mathrm{n}, \alpha), \mathrm{E}$ $\approx 8.3 \mathrm{MeV}$; measured activation $\sigma$. Spectrum average technique, comparison with previous results. JOUR RAACA 9263

2005CH62 NUCLEAR REACTIONS ${ }^{103} \mathrm{Rh}\left(\gamma, \gamma^{\prime}\right), \mathrm{E} \approx 40 \mathrm{keV}$; measured $\mathrm{E} \gamma$, $\mathrm{X}$-ray spectra; deduced ICC. Isomer production via bremsstrahlung spectra. JOUR CPLEE 222530

${ }^{103} \mathrm{Ag}$ 2004HE35 NUCLEAR REACTIONS Pd(p, xn) ${ }^{103} \mathrm{Ag}, \mathrm{E} \approx 15-37 \mathrm{MeV} ; \mathrm{Pd}(\mathrm{d}$, $\mathrm{xn})^{103} \mathrm{Ag}, \mathrm{E} \approx 5-20 \mathrm{MeV}$; measured excitation functions. Stacked-foil activation technique. JOUR RAACA 92215

${ }^{103}$ In $\quad$ 2005KA48

RADIOACTIVITY ${ }^{103} \mathrm{Sn}\left(\beta^{+}\right)$, (EC) [from ${ }^{58} \mathrm{Ni}\left({ }^{50} \mathrm{Cr}, \mathrm{X}\right), \mathrm{E}=5 \mathrm{MeV} /$ nucleon]; measured $\mathrm{E} \gamma, \mathrm{I} \gamma, \gamma \gamma-, \beta \gamma$-coin, $\mathrm{E} \beta, \mathrm{B}(\mathrm{GT}), \mathrm{T}_{1 / 2} \cdot{ }^{103} \mathrm{In}$ deduced levels, $\mathrm{J}, \pi$, hindrance factor. ${ }^{105} \mathrm{Sn}\left(\beta^{+}\right)$, (EC) [from ${ }^{58} \mathrm{Ni}\left({ }^{50} \mathrm{Cr}, \mathrm{X}\right), \mathrm{E}=5 \mathrm{MeV}$ / nucleon]; analyzed data; deduced $\mathrm{B}(\mathrm{GT})$, hindrance factor. JOUR ZAANE 25 s01 139

${ }^{103} \mathrm{Sn}$ 2005KA47 NUCLEAR REACTIONS ${ }^{58} \mathrm{Ni}\left({ }^{50} \mathrm{Cr}, \mathrm{X}\right){ }^{101} \mathrm{Sn} /{ }^{102} \mathrm{Sn} /{ }^{103} \mathrm{Sn} /{ }^{104} \mathrm{Sn} /$ ${ }^{105} \mathrm{Sn}, \mathrm{E} \approx 5 \mathrm{MeV} /$ nucleon; measured production $\sigma .{ }^{58} \mathrm{Ni}\left({ }^{50} \mathrm{Cr}\right.$, $\mathrm{X})^{100} \mathrm{Sn}, \mathrm{E}=5.8 \mathrm{MeV} /$ nucleon; deduced approximate production $\sigma$. JOUR ZAANE 25 s01 135

2005KA48 RADIOACTIVITY ${ }^{103} \mathrm{Sn}\left(\beta^{+}\right),(\mathrm{EC})$ [from ${ }^{58} \mathrm{Ni}\left({ }^{50} \mathrm{Cr}, \mathrm{X}\right), \mathrm{E}=5 \mathrm{MeV} /$ nucleon]; measured $\mathrm{E} \gamma, \mathrm{I} \gamma, \gamma \gamma$-, $\beta \gamma$-coin, $\mathrm{E} \beta, \mathrm{B}(\mathrm{GT}), \mathrm{T}_{1 / 2} .{ }^{103} \mathrm{In}$ deduced levels, $\mathrm{J}, \pi$, hindrance factor. ${ }^{105} \mathrm{Sn}\left(\beta^{+}\right)$, (EC) [from ${ }^{58} \mathrm{Ni}\left({ }^{50} \mathrm{Cr}, \mathrm{X}\right), \mathrm{E}=5 \mathrm{MeV}$ / nucleon]; analyzed data; deduced $\mathrm{B}(\mathrm{GT})$, hindrance factor. JOUR ZAANE 25 s01 139 


\section{$\mathrm{A}=104$}

\begin{tabular}{|c|c|c|}
\hline \multirow[t]{2}{*}{${ }^{104} \mathrm{Zr}$} & 2005F017 & $\begin{array}{l}\text { RADIOACTIVITY }{ }^{252} \mathrm{Cf}(\mathrm{SF}) ; \text { measured } \mathrm{E} \gamma, \mathrm{I} \gamma, \gamma \gamma \text {-coin. }{ }^{98} \mathrm{Sr} \\
{ }^{102,104} \mathrm{Zr},{ }^{137} \mathrm{Xe},{ }^{143} \mathrm{Ba},{ }^{152} \mathrm{Ce} \text { levels deduced } \mathrm{T}_{1 / 2} \text {. Gammasphere }\end{array}$ \\
\hline & 2005J022 & $\begin{array}{l}\text { array, time-gated triple-coincidence method. JOUR ZAANE } 25 \text { s01 } 465 \\
\text { ATOMIC MASSES 98,99,100,101,102,103,104,105 Zr; measured masses. } \\
\text { Penning trap mass spectrometer. JOUR ZAANE } 25 \text { s01 } 27\end{array}$ \\
\hline${ }^{104} \mathrm{Mo}$ & 2005SM08 & $\begin{array}{l}\text { RADIOACTIVITY }{ }^{252} \mathrm{Cf}(\mathrm{SF}) \text {; measured } \mathrm{E} \gamma, \mathrm{I} \gamma(\theta, \mathrm{H}, \mathrm{t}), \gamma \gamma \text {-coin. } \\
96,100,102 \mathrm{Zr},{ }^{102,104,106,108} \mathrm{Mo},{ }_{106,108,110,112} \mathrm{Ru},{ }^{110,114,116} \mathrm{Pd} \text { levels } \\
\text { deduced g factors, } \mathrm{B}(\mathrm{E} 2) \text {. Gammasphere array, time-integral perturbed } \\
\text { angular correlation technique. Comparison with interacting boson } \\
\text { model predictions. JOUR JPGPE } 31 \mathrm{~S} 1433\end{array}$ \\
\hline${ }^{104} \mathrm{Rh}$ & 2005WI23 & $\begin{array}{l}\text { NUCLEAR REACTIONS }{ }^{100} \mathrm{Mo}\left({ }^{11} \mathrm{~B} \text {, xnypz } \alpha\right)^{104} \mathrm{Rh} /{ }^{105} \mathrm{Rh} /{ }^{107} \mathrm{Pd} / \\
{ }^{108} \mathrm{Pd}, \mathrm{E}=43 \mathrm{MeV} ;{ }^{51} \mathrm{~V}\left({ }^{16} \mathrm{O} \text {, xnypz } \alpha\right)^{60} \mathrm{Ni} /{ }^{61} \mathrm{Ni} /{ }^{61} \mathrm{Cu} /{ }^{62} \mathrm{Cu}, \mathrm{E}=70 \\
\mathrm{MeV} ; \text { measured } \mathrm{E} \gamma, \mathrm{I} \gamma, \gamma \gamma-\text {, (charged particle }) \gamma \text {-coin; deduced } \gamma \text {-ray } \\
\text { yield ratios. Application to exit channel determination discussed. } \\
\text { JOUR BJPHE } 35898\end{array}$ \\
\hline \multirow[t]{2}{*}{${ }^{104} \mathrm{Pd}$} & 2004R048 & $\begin{array}{l}\text { NUCLEAR REACTIONS }{ }^{104,106,108,110} \mathrm{Pd}(\mathrm{d}, \mathrm{d} \text { '), } \mathrm{E}=13 \mathrm{MeV} \text {; } \\
\text { measured } \sigma(\mathrm{E}, \theta) .{ }^{104,106,108,110} \mathrm{Pd} \text { levels deduced } \mathrm{B}(\mathrm{E} 2) \text {, deformation } \\
\text { lengths, Coulomb-nuclear interference parameters. DWBA-deformed } \\
\text { optical model analysis. JOUR BJPHE } 34777\end{array}$ \\
\hline & 2005BEZS & $\begin{array}{l}\text { NUCLEAR REACTIONS }{ }^{108} \mathrm{Pd}\left({ }^{122} \mathrm{Cd},{ }^{122} \mathrm{Cd}\right),{ }^{104} \mathrm{Pd}\left({ }^{124} \mathrm{Cd},{ }^{124} \mathrm{Cd}\right) \\
\left({ }^{126} \mathrm{Cd},{ }^{126} \mathrm{Cd}\right) \text {, E not given; measured } \mathrm{E} \gamma, \mathrm{I} \gamma \text {, (particle) } \gamma \text {-coin } \\
\text { following projectile Coulomb excitation. }{ }^{122,124} \mathrm{Cd} \text { levels deduced } \\
\text { excitation B(E2). REPT MLL } 2004 \text { Annual,P14,Behrens }\end{array}$ \\
\hline${ }^{104} \mathrm{In}$ & 2005CL08 & 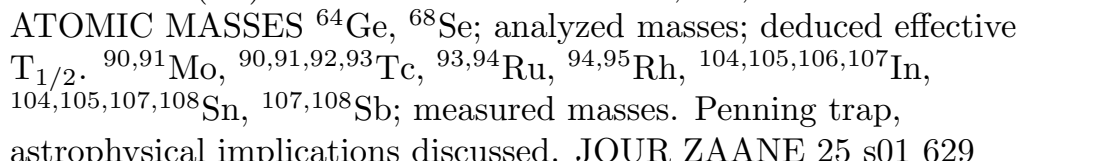 \\
\hline \multirow[t]{3}{*}{${ }^{104} \mathrm{Sn}$} & 2005CL08 & $\begin{array}{l}\text { ATOMIC MASSES }{ }^{64} \mathrm{Ge},{ }^{68} \mathrm{Se} \text {; analyzed masses; deduced effective } \\
\mathrm{T}_{1 / 2} \cdot{ }^{90,91} \mathrm{Mo},{ }^{90,91,92,93} \mathrm{Tc},{ }^{93,94} \mathrm{Ru},{ }^{94,95} \mathrm{Rh},{ }^{104,105,106,107} \mathrm{In}, \\
{ }^{104,105,107,108} \mathrm{Sn},{ }^{107,108} \mathrm{Sb} \text {; measured masses. Penning trap, }\end{array}$ \\
\hline & $2005 \mathrm{KA} 47$ & $\begin{array}{l}\text { astrophysical implications discussed. JOUR ZAANE } 25 \text { s01 } 629 \\
\text { NUCLEAR REACTIONS }{ }^{58} \mathrm{Ni}\left({ }^{50} \mathrm{Cr}, \mathrm{X}\right){ }^{101} \mathrm{Sn} /{ }^{102} \mathrm{Sn} /{ }^{103} \mathrm{Sn} /{ }^{104} \mathrm{Sn} / \\
{ }^{105} \mathrm{Sn}, \mathrm{E} \approx 5 \mathrm{MeV} / \text { nucleon; measured production } \sigma .{ }^{58} \mathrm{Ni}\left({ }^{50} \mathrm{Cr} \text {, }\right. \\
\mathrm{X})^{100} \mathrm{Sn}, \mathrm{E}=5.8 \mathrm{MeV} / \text { nucleon; deduced approximate production } \sigma . \\
\text { JOUR ZAANE } 25 \mathrm{~s} 01135\end{array}$ \\
\hline & 2005LI47 & $\begin{array}{l}\text { RADIOACTIVITY }{ }^{105} \mathrm{Sb}(\mathrm{p})\left[\text { from }{ }^{50} \mathrm{Cr}\left({ }^{58} \mathrm{Ni}, 2 \mathrm{np}\right)\right] ; \text { measured } \mathrm{Ep} ; \\
\text { deduced upper limit for proton decay branching ratio. JOUR PRVCA } \\
72047301\end{array}$ \\
\hline
\end{tabular}

\section{$\mathrm{A}=\mathbf{1 0 5}$}

$\begin{array}{ll}{ }^{105} \mathrm{Zr} & \text { 2005J022 } \\ { }^{105} \mathrm{Nb} & \text { 2005LU21 }\end{array}$

ATOMIC MASSES 98,99,100,101,102,103,104,105 Zr; measured masses. Penning trap mass spectrometer. JOUR ZAANE 25 s01 27 RADIOACTIVITY ${ }^{252} \mathrm{Cf}(\mathrm{SF})$; measured $\mathrm{E} \gamma, \mathrm{I} \gamma, \gamma \gamma$-coin. ${ }^{99,101} \mathrm{Y}$, ${ }^{101,105} \mathrm{Nb}$ deduced levels, J, $\pi$, configurations, rotational bands, shape transition features. Gammasphere array, triaxial-rotor-plus-quasiparticle calculations. JOUR JPGPE 311303 


\section{$\mathrm{A}=105$ (continued)}

2005LU24 RADIOACTIVITY ${ }^{252} \mathrm{Cf}(\mathrm{SF})$; measured $\mathrm{E} \gamma, \mathrm{I} \gamma, \gamma \gamma$-coin. ${ }^{99,101} \mathrm{Y}$, ${ }^{101,105} \mathrm{Nb}$ deduced levels, $\mathrm{J}, \pi$, configurations, deformation.

Gammasphere array, triaxial-rotor-plus-particle calculations. JOUR ZAANE 25 s01 469

${ }^{105} \mathrm{Rh}$ 2004AL43 NUCLEAR REACTIONS ${ }^{100} \mathrm{Mo}\left({ }^{11} \mathrm{~B}, 2 \mathrm{n} \alpha\right), \mathrm{E}=43 \mathrm{MeV}$; measured $\mathrm{E} \gamma$, $\mathrm{I} \gamma, \gamma \gamma$-, (charged particle) $\gamma$-coin. ${ }^{105} \mathrm{Rh}$ deduced levels, J, $\pi$, possible chiral bands. Tilted axis cranking model calculations. JOUR BJPHE 34999

2005WI23 NUCLEAR REACTIONS ${ }^{100} \mathrm{Mo}\left({ }^{11} \mathrm{~B}\right.$, xnypz $\left.\alpha\right){ }^{104} \mathrm{Rh} /{ }^{105} \mathrm{Rh} /{ }^{107} \mathrm{Pd} /$ ${ }^{108} \mathrm{Pd}, \mathrm{E}=43 \mathrm{MeV} ;{ }^{51} \mathrm{~V}\left({ }^{16} \mathrm{O}\right.$, xnypz $\left.\alpha\right){ }^{60} \mathrm{Ni} /{ }^{61} \mathrm{Ni} /{ }^{61} \mathrm{Cu} /{ }^{62} \mathrm{Cu}, \mathrm{E}=70$ $\mathrm{MeV}$; measured $\mathrm{E} \gamma, \mathrm{I} \gamma, \gamma \gamma$-, (charged particle) $\gamma$-coin; deduced $\gamma$-ray yield ratios. Application to exit channel determination discussed. JOUR BJPHE 35898

${ }^{105} \mathrm{Ag} \quad$ 2005HA56 NUCLEAR REACTIONS ${ }^{104} \mathrm{Pd}(\mathrm{p}, \gamma), \mathrm{E}(\mathrm{cm})=2-8 \mathrm{MeV} ;{ }^{118} \mathrm{Sn}(\alpha, \gamma)$, $\mathrm{E}(\mathrm{cm})=10-11 \mathrm{MeV}$; measured $\mathrm{E} \gamma, \mathrm{I} \gamma, \sigma$. Comparison with model predictions. JOUR JPGPE 31 S1417

${ }^{105} \mathrm{In} \quad$ 2005CL08 ATOMIC MASSES ${ }^{64} \mathrm{Ge},{ }^{68} \mathrm{Se}$; analyzed masses; deduced effective $\mathrm{T}_{1 / 2} .{ }^{90,91} \mathrm{Mo},{ }^{90,91,92,93} \mathrm{Tc},{ }^{93,94} \mathrm{Ru},{ }^{94,95} \mathrm{Rh},{ }^{104,105,106,107} \mathrm{In}$, $104,105,107,108 \mathrm{Sn},{ }^{107,108} \mathrm{Sb}$; measured masses. Penning trap, astrophysical implications discussed. JOUR ZAANE 25 s01 629 2005KA48 RADIOACTIVITY ${ }^{103} \mathrm{Sn}\left(\beta^{+}\right)$, (EC) [from ${ }^{58} \mathrm{Ni}\left({ }^{50} \mathrm{Cr}, \mathrm{X}\right), \mathrm{E}=5 \mathrm{MeV} /$ nucleon]; measured $\mathrm{E} \gamma, \mathrm{I} \gamma, \gamma \gamma-, \beta \gamma$-coin, $\mathrm{E} \beta, \mathrm{B}(\mathrm{GT}), \mathrm{T}_{1 / 2} .{ }^{103} \mathrm{In}$ deduced levels, $\mathrm{J}, \pi$, hindrance factor. ${ }^{105} \mathrm{Sn}\left(\beta^{+}\right)$, (EC) [from ${ }^{58} \mathrm{Ni}\left({ }^{50} \mathrm{Cr}, \mathrm{X}\right), \mathrm{E}=5 \mathrm{MeV} /$ nucleon]; analyzed data; deduced $\mathrm{B}(\mathrm{GT})$, hindrance factor. JOUR ZAANE 25 s01 139

${ }^{105} \mathrm{Sn} \quad$ 2005CL08 ATOMIC MASSES ${ }^{64} \mathrm{Ge},{ }^{68} \mathrm{Se}$; analyzed masses; deduced effective $\mathrm{T}_{1 / 2} .{ }^{90,91} \mathrm{Mo},{ }^{90,91,92,93} \mathrm{Tc},{ }^{93,94} \mathrm{Ru},{ }^{94,95} \mathrm{Rh},{ }^{104,105,106,107} \mathrm{In}$, ${ }_{104,105,107,108} \mathrm{Sn},{ }^{107,108} \mathrm{Sb}$; measured masses. Penning trap, astrophysical implications discussed. JOUR ZAANE 25 s01 629 2005KA47 NUCLEAR REACTIONS ${ }^{58} \mathrm{Ni}\left({ }^{50} \mathrm{Cr}, \mathrm{X}\right){ }^{101} \mathrm{Sn} /{ }^{102} \mathrm{Sn} /{ }^{103} \mathrm{Sn} /{ }^{104} \mathrm{Sn} /$ ${ }^{105} \mathrm{Sn}, \mathrm{E} \approx 5 \mathrm{MeV} /$ nucleon; measured production $\sigma \cdot{ }^{58} \mathrm{Ni}\left({ }^{50} \mathrm{Cr}\right.$, $\mathrm{X})^{100} \mathrm{Sn}, \mathrm{E}=5.8 \mathrm{MeV} /$ nucleon; deduced approximate production $\sigma$. JOUR ZAANE 25 s01 135

2005KA48 RADIOACTIVITY ${ }^{103} \mathrm{Sn}\left(\beta^{+}\right)$, (EC) [from ${ }^{58} \mathrm{Ni}\left({ }^{50} \mathrm{Cr}, \mathrm{X}\right), \mathrm{E}=5 \mathrm{MeV} /$ nucleon]; measured $\mathrm{E} \gamma, \mathrm{I} \gamma, \gamma \gamma-, \beta \gamma$-coin, $\mathrm{E} \beta, \mathrm{B}(\mathrm{GT}), \mathrm{T}_{1 / 2} \cdot{ }^{103} \mathrm{In}$ deduced levels, $\mathrm{J}, \pi$, hindrance factor. ${ }^{105} \mathrm{Sn}\left(\beta^{+}\right)$, (EC) [from ${ }^{58} \mathrm{Ni}\left({ }^{50} \mathrm{Cr}, \mathrm{X}\right), \mathrm{E}=5 \mathrm{MeV} /$ nucleon]; analyzed data; deduced $\mathrm{B}(\mathrm{GT})$, hindrance factor. JOUR ZAANE 25 s01 139

${ }^{105} \mathrm{Sb} 2005 \mathrm{LI} 47$ NUCLEAR REACTIONS ${ }^{50} \mathrm{Cr}\left({ }^{58} \mathrm{Ni}, 2 \mathrm{np}\right), \mathrm{E}=222,255 \mathrm{MeV}$; measured delayed Ep. ${ }^{105} \mathrm{Sb}$ deduced upper limit for proton decay branching ratio. JOUR PRVCA 72047301

2005LI47 RADIOACTIVITY ${ }^{105} \mathrm{Sb}$ (p) [from $\left.{ }^{50} \mathrm{Cr}\left({ }^{58} \mathrm{Ni}, 2 \mathrm{np}\right)\right]$; measured Ep; deduced upper limit for proton decay branching ratio. JOUR PRVCA 72047301 


\section{$\mathrm{A}=106$}

${ }^{106} \mathrm{Mo} \quad$ 2005SM08 RADIOACTIVITY ${ }^{252} \mathrm{Cf}(\mathrm{SF}) ;$ measured $\mathrm{E} \gamma, \mathrm{I} \gamma(\theta, \mathrm{H}, \mathrm{t}), \gamma \gamma$-coin. 96,100,102 Zr, ${ }^{102,104,106,108} \mathrm{Mo},{ }^{106,108,110,112} \mathrm{Ru},{ }^{110,114,116} \mathrm{Pd}$ levels deduced $\mathrm{g}$ factors, B(E2). Gammasphere array, time-integral perturbed angular correlation technique. Comparison with interacting boson model predictions. JOUR JPGPE 31 S1433

$2005 Z$ RADIOACTIVITY ${ }^{252} \mathrm{Cf}(\mathrm{SF})$; measured E $\gamma, \mathrm{I} \gamma, \gamma \gamma$-coin. ${ }^{106}$ Mo deduced high-spin levels, J, $\pi$, chiral vibrational bands. Gammasphere array, tilted-axis cranking model analysis. JOUR ZAANE 25 s01 459

${ }^{106} \mathrm{Ru} \quad 2005$ SM08

RADIOACTIVITY ${ }^{252} \mathrm{Cf}(\mathrm{SF})$; measured $\mathrm{E} \gamma, \mathrm{I} \gamma(\theta, \mathrm{H}, \mathrm{t}), \gamma \gamma$-coin. 96,100,102 Zr, ${ }^{102,104,106,108} \mathrm{Mo},{ }^{106,108,110,112} \mathrm{Ru},{ }^{110,114,116} \mathrm{Pd}$ levels

deduced $\mathrm{g}$ factors, B(E2). Gammasphere array, time-integral perturbed angular correlation technique. Comparison with interacting boson model predictions. JOUR JPGPE 31 S1433

${ }^{106} \mathrm{Pd}$ 2004R048 NUCLEAR REACTIONS ${ }^{104,106,108,110} \mathrm{Pd}(\mathrm{d}, \mathrm{d}$ '), E=13 $\mathrm{MeV}$; measured $\sigma(\mathrm{E}, \theta) .{ }^{104,106,108,110} \mathrm{Pd}$ levels deduced $\mathrm{B}(\mathrm{E} 2)$, deformation lengths, Coulomb-nuclear interference parameters. DWBA-deformed optical model analysis. JOUR BJPHE 34777

${ }^{106} \mathrm{Ag} \quad$ 2005J020 NUCLEAR REACTIONS ${ }^{100} \mathrm{Mo}\left({ }^{10} \mathrm{~B}, 4 \mathrm{n}\right), \mathrm{E}=42 \mathrm{MeV}$; measured $\mathrm{E} \gamma$, $\mathrm{I} \gamma, \gamma \gamma$-coin. ${ }^{106} \mathrm{Ag}$ deduced high-spin levels, J, $\pi$, configurations, possible triaxial rotation. Gammasphere array. JOUR JPGPE 31 $\mathrm{S} 1895$

${ }^{106}$ In $\quad 2005$ CL08
ATOMIC MASSES ${ }^{64} \mathrm{Ge},{ }^{68} \mathrm{Se}$; analyzed masses; deduced effective $\mathrm{T}_{1 / 2} \cdot{ }^{90,91} \mathrm{Mo},{ }^{90,91,92,93} \mathrm{Tc},{ }^{93,94} \mathrm{Ru},{ }^{94,95} \mathrm{Rh},{ }^{104,105,106,107} \mathrm{In}$, $104,105,107,108 \mathrm{Sn},{ }^{107,108} \mathrm{Sb}$; measured masses. Penning trap, astrophysical implications discussed. JOUR ZAANE 25 s01 629

${ }^{106} \mathrm{Te} \quad 2005 \mathrm{HA} 57$ NUCLEAR REACTIONS ${ }^{54} \mathrm{Fe}\left({ }^{54} \mathrm{Fe}, 2 \mathrm{n}\right), \mathrm{E}=182 \mathrm{MeV}$; measured E $\gamma$, $\mathrm{I} \gamma,($ recoil $) \gamma$-coin; deduced $\sigma .{ }^{106} \mathrm{Te}$ deduced levels, possible vibrational excitation. Recoil-decay tagging, level systematics in Te isotopes discussed. JOUR PRVCA 72041303

2005HA57 RADIOACTIVITY ${ }^{106} \mathrm{Te}(\alpha)\left[\right.$ from $\left.{ }^{54} \mathrm{Fe}\left({ }^{54} \mathrm{Fe}, 2 \mathrm{n}\right)\right]$; measured $\mathrm{E} \alpha, \mathrm{T}_{1 / 2}$. JOUR PRVCA 72041303

\section{$\mathrm{A}=107$}

\begin{tabular}{|c|c|c|}
\hline${ }^{107} \mathrm{Pd}$ & 2005WI23 & $\begin{array}{l}\text { NUCLEAR REACTIONS }{ }^{100} \mathrm{Mo}\left({ }^{11} \mathrm{~B}, \text { xnypz } \alpha\right){ }^{104} \mathrm{Rh} /{ }^{105} \mathrm{Rh} /{ }^{107} \mathrm{Pd} / \\
{ }^{108} \mathrm{Pd}, \mathrm{E}=43 \mathrm{MeV} ;{ }^{11} \mathrm{~V}\left({ }^{16} \mathrm{O}, \text { xnypz } \alpha\right)^{60} \mathrm{Ni} /{ }^{61} \mathrm{Ni} /{ }^{61} \mathrm{Cu} /{ }^{62} \mathrm{Cu}, \mathrm{E}=70 \\
\mathrm{MeV} \text {; measured E } \gamma, \mathrm{I} \gamma, \gamma \gamma-,(\text { charged particle }) \gamma \text {-coin; deduced } \gamma \text {-ray } \\
\text { yield ratios. Application to exit channel determination discussed. } \\
\text { JOUR BJPHE } 35898\end{array}$ \\
\hline${ }^{107} \mathrm{Cd}$ & 2005AN26 & $\begin{array}{l}\text { NUCLEAR REACTIONS }{ }^{98} \mathrm{Mo}\left({ }^{12} \mathrm{C}, 3 \mathrm{n}\right), \mathrm{E}=60 \mathrm{MeV} \text {; measured } \\
\text { Doppler-shifted } \mathrm{E} \gamma, \mathrm{I} \gamma, \gamma \gamma \text {-coin. }{ }^{107} \mathrm{Cd} \text { levels deduced } \mathrm{T}_{1 / 2}, \mathrm{~B}(\mathrm{E} 2) \text {. } \\
\text { Recoil-distance method. JOUR JPGPE } 31 \mathrm{~S} 1563\end{array}$ \\
\hline${ }^{107} \mathrm{In}$ & 2005CL08 & 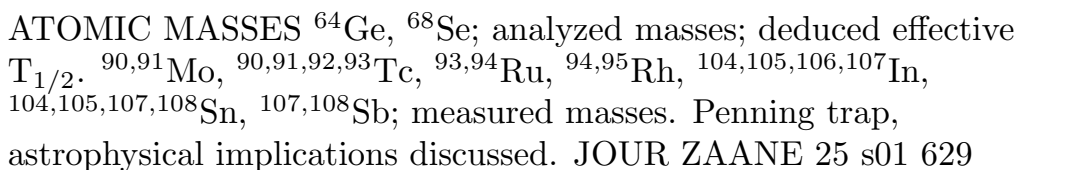 \\
\hline
\end{tabular}




\section{$\mathrm{A}=107$ (continued)}

\begin{tabular}{|c|c|c|}
\hline \multirow[b]{2}{*}{${ }^{107} \mathrm{Sn}$} & \multirow[b]{2}{*}{ 2005CL08 } & \\
\hline & & 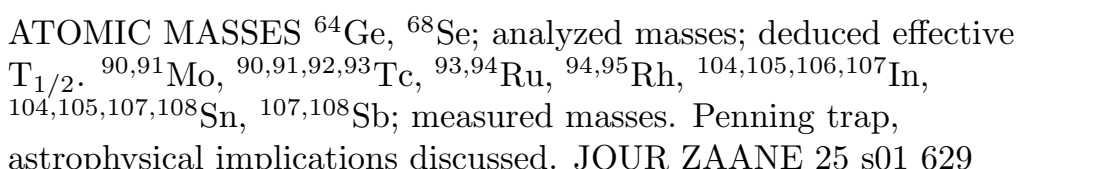 \\
\hline \multirow[t]{2}{*}{${ }^{107} \mathrm{Sb}$} & \multirow[t]{2}{*}{ 2005CL08 } & $\begin{array}{l}\text { ATOMIC MASSES }{ }^{64} \mathrm{Ge},{ }^{68} \mathrm{Se} \text {; analyzed masses; deduced effective } \\
\mathrm{T}_{1 / 2} \cdot{ }^{90,91} \mathrm{Mo},{ }^{90,91,92,93} \mathrm{Tc},{ }^{93,94} \mathrm{Ru},{ }^{94,95} \mathrm{Rh},{ }^{104,105,106,107} \mathrm{In}, \\
104,105,107,108 \mathrm{Sn},{ }^{107,108} \mathrm{Sb} \text {; measured masses. Penning trap, } \\
\text { astrophysical implications discussed. JOUR ZAANE } 25 \text { s01 } 629\end{array}$ \\
\hline & & $A=108$ \\
\hline${ }^{108} \mathrm{Mo}$ & 2005SM08 & $\begin{array}{l}\text { RADIOACTIVITY }{ }^{252} \mathrm{Cf}(\mathrm{SF}) ; \text { measured } \mathrm{E} \gamma, \mathrm{I} \gamma(\theta, \mathrm{H}, \mathrm{t}), \gamma \gamma \text {-coin. } \\
96,100,102 \mathrm{Zr},{ }^{102,104,106,108} \mathrm{Mo}, 106,108,110,112 \mathrm{Ru},{ }^{110,114,116} \mathrm{Pd} \text { levels } \\
\text { deduced g factors, } \mathrm{B}(\mathrm{E} 2) \text {. Gammasphere array, time-integral perturbed } \\
\text { angular correlation technique. Comparison with interacting boson } \\
\text { model predictions. JOUR JPGPE } 31 \mathrm{~S} 1433\end{array}$ \\
\hline${ }^{108} \mathrm{Tc}$ & 2005HW06 & $\begin{array}{l}\text { RADIOACTIVITY }{ }^{252} \mathrm{Cf}(\mathrm{SF}) ; \text { measured } \mathrm{E} \gamma, \mathrm{I} \gamma, \gamma \gamma \text {-coin. }{ }^{95,97} \mathrm{Sr},{ }^{99} \mathrm{Zr} \text {, } \\
{ }^{108} \mathrm{Tc},{ }^{133,134} \mathrm{Te},{ }^{137} \mathrm{Xe} \text { levels deduced } \mathrm{T}_{1 / 2} . \text { Gammasphere array, } \\
\text { time-gated triple-coincidence method. JOUR ZAANE } 25 \text { s01 } 463\end{array}$ \\
\hline \multirow[t]{2}{*}{${ }^{108} \mathrm{Ru}$} & \multirow[t]{2}{*}{ 2005SM08 } & $\begin{array}{l}\text { RADIOACTIVITY }{ }^{252} \mathrm{Cf}(\mathrm{SF}) ; \text { measured } \mathrm{E} \gamma, \mathrm{I} \gamma(\theta, \mathrm{H}, \mathrm{t}), \gamma \gamma \text {-coin. } \\
96,100,102 \mathrm{Zr},{ }^{102,104,106,108} \mathrm{Mo},{ }^{106,108,110,112} \mathrm{Ru},{ }^{110,114,116} \mathrm{Pd} \text { levels }\end{array}$ \\
\hline & & $\begin{array}{l}\text { deduced } \mathrm{g} \text { factors, } \mathrm{B}(\mathrm{E} 2) \text {. Gammasphere array, time-integral perturbed } \\
\text { angular correlation technique. Comparison with interacting boson } \\
\text { model predictions. JOUR JPGPE } 31 \text { S1433 }\end{array}$ \\
\hline \multirow[t]{5}{*}{${ }^{108} \mathrm{Pd}$} & 2004AL44 & $\begin{array}{l}\text { NUCLEAR REACTIONS }{ }^{100} \mathrm{Mo}\left({ }^{11} \mathrm{~B}, 2 \mathrm{np}\right), \mathrm{E}=43 \mathrm{MeV} \text {; measured } \mathrm{E} \gamma \text {, } \\
\mathrm{I} \gamma, \gamma \gamma-\text {, (charged particle) } \gamma \text {-coin. }{ }^{108} \mathrm{Pd} \text { deduced levels, J, } \pi \text {, } \\
\text { configurations. Cranking model analysis. JOUR BJPHE } 341005\end{array}$ \\
\hline & 2004R048 & $\begin{array}{l}\text { NUCLEAR REACTIONS }{ }^{104,106,108,110} \mathrm{Pd}\left(\mathrm{d}, \mathrm{d}^{\prime}\right), \mathrm{E}=13 \mathrm{MeV} \text {; } \\
\text { measured } \sigma(\mathrm{E}, \theta) .{ }^{104,106,108,110} \mathrm{Pd} \text { levels deduced } \mathrm{B}(\mathrm{E} 2) \text {, deformation } \\
\text { lengths, Coulomb-nuclear interference parameters. DWBA-deformed } \\
\text { optical model analysis. JOUR BJPHE } 34777\end{array}$ \\
\hline & 2005BEZS & $\begin{array}{l}\text { NUCLEAR REACTIONS }{ }^{108} \mathrm{Pd}\left({ }^{122} \mathrm{Cd},{ }^{122} \mathrm{Cd}\right),{ }^{104} \mathrm{Pd}\left({ }^{124} \mathrm{Cd},{ }^{124} \mathrm{Cd}{ }^{\prime}\right) \text {, } \\
\left({ }^{126} \mathrm{Cd},{ }^{126} \mathrm{Cd} \text { '), E not given; measured } \mathrm{E} \gamma, \mathrm{I} \gamma,(\text { particle }) \gamma \text {-coin }\right. \\
\text { following projectile Coulomb excitation. }{ }^{122,124} \mathrm{Cd} \text { levels deduced } \\
\text { excitation B(E2). REPT MLL } 2004 \text { Annual,P14,Behrens }\end{array}$ \\
\hline & 2005WI23 & $\begin{array}{l}\text { NUCLEAR REACTIONS }{ }^{100} \mathrm{Mo}\left({ }^{11} \mathrm{~B} \text {, xnypz } \alpha\right)^{104} \mathrm{Rh} /{ }^{105} \mathrm{Rh} /{ }^{107} \mathrm{Pd} / \\
{ }^{108} \mathrm{Pd}, \mathrm{E}=43 \mathrm{MeV} ;{ }^{51} \mathrm{~V}\left({ }^{16} \mathrm{O}, \text { xnypz } \alpha\right){ }^{60} \mathrm{Ni} /{ }^{61} \mathrm{Ni} /{ }^{61} \mathrm{Cu} /{ }^{62} \mathrm{Cu}, \mathrm{E}=70\end{array}$ \\
\hline & & $\begin{array}{l}\mathrm{MeV} \text {; measured } \mathrm{E} \gamma, \mathrm{I} \gamma, \gamma \gamma-\text {, (charged particle) } \gamma \text {-coin; deduced } \gamma \text {-ray } \\
\text { yield ratios. Application to exit channel determination discussed. } \\
\text { JOUR BJPHE } 35898\end{array}$ \\
\hline${ }^{108} \mathrm{Sn}$ & 2005CL08 & $\begin{array}{l}\text { ATOMIC MASSES }{ }^{64} \mathrm{Ge},{ }^{68} \mathrm{Se} \text {; analyzed masses; deduced effective } \\
\mathrm{T}_{1 / 2 \cdot} \cdot{ }^{90,91} \mathrm{Mo},{ }^{90,91,92,93} \mathrm{Tc},{ }^{93,94} \mathrm{Ru},{ }^{94,95} \mathrm{Rh},{ }^{104,105,106,107} \mathrm{In}, \\
104,105,107,108 \mathrm{Sn},{ }^{107,108} \mathrm{Sb} \text {; measured masses. Penning trap, }\end{array}$ \\
\hline${ }^{108} \mathrm{Sb}$ & 2005CL08 & $\begin{array}{l}\text { ATOMIC MASSES }{ }^{64} \mathrm{Ge},{ }^{68} \mathrm{Se} \text {; analyzed masses; deduced effective } \\
\mathrm{T}_{1 / 2} \cdot{ }^{90,91} \mathrm{Mo},{ }^{90,91,92,93} \mathrm{Tc},{ }^{93,94} \mathrm{Ru},{ }^{94,95} \mathrm{Rh},{ }^{104,105,106,107} \mathrm{In}, \\
{ }_{104,105,107,108} \mathrm{Sn},{ }^{107,108} \mathrm{Sb} \text {; measured masses. Penning trap, } \\
\text { astrophysical implications discussed. JOUR ZAANE } 25 \text { s01 } 629\end{array}$ \\
\hline
\end{tabular}




\section{$\mathrm{A}=109$}

No references found

$$
\mathrm{A}=\mathbf{1 1 0}
$$

${ }^{110} \mathrm{Ru} \quad$ 2005SM08

${ }^{110} \mathrm{Pd} \quad 2004 \mathrm{RO} 48$

2005SM08

${ }^{110} \mathrm{Cd} \quad 2005 K 032$

2005SH53

${ }^{111} \mathrm{Te}$

2005SH53

2005K032

NUCLEAR REACTIONS ${ }^{110,111,112,114,116} \mathrm{Cd}\left(\gamma, \gamma^{\prime}\right), \mathrm{E} \approx 2.7-4.1 \mathrm{MeV}$ bremsstrahlung; measured $\mathrm{E} \gamma, \mathrm{I} \gamma, \gamma$-ray polarization.

${ }_{110,111,112,114,116} \mathrm{Cd}$ deduced levels, J, $\pi$, excitation B(M1), B(E1). JOUR PRVCA 72034302

RADIOACTIVITY ${ }^{111} \mathrm{Te}\left(\beta^{+}\right)$[from $\left.{ }^{58} \mathrm{Ni}\left({ }^{56} \mathrm{Fe}, 2 \mathrm{pn}\right)\right] ;{ }^{135} \mathrm{Sn}\left(\beta^{-}\right)$, $\left(\beta^{-} \mathrm{n}\right)$ [from $\left.\mathrm{U}(\mathrm{p}, \mathrm{F}), \mathrm{E}=1.4 \mathrm{GeV}\right]$; measured $\mathrm{E} \gamma, \mathrm{I} \gamma, \gamma \gamma$-coin following decay of mass-separated sources. ${ }^{111,134,135} \mathrm{Sb}$ deduced levels, J, $\pi$. Comparison with model calculations. JOUR ZAANE 25 s01 121 RADIOACTIVITY ${ }^{111} \mathrm{Te}\left(\beta^{+}\right)$[from $\left.{ }^{58} \mathrm{Ni}\left({ }^{56} \mathrm{Fe}, 2 \mathrm{pn}\right)\right] ;{ }^{135} \mathrm{Sn}\left(\beta^{-}\right)$, $\left(\beta^{-} \mathrm{n}\right)$ [from $\left.\mathrm{U}(\mathrm{p}, \mathrm{F}), \mathrm{E}=1.4 \mathrm{GeV}\right]$; measured $\mathrm{E} \gamma, \mathrm{I} \gamma, \gamma \gamma$-coin following decay of mass-separated sources. ${ }^{111,134,135} \mathrm{Sb}$ deduced levels, J, $\pi$. Comparison with model calculations. JOUR ZAANE 25 s01 121

\section{$\mathrm{A}=112$}

${ }^{112} \mathrm{Ru} \quad$ 2005SM08

RADIOACTIVITY ${ }^{252} \mathrm{Cf}(\mathrm{SF})$; measured $\mathrm{E} \gamma, \mathrm{I} \gamma(\theta, \mathrm{H}, \mathrm{t}), \gamma \gamma$-coin. deduced $\mathrm{g}$ factors, B(E2). Gammasphere array, time-integral perturbed angular correlation technique. Comparison with interacting boson measured $\sigma(\mathrm{E}, \theta)$. ${ }^{104,106,108,110} \mathrm{Pd}$ levels deduced $\mathrm{B}(\mathrm{E} 2)$, deformation lengths, Coulomb-nuclear interference parameters. DWBA-deformed optical model analysis. JOUR BJPHE 34777 96,100,102 Zr, 102,104,106,108 Mo, 106,108,110,112 Ru, 110,114,116 Pd levels deduced $\mathrm{g}$ factors, $\mathrm{B}(\mathrm{E} 2)$. Gammasphere array, time-integral perturbed ular correlation technique. Comparison with interacting boson

bremsstrahlung; measured $\mathrm{E} \gamma, \mathrm{I} \gamma, \gamma$-ray polarization.

110,111,112,114,116 Cd deduced levels, J, $\pi$, excitation B(M1), B(E1). JOUR PRVCA 72034302

\section{$\mathrm{A}=\mathbf{1 1 1}$}

RADIOACTIVITY ${ }^{252} \mathrm{Cf}(\mathrm{SF})$; measured $\mathrm{E} \gamma, \mathrm{I} \gamma(\theta, \mathrm{H}, \mathrm{t}), \gamma \gamma$-coin. 96,100,102 Zr, ${ }^{102,104,106,108} \mathrm{Mo},{ }^{106,108,110,112} \mathrm{Ru},{ }^{110,114,116} \mathrm{Pd}$ levels deduced $\mathrm{g}$ factors, $\mathrm{B}(\mathrm{E} 2)$. Gammasphere array, time-integral perturbed angular correlation technique. Comparison with interacting boson model predictions. JOUR JPGPE 31 S1433 


\section{$\mathrm{A}=112$ (continued)}

\begin{tabular}{|c|c|c|}
\hline${ }^{112} \mathrm{Cd}$ & $2005 \mathrm{~K} 032$ & $\begin{array}{l}\text { NUCLEAR REACTIONS } 110,111,112,114,116 \mathrm{Cd}\left(\gamma, \gamma^{\prime}\right), \mathrm{E} \approx 2.7-4.1 \mathrm{MeV} \\
\text { bremsstrahlung; measured } \mathrm{E} \gamma, \mathrm{I} \gamma, \gamma \text {-ray polarization. } \\
{ }_{110,111,112,114,116} \mathrm{Cd} \text { deduced levels, J, } \pi \text {, excitation } \mathrm{B}(\mathrm{M} 1), \mathrm{B}(\mathrm{E} 1)\end{array}$ \\
\hline${ }^{112} \mathrm{Sn}$ & $2005 \mathrm{KU} 28$ & $\begin{array}{l}\text { NUCLEAR REACTIONS }{ }^{112} \mathrm{Sn}(\mathrm{n}, \mathrm{n} \gamma), \mathrm{E}=2.5-4.0 \mathrm{MeV} \text {; measured } \mathrm{E} \gamma \text {, } \\
\mathrm{I} \gamma, \gamma \gamma \text {-coin, angular distributions, excitation functions, DSA. }{ }^{112} \mathrm{Sn} \\
\text { deduced levels, } \mathrm{J}, \pi, \delta, \mathrm{T}_{1 / 2}, \mathrm{~B}(\mathrm{M} 1) \text {, B(E2). JOUR PRVCA } 72034313\end{array}$ \\
\hline & $2005 \mathrm{KU} 37$ & $\begin{array}{l}\text { NUCLEAR REACTIONS }{ }^{112} \mathrm{Sn}(\mathrm{n}, \mathrm{n} \text { ' } \gamma), \mathrm{E}=2.5-4.0 \mathrm{MeV} \text {; measured } \mathrm{E} \gamma \text {, } \\
\mathrm{I} \gamma, \gamma \gamma \text {-coin, DSA, excitation functions, angular distributions. }{ }^{112} \mathrm{Sn} \\
\text { deduced levels, } \mathrm{J}, \pi, \mathrm{T}_{1 / 2} \text {. JOUR ZAANE } 25 \mathrm{~s} 01443\end{array}$ \\
\hline & 2005PYZZ & $\begin{array}{l}\text { NUCLEAR REACTIONS }{ }^{112} \mathrm{Sn}\left(\gamma, \gamma^{\prime}\right), \mathrm{E}=3.8 \mathrm{MeV} \text { bremsstrahlung; } \\
\text { measured } \mathrm{E} \gamma, \mathrm{I} \gamma .{ }^{112} \mathrm{Sn} \text { level deduced } \mathrm{B}(\mathrm{E} 1) \text {, decay width, two-phonon } \\
\text { configuration. PREPRINT nucl-ex/0512013,12/8/2005 }\end{array}$ \\
\hline
\end{tabular}

\section{$\mathrm{A}=113$}

${ }^{113} \mathrm{In} \quad$ 2005NA37 NUCLEAR REACTIONS ${ }^{100} \mathrm{Mo}\left({ }^{18} \mathrm{O}, 4 \mathrm{np}\right), \mathrm{E}=95 \mathrm{MeV} ;{ }^{110} \mathrm{Pd}\left({ }^{7} \mathrm{Li}\right.$ $4 \mathrm{n}), \mathrm{E}=36 \mathrm{MeV}$; measured $\mathrm{E} \gamma, \mathrm{I} \gamma, \gamma \gamma$-coin. ${ }^{113} \mathrm{In}$ deduced high-spin levels, J, $\pi$, configurations, shape coexistence. Cranked mean-field calculations. JOUR PRVCA 72044304

\section{$\mathrm{A}=\mathbf{1 1 4}$}

${ }^{114} \mathrm{Pd} \quad$ 2005SM08 RADIOACTIVITY ${ }^{252} \mathrm{Cf}(\mathrm{SF}) ;$ measured $\mathrm{E} \gamma, \mathrm{I} \gamma(\theta, \mathrm{H}, \mathrm{t}), \gamma \gamma$-coin. ${ }^{96,100,102} \mathrm{Zr},{ }^{102,104,106,108} \mathrm{Mo},{ }^{106,108,110,112} \mathrm{Ru},{ }^{110,114,116} \mathrm{Pd}$ levels deduced $\mathrm{g}$ factors, $\mathrm{B}(\mathrm{E} 2)$. Gammasphere array, time-integral perturbed angular correlation technique. Comparison with interacting boson model predictions. JOUR JPGPE 31 S1433

${ }^{114} \mathrm{Cd} \quad$ 2005K032 NUCLEAR REACTIONS ${ }^{110,111,112,114,116} \mathrm{Cd}\left(\gamma, \gamma^{\prime}\right), \mathrm{E} \approx 2.7-4.1 \mathrm{MeV}$ bremsstrahlung; measured $\mathrm{E} \gamma, \mathrm{I} \gamma, \gamma$-ray polarization.

${ }_{110,111,112,114,116} \mathrm{Cd}$ deduced levels, J, $\pi$, excitation B(M1), B(E1). JOUR PRVCA 72034302

\section{$\mathrm{A}=\mathbf{1 1 5}$}

${ }^{115} \mathrm{In}$ 2004AG09 NUCLEAR REACTIONS ${ }^{103} \mathrm{Rh}\left(\mathrm{n}, \mathrm{n}^{\prime}\right){ }^{103 m} \mathrm{Rh}, \mathrm{E} \approx 4.8 \mathrm{MeV} ;{ }^{115} \mathrm{In}(\mathrm{n}$, $\left.\mathrm{n}^{\prime}\right){ }^{115 m} \mathrm{In}, \mathrm{E} \approx 5 \mathrm{MeV} ;{ }^{232} \mathrm{Th},{ }^{238} \mathrm{U}(\mathrm{n}, \mathrm{F}), \mathrm{E} \approx 5 \mathrm{MeV} ;{ }^{24} \mathrm{Mg},{ }^{27} \mathrm{Al}$, ${ }^{46,47,48} \mathrm{Ti},{ }^{54,56} \mathrm{Fe},{ }^{58} \mathrm{Ni},{ }^{64} \mathrm{Zn}(\mathrm{n}, \mathrm{p}), \mathrm{E} \approx 2-8 \mathrm{MeV} ;{ }^{27} \mathrm{Al},{ }^{59} \mathrm{Co}(\mathrm{n}, \alpha), \mathrm{E}$ $\approx 8.3 \mathrm{MeV}$; measured activation $\sigma$. Spectrum average technique, comparison with previous results. JOUR RAACA 9263 


\section{$\mathrm{A}=\mathbf{1 1 6}$}

\begin{tabular}{|c|c|c|}
\hline \multirow[t]{2}{*}{${ }^{116} \mathrm{Pd}$} & 2005SM08 & 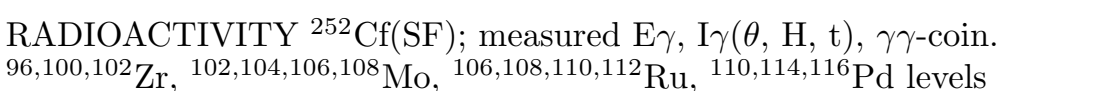 \\
\hline & & $\begin{array}{l}\text { deduced } g \text { factors, } \mathrm{B}(\mathrm{E} 2) \text {. Gammasphere array, time-integral perturbed } \\
\text { angular correlation technique. Comparison with interacting boson } \\
\text { model predictions. JOUR JPGPE } 31 \mathrm{~S} 1433\end{array}$ \\
\hline \multirow[t]{2}{*}{${ }^{116} \mathrm{Ag}$} & 2005BA94 & $\begin{array}{l}\left.\text { RADIOACTIVITY }{ }^{116 m} \mathrm{Ag}(\mathrm{IT}),{ }^{116} \mathrm{Ag}\left(\beta^{-}\right) \text {[from } \mathrm{U}(\mathrm{p}, \mathrm{F})\right] ; \text { measured } \\
\mathrm{E}(\mathrm{ce}), \mathrm{I}(\mathrm{ce}), \mathrm{E} \gamma, \mathrm{I} \gamma .{ }^{116} \mathrm{Ag} \text { deduced levels, J, } \pi, \mathrm{ICC} \text {, isomer } \mathrm{T}_{1 / 2} . \\
{ }^{116} \mathrm{Cd} \text { deduced transitions. JOUR PRVCA } 72044306\end{array}$ \\
\hline & 2005RI19 & $\begin{array}{l}\text { RADIOACTIVITY }{ }^{116,118,120} \mathrm{Ag}\left(\beta^{-}\right) ; \text {measured } \mathrm{E} \gamma, \mathrm{I} \gamma, \beta \gamma \text {-coin. } \\
116,118,120 \mathrm{Cd} \text { deduced levels, J, } \pi \text {. Three-phonon states discussed. }\end{array}$ \\
\hline \multirow[t]{3}{*}{${ }^{116} \mathrm{Cd}$} & 2005BA94 & $\begin{array}{l}\left.\text { RADIOACTIVITY }{ }^{116 m} \mathrm{Ag}(\mathrm{IT}),{ }^{116} \mathrm{Ag}\left(\beta^{-}\right) \text {from } \mathrm{U}(\mathrm{p}, \mathrm{F})\right] \text {; measured } \\
\mathrm{E}(\mathrm{ce}), \mathrm{I}(\mathrm{ce}), \mathrm{E} \gamma, \mathrm{I} \gamma \cdot{ }^{116} \mathrm{Ag} \text { deduced levels, J, } \pi, \mathrm{ICC} \text {, isomer } \mathrm{T}_{1 / 2} . \\
{ }^{116} \mathrm{Cd} \text { deduced transitions. JOUR PRVCA } 72044306\end{array}$ \\
\hline & $2005 \mathrm{~K} 032$ & $\begin{array}{l}\text { NUCLEAR REACTIONS }{ }^{110,111,112,114,116} \mathrm{Cd}\left(\gamma, \gamma^{\prime}\right), \mathrm{E} \approx 2.7-4.1 \mathrm{MeV} \\
\text { bremsstrahlung; measured } \mathrm{E} \gamma, \mathrm{I} \gamma, \gamma \text {-ray polarization. } \\
110,111,112,114,116 \mathrm{Cd} \text { deduced levels, J, } \pi \text {, excitation } \mathrm{B}(\mathrm{M} 1), \mathrm{B}(\mathrm{E} 1) .\end{array}$ \\
\hline & 2005RI19 & $\begin{array}{l}\text { JOUR PRVCA } 72034302 \\
\text { RADIOACTIVITY } 116,118,120 \mathrm{Ag}\left(\beta^{-}\right) \text {; measured } \mathrm{E} \gamma, \mathrm{I} \gamma, \beta \gamma \text {-coin. } \\
{ }_{116,118,120} \text { Cd deduced levels, J, } \pi \text {. Three-phonon states discussed. } \\
\text { JOUR ZAANE } 25 \text { s01 } 119\end{array}$ \\
\hline
\end{tabular}

$$
\mathrm{A}=\mathbf{1 1 7}
$$

No references found

$$
\mathrm{A}=\mathbf{1 1 8}
$$

${ }^{118} \mathrm{Ag} \quad$ 2005RI19 RADIOACTIVITY ${ }^{116,118,120} \mathrm{Ag}\left(\beta^{-}\right) ;$measured $\mathrm{E} \gamma, \mathrm{I} \gamma, \boldsymbol{\beta} \gamma$-coin. ${ }^{116,118,120} \mathrm{Cd}$ deduced levels, J, $\pi$. Three-phonon states discussed. JOUR ZAANE 25 s01 119

${ }^{118} \mathrm{Cd} \quad$ 2005RI19 RADIOACTIVITY ${ }^{116,118,120} \mathrm{Ag}\left(\beta^{-}\right)$; measured $\mathrm{E} \gamma, \mathrm{I} \gamma, \beta \gamma$-coin. $116,118,120 \mathrm{Cd}$ deduced levels, J, $\pi$. Three-phonon states discussed. JOUR ZAANE 25 s01 119

$$
\mathrm{A}=\mathbf{1 1 9}
$$

${ }^{119}$ In $\quad 2005 G U 32$

NUCLEAR REACTIONS ${ }^{122} \mathrm{Sn},{ }^{123} \mathrm{Sb}$ (polarized $\mathrm{p}, \alpha$ ), $\mathrm{E}=24 \mathrm{MeV}$; measured $\mathrm{E} \alpha, \sigma(\theta), \operatorname{Ay}(\theta) .{ }^{119} \mathrm{In},{ }^{120} \mathrm{Sn}$ deduced homologous states features. JOUR PRVCA 72044604 


\begin{tabular}{|c|c|c|}
\hline & & $A=120$ \\
\hline${ }^{120} \mathrm{Ag}$ & 2005RI19 & $\begin{array}{l}\text { RADIOACTIVITY }{ }^{116,118,120} \mathrm{Ag}\left(\beta^{-}\right) \text {; measured } \mathrm{E} \gamma, \mathrm{I} \gamma, \beta \gamma \text {-coin. } \\
{ }^{116,118,120} \mathrm{Cd} \text { deduced levels, } \mathrm{J}, \pi \text {. Three-phonon states discussed. } \\
\text { JOUR ZAANE } 25 \text { s01 } 119\end{array}$ \\
\hline${ }^{120} \mathrm{Cd}$ & $2005 R I 19$ & $\begin{array}{l}\text { RADIOACTIVITY }{ }^{116,118,120} \mathrm{Ag}\left(\beta^{-}\right) \text {; measured } \mathrm{E} \gamma, \mathrm{I} \gamma, \beta \gamma \text {-coin. } \\
{ }^{116,118,120} \mathrm{Cd} \text { deduced levels, } \mathrm{J}, \pi \text {. Three-phonon states discussed. }\end{array}$ \\
\hline${ }^{120} \mathrm{Sn}$ & 2005GU32 & $\begin{array}{l}\text { JOUR ZAANE } 25 \text { s01 } 119 \\
\text { NUCLEAR REACTIONS }{ }^{122} \mathrm{Sn},{ }^{123} \mathrm{Sb}(\text { polarized } \mathrm{p}, \alpha), \mathrm{E}=24 \mathrm{MeV} \text {; } \\
\text { measured } \mathrm{E} \alpha, \sigma(\theta), \mathrm{Ay}(\theta) .{ }^{119} \mathrm{In},{ }^{120} \mathrm{Sn} \text { deduced homologous states } \\
\text { features. JOUR PRVCA } 72{ }^{044604}\end{array}$ \\
\hline
\end{tabular}

$$
\mathrm{A}=121
$$

No references found

\section{$\mathrm{A}=122$}

${ }^{122} \mathrm{Cd} \quad$ 2005BEZS

NUCLEAR REACTIONS ${ }^{108} \mathrm{Pd}\left({ }^{122} \mathrm{Cd},{ }^{122} \mathrm{Cd}\right),{ }^{104} \mathrm{Pd}\left({ }^{124} \mathrm{Cd},{ }^{124} \mathrm{Cd}\right.$ '), $\left({ }^{126} \mathrm{Cd},{ }^{126} \mathrm{Cd}\right.$ '), E not given; measured $\mathrm{E} \gamma, \mathrm{I} \gamma$, (particle) $\gamma$-coin following projectile Coulomb excitation. ${ }^{122,124} \mathrm{Cd}$ levels deduced excitation B(E2). REPT MLL 2004 Annual,P14,Behrens
${ }^{122} \mathrm{Te}$ 2005HA56 NUCLEAR REACTIONS ${ }^{104} \mathrm{Pd}(\mathrm{p}, \gamma), \mathrm{E}(\mathrm{cm})=2-8 \mathrm{MeV} ;{ }^{118} \operatorname{Sn}(\alpha, \gamma)$, $\mathrm{E}(\mathrm{cm})=10-11 \mathrm{MeV}$; measured $\mathrm{E} \gamma, \mathrm{I} \gamma, \sigma$. Comparison with model predictions. JOUR JPGPE 31 S1417

${ }^{122} \mathrm{Cs} \quad 2005 \mathrm{KU} 34$ NUCLEAR REACTIONS ${ }^{107} \mathrm{Ag}\left({ }^{19} \mathrm{~F}, 3 \mathrm{np}\right), \mathrm{E}=93 \mathrm{MeV}$; measured $\mathrm{E} \gamma$, $\mathrm{I} \gamma, \gamma \gamma$-coin. ${ }^{122} \mathrm{Cs}$ deduced levels, $\mathrm{J}, \pi$, configurations. Comparison with model predictions. JOUR PRVCA 72044319

\section{$\mathrm{A}=123$}

${ }^{123} \mathrm{I}$ 2004GL10
NUCLEAR REACTIONS ${ }^{124} \mathrm{Te}(\mathrm{p}, \mathrm{n}),(\mathrm{p}, 2 \mathrm{n}), \mathrm{E} \approx 8-19 \mathrm{MeV} ;$
measured thick-target yields. JOUR RAACA 92951

2006HA01 NUCLEAR REACTIONS Sn $(\alpha, \mathrm{xn}){ }^{123} \mathrm{I} /{ }^{124} \mathrm{I} /{ }^{125} \mathrm{I} /{ }^{126} \mathrm{I}, \mathrm{E}=8-26$ $\mathrm{MeV} ;{ }^{121} \mathrm{Sb}(\alpha, \mathrm{n}),(\alpha, 2 \mathrm{n}), \mathrm{E}=8-26 \mathrm{MeV}$; measured $\sigma$. Stacked-foil activation, comparison with previous results. JOUR ARISE 64101 ${ }^{123} \mathrm{Cs} \quad 2005 \mathrm{SI} 31$ NUCLEAR REACTIONS ${ }^{100} \mathrm{Mo}\left({ }^{28} \mathrm{Si}, 4 \mathrm{np}\right), \mathrm{E}=130 \mathrm{MeV}$; measured $\mathrm{E} \gamma, \mathrm{I} \gamma, \gamma \gamma$-coin. ${ }^{123} \mathrm{Cs}$ deduced high-spin levels, J, $\pi$, configurations. Total Routhian surface calculations. JOUR ZAANE 25345

\section{$\mathrm{A}=124$}

${ }^{124} \mathrm{Ag} \quad$ 2005KA45 RADIOACTIVITY ${ }^{124,126} \mathrm{Ag}\left(\beta^{-}\right)$; measured $\mathrm{E} \gamma, \mathrm{I} \gamma, \gamma \gamma$-coin following decay of mass-separated sources. ${ }^{124,126} \mathrm{Cd}$ deduced levels, $\mathrm{J}, \pi$. Comparison with shell-model predictions. JOUR ZAANE 25 s01 117 


\section{$\mathrm{A}=124$ (continued)}

${ }^{124} \mathrm{Cd}$ 2005BEzS NUCLEAR REACTIONS ${ }^{108} \mathrm{Pd}\left({ }^{122} \mathrm{Cd},{ }^{122} \mathrm{Cd}\right.$ ' $),{ }^{104} \mathrm{Pd}\left({ }^{124} \mathrm{Cd},{ }^{124} \mathrm{Cd}\right.$ ' $)$, $\left({ }^{126} \mathrm{Cd},{ }^{126} \mathrm{Cd}\right.$ '), E not given; measured $\mathrm{E} \gamma, \mathrm{I} \gamma$, (particle) $\gamma$-coin following projectile Coulomb excitation. ${ }^{122,124} \mathrm{Cd}$ levels deduced excitation B(E2). REPT MLL 2004 Annual,P14,Behrens

2005KA45 RADIOACTIVITY ${ }^{124,126} \mathrm{Ag}\left(\beta^{-}\right)$; measured $\mathrm{E} \gamma, \mathrm{I} \gamma, \gamma \gamma$-coin following decay of mass-separated sources. ${ }^{124,126} \mathrm{Cd}$ deduced levels, $\mathrm{J}, \pi$.

Comparison with shell-model predictions. JOUR ZAANE 25 s01 117

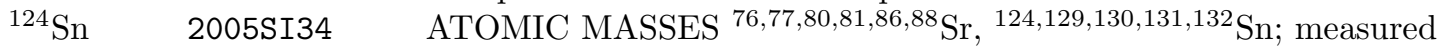
masses. Penning trap mass spectrometer, comparison with previous results. JOUR NUPAB 76345

${ }^{124} \mathrm{I} \quad$ 2004GL10 NUCLEAR REACTIONS ${ }^{124} \mathrm{Te}(\mathrm{p}, \mathrm{n}),(\mathrm{p}, 2 \mathrm{n}), \mathrm{E} \approx 8-19 \mathrm{MeV}$; measured thick-target yields. JOUR RAACA 92951

2006HA01 NUCLEAR REACTIONS Sn $(\alpha, \mathrm{xn}){ }^{123} \mathrm{I} /{ }^{124} \mathrm{I} /{ }^{125} \mathrm{I} /{ }^{126} \mathrm{I}, \mathrm{E}=8-26$ $\mathrm{MeV} ;{ }^{121} \mathrm{Sb}(\alpha, \mathrm{n}),(\alpha, 2 \mathrm{n}), \mathrm{E}=8-26 \mathrm{MeV}$; measured $\sigma$. Stacked-foil activation, comparison with previous results. JOUR ARISE 64101

${ }^{124} \mathrm{Cs} \quad 2005 \mathrm{GU} 37$ ATOMIC MASSES ${ }^{56,57} \mathrm{Mn},{ }^{82 m} \mathrm{Rb},{ }^{92} \mathrm{Sr},{ }^{124,127} \mathrm{Cs},{ }^{130} \mathrm{Ba}$; measured masses. Penning trap mass spectrometer. JOUR ZAANE 25 s01 35 NUCLEAR REACTIONS ${ }^{64} \mathrm{Ni}\left({ }^{64} \mathrm{Ni}, 3 \mathrm{n}\right),\left({ }^{64} \mathrm{Ni}, 4 \mathrm{n}\right), \mathrm{E}=255-261 \mathrm{MeV}$; measured $\mathrm{E} \gamma, \mathrm{I} \gamma, \gamma \gamma$-coin. ${ }^{124,125} \mathrm{Ba}$ deduced levels, $\mathrm{J}, \pi$, octupole correlations. Euroball and Diamant arrays. JOUR JPGPE 31 S1729

$$
\mathrm{A}=125
$$

${ }^{125} \mathrm{Sn} \quad$ 2005J023

NUCLEAR REACTIONS ${ }^{2} \mathrm{H}\left({ }^{124} \mathrm{Sn}, \mathrm{p}\right), \mathrm{E}=4.5 \mathrm{MeV} /$ nucleon; measured $\sigma(\theta) .{ }^{125} \mathrm{Sn}$ levels deduced spectroscopic factors. DWBA analysis. JOUR ZAANE 25 s01 283

2005LE34 NUCLEAR MOMENTS

$125,125 m, 126,127,127 m, 128,129,129 m, 130,130 m, 131,131 m, 132 \mathrm{Sn}$; measured isotope

shifts; deduced charge radii, dynamical effects.

$125,125 m, 127,127 m, 129,129 m, 130 m, 131,131 m \mathrm{Sn}$; measured $\mu$, quadrupole moments. Laser spectroscopy, mean-field calculations. JOUR PRVCA 72034305

${ }^{125} \mathrm{Sb} \quad$ 2005JU12 NUCLEAR REACTIONS ${ }^{124} \mathrm{Sn}\left({ }^{7} \mathrm{Li}, 2 \mathrm{n} \alpha\right), \mathrm{E}=37 \mathrm{MeV}$; measured delayed $\mathrm{E} \gamma, \mathrm{I} \gamma, \mathrm{E}(\mathrm{ce}), \mathrm{I}(\mathrm{ce}) .{ }^{125} \mathrm{Sb}$ deduced levels, J, $\pi$, configurations, isomeric states $\mathrm{T}_{1 / 2}$, ICC. Level systematics in neighboring isotopes compared. JOUR JPGPE 31 S1899

${ }^{125} \mathrm{Te} \quad 2004 \mathrm{G059}$ RADIOACTIVITY ${ }^{125 m} \mathrm{Te}(\mathrm{IT}) ;$ measured $\mathrm{T}_{1 / 2}$, non-exponential decay features. JOUR BRSPE 681335

${ }^{125} \mathrm{I} \quad$ 2006HA01 NUCLEAR REACTIONS $\operatorname{Sn}(\alpha, \mathrm{xn}){ }^{123} \mathrm{I} /{ }^{124} \mathrm{I} /{ }^{125} \mathrm{I} /{ }^{126} \mathrm{I}, \mathrm{E}=8-26$ $\mathrm{MeV} ;{ }^{121} \mathrm{Sb}(\alpha, \mathrm{n}),(\alpha, 2 \mathrm{n}), \mathrm{E}=8-26 \mathrm{MeV}$; measured $\sigma$. Stacked-foil activation, comparison with previous results. JOUR ARISE 64101 NUCLEAR REACTIONS ${ }^{64} \mathrm{Ni}\left({ }^{64} \mathrm{Ni}, 3 \mathrm{n}\right),\left({ }^{64} \mathrm{Ni}, 4 \mathrm{n}\right), \mathrm{E}=255-261 \mathrm{MeV}$; measured $\mathrm{E} \gamma$, I $\gamma, \gamma \gamma$-coin. ${ }^{124,125} \mathrm{Ba}$ deduced levels, $\mathrm{J}, \pi$, octupole correlations. Euroball and Diamant arrays. JOUR JPGPE 31 S1729 


\begin{tabular}{|c|c|c|}
\hline \multicolumn{3}{|r|}{$A=126$} \\
\hline${ }^{126} \mathrm{Ag}$ & 2005KA45 & $\begin{array}{l}\text { RADIOACTIVITY }{ }^{124,126} \mathrm{Ag}\left(\beta^{-}\right) \text {; measured } \mathrm{E} \gamma, \mathrm{I} \gamma, \gamma \gamma \text {-coin following } \\
\text { decay of mass-separated sources. }{ }^{124,126} \mathrm{Cd} \text { deduced levels, J, } \pi \text {. } \\
\text { Comparison with shell-model predictions. JOUR ZAANE } 25 \text { s01 } 117\end{array}$ \\
\hline${ }^{126} \mathrm{Cd}$ & $2005 \mathrm{KA} 45$ & $\begin{array}{l}\text { RADIOACTIVITY }{ }^{124,126} \mathrm{Ag}\left(\beta^{-}\right) \text {; measured } \mathrm{E} \gamma, \mathrm{I} \gamma, \gamma \gamma \text {-coin following } \\
\text { decay of mass-separated sources. }{ }^{124,126} \mathrm{Cd} \text { deduced levels, J, } \pi \text {. } \\
\text { Comparison with shell-model predictions. JOUR ZAANE } 25 \text { s01 } 117\end{array}$ \\
\hline \multirow[t]{3}{*}{${ }^{126} \mathrm{Sn}$} & 2005LE34 & $\begin{array}{l}\text { NUCLEAR MOMENTS } \\
125,125 m, 126,127,127 m, 128,129,129 m, 130,130 m, 131,131 m, 132 \mathrm{Sn} ; \text { measured isotope }\end{array}$ \\
\hline & & $\begin{array}{l}\text { shifts; deduced charge radii, dynamical effects. } \\
125,125 \mathrm{~m}, 127,127 \mathrm{~m}, 129,129 \mathrm{~m}, 130 \mathrm{~m}, 131,131 \mathrm{~m} \mathrm{Sn} \text {; measured } \mu \text {, quadrupole } \\
\text { moments. Laser spectroscopy, mean-field calculations. JOUR PRVCA } \\
72034305\end{array}$ \\
\hline & 2005RA32 & 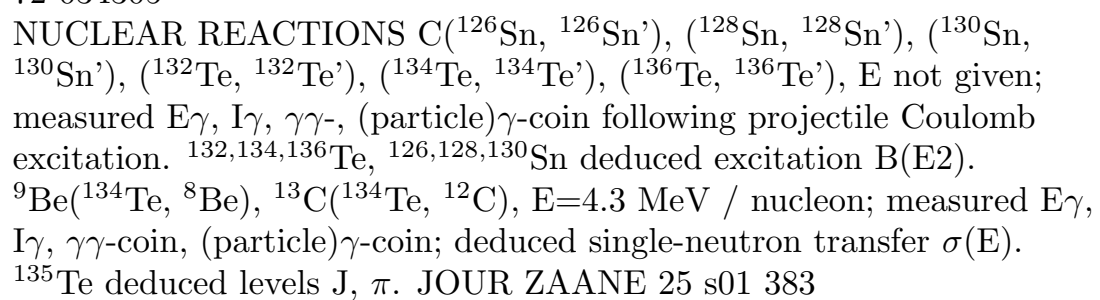 \\
\hline \multirow[t]{2}{*}{${ }^{126} \mathrm{I}$} & 2006HA01 & $\begin{array}{l}\text { NUCLEAR REACTIONS } \mathrm{Sn}(\alpha, \mathrm{xn}){ }^{123} \mathrm{I} /{ }^{124} \mathrm{I} /{ }^{125} \mathrm{I} /{ }^{126} \mathrm{I}, \mathrm{E}=8-26 \\
\mathrm{MeV} ;{ }^{121} \mathrm{Sb}(\alpha, \mathrm{n}),(\alpha, 2 \mathrm{n}), \mathrm{E}=8-26 \mathrm{MeV} ; \text { measured } \sigma . \text { Stacked-foil } \\
\text { activation, comparison with previous results. JOUR ARISE } 64101\end{array}$ \\
\hline & & $A=127$ \\
\hline \multirow[t]{2}{*}{${ }^{127} \mathrm{Sn}$} & 2005LE34 & $\begin{array}{l}\text { NUCLEAR MOMENTS } \\
125,125 m, 126,127,127 m, 128,129,129 m, 130,130 m, 131,131 m, 132 \mathrm{Sn}: \text { measured isotope }\end{array}$ \\
\hline & & $\begin{array}{l}\text { shifts; deduced charge radii, dynamical effects. } \\
125,125 m, 127,127 m, 129,129 m, 130 m, 131,131 m \text { Sn; measured } \mu \text {, quadrupole } \\
\text { moments. Laser spectroscopy, mean-field calculations. JOUR PRVCA } \\
72034305\end{array}$ \\
\hline \multirow[t]{2}{*}{${ }^{127} \mathrm{Cs}$} & 2005GU37 & $\begin{array}{l}\text { ATOMIC MASSES }{ }^{56,57} \mathrm{Mn},{ }^{82 m} \mathrm{Rb},{ }^{92} \mathrm{Sr},{ }^{124,127} \mathrm{Cs},{ }^{130} \mathrm{Ba} \text {; measured } \\
\text { masses. Penning trap mass spectrometer. JOUR ZAANE } 25 \text { s01 } 35\end{array}$ \\
\hline & & $\mathrm{A}=128$ \\
\hline \multirow[t]{2}{*}{${ }^{128} \mathrm{Sn}$} & 2005LE34 & $\begin{array}{l}\text { NUCLEAR MOMENTS } \\
125,125 m, 126,127,127 m, 128,129,129 m, 130,130 m, 131,131 m, 132 \mathrm{Sn} ; \text { measured isotope }\end{array}$ \\
\hline & & $\begin{array}{l}\text { shifts; deduced charge radii, dynamical effects. } \\
125,125 m, 127,127 m, 129,129 m, 130 m, 131,131 m \mathrm{Sn} \text {; measured } \mu \text {, quadrupole } \\
\text { moments. Laser spectroscopy, mean-field calculations. JOUR PRVCA } \\
72034305\end{array}$ \\
\hline
\end{tabular}




\section{$\mathrm{A}=128$ (continued)}

2005RA32 NUCLEAR REACTIONS C $\left({ }^{126} \mathrm{Sn},{ }^{126} \mathrm{Sn}^{\prime}\right),\left({ }^{128} \mathrm{Sn},{ }^{128} \mathrm{Sn}^{\prime}\right),\left({ }^{130} \mathrm{Sn}\right.$, ${ }^{130} \mathrm{Sn}$ '), $\left({ }^{132} \mathrm{Te},{ }^{132} \mathrm{Te}\right.$ '), $\left({ }^{134} \mathrm{Te},{ }^{134} \mathrm{Te}\right),\left({ }^{136} \mathrm{Te},{ }^{136} \mathrm{Te}\right.$ '), E not given; measured $\mathrm{E} \gamma, \mathrm{I} \gamma, \gamma \gamma$-, (particle) $\gamma$-coin following projectile Coulomb excitation. ${ }^{132,134,136} \mathrm{Te},{ }^{126,128,130} \mathrm{Sn}$ deduced excitation $\mathrm{B}(\mathrm{E} 2)$. ${ }^{9} \mathrm{Be}\left({ }^{134} \mathrm{Te},{ }^{8} \mathrm{Be}\right),{ }^{13} \mathrm{C}\left({ }^{134} \mathrm{Te},{ }^{12} \mathrm{C}\right), \mathrm{E}=4.3 \mathrm{MeV} /$ nucleon; measured $\mathrm{E} \gamma$, $\mathrm{I} \gamma, \gamma \gamma$-coin, (particle) $\gamma$-coin; deduced single-neutron transfer $\sigma(\mathrm{E})$.

${ }^{135}$ Te deduced levels J, $\pi$. JOUR ZAANE 25 s01 383

$$
\mathrm{A}=129
$$

\section{${ }^{129} \mathrm{Sn} \quad$ 2005LE34 NUCLEAR MOMENTS}

$125,125 m, 126,127,127 m, 128,129,129 m, 130,130 m, 131,131 m, 132 \mathrm{Sn}$; measured isotope shifts; deduced charge radii, dynamical effects.

$125,125 m, 127,127 m, 129,129 m, 130 m, 131,131 m \mathrm{Sn}$; measured $\mu$, quadrupole moments. Laser spectroscopy, mean-field calculations. JOUR PRVCA 72034305

2005SI34 ATOMIC MASSES $76,77,80,81,86,88 \mathrm{Sr},{ }^{124,129,130,131,132} \mathrm{Sn}$; measured masses. Penning trap mass spectrometer, comparison with previous results. JOUR NUPAB 76345

${ }^{129} \mathrm{Sb} 2005$ YU07 NUCLEAR REACTIONS ${ }^{50} \mathrm{Ti}\left({ }^{129} \mathrm{Sb},{ }^{129} \mathrm{Sb}\right),\left({ }^{129} \mathrm{Te},{ }^{129} \mathrm{Te}\right.$ '), E=400 $\mathrm{MeV}$; measured $\mathrm{E} \gamma, \mathrm{I} \gamma$, (particle) $\gamma$-coin following projectile Coulomb excitation. ${ }^{129} \mathrm{Te},{ }^{129} \mathrm{Sb}$ deduced transitions B(E2). Clarion, Hyball arrays. JOUR ZAANE 25 s01 395

${ }^{129} \mathrm{Te} 2005 \mathrm{YU} 07$ NUCLEAR REACTIONS ${ }^{50} \mathrm{Ti}\left({ }^{129} \mathrm{Sb},{ }^{129} \mathrm{Sb}\right),\left({ }^{129} \mathrm{Te},{ }^{129} \mathrm{Te}\right.$ '), E $=400$ $\mathrm{MeV}$; measured $\mathrm{E} \gamma, \mathrm{I} \gamma$, (particle) $\gamma$-coin following projectile Coulomb excitation. ${ }^{129} \mathrm{Te},{ }^{129} \mathrm{Sb}$ deduced transitions B(E2). Clarion, Hyball arrays. JOUR ZAANE 25 s01 395

\section{$\mathrm{A}=130$}

${ }^{130} \mathrm{Sn} \quad$ 2005LE34 NUCLEAR MOMENTS

$125,125 m, 126,127,127 m, 128,129,129 m, 130,130 m, 131,131 m, 132 \mathrm{Sn}$; measured isotope shifts; deduced charge radii, dynamical effects.

$125,125 m, 127,127 m, 129,129 m, 130 m, 131,131 m \mathrm{Sn}$; measured $\mu$, quadrupole moments. Laser spectroscopy, mean-field calculations. JOUR PRVCA 72034305

2005RA32 NUCLEAR REACTIONS C( $\left.{ }^{126} \mathrm{Sn},{ }^{126} \mathrm{Sn}\right),\left({ }^{128} \mathrm{Sn},{ }^{128} \mathrm{Sn}\right),\left({ }^{130} \mathrm{Sn}\right.$, $\left.{ }^{130} \mathrm{Sn}^{\prime}\right),\left({ }^{132} \mathrm{Te},{ }^{132} \mathrm{Te}\right),\left({ }^{134} \mathrm{Te},{ }^{134} \mathrm{Te}\right),\left({ }^{136} \mathrm{Te},{ }^{136} \mathrm{Te}\right), \mathrm{E}$ not given; measured $\mathrm{E} \gamma, \mathrm{I} \gamma, \gamma \gamma$-, (particle) $\gamma$-coin following projectile Coulomb excitation. ${ }^{132,134,136} \mathrm{Te},{ }^{126,128,130} \mathrm{Sn}$ deduced excitation $\mathrm{B}(\mathrm{E} 2)$.

${ }^{9} \mathrm{Be}\left({ }^{134} \mathrm{Te},{ }^{8} \mathrm{Be}\right),{ }^{13} \mathrm{C}\left({ }^{134} \mathrm{Te},{ }^{12} \mathrm{C}\right), \mathrm{E}=4.3 \mathrm{MeV} /$ nucleon; measured $\mathrm{E} \gamma$, $\mathrm{I} \gamma, \gamma \gamma$-coin, (particle) $\gamma$-coin; deduced single-neutron transfer $\sigma(\mathrm{E})$.

${ }^{135}$ Te deduced levels J, $\pi$. JOUR ZAANE 25 s01 383

2005SI34 ATOMIC MASSES ${ }^{76,77,80,81,86,88}$ Sr, ${ }^{124,129,130,131,132} \mathrm{Sn}$; measured masses. Penning trap mass spectrometer, comparison with previous results. JOUR NUPAB 76345 


\section{$\mathrm{A}=130$ (continued)}

$\begin{array}{ll}{ }^{130} \mathrm{Te} & 2005 \mathrm{AR} 25 \\ { }^{130} \mathrm{Xe} & 2005 \mathrm{AR} 25 \\ { }^{130} \mathrm{Ba} & 2005 \mathrm{GU} 37\end{array}$

${ }^{131} \mathrm{Sn} \quad$ 2005LE34 $125,125 m, 126,127,127 m, 128,129,129 m, 130,130 m, 131,131 m, 132 \mathrm{Sn}$; measured isotope shifts; deduced charge radii, dynamical effects.

$125,125 m, 127,127 m, 129,129 m, 130 m, 131,131 m \mathrm{Sn}$; measured $\mu$, quadrupole moments. Laser spectroscopy, mean-field calculations. JOUR PRVCA 72034305

2005SI34 ATOMIC MASSES $76,77,80,81,86,88 \mathrm{Sr},{ }^{124,129,130,131,132} \mathrm{Sn}$; measured masses. Penning trap mass spectrometer, comparison with previous results. JOUR NUPAB 76345

$$
\mathrm{A}=132
$$

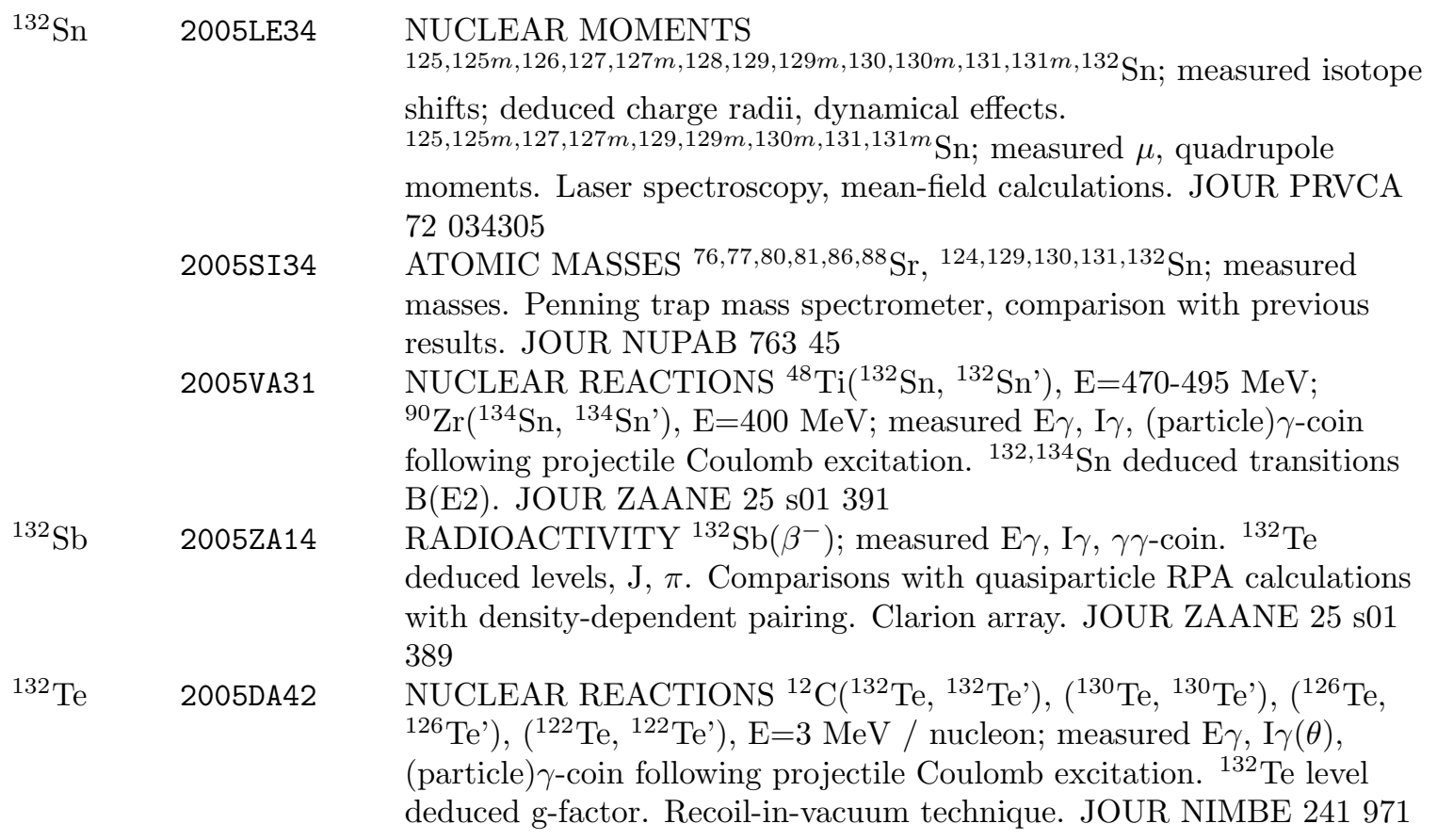

RADIOACTIVITY ${ }^{130} \mathrm{Te}\left(2 \beta^{-}\right)$; measured $0 \nu \beta \beta$-decay $\mathrm{T}_{1 / 2}$ lower limit. JOUR PRLTA 95142501 limit. JOUR PRLTA 95142501 ATOMIC MASSES ${ }^{56,57} \mathrm{Mn},{ }^{82 m} \mathrm{Rb},{ }^{92} \mathrm{Sr},{ }^{124,127} \mathrm{Cs},{ }^{130} \mathrm{Ba}$; measured masses. Penning trap mass spectrometer. JOUR ZAANE 25 s01 35

\section{$A=131$}




\section{$\mathrm{A}=132$ (continued)}

2005GR25

NUCLEAR REACTIONS ${ }^{64} \mathrm{Ni}\left({ }^{132} \mathrm{Sn}, \mathrm{X}\right),\left({ }^{134} \mathrm{Sn}, \mathrm{X}\right), \mathrm{E}=450-620 \mathrm{MeV}$; measured fusion $\sigma$. C $\left({ }^{130} \mathrm{Te},{ }^{130} \mathrm{Te}\right),\left({ }^{132} \mathrm{Te},{ }^{132} \mathrm{Te}\right), \mathrm{E}=3 \mathrm{MeV} /$ nucleon; measured $\mathrm{E} \gamma, \mathrm{I} \gamma$, (particle) $\gamma$-coin following projectile Coulomb excitation. ${ }^{132} \mathrm{Te}$ level deduced g factor. ${ }^{13} \mathrm{C}\left({ }^{134} \mathrm{Te},{ }^{135} \mathrm{Te}\right), \mathrm{E}=550$ $\mathrm{MeV} ;$ measured $\mathrm{E} \gamma, \mathrm{I} \gamma$. ${ }^{135} \mathrm{Te}$ level deduced J, $\pi$. JOUR JPGPE 31 S1639

2005RA32 NUCLEAR REACTIONS C ( $\left.{ }^{126} \mathrm{Sn},{ }^{126} \mathrm{Sn}\right),\left({ }^{128} \mathrm{Sn},{ }^{128} \mathrm{Sn}\right),\left({ }^{130} \mathrm{Sn}\right.$, ${ }^{130} \mathrm{Sn}$ '), $\left({ }^{132} \mathrm{Te},{ }^{132} \mathrm{Te}\right.$ '), $\left({ }^{134} \mathrm{Te},{ }^{134} \mathrm{Te}\right),\left({ }^{136} \mathrm{Te},{ }^{136} \mathrm{Te}\right), \mathrm{E}$ not given; measured $\mathrm{E} \gamma, \mathrm{I} \gamma, \gamma \gamma-$, (particle) $\gamma$-coin following projectile Coulomb excitation. ${ }^{132,134,136} \mathrm{Te},{ }^{126,128,130} \mathrm{Sn}$ deduced excitation $\mathrm{B}(\mathrm{E} 2)$. ${ }^{9} \mathrm{Be}\left({ }^{134} \mathrm{Te},{ }^{8} \mathrm{Be}\right),{ }^{13} \mathrm{C}\left({ }^{134} \mathrm{Te},{ }^{12} \mathrm{C}\right), \mathrm{E}=4.3 \mathrm{MeV} /$ nucleon; measured $\mathrm{E} \gamma$, $\mathrm{I} \gamma, \gamma \gamma$-coin, (particle) $\gamma$-coin; deduced single-neutron transfer $\sigma(\mathrm{E})$. ${ }^{135}$ Te deduced levels J, $\pi$. JOUR ZAANE 25 s01 383 2005ST33 NUCLEAR REACTIONS C $\left({ }^{132} \mathrm{Te},{ }^{132} \mathrm{Te}\right.$ '), $\left({ }^{122} \mathrm{Te},{ }^{122} \mathrm{Te}\right),\left({ }^{126} \mathrm{Te}\right.$, $\left.{ }^{126} \mathrm{Te}^{\prime}\right),\left({ }^{130} \mathrm{Te},{ }^{130} \mathrm{Te}\right), \mathrm{E}=3 \mathrm{MeV} /$ nucleon; measured $\mathrm{E} \gamma, \mathrm{I} \gamma(\theta, \phi)$, (particle) $\gamma$-coin following projectile Coulomb excitation; deduced parameters. ${ }^{132} \mathrm{Te}$ level deduced g factor. Clarion, Hyball arrays, recoil-in-vacuum technique. JOUR ZAANE 25 s01 205

2005ZA14 RADIOACTIVITY ${ }^{132} \mathrm{Sb}\left(\beta^{-}\right)$; measured $\mathrm{E} \gamma, \mathrm{I} \gamma, \gamma \gamma$-coin. ${ }^{132} \mathrm{Te}$ deduced levels, J, $\pi$. Comparisons with quasiparticle RPA calculations with density-dependent pairing. Clarion array. JOUR ZAANE 25 s01 389

${ }^{132} \mathrm{Ce} \quad$ 2005WI19 NUCLEAR REACTIONS ${ }^{68} \mathrm{Zn}\left({ }^{64} \mathrm{Ni}, \mathrm{X}\right), \mathrm{E}=300,400,500 \mathrm{MeV}$; measured $\mathrm{E} \gamma, \mathrm{I} \gamma$, (particle) $\gamma$-coin. ${ }^{132} \mathrm{Ce}$ deduced GDR parameters. JOUR JPGPE 31 S1973

\section{$\mathrm{A}=133$}

${ }^{133} \mathrm{Te} \quad 2005 \mathrm{HW} 06$

${ }^{133} \mathrm{Cs} \quad$ 2004GE20

${ }^{133} \mathrm{Ba} \quad 2004 \mathrm{GE} 20$

${ }^{133} \mathrm{Nd} \quad 2005 \mathrm{PE} 18$
RADIOACTIVITY ${ }^{252} \mathrm{Cf}(\mathrm{SF})$; measured $\mathrm{E} \gamma$, I $\gamma, \gamma \gamma$-coin. ${ }^{95,97} \mathrm{Sr},{ }^{99} \mathrm{Zr}$, ${ }^{108} \mathrm{Tc},{ }^{133,134} \mathrm{Te},{ }^{137} \mathrm{Xe}$ levels deduced $\mathrm{T}_{1 / 2}$. Gammasphere array, time-gated triple-coincidence method. JOUR ZAANE 25 s01 463 RADIOACTIVITY ${ }^{155} \mathrm{Sm}\left(\beta^{-}\right)$[from ${ }^{154} \mathrm{Sm}(\mathrm{n}, \gamma)$ ]; ${ }^{60} \mathrm{Co},{ }^{133} \mathrm{Ba},{ }^{152} \mathrm{Eu}$; measured $\gamma$-ray angular correlations. ${ }^{155} \mathrm{Eu},{ }^{60} \mathrm{Ni},{ }^{133} \mathrm{Cs},{ }^{152} \mathrm{Gd}$ transitions deduced $\delta$. Comparison with previous results. JOUR BJPHE 34722 NUCLEAR MOMENTS ${ }^{133} \mathrm{Cs}$; measured hfs; deduced constants. JOUR EULEE 72740 RADIOACTIVITY ${ }^{155} \mathrm{Sm}\left(\beta^{-}\right)$[from ${ }^{154} \mathrm{Sm}(\mathrm{n}, \gamma)$ ]; ${ }^{60} \mathrm{Co},{ }^{133} \mathrm{Ba},{ }^{152} \mathrm{Eu}$; measured $\gamma$-ray angular correlations. ${ }^{155} \mathrm{Eu},{ }^{60} \mathrm{Ni},{ }^{133} \mathrm{Cs},{ }^{152} \mathrm{Gd}$ transitions deduced $\delta$. Comparison with previous results. JOUR BJPHE 34722

NUCLEAR REACTIONS ${ }^{104} \mathrm{Pd}\left({ }^{32} \mathrm{~S}, \mathrm{n} 2 \mathrm{p}\right), \mathrm{E}=135 \mathrm{MeV}$; measured Doppler-shifted $\mathrm{E} \gamma, \mathrm{I} \gamma, \gamma \gamma$-coin. ${ }^{133} \mathrm{Nd}$ levels deduced $\mathrm{T}_{1 / 2}, \mathrm{~B}(\mathrm{E} 2)$, decay-out mechanism for highly deformed rotational band. GASP array, recoil-distance method. JOUR PRVCA 72031304 


\section{$\mathrm{A}=134$}

\begin{tabular}{|c|c|c|}
\hline${ }^{134} \mathrm{Sn}$ & 2005VA31 & $\begin{array}{l}\text { NUCLEAR REACTIONS }{ }^{48} \mathrm{Ti}\left({ }^{132} \mathrm{Sn},{ }^{132} \mathrm{Sn}\right), \mathrm{E}=470-495 \mathrm{MeV} \text {; } \\
{ }^{90} \mathrm{Zr}\left({ }^{134} \mathrm{Sn},{ }^{134} \mathrm{Sn}\right), \mathrm{E}=400 \mathrm{MeV} \text {; measured } \mathrm{E} \gamma, \mathrm{I} \gamma \text {, (particle) } \gamma \text {-coin } \\
\text { following projectile Coulomb excitation. }{ }^{132,134} \mathrm{Sn} \text { deduced transitions } \\
\mathrm{B}(\mathrm{E} 2) . \text { JOUR ZAANE } 25 \mathrm{~s} 01391\end{array}$ \\
\hline${ }^{134} \mathrm{Sb}$ & 2005SH53 & $\begin{array}{l}\text { RADIOACTIVITY }{ }^{111} \mathrm{Te}\left(\beta^{+}\right)\left[\text {from }{ }^{58} \mathrm{Ni}\left({ }^{56} \mathrm{Fe}, 2 \mathrm{pn}\right)\right] ;{ }^{135} \mathrm{Sn}\left(\beta^{-}\right) \text {, } \\
\left(\beta^{-} \mathrm{n}\right)[\text { from U }(\mathrm{p}, \mathrm{F}), \mathrm{E}=1.4 \mathrm{GeV}] \text {; measured } \mathrm{E} \gamma, \mathrm{I} \gamma, \gamma \gamma \text {-coin following } \\
\text { decay of mass-separated sources. } \\
\text { Comparison with model calculations. JOUR ZAANE } 25 \text { s01 } 121\end{array}$ \\
\hline${ }^{134} \mathrm{Te}$ & 2005HW06 & $\begin{array}{l}\text { RADIOACTIVITY }{ }^{252} \mathrm{Cf}(\mathrm{SF}) \text {; measured } \mathrm{E} \gamma, \mathrm{I} \gamma, \gamma \gamma \text {-coin. }{ }^{95,97} \mathrm{Sr},{ }^{99} \mathrm{Zr} \text {, } \\
{ }^{108} \mathrm{Tc},{ }^{133,134} \mathrm{Te},{ }^{137} \mathrm{Xe} \text { levels deduced } \mathrm{T}_{1 / 2} . \text { Gammasphere array, } \\
\text { time-gated triple-coincidence method. JOUR ZAANE } 25 \text { s01 } 463\end{array}$ \\
\hline & 2005RA32 & $\begin{array}{l}\text { NUCLEAR REACTIONS C }\left({ }^{126} \mathrm{Sn},{ }^{126} \mathrm{Sn}^{\prime}\right),\left({ }^{128} \mathrm{Sn},{ }^{128} \mathrm{Sn}{ }^{\prime}\right),\left({ }^{130} \mathrm{Sn},\right. \\
\left.{ }^{130} \mathrm{Sn}\right),\left({ }^{132} \mathrm{Te},{ }^{132} \mathrm{Te}\right),\left({ }^{134} \mathrm{Te},{ }^{134} \mathrm{Te}\right),\left({ }^{136} \mathrm{Te},{ }^{136} \mathrm{Te}\right), \mathrm{E} \text { not given; } \\
\text { measured } \mathrm{E} \gamma, \mathrm{I} \gamma, \gamma \gamma-,(\text { particle}) \gamma \text {-coin following projectile Coulomb } \\
\text { excitation. }{ }^{132,134,136} \mathrm{Te},{ }^{126,128,130} \mathrm{Sn} \text { deduced excitation } \mathrm{B}(\mathrm{E} 2) \text {. } \\
{ }^{9} \mathrm{Be}\left({ }^{134} \mathrm{Te},{ }^{8} \mathrm{Be}\right),{ }^{13} \mathrm{C}\left({ }^{134} \mathrm{Te},{ }^{12} \mathrm{C}\right), \mathrm{E}=4.3 \mathrm{MeV} / \text { nucleon; measured E} \gamma, \\
\mathrm{I} \gamma, \gamma \gamma \text {-coin, (particle) } \gamma \text {-coin; deduced single-neutron transfer } \sigma(\mathrm{E}) \text {. } \\
{ }^{135} \mathrm{Te} \text { deduced levels J, } \pi \text {. JOUR ZAANE } 25 \text { s01 } 383\end{array}$ \\
\hline${ }^{134} \mathrm{Pr}$ & 2005 T022 & $\begin{array}{l}\text { NUCLEAR REACTIONS }{ }^{119} \mathrm{Sn}\left({ }^{19} \mathrm{~F}, 4 \mathrm{n}\right), \mathrm{E}=83,87 \mathrm{MeV} \text {; measured } \\
\mathrm{E} \gamma, \mathrm{I} \gamma, \gamma \gamma \text {-coin, DSA. }{ }^{134} \mathrm{Pr} \text { levels deduced } \mathrm{T}_{1 / 2}, \mathrm{~B}(\mathrm{M} 1), \mathrm{B}(\mathrm{E} 2) \text {, } \\
\text { mixing ratios. Euroball IV array, recoil-distance and Doppler-shift } \\
\text { attenuation techniques. JOUR ZAANE } 25 \mathrm{~s} 01447\end{array}$ \\
\hline
\end{tabular}

\section{$\mathrm{A}=135$}

${ }^{135} \mathrm{Sn} \quad 2005 K 040$

2005SH53

${ }^{135} \mathrm{Sb} \quad 2005 \mathrm{~K} 040$

2005SH53

${ }^{135} \mathrm{Te} \quad 2005 \mathrm{GR} 25$
RADIOACTIVITY ${ }^{135} \mathrm{Sn}\left(\beta^{-}\right)$[from ${ }^{235} \mathrm{U}(\mathrm{n}, \mathrm{F}), \mathrm{E}=$ thermal]; measured $\mathrm{E} \gamma, \mathrm{I} \gamma, \beta \gamma$-coin following decay of mass-separated sources. ${ }^{135} \mathrm{Sb}$ deduced levels, J, $\pi, \mathrm{T}_{1 / 2}, \mathrm{~B}(\mathrm{M} 1)$, configurations. Comparison with shell model calculations. JOUR ZAANE 25 s01 123 RADIOACTIVITY ${ }^{111} \mathrm{Te}\left(\beta^{+}\right)$[from $\left.{ }^{58} \mathrm{Ni}\left({ }^{56} \mathrm{Fe}, 2 \mathrm{pn}\right)\right] ;{ }^{135} \mathrm{Sn}\left(\beta^{-}\right)$, $\left(\beta^{-} \mathrm{n}\right)$ [from $\left.\mathrm{U}(\mathrm{p}, \mathrm{F}), \mathrm{E}=1.4 \mathrm{GeV}\right]$; measured $\mathrm{E} \gamma, \mathrm{I} \gamma, \gamma \gamma$-coin following decay of mass-separated sources. ${ }^{111,134,135} \mathrm{Sb}$ deduced levels, J, $\pi$. Comparison with model calculations. JOUR ZAANE 25 s01 121 RADIOACTIVITY ${ }^{135} \mathrm{Sn}\left(\beta^{-}\right)$[from ${ }^{235} \mathrm{U}(\mathrm{n}, \mathrm{F}), \mathrm{E}=$ thermal]; measured $\mathrm{E} \gamma, \mathrm{I} \gamma, \beta \gamma$-coin following decay of mass-separated sources. ${ }^{135} \mathrm{Sb}$ deduced levels, $\mathrm{J}, \pi, \mathrm{T}_{1 / 2}, \mathrm{~B}(\mathrm{M} 1)$, configurations. Comparison with shell model calculations. JOUR ZAANE 25 s01 123 RADIOACTIVITY ${ }^{111} \mathrm{Te}\left(\beta^{+}\right)$[from $\left.{ }^{58} \mathrm{Ni}\left({ }^{56} \mathrm{Fe}, 2 \mathrm{pn}\right)\right] ;{ }^{135} \mathrm{Sn}\left(\beta^{-}\right)$, $\left(\beta^{-} \mathrm{n}\right)$ from $\mathrm{U}(\mathrm{p}, \mathrm{F}), \mathrm{E}=1.4 \mathrm{GeV}$ ]; measured $\mathrm{E} \gamma, \mathrm{I} \gamma, \gamma \gamma$-coin following decay of mass-separated sources. ${ }^{111,134,135} \mathrm{Sb}$ deduced levels, J, $\pi$. Comparison with model calculations. JOUR ZAANE 25 s01 121 NUCLEAR REACTIONS ${ }^{64} \mathrm{Ni}\left({ }^{132} \mathrm{Sn}, \mathrm{X}\right),\left({ }^{134} \mathrm{Sn}, \mathrm{X}\right), \mathrm{E}=450-620 \mathrm{MeV}$; measured fusion $\sigma . \mathrm{C}\left({ }^{130} \mathrm{Te},{ }^{130} \mathrm{Te}\right),\left({ }^{132} \mathrm{Te},{ }^{132} \mathrm{Te}\right), \mathrm{E}=3 \mathrm{MeV} /$ nucleon; measured $\mathrm{E} \gamma, \mathrm{I} \gamma$, (particle) $\gamma$-coin following projectile Coulomb excitation. ${ }^{132} \mathrm{Te}$ level deduced g factor. ${ }^{13} \mathrm{C}\left({ }^{134} \mathrm{Te},{ }^{135} \mathrm{Te}\right), \mathrm{E}=550$ $\mathrm{MeV}$; measured $\mathrm{E} \gamma, \mathrm{I} \gamma$. ${ }^{135}$ Te level deduced J, $\pi$. JOUR JPGPE 31 S1639 


\section{$\mathrm{A}=135$ (continued)}

2005RA32 NUCLEAR REACTIONS C $\left({ }^{126} \mathrm{Sn},{ }^{126} \mathrm{Sn}^{\prime}\right),\left({ }^{128} \mathrm{Sn},{ }^{128} \mathrm{Sn}^{\prime}\right),\left({ }^{130} \mathrm{Sn}\right.$, ${ }^{130} \mathrm{Sn}$ '), $\left({ }^{132} \mathrm{Te},{ }^{132} \mathrm{Te}\right.$ '), $\left({ }^{134} \mathrm{Te},{ }^{134} \mathrm{Te}\right.$ '), $\left({ }^{136} \mathrm{Te},{ }^{136} \mathrm{Te}\right.$ '), E not given; measured $\mathrm{E} \gamma, \mathrm{I} \gamma, \gamma \gamma$-, (particle) $\gamma$-coin following projectile Coulomb excitation. ${ }^{132,134,136} \mathrm{Te},{ }^{126,128,130} \mathrm{Sn}$ deduced excitation $\mathrm{B}(\mathrm{E} 2)$. ${ }^{9} \mathrm{Be}\left({ }^{134} \mathrm{Te},{ }^{8} \mathrm{Be}\right),{ }^{13} \mathrm{C}\left({ }^{134} \mathrm{Te},{ }^{12} \mathrm{C}\right), \mathrm{E}=4.3 \mathrm{MeV} /$ nucleon; measured $\mathrm{E} \gamma$, $\mathrm{I} \gamma, \gamma \gamma$-coin, (particle) $\gamma$-coin; deduced single-neutron transfer $\sigma(\mathrm{E})$.

${ }^{135}$ Te deduced levels J, $\pi$. JOUR ZAANE 25 s01 383

${ }^{135} \mathrm{Xe} \quad 2004 \mathrm{GA} 60$ NUCLEAR REACTIONS ${ }^{237} \mathrm{~Np}(\gamma, \mathrm{F}){ }^{135} \mathrm{Xe} /{ }^{137} \mathrm{Xe} /{ }^{138} \mathrm{Xe} /{ }^{139} \mathrm{Xe} /$ ${ }^{140} \mathrm{Xe} /{ }^{141} \mathrm{Xe} /{ }^{142} \mathrm{Xe} /{ }^{89} \mathrm{Kr} /{ }^{91} \mathrm{Kr} /{ }^{92} \mathrm{Kr} /{ }^{93} \mathrm{Kr}, \mathrm{E}=25 \mathrm{MeV}$ bremsstrahlung; measured fission yields, isotopic distribution parameters. Comparison with results from other targets. JOUR BRSPE 681298 2005GA50 NUCLEAR REACTIONS ${ }^{237} \mathrm{~Np},{ }^{243} \mathrm{Am}(\gamma, \mathrm{F}){ }^{135} \mathrm{Xe} /{ }^{137} \mathrm{Xe} /{ }^{138} \mathrm{Xe} /$ ${ }^{139} \mathrm{Xe} /{ }^{140} \mathrm{Xe} /{ }^{141} \mathrm{Xe} /{ }^{142} \mathrm{Xe} /{ }^{89} \mathrm{Kr} /{ }^{91} \mathrm{Kr} /{ }^{92} \mathrm{Kr} /{ }^{93} \mathrm{Kr}, \mathrm{E}=25 \mathrm{MeV}$ bremsstrahlung; measured fission yields, isotopic distribution parameters. JOUR YAFIA 681475

\section{$\mathrm{A}=\mathbf{1 3 6}$}

${ }^{136} \mathrm{Te} \quad$ 2005RA32

NUCLEAR REACTIONS C( $\left.{ }^{126} \mathrm{Sn},{ }^{126} \mathrm{Sn}\right),\left({ }^{128} \mathrm{Sn},{ }^{128} \mathrm{Sn}\right),\left({ }^{130} \mathrm{Sn}\right.$, ${ }^{130} \mathrm{Sn}$ '), $\left({ }^{132} \mathrm{Te},{ }^{132} \mathrm{Te}\right),\left({ }^{134} \mathrm{Te},{ }^{134} \mathrm{Te}\right),\left({ }^{136} \mathrm{Te},{ }^{136} \mathrm{Te}\right)$, E not given; measured $\mathrm{E} \gamma, \mathrm{I} \gamma, \gamma \gamma-$, (particle) $\gamma$-coin following projectile Coulomb excitation. ${ }^{132,134,136} \mathrm{Te},{ }^{126,128,130} \mathrm{Sn}$ deduced excitation $\mathrm{B}(\mathrm{E} 2)$. ${ }^{9} \mathrm{Be}\left({ }^{134} \mathrm{Te},{ }^{8} \mathrm{Be}\right),{ }^{13} \mathrm{C}\left({ }^{134} \mathrm{Te},{ }^{12} \mathrm{C}\right), \mathrm{E}=4.3 \mathrm{MeV} /$ nucleon; measured $\mathrm{E} \gamma$, $\mathrm{I} \gamma, \gamma \gamma$-coin, (particle) $\gamma$-coin; deduced single-neutron transfer $\sigma(\mathrm{E})$.

${ }^{135}$ Te deduced levels J, $\pi$. JOUR ZAANE 25 s01 383

${ }^{136} \mathrm{Xe} \quad$ 2005GAZU RADIOACTIVITY ${ }^{136} \mathrm{Xe}\left(2 \beta^{-}\right)$; measured $0 \nu \beta \beta$-decay and $2 \nu \beta \beta$-decay $\mathrm{T}_{1 / 2}$ lower limits. PREPRINT nucl-ex/0510071,10/26/2005

${ }^{136} \mathrm{Ba} \quad$ 2005GAZU

RADIOACTIVITY ${ }^{136} \mathrm{Xe}\left(2 \beta^{-}\right)$; measured $0 \nu \beta \beta$-decay and $2 \nu \beta \beta$-decay $\mathrm{T}_{1 / 2}$ lower limits. PREPRINT nucl-ex/0510071,10/26/2005

${ }^{136} \mathrm{Ce} \quad$ 2005LA29 NUCLEAR REACTIONS ${ }^{124} \mathrm{Sn}\left({ }^{16} \mathrm{O}, 4 \mathrm{n}\right), \mathrm{E}=80 \mathrm{MeV}$; measured $\mathrm{E} \gamma$, $\mathrm{I} \gamma, \gamma \gamma$-coin, $\gamma$-ray polarization, DSA. ${ }^{136} \mathrm{Ce}$ deduced high-spin levels, I, $\pi, \mathrm{T}_{1 / 2}, \mathrm{~B}(\mathrm{M} 1), \mathrm{B}(\mathrm{E} 2)$, transition quadrupole moments. ${ }^{124} \mathrm{Sn}\left({ }^{16} \mathrm{O}\right.$, $4 \mathrm{n}), \mathrm{E}=65-98 \mathrm{MeV}$; measured $\mathrm{E} \gamma$, excitation functions. Comparisons with cranking model predictions. JOUR NUPAB 7611

\section{$\mathrm{A}=137$}

${ }^{137} \mathrm{Xe} \quad 2004 \mathrm{GA} 60$

NUCLEAR REACTIONS ${ }^{237} \mathrm{~Np}(\gamma, \mathrm{F}){ }^{135} \mathrm{Xe} /{ }^{137} \mathrm{Xe} /{ }^{138} \mathrm{Xe} /{ }^{139} \mathrm{Xe} /$ ${ }^{140} \mathrm{Xe} /{ }^{141} \mathrm{Xe} /{ }^{142} \mathrm{Xe} /{ }^{89} \mathrm{Kr} /{ }^{91} \mathrm{Kr} /{ }^{92} \mathrm{Kr} /{ }^{93} \mathrm{Kr}, \mathrm{E}=25 \mathrm{MeV}$ bremsstrahlung; measured fission yields, isotopic distribution parameters. Comparison with results from other targets. JOUR BRSPE 681298

2005F017 RADIOACTIVITY ${ }^{252} \mathrm{Cf}(\mathrm{SF})$; measured E $\gamma, \mathrm{I} \gamma, \gamma \gamma$-coin. ${ }^{98} \mathrm{Sr}$, ${ }^{102,104} \mathrm{Zr},{ }^{137} \mathrm{Xe},{ }^{143} \mathrm{Ba},{ }^{152} \mathrm{Ce}$ levels deduced $\mathrm{T}_{1 / 2}$. Gammasphere array, time-gated triple-coincidence method. JOUR ZAANE 25 s01 465 


\section{$\mathrm{A}=137$ (continued)}

2005GA50 NUCLEAR REACTIONS ${ }^{237} \mathrm{~Np},{ }^{243} \mathrm{Am}(\gamma, \mathrm{F}){ }^{135} \mathrm{Xe} /{ }^{137} \mathrm{Xe} /{ }^{138} \mathrm{Xe} /$ ${ }^{139} \mathrm{Xe} /{ }^{140} \mathrm{Xe} /{ }^{141} \mathrm{Xe} /{ }^{142} \mathrm{Xe} /{ }^{89} \mathrm{Kr} /{ }^{91} \mathrm{Kr} /{ }^{92} \mathrm{Kr} /{ }^{93} \mathrm{Kr}, \mathrm{E}=25 \mathrm{MeV}$ bremsstrahlung; measured fission yields, isotopic distribution parameters. JOUR YAFIA 681475

2005HW06 RADIOACTIVITY ${ }^{252} \mathrm{Cf}(\mathrm{SF})$; measured $\mathrm{E} \gamma, \mathrm{I} \gamma, \gamma \gamma$-coin. ${ }^{95,97} \mathrm{Sr},{ }^{99} \mathrm{Zr}$, ${ }^{108} \mathrm{Tc},{ }^{133,134} \mathrm{Te},{ }^{137} \mathrm{Xe}$ levels deduced $\mathrm{T}_{1 / 2}$. Gammasphere array, time-gated triple-coincidence method. JOUR ZAANE 25 s01 463

$$
\mathrm{A}=138
$$

${ }^{138} \mathrm{Xe} \quad 2004 \mathrm{GA} 60$

NUCLEAR REACTIONS ${ }^{237} \mathrm{~Np}(\gamma, \mathrm{F}){ }^{135} \mathrm{Xe} /{ }^{137} \mathrm{Xe} /{ }^{138} \mathrm{Xe} /{ }^{139} \mathrm{Xe} /$ ${ }^{140} \mathrm{Xe} /{ }^{141} \mathrm{Xe} /{ }^{142} \mathrm{Xe} /{ }^{89} \mathrm{Kr} /{ }^{91} \mathrm{Kr} /{ }^{92} \mathrm{Kr} /{ }^{93} \mathrm{Kr}, \mathrm{E}=25 \mathrm{MeV}$ bremsstrahlung; measured fission yields, isotopic distribution parameters. Comparison with results from other targets. JOUR BRSPE 681298

2005GA50 NUCLEAR REACTIONS ${ }^{237} \mathrm{~Np},{ }^{243} \mathrm{Am}(\gamma, \mathrm{F}){ }^{135} \mathrm{Xe} /{ }^{137} \mathrm{Xe} /{ }^{138} \mathrm{Xe} /$ ${ }^{139} \mathrm{Xe} /{ }^{140} \mathrm{Xe} /{ }^{141} \mathrm{Xe} /{ }^{142} \mathrm{Xe} /{ }^{89} \mathrm{Kr} /{ }^{91} \mathrm{Kr} /{ }^{92} \mathrm{Kr} /{ }^{93} \mathrm{Kr}, \mathrm{E}=25 \mathrm{MeV}$ bremsstrahlung; measured fission yields, isotopic distribution parameters. JOUR YAFIA 681475

$$
\mathrm{A}=\mathbf{1 3 9}
$$

${ }^{139} \mathrm{Xe} \quad 2004 \mathrm{GA} 60$

NUCLEAR REACTIONS ${ }^{237} \mathrm{~Np}(\gamma, \mathrm{F}){ }^{135} \mathrm{Xe} /{ }^{137} \mathrm{Xe} /{ }^{138} \mathrm{Xe} /{ }^{139} \mathrm{Xe} /$ ${ }^{140} \mathrm{Xe} /{ }^{141} \mathrm{Xe} /{ }^{142} \mathrm{Xe} /{ }^{89} \mathrm{Kr} /{ }^{91} \mathrm{Kr} /{ }^{92} \mathrm{Kr} /{ }^{93} \mathrm{Kr}, \mathrm{E}=25 \mathrm{MeV}$ bremsstrahlung; measured fission yields, isotopic distribution parameters. Comparison with results from other targets. JOUR BRSPE 681298

2005GA50 NUCLEAR REACTIONS ${ }^{237} \mathrm{~Np},{ }^{243} \mathrm{Am}(\gamma, \mathrm{F}){ }^{135} \mathrm{Xe} /{ }^{137} \mathrm{Xe} /{ }^{138} \mathrm{Xe} /$ ${ }^{139} \mathrm{Xe} /{ }^{140} \mathrm{Xe} /{ }^{141} \mathrm{Xe} /{ }^{142} \mathrm{Xe} /{ }^{89} \mathrm{Kr} /{ }^{91} \mathrm{Kr} /{ }^{92} \mathrm{Kr} /{ }^{93} \mathrm{Kr}, \mathrm{E}=25 \mathrm{MeV}$ bremsstrahlung; measured fission yields, isotopic distribution parameters. JOUR YAFIA 681475

$$
\mathrm{A}=\mathbf{1 4 0}
$$

${ }^{140} \mathrm{Xe} \quad 2004 \mathrm{GA} 60$

NUCLEAR REACTIONS ${ }^{237} \mathrm{~Np}(\gamma, \mathrm{F}){ }^{135} \mathrm{Xe} /{ }^{137} \mathrm{Xe} /{ }^{138} \mathrm{Xe} /{ }^{139} \mathrm{Xe} /$ ${ }^{140} \mathrm{Xe} /{ }^{141} \mathrm{Xe} /{ }^{142} \mathrm{Xe} /{ }^{89} \mathrm{Kr} /{ }^{91} \mathrm{Kr} /{ }^{92} \mathrm{Kr} /{ }^{93} \mathrm{Kr}, \mathrm{E}=25 \mathrm{MeV}$ bremsstrahlung; measured fission yields, isotopic distribution parameters. Comparison with results from other targets. JOUR BRSPE 681298

2005GA50 NUCLEAR REACTIONS ${ }^{237} \mathrm{~Np},{ }^{243} \mathrm{Am}(\gamma, \mathrm{F}){ }^{135} \mathrm{Xe} /{ }^{137} \mathrm{Xe} /{ }^{138} \mathrm{Xe} /$ ${ }^{139} \mathrm{Xe} /{ }^{140} \mathrm{Xe} /{ }^{141} \mathrm{Xe} /{ }^{142} \mathrm{Xe} /{ }^{89} \mathrm{Kr} /{ }^{91} \mathrm{Kr} /{ }^{92} \mathrm{Kr} /{ }^{93} \mathrm{Kr}, \mathrm{E}=25 \mathrm{MeV}$ bremsstrahlung; measured fission yields, isotopic distribution parameters. JOUR YAFIA 681475 


\section{$\mathrm{A}=140$ (continued)}

\begin{tabular}{|c|c|c|}
\hline${ }^{140} \mathrm{Eu}$ & $2005 \mathrm{TA} 31$ & $\begin{array}{l}\text { RADIOACTIVITY }{ }^{140 m} \mathrm{Eu},{ }^{142 m} \mathrm{~Tb},{ }^{144 m} \mathrm{Ho}(\mathrm{IT})\left[\text { from }{ }^{54} \mathrm{Fe}\left({ }^{92} \mathrm{Mo}, \mathrm{X}\right)\right] \text {; } \\
\text { measured X-ray spectra, E } \gamma, \gamma \gamma \text {-coin, } \mathrm{E}(\mathrm{ce}), \mathrm{T}_{1 / 2} \cdot{ }^{140} \mathrm{Eu},{ }^{142} \mathrm{~Tb},{ }^{144} \mathrm{Ho} \\
\text { dlevels, J, } \pi \text {, configurations. Mass-separated sources. JOUR ZAANE } \\
25 \mathrm{~s} 01151\end{array}$ \\
\hline${ }^{140}$ Dy & 2005BI24 & $\begin{array}{l}\text { RADIOACTIVITY }{ }^{141} \mathrm{Ho},{ }^{144,145,146} \mathrm{Tm}(\mathrm{p})\left[\text { from }{ }^{92} \mathrm{Mo}\left({ }^{54} \mathrm{Fe} \text {, xnyp }\right)\right. \\
\left.\text { and }{ }^{92} \mathrm{Mo}\left({ }^{58} \mathrm{Ni} \text {, xnyp }\right)\right] ; \text { measured Ep, } \mathrm{T}_{1 / 2} ; \text { deduced branching ratios. } \\
{ }^{141} \mathrm{Ho},{ }^{140} \mathrm{Dy},{ }^{145,146} \mathrm{Tm},{ }^{144,145} \mathrm{Er} \text { deduced levels, configurations. } \\
\text { JOUR NIMBE } 241185\end{array}$ \\
\hline
\end{tabular}

$$
\mathrm{A}=141
$$

${ }^{141} \mathrm{Xe} \quad$ 2004Ga60 NUCLEAR REACTIONS ${ }^{237} \mathrm{~Np}(\gamma, \mathrm{F}){ }^{135} \mathrm{Xe} /{ }^{137} \mathrm{Xe} /{ }^{138} \mathrm{Xe} /{ }^{139} \mathrm{Xe} /$ ${ }^{140} \mathrm{Xe} /{ }^{141} \mathrm{Xe} /{ }^{142} \mathrm{Xe} /{ }^{89} \mathrm{Kr} /{ }^{91} \mathrm{Kr} /{ }^{92} \mathrm{Kr} /{ }^{93} \mathrm{Kr}, \mathrm{E}=25 \mathrm{MeV}$ bremsstrahlung; measured fission yields, isotopic distribution parameters. Comparison with results from other targets. JOUR BRSPE 681298

2005GA50 NUCLEAR REACTIONS ${ }^{237} \mathrm{~Np},{ }^{243} \mathrm{Am}(\gamma, \mathrm{F}){ }^{135} \mathrm{Xe} /{ }^{137} \mathrm{Xe} /{ }^{138} \mathrm{Xe} /$ ${ }^{139} \mathrm{Xe} /{ }^{140} \mathrm{Xe} /{ }^{141} \mathrm{Xe} /{ }^{142} \mathrm{Xe} /{ }^{89} \mathrm{Kr} /{ }^{91} \mathrm{Kr} /{ }^{92} \mathrm{Kr} /{ }^{93} \mathrm{Kr}, \mathrm{E}=25 \mathrm{MeV}$ bremsstrahlung; measured fission yields, isotopic distribution parameters. JOUR YAFIA 681475

${ }^{141} \mathrm{~Tb}$ 2004ME25 NUCLEAR REACTIONS ${ }^{92} \mathrm{Mo}\left({ }^{54} \mathrm{Fe}, \mathrm{p} \alpha\right), \mathrm{E}=240 \mathrm{MeV}$; measured $\mathrm{E} \gamma$, $\mathrm{I} \gamma, \gamma \gamma$-, (charged particle) $\gamma$-coin. ${ }^{141} \mathrm{~Tb}$ deduced high-spin levels, $\mathrm{J}, \pi$, configurations. GASP, ISIS arrays, cranking model analysis. JOUR BJPHE 341002

${ }^{141} \mathrm{Ho} \quad$ 2005BI24 RADIOACTIVITY ${ }^{141} \mathrm{Ho},{ }^{144,145,146} \mathrm{Tm}(\mathrm{p})\left[\right.$ from ${ }^{92} \mathrm{Mo}\left({ }^{54} \mathrm{Fe}\right.$, xnyp $)$ and ${ }^{92} \mathrm{Mo}\left({ }^{58} \mathrm{Ni}\right.$, xnyp)]; measured $\mathrm{Ep}, \mathrm{T}_{1 / 2}$; deduced branching ratios. ${ }^{141} \mathrm{Ho},{ }^{140} \mathrm{Dy},{ }^{145,146} \mathrm{Tm},{ }^{144,145} \mathrm{Er}$ deduced levels, configurations. JOUR NIMBE 241185

\section{$\mathrm{A}=142$}

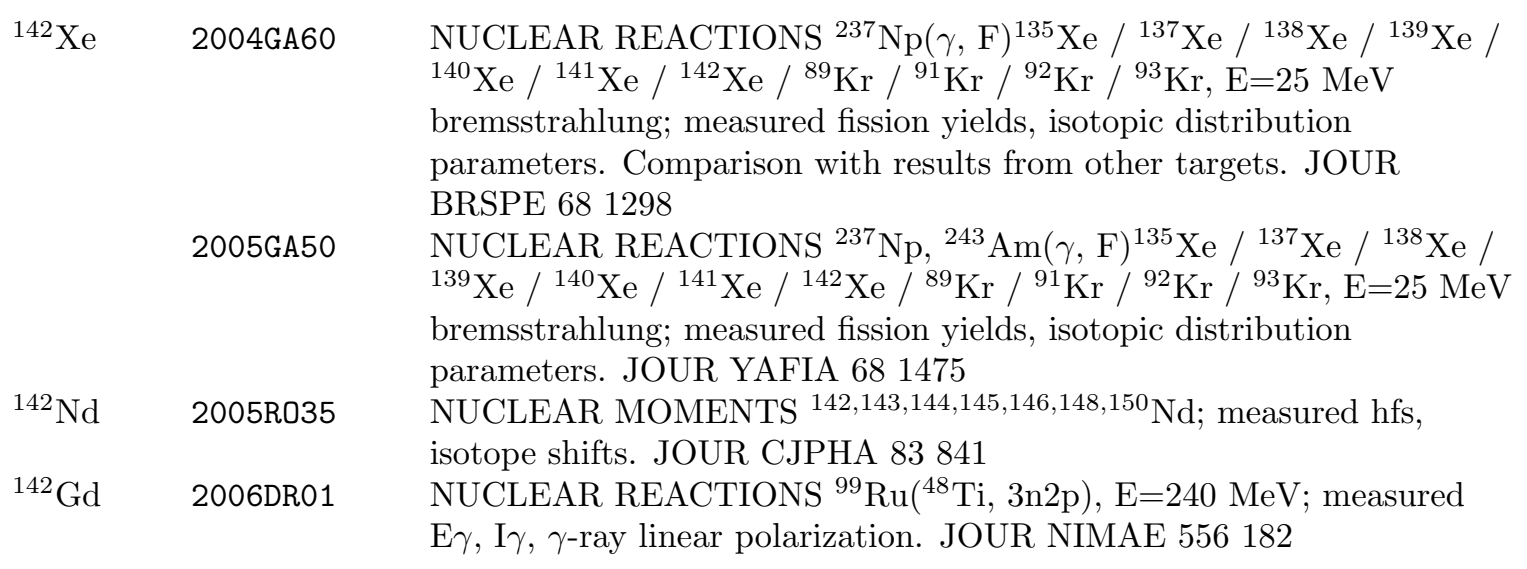




\section{$\mathrm{A}=142$ (continued)}

${ }^{142} \mathrm{~Tb} \quad 2005 \mathrm{RI} 17$

2005TA31

${ }^{143} \mathrm{Ba}$

${ }^{143} \mathrm{Nd} \quad$ 2005R035

${ }^{143}$ Dy $\quad 2005$ RI17

${ }^{143} \mathrm{Er}$

2005BI24

2005GR32

${ }^{144} \mathrm{Ba}$

2005SH49

${ }^{144} \mathrm{Nd} \quad 2005 \mathrm{R} 035$

${ }^{144} \mathrm{Ho} \quad$ 2005TA31

${ }^{144} \mathrm{Er}$

2005BI24

2005SE26
NUCLEAR REACTIONS ${ }^{92} \mathrm{Mo}\left({ }^{54} \mathrm{Fe}\right.$, xnypz $\left.\alpha\right), \mathrm{E}=245 \mathrm{MeV}$; measured prompt and delayed $\mathrm{E} \gamma, \mathrm{I} \gamma, \gamma \gamma$-, (recoil) $\gamma$-coin. ${ }^{142} \mathrm{~Tb},{ }^{163} \mathrm{Dy}$ deduced transitions. ${ }^{143}$ Dy deduced isomeric state $\mathrm{T}_{1 / 2}$. Jurogam array. JOUR JPGPE 31 S1949 RADIOACTIVITY ${ }^{140 m} \mathrm{Eu},{ }^{142 m} \mathrm{~Tb},{ }^{144 m} \mathrm{Ho}$ (IT) [from ${ }^{54} \mathrm{Fe}\left({ }^{92} \mathrm{Mo}, \mathrm{X}\right)$ ]; measured X-ray spectra, E $\gamma, \gamma \gamma$-coin, $\mathrm{E}(\mathrm{ce}), \mathrm{T}_{1 / 2} \cdot{ }^{140} \mathrm{Eu},{ }^{142} \mathrm{~Tb},{ }^{144} \mathrm{Ho}$ dlevels, J, $\pi$, configurations. Mass-separated sources. JOUR ZAANE $25 \mathrm{~s} 01151$

\section{$\mathrm{A}=143$}

RADIOACTIVITY ${ }^{252} \mathrm{Cf}(\mathrm{SF})$; measured E $\gamma, \mathrm{I} \gamma, \gamma \gamma$-coin. ${ }^{98} \mathrm{Sr}$, ${ }^{102,104} \mathrm{Zr},{ }^{137} \mathrm{Xe},{ }^{143} \mathrm{Ba},{ }^{152} \mathrm{Ce}$ levels deduced $\mathrm{T}_{1 / 2}$. Gammasphere array, time-gated triple-coincidence method. JOUR ZAANE 25 s01 465 NUCLEAR MOMENTS ${ }^{142,143,144,145,146,148,150} \mathrm{Nd}$; measured hfs, isotope shifts. JOUR CJPHA 83841

NUCLEAR REACTIONS ${ }^{92} \mathrm{Mo}\left({ }^{54} \mathrm{Fe}\right.$, xnypz $\left.\alpha\right), \mathrm{E}=245 \mathrm{MeV}$; measured prompt and delayed $\mathrm{E} \gamma, \mathrm{I} \gamma, \gamma \gamma-$, (recoil) $\gamma$-coin. ${ }^{142} \mathrm{~Tb},{ }^{163} \mathrm{Dy}$ deduced transitions. ${ }^{143}$ Dy deduced isomeric state $\mathrm{T}_{1 / 2}$. Jurogam array. JOUR JPGPE 31 S1949

RADIOACTIVITY ${ }^{141} \mathrm{Ho},{ }^{144,145,146} \mathrm{Tm}$ (p) [from ${ }^{92} \mathrm{Mo}\left({ }^{54} \mathrm{Fe}\right.$, xnyp) and ${ }^{92} \mathrm{Mo}\left({ }^{58} \mathrm{Ni}\right.$, xnyp)]; measured $\mathrm{Ep}, \mathrm{T}_{1 / 2}$; deduced branching ratios. ${ }^{141} \mathrm{Ho},{ }^{140} \mathrm{Dy},{ }^{145,146} \mathrm{Tm},{ }^{144,145} \mathrm{Er}$ deduced levels, configurations. JOUR NIMBE 241185 RADIOACTIVITY ${ }^{144} \mathrm{Tm}(\mathrm{p})$ [from ${ }^{58} \mathrm{Ni}\left({ }^{92} \mathrm{Mo}, \mathrm{p} 5 \mathrm{n}\right), \mathrm{E}=340 \mathrm{MeV}$; measured proton spectra, $\mathrm{T}_{1 / 2}$; deduced fine structure. JOUR ZAANE $25 \mathrm{~s} 01145$

\section{$\mathrm{A}=144$}

RADIOACTIVITY ${ }^{252} \mathrm{Cf}(\mathrm{SF})$; measured Doppler-shifted $\mathrm{E} \gamma, \mathrm{I} \gamma, \gamma \gamma$-, (fragment) $\gamma$-coin. ${ }^{144} \mathrm{Ba}$ deduced transitions $\mathrm{T}_{1 / 2}, \mathrm{~B}(\mathrm{E} 2)$, transition dipole, quadrupole, and octupole moments for alternating-parity band. Gammasphere array, cluster-model analysis. JOUR ZAANE 25387 NUCLEAR MOMENTS $142,143,144,145,146,148,150 \mathrm{Nd}$; measured hfs, isotope shifts. JOUR CJPHA 83841 RADIOACTIVITY ${ }^{140 m} \mathrm{Eu},{ }^{142 m} \mathrm{~Tb},{ }^{144 m} \mathrm{Ho}(\mathrm{IT})$ [from ${ }^{54} \mathrm{Fe}\left({ }^{92} \mathrm{Mo}, \mathrm{X}\right)$ ]; measured X-ray spectra, E $\gamma, \gamma \gamma$-coin, $\mathrm{E}(\mathrm{ce}), \mathrm{T}_{1 / 2} \cdot{ }^{140} \mathrm{Eu},{ }^{142} \mathrm{~Tb},{ }^{144} \mathrm{Ho}$ dlevels, $\mathrm{J}, \pi$, configurations. Mass-separated sources. JOUR ZAANE $25 \mathrm{~s} 01151$

RADIOACTIVITY ${ }^{141} \mathrm{Ho},{ }^{144,145,146} \mathrm{Tm}$ (p) [from ${ }^{92} \mathrm{Mo}\left({ }^{54} \mathrm{Fe}\right.$, xnyp) and ${ }^{92} \mathrm{Mo}\left({ }^{58} \mathrm{Ni}\right.$, xnyp)]; measured $\mathrm{Ep}, \mathrm{T}_{1 / 2}$; deduced branching ratios. ${ }^{141} \mathrm{Ho},{ }^{140} \mathrm{Dy},{ }^{145,146} \mathrm{Tm},{ }^{144,145} \mathrm{Er}$ deduced levels, configurations. JOUR NIMBE 241185 RADIOACTIVITY ${ }^{145} \mathrm{Tm}(\mathrm{p})$ [from ${ }^{58} \mathrm{Ni}\left({ }^{92} \mathrm{Mo}, 4 \mathrm{np}\right)$ ]; measured Ep, E $\gamma$, p $\gamma$-coin. JOUR ZAANE 25 s01 159 


\section{$\mathrm{A}=144$ (continued)}

\begin{tabular}{|c|c|c|}
\hline${ }^{144} \mathrm{Tm}$ & 2005BI24 & $\begin{array}{l}\text { RADIOACTIVITY }{ }^{141} \mathrm{Ho},{ }^{144,145,146} \mathrm{Tm}(\mathrm{p})\left[\text { from }{ }^{92} \mathrm{Mo}\left({ }^{54} \mathrm{Fe} \text {, xnyp }\right)\right. \\
\left.\text { and }{ }^{92} \mathrm{Mo}\left({ }^{58} \mathrm{Ni} \text {, xnyp }\right)\right] ; \text { measured Ep, } \mathrm{T}_{1 / 2} \text {; deduced branching ratios. } \\
{ }^{141} \mathrm{Ho},{ }^{140} \mathrm{Dy},{ }^{145,146} \mathrm{Tm},{ }^{144,145} \mathrm{Er} \text { deduced levels, configurations. } \\
\text { JOUR NIMBE } 241185\end{array}$ \\
\hline & 2005GR32 & $\begin{array}{l}\text { RADIOACTIVITY }{ }^{144} \mathrm{Tm}(\mathrm{p})\left[\text { from }{ }^{58} \mathrm{Ni}\left({ }^{92} \mathrm{Mo}, \mathrm{p} 5 \mathrm{n}\right), \mathrm{E}=340 \mathrm{MeV}\right] \\
\text { measured proton spectra, } \mathrm{T}_{1 / 2} \text {; deduced fine structure. JOUR ZAANE } \\
25 \mathrm{~s} 01145\end{array}$ \\
\hline
\end{tabular}

\section{$\mathrm{A}=145$}

${ }^{145} \mathrm{Nd} \quad 2005 \mathrm{R} 035$

${ }^{145} \mathrm{Er} \quad 2005 \mathrm{BB} 02$

2005BI24

2005R040

${ }^{145} \mathrm{Tm}$

2005BI24

2005SE26

2005SE26

${ }^{146} \mathrm{Nd} \quad 2005 \mathrm{R} 035$

${ }^{146} \mathrm{Tm} \quad 2005 \mathrm{BB} 02$

2005BI24
NUCLEAR MOMENTS ${ }^{142,143,144,145,146,148,150} \mathrm{Nd}$; measured hfs, isotope shifts. JOUR CJPHA 83841

RADIOACTIVITY ${ }^{146} \mathrm{Tm}$ (p) [from ${ }^{58} \mathrm{Ni}\left({ }^{92} \mathrm{Mo}, \mathrm{p} 3 \mathrm{n}\right), \mathrm{E}=297 \mathrm{MeV}$; measured proton spectra, $\mathrm{T}_{1 / 2}$; deduced fine structure, decay branching ratios. ${ }^{145}$ Er deduced levels, configurations. JOUR ZAANE 25 s01 149 RADIOACTIVITY ${ }^{141} \mathrm{Ho},{ }^{144,145,146} \mathrm{Tm}$ (p) [from ${ }^{92} \mathrm{Mo}\left({ }^{54} \mathrm{Fe}\right.$, xnyp) and ${ }^{92} \mathrm{Mo}\left({ }^{58} \mathrm{Ni}\right.$, xnyp) $]$; measured Ep, $\mathrm{T}_{1 / 2}$; deduced branching ratios. ${ }^{141} \mathrm{Ho},{ }^{140} \mathrm{Dy},{ }^{145,146} \mathrm{Tm},{ }^{144,145} \mathrm{Er}$ deduced levels, configurations. JOUR NIMBE 241185 RADIOACTIVITY ${ }^{146} \mathrm{Tm}(\mathrm{p})$ [from ${ }^{58} \mathrm{Ni}\left({ }^{92} \mathrm{Mo}, \mathrm{X}\right)$ ]; measured $\mathrm{E} \gamma, \mathrm{Ep}$, $\mathrm{T}_{1 / 2}$ following proton decay from ground and excited states. ${ }^{146} \mathrm{Tm}$, ${ }^{145}$ Er deduced levels, J, $\pi$, configurations. JOUR ZAANE 25 s01 155 RADIOACTIVITY ${ }^{141} \mathrm{Ho},{ }^{144,145,146} \mathrm{Tm}(\mathrm{p})\left[\right.$ from ${ }^{92} \mathrm{Mo}\left({ }^{54} \mathrm{Fe}\right.$, xnyp) and ${ }^{92} \mathrm{Mo}\left({ }^{58} \mathrm{Ni}\right.$, xnyp $\left.)\right]$; measured $\mathrm{Ep}, \mathrm{T}_{1 / 2}$; deduced branching ratios. ${ }^{141} \mathrm{Ho},{ }^{140} \mathrm{Dy},{ }^{145,146} \mathrm{Tm},{ }^{144,145} \mathrm{Er}$ deduced levels, configurations. JOUR NIMBE 241185

NUCLEAR REACTIONS ${ }^{58} \mathrm{Ni}\left({ }^{92} \mathrm{Mo}, 2 \mathrm{np}\right), \mathrm{E}=512 \mathrm{MeV} ;{ }^{58} \mathrm{Ni}\left({ }^{92} \mathrm{Mo}\right.$, $3 \mathrm{np}), \mathrm{E}=460 \mathrm{MeV} ;{ }^{58} \mathrm{Ni}\left({ }^{92} \mathrm{Mo}, 4 \mathrm{np}\right), \mathrm{E}=417 \mathrm{MeV}$; measured $\mathrm{E} \gamma, \mathrm{I} \gamma$, $\gamma \gamma-$, (recoil) $\gamma$-coin. ${ }^{145,146,147} \mathrm{Tm}$ deduced levels, J, $\pi$, proton-decay features. Gammasphere array, recoil-decay tagging. Comparison with Particle Rotor model predictions. JOUR ZAANE 25 s01 159 RADIOACTIVITY ${ }^{145} \mathrm{Tm}(\mathrm{p})$ [from ${ }^{58} \mathrm{Ni}\left({ }^{92} \mathrm{Mo}, 4 \mathrm{np}\right)$ ]; measured Ep, $\mathrm{E} \gamma, \mathrm{p} \gamma$-coin. JOUR ZAANE 25 s01 159

\section{$\mathrm{A}=146$}

NUCLEAR MOMENTS $142,143,144,145,146,148,150 \mathrm{Nd}$; measured hfs, isotope shifts. JOUR CJPHA 83841

RADIOACTIVITY ${ }^{146} \mathrm{Tm}(\mathrm{p})$ [from ${ }^{58} \mathrm{Ni}\left({ }^{92} \mathrm{Mo}, \mathrm{p} 3 \mathrm{n}\right), \mathrm{E}=297 \mathrm{MeV}$ ]; measured proton spectra, $\mathrm{T}_{1 / 2}$; deduced fine structure, decay branching ratios. ${ }^{145}$ Er deduced levels, configurations. JOUR ZAANE 25 s01 149 RADIOACTIVITY ${ }^{141} \mathrm{Ho},{ }^{144,145,146} \mathrm{Tm}(\mathrm{p})$ [from ${ }^{92} \mathrm{Mo}\left({ }^{54} \mathrm{Fe}\right.$, xnyp) and ${ }^{92} \mathrm{Mo}\left({ }^{58} \mathrm{Ni}\right.$, xnyp $\left.)\right]$; measured $\mathrm{Ep}, \mathrm{T}_{1 / 2}$; deduced branching ratios. ${ }^{141} \mathrm{Ho},{ }^{140} \mathrm{Dy},{ }^{145,146} \mathrm{Tm},{ }^{144,145} \mathrm{Er}$ deduced levels, configurations. JOUR NIMBE 241185 


\section{$\mathrm{A}=146$ (continued)}

2005R040 NUCLEAR REACTIONS ${ }^{58} \mathrm{Ni}\left({ }^{92} \mathrm{Mo}\right.$, 3np), E not given; measured E $\gamma$, $\mathrm{I} \gamma, \gamma \gamma$-, (recoil) $\gamma$-coin. ${ }^{146} \mathrm{Tm}$ deduced levels, $\mathrm{J}, \pi$. Gammasphere array, recoil-decay tagging. JOUR ZAANE 25 s01 155

2005R040 RADIOACTIVITY ${ }^{146} \mathrm{Tm}(\mathrm{p})$ [from $\left.{ }^{58} \mathrm{Ni}\left({ }^{92} \mathrm{Mo}, \mathrm{X}\right)\right]$; measured E $\gamma, \mathrm{Ep}$, $\mathrm{T}_{1 / 2}$ following proton decay from ground and excited states. ${ }^{146} \mathrm{Tm}$, ${ }^{145}$ Er deduced levels, J, $\pi$, configurations. JOUR ZAANE 25 s01 155 2005SE26 NUCLEAR REACTIONS ${ }^{58} \mathrm{Ni}\left({ }^{92} \mathrm{Mo}, 2 \mathrm{np}\right), \mathrm{E}=512 \mathrm{MeV} ;{ }^{58} \mathrm{Ni}\left({ }^{92} \mathrm{Mo}\right.$, 3np), $\mathrm{E}=460 \mathrm{MeV} ;{ }^{58} \mathrm{Ni}\left({ }^{92} \mathrm{Mo}, 4 \mathrm{np}\right), \mathrm{E}=417 \mathrm{MeV}$; measured $\mathrm{E} \gamma, \mathrm{I} \gamma$, $\gamma \gamma$-, (recoil) $\gamma$-coin. ${ }^{145,146,147} \mathrm{Tm}$ deduced levels, J, $\pi$, proton-decay features. Gammasphere array, recoil-decay tagging. Comparison with Particle Rotor model predictions. JOUR ZAANE 25 s01 159

\section{$\mathrm{A}=147$}

${ }^{147}$ Dy $\quad 2005$ GE10

ATOMIC MASSES ${ }^{147,147 m}$ Dy; measured masses for ground and isomeric states. Schottky mass spectrometry. JOUR JPGPE 31 S1779

${ }^{147} \mathrm{Tm} \quad$ 2005SE26 NUCLEAR REACTIONS ${ }^{58} \mathrm{Ni}\left({ }^{92} \mathrm{Mo}, 2 \mathrm{np}\right), \mathrm{E}=512 \mathrm{MeV} ;{ }^{58} \mathrm{Ni}\left({ }^{92} \mathrm{Mo}\right.$, $3 \mathrm{np}), \mathrm{E}=460 \mathrm{MeV} ;{ }^{58} \mathrm{Ni}\left({ }^{92} \mathrm{Mo}, 4 \mathrm{np}\right), \mathrm{E}=417 \mathrm{MeV}$; measured $\mathrm{E} \gamma, \mathrm{I} \gamma$, $\gamma \gamma-$, (recoil) $\gamma$-coin. ${ }^{145,146,147} \mathrm{Tm}$ deduced levels, J, $\pi$, proton-decay features. Gammasphere array, recoil-decay tagging. Comparison with Particle Rotor model predictions. JOUR ZAANE 25 s01 159

\section{$\mathrm{A}=148$}

${ }^{148} \mathrm{Nd}$ 2005R035 NUCLEAR MOMENTS $142,143,144,145,146,148,150 \mathrm{Nd}$; measured hfs, isotope shifts. JOUR CJPHA 83841

${ }^{148} \mathrm{Gd}$ 2005KE07 NUCLEAR REACTIONS W, Ta, Au(p, X) ${ }^{148} \mathrm{Gd}, \mathrm{E}=600,800 \mathrm{MeV}$; measured cumulative production $\sigma$. Comparison with previous results, model predictions. JOUR NUPAB 760225

$$
\mathrm{A}=149
$$

No references found

$$
\mathrm{A}=\mathbf{1 5 0}
$$

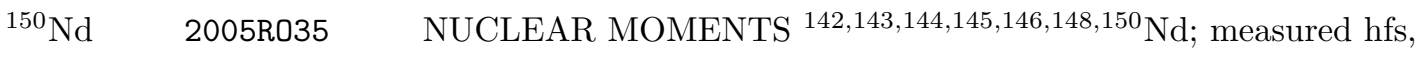
isotope shifts. JOUR CJPHA 83841

$$
\mathrm{A}=151
$$

No references found 


\section{$\mathrm{A}=152$}

\begin{tabular}{|c|c|c|}
\hline \multirow[t]{2}{*}{${ }^{152} \mathrm{Ce}$} & $2005 F 017$ & 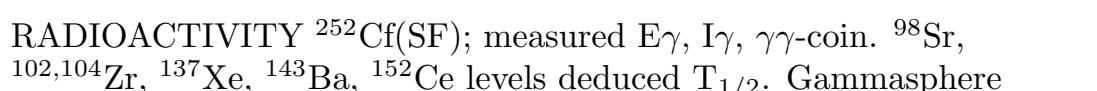 \\
\hline & & array, time-gated triple-coincidence method. JOUR ZAANE 25 s01 465 \\
\hline \multirow[t]{3}{*}{${ }^{152} \mathrm{Sm}$} & $2004 K U 35$ & $\begin{array}{l}\text { RADIOACTIVITY }{ }^{238} \mathrm{Pu},{ }^{226} \mathrm{Ra}(\alpha) ;{ }^{152} \mathrm{Eu}(\mathrm{EC}) ; \text { measured low-energy } \\
\text { electron spectra, angular distributions, (electron) } \alpha-\text {, (electron) } \gamma-\text {, } \\
\text { (electron)(X-ray)-coin. JOUR BRSPE } 681358\end{array}$ \\
\hline & $2005 \mathrm{GA} 47$ & $\begin{array}{l}\text { NUCLEAR REACTIONS }{ }^{150} \mathrm{Nd}(\alpha, 2 \mathrm{n}), \mathrm{E}=22.5 \mathrm{MeV} ;{ }^{152} \mathrm{Sm}\left(\mathrm{n}, \mathrm{n}{ }^{\prime}\right), \mathrm{E} \\
\text { not given; measured } \mathrm{E} \gamma, \mathrm{I} \gamma, \gamma \gamma \text {-coin. }{ }^{152} \mathrm{Sm} \text { deduced levels, J, } \pi \text {, } \\
\text { octupole and hexadecapole bands. JOUR JPGPE } 31 \mathrm{~S} 1855\end{array}$ \\
\hline & 2005WI20 & $\begin{array}{l}\text { RADIOACTIVITY }{ }^{26} \mathrm{Na}\left(\beta^{-}\right) ;{ }^{152} \mathrm{Eu}\left(\beta^{-}\right),(\mathrm{EC}) ; \text { measured } \mathrm{E} \gamma, \mathrm{I} \gamma, \gamma \gamma- \\
\beta \gamma \text {-coin. }{ }^{152} \mathrm{Sm} \text { level deduced } \mathrm{T}_{1 / 2} \text {. JOUR JPGPE } 31 \mathrm{~S} 1979\end{array}$ \\
\hline \multirow[t]{3}{*}{${ }^{152} \mathrm{Eu}$} & 2004GE20 & $\begin{array}{l}\left.\text { RADIOACTIVITY }{ }^{155} \mathrm{Sm}\left(\beta^{-}\right) \text {[from }{ }^{154} \mathrm{Sm}(\mathrm{n}, \gamma)\right] ;{ }^{60} \mathrm{Co},{ }^{133} \mathrm{Ba},{ }^{152} \mathrm{Eu} \text {; } \\
\text { measured } \gamma \text {-ray angular correlations. }{ }^{155} \mathrm{Eu},{ }^{60} \mathrm{Ni},{ }^{133} \mathrm{Cs},{ }^{152} \mathrm{Gd} \\
\text { transitions deduced } \delta \text {. Comparison with previous results. JOUR } \\
\text { BJPHE } 34722\end{array}$ \\
\hline & 2004 KU35 & $\begin{array}{l}\text { RADIOACTIVITY }{ }^{238} \mathrm{Pu},{ }^{226} \mathrm{Ra}(\alpha) ;{ }^{152} \mathrm{Eu}(\mathrm{EC}) ; \text { measured low-energy } \\
\text { electron spectra, angular distributions, (electron) } \alpha \text {-, (electron) } \gamma-\text {, } \\
\text { (electron)(X-ray)-coin. JOUR BRSPE } 681358\end{array}$ \\
\hline & 2005WI20 & $\begin{array}{l}\text { RADIOACTIVITY }{ }^{26} \mathrm{Na}\left(\beta^{-}\right) ;{ }^{152} \mathrm{Eu}\left(\beta^{-}\right),(\mathrm{EC}) ; \text { measured } \mathrm{E} \gamma, \mathrm{I} \gamma, \gamma \gamma-\text {, } \\
\beta \gamma \text {-coin. }{ }^{152} \mathrm{Sm} \text { level deduced } \mathrm{T}_{1 / 2} \text {. JOUR JPGPE } 31 \mathrm{~S} 1979\end{array}$ \\
\hline \multirow[t]{2}{*}{${ }^{152} \mathrm{Gd}$} & 2004GE20 & $\begin{array}{l}\left.\text { RADIOACTIVITY }{ }^{155} \mathrm{Sm}\left(\beta^{-}\right) \text {[from }{ }^{154} \mathrm{Sm}(\mathrm{n}, \gamma)\right] ;{ }^{60} \mathrm{Co},{ }^{133} \mathrm{Ba},{ }^{152} \mathrm{Eu} \text {; } \\
\text { measured } \gamma \text {-ray angular correlations. }{ }^{155} \mathrm{Eu},{ }^{60} \mathrm{Ni},{ }^{133} \mathrm{Cs},{ }^{152} \mathrm{Gd} \\
\text { transitions deduced } \delta \text {. Comparison with previous results. JOUR } \\
\text { BJPHE } 34722\end{array}$ \\
\hline & $2005 W I 20$ & $\begin{array}{l}\text { RADIOACTIVITY }{ }^{26} \mathrm{Na}\left(\beta^{-}\right) ;{ }^{152} \mathrm{Eu}\left(\beta^{-}\right),(\mathrm{EC}) ; \text { measured } \mathrm{E} \gamma, \mathrm{I} \gamma, \\
\beta \gamma \text {-coin. }{ }^{152} \mathrm{Sm} \text { level deduced } \mathrm{T}_{1 / 2} . \text { JOUR JPGPE } 31 \mathrm{~S} 1979\end{array}$ \\
\hline
\end{tabular}

$$
\mathrm{A}=153
$$

No references found

$$
\mathrm{A}=154
$$

No references found

\section{$\mathrm{A}=155$}

${ }^{155} \mathrm{Sm} \quad 2004 \mathrm{GE} 20$

RADIOACTIVITY ${ }^{155} \mathrm{Sm}\left(\beta^{-}\right)$[from ${ }^{154} \mathrm{Sm}(\mathrm{n}, \gamma)$ ]; ${ }^{60} \mathrm{Co},{ }^{133} \mathrm{Ba},{ }^{152} \mathrm{Eu}$; measured $\gamma$-ray angular correlations. ${ }^{155} \mathrm{Eu},{ }^{60} \mathrm{Ni},{ }^{133} \mathrm{Cs},{ }^{152} \mathrm{Gd}$ transitions deduced $\delta$. Comparison with previous results. JOUR BJPHE 34722

2005RA33 RADIOACTIVITY ${ }^{155} \mathrm{Sm}\left(\beta^{-}\right)$[from $\left.{ }^{154} \mathrm{Sm}(\mathrm{n}, \gamma)\right]$; measured $\mathrm{E} \gamma$, I $\gamma$, $\gamma \gamma$-coin; deduced log ft. ${ }^{155} \mathrm{Eu}$ deduced levels, J, $\pi, \beta$-feeding intensities. JOUR BJPHE 35839 


\section{$\mathrm{A}=155$ (continued)}

${ }^{155} \mathrm{Eu} \quad$ 2004GE20 RADIOACTIVITY ${ }^{155} \mathrm{Sm}\left(\beta^{-}\right)\left[\right.$from $\left.{ }^{154} \mathrm{Sm}(\mathrm{n}, \gamma)\right] ;{ }^{60} \mathrm{Co},{ }^{133} \mathrm{Ba},{ }^{152} \mathrm{Eu}$ measured $\gamma$-ray angular correlations. ${ }^{155} \mathrm{Eu},{ }^{60} \mathrm{Ni},{ }^{133} \mathrm{Cs},{ }^{152} \mathrm{Gd}$ transitions deduced $\delta$. Comparison with previous results. JOUR BJPHE 34722

2005RA33 RADIOACTIVITY ${ }^{155} \mathrm{Sm}\left(\beta^{-}\right)$[from $\left.{ }^{154} \mathrm{Sm}(\mathrm{n}, \gamma)\right]$; measured $\mathrm{E} \gamma$, I $\gamma$, $\gamma \gamma$-coin; deduced log ft. ${ }^{155} \mathrm{Eu}$ deduced levels, J, $\pi$, $\beta$-feeding intensities. JOUR BJPHE 35839

$$
\mathrm{A}=156
$$

No references found

$$
\mathrm{A}=\mathbf{1 5 7}
$$

${ }^{157} \mathrm{Er} \quad 2005 \mathrm{RI} 16$

NUCLEAR REACTIONS ${ }^{114} \mathrm{Cd}\left({ }^{48} \mathrm{Ca}, 5 \mathrm{n}\right), \mathrm{E}=215 \mathrm{MeV}$; measured $\mathrm{E} \gamma$, $\mathrm{I} \gamma, \gamma \gamma$-coin. ${ }^{157} \mathrm{Er}$ deduced high-spin levels, $\mathrm{J}, \pi$, band termination features. Gammasphere array. JOUR JPGPE 31 S1735

${ }^{157} \mathrm{Lu} \quad$ 2005SC22 RADIOACTIVITY ${ }^{167,167 m, 169,169 m} \mathrm{Ir},{ }^{165,165 m} \mathrm{Re},{ }^{161} \mathrm{Ta}(\alpha)[$ from ${ }^{92} \mathrm{Mo}\left({ }^{78} \mathrm{Kr}, 2 \mathrm{np}\right)$ and ${ }^{112} \mathrm{Sn}\left({ }^{58} \mathrm{Ni}, \mathrm{p}\right)$ and subsequent decay]; measured $\mathrm{E} \alpha, \mathrm{E} \gamma, \alpha \gamma$-coin, $\mathrm{T}_{1 / 2}$; deduced spectroscopic factors. ${ }^{167,167 m} \operatorname{Ir}(\mathrm{p})$ [from $\left.{ }^{112} \mathrm{Sn}\left({ }^{58} \mathrm{Ni}, 2 \mathrm{np}\right)\right]$; measured $\mathrm{Ep}, \mathrm{T}_{1 / 2}$; deduced spectroscopic factors. Jurogam array, mass separator. JOUR JPGPE 31 S1719

\section{$\mathrm{A}=\mathbf{1 5 8}$}

${ }^{158} \mathrm{Gd}$ 2005ME19 NUCLEAR REACTIONS ${ }^{160} \mathrm{Gd},{ }^{164} \mathrm{Dy},{ }^{170} \mathrm{Er},{ }^{178} \mathrm{Hf},{ }^{186} \mathrm{~W},{ }^{192} \mathrm{Os}(\mathrm{p}$, t), $\mathrm{E}=25 \mathrm{MeV}$; measured triton spectra, $\sigma(\theta) .{ }^{158} \mathrm{Gd},{ }^{162} \mathrm{Dy},{ }^{168} \mathrm{Er}$, ${ }^{176} \mathrm{Hf},{ }^{184} \mathrm{~W},{ }^{190}$ Os deduced $0^{+}$level energies. JOUR JPGPE $31 \mathrm{~S} 1399$ 2005MI28 NUCLEAR REACTIONS ${ }^{158} \mathrm{Gd}$ (X-ray, X-ray), E $\approx 79.5 \mathrm{keV}$; measured delayed X-ray spectrum. ${ }^{158} \mathrm{Gd}$ deduced excited state energy, $\mathrm{T}_{1 / 2}$. Synchrotron radiation, comparison with previous results. JOUR JUPSA 743122

$$
\mathrm{A}=\mathbf{1 5 9}
$$

No references found

$$
\mathrm{A}=\mathbf{1 6 0}
$$

${ }^{160} \mathrm{Tm}$ 2005LA32 NUCLEAR REACTIONS ${ }^{130} \mathrm{Te}\left({ }^{35} \mathrm{Cl}, 5 \mathrm{n}\right), \mathrm{E}=170 \mathrm{MeV}$; measured $\mathrm{E} \gamma$, $\mathrm{I} \gamma, \gamma \gamma$-coin. ${ }^{160} \mathrm{Tm}$ deduced high-spin levels, $\mathrm{J}, \pi$, configurations. Euroball array. JOUR PRVCA 72057303 


\section{$\mathrm{A}=160$ (continued)}

${ }^{160} \mathrm{Yb}$ 2005BA88 NUCLEAR REACTIONS ${ }^{208} \mathrm{~Pb}(\mathrm{p}, \gamma), \mathrm{E}=11.9 \mathrm{MeV}$; measured $\mathrm{E} \gamma, \mathrm{I} \gamma$. ${ }^{147} \mathrm{Sm}\left({ }^{16} \mathrm{O}, 3 \mathrm{n}\right), \mathrm{E}=73 \mathrm{MeV}$; measured $\mathrm{E} \gamma, \mathrm{I} \gamma, \gamma \gamma$-coin. ${ }^{160} \mathrm{Yb}$ deduced high-spin levels, J, $\pi$. Afrodite array. JOUR JPGPE 31 S1747

\section{$\mathrm{A}=161$}

${ }^{161} \mathrm{Yb} \quad$ 2005DU23

NUCLEAR REACTIONS Ge( $\left.{ }^{18} \mathrm{O}, \mathrm{X}\right)^{83 m} \mathrm{Sr} /{ }^{83} \mathrm{Y} /{ }^{84 m} \mathrm{Y} /{ }^{88 m} \mathrm{Y} /$ ${ }^{85} \mathrm{Zr} /{ }^{87} \mathrm{Zr}, \mathrm{E}=82.8 \mathrm{GeV} ;{ }^{84} \mathrm{Se}\left({ }^{18} \mathrm{O}, \mathrm{X}\right){ }^{86 m} \mathrm{Y} /{ }^{85} \mathrm{Zr} /{ }^{87} \mathrm{Nb} /{ }^{87 m} \mathrm{Nb} /$ ${ }^{88} \mathrm{Nb} /{ }^{88} \mathrm{Mo}, \mathrm{E}=82.7 \mathrm{MeV} ;{ }^{124} \mathrm{Sn}\left({ }^{50} \mathrm{Ti}, \mathrm{X}\right){ }^{168 m} \mathrm{Lu} /{ }^{167} \mathrm{Hf} /{ }^{168} \mathrm{Hf}$, $\mathrm{E}=223.7 \mathrm{MeV} ;{ }^{116} \mathrm{Sn}\left({ }^{50} \mathrm{Ti}, \mathrm{X}\right){ }^{162} \mathrm{Tm} /{ }^{161} \mathrm{Yb} /{ }^{162} \mathrm{Yb} /{ }^{163} \mathrm{Yb} /{ }^{162} \mathrm{Lu} /$ ${ }^{162} \mathrm{Hf}, \mathrm{E}=224.4 \mathrm{MeV}$; measured delayed $\mathrm{E} \gamma, \mathrm{I} \gamma$ following residual nucleus decay. Physical preseparation technique. JOUR NIMAE 551 528

${ }^{161} \mathrm{Ta} \quad 2005 \mathrm{SC} 22 \quad$ RADIOACTIVITY ${ }^{167,167 m, 169,169 m} \mathrm{Ir},{ }^{165,165 m} \mathrm{Re},{ }^{161} \mathrm{Ta}(\alpha)$ [from ${ }^{92} \mathrm{Mo}\left({ }^{78} \mathrm{Kr}, 2 \mathrm{np}\right)$ and ${ }^{112} \mathrm{Sn}\left({ }^{58} \mathrm{Ni}, \mathrm{p}\right)$ and subsequent decay]; measured $\mathrm{E} \alpha, \mathrm{E} \gamma, \alpha \gamma$-coin, $\mathrm{T}_{1 / 2}$; deduced spectroscopic factors. ${ }^{167,167 m} \operatorname{Ir}(\mathrm{p})$ [from ${ }^{112} \mathrm{Sn}\left({ }^{58} \mathrm{Ni}, 2 \mathrm{np}\right)$ ]; measured $\mathrm{Ep}, \mathrm{T}_{1 / 2}$; deduced spectroscopic factors. Jurogam array, mass separator. JOUR JPGPE 31 S1719

\section{$\mathrm{A}=162$}

${ }^{162} \mathrm{Gd}$ 2005J024 RADIOACTIVITY ${ }^{252} \mathrm{Cf}(\mathrm{SF}) ;$ measured E $\gamma, \mathrm{I} \gamma, \gamma \gamma$-coin. ${ }^{162,164} \mathrm{Gd}$ deduced levels, J, $\pi$. Gammasphere array, level systematics in neighboring nuclides discussed. JOUR ZAANE 25 s01 467 ${ }^{162} \mathrm{Dy}$ 2005ME19 NUCLEAR REACTIONS ${ }^{160} \mathrm{Gd},{ }^{164} \mathrm{Dy},{ }^{170} \mathrm{Er},{ }^{178} \mathrm{Hf},{ }^{186} \mathrm{~W},{ }^{192} \mathrm{Os}(\mathrm{p}$, t), $\mathrm{E}=25 \mathrm{MeV}$; measured triton spectra, $\sigma(\theta) .{ }^{158} \mathrm{Gd},{ }^{162} \mathrm{Dy},{ }^{168} \mathrm{Er}$, ${ }^{176} \mathrm{Hf},{ }^{184} \mathrm{~W},{ }^{190}$ Os deduced $0^{+}$level energies. JOUR JPGPE $31 \mathrm{~S} 1399$ ${ }^{162} \mathrm{Tm}$ 2005DU23 NUCLEAR REACTIONS Ge( $\left.{ }^{18} \mathrm{O}, \mathrm{X}\right)^{83 m} \mathrm{Sr} /{ }^{83} \mathrm{Y} /{ }^{84 m} \mathrm{Y} /{ }^{88 m} \mathrm{Y} /$ ${ }^{85} \mathrm{Zr} /{ }^{87} \mathrm{Zr}, \mathrm{E}=82.8 \mathrm{GeV} ;{ }^{84} \mathrm{Se}\left({ }^{18} \mathrm{O}, \mathrm{X}\right){ }^{86 m} \mathrm{Y} /{ }^{85} \mathrm{Zr} /{ }^{87} \mathrm{Nb} /{ }^{87 m} \mathrm{Nb} /$ ${ }^{88} \mathrm{Nb} /{ }^{88} \mathrm{Mo}, \mathrm{E}=82.7 \mathrm{MeV} ;{ }^{124} \mathrm{Sn}\left({ }^{50} \mathrm{Ti}, \mathrm{X}\right){ }^{168 m} \mathrm{Lu} /{ }^{167} \mathrm{Hf} /{ }^{168} \mathrm{Hf}$, $\mathrm{E}=223.7 \mathrm{MeV} ;{ }^{116} \mathrm{Sn}\left({ }^{50} \mathrm{Ti}, \mathrm{X}\right){ }^{162} \mathrm{Tm} /{ }^{161} \mathrm{Yb} /{ }^{162} \mathrm{Yb} /{ }^{163} \mathrm{Yb} /{ }^{162} \mathrm{Lu} /$ ${ }^{162} \mathrm{Hf}, \mathrm{E}=224.4 \mathrm{MeV}$; measured delayed $\mathrm{E} \gamma$, I $\gamma$ following residual nucleus decay. Physical preseparation technique. JOUR NIMAE 551 528

${ }^{162} \mathrm{Yb}$ 2005DU23 NUCLEAR REACTIONS Ge $\left({ }^{18} \mathrm{O}, \mathrm{X}\right){ }^{83 m} \mathrm{Sr} /{ }^{83} \mathrm{Y} /{ }^{84 m} \mathrm{Y} /{ }^{88 m} \mathrm{Y} /$ ${ }^{85} \mathrm{Zr} /{ }^{87} \mathrm{Zr}, \mathrm{E}=82.8 \mathrm{GeV} ;{ }^{84} \mathrm{Se}\left({ }^{18} \mathrm{O}, \mathrm{X}\right){ }^{86 m} \mathrm{Y} /{ }^{85} \mathrm{Zr} /{ }^{87} \mathrm{Nb} /{ }^{87 m} \mathrm{Nb} /$ ${ }^{88} \mathrm{Nb} /{ }^{88} \mathrm{Mo}, \mathrm{E}=82.7 \mathrm{MeV} ;{ }^{124} \mathrm{Sn}\left({ }^{50} \mathrm{Ti}, \mathrm{X}\right){ }^{168 m} \mathrm{Lu} /{ }^{167} \mathrm{Hf} /{ }^{168} \mathrm{Hf}$, $\mathrm{E}=223.7 \mathrm{MeV} ;{ }^{116} \mathrm{Sn}\left({ }^{50} \mathrm{Ti}, \mathrm{X}\right){ }^{162} \mathrm{Tm} /{ }^{161} \mathrm{Yb} /{ }^{162} \mathrm{Yb} /{ }^{163} \mathrm{Yb} /{ }^{162} \mathrm{Lu} /$ ${ }^{162} \mathrm{Hf}, \mathrm{E}=224.4 \mathrm{MeV}$; measured delayed $\mathrm{E} \gamma$, I $\gamma$ following residual nucleus decay. Physical preseparation technique. JOUR NIMAE 551 528 


\section{$\mathrm{A}=162$ (continued)}

\begin{tabular}{|c|c|c|}
\hline${ }^{162} \mathrm{Lu}$ & 2005DU23 & $\begin{array}{l}\text { NUCLEAR REACTIONS Ge }\left({ }^{18} \mathrm{O}, \mathrm{X}\right){ }^{83 m} \mathrm{Sr} /{ }^{83} \mathrm{Y} /{ }^{84 m} \mathrm{Y} /{ }^{88 m} \mathrm{Y} / \\
\\
{ }^{85} \mathrm{Zr} /{ }^{87} \mathrm{Zr}, \mathrm{E}=82.8 \mathrm{GeV} ;{ }^{84} \mathrm{Se}\left({ }^{18} \mathrm{O}, \mathrm{X}\right){ }^{86 m} \mathrm{Y} /{ }^{85} \mathrm{Zr} /{ }^{87} \mathrm{Nb} /{ }^{87 m} \mathrm{Nb} / \\
{ }^{88} \mathrm{Nb} /{ }^{88} \mathrm{Mo}, \mathrm{E}=82.7 \mathrm{MeV} ;{ }^{124} \mathrm{Sn}\left({ }^{50} \mathrm{Ti}, \mathrm{X}\right){ }^{168 m} \mathrm{Lu} /{ }^{167} \mathrm{Hf} /{ }^{168} \mathrm{Hf}, \\
\mathrm{E}=223.7 \mathrm{MeV} ;{ }^{116} \mathrm{Sn}\left({ }^{50} \mathrm{Ti}, \mathrm{X}\right){ }^{162} \mathrm{Tm} /{ }^{161} \mathrm{Yb} /{ }^{162} \mathrm{Yb} /{ }^{163} \mathrm{Yb} /{ }^{162} \mathrm{Lu} / \\
{ }^{162} \mathrm{Hf}, \mathrm{E}=224.4 \mathrm{MeV} ; \text { measured delayed } \mathrm{E} \gamma, \mathrm{I} \gamma \text { following residual } \\
\text { nucleus decay. Physical preseparation technique. JOUR NIMAE } 551 \\
528\end{array}$ \\
\hline${ }^{162} \mathrm{Hf}$ & 2005DU23 & $\begin{array}{l}\text { NUCLEAR REACTIONS Ge }\left({ }^{18} \mathrm{O}, \mathrm{X}\right){ }^{83 m} \mathrm{Sr} /{ }^{83} \mathrm{Y} /{ }^{84 m} \mathrm{Y} /{ }^{88 m} \mathrm{Y} / \\
\left.{ }^{85} \mathrm{Zr} /{ }^{87} \mathrm{Zr}, \mathrm{E}=82.8 \mathrm{GeV} ;{ }^{84} \mathrm{Se}{ }^{18} \mathrm{O}, \mathrm{X}\right){ }^{86 m} \mathrm{Y} /{ }^{85} \mathrm{Zr} /{ }^{87} \mathrm{Nb} /{ }^{87 m} \mathrm{Nb} / \\
{ }^{88} \mathrm{Nb} /{ }^{88} \mathrm{Mo}, \mathrm{E}=82.7 \mathrm{MeV} ;{ }^{124} \mathrm{Sn}\left({ }^{50} \mathrm{Ti}, \mathrm{X}\right){ }^{168 m} \mathrm{Lu} /{ }^{167} \mathrm{Hf} /{ }^{168} \mathrm{Hf}, \\
\mathrm{E}=223.7 \mathrm{MeV} ;{ }^{116} \mathrm{Sn}\left({ }^{50} \mathrm{Ti}, \mathrm{X}\right){ }^{162} \mathrm{Tm} /{ }^{161} \mathrm{Yb} /{ }^{162} \mathrm{Yb} /{ }^{163} \mathrm{Yb} /{ }^{162} \mathrm{Lu} / \\
{ }^{162} \mathrm{Hf}, \mathrm{E}=224.4 \mathrm{MeV} ; \text { measured delayed } \mathrm{E} \gamma, \mathrm{I} \gamma \text { following residual } \\
\text { nucleus decay. Physical preseparation technique. JOUR NIMAE } 551 \\
528\end{array}$ \\
\hline
\end{tabular}

\section{$\mathrm{A}=163$}

${ }^{163}$ Dy $\quad 2005 R I 17$

NUCLEAR REACTIONS ${ }^{92} \mathrm{Mo}\left({ }^{54} \mathrm{Fe}\right.$, xnypz $\left.\alpha\right), \mathrm{E}=245 \mathrm{MeV}$; measured prompt and delayed $\mathrm{E} \gamma, \mathrm{I} \gamma, \gamma \gamma$-, (recoil) $\gamma$-coin. ${ }^{142} \mathrm{~Tb},{ }^{163} \mathrm{Dy}$ deduced transitions. ${ }^{143}$ Dy deduced isomeric state $\mathrm{T}_{1 / 2}$. Jurogam array. JOUR JPGPE 31 S1949

${ }^{163} \mathrm{Er}$ 2005LE35 NUCLEAR REACTIONS ${ }^{150} \mathrm{Nd}\left({ }^{18} \mathrm{O}, 5 \mathrm{n}\right), \mathrm{E}=87,93 \mathrm{MeV}$; measured $\mathrm{E} \gamma, \mathrm{I} \gamma, \gamma \gamma$-coin. ${ }^{163} \mathrm{Er}$ deduced quasi-continuum high-spin spectra, rotational bands excitation energy, compound and rotational damping widths vs K-quantum number, order-to-chaos transition features. Euroball array, comparison with model predictions. JOUR PRVCA 72 034307

${ }^{163} \mathrm{Yb}$ 2005DU23 NUCLEAR REACTIONS Ge( $\left.{ }^{18} \mathrm{O}, \mathrm{X}\right)^{83 m} \mathrm{Sr} /{ }^{83} \mathrm{Y} /{ }^{84 m} \mathrm{Y} /{ }^{88 m} \mathrm{Y} /$ ${ }^{85} \mathrm{Zr} /{ }^{87} \mathrm{Zr}, \mathrm{E}=82.8 \mathrm{GeV} ;{ }^{84} \mathrm{Se}\left({ }^{18} \mathrm{O}, \mathrm{X}\right){ }^{86 m} \mathrm{Y} /{ }^{85} \mathrm{Zr} /{ }^{87} \mathrm{Nb} /{ }^{87 m} \mathrm{Nb} /$ ${ }^{88} \mathrm{Nb} /{ }^{88} \mathrm{Mo}, \mathrm{E}=82.7 \mathrm{MeV} ;{ }^{124} \mathrm{Sn}\left({ }^{50} \mathrm{Ti}, \mathrm{X}\right){ }^{168 m} \mathrm{Lu} /{ }^{167} \mathrm{Hf} /{ }^{168} \mathrm{Hf}$, $\mathrm{E}=223.7 \mathrm{MeV} ;{ }^{116} \mathrm{Sn}\left({ }^{50} \mathrm{Ti}, \mathrm{X}\right){ }^{162} \mathrm{Tm} /{ }^{161} \mathrm{Yb} /{ }^{162} \mathrm{Yb} /{ }^{163} \mathrm{Yb} /{ }^{162} \mathrm{Lu} /$ ${ }^{162} \mathrm{Hf}, \mathrm{E}=224.4 \mathrm{MeV}$; measured delayed $\mathrm{E} \gamma$, I $\gamma$ following residual nucleus decay. Physical preseparation technique. JOUR NIMAE 551 528

${ }^{163} \operatorname{Re} \quad 2005 \mathrm{SC} 22 \quad$ RADIOACTIVITY ${ }^{167,167 m, 169,169 m} \operatorname{Ir},{ }^{165,165 m} \operatorname{Re},{ }^{161} \mathrm{Ta}(\alpha)$ [from ${ }^{92} \mathrm{Mo}\left({ }^{78} \mathrm{Kr}, 2 \mathrm{np}\right)$ and ${ }^{112} \mathrm{Sn}\left({ }^{58} \mathrm{Ni}, \mathrm{p}\right)$ and subsequent decay]; measured $\mathrm{E} \alpha, \mathrm{E} \gamma, \alpha \gamma$-coin, $\mathrm{T}_{1 / 2}$; deduced spectroscopic factors. ${ }^{167,167 m} \operatorname{Ir}(\mathrm{p})$ [from $\left.{ }^{112} \mathrm{Sn}\left({ }^{58} \mathrm{Ni}, 2 \mathrm{np}\right)\right]$; measured Ep, $\mathrm{T}_{1 / 2}$; deduced spectroscopic factors. Jurogam array, mass separator. JOUR JPGPE 31 S1719

\section{$\mathrm{A}=164$}

${ }^{164} \mathrm{Gd} \quad$ 2005J024 RADIOACTIVITY ${ }^{252} \mathrm{Cf}(\mathrm{SF}) ;$ measured $\mathrm{E} \gamma, \mathrm{I} \gamma, \gamma \gamma$-coin. ${ }^{162,164} \mathrm{Gd}$ deduced levels, J, $\pi$. Gammasphere array, level systematics in neighboring nuclides discussed. JOUR ZAANE 25 s01 467 


\section{$\mathrm{A}=\mathbf{1 6 5}$}

\begin{tabular}{|c|c|c|}
\hline${ }^{165} \mathrm{Er}$ & 2004BE58 & $\begin{array}{l}\text { NUCLEAR REACTIONS }{ }^{165} \mathrm{Ho}(\mathrm{p}, \mathrm{n}), \mathrm{E} \approx 8-18 \mathrm{MeV} \text {; measured } \\
\text { excitation function; deduced thick-target yield. Activation technique. } \\
\text { JOUR RAACA } 92219\end{array}$ \\
\hline${ }^{165} \mathrm{Re}$ & $2005 \mathrm{SC} 22$ & $\begin{array}{l}\text { RADIOACTIVITY }{ }^{167,167 m, 169,169 m} \mathrm{Ir},{ }^{165,165 m} \mathrm{Re},{ }^{161} \mathrm{Ta}(\alpha) \text { [from } \\
{ }^{12} \mathrm{Mo}\left({ }^{78} \mathrm{Kr}, 2 \mathrm{np}\right) \text { and }{ }^{112} \mathrm{Sn}\left({ }^{58} \mathrm{Ni}, \mathrm{p}\right) \text { and subsequent decay]; measured } \\
\mathrm{E} \alpha, \mathrm{E} \gamma, \alpha \gamma \text {-coin, } \mathrm{T}_{1 / 2} ; \text { deduced spectroscopic factors. }{ }^{167,167 m} \mathrm{Ir}(\mathrm{p}) \\
{\left[\text { from }{ }^{112} \mathrm{Sn}\left({ }^{58} \mathrm{Ni}, 2 \mathrm{np}\right)\right] ; \text { measured Ep, } \mathrm{T}_{1 / 2} \text {; deduced spectroscopic }} \\
\text { factors. Jurogam array, mass separator. JOUR JPGPE } 31 \mathrm{~S} 1719\end{array}$ \\
\hline
\end{tabular}

\section{$\mathrm{A}=\mathbf{1 6 6}$}

${ }^{166} \mathrm{Er}$

2005BU37

${ }^{166} \mathrm{Os}$

$2005 \mathrm{SC} 22$

2005GU28

${ }^{167} \mathrm{Hf}$

2005DU23

${ }^{167} \mathrm{Ir} \quad 2005 \mathrm{SC} 22$

2005SC22

\section{$\mathrm{A}=167$}

NUCLEAR REACTIONS ${ }^{164} \mathrm{Dy}\left({ }^{7} \mathrm{Li}\right.$, xnyp), E $=55 \mathrm{MeV}$; measured $\mathrm{E} \gamma$, $\mathrm{I} \gamma, \gamma \gamma-$, (charged particle) $\gamma$-coin. ${ }^{167} \mathrm{Tm}$ deduced high-spin levels, J, $\pi$, configurations. ${ }^{166} \mathrm{Er}$ deduced rotational band features. GASP, ISIS arrays. JOUR JPGPE 31 S1827

NUCLEAR REACTIONS ${ }^{123} \mathrm{Sb}\left({ }^{48} \mathrm{Ca}, 4 \mathrm{n}\right), \mathrm{E}=203 \mathrm{MeV}$; measured $\mathrm{E} \gamma$, $\mathrm{I} \gamma, \gamma \gamma$-coin, DSA. ${ }^{167} \mathrm{Lu}$ deduced triaxial superdeformed band transition quadrupole moment. Gammasphere array. JOUR JPGPE 31 S1873

NUCLEAR REACTIONS Ge $\left({ }^{18} \mathrm{O}, \mathrm{X}\right){ }^{83 m} \mathrm{Sr} /{ }^{83} \mathrm{Y} /{ }^{84 m} \mathrm{Y} /{ }^{88 m} \mathrm{Y} /$ ${ }^{85} \mathrm{Zr} /{ }^{87} \mathrm{Zr}, \mathrm{E}=82.8 \mathrm{GeV} ;{ }^{84} \mathrm{Se}\left({ }^{18} \mathrm{O}, \mathrm{X}\right){ }^{86 m} \mathrm{Y} /{ }^{85} \mathrm{Zr} /{ }^{87} \mathrm{Nb} /{ }^{87 m} \mathrm{Nb} /$ ${ }^{88} \mathrm{Nb} /{ }^{88} \mathrm{Mo}, \mathrm{E}=82.7 \mathrm{MeV} ;{ }^{124} \mathrm{Sn}\left({ }^{50} \mathrm{Ti}, \mathrm{X}\right){ }^{168 m} \mathrm{Lu} /{ }^{167} \mathrm{Hf} /{ }^{168} \mathrm{Hf}$, $\mathrm{E}=223.7 \mathrm{MeV} ;{ }^{116} \mathrm{Sn}\left({ }^{50} \mathrm{Ti}, \mathrm{X}\right){ }^{162} \mathrm{Tm} /{ }^{161} \mathrm{Yb} /{ }^{162} \mathrm{Yb} /{ }^{163} \mathrm{Yb} /{ }^{162} \mathrm{Lu} /$ ${ }^{162} \mathrm{Hf}, \mathrm{E}=224.4 \mathrm{MeV}$; measured delayed $\mathrm{E} \gamma, \mathrm{I} \gamma$ following residual nucleus decay. Physical preseparation technique. JOUR NIMAE 551 528

NUCLEAR REACTIONS ${ }^{92} \mathrm{Mo}\left({ }^{78} \mathrm{Kr}, 2 \mathrm{np}\right), \mathrm{E}=360 \mathrm{MeV} ;{ }^{112} \mathrm{Sn}\left({ }^{58} \mathrm{Ni}\right.$, p), $\mathrm{E}=266 \mathrm{MeV}$; measured $\mathrm{E} \gamma, \mathrm{I} \gamma, \gamma \gamma-$, (recoil) $\gamma$-coin. ${ }^{167,169} \mathrm{Ir}$ deduced transitions. Recoil-decay tagging, Jurogam array. JOUR JPGPE 31 S1719

RADIOACTIVITY ${ }^{167,167 m, 169,169 m} \operatorname{Ir},{ }^{165,165 m} \mathrm{Re},{ }^{161} \mathrm{Ta}(\alpha)$ [from ${ }^{92} \mathrm{Mo}\left({ }^{78} \mathrm{Kr}, 2 \mathrm{np}\right)$ and ${ }^{112} \mathrm{Sn}\left({ }^{58} \mathrm{Ni}, \mathrm{p}\right)$ and subsequent decay]; measured $\mathrm{E} \alpha, \mathrm{E} \gamma, \alpha \gamma$-coin, $\mathrm{T}_{1 / 2}$; deduced spectroscopic factors. ${ }^{167,167 m} \operatorname{Ir}(\mathrm{p})$ [from $\left.{ }^{112} \mathrm{Sn}\left({ }^{58} \mathrm{Ni}, 2 \mathrm{np}\right)\right]$; measured $\mathrm{Ep}, \mathrm{T}_{1 / 2}$; deduced spectroscopic factors. Jurogam array, mass separator. JOUR JPGPE 31 S1719 


\section{$\mathrm{A}=168$}

${ }^{168} \mathrm{Er} \quad 2005 \mathrm{BUZZ} \quad$ NUCLEAR REACTIONS ${ }^{170} \mathrm{Er}(\mathrm{p}, \mathrm{t}), \mathrm{E}=25.0 \mathrm{MeV}$; measured $\mathrm{E} \gamma, \mathrm{I} \gamma$, $\sigma(\theta) .{ }^{168} \mathrm{Er}$ deduced $0^{+}$states energies. REPT MLL 2004

Annual,P16,Bucurescu

2005ME19 NUCLEAR REACTIONS ${ }^{160} \mathrm{Gd},{ }^{164} \mathrm{Dy},{ }^{170} \mathrm{Er},{ }^{178} \mathrm{Hf},{ }^{186} \mathrm{~W},{ }^{192} \mathrm{Os}(\mathrm{p}$, $\mathrm{t}), \mathrm{E}=25 \mathrm{MeV}$; measured triton spectra, $\sigma(\theta) .{ }^{158} \mathrm{Gd},{ }^{162} \mathrm{Dy},{ }^{168} \mathrm{Er}$, ${ }^{176} \mathrm{Hf},{ }^{184} \mathrm{~W},{ }^{190} \mathrm{Os}$ deduced $0^{+}$level energies. JOUR JPGPE $31 \mathrm{~S} 1399$

${ }^{168} \mathrm{Lu}$ 2005DU23 NUCLEAR REACTIONS Ge $\left({ }^{18} \mathrm{O}, \mathrm{X}\right){ }^{83 m} \mathrm{Sr} /{ }^{83} \mathrm{Y} /{ }^{84 m} \mathrm{Y} /{ }^{88 m} \mathrm{Y} /$ ${ }^{85} \mathrm{Zr} /{ }^{87} \mathrm{Zr}, \mathrm{E}=82.8 \mathrm{GeV} ;{ }^{84} \mathrm{Se}\left({ }^{18} \mathrm{O}, \mathrm{X}\right){ }^{86 m} \mathrm{Y} /{ }^{85} \mathrm{Zr} /{ }^{87} \mathrm{Nb} /{ }^{87 m} \mathrm{Nb} /$ ${ }^{88} \mathrm{Nb} /{ }^{88} \mathrm{Mo}, \mathrm{E}=82.7 \mathrm{MeV} ;{ }^{124} \mathrm{Sn}\left({ }^{50} \mathrm{Ti}, \mathrm{X}\right){ }^{168 m} \mathrm{Lu} /{ }^{167} \mathrm{Hf} /{ }^{168} \mathrm{Hf}$, $\mathrm{E}=223.7 \mathrm{MeV} ;{ }^{116} \mathrm{Sn}\left({ }^{50} \mathrm{Ti}, \mathrm{X}\right){ }^{162} \mathrm{Tm} /{ }^{161} \mathrm{Yb} /{ }^{162} \mathrm{Yb} /{ }^{163} \mathrm{Yb} /{ }^{162} \mathrm{Lu} /$ ${ }^{162} \mathrm{Hf}, \mathrm{E}=224.4 \mathrm{MeV}$; measured delayed $\mathrm{E} \gamma$, I $\gamma$ following residual nucleus decay. Physical preseparation technique. JOUR NIMAE 551 528

${ }^{168} \mathrm{Hf}$ 2005DU23 NUCLEAR REACTIONS Ge $\left({ }^{18} \mathrm{O}, \mathrm{X}\right)^{83 m} \mathrm{Sr} /{ }^{83} \mathrm{Y} /{ }^{84 m} \mathrm{Y} /{ }^{88 m} \mathrm{Y} /$ ${ }^{85} \mathrm{Zr} /{ }^{87} \mathrm{Zr}, \mathrm{E}=82.8 \mathrm{GeV} ;{ }^{84} \mathrm{Se}\left({ }^{18} \mathrm{O}, \mathrm{X}\right)^{86 m} \mathrm{Y} /{ }^{85} \mathrm{Zr} /{ }^{87} \mathrm{Nb} /{ }^{87 m} \mathrm{Nb} /$ ${ }^{88} \mathrm{Nb} /{ }^{88} \mathrm{Mo}, \mathrm{E}=82.7 \mathrm{MeV} ;{ }^{124} \mathrm{Sn}\left({ }^{50} \mathrm{Ti}, \mathrm{X}\right){ }^{168 m} \mathrm{Lu} /{ }^{167} \mathrm{Hf} /{ }^{168} \mathrm{Hf}$, $\mathrm{E}=223.7 \mathrm{MeV} ;{ }^{116} \mathrm{Sn}\left({ }^{50} \mathrm{Ti}, \mathrm{X}\right){ }^{162} \mathrm{Tm} /{ }^{161} \mathrm{Yb} /{ }^{162} \mathrm{Yb} /{ }^{163} \mathrm{Yb} /{ }^{162} \mathrm{Lu} /$ ${ }^{162} \mathrm{Hf}, \mathrm{E}=224.4 \mathrm{MeV}$; measured delayed $\mathrm{E} \gamma, \mathrm{I} \gamma$ following residual nucleus decay. Physical preseparation technique. JOUR NIMAE 551 528

\section{$\mathrm{A}=\mathbf{1 6 9}$}

${ }^{169} \mathrm{Ir} \quad$ 2005SC22 NUCLEAR REACTIONS ${ }^{92} \mathrm{Mo}\left({ }^{78} \mathrm{Kr}, 2 \mathrm{np}\right), \mathrm{E}=360 \mathrm{MeV} ;{ }^{112} \mathrm{Sn}\left({ }^{58} \mathrm{Ni}\right.$, p), $\mathrm{E}=266 \mathrm{MeV}$; measured $\mathrm{E} \gamma, \mathrm{I} \gamma, \gamma \gamma-$, (recoil) $\gamma$-coin. ${ }^{167,169} \mathrm{Ir}$ deduced transitions. Recoil-decay tagging, Jurogam array. JOUR JPGPE 31 S1719

2005SC22 RADIOACTIVITY ${ }^{167,167 m, 169,169 m} \operatorname{Ir},{ }^{165,165 m} \mathrm{Re},{ }^{161} \mathrm{Ta}(\alpha)$ [from ${ }^{92} \mathrm{Mo}\left({ }^{78} \mathrm{Kr}, 2 \mathrm{np}\right)$ and ${ }^{112} \mathrm{Sn}\left({ }^{58} \mathrm{Ni}, \mathrm{p}\right)$ and subsequent decay]; measured $\mathrm{E} \alpha, \mathrm{E} \gamma, \alpha \gamma$-coin, $\mathrm{T}_{1 / 2}$; deduced spectroscopic factors. ${ }^{167,167 m} \operatorname{Ir}(\mathrm{p})$ [from $\left.{ }^{112} \mathrm{Sn}\left({ }^{58} \mathrm{Ni}, 2 \mathrm{np}\right)\right]$; measured Ep, $\mathrm{T}_{1 / 2}$; deduced spectroscopic factors. Jurogam array, mass separator. JOUR JPGPE 31 S1719 ${ }^{169} \mathrm{Pt}$ 2005J018 NUCLEAR REACTIONS $\mathrm{Sn}\left({ }^{58} \mathrm{Ni}, \mathrm{xn}\right){ }^{169} \mathrm{Pt} /{ }^{170} \mathrm{Pt} /{ }^{171} \mathrm{Pt} /{ }^{172} \mathrm{Pt} /$ ${ }^{173} \mathrm{Pt}, \mathrm{E}=266 \mathrm{MeV}$; measured $\mathrm{E} \gamma, \mathrm{I} \gamma, \gamma \gamma-$, (recoil) $\gamma$-coin. ${ }_{169,170,171,172,173} \mathrm{Pt}$ deduced levels, J, $\pi$. Recoil-decay tagging. JOUR JPGPE 31 S1715

\section{$\mathrm{A}=\mathbf{1 7 0}$}

${ }^{170} \mathrm{Pt}$ 2005J018 NUCLEAR REACTIONS Sn $\left({ }^{58} \mathrm{Ni}, \mathrm{xn}\right){ }^{169} \mathrm{Pt} /{ }^{170} \mathrm{Pt} /{ }^{171} \mathrm{Pt} /{ }^{172} \mathrm{Pt} /$ ${ }^{173} \mathrm{Pt}, \mathrm{E}=266 \mathrm{MeV}$; measured $\mathrm{E} \gamma, \mathrm{I} \gamma, \gamma \gamma-$, (recoil) $\gamma$-coin. ${ }_{169,170,171,172,173} \mathrm{Pt}$ deduced levels, J, $\pi$. Recoil-decay tagging. JOUR JPGPE 31 S1715 


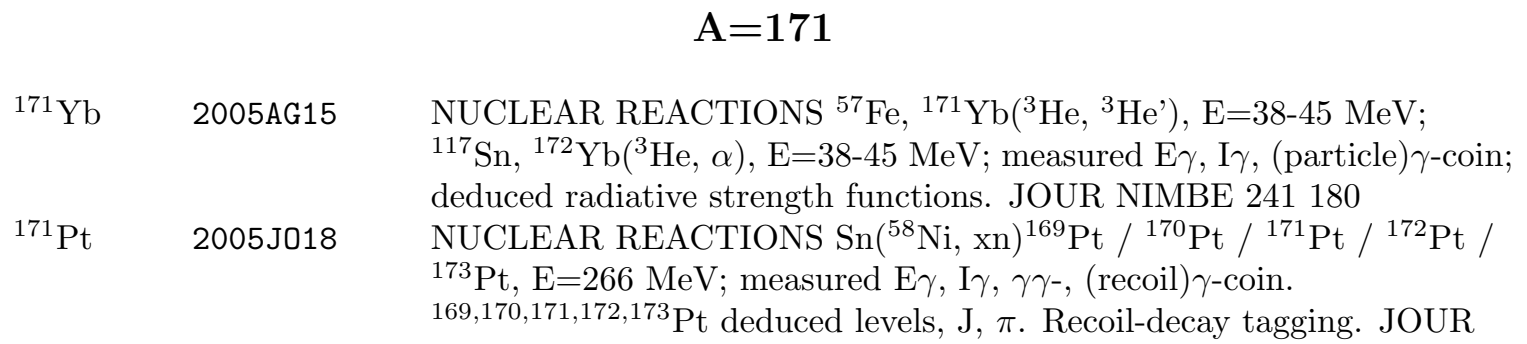

\section{$\mathrm{A}=172$}

${ }^{172} \mathrm{Yb} \quad 2005 \mathrm{VE} 07$

NUCLEAR REACTIONS ${ }^{170} \operatorname{Er}\left({ }^{7} \mathrm{Li}, 3 \mathrm{np}\right),\left({ }^{7} \mathrm{Li}, 4 \mathrm{np}\right),\left({ }^{7} \mathrm{Li}, 3 \mathrm{nd}\right),\left({ }^{7} \mathrm{Li}\right.$, 2nt), $\mathrm{E}=51 \mathrm{MeV}$; measured $\mathrm{E} \gamma, \mathrm{I} \gamma$, (charged particle) $\gamma$-coin. ${ }^{172,173} \mathrm{Yb}$ deduced high-spin levels, $\mathrm{J}, \pi$, configurations, absence of a static pair field. GASP, ISIS arrays. JOUR ZAANE 2619

${ }^{172} \mathrm{Hf} \quad$ 2005KA52

NUCLEAR REACTIONS ${ }^{177} \mathrm{Hf}(\mathrm{n}, \gamma), \mathrm{E}=$ thermal, resonance; ${ }^{178} \mathrm{Hf}(\mathrm{n}$, n' $\gamma), \mathrm{E}>3 \mathrm{MeV}$; measured isomer production $\sigma$. Ta, W, ${ }^{186} \mathrm{~W}, \operatorname{Re}(\mathrm{p}$, $\mathrm{X}){ }^{179 m} \mathrm{Hf} /{ }^{178 m} \mathrm{Hf} /{ }^{177 m} \mathrm{Lu} /{ }^{178} \mathrm{~W} /{ }^{175} \mathrm{Hf} /{ }^{172} \mathrm{Hf} /{ }^{173} \mathrm{Lu}, \mathrm{E}=650$ $\mathrm{MeV}$; analyzed yields, isomer ratios. ${ }^{176} \mathrm{Yb}(\alpha, 2 \mathrm{n}), \mathrm{E}<36 \mathrm{MeV}$; measured isomer yield. Other reactions discussed. JOUR YAFIA 68 1827

${ }^{172} \mathrm{Pt}$ 2005J018 NUCLEAR REACTIONS Sn $\left({ }^{58} \mathrm{Ni}, \mathrm{xn}\right){ }^{169} \mathrm{Pt} /{ }^{170} \mathrm{Pt} /{ }^{171} \mathrm{Pt} /{ }^{172} \mathrm{Pt} /$ ${ }^{173} \mathrm{Pt}, \mathrm{E}=266 \mathrm{MeV}$; measured $\mathrm{E} \gamma, \mathrm{I} \gamma, \gamma \gamma-$, (recoil) $\gamma$-coin. ${ }_{169,170,171,172,173} \mathrm{Pt}$ deduced levels, J, $\pi$. Recoil-decay tagging. JOUR JPGPE 31 S1715

\section{$\mathrm{A}=173$}

${ }^{173} \mathrm{Yb} \quad 2005 \mathrm{TE04}$

NUCLEAR REACTIONS ${ }^{172,173} \mathrm{Yb}(\mathrm{n}, \gamma), \mathrm{E}=$ resonance; measured $\mathrm{E} \gamma$, $\mathrm{I} \gamma$, capture yields. ${ }^{173} \mathrm{Yb}$ deduced resonance energies, $\mathrm{J}, \pi .{ }^{173,174} \mathrm{Yb}$ deduced levels, J, $\pi$. JOUR NUPAB 76331

2005VE07 NUCLEAR REACTIONS ${ }^{170} \mathrm{Er}\left({ }^{7} \mathrm{Li}, 3 \mathrm{np}\right),\left({ }^{7} \mathrm{Li}, 4 \mathrm{np}\right),\left({ }^{7} \mathrm{Li}, 3 \mathrm{nd}\right),\left({ }^{7} \mathrm{Li}\right.$, 2nt), $\mathrm{E}=51 \mathrm{MeV}$; measured $\mathrm{E} \gamma, \mathrm{I} \gamma$, (charged particle) $\gamma$-coin. ${ }^{172,173} \mathrm{Yb}$ deduced high-spin levels, J, $\pi$, configurations, absence of a static pair field. GASP, ISIS arrays. JOUR ZAANE 2619

${ }^{173} \mathrm{Lu}$ 2005KA52 NUCLEAR REACTIONS ${ }^{177} \mathrm{Hf}(\mathrm{n}, \gamma), \mathrm{E}=$ thermal, resonance; ${ }^{178} \mathrm{Hf}(\mathrm{n}$, n' $\gamma), \mathrm{E}>3 \mathrm{MeV}$; measured isomer production $\sigma$. Ta, W, ${ }^{186} \mathrm{~W}, \operatorname{Re}(\mathrm{p}$, $\mathrm{X}){ }^{179 m} \mathrm{Hf} /{ }^{178 m} \mathrm{Hf} /{ }^{177 m} \mathrm{Lu} /{ }^{178} \mathrm{~W} /{ }^{175} \mathrm{Hf} /{ }^{172} \mathrm{Hf} /{ }^{173} \mathrm{Lu}, \mathrm{E}=650$ $\mathrm{MeV}$; analyzed yields, isomer ratios. ${ }^{176} \mathrm{Yb}(\alpha, 2 \mathrm{n}), \mathrm{E}<36 \mathrm{MeV}$; measured isomer yield. Other reactions discussed. JOUR YAFIA 68 1827

${ }^{173} \mathrm{Ir} \quad$ 2005CA43 NUCLEAR REACTIONS ${ }^{92} \mathrm{Mo}\left({ }^{84} \mathrm{Sr}, \mathrm{n} 2 \mathrm{p}\right),\left({ }^{84} \mathrm{Sr}, 3 \mathrm{p}\right),\left({ }^{84} \mathrm{Sr}, 2 \mathrm{np}\right)$, ${ }^{104} \mathrm{Ru}\left({ }^{84} \mathrm{Kr}, 2 \mathrm{np}\right),{ }^{90} \mathrm{Zr}\left({ }^{90} \mathrm{Zr}, \mathrm{n}\right),\left({ }^{90} \mathrm{Zr}, \mathrm{p}\right)$, E not given; ${ }^{92} \mathrm{Mo}\left({ }^{90} \mathrm{Zr}, \mathrm{n}\right)$, $\left({ }^{90} \mathrm{Zr}, \mathrm{p}\right), \mathrm{E}=385 \mathrm{MeV}$; measured $\mathrm{E} \gamma, \mathrm{I} \gamma, \gamma \gamma-$, (recoil) $\gamma$-coin. ${ }^{179} \mathrm{Hg}$ deduced high-spin levels, J, $\pi$. Gammasphere array, fragment separator. JOUR JPGPE 31 S1599 


\section{$\mathrm{A}=173$ (continued)}

\begin{tabular}{|c|c|c|}
\hline \multirow[t]{2}{*}{${ }^{173} \mathrm{Pt}$} & $2005 \mathrm{CA} 43$ & $\begin{array}{l}\text { NUCLEAR REACTIONS }{ }^{92} \mathrm{Mo}\left({ }^{84} \mathrm{Sr}, \mathrm{n} 2 \mathrm{p}\right),\left({ }^{84} \mathrm{Sr}, 3 \mathrm{p}\right),\left({ }^{84} \mathrm{Sr}, 2 \mathrm{np}\right), \\
{ }^{104} \mathrm{Ru}\left({ }^{84} \mathrm{Kr}, 2 \mathrm{np}\right),{ }^{90} \mathrm{Zr}\left({ }^{90} \mathrm{Zr}, \mathrm{n}\right),\left({ }^{90} \mathrm{Zr}, \mathrm{p}\right) \text {, E not given; }{ }^{92} \mathrm{Mo}\left({ }^{90} \mathrm{Zr}, \mathrm{n}\right) \text {, } \\
\left.\left({ }^{90} \mathrm{Zr}, \mathrm{p}\right), \mathrm{E}=385 \mathrm{MeV} ; \text { measured } \mathrm{E} \gamma, \mathrm{I} \gamma, \gamma \gamma-\text {, (recoil }\right) \gamma \text {-coin. }{ }^{179} \mathrm{Hg} \\
\text { deduced high-spin levels, J, } \pi \text {. Gammasphere array, fragment } \\
\text { separator. JOUR JPGPE } 31 \mathrm{~S} 1599\end{array}$ \\
\hline & 2005J018 & $\begin{array}{l}\text { NUCLEAR REACTIONS Sn }\left({ }^{58} \mathrm{Ni}, \mathrm{xn}\right){ }^{169} \mathrm{Pt} /{ }^{170} \mathrm{Pt} /{ }^{171} \mathrm{Pt} /{ }^{172} \mathrm{Pt} / \\
\left.{ }^{173} \mathrm{Pt}, \mathrm{E}=266 \mathrm{MeV} ; \text { measured } \mathrm{E} \gamma, \mathrm{I} \gamma, \gamma \gamma-, \text { (recoil }\right) \gamma \text {-coin. } \\
{ }^{169,170,171,172,173} \mathrm{Pt} \text { deduced levels, J, } \pi \text {. Recoil-decay tagging. JOUR }\end{array}$ \\
\hline${ }^{3} \mathrm{Au}$ & $2005 \mathrm{CA} 43$ & $\begin{array}{l}\text { JPGPE } 31 \text { S1715 } \\
\text { NUCLEAR REACTIONS }{ }^{92} \mathrm{Mo}\left({ }^{84} \mathrm{Sr}, \mathrm{n} 2 \mathrm{p}\right),\left({ }^{84} \mathrm{Sr}, 3 \mathrm{p}\right),\left({ }^{84} \mathrm{Sr}, 2 \mathrm{np}\right), \\
{ }^{104} \mathrm{Ru}\left({ }^{84} \mathrm{Kr}, 2 \mathrm{np}\right),{ }^{90} \mathrm{Zr}\left({ }^{90} \mathrm{Zr}, \mathrm{n}\right),\left({ }^{90} \mathrm{Zr}, \mathrm{p}\right), \mathrm{E} \text { not given; }{ }^{92} \mathrm{Mo}\left({ }^{90} \mathrm{Zr}, \mathrm{n}\right) \text {, } \\
\left({ }^{90} \mathrm{Zr}, \mathrm{p}\right), \mathrm{E}=385 \mathrm{MeV} ; \text { measured } \mathrm{E} \gamma, \mathrm{I} \gamma, \gamma \gamma-,(\text { recoil }) \gamma \text {-coin. }{ }^{179} \mathrm{Hg} \\
\text { deduced high-spin levels, J, } \pi . \text { Gammasphere array, fragment } \\
\text { separator. JOUR JPGPE } 31 \mathrm{~S} 1599\end{array}$ \\
\hline
\end{tabular}

\section{$\mathrm{A}=\mathbf{1 7 4}$}

${ }^{174} \mathrm{Tm} \quad 2005 \mathrm{CH} 67$

RADIOACTIVITY ${ }^{174} \mathrm{Tm}$ (IT) [from Ta(p, X), E=500 MeV]; measured $\mathrm{E} \gamma, \mathrm{I} \gamma, \gamma \gamma$-coin following decay of mass-separated sources; deduced $\mathrm{T}_{1 / 2}$. Discussed K-hindrance and Nilsson configuration of new level. JOUR ZAANE 25 s01 125

${ }^{174} \mathrm{Yb}$ 2005TE04 NUCLEAR REACTIONS ${ }^{172,173} \mathrm{Yb}(\mathrm{n}, \gamma), \mathrm{E}=$ resonance; measured E $\gamma$, I $\gamma$, capture yields. ${ }^{173} \mathrm{Yb}$ deduced resonance energies, $\mathrm{J}, \pi .{ }^{173,174} \mathrm{Yb}$ deduced levels, J, $\pi$. JOUR NUPAB 76331

${ }^{174} \mathrm{Re} \quad 2005 \mathrm{ZH} 32 \quad$ NUCLEAR REACTIONS ${ }^{152} \mathrm{Sm}\left({ }^{27} \mathrm{Al}, 5 \mathrm{n}\right), \mathrm{E}=125,132,140 \mathrm{MeV}$; measured $\mathrm{E} \gamma, \mathrm{I} \gamma, \gamma \gamma$-coin; deduced excitation functions. ${ }^{174} \mathrm{Re}$ deduced high-spin levels, $\mathrm{J}, \pi$, configurations, signature inversion. Level systematics in neighboring nuclides discussed. JOUR CPLEE 222788

\section{$\mathrm{A}=\mathbf{1 7 5}$}

${ }^{175} \mathrm{Yb}$ 2005NC01 NUCLEAR REACTIONS ${ }^{176} \mathrm{Yb}\left({ }^{136} \mathrm{Xe}, \mathrm{X}\right){ }^{175} \mathrm{Yb} /{ }^{176} \mathrm{Yb} /{ }^{177} \mathrm{Yb}$, $\mathrm{E}=750 \mathrm{MeV}$; measured $\mathrm{E} \gamma, \mathrm{I} \gamma, \gamma \gamma$-coin. ${ }^{175,176,177} \mathrm{Yb}$ deduced high-spin levels, J, $\pi$, configurations, gK-gR. Afrodite array. JOUR ZAANE 26265

${ }^{175} \mathrm{Hf}$ 2005KA52 NUCLEAR REACTIONS ${ }^{177} \mathrm{Hf}(\mathrm{n}, \gamma), \mathrm{E}=$ thermal, resonance; ${ }^{178} \mathrm{Hf}(\mathrm{n}$, n' $\gamma), \mathrm{E}>3 \mathrm{MeV}$; measured isomer production $\sigma$. Ta, W, ${ }^{186} \mathrm{~W}, \operatorname{Re}(\mathrm{p}$, $\mathrm{X})^{179 m} \mathrm{Hf} /{ }^{178 m} \mathrm{Hf} /{ }^{177 m} \mathrm{Lu} /{ }^{178} \mathrm{~W} /{ }^{175} \mathrm{Hf} /{ }^{172} \mathrm{Hf} /{ }^{173} \mathrm{Lu}, \mathrm{E}=650$ $\mathrm{MeV}$; analyzed yields, isomer ratios. ${ }^{176} \mathrm{Yb}(\alpha, 2 \mathrm{n}), \mathrm{E}<36 \mathrm{MeV}$; measured isomer yield. Other reactions discussed. JOUR YAFIA 68 1827 


\section{$\mathrm{A}=176$}

${ }^{176} \mathrm{Yb} \quad 2005 B I 25$

2005NC01

${ }^{176} \mathrm{Hf} \quad 2005 \mathrm{ME} 19$

${ }^{176}$ Os 2005DE48

2005M033

${ }^{177} \mathrm{Yb}$

2005NC01

${ }^{177} \mathrm{Lu}$

2005KA52

\section{$\mathrm{A}=178$ \\ $\mathrm{A}=178$}

${ }^{178} \mathrm{Hf} \quad$ 2005KA52 ZAANE 26265 PRVCA 72034306

\section{$\mathrm{A}=\mathbf{1 7 7}$} ZAANE 26265 1827
NUCLEAR MOMENTS 86,87,88,89,90,91,92,93,94,95,96,97,98,99,100,101,102 Zr; measured charge radii. ${ }^{176} \mathrm{Yb}$; measured isomer shift. Ion-beam cooler, laser spectroscopy. JOUR ZAANE 25 s01 187 NUCLEAR REACTIONS ${ }^{176} \mathrm{Yb}\left({ }^{136} \mathrm{Xe}, \mathrm{X}\right){ }^{175} \mathrm{Yb} /{ }^{176} \mathrm{Yb} /{ }^{177} \mathrm{Yb}$, $\mathrm{E}=750 \mathrm{MeV}$; measured $\mathrm{E} \gamma, \mathrm{I} \gamma, \gamma \gamma$-coin. ${ }^{175,176,177} \mathrm{Yb}$ deduced high-spin levels, J, $\pi$, configurations, gK-gR. Afrodite array. JOUR NUCLEAR REACTIONS ${ }^{160} \mathrm{Gd},{ }^{164} \mathrm{Dy},{ }^{170} \mathrm{Er},{ }^{178} \mathrm{Hf},{ }^{186} \mathrm{~W},{ }^{192} \mathrm{Os}(\mathrm{p}$, $\mathrm{t}), \mathrm{E}=25 \mathrm{MeV}$; measured triton spectra, $\sigma(\theta) .{ }^{158} \mathrm{Gd},{ }^{162} \mathrm{Dy},{ }^{168} \mathrm{Er}$, ${ }^{176} \mathrm{Hf},{ }^{184} \mathrm{~W},{ }^{190}$ Os deduced $0^{+}$level energies. JOUR JPGPE 31 S1399 NUCLEAR REACTIONS ${ }^{164,166,168} \operatorname{Er}\left({ }^{16} \mathrm{O}, 4 \mathrm{n}\right), \mathrm{E}=80 \mathrm{MeV}$; measured prompt and delayed $\mathrm{E} \gamma, \mathrm{I} \gamma \cdot{ }^{154} \mathrm{Sm}\left({ }^{29} \mathrm{Si}, 5 \mathrm{n}\right), \mathrm{E}=158 \mathrm{MeV}$; measured Doppler-shifted $\mathrm{E} \gamma, \mathrm{I} \gamma, \gamma \gamma$-coin. ${ }^{176,178,180}$ Os deduced levels $\mathrm{T}_{1 / 2}$, transition quadrupole moments, symmetry features. Electronic timing and recoil distance techniques, GASP array, interacting boson model and general collective model predictions. JOUR JPGPE 31 S1427 NUCLEAR REACTIONS ${ }^{164,166,168} \operatorname{Er}\left({ }^{16} \mathrm{O}, 4 \mathrm{n}\right), \mathrm{E}=80 \mathrm{MeV}$; measured prompt and delayed $\mathrm{E} \gamma, \mathrm{I} \gamma, \gamma \gamma$-coin. ${ }^{166} \operatorname{Er}\left({ }^{16} \mathrm{O}, 4 \mathrm{n}\right), \mathrm{E}=80 \mathrm{MeV}$; measured Doppler-shifted E $\gamma, \mathrm{I} \gamma, \gamma \gamma$-coin. ${ }^{176,178,180}$ Os deduced levels, $\mathrm{J}, \pi, \mathrm{T}_{1 / 2}, \mathrm{~B}(\mathrm{E} 2)$. Pulsed-beam and recoil-distance techniques. JOUR

NUCLEAR REACTIONS ${ }^{176} \mathrm{Yb}\left({ }^{136} \mathrm{Xe}, \mathrm{X}\right){ }^{175} \mathrm{Yb} /{ }^{176} \mathrm{Yb} /{ }^{177} \mathrm{Yb}$, $\mathrm{E}=750 \mathrm{MeV}$; measured $\mathrm{E} \gamma, \mathrm{I} \gamma, \gamma \gamma$-coin. ${ }^{175,176,177} \mathrm{Yb}$ deduced high-spin levels, J, $\pi$, configurations, gK-gR. Afrodite array. JOUR

NUCLEAR REACTIONS ${ }^{177} \mathrm{Hf}(\mathrm{n}, \gamma), \mathrm{E}=$ thermal, resonance; ${ }^{178} \mathrm{Hf}(\mathrm{n}$, n' $\gamma), \mathrm{E}>3 \mathrm{MeV}$; measured isomer production $\sigma$. Ta, W, ${ }^{186} \mathrm{~W}, \operatorname{Re}(\mathrm{p}$, $\mathrm{X})^{179 m} \mathrm{Hf} /{ }^{178 m} \mathrm{Hf} /{ }^{177 m} \mathrm{Lu} /{ }^{178} \mathrm{~W} /{ }^{175} \mathrm{Hf} /{ }^{172} \mathrm{Hf} /{ }^{173} \mathrm{Lu}, \mathrm{E}=650$ $\mathrm{MeV}$; analyzed yields, isomer ratios. ${ }^{176} \mathrm{Yb}(\alpha, 2 \mathrm{n}), \mathrm{E}<36 \mathrm{MeV}$; measured isomer yield. Other reactions discussed. JOUR YAFIA 68

NUCLEAR REACTIONS ${ }^{177} \mathrm{Hf}(\mathrm{n}, \gamma), \mathrm{E}=$ thermal, resonance; ${ }^{178} \mathrm{Hf}(\mathrm{n}$, n' $\gamma), \mathrm{E}>3 \mathrm{MeV}$; measured isomer production $\sigma$. Ta, W, ${ }^{186} \mathrm{~W}, \operatorname{Re}(\mathrm{p}$, $\mathrm{X})^{179 m} \mathrm{Hf} /{ }^{178 m} \mathrm{Hf} /{ }^{177 m} \mathrm{Lu} /{ }^{178} \mathrm{~W} /{ }^{175} \mathrm{Hf} /{ }^{172} \mathrm{Hf} /{ }^{173} \mathrm{Lu}, \mathrm{E}=650$ $\mathrm{MeV}$; analyzed yields, isomer ratios. ${ }^{176} \mathrm{Yb}(\alpha, 2 \mathrm{n}), \mathrm{E}<36 \mathrm{MeV}$; measured isomer yield. Other reactions discussed. JOUR YAFIA 68 1827 


\section{$\mathrm{A}=178$ (continued)}

\begin{tabular}{|c|c|c|}
\hline${ }^{178} \mathrm{~W}$ & 2005KA52 & $\begin{array}{l}\text { NUCLEAR REACTIONS }{ }^{177} \mathrm{Hf}(\mathrm{n}, \gamma), \mathrm{E}=\text { thermal, resonance; }{ }^{178} \mathrm{Hf}(\mathrm{n} \text {, } \\
\mathrm{n} \gamma), \mathrm{E}>3 \mathrm{MeV} \text {; measured isomer production } \sigma . \mathrm{Ta}, \mathrm{W},{ }^{186} \mathrm{~W}, \mathrm{Re}(\mathrm{p} \text {, } \\
\mathrm{X}){ }^{179 m} \mathrm{Hf} /{ }^{178 m} \mathrm{Hf} /{ }^{177 m} \mathrm{Lu} /{ }^{178} \mathrm{~W} /{ }^{175} \mathrm{Hf} /{ }^{172} \mathrm{Hf} /{ }^{173} \mathrm{Lu}, \mathrm{E}=650 \\
\mathrm{MeV} \text {; analyzed yields, isomer ratios. }{ }^{176} \mathrm{Yb}(\alpha, 2 \mathrm{n}), \mathrm{E}<36 \mathrm{MeV} ; \\
\text { measured isomer yield. Other reactions discussed. JOUR YAFIA } 68 \\
1827\end{array}$ \\
\hline${ }^{178} \mathrm{Os}$ & 2005DE48 & $\begin{array}{l}\text { NUCLEAR REACTIONS }{ }^{164,166,168} \mathrm{Er}\left({ }^{16} \mathrm{O}, 4 \mathrm{n}\right), \mathrm{E}=80 \mathrm{MeV} \text {; measured } \\
\text { prompt and delayed } \mathrm{E} \gamma, \mathrm{I} \gamma \cdot{ }^{154} \mathrm{Sm}\left({ }^{29} \mathrm{Si}, 5 \mathrm{n}\right), \mathrm{E}=158 \mathrm{MeV} \text {; measured } \\
\text { Doppler-shifted } \mathrm{E} \gamma, \mathrm{I} \gamma, \gamma \gamma \text {-coin. }{ }^{176,178,180} \mathrm{Os} \text { deduced levels } \mathrm{T}_{1 / 2} \text {, } \\
\text { transition quadrupole moments, symmetry features. Electronic timing } \\
\text { and recoil distance techniques, GASP array, interacting boson model } \\
\text { and general collective model predictions. JOUR JPGPE } 31 \mathrm{~S} 1427\end{array}$ \\
\hline & 2005 MO33 & $\begin{array}{l}\text { NUCLEAR REACTIONS }{ }^{164,166,168} \operatorname{Er}\left({ }^{16} \mathrm{O}, 4 \mathrm{n}\right), \mathrm{E}=80 \mathrm{MeV} \text {; measured } \\
\text { prompt and delayed } \mathrm{E} \gamma, \mathrm{I} \gamma, \gamma \gamma \text {-coin. }{ }^{166} \operatorname{Er}\left({ }^{16} \mathrm{O}, 4 \mathrm{n}\right), \mathrm{E}=80 \mathrm{MeV} \text {; } \\
\text { measured Doppler-shifted } \mathrm{E} \gamma, \mathrm{I} \gamma, \gamma \gamma \text {-coin. }{ }^{176,178,180} \mathrm{Os} \text { deduced levels, } \\
\mathrm{J}, \pi, \mathrm{T}_{1 / 2}, \mathrm{~B}(\mathrm{E} 2) . \text { Pulsed-beam and recoil-distance techniques. JOUR } \\
\text { PRVCA } 72034306\end{array}$ \\
\hline
\end{tabular}

$$
\mathrm{A}=\mathbf{1 7 9}
$$

${ }^{179} \mathrm{Hf} \quad$ 2005KA52

NUCLEAR REACTIONS ${ }^{177} \mathrm{Hf}(\mathrm{n}, \gamma), \mathrm{E}=$ thermal, resonance; ${ }^{178} \mathrm{Hf}(\mathrm{n}$, n' $\gamma)$, E $>3 \mathrm{MeV}$; measured isomer production $\sigma$. Ta, W, ${ }^{186} \mathrm{~W}, \operatorname{Re}(\mathrm{p}$, $\mathrm{X}){ }^{179 m} \mathrm{Hf} /{ }^{178 m} \mathrm{Hf} /{ }^{177 m} \mathrm{Lu} /{ }^{178} \mathrm{~W} /{ }^{175} \mathrm{Hf} /{ }^{172} \mathrm{Hf} /{ }^{173} \mathrm{Lu}, \mathrm{E}=650$ $\mathrm{MeV}$; analyzed yields, isomer ratios. ${ }^{176} \mathrm{Yb}(\alpha, 2 \mathrm{n}), \mathrm{E}<36 \mathrm{MeV}$; measured isomer yield. Other reactions discussed. JOUR YAFIA 68 1827

${ }^{179} \mathrm{Au} \quad$ 2005CA43 NUCLEAR REACTIONS ${ }^{92} \mathrm{Mo}\left({ }^{84} \mathrm{Sr}, \mathrm{n} 2 \mathrm{p}\right),\left({ }^{84} \mathrm{Sr}, 3 \mathrm{p}\right),\left({ }^{84} \mathrm{Sr}, 2 \mathrm{np}\right)$, ${ }^{104} \mathrm{Ru}\left({ }^{84} \mathrm{Kr}, 2 \mathrm{np}\right),{ }^{90} \mathrm{Zr}\left({ }^{90} \mathrm{Zr}, \mathrm{n}\right),\left({ }^{90} \mathrm{Zr}, \mathrm{p}\right)$, E not given; ${ }^{92} \mathrm{Mo}\left({ }^{90} \mathrm{Zr}, \mathrm{n}\right)$, $\left({ }^{90} \mathrm{Zr}, \mathrm{p}\right), \mathrm{E}=385 \mathrm{MeV}$; measured $\mathrm{E} \gamma, \mathrm{I} \gamma, \gamma \gamma-$, (recoil) $\gamma$-coin. ${ }^{179} \mathrm{Hg}$ deduced high-spin levels, $\mathrm{J}, \pi$. Gammasphere array, fragment separator. JOUR JPGPE 31 S1599

${ }^{179} \mathrm{Hg} \quad$ 2005CA43 NUCLEAR REACTIONS ${ }^{92} \mathrm{Mo}\left({ }^{84} \mathrm{Sr}, \mathrm{n} 2 \mathrm{p}\right),\left({ }^{84} \mathrm{Sr}, 3 \mathrm{p}\right),\left({ }^{84} \mathrm{Sr}, 2 \mathrm{np}\right)$, ${ }^{104} \mathrm{Ru}\left({ }^{84} \mathrm{Kr}, 2 \mathrm{np}\right),{ }^{90} \mathrm{Zr}\left({ }^{90} \mathrm{Zr}, \mathrm{n}\right),\left({ }^{90} \mathrm{Zr}, \mathrm{p}\right)$, E not given; ${ }^{92} \mathrm{Mo}\left({ }^{90} \mathrm{Zr}, \mathrm{n}\right)$, $\left({ }^{90} \mathrm{Zr}, \mathrm{p}\right), \mathrm{E}=385 \mathrm{MeV}$; measured $\mathrm{E} \gamma, \mathrm{I} \gamma, \gamma \gamma-$, (recoil) $\gamma$-coin. ${ }^{179} \mathrm{Hg}$ deduced high-spin levels, J, $\pi$. Gammasphere array, fragment separator. JOUR JPGPE 31 S1599

\section{$\mathrm{A}=\mathbf{1 8 0}$}

${ }^{180} \mathrm{Re}$ 2005EL10 NUCLEAR REACTIONS ${ }^{174} \mathrm{Yb}\left({ }^{11} \mathrm{~B}, 5 \mathrm{n}\right), \mathrm{E}=71 \mathrm{MeV}$; measured $\mathrm{E} \gamma$, $\mathrm{I} \gamma, \mathrm{E}(\mathrm{ce}), \mathrm{I}(\mathrm{ce}), \gamma \gamma-$, (ce) $\gamma$-coin. ${ }^{180}$ Re deduced high-spin levels, $\mathrm{J}, \pi$, ICC, configurations, K-forbidden transitions. Potential energy surface calculations. JOUR PRVCA 72054306 


\section{$\mathrm{A}=180$ (continued)}

${ }^{180} \mathrm{Os} \quad 2005 \mathrm{DE} 48$

2005M033

${ }^{181} \mathrm{~Pb}$

2005CA43

NUCLEAR REACTIONS ${ }^{164,166,168} \operatorname{Er}\left({ }^{16} \mathrm{O}, 4 \mathrm{n}\right), \mathrm{E}=80 \mathrm{MeV}$; measured prompt and delayed $\mathrm{E} \gamma, \mathrm{I} \gamma \cdot{ }^{154} \mathrm{Sm}\left({ }^{29} \mathrm{Si}, 5 \mathrm{n}\right), \mathrm{E}=158 \mathrm{MeV}$; measured Doppler-shifted E $\gamma$, I $\gamma, \gamma \gamma$-coin. ${ }^{176,178,180}$ Os deduced levels $\mathrm{T}_{1 / 2}$, transition quadrupole moments, symmetry features. Electronic timing and recoil distance techniques, GASP array, interacting boson model and general collective model predictions. JOUR JPGPE 31 S1427 NUCLEAR REACTIONS ${ }^{164,166,168} \operatorname{Er}\left({ }^{16} \mathrm{O}, 4 \mathrm{n}\right), \mathrm{E}=80 \mathrm{MeV}$; measured prompt and delayed $\mathrm{E} \gamma$, I $\gamma, \gamma \gamma$-coin. ${ }^{166} \operatorname{Er}\left({ }^{16} \mathrm{O}, 4 \mathrm{n}\right), \mathrm{E}=80 \mathrm{MeV}$; measured Doppler-shifted $\mathrm{E} \gamma, \mathrm{I} \gamma, \gamma \gamma$-coin. ${ }^{176,178,180}$ Os deduced levels, $\mathrm{J}, \pi, \mathrm{T}_{1 / 2}, \mathrm{~B}(\mathrm{E} 2)$. Pulsed-beam and recoil-distance techniques. JOUR PRVCA 72034306

\section{$\mathrm{A}=181$}

NUCLEAR REACTIONS ${ }^{150} \mathrm{Nd}\left({ }^{36} \mathrm{~S}, 3 \mathrm{n}\right),\left({ }^{36} \mathrm{~S}, 5 \mathrm{n}\right)$, E not given; measured prompt and delayed $\mathrm{E} \gamma, \mathrm{I} \gamma, \gamma \gamma$-coin. ${ }^{181,183}$ Os deduced levels, $\mathrm{J}, \pi$, configurations. Comparison with tilted axis cranking model predictions. JOUR JPGPE 31 S1709

2005CA43 NUCLEAR REACTIONS ${ }^{92} \mathrm{Mo}\left({ }^{84} \mathrm{Sr}, \mathrm{n} 2 \mathrm{p}\right),\left({ }^{84} \mathrm{Sr}, 3 \mathrm{p}\right),\left({ }^{84} \mathrm{Sr}, 2 \mathrm{np}\right)$, ${ }^{104} \mathrm{Ru}\left({ }^{84} \mathrm{Kr}, 2 \mathrm{np}\right),{ }^{90} \mathrm{Zr}\left({ }^{90} \mathrm{Zr}, \mathrm{n}\right),\left({ }^{90} \mathrm{Zr}, \mathrm{p}\right)$, E not given; ${ }^{92} \mathrm{Mo}\left({ }^{90} \mathrm{Zr}, \mathrm{n}\right)$, $\left({ }^{90} \mathrm{Zr}, \mathrm{p}\right), \mathrm{E}=385 \mathrm{MeV}$; measured $\mathrm{E} \gamma$, I $\gamma, \gamma \gamma-$, (recoil) $\gamma$-coin. ${ }^{179} \mathrm{Hg}$ deduced high-spin levels, J, $\pi$. Gammasphere array, fragment separator. JOUR JPGPE 31 S1599 NUCLEAR REACTIONS ${ }^{92} \mathrm{Mo}\left({ }^{84} \mathrm{Sr}, \mathrm{n} 2 \mathrm{p}\right),\left({ }^{84} \mathrm{Sr}, 3 \mathrm{p}\right),\left({ }^{84} \mathrm{Sr}, 2 \mathrm{np}\right)$, ${ }^{104} \mathrm{Ru}\left({ }^{84} \mathrm{Kr}, 2 \mathrm{np}\right),{ }^{90} \mathrm{Zr}\left({ }^{90} \mathrm{Zr}, \mathrm{n}\right),\left({ }^{90} \mathrm{Zr}, \mathrm{p}\right)$, E not given; ${ }^{92} \mathrm{Mo}\left({ }^{90} \mathrm{Zr}, \mathrm{n}\right)$, $\left({ }^{90} \mathrm{Zr}, \mathrm{p}\right), \mathrm{E}=385 \mathrm{MeV}$; measured $\mathrm{E} \gamma, \mathrm{I} \gamma, \gamma \gamma-$, (recoil $) \gamma$-coin. ${ }^{179} \mathrm{Hg}$ deduced high-spin levels, J, $\pi$. Gammasphere array, fragment separator. JOUR JPGPE 31 S1599

\section{$\mathrm{A}=182$}

No references found

\section{$\mathrm{A}=\mathbf{1 8 3}$}

${ }^{183} \mathrm{Re} \quad$ 2005CL07 NUCLEAR REACTIONS ${ }^{184} \mathrm{~W}\left({ }^{7} \mathrm{Li}, \mathrm{xn}\right),\left({ }^{7} \mathrm{Li}, \mathrm{xnp}\right),\left({ }^{7} \mathrm{Li}, \mathrm{xn} \alpha\right)$, $\mathrm{E}=35-70 \mathrm{MeV}$; calculated $\sigma \cdot{ }^{184} \mathrm{~W}\left({ }^{7} \mathrm{Li}, \mathrm{X}\right){ }^{184} \mathrm{Os} /{ }^{185} \mathrm{Os} /{ }^{186} \mathrm{Os} /$ ${ }^{188} \mathrm{Os} /{ }^{184} \operatorname{Ir} /{ }^{185} \operatorname{Ir} /{ }^{186} \operatorname{Ir} /{ }^{183} \operatorname{Re} /{ }^{185} \mathrm{Re}, \mathrm{E}=40-70 \mathrm{MeV}$; measured $\mathrm{E} \gamma, \mathrm{I} \gamma, \gamma \gamma-$, (charged particle) $\gamma$-coin, particle yield ratios. ${ }^{160} \mathrm{Gd}\left({ }^{7} \mathrm{Li}\right.$, xnp), $\mathrm{E}=35-65 \mathrm{MeV}$; analyzed $\sigma$. Liberace, Stars arrays. JOUR PRVCA 72054605

${ }^{183} \mathrm{Os} \quad 2005 \mathrm{CU} 05 \quad$ NUCLEAR REACTIONS ${ }^{150} \mathrm{Nd}\left({ }^{36} \mathrm{~S}, 3 \mathrm{n}\right),\left({ }^{36} \mathrm{~S}, 5 \mathrm{n}\right)$, E not given; measured prompt and delayed $\mathrm{E} \gamma, \mathrm{I} \gamma, \gamma \gamma$-coin. ${ }^{181,183}$ Os deduced levels, J, $\pi$, configurations. Comparison with tilted axis cranking model predictions. JOUR JPGPE 31 S1709 


\section{$\mathrm{A}=184$}

${ }^{184} \mathrm{~W}$ 2004L022 NUCLEAR REACTIONS ${ }^{183} \mathrm{~W}(\mathrm{n}, \gamma), \mathrm{E}=$ thermal; measured E $\gamma, \mathrm{I} \gamma$ ${ }^{184} \mathrm{~W}$ deduced levels, J, $\pi$, neutron binding energy. JOUR BRSPE 68 1292

2005ME19 NUCLEAR REACTIONS ${ }^{160} \mathrm{Gd},{ }^{164} \mathrm{Dy},{ }^{170} \mathrm{Er},{ }^{178} \mathrm{Hf},{ }^{186} \mathrm{~W},{ }^{192} \mathrm{Os}(\mathrm{p}$, $\mathrm{t}), \mathrm{E}=25 \mathrm{MeV}$; measured triton spectra, $\sigma(\theta) .{ }^{158} \mathrm{Gd},{ }^{162} \mathrm{Dy},{ }^{168} \mathrm{Er}$, ${ }^{176} \mathrm{Hf},{ }^{184} \mathrm{~W},{ }^{190}$ Os deduced $0^{+}$level energies. JOUR JPGPE 31 S1399

${ }^{184} \mathrm{Re} \quad 2005 \mathrm{WH} 04$ NUCLEAR REACTIONS ${ }^{180} \mathrm{Hf}\left({ }^{7} \mathrm{Li}, 3 \mathrm{n}\right), \mathrm{E}=30 \mathrm{MeV}$; measured prompt and delayed $\mathrm{E} \gamma, \mathrm{I} \gamma, \gamma \gamma$-coin, DCO ratios. ${ }^{184}$ Re deduced levels, $\mathrm{J}, \pi, \mathrm{T}_{1 / 2}$, gK - gR, configurations, rotational bands. Comparison with Nilsson-type blocked BCS calculations. JOUR NUPAB 7631

${ }^{184} \mathrm{Os} \quad 2005 \mathrm{CL} 07 \quad$ NUCLEAR REACTIONS ${ }^{184} \mathrm{~W}\left({ }^{7} \mathrm{Li}, \mathrm{xn}\right),\left({ }^{7} \mathrm{Li}, \mathrm{xnp}\right),\left({ }^{7} \mathrm{Li}, \mathrm{xn} \alpha\right)$, $\mathrm{E}=35-70 \mathrm{MeV}$; calculated $\sigma .{ }^{184} \mathrm{~W}\left({ }^{7} \mathrm{Li}, \mathrm{X}\right){ }^{184} \mathrm{Os} /{ }^{185} \mathrm{Os} /{ }^{186} \mathrm{Os} /$ ${ }^{188} \mathrm{Os} /{ }^{184} \mathrm{Ir} /{ }^{185} \mathrm{Ir} /{ }^{186} \mathrm{Ir} /{ }^{183} \mathrm{Re} /{ }^{185} \mathrm{Re}, \mathrm{E}=40-70 \mathrm{MeV}$; measured $\mathrm{E} \gamma, \mathrm{I} \gamma, \gamma \gamma-$, (charged particle) $\gamma$-coin, particle yield ratios. ${ }^{160} \mathrm{Gd}\left({ }^{7} \mathrm{Li}\right.$, xnp), E=35-65 MeV; analyzed $\sigma$. Liberace, Stars arrays. JOUR PRVCA 72054605

${ }^{184} \mathrm{Ir} \quad 2005 \mathrm{CL} 07$

NUCLEAR REACTIONS ${ }^{184} \mathrm{~W}\left({ }^{7} \mathrm{Li}, \mathrm{xn}\right),\left({ }^{7} \mathrm{Li}, \mathrm{xnp}\right),\left({ }^{7} \mathrm{Li}, \mathrm{xn} \alpha\right)$, $\mathrm{E}=35-70 \mathrm{MeV}$; calculated $\sigma .{ }^{184} \mathrm{~W}\left({ }^{7} \mathrm{Li}, \mathrm{X}\right){ }^{184} \mathrm{Os} /{ }^{185} \mathrm{Os} /{ }^{186} \mathrm{Os} /$ ${ }^{188} \mathrm{Os} /{ }^{184} \mathrm{Ir} /{ }^{185} \mathrm{Ir} /{ }^{186} \mathrm{Ir} /{ }^{183} \mathrm{Re} /{ }^{185} \mathrm{Re}, \mathrm{E}=40-70 \mathrm{MeV}$; measured $\mathrm{E} \gamma, \mathrm{I} \gamma, \gamma \gamma$-, (charged particle) $\gamma$-coin, particle yield ratios. ${ }^{160} \mathrm{Gd}\left({ }^{7} \mathrm{Li}\right.$, xnp), E=35-65 MeV; analyzed $\sigma$. Liberace, Stars arrays. JOUR PRVCA 72054605

${ }^{184} \mathrm{Au} \quad 2005 \mathrm{ZH} 30 \quad$ NUCLEAR REACTIONS ${ }^{159} \mathrm{~Tb}\left({ }^{29} \mathrm{Si}, 4 \mathrm{n}\right), \mathrm{E}=140 \mathrm{MeV}$; measured E $\gamma$, $\mathrm{I} \gamma, \gamma \gamma$-coin. ${ }^{184} \mathrm{Au}$ deduced high-spin levels, J, $\pi$, configurations, signature inversion. GASP array. JOUR JPGPE 31 S1545

${ }^{184} \mathrm{~Pb} 2005 \mathrm{UU} 03$ RADIOACTIVITY ${ }^{188,190,192,194,196,198,200,202,204} \mathrm{Po}$, 191,193,195,197,199 At, ${ }^{196,198,200,202,204,206} \mathrm{Rn},{ }^{199,201,203,205,207} \operatorname{Fr}(\alpha)$; measured reduced widths using gas filled recoil separator; deduced hindrance factors, proton intruder states and deformation effects.

JOUR ZAANE 25 s01 179

\section{$\mathrm{A}=185$}

${ }^{185} \mathrm{~W} \quad 2005 \mathrm{~B} 047$

NUCLEAR REACTIONS ${ }^{184} \mathrm{~W}(\mathrm{n}, \gamma), \mathrm{E}=$ thermal; measured prompt and delayed $\mathrm{E} \gamma, \mathrm{I} \gamma, \gamma \gamma$-coin. ${ }^{184} \mathrm{~W}$ (polarized d, p), $\mathrm{E}=18,21 \mathrm{MeV}$; ${ }^{186} \mathrm{~W}$ (polarized d, t), E=22 MeV; measured particle spectra, $\sigma(\theta)$, asymmetry. ${ }^{185} \mathrm{~W}$ deduced levels, J, $\pi, \gamma$-branching ratios, cross sections, binding energy, spectroscopic factors. DWBA analysis, quasiparticle-phonon model calculation. Enriched targets, Ge detectors, Q3D magnetic spectrograph. JOUR NUPAB 762167

${ }^{185} \mathrm{Re} \quad 2005 \mathrm{CL} 07 \quad$ NUCLEAR REACTIONS ${ }^{184} \mathrm{~W}\left({ }^{7} \mathrm{Li}, \mathrm{xn}\right),\left({ }^{7} \mathrm{Li}, \mathrm{xnp}\right),\left({ }^{7} \mathrm{Li}, \mathrm{xn} \alpha\right)$, $\mathrm{E}=35-70 \mathrm{MeV}$; calculated $\sigma .{ }^{184} \mathrm{~W}\left({ }^{7} \mathrm{Li}, \mathrm{X}\right){ }^{184} \mathrm{Os} /{ }^{185} \mathrm{Os} /{ }^{186} \mathrm{Os} /$ ${ }^{188} \mathrm{Os} /{ }^{184} \mathrm{Ir} /{ }^{185} \mathrm{Ir} /{ }^{186} \mathrm{Ir} /{ }^{183} \mathrm{Re} /{ }^{185} \mathrm{Re}, \mathrm{E}=40-70 \mathrm{MeV}$; measured $\mathrm{E} \gamma, \mathrm{I} \gamma, \gamma \gamma-$, (charged particle) $\gamma$-coin, particle yield ratios. ${ }^{160} \mathrm{Gd}\left({ }^{7} \mathrm{Li}\right.$, xnp), E=35-65 MeV; analyzed $\sigma$. Liberace, Stars arrays. JOUR PRVCA 72054605 


\section{$\mathrm{A}=185$ (continued)}

\begin{tabular}{|c|c|c|}
\hline \multirow[t]{2}{*}{${ }^{185} \mathrm{Os}$} & 2005CL07 & $\begin{array}{l}\text { NUCLEAR REACTIONS }{ }^{184} \mathrm{~W}\left({ }^{7} \mathrm{Li}, \mathrm{xn}\right),\left({ }^{7} \mathrm{Li} \text {, xnp }\right),\left({ }^{7} \mathrm{Li}, \mathrm{xn} \alpha\right) \text {, } \\
\mathrm{E}=35-70 \mathrm{MeV} \text {; calculated } \sigma .{ }^{184} \mathrm{~W}\left({ }^{7} \mathrm{Li}, \mathrm{X}\right){ }^{184} \mathrm{Os} /{ }^{185} \mathrm{Os} /{ }^{186} \mathrm{Os} / \\
{ }^{188} \mathrm{Os} /{ }^{184} \mathrm{Ir} /{ }^{185} \mathrm{Ir} /{ }^{186} \mathrm{Ir} /{ }^{183} \mathrm{Re} /{ }^{185} \mathrm{Re}, \mathrm{E}=40-70 \mathrm{MeV} \text {; measured } \\
\mathrm{E} \gamma, \mathrm{I} \gamma, \gamma \gamma-, \text { (charged particle) } \gamma \text {-coin, particle yield ratios. }{ }^{160} \mathrm{Gd}\left({ }^{7} \mathrm{Li} \text {, }\right. \\
\text { xnp), E }=35-65 \mathrm{MeV} \text {; analyzed } \sigma . \text { Liberace, Stars arrays. JOUR } \\
\text { PRVCA } 72054605\end{array}$ \\
\hline & 2005 TA26 & $\begin{array}{l}\text { NUCLEAR REACTIONS Ir }(\mathrm{p}, \mathrm{xnyp}))^{188} \mathrm{Pt} /{ }^{189} \mathrm{Pt} /{ }^{191} \mathrm{Pt} /{ }^{185} \mathrm{Ir} / \\
{ }^{186} \mathrm{Ir} /{ }^{188} \mathrm{Ir} /{ }^{189} \mathrm{Ir} /{ }^{190} \mathrm{Ir} /{ }^{192} \mathrm{Ir} /{ }^{185} \mathrm{Os}, \mathrm{E} \approx 3-70 \mathrm{MeV} ; \text { measured } \sigma ; \\
\text { deduced integral yields. Stacked-foil activation technique. JOUR } \\
\text { NIMBE } 239293\end{array}$ \\
\hline \multirow[t]{2}{*}{${ }^{185} \mathrm{Ir}$} & 2005CL07 & $\begin{array}{l}\text { NUCLEAR REACTIONS }{ }^{184} \mathrm{~W}\left({ }^{7} \mathrm{Li}, \mathrm{xn}\right),\left({ }^{7} \mathrm{Li}, \mathrm{xnp}\right),\left({ }^{7} \mathrm{Li}, \mathrm{xn} \alpha\right) \text {, } \\
\mathrm{E}=35-70 \mathrm{MeV} \text {; calculated } \sigma .{ }^{184} \mathrm{~W}\left({ }^{7} \mathrm{Li}, \mathrm{X}\right){ }^{184} \mathrm{Os} /{ }^{185} \mathrm{Os} /{ }^{186} \mathrm{Os} / \\
{ }^{188} \mathrm{Os} /{ }^{184} \mathrm{Ir} /{ }^{185} \mathrm{Ir} /{ }^{186} \mathrm{Ir} /{ }^{183} \mathrm{Re} /{ }^{185} \mathrm{Re}, \mathrm{E}=40-70 \mathrm{MeV} ; \text { measured } \\
\mathrm{E} \gamma, \mathrm{I} \gamma, \gamma \gamma-,(\text { charged particle }) \gamma \text {-coin, particle yield ratios. }{ }^{160} \mathrm{Gd}\left({ }^{7} \mathrm{Li} \text {, }\right. \\
\text { xnp}), \mathrm{E}=35-65 \mathrm{MeV} \text {; analyzed } \sigma . \text { Liberace, Stars arrays. JOUR } \\
\text { PRVCA } 72054605\end{array}$ \\
\hline & 2005 TA26 & $\begin{array}{l}\text { NUCLEAR REACTIONS } \operatorname{Ir}(\mathrm{p}, \mathrm{xnyp}){ }^{188} \mathrm{Pt} /{ }^{189} \mathrm{Pt} /{ }^{191} \mathrm{Pt} /{ }^{185} \mathrm{Ir} / \\
{ }^{186} \mathrm{Ir} /{ }^{188} \mathrm{Ir} /{ }^{189} \mathrm{Ir} /{ }^{190} \mathrm{Ir} /{ }^{192} \mathrm{Ir} /{ }^{185} \mathrm{Os}, \mathrm{E} \approx 3-70 \mathrm{MeV} ; \text { measured } \sigma ; \\
\text { deduced integral yields. Stacked-foil activation technique. JOUR } \\
\text { NIMBE } 239293\end{array}$ \\
\hline${ }^{185} \mathrm{Au}$ & $2005 \mathrm{CA} 43$ & $\begin{array}{l}\text { NUCLEAR REACTIONS }{ }^{92} \mathrm{Mo}\left({ }^{84} \mathrm{Sr}, \mathrm{n} 2 \mathrm{p}\right),\left({ }^{84} \mathrm{Sr}, 3 \mathrm{p}\right),\left({ }^{84} \mathrm{Sr}, 2 \mathrm{np}\right) \text {, } \\
{ }^{104} \mathrm{Ru}\left({ }^{84} \mathrm{Kr}, 2 \mathrm{np}\right),{ }^{90} \mathrm{Zr}\left({ }^{90} \mathrm{Zr}, \mathrm{n}\right),\left({ }^{90} \mathrm{Zr}, \mathrm{p}\right), \mathrm{E} \text { not given; }{ }^{92} \mathrm{Mo}\left({ }^{90} \mathrm{Zr}, \mathrm{n}\right) \text {, } \\
\left.\left({ }^{90} \mathrm{Zr}, \mathrm{p}\right), \mathrm{E}=385 \mathrm{MeV} \text { measured } \mathrm{E} \gamma, \mathrm{I} \gamma, \gamma \gamma-\text {, (recoil }\right) \gamma \text {-coin. }{ }^{179} \mathrm{Hg} \\
\text { deduced high-spin levels, J, } \pi \text {. Gammasphere array, fragment } \\
\text { separator. JOUR JPGPE } 31 \text { S1599 }\end{array}$ \\
\hline${ }^{185} \mathrm{Bi}$ & 2005GEZW & $\begin{array}{l}\text { ATOMIC MASSES }{ }^{235} \mathrm{Ac} ; \text { measured mass, } \mathrm{T}_{1 / 2} \text {. } \\
\text { 185,186,187,188,189,190,191,192,193,194,195,196} \mathrm{Bi} \text {; measured masses, proton } \\
\text { separation energies. }{ }^{207 m} \mathrm{Tl} \text {; measured } \mathrm{T}_{1 / 2} . \text { Stored beams, Schottky } \\
\text { mass spectrometry. PREPRINT nucl-ex/0510009,10/4/2005 }\end{array}$ \\
\hline
\end{tabular}

\section{$\mathrm{A}=\mathbf{1 8 6}$}

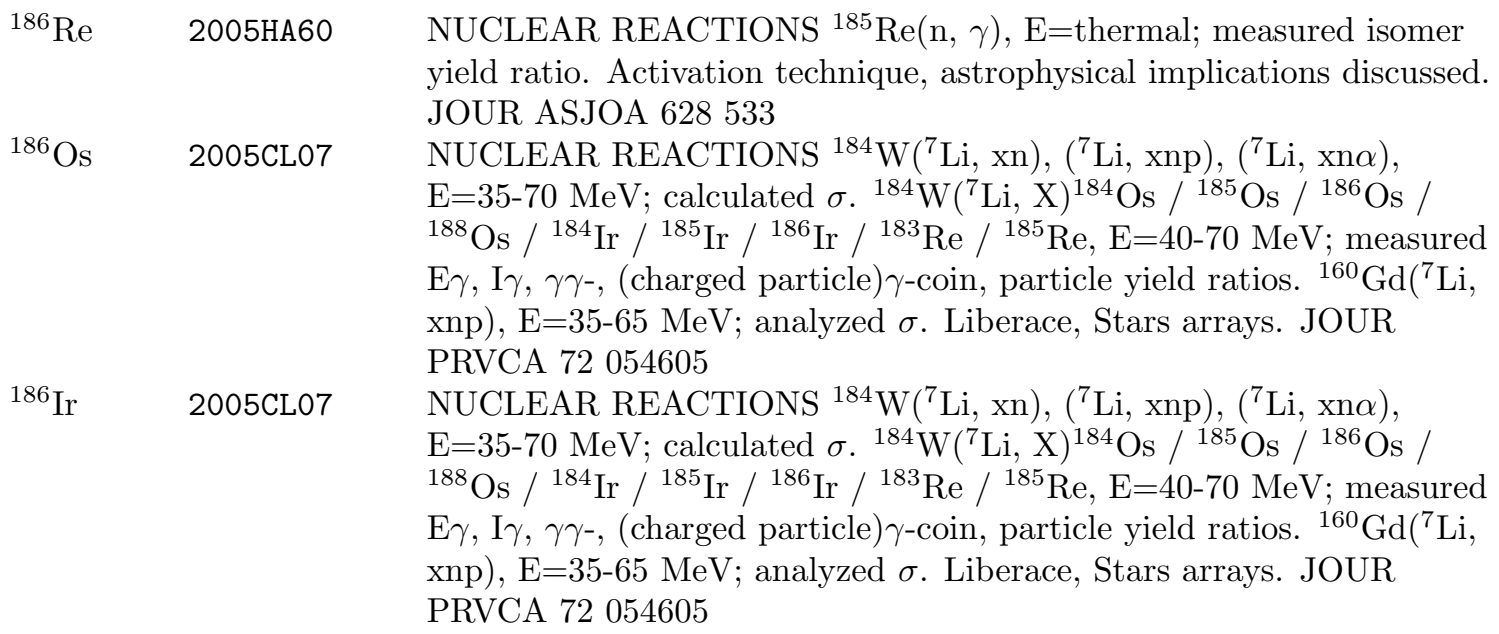




\section{$\mathrm{A}=186$ (continued)}

2005 TA26 NUCLEAR REACTIONS Ir $\left(\mathrm{p}\right.$, xnyp) ${ }^{188} \mathrm{Pt} /{ }^{189} \mathrm{Pt} /{ }^{191} \mathrm{Pt} /{ }^{185} \mathrm{Ir} /$ ${ }^{186} \operatorname{Ir} /{ }^{188} \operatorname{Ir} /{ }^{189} \operatorname{Ir} /{ }^{190} \operatorname{Ir} /{ }^{192} \operatorname{Ir} /{ }^{185} \mathrm{Os}, \mathrm{E} \approx 3-70 \mathrm{MeV}$; measured $\sigma$; deduced integral yields. Stacked-foil activation technique. JOUR NIMBE 239293

${ }^{186} \mathrm{~Pb}$ 2005PA69 NUCLEAR REACTIONS ${ }^{106} \mathrm{Pd}\left({ }^{83} \mathrm{Kr}, 3 \mathrm{n}\right), \mathrm{E}=355 \mathrm{MeV}$; measured $\mathrm{E} \gamma$, $\mathrm{I} \gamma, \gamma \gamma$-, (recoil) $\gamma$-coin; deduced production $\sigma .{ }^{186} \mathrm{~Pb}$ deduced levels, J, $\pi$, deformation. Jurogam array, recoil-decay tagging. JOUR ZAANE 25 s01 449

2005UU03 RADIOACTIVITY ${ }^{188,190,192,194,196,198,200,202,204} \mathrm{Po}$, ${ }_{191,193,195,197,199}$ At, ${ }^{196,198,200,202,204,206} \mathrm{Rn},{ }^{199,201,203,205,207} \operatorname{Fr}(\alpha)$; measured reduced widths using gas filled recoil separator; deduced hindrance factors, proton intruder states and deformation effects. JOUR ZAANE 25 s01 179

${ }^{186} \mathrm{Bi} \quad$ 2005GEZW ATOMIC MASSES ${ }^{235}$ Ac; measured mass, $\mathrm{T}_{1 / 2}$. $185,186,187,188,189,190,191,192,193,194,195,196 \mathrm{Bi}$; measured masses, proton separation energies. ${ }^{207 m} \mathrm{Tl}$; measured $\mathrm{T}_{1 / 2}$. Stored beams, Schottky mass spectrometry. PREPRINT nucl-ex/0510009,10/4/2005

\section{$\mathrm{A}=187$}

${ }^{187} \mathrm{~Pb} \quad$ 2005WE11

ATOMIC MASSES ${ }^{187,187 m} \mathrm{~Pb}$; measured masses. ${ }^{187} \mathrm{~Pb}$ deduced isomeric state energy. Penning trap mass spectrometer. JOUR PYLAA 34781

${ }^{187} \mathrm{Bi} \quad$ 2005GEZW ATOMIC MASSES ${ }^{235}$ Ac; measured mass, $\mathrm{T}_{1 / 2}$. $185,186,187,188,189,190,191,192,193,194,195,196 \mathrm{Bi}$; measured masses, proton separation energies. ${ }^{207 m} \mathrm{Tl}$; measured $\mathrm{T}_{1 / 2}$. Stored beams, Schottky mass spectrometry. PREPRINT nucl-ex/0510009,10/4/2005

2005KE10 RADIOACTIVITY ${ }^{191,193,195} \operatorname{At}(\alpha)$; measured $\mathrm{E} \alpha, \mathrm{E} \gamma, \gamma \alpha$-coin. ${ }^{191,193,195}$ At deduced levels, J, $\pi$, configurations, proton separation energies. ${ }^{187,189,191} \mathrm{Bi}$ deduced levels $\mathrm{J}$, $\pi$, configurations. Comparison with theory. JOUR ZAANE 25 s01 181

2005UU03 RADIOACTIVITY 188,190,192,194,196,198,200,202,204 Po, $191,193,195,197,199$ At, ${ }^{196,198,200,202,204,206} \mathrm{Rn},{ }^{199,201,203,205,207} \operatorname{Fr}(\alpha)$; measured reduced widths using gas filled recoil separator; deduced hindrance factors, proton intruder states and deformation effects. JOUR ZAANE 25 s01 179

\section{$\mathrm{A}=\mathbf{1 8 8}$}

${ }^{188} \mathrm{Os}$ 2004M054 NUCLEAR REACTIONS ${ }^{192} \mathrm{Os}\left({ }^{82} \mathrm{Se}, \mathrm{X}\right){ }^{188} \mathrm{Os} /{ }^{190} \mathrm{Os}, \mathrm{E}=460 \mathrm{MeV}$; measured $\mathrm{E} \gamma, \mathrm{I} \gamma, \gamma \gamma$-coin. ${ }^{188,190}$ Os deduced high-spin levels, J, $\pi$. GASP array. JOUR BJPHE 34792 


\section{$\mathrm{A}=188$ (continued)}

2005CL07 NUCLEAR REACTIONS ${ }^{184} \mathrm{~W}\left({ }^{7} \mathrm{Li}, \mathrm{xn}\right),\left({ }^{7} \mathrm{Li}, \mathrm{xnp}\right),\left({ }^{7} \mathrm{Li}, \mathrm{xn} \alpha\right)$, $\mathrm{E}=35-70 \mathrm{MeV}$; calculated $\sigma .{ }^{184} \mathrm{~W}\left({ }^{7} \mathrm{Li}, \mathrm{X}\right){ }^{184} \mathrm{Os} /{ }^{185} \mathrm{Os} /{ }^{186} \mathrm{Os} /$ ${ }^{188} \mathrm{Os} /{ }^{184} \mathrm{Ir} /{ }^{185} \mathrm{Ir} /{ }^{186} \mathrm{Ir} /{ }^{183} \mathrm{Re} /{ }^{185} \mathrm{Re}, \mathrm{E}=40-70 \mathrm{MeV}$; measured $\mathrm{E} \gamma, \mathrm{I} \gamma, \gamma \gamma-$, (charged particle) $\gamma$-coin, particle yield ratios. ${ }^{160} \mathrm{Gd}\left({ }^{7} \mathrm{Li}\right.$, xnp), $\mathrm{E}=35-65 \mathrm{MeV}$; analyzed $\sigma$. Liberace, Stars arrays. JOUR PRVCA 72054605

${ }^{188} \operatorname{Ir} \quad 2005 T$ A26

NUCLEAR REACTIONS Ir(p, xnyp) ${ }^{188} \mathrm{Pt} /{ }^{189} \mathrm{Pt} /{ }^{191} \mathrm{Pt} /{ }^{185} \mathrm{Ir} /$ ${ }^{186} \operatorname{Ir} /{ }^{188} \operatorname{Ir} /{ }^{189} \operatorname{Ir} /{ }^{190} \operatorname{Ir} /{ }^{192} \operatorname{Ir} /{ }^{185} \mathrm{Os}, \mathrm{E} \approx 3-70 \mathrm{MeV}$; measured $\sigma$; deduced integral yields. Stacked-foil activation technique. JOUR NIMBE 239293

${ }^{188} \mathrm{Pt}$ 2005TA26 NUCLEAR REACTIONS Ir $(\mathrm{p}, \mathrm{xnyp}){ }^{188} \mathrm{Pt} /{ }^{189} \mathrm{Pt} /{ }^{191} \mathrm{Pt} /{ }^{185} \mathrm{Ir} /$ ${ }^{186} \operatorname{Ir} /{ }^{188} \operatorname{Ir} /{ }^{189} \operatorname{Ir} /{ }^{190} \operatorname{Ir} /{ }^{192} \operatorname{Ir} /{ }^{185} \mathrm{Os}, \mathrm{E} \approx 3-70 \mathrm{MeV}$; measured $\sigma$; deduced integral yields. Stacked-foil activation technique. JOUR NIMBE 239293

${ }^{188} \mathrm{~Pb}$ 2005GR35 NUCLEAR REACTIONS ${ }^{108} \mathrm{Pd}\left({ }^{83} \mathrm{Kr}, 3 \mathrm{n}\right), \mathrm{E}=340 \mathrm{MeV}$; measured Doppler-shifted $\mathrm{E} \gamma$, I $\gamma$, (recoil) $\gamma$-coin. ${ }^{188} \mathrm{~Pb}$ levels deduced $\mathrm{T}_{1 / 2}$, $\mathrm{B}(\mathrm{E} 2)$, deformation. Jurogam array, mass separator, recoil-distance technique. JOUR ZAANE 25 s01 441

2005 UU03 RADIOACTIVITY 188,190,192,194,196,198,200,202,204 Po, $191,193,195,197,199$ At, ${ }^{196,198,200,202,204,206} \mathrm{Rn},{ }^{199,201,203,205,207} \operatorname{Fr}(\alpha)$; measured reduced widths using gas filled recoil separator; deduced hindrance factors, proton intruder states and deformation effects. JOUR ZAANE 25 s01 179

\section{${ }^{188} \mathrm{Bi} \quad$ 2005GEZW ATOMIC MASSES ${ }^{235}$ Ac; measured mass, $\mathrm{T}_{1 / 2}$.} 185,186,187,188,189,190,191,192,193,194,195,196 $\mathrm{Bi}$; measured masses, proton separation energies. ${ }^{207 \mathrm{~m}} \mathrm{Tl}$; measured $\mathrm{T}_{1 / 2}$. Stored beams, Schottky mass spectrometry. PREPRINT nucl-ex/0510009,10/4/2005

${ }^{188} \mathrm{Po} \quad 2005 \mathrm{UUU} 03 \quad$ RADIOACTIVITY ${ }^{188,190,192,194,196,198,200,202,204} \mathrm{Po}$, $191,193,195,197,199$ At, ${ }^{196,198,200,202,204,206} \mathrm{Rn},{ }^{199,201,203,205,207} \operatorname{Fr}(\alpha)$; measured reduced widths using gas filled recoil separator; deduced hindrance factors, proton intruder states and deformation effects. JOUR ZAANE 25 s01 179

$$
\mathrm{A}=\mathbf{1 8 9}
$$

${ }^{189} \mathrm{Ir}$ 2005TA26 NUCLEAR REACTIONS Ir(p, xnyp) ${ }^{188} \mathrm{Pt} /{ }^{189} \mathrm{Pt} /{ }^{191} \mathrm{Pt} /{ }^{185} \mathrm{Ir} /$ ${ }^{186} \operatorname{Ir} /{ }^{188} \operatorname{Ir} /{ }^{189} \operatorname{Ir} /{ }^{190} \operatorname{Ir} /{ }^{192} \operatorname{Ir} /{ }^{185} \mathrm{Os}, \mathrm{E} \approx 3-70 \mathrm{MeV}$; measured $\sigma ;$ deduced integral yields. Stacked-foil activation technique. JOUR NIMBE 239293

${ }^{189} \mathrm{Pt}$ 2005TA26 NUCLEAR REACTIONS Ir(p, xnyp) ${ }^{188} \mathrm{Pt} /{ }^{189} \mathrm{Pt} /{ }^{191} \mathrm{Pt} /{ }^{185} \mathrm{Ir} /$ ${ }^{186} \operatorname{Ir} /{ }^{188} \operatorname{Ir} /{ }^{189} \operatorname{Ir} /{ }^{190} \operatorname{Ir} /{ }^{192} \operatorname{Ir} /{ }^{185} \mathrm{Os}, \mathrm{E} \approx 3-70 \mathrm{MeV}$; measured $\sigma$; deduced integral yields. Stacked-foil activation technique. JOUR NIMBE 239293

${ }^{189} \mathrm{Bi} \quad$ 2005GEZW ATOMIC MASSES ${ }^{235}$ Ac; measured mass, $\mathrm{T}_{1 / 2}$. 185,186,187,188,189,190,191,192,193,194,195,196 $\mathrm{Bi}$; measured masses, proton separation energies. ${ }^{207 m} \mathrm{Tl}$; measured $\mathrm{T}_{1 / 2}$. Stored beams, Schottky mass spectrometry. PREPRINT nucl-ex/0510009,10/4/2005 


\section{$\mathrm{A}=189$ (continued)}

2005KE10 RADIOACTIVITY ${ }^{191,193,195} \operatorname{At}(\alpha)$; measured E $\alpha, \mathrm{E} \gamma, \gamma \alpha$-coin. 191,193,195 At deduced levels, J, $\pi$, configurations, proton separation energies. ${ }^{187,189,191} \mathrm{Bi}$ deduced levels $\mathrm{J}, \pi$, configurations. Comparison with theory. JOUR ZAANE 25 s01 181

2005UU03 RADIOACTIVITY ${ }^{188,190,192,194,196,198,200,202,204} \mathrm{Po}$, $191,193,195,197,199$ At, ${ }^{196,198,200,202,204,206} \mathrm{Rn},{ }^{199,201,203,205,207} \operatorname{Fr}(\alpha)$; measured reduced widths using gas filled recoil separator; deduced hindrance factors, proton intruder states and deformation effects. JOUR ZAANE 25 s01 179

$$
\mathrm{A}=\mathbf{1 9 0}
$$

${ }^{190} \mathrm{Os} \quad 2004 \mathrm{MO54}$

2005ME19

${ }^{190} \mathrm{Ir} \quad$ 2005TA26

${ }^{190} \mathrm{Tl} \quad$ 2005XI06

2005ZH31

${ }^{190} \mathrm{~Pb} \quad 2005 \mathrm{UU} 03$

${ }^{190} \mathrm{Bi} \quad$ 2005GEZW

${ }^{190} \mathrm{Po} \quad 2005 \mathrm{UU} 03$
NUCLEAR REACTIONS ${ }^{192} \mathrm{Os}\left({ }^{82} \mathrm{Se}, \mathrm{X}\right){ }^{188} \mathrm{Os} /{ }^{190} \mathrm{Os}, \mathrm{E}=460 \mathrm{MeV}$; measured $\mathrm{E} \gamma, \mathrm{I} \gamma, \gamma \gamma$-coin. ${ }^{188,190}$ Os deduced high-spin levels, J, $\pi$. GASP array. JOUR BJPHE 34792 NUCLEAR REACTIONS ${ }^{160} \mathrm{Gd},{ }^{164} \mathrm{Dy},{ }^{170} \mathrm{Er},{ }^{178} \mathrm{Hf},{ }^{186} \mathrm{~W},{ }^{192} \mathrm{Os}(\mathrm{p}$, t), $\mathrm{E}=25 \mathrm{MeV}$; measured triton spectra, $\sigma(\theta) .{ }^{158} \mathrm{Gd},{ }^{162} \mathrm{Dy},{ }^{168} \mathrm{Er}$, ${ }^{176} \mathrm{Hf},{ }^{184} \mathrm{~W},{ }^{190} \mathrm{Os}$ deduced $0^{+}$level energies. JOUR JPGPE $31 \mathrm{~S} 1399$ NUCLEAR REACTIONS Ir(p, xnyp) ${ }^{188} \mathrm{Pt} /{ }^{189} \mathrm{Pt} /{ }^{191} \mathrm{Pt} /{ }^{185} \mathrm{Ir} /$ ${ }^{186} \operatorname{Ir} /{ }^{188} \operatorname{Ir} /{ }^{189} \operatorname{Ir} /{ }^{190} \operatorname{Ir} /{ }^{192} \operatorname{Ir} /{ }^{185} \mathrm{Os}, \mathrm{E} \approx 3-70 \mathrm{MeV}$; measured $\sigma$; deduced integral yields. Stacked-foil activation technique. JOUR NIMBE 239293

NUCLEAR REACTIONS ${ }^{160} \mathrm{Gd}\left({ }^{35} \mathrm{Cl}, 5 \mathrm{n}\right), \mathrm{E}=167,175 \mathrm{MeV}$; measured $\mathrm{E} \gamma, \mathrm{I} \gamma, \gamma \gamma$-coin. ${ }^{190} \mathrm{Tl}$ deduced levels, $\mathrm{J}, \pi$, configurations, rotational band, signature inversion. Total Routhian surface calculations. JOUR PRVCA 72044302

NUCLEAR REACTIONS ${ }^{160} \mathrm{Gd}\left({ }^{35} \mathrm{Cl}, 5 \mathrm{n}\right), \mathrm{E}=167 \mathrm{MeV}$; measured $\mathrm{E} \gamma$, $\mathrm{I} \gamma, \gamma \gamma$-coin. ${ }^{190} \mathrm{Tl}$ deduced levels, J, $\pi$, configurations, rotational band signature inversion. Level systematics in neighboring isotopes discussed. JOUR JPGPE 31 S1985

RADIOACTIVITY ${ }^{188,190,192,194,196,198,200,202,204} \mathrm{Po}$, $191,193,195,197,199$ At, ${ }^{196,198,200,202,204,206} \mathrm{Rn},{ }^{199,201,203,205,207} \operatorname{Fr}(\alpha)$; measured reduced widths using gas filled recoil separator; deduced hindrance factors, proton intruder states and deformation effects.

JOUR ZAANE 25 s01 179 ATOMIC MASSES ${ }^{235}$ Ac; measured mass, $\mathrm{T}_{1 / 2}$. $185,186,187,188,189,190,191,192,193,194,195,196 \mathrm{Bi}$; measured masses, proton separation energies. ${ }^{207 m} \mathrm{Tl}$; measured $\mathrm{T}_{1 / 2}$. Stored beams, Schottky mass spectrometry. PREPRINT nucl-ex/0510009,10/4/2005

RADIOACTIVITY 188,190,192,194,196,198,200,202,204 Po, ${ }_{191,193,195,197,199}$ At, ${ }^{196,198,200,202,204,206} \mathrm{Rn},{ }^{199,201,203,205,207} \operatorname{Fr}(\alpha)$; measured reduced widths using gas filled recoil separator; deduced hindrance factors, proton intruder states and deformation effects. JOUR ZAANE 25 s01 179 


\section{$\mathrm{A}=191$}

${ }^{191} \mathrm{Os}$ 2005J019 NUCLEAR REACTIONS ${ }^{192} \mathrm{Os}\left({ }^{82} \mathrm{Se}, \mathrm{X}\right){ }^{191} \mathrm{Os}, \mathrm{E}=460 \mathrm{MeV}$; measured $\mathrm{E} \gamma, \mathrm{I} \gamma, \gamma \gamma$-coin. ${ }^{191}$ Os deduced levels, J, $\pi$, branching ratios, configurations, isomeric state features. GASP array. JOUR JPGPE 31 S1891

${ }^{191} \mathrm{Pt} \quad$ 2005TA26 NUCLEAR REACTIONS $\operatorname{Ir}(\mathrm{p}, \mathrm{xnyp}){ }^{188} \mathrm{Pt} /{ }^{189} \mathrm{Pt} /{ }^{191} \mathrm{Pt} /{ }^{185} \mathrm{Ir} /$ ${ }^{186} \mathrm{Ir} /{ }^{188} \mathrm{Ir} /{ }^{189} \mathrm{Ir} /{ }^{190} \mathrm{Ir} /{ }^{192} \mathrm{Ir} /{ }^{185} \mathrm{Os}$, E $\approx 3-70 \mathrm{MeV}$; measured $\sigma$; deduced integral yields. Stacked-foil activation technique. JOUR NIMBE 239293

2006DI01 NUCLEAR REACTIONS Pt(d, X) ${ }^{191} \mathrm{Au} /{ }^{192} \mathrm{Au} /{ }^{193} \mathrm{Au} /{ }^{194} \mathrm{Au} /$ ${ }^{195} \mathrm{Au} /{ }^{196} \mathrm{Au} /{ }^{196 m} \mathrm{Au} /{ }^{198} \mathrm{Au} /{ }^{199} \mathrm{Au} /{ }^{191} \mathrm{Pt} /{ }^{195 m} \mathrm{Pt} /{ }^{197} \mathrm{Pt} /$ ${ }^{192} \mathrm{Ir}, \mathrm{E} \approx 10-40 \mathrm{MeV}$; measured production $\sigma$. Stacked-foil activation technique, comparison with model predictions. JOUR NIMBE 24320 ${ }^{191} \mathrm{Au}$ 2006Di01 NUCLEAR REACTIONS Pt $(\mathrm{d}, \mathrm{X}){ }^{191} \mathrm{Au} /{ }^{192} \mathrm{Au} /{ }^{193} \mathrm{Au} /{ }^{194} \mathrm{Au} /$ ${ }^{195} \mathrm{Au} /{ }^{196} \mathrm{Au} /{ }^{196 m} \mathrm{Au} /{ }^{198} \mathrm{Au} /{ }^{199} \mathrm{Au} /{ }^{191} \mathrm{Pt} /{ }^{195 m} \mathrm{Pt} /{ }^{197} \mathrm{Pt} /$ ${ }^{192} \mathrm{Ir}, \mathrm{E} \approx 10-40 \mathrm{MeV}$; measured production $\sigma$. Stacked-foil activation technique, comparison with model predictions. JOUR NIMBE 24320

${ }^{191} \mathrm{Bi} \quad 2005 \mathrm{GEZW}$ ATOMIC MASSES ${ }^{235}$ Ac; measured mass, $\mathrm{T}_{1 / 2}$. $185,186,187,188,189,190,191,192,193,194,195,196 \mathrm{Bi}$; measured masses, proton separation energies. ${ }^{207 m} \mathrm{Tl}$; measured $\mathrm{T}_{1 / 2}$. Stored beams, Schottky mass spectrometry. PREPRINT nucl-ex/0510009,10/4/2005

2005KE10 RADIOACTIVITY ${ }^{191,193,195} \operatorname{At}(\alpha)$; measured E $\alpha, \mathrm{E} \gamma, \gamma \alpha$-coin. ${ }^{191,193,195}$ At deduced levels, J, $\pi$, configurations, proton separation energies. ${ }^{187,189,191} \mathrm{Bi}$ deduced levels $\mathrm{J}, \pi$, configurations. Comparison with theory. JOUR ZAANE 25 s01 181

2005UU03 RADIOACTIVITY ${ }^{188,190,192,194,196,198,200,202,204}$ Po, 191,193,195,197,199 At, ${ }^{196,198,200,202,204,206} \mathrm{Rn},{ }^{199,201,203,205,207} \operatorname{Fr}(\alpha)$; measured reduced widths using gas filled recoil separator; deduced hindrance factors, proton intruder states and deformation effects.

JOUR ZAANE 25 s01 179

${ }^{191} \mathrm{At} \quad$ 2005KE10 NUCLEAR REACTIONS ${ }^{142} \mathrm{Nd}\left({ }^{56} \mathrm{Fe}, 2 \mathrm{np}\right), \mathrm{E}=262 \mathrm{MeV} ;{ }^{141} \mathrm{Pr}\left({ }^{56} \mathrm{Fe}\right.$, $4 \mathrm{n}), \mathrm{E}=266 \mathrm{MeV} ;{ }^{141} \operatorname{Pr}\left({ }^{54} \mathrm{Fe}, 4 \mathrm{n}\right), \mathrm{E}=260 \mathrm{MeV}$; measured production $\sigma$. JOUR ZAANE 25 s01 181

2005KE10 RADIOACTIVITY ${ }^{191,193,195} \operatorname{At}(\alpha)$; measured E $\alpha, \mathrm{E} \gamma, \gamma \alpha$-coin. 191,193,195 At deduced levels, J, $\pi$, configurations, proton separation energies. ${ }^{187,189,191} \mathrm{Bi}$ deduced levels $\mathrm{J}, \pi$, configurations. Comparison with theory. JOUR ZAANE 25 s01 181

2005UU03 RADIOACTIVITY 188,190,192,194,196,198,200,202,204Po, ${ }_{191,193,195,197,199}$ At, ${ }^{196,198,200,202,204,206} \mathrm{Rn},{ }^{199,201,203,205,207} \operatorname{Fr}(\alpha)$; measured reduced widths using gas filled recoil separator; deduced hindrance factors, proton intruder states and deformation effects.

JOUR ZAANE 25 s01 179

$$
\mathrm{A}=192
$$

${ }^{192} \mathrm{Ir} \quad$ 2005TA26 NUCLEAR REACTIONS $\operatorname{Ir}(\mathrm{p}, \mathrm{xnyp}){ }^{188} \mathrm{Pt} /{ }^{189} \mathrm{Pt} /{ }^{191} \mathrm{Pt} /{ }^{185} \mathrm{Ir} /$ ${ }^{186} \mathrm{Ir} /{ }^{188} \mathrm{Ir} /{ }^{189} \mathrm{Ir} /{ }^{190} \mathrm{Ir} /{ }^{192} \mathrm{Ir} /{ }^{185} \mathrm{Os}, \mathrm{E} \approx 3-70 \mathrm{MeV}$; measured $\sigma$; deduced integral yields. Stacked-foil activation technique. JOUR NIMBE 239293 


\section{$\mathrm{A}=192$ (continued)}

2006DI01 NUCLEAR REACTIONS Pt(d, X) $)^{191} \mathrm{Au} /{ }^{192} \mathrm{Au} /{ }^{193} \mathrm{Au} /{ }^{194} \mathrm{Au} /$ ${ }^{195} \mathrm{Au} /{ }^{196} \mathrm{Au} /{ }^{196 m} \mathrm{Au} /{ }^{198} \mathrm{Au} /{ }^{199} \mathrm{Au} /{ }^{191} \mathrm{Pt} /{ }^{195 m} \mathrm{Pt} /{ }^{197} \mathrm{Pt} /$

${ }^{192} \mathrm{Ir}, \mathrm{E} \approx 10-40 \mathrm{MeV}$; measured production $\sigma$. Stacked-foil activation technique, comparison with model predictions. JOUR NIMBE 24320

\begin{tabular}{|c|c|c|}
\hline${ }^{192} \mathrm{Au}$ & 2006DI01 & $\begin{array}{l}\text { NUCLEAR REACTIONS Pt(d, X) }{ }^{191} \mathrm{Au} /{ }^{192} \mathrm{Au} /{ }^{193} \mathrm{Au} /{ }^{194} \mathrm{Au} / \\
{ }^{195} \mathrm{Au} /{ }^{196} \mathrm{Au} /{ }^{196 m} \mathrm{Au} /{ }^{198} \mathrm{Au} /{ }^{199} \mathrm{Au} /{ }^{191} \mathrm{Pt} /{ }^{195 m} \mathrm{Pt} /{ }^{197} \mathrm{Pt} / \\
\\
\text { tech } \mathrm{Ir}, \mathrm{E} \approx 10-40 \mathrm{MeV} \text {; measured production } \sigma . \text { Stacked-foil activation } \\
\text { technique, comparison with model predictions. JOUR NIMBE } 24320\end{array}$ \\
\hline \multirow[t]{2}{*}{${ }^{192} \mathrm{~Pb}$} & 2005UU03 & $\begin{array}{l}\text { RADIOACTIVITY }{ }^{188,190,192,194,196,198,200,202,204} \mathrm{Po}, \\
191,193,195,197,199 \text { At, }{ }^{196,198,200,202,204,206} \mathrm{Rn},{ }^{199,201,203,205,207} \operatorname{Fr}(\alpha)\end{array}$ \\
\hline & & $\begin{array}{l}\text { measured reduced widths using gas filled recoil separator; deduced } \\
\text { hindrance factors, proton intruder states and deformation effects. } \\
\text { JOUR ZAANE } 25 \text { s01 } 179\end{array}$ \\
\hline \multirow[t]{2}{*}{${ }^{192} \mathrm{Bi}$} & 2005GEZW & $\begin{array}{l}\text { ATOMIC MASSES }{ }^{235} \text { Ac; measured mass, } T_{1 / 2} . \\
185,186,187,188,189,190,191,192,193,194,195,196 \mathrm{Bi} \text {; measured masses, proton }\end{array}$ \\
\hline & & $\begin{array}{l}\text { separation energies. }{ }^{207 m} \mathrm{Tl} \text {; measured } \mathrm{T}_{1 / 2} . \text { Stored beams, Schottky } \\
\text { mass spectrometry. PREPRINT nucl-ex/0510009,10/4/2005 }\end{array}$ \\
\hline \multirow[t]{2}{*}{${ }^{192} \mathrm{Po}$} & 2005UU03 & $\begin{array}{l}\text { RADIOACTIVITY }{ }^{188,190,192,194,196,198,200,202,204} \mathrm{Po}, \\
191,193,195,197,199 \text { At, } 196,198,200,202,204,206 \mathrm{Rn},{ }^{199,201,203,205,207} \operatorname{Fr}(\alpha) \text {; }\end{array}$ \\
\hline & & $\begin{array}{l}\text { measured reduced widths using gas filled recoil separator; deduced } \\
\text { hindrance factors, proton intruder states and deformation effects. } \\
\text { JOUR ZAANE } 25 \text { s01 } 179\end{array}$ \\
\hline
\end{tabular}

\section{$\mathrm{A}=193$}

${ }^{193} \mathrm{Os} \quad$ 2004ZA15 RADIOACTIVITY ${ }^{193} \mathrm{Os}\left(\beta^{-}\right)$[from $\left.{ }^{192} \mathrm{Os}(\mathrm{n}, \gamma)\right]$; measured $\mathrm{E} \gamma, \mathrm{I} \gamma$, $\gamma \gamma$-coin. ${ }^{193} \mathrm{Ir}$ deduced levels, transition intensities. JOUR BJPHE 34 719

2005ZA15 RADIOACTIVITY ${ }^{193} \mathrm{Os}\left(\beta^{-}\right)$[from $\left.{ }^{192} \mathrm{Os}(\mathrm{n}, \gamma)\right]$; measured $\mathrm{E} \gamma, \mathrm{I} \gamma$, $\gamma \gamma$-coin. ${ }^{193}$ Ir deduced levels, J, $\pi$. JOUR BJPHE 35843

${ }^{193} \mathrm{Ir} \quad$ 2004ZA15 RADIOACTIVITY ${ }^{193} \mathrm{Os}\left(\beta^{-}\right)\left[\right.$from $\left.{ }^{192} \mathrm{Os}(\mathrm{n}, \gamma)\right]$; measured $\mathrm{E} \gamma, \mathrm{I} \gamma$, $\gamma \gamma$-coin. ${ }^{193} \mathrm{Ir}$ deduced levels, transition intensities. JOUR BJPHE 34 719

2005ZA15 RADIOACTIVITY ${ }^{193} \mathrm{Os}\left(\beta^{-}\right)$[from ${ }^{192} \mathrm{Os}(\mathrm{n}, \gamma)$ ]; measured $\mathrm{E} \gamma$, I $\gamma$, $\gamma \gamma$-coin. ${ }^{193} \mathrm{Ir}$ deduced levels, J, $\pi$. JOUR BJPHE 35843

${ }^{193} \mathrm{Au}$ 2006Di01 NUCLEAR REACTIONS Pt(d, X) ${ }^{191} \mathrm{Au} /{ }^{192} \mathrm{Au} /{ }^{193} \mathrm{Au} /{ }^{194} \mathrm{Au} /$ ${ }^{195} \mathrm{Au} /{ }^{196} \mathrm{Au} /{ }^{196 m} \mathrm{Au} /{ }^{198} \mathrm{Au} /{ }^{199} \mathrm{Au} /{ }^{191} \mathrm{Pt} /{ }^{195 m} \mathrm{Pt} /{ }^{197} \mathrm{Pt} /$

${ }^{192} \mathrm{Ir}, \mathrm{E} \approx 10-40 \mathrm{MeV}$; measured production $\sigma$. Stacked-foil activation technique, comparison with model predictions. JOUR NIMBE 24320

${ }^{193} \mathrm{~Pb} 2005 \mathrm{GL} 09$ NUCLEAR REACTIONS ${ }^{170} \mathrm{Er}\left({ }^{28} \mathrm{Si}, 5 \mathrm{n}\right), \mathrm{E}=149 \mathrm{MeV}$; measured $\mathrm{E} \gamma$, $\mathrm{I} \gamma, \gamma \gamma$-coin, DSA. ${ }^{193} \mathrm{~Pb}$ deduced magnetic rotational band levels $\mathrm{T}_{1 / 2}$. GASP array, recoil-distance and Doppler-shift attenuation methods used. JOUR JPGPE 31 S1559

${ }^{193} \mathrm{Bi} \quad$ 2005GEZW $\quad$ ATOMIC MASSES ${ }^{235}$ Ac; measured mass, $\mathrm{T}_{1 / 2}$. 185,186,187,188,189,190,191,192,193,194,195,196 $\mathrm{Bi}$; measured masses, proton separation energies. ${ }^{207 m} \mathrm{Tl}$; measured $\mathrm{T}_{1 / 2}$. Stored beams, Schottky mass spectrometry. PREPRINT nucl-ex/0510009,10/4/2005 


\section{$\mathrm{A}=193$ (continued)}

2005 UU03 RADIOACTIVITY 188,190,192,194,196,198,200,202,204 Po, 191,193,195,197,199 At, ${ }^{196,198,200,202,204,206} \mathrm{Rn},{ }^{199,201,203,205,207} \operatorname{Fr}(\alpha)$; measured reduced widths using gas filled recoil separator; deduced hindrance factors, proton intruder states and deformation effects.

JOUR ZAANE 25 s01 179

${ }^{193} \mathrm{At}$ 2005KE10 NUCLEAR REACTIONS ${ }^{142} \mathrm{Nd}\left({ }^{56} \mathrm{Fe}, 2 \mathrm{np}\right), \mathrm{E}=262 \mathrm{MeV} ;{ }^{141} \mathrm{Pr}\left({ }^{56} \mathrm{Fe}\right.$, $4 \mathrm{n}), \mathrm{E}=266 \mathrm{MeV} ;{ }^{141} \operatorname{Pr}\left({ }^{54} \mathrm{Fe}, 4 \mathrm{n}\right), \mathrm{E}=260 \mathrm{MeV}$; measured production $\sigma$. JOUR ZAANE 25 s01 181

2005KE10 RADIOACTIVITY ${ }^{191,193,195} \operatorname{At}(\alpha)$; measured $\mathrm{E} \alpha, \mathrm{E} \gamma, \gamma \alpha$-coin. 191,193,195 At deduced levels, J, $\pi$, configurations, proton separation energies. ${ }^{187,189,191} \mathrm{Bi}$ deduced levels J, $\pi$, configurations. Comparison with theory. JOUR ZAANE 25 s01 181

2005UU03 RADIOACTIVITY ${ }^{188,190,192,194,196,198,200,202,204}$ Po, $191,193,195,197,199$ At, ${ }^{196,198,200,202,204,206} \mathrm{Rn},{ }^{199,201,203,205,207} \operatorname{Fr}(\alpha)$; measured reduced widths using gas filled recoil separator; deduced hindrance factors, proton intruder states and deformation effects. JOUR ZAANE 25 s01 179

\section{$\mathrm{A}=194$}

\begin{tabular}{|c|c|c|}
\hline${ }^{4} \mathrm{Pt}$ & 2005SH52 & $\begin{array}{l}\text { ATOMIC MASSES }{ }^{194,195,196,198} \mathrm{Pt} \text {; measured masses. Penning trap } \\
\text { mass spectrometer. JOUR ZAANE } 25 \mathrm{~s} 0145\end{array}$ \\
\hline${ }^{194} \mathrm{Au}$ & 2006DI01 & $\begin{array}{l}\text { NUCLEAR REACTIONS Pt }(\mathrm{d}, \mathrm{X}){ }^{191} \mathrm{Au} /{ }^{192} \mathrm{Au} /{ }^{193} \mathrm{Au} /{ }^{194} \mathrm{Au} / \\
{ }^{195} \mathrm{Au} /{ }^{196} \mathrm{Au} /{ }^{196 m} \mathrm{Au} /{ }^{198} \mathrm{Au} /{ }^{199} \mathrm{Au} /{ }^{191} \mathrm{Pt} /{ }^{195 m} \mathrm{Pt} /{ }^{197} \mathrm{Pt} / \\
{ }^{192} \mathrm{Ir}, \mathrm{E} \approx 10-40 \mathrm{MeV} \text {; measured production } \sigma . \text { Stacked-foil activation } \\
\text { technique, comparison with model predictions. JOUR NIMBE } 24320\end{array}$ \\
\hline \multirow[t]{4}{*}{${ }^{194} \mathrm{~Pb}$} & 2005DRZW & $\begin{array}{l}\text { NUCLEAR REACTIONS }{ }^{170} \operatorname{Er}\left({ }^{29} \mathrm{Si}, 5 \mathrm{n}\right), \mathrm{E}=147 \mathrm{MeV} ;{ }^{170} \mathrm{Er}\left({ }^{30} \mathrm{Si} \text {, }\right. \\
4 \mathrm{n}), \mathrm{E}=138 \mathrm{MeV} \text {; measured prompt and delayed } \mathrm{E} \gamma, \mathrm{I} \gamma, \gamma \gamma \text {-coin. } \\
{ }_{194,196} \mathrm{~Pb} \text { deduced levels, J, } \pi \text {, configurations, isomers } \mathrm{T}_{1 / 2} \text { and decay }\end{array}$ \\
\hline & & $\begin{array}{l}\text { B(E1), B(E2), B(E3). Caesar array, potential energy surface } \\
\text { calculations. PREPRINT ANU-P/1662,Dracoulis }\end{array}$ \\
\hline & 2005UU03 & $\begin{array}{l}\text { RADIOACTIVITY }{ }^{188,190,192,194,196,198,200,202,204} \mathrm{Po}, \\
{ }^{191,193,195,197,199} \text { At, }{ }^{196,198,200,202,204,206} \mathrm{Rn},{ }^{199,201,203,205,207} \operatorname{Fr}(\alpha)\end{array}$ \\
\hline & & $\begin{array}{l}\text { measured reduced widths using gas filled recoil separator; deduced } \\
\text { hindrance factors, proton intruder states and deformation effects. } \\
\text { JOUR ZAANE } 25 \text { s01 } 179\end{array}$ \\
\hline \multirow[t]{2}{*}{${ }^{194} \mathrm{Bi}$} & 2005GEZW & $\begin{array}{l}\text { ATOMIC MASSES }{ }^{235} \text { Ac; measured mass, } T_{1 / 2} . \\
185,186,187,188,189,190,191,192,193,194,195,196 \mathrm{Bi} ; \text { measured masses, proton }\end{array}$ \\
\hline & & $\begin{array}{l}\text { separation energies. }{ }^{207 m} \mathrm{Tl} \text {; measured } \mathrm{T}_{1 / 2} \text {. Stored beams, Schottky } \\
\text { mass spectrometry. PREPRINT nucl-ex/0510009,10/4/2005 }\end{array}$ \\
\hline \multirow[t]{2}{*}{${ }^{194} \mathrm{Po}$} & 2005UU03 & $\begin{array}{l}\text { RADIOACTIVITY }{ }^{188,190,192,194,196,198,200,202,204} \mathrm{Po}, \\
191,193,195,197,199 \text { At, }{ }^{196,198,200,202,204,206} \mathrm{Rn},{ }^{199,201,203,205,207} \operatorname{Fr}(\alpha)\end{array}$ \\
\hline & & $\begin{array}{l}\text { measured reduced widths using gas filled recoil separator; deduced } \\
\text { hindrance factors, proton intruder states and deformation effects. } \\
\text { JOUR ZAANE } 25 \text { s01 } 179\end{array}$ \\
\hline
\end{tabular}

JOUR ZAANE 25 s01 179 


\section{$\mathrm{A}=195$}

\begin{tabular}{|c|c|c|}
\hline \multirow[t]{2}{*}{${ }^{195} \mathrm{Pt}$} & 2005SH52 & $\begin{array}{l}\text { ATOMIC MASSES }{ }^{194,195,196,198} \text { Pt; measured masses. Penning trap } \\
\text { mass spectrometer. JOUR ZAANE } 25 \text { s01 } 45\end{array}$ \\
\hline & 2006DI01 & $\begin{array}{l}\text { NUCLEAR REACTIONS Pt }(\mathrm{d}, \mathrm{X}){ }^{191} \mathrm{Au} /{ }^{192} \mathrm{Au} /{ }^{193} \mathrm{Au} /{ }^{194} \mathrm{Au} / \\
{ }^{195} \mathrm{Au} /{ }^{196} \mathrm{Au} /{ }^{196 m} \mathrm{Au} /{ }^{198} \mathrm{Au} /{ }^{199} \mathrm{Au} /{ }^{191} \mathrm{Pt} /{ }^{195 m} \mathrm{Pt} /{ }^{197} \mathrm{Pt} / \\
{ }^{192} \mathrm{Ir}, \mathrm{E} \approx 10-40 \mathrm{MeV} \text {; measured production } \sigma . \text { Stacked-foil activation } \\
\text { technique, comparison with model predictions. JOUR NIMBE } 24320\end{array}$ \\
\hline${ }^{195} \mathrm{Au}$ & 2006DI01 & $\begin{array}{l}\text { NUCLEAR REACTIONS Pt }(\mathrm{d}, \mathrm{X}){ }^{191} \mathrm{Au} /{ }^{192} \mathrm{Au} /{ }^{193} \mathrm{Au} /{ }^{194} \mathrm{Au} / \\
{ }^{195} \mathrm{Au} /{ }^{196} \mathrm{Au} /{ }^{196 m} \mathrm{Au} /{ }^{198} \mathrm{Au} /{ }^{199} \mathrm{Au} /{ }^{191} \mathrm{Pt} /{ }^{195 m} \mathrm{Pt} /{ }^{197} \mathrm{Pt} / \\
{ }^{192} \mathrm{Ir}, \mathrm{E} \approx 10-40 \mathrm{MeV} \text {; measured production } \sigma . \text { Stacked-foil activation } \\
\text { technique, comparison with model predictions. JOUR NIMBE } 24320\end{array}$ \\
\hline \multirow[t]{5}{*}{${ }^{195} \mathrm{Bi}$} & 2005GEZW & $\begin{array}{l}\text { ATOMIC MASSES }{ }^{235} \text { Ac; measured mass, } T_{1 / 2} \cdot \\
185,186,187,188,189,190,191,192,193,194,195,196 \mathrm{Bi} \text {; measured masses, proton }\end{array}$ \\
\hline & & $\begin{array}{l}\text { separation energies. }{ }^{207 m} \mathrm{Tl} \text {; measured } \mathrm{T}_{1 / 2} . \text { Stored beams, Schottky } \\
\text { mass spectrometry. PREPRINT nucl-ex/0510009,10/4/2005 }\end{array}$ \\
\hline & 2005UU03 & RADIOACTIVITY 188,190,192,194,196,198,200,202,204Po, \\
\hline & & ${ }^{191,193,195,197,199}$ At, ${ }^{196,198,200,202,204,206} \mathrm{Rn},{ }^{199,201,203,205,207} \operatorname{Fr}(\alpha)$ \\
\hline & & $\begin{array}{l}\text { measured reduced widths using gas filled recoil separator; deduced } \\
\text { hindrance factors, proton intruder states and deformation effects. } \\
\text { JOUR ZAANE } 25 \text { s01 } 179\end{array}$ \\
\hline \multirow[t]{5}{*}{${ }^{195} \mathrm{At}$} & 2005KE10 & $\begin{array}{l}\text { NUCLEAR REACTIONS }{ }^{142} \mathrm{Nd}\left({ }^{56} \mathrm{Fe}, 2 \mathrm{np}\right), \mathrm{E}=262 \mathrm{MeV} ;{ }^{141} \operatorname{Pr}\left({ }^{56} \mathrm{Fe},\right. \\
4 \mathrm{n}), \mathrm{E}=266 \mathrm{MeV} ;{ }^{141} \operatorname{Pr}\left({ }^{54} \mathrm{Fe}, 4 \mathrm{n}\right), \mathrm{E}=260 \mathrm{MeV} \text {; measured production } \\
\sigma . \text { JOUR ZAANE } 25 \mathrm{~s} 01181\end{array}$ \\
\hline & 2005KE10 & RADIOACTIVITY ${ }^{191,193,195} \operatorname{At}(\alpha) ;$ measured $\mathrm{E} \alpha, \mathrm{E} \gamma, \gamma \alpha$-coin. \\
\hline & & $\begin{array}{l}{ }^{191,193,195} \text { At deduced levels, J, } \pi \text {, configurations, proton separation } \\
\text { energies. }{ }^{187,189,191} \mathrm{Bi} \text { deduced levels } \mathrm{J}, \pi \text {, configurations. Comparison } \\
\text { with theory. JOUR ZAANE } 25 \text { s01 } 181\end{array}$ \\
\hline & 2005UU03 & RADIOACTIVITY ${ }^{188,190,192,194,196,198,200,202,204} \mathrm{Po}$ \\
\hline & & $\begin{array}{l}191,193,195,197,199 \text { At, }{ }^{196,198,200,202,204,206} \mathrm{Rn},{ }^{199,201,203,205,207} \operatorname{Fr}(\alpha) \\
\text { measured reduced widths using gas filled recoil separator; deduced } \\
\text { hindrance factors, proton intruder states and deformation effects. } \\
\text { JOUR ZAANE } 25 \text { s01 } 179\end{array}$ \\
\hline
\end{tabular}

\section{$\mathrm{A}=196$}

${ }^{196} \mathrm{Pt} \quad$ 2005SH52 ATOMIC MASSES ${ }^{194,195,196,198} \mathrm{Pt}$; measured masses. Penning trap mass spectrometer. JOUR ZAANE 25 s01 45

${ }^{196} \mathrm{Au}$ 2005WA31 NUCLEAR REACTIONS ${ }^{92,98,100} \mathrm{Mo}\left(\gamma, \gamma^{\prime}\right), \mathrm{E}=13.2 \mathrm{MeV}$ bremsstrahlung; measured $\mathrm{E} \gamma, \mathrm{I} \gamma .{ }^{92,100} \mathrm{Mo},{ }^{197} \mathrm{Au}(\gamma, \mathrm{n}),{ }^{92} \mathrm{Mo}(\gamma, \mathrm{p})$, $(\gamma, \alpha), \mathrm{E} \approx 11.8-16.5 \mathrm{MeV}$ bremsstrahlung; measured integrated $\sigma$. JOUR JPGPE 31 S1969

2006DI01 NUCLEAR REACTIONS Pt(d, X) ${ }^{191} \mathrm{Au} /{ }^{192} \mathrm{Au} /{ }^{193} \mathrm{Au} /{ }^{194} \mathrm{Au} /$ ${ }^{195} \mathrm{Au} /{ }^{196} \mathrm{Au} /{ }^{196 m} \mathrm{Au} /{ }^{198} \mathrm{Au} /{ }^{199} \mathrm{Au} /{ }^{191} \mathrm{Pt} /{ }^{195 m} \mathrm{Pt} /{ }^{197} \mathrm{Pt} /$

${ }^{192} \mathrm{Ir}, \mathrm{E} \approx 10-40 \mathrm{MeV}$; measured production $\sigma$. Stacked-foil activation technique, comparison with model predictions. JOUR NIMBE 24320 


\section{$\mathrm{A}=196$ (continued)}

$196 \mathrm{~Pb}$

2005DRZW

2005UU03

2005WIZY

${ }^{196} \mathrm{Bi}$

2005GEZW

${ }^{196} \mathrm{Po}$

2005UU03

$2005 U$ U03
NUCLEAR REACTIONS ${ }^{170} \operatorname{Er}\left({ }^{29} \mathrm{Si}, 5 \mathrm{n}\right), \mathrm{E}=147 \mathrm{MeV} ;{ }^{170} \operatorname{Er}\left({ }^{30} \mathrm{Si}\right.$, $4 \mathrm{n}), \mathrm{E}=138 \mathrm{MeV}$; measured prompt and delayed $\mathrm{E} \gamma, \mathrm{I} \gamma, \gamma \gamma$-coin. ${ }_{194,196} \mathrm{~Pb}$ deduced levels, $\mathrm{J}, \pi$, configurations, isomers $\mathrm{T}_{1 / 2}$ and decay $\mathrm{B}(\mathrm{E} 1), \mathrm{B}(\mathrm{E} 2), \mathrm{B}(\mathrm{E} 3)$. Caesar array, potential energy surface calculations. PREPRINT ANU-P/1662,Dracoulis RADIOACTIVITY 188,190,192,194,196,198,200,202,204 Po, 191,193,195,197,199 At, ${ }^{196,198,200,202,204,206}$ Rn, ${ }^{199,201,203,205,207} \operatorname{Fr}(\alpha)$; measured reduced widths using gas filled recoil separator; deduced hindrance factors, proton intruder states and deformation effects. JOUR ZAANE 25 s01 179 NUCLEAR REACTIONS ${ }^{170} \operatorname{Er}\left({ }^{30} \mathrm{Si}, 4 \mathrm{n}\right), \mathrm{E}=144 \mathrm{MeV}$; measured prompt and delayed $\mathrm{E} \gamma, \mathrm{I} \gamma, \gamma \gamma$-coin. ${ }^{196} \mathrm{~Pb}$ deduced superdeformed band excitation energy, J, $\pi$. Euroball IV array, time-correlated spectroscopy. JOUR PRLTA 95182501 NUCLEAR REACTIONS ${ }^{170} \operatorname{Er}\left({ }^{30} \mathrm{Si}, 4 \mathrm{n}\right), \mathrm{E}=144 \mathrm{MeV}$; measured prompt and delayed $\mathrm{E} \gamma, \mathrm{I} \gamma, \gamma \gamma$-coin. ${ }^{196} \mathrm{~Pb}$ deduced superdeformed band excitation energy. Euroball IV array, time-correlated spectroscopy. Level systematics in neighboring nuclides discussed. PREPRINT ANU-P/1667,Wilson ATOMIC MASSES ${ }^{235}$ Ac; measured mass, $\mathrm{T}_{1 / 2}$. 185,186,187,188,189,190,191,192,193,194,195,196 $\mathrm{Bi}$; measured masses, proton separation energies. ${ }^{207 \mathrm{~m}} \mathrm{Tl}$; measured $\mathrm{T}_{1 / 2}$. Stored beams, Schottky mass spectrometry. PREPRINT nucl-ex/0510009,10/4/2005 RADIOACTIVITY 188,190,192,194,196,198,200,202,204 Po, 191,193,195,197,199 At, ${ }^{196,198,200,202,204,206} \mathrm{Rn},{ }^{199,201,203,205,207} \operatorname{Fr}(\alpha)$; measured reduced widths using gas filled recoil separator; deduced hindrance factors, proton intruder states and deformation effects. JOUR ZAANE 25 s01 179

RADIOACTIVITY ${ }^{188,190,192,194,196,198,200,202,204} \mathrm{Po}$ 191,193,195,197,199 At, ${ }^{196,198,200,202,204,206}$ Rn, ${ }^{199,201,203,205,207} \operatorname{Fr}(\alpha)$; measured reduced widths using gas filled recoil separator; deduced hindrance factors, proton intruder states and deformation effects. JOUR ZAANE 25 s01 179

$$
\mathrm{A}=197
$$

${ }^{197} \mathrm{Pt} \quad 2006 \mathrm{DI01}$

NUCLEAR REACTIONS Pt(d, X) ${ }^{191} \mathrm{Au} /{ }^{192} \mathrm{Au} /{ }^{193} \mathrm{Au} /{ }^{194} \mathrm{Au} /$ ${ }^{195} \mathrm{Au} /{ }^{196} \mathrm{Au} /{ }^{196 m} \mathrm{Au} /{ }^{198} \mathrm{Au} /{ }^{199} \mathrm{Au} /{ }^{191} \mathrm{Pt} /{ }^{195 m} \mathrm{Pt} /{ }^{197} \mathrm{Pt} /$ ${ }^{192} \mathrm{Ir}, \mathrm{E} \approx 10-40 \mathrm{MeV}$; measured production $\sigma$. Stacked-foil activation technique, comparison with model predictions. JOUR NIMBE 24320 NUCLEAR REACTIONS ${ }^{209} \mathrm{Bi}\left({ }^{26} \mathrm{Mg},{ }^{26} \mathrm{Mg}\right.$ ') $\mathrm{E}=78.6 \mathrm{MeV} /$ nucleon; ${ }^{197} \mathrm{Au}\left({ }^{32} \mathrm{Mg},{ }^{32} \mathrm{Mg}\right.$ '), $\mathrm{E}=81.1 \mathrm{MeV} /$ nucleon; ${ }^{209} \mathrm{Bi}\left({ }^{34} \mathrm{Mg},{ }^{34} \mathrm{Mg}{ }^{\prime}\right)$, $\mathrm{E}=76.4 \mathrm{MeV} /$ nucleon; measured $\mathrm{E} \gamma, \mathrm{I} \gamma$, (particle) $\gamma$-coin following projectile Coulomb excitation. ${ }^{26,32,34} \mathrm{Mg}$ deduced transitions $\mathrm{B}(\mathrm{E} 2)$, deformation parameters. Comparison with previous work, model predictions. JOUR PRVCA 72054320 


\begin{abstract}
${ }^{197}$ At 2005UU03 RADIOACTIVITY ${ }^{188,190,192,194,196,198,200,202,204}$ Po, 191,193,195,197,199 At, ${ }^{196,198,200,202,204,206}$ Rn, ${ }^{199,201,203,205,207} \operatorname{Fr}(\alpha)$; measured reduced widths using gas filled recoil separator; deduced hindrance factors, proton intruder states and deformation effects. JOUR ZAANE 25 s01 179
\end{abstract}

\section{$\mathrm{A}=198$}

$\begin{array}{ll}{ }^{198} \mathrm{Pt} & 2005 \mathrm{SH} 52 \\ { }^{198} \mathrm{Au} & \text { 2004TA46 }\end{array}$

ATOMIC MASSES ${ }^{194,195,196,198}$ Pt; measured masses. Penning trap mass spectrometer. JOUR ZAANE 25 s01 45

NUCLEAR REACTIONS ${ }^{198} \operatorname{Pt}(\mathrm{p}, \mathrm{n}), \mathrm{E} \approx 6-37 \mathrm{MeV} ;{ }^{198} \operatorname{Pt}(\mathrm{d}, \mathrm{n}),(\mathrm{d}$, $2 \mathrm{n}), \mathrm{E} \approx 5-20 \mathrm{MeV}$; measured excitation functions. Activation technique. JOUR RAACA 92223

2005SE23 NUCLEAR REACTIONS ${ }^{197} \mathrm{Au}(\mathrm{n}, \gamma), \mathrm{E}=$ spectrum; measured $\mathrm{E} \gamma, \mathrm{I} \gamma$; deduced neutron flux. ${ }^{7} \operatorname{Li}(\mathrm{p}, \mathrm{n})$, E not given; deduced neutron spectrum. ${ }^{62} \mathrm{Ni}(\mathrm{n}, \gamma), \mathrm{E} \approx 5.5-20 \mathrm{keV}$; measured $\sigma$; deduced Maxwellian-averaged $\sigma$. JOUR JUPSA 742981

2006DI01 NUCLEAR REACTIONS Pt(d, X) ${ }^{191} \mathrm{Au} /{ }^{192} \mathrm{Au} /{ }^{193} \mathrm{Au} /{ }^{194} \mathrm{Au} /$ ${ }^{195} \mathrm{Au} /{ }^{196} \mathrm{Au} /{ }^{196 m} \mathrm{Au} /{ }^{198} \mathrm{Au} /{ }^{199} \mathrm{Au} /{ }^{191} \mathrm{Pt} /{ }^{195 m} \mathrm{Pt} /{ }^{197} \mathrm{Pt} /$ ${ }^{192} \mathrm{Ir}, \mathrm{E} \approx 10-40 \mathrm{MeV}$; measured production $\sigma$. Stacked-foil activation technique, comparison with model predictions. JOUR NIMBE 24320

${ }^{198} \mathrm{~Pb}$

2005 UU03

RADIOACTIVITY 188,190,192,194,196,198,200,202,204 Po, ${ }_{191,193,195,197,199}$ At, ${ }^{196,198,200,202,204,206} \mathrm{Rn},{ }^{199,201,203,205,207} \operatorname{Fr}(\alpha)$; measured reduced widths using gas filled recoil separator; deduced hindrance factors, proton intruder states and deformation effects. JOUR ZAANE 25 s01 179

${ }^{198} \mathrm{Po} \quad 2005 \mathrm{UU} 03$

RADIOACTIVITY 188,190,192,194,196,198,200,202,204 Po, 191,193,195,197,199 At, ${ }^{196,198,200,202,204,206} \mathrm{Rn},{ }^{199,201,203,205,207} \operatorname{Fr}(\alpha)$; measured reduced widths using gas filled recoil separator; deduced hindrance factors, proton intruder states and deformation effects. JOUR ZAANE 25 s01 179

${ }^{198} \mathrm{Rn} \quad 2005 \mathrm{UU} 03$ RADIOACTIVITY ${ }^{188,190,192,194,196,198,200,202,204}$ Po, 191,193,195,197,199 At, ${ }^{196,198,200,202,204,206} \mathrm{Rn},{ }^{199,201,203,205,207} \operatorname{Fr}(\alpha)$; measured reduced widths using gas filled recoil separator; deduced hindrance factors, proton intruder states and deformation effects. JOUR ZAANE 25 s01 179

\section{$\mathrm{A}=199$}

${ }^{199} \mathrm{Au} \quad 2004 \mathrm{TA} 46$

NUCLEAR REACTIONS ${ }^{198} \mathrm{Pt}(\mathrm{p}, \mathrm{n}), \mathrm{E} \approx 6-37 \mathrm{MeV} ;{ }^{198} \mathrm{Pt}(\mathrm{d}, \mathrm{n}),(\mathrm{d}$, $2 \mathrm{n}), \mathrm{E} \approx 5-20 \mathrm{MeV}$; measured excitation functions. Activation technique. JOUR RAACA 92223

2006DI01 NUCLEAR REACTIONS Pt(d, X) ${ }^{191} \mathrm{Au} /{ }^{192} \mathrm{Au} /{ }^{193} \mathrm{Au} /{ }^{194} \mathrm{Au} /$ ${ }^{195} \mathrm{Au} /{ }^{196} \mathrm{Au} /{ }^{196 m} \mathrm{Au} /{ }^{198} \mathrm{Au} /{ }^{199} \mathrm{Au} /{ }^{191} \mathrm{Pt} /{ }^{195 m} \mathrm{Pt} /{ }^{197} \mathrm{Pt} /$

${ }^{192} \mathrm{Ir}, \mathrm{E} \approx 10-40 \mathrm{MeV}$; measured production $\sigma$. Stacked-foil activation technique, comparison with model predictions. JOUR NIMBE 24320 


\begin{tabular}{|c|c|c|}
\hline \multirow[b]{2}{*}{${ }^{199} \mathrm{At}$} & \multicolumn{2}{|r|}{$A=199$ (continued) } \\
\hline & 2005UU03 & $\begin{array}{l}\text { RADIOACTIVITY } 188,190,192,194,196,198,200,202,204 \mathrm{Po} \\
191,193,195,197,199 \text { At, } 196,198,200,202,204,206 \mathrm{Rn}, 199,201,203,205,207 \operatorname{Fr}(\alpha)\end{array}$ \\
\hline & & $\begin{array}{l}\text { measured reduced widths using gas filled recoil separator; deduced } \\
\text { hindrance factors, proton intruder states and deformation effects. } \\
\text { JOUR ZAANE } 25 \text { s01 179 }\end{array}$ \\
\hline${ }^{199} \mathrm{Fr}$ & 2005UU03 & $\begin{array}{l}\text { RADIOACTIVITY }{ }^{188,190,192,194,196,198,200,202,204} \mathrm{Po} \\
{ }_{191,193,195,197,199} \text { At, }{ }^{196,198,200,202,204,206} \mathrm{Rn},{ }^{199,201,203,205,207} \operatorname{Fr}(\alpha)\end{array}$ \\
\hline & & $\begin{array}{l}\text { measured reduced widths using gas filled recoil separator; deduced } \\
\text { hindrance factors, proton intruder states and deformation effects. } \\
\text { JOUR ZAANE } 25 \text { s01 } 179\end{array}$ \\
\hline
\end{tabular}

\section{$\mathrm{A}=\mathbf{2 0 0}$}

${ }^{200} \mathrm{~Pb} \quad 2005 \mathrm{UU} 03$

RADIOACTIVITY 188,190,192,194,196,198,200,202,204 Po, 191,193,195,197,199 At, ${ }^{196,198,200,202,204,206} \mathrm{Rn},{ }^{199,201,203,205,207} \operatorname{Fr}(\alpha)$; measured reduced widths using gas filled recoil separator; deduced hindrance factors, proton intruder states and deformation effects. JOUR ZAANE 25 s01 179

$\begin{array}{ll}{ }^{200} \mathrm{Po} 2005 \mathrm{UU} 03 & \text { RADIOACTIVITY }{ }^{188,190,192,194,196,198,200,202,204} \mathrm{Po}, \\ & 191,193,195,197,199 \text { At, }{ }^{196,198,200,202,204,206} \mathrm{Rn},{ }^{199,201,203,205,207} \operatorname{Fr}(\alpha) ;\end{array}$ measured reduced widths using gas filled recoil separator; deduced hindrance factors, proton intruder states and deformation effects.

JOUR ZAANE 25 s01 179

${ }^{200} \mathrm{Rn} \quad 2005 \mathrm{UU} 03 \quad$ RADIOACTIVITY ${ }^{188,190,192,194,196,198,200,202,204} \mathrm{Po}$, 191,193,195,197,199 At, ${ }^{196,198,200,202,204,206} \mathrm{Rn},{ }^{199,201,203,205,207} \operatorname{Fr}(\alpha)$; measured reduced widths using gas filled recoil separator; deduced hindrance factors, proton intruder states and deformation effects. JOUR ZAANE 25 s01 179

\section{$\mathrm{A}=\mathbf{2 0 1}$}

\begin{tabular}{|c|c|c|}
\hline${ }^{201} \mathrm{At}$ & 2005UU03 & $\begin{array}{l}\text { RADIOACTIVITY }{ }^{188,190,192,194,196,198,200,202,204} \mathrm{Po} \\
{ }_{191,193,195,197,199} \text { At, }{ }^{196,198,200,202,204,206} \mathrm{Rn},{ }^{199,201,203,205,207} \operatorname{Fr}(\alpha)\end{array}$ \\
\hline & & $\begin{array}{l}\text { measured reduced widths using gas filled recoil separator; deduced } \\
\text { hindrance factors, proton intruder states and deformation effects. } \\
\text { JOUR ZAANE } 25 \text { s01 } 179\end{array}$ \\
\hline${ }^{201} \mathrm{Fr}$ & 2005UU03 & $\begin{array}{l}\text { RADIOACTIVITY }{ }^{188,190,192,194,196,198,200,202,204} \mathrm{Po}, \\
191,193,195,197,199 \text { At, }{ }^{196,198,200,202,204,206} \mathrm{Rn},{ }^{199,201,203,205,207} \operatorname{Fr}(\alpha)\end{array}$ \\
\hline & & $\begin{array}{l}\text { measured reduced widths using gas filled recoil separator; deduced } \\
\text { hindrance factors, proton intruder states and deformation effects. } \\
\text { JOUR ZAANE } 25 \text { s01 } 179\end{array}$ \\
\hline
\end{tabular}




\begin{tabular}{|c|c|c|}
\hline \multirow[b]{2}{*}{${ }^{202} \mathrm{Po}$} & \multicolumn{2}{|r|}{$A=202$} \\
\hline & 2005UU03 & $\begin{array}{l}\text { RADIOACTIVITY } 188,190,192,194,196,198,200,202,204 \mathrm{Po}, \\
{ }_{191,193,195,197,199} \mathrm{At},{ }^{196,198,200,202,204,206} \mathrm{Rn},{ }^{199,201,203,205,207} \mathrm{Fr}(\alpha)\end{array}$ \\
\hline & & $\begin{array}{l}\text { measured reduced widths using gas filled recoil separator; deduced } \\
\text { hindrance factors, proton intruder states and deformation effects. } \\
\text { JOUR ZAANE } 25 \text { s01 } 179\end{array}$ \\
\hline${ }^{202} \mathrm{Rn}$ & 2005UU03 & $\begin{array}{l}\text { RADIOACTIVITY }{ }^{188,190,192,194,196,198,200,202,204} \mathrm{Po}, \\
{ }^{191,193,195,197,199} \text { At, }{ }^{196,198,200,202,204,206} \mathrm{Rn},{ }^{199,201,203,205,207} \operatorname{Fr}(\alpha) \text {; }\end{array}$ \\
\hline & & $\begin{array}{l}\text { measured reduced widths using gas filled recoil separator; deduced } \\
\text { hindrance factors, proton intruder states and deformation effects. } \\
\text { JOUR ZAANE } 25 \text { s01 } 179\end{array}$ \\
\hline
\end{tabular}

\section{$\mathrm{A}=\mathbf{2 0 3}$}

$\begin{array}{ll}{ }^{203} \mathrm{At} & \\ & 2005 \mathrm{UU} 03 \\ { }^{203} \mathrm{Fr} & 2005 \mathrm{UU} 03\end{array}$

RADIOACTIVITY $188,190,192,194,196,198,200,202,204 \mathrm{Po}$, 191,193,195,197,199 At, ${ }^{196,198,200,202,204,206} \mathrm{Rn},{ }^{199,201,203,205,207} \operatorname{Fr}(\alpha)$; measured reduced widths using gas filled recoil separator; deduced hindrance factors, proton intruder states and deformation effects. JOUR ZAANE 25 s01 179

RADIOACTIVITY ${ }^{188,190,192,194,196,198,200,202,204} \mathrm{Po}$, $191,193,195,197,199$ At, ${ }^{196,198,200,202,204,206} \mathrm{Rn},{ }^{199,201,203,205,207} \operatorname{Fr}(\alpha)$; measured reduced widths using gas filled recoil separator; deduced hindrance factors, proton intruder states and deformation effects. JOUR ZAANE 25 s01 179

\section{$\mathrm{A}=\mathbf{2 0 4}$}

$\begin{array}{ll}{ }^{204} \mathrm{~Pb} & 2005 W A 34 \\ { }^{204} \mathrm{Po} & 2005 \mathrm{UU} 03\end{array}$

${ }^{204} \mathrm{Rn}$

$2005 U U 03$
NUCLEAR MOMENTS ${ }^{204,206,207,208} \mathrm{~Pb}$; measured hfs, isotope shifts. JOUR ZDDNE 36249
RADIOACTIVITY $188,190,192,194,196,198,200,202,204 \mathrm{Po}$, $191,193,195,197,199$ At, ${ }^{196,198,200,202,204,206} \mathrm{Rn},{ }^{199,201,203,205,207} \operatorname{Fr}(\alpha)$; measured reduced widths using gas filled recoil separator; deduced hindrance factors, proton intruder states and deformation effects. JOUR ZAANE 25 s01 179 RADIOACTIVITY 188,190,192,194,196,198,200,202,204 Po, 191,193,195,197,199 At, ${ }^{196,198,200,202,204,206} \mathrm{Rn},{ }^{199,201,203,205,207} \operatorname{Fr}(\alpha)$; measured reduced widths using gas filled recoil separator; deduced hindrance factors, proton intruder states and deformation effects. JOUR ZAANE 25 s01 179

\section{$\mathrm{A}=\mathbf{2 0 5}$}

$\begin{array}{ll}{ }^{205} \mathrm{~Pb} & 2004 \mathrm{KU} 33 \\ { }^{205} \mathrm{Bi} & 2004 \mathrm{KU} 33\end{array}$

RADIOACTIVITY ${ }^{205} \mathrm{Bi}(\mathrm{EC})$ [from Pb, Bi(p, X)]; measured $\mathrm{T}_{1 / 2}$. Comparison with previous results. JOUR RAACA 92233

RADIOACTIVITY ${ }^{205} \mathrm{Bi}(\mathrm{EC})$ [from $\mathrm{Pb}, \mathrm{Bi}(\mathrm{p}, \mathrm{X})$ ]; measured $\mathrm{T}_{1 / 2}$. Comparison with previous results. JOUR RAACA 92233 
$\mathrm{A}=\mathbf{2 0 5}$ (continued)

$\begin{array}{ll}{ }^{205} \mathrm{Fr} & \text { 2005UU03 } \\ & \text { RADIOACTIVITY }{ }^{188,190,192,194,196,198,200,202,204} \mathrm{Po}, \\ & \text { measured reduced widths using gas filled recoil separator; deduced } \\ & \text { hindrance factors, proton intruder states and deformation effects. } \\ & \text { JOUR ZAANE 25 s01 } 179\end{array}$

$\mathrm{A}=206$

${ }^{206} \mathrm{~Pb} \quad 2005 \mathrm{CO} 25 \quad$ NUCLEAR REACTIONS ${ }^{208} \mathrm{~Pb}\left({ }^{40} \mathrm{Ca},{ }^{42} \mathrm{Ca}\right), \mathrm{E}=225 \mathrm{MeV}$; measured $\sigma(\mathrm{E}, \theta) .{ }^{42} \mathrm{Ca}$ deduced excited states configurations. ${ }^{208} \mathrm{~Pb}\left({ }^{90} \mathrm{Zr}, \mathrm{X}\right)$, $\mathrm{E}=560 \mathrm{MeV}$; measured $\mathrm{E} \gamma, \mathrm{I} \gamma$, (fragment) $\gamma$-coin, isotopic yields for projectile-like fragments. ${ }^{90} \mathrm{Zr}$ deduced transitions. JOUR ZAANE 25 s01 427

2005WA34 NUCLEAR MOMENTS ${ }^{204,206,207,208} \mathrm{~Pb}$; measured hfs, isotope shifts. JOUR ZDDNE 36249

${ }^{206} \mathrm{Rn} \quad 2005$ UUO3 $\quad$ RADIOACTIVITY ${ }^{188,190,192,194,196,198,200,202,204} \mathrm{Po}$, $191,193,195,197,199$ At, ${ }^{196,198,200,202,204,206} \mathrm{Rn},{ }^{199,201,203,205,207} \operatorname{Fr}(\alpha)$; measured reduced widths using gas filled recoil separator; deduced hindrance factors, proton intruder states and deformation effects. JOUR ZAANE 25 s01 179

\section{$\mathrm{A}=\mathbf{2 0 7}$}

${ }^{207} \mathrm{Tl}$ 2005GEZW ATOMIC MASSES ${ }^{235}$ Ac; measured mass, $\mathrm{T}_{1 / 2}$.

$185,186,187,188,189,190,191,192,193,194,195,196 \mathrm{Bi}$; measured masses, proton separation energies. ${ }^{207 m} \mathrm{Tl}$; measured $\mathrm{T}_{1 / 2}$. Stored beams, Schottky mass spectrometry. PREPRINT nucl-ex/0510009,10/4/2005

${ }^{207} \mathrm{~Pb}$ 2005BOZT NUCLEAR REACTIONS ${ }^{206} \mathrm{~Pb}(\mathrm{n}, \mathrm{X}),(\mathrm{n}, \gamma), \mathrm{E}=0-600 \mathrm{keV}$; measured total and capture $\sigma$; deduced resonance parameters. ${ }^{206} \mathrm{~Pb},{ }^{209} \mathrm{Bi}(\mathrm{n}, \gamma)$, $\mathrm{E}=$ thermal; measured $\sigma$. THESIS A Borella,Gent Univ

2005WA34 NUCLEAR MOMENTS ${ }^{204,206,207,208} \mathrm{~Pb}$; measured hfs, isotope shifts. JOUR ZDDNE 36249

${ }^{207} \mathrm{Fr} \quad 2005$ UUO3 $\quad$ RADIOACTIVITY ${ }^{188,190,192,194,196,198,200,202,204} \mathrm{Po}$, $191,193,195,197,199$ At, ${ }^{196,198,200,202,204,206} \mathrm{Rn},{ }^{199,201,203,205,207} \operatorname{Fr}(\alpha)$; measured reduced widths using gas filled recoil separator; deduced hindrance factors, proton intruder states and deformation effects. JOUR ZAANE 25 s01 179

\section{$\mathrm{A}=\mathbf{2 0 8}$}

${ }^{208} \mathrm{Tl}$ 2005GR28 NUCLEAR REACTIONS ${ }^{1} \mathrm{H}\left(\pi^{-}, \pi^{+} \pi^{-}\right),\left(\pi^{+}, 2 \pi^{+}\right), \mathrm{E}=243,264,284$, $305 \mathrm{MeV} ;{ }^{2} \mathrm{H},{ }^{12} \mathrm{C},{ }^{40} \mathrm{Ca},{ }^{208} \mathrm{~Pb}\left(\pi^{+}, 2 \pi^{+}\right),\left(\pi^{+}, \pi^{+} \pi^{-}\right), \mathrm{E}=283 \mathrm{MeV}$; $\mathrm{Sc}\left(\pi^{+}, 2 \pi^{+} \mathrm{X}\right),\left(\pi^{+}, \pi^{+} \pi^{-} \mathrm{X}\right), \mathrm{E}=243,264,284,305 \mathrm{MeV}$; measured invariant mass distributions, $\sigma(\theta)$, correlations; deduced partial chiral symmetry restoration. JOUR NUPAB 76380 


\section{$\mathrm{A}=208$ (continued)}

${ }^{208} \mathrm{~Pb}$ 2005G034 NUCLEAR REACTIONS ${ }^{208} \mathrm{~Pb}\left({ }^{23} \mathrm{Al}, \mathrm{p}^{22} \mathrm{Mg}\right), \mathrm{E}=50 \mathrm{MeV} /$ nucleon; measured relative energy spectrum, $\sigma(\theta)$. ${ }^{23} \mathrm{Al}$ deduced excited state radiative width. Astrophysical implications discussed. JOUR JPGPE $31 \mathrm{~S} 1517$

20050R02 NUCLEAR REACTIONS ${ }^{208} \mathrm{~Pb}(\mathrm{p}, \mathrm{p}$ '), E=17.3 MeV; measured Ep, $\mathrm{E}(\mathrm{ce}),(\mathrm{ce}) \mathrm{p}$-coin. ${ }^{208} \mathrm{~Pb}$ deduced levels, electric monopole transitions, E3 / E0 branching ratio. JOUR JPGPE 31 S1705

2005R042 NUCLEAR REACTIONS ${ }^{208} \mathrm{~Pb}\left({ }^{17} \mathrm{~F},{ }^{17} \mathrm{~F}\right),\left({ }^{17} \mathrm{~F},{ }^{16} \mathrm{OX}\right), \mathrm{E}=90.4 \mathrm{Mev}$; measured $\sigma(\theta)$. JOUR ZAANE 25 s01 289

2005SA52 NUCLEAR REACTIONS ${ }^{208} \mathrm{~Pb}\left({ }^{6} \mathrm{He},{ }^{6} \mathrm{He}\right),\left({ }^{6} \mathrm{He}, \alpha\right), \mathrm{E}=14,16,17,18$, $22 \mathrm{MeV}$; measured $\sigma(\theta)$; deduced reaction mechanism features. JOUR JPGPE 31 S1953

2005WA34 NUCLEAR MOMENTS ${ }^{204,206,207,208} \mathrm{~Pb}$; measured hfs, isotope shifts. JOUR ZDDNE 36249

2005YAZW NUCLEAR REACTIONS ${ }^{208} \mathrm{~Pb}(\mathrm{n}, \mathrm{n} \gamma), \mathrm{E}=6.5 \mathrm{MeV}$; measured $\mathrm{E} \gamma$, $\mathrm{I} \gamma .{ }^{208} \mathrm{~Pb}$ deduced levels, $\mathrm{J}, \pi, \mathrm{T}_{1 / 2}, \delta, \mathrm{B}(\mathrm{Ee}) / \mathrm{B}(\mathrm{M} 1) . \mathrm{PC}$

Yates, $11 / 29 / 2005$

${ }^{208} \mathrm{Bi} \quad$ 2005GR28

NUCLEAR REACTIONS ${ }^{1} \mathrm{H}\left(\pi^{-}, \pi^{+} \pi^{-}\right),\left(\pi^{+}, 2 \pi^{+}\right), \mathrm{E}=243,264,284$, $305 \mathrm{MeV} ;{ }^{2} \mathrm{H},{ }^{12} \mathrm{C},{ }^{40} \mathrm{Ca},{ }^{208} \mathrm{~Pb}\left(\pi^{+}, 2 \pi^{+}\right),\left(\pi^{+}, \pi^{+} \pi^{-}\right), \mathrm{E}=283 \mathrm{MeV}$; $\mathrm{Sc}\left(\pi^{+}, 2 \pi^{+} \mathrm{X}\right),\left(\pi^{+}, \pi^{+} \pi^{-} \mathrm{X}\right), \mathrm{E}=243,264,284,305 \mathrm{MeV}$; measured invariant mass distributions, $\sigma(\theta)$, correlations; deduced partial chiral symmetry restoration. JOUR NUPAB 76380

${ }^{208} \mathrm{Ra}$ 2005RE23 NUCLEAR REACTIONS ${ }^{182,184} \mathrm{~W}\left({ }^{30} \mathrm{Si}, 4 \mathrm{n}\right), \mathrm{E}=148 \mathrm{MeV}$; measured delayed $\mathrm{E} \gamma$, I $\gamma, \gamma \gamma$-, (recoil) $\gamma$-coin. ${ }^{208,210} \mathrm{Ra}$ deduced levels, $\mathrm{J}, \pi$, isomers $\mathrm{T}_{1 / 2}, \mathrm{~B}(\mathrm{E} 2)$. Mass separator. JOUR JPGPE $31 \mathrm{~S} 1605$

\section{$\mathrm{A}=\mathbf{2 0 9}$}

${ }^{209} \mathrm{Bi} \quad$ 2005BA88

NUCLEAR REACTIONS ${ }^{208} \mathrm{~Pb}(\mathrm{p}, \gamma), \mathrm{E}=11.9 \mathrm{MeV} ;$ measured $\mathrm{E} \gamma, \mathrm{I} \gamma$. ${ }^{147} \mathrm{Sm}\left({ }^{16} \mathrm{O}, 3 \mathrm{n}\right), \mathrm{E}=73 \mathrm{MeV}$; measured $\mathrm{E} \gamma, \mathrm{I} \gamma, \gamma \gamma$-coin. ${ }^{160} \mathrm{Yb}$ deduced high-spin levels, J, $\pi$. Afrodite array. JOUR JPGPE 31 S1747

2005CH66 NUCLEAR REACTIONS ${ }^{209} \mathrm{Bi}\left({ }^{26} \mathrm{Mg},{ }^{26} \mathrm{Mg}\right.$ '), E=78.6 MeV / nucleon; ${ }^{197} \mathrm{Au}\left({ }^{32} \mathrm{Mg},{ }^{32} \mathrm{Mg}\right.$ '), E=81.1 MeV / nucleon; ${ }^{209} \mathrm{Bi}\left({ }^{34} \mathrm{Mg},{ }^{34} \mathrm{Mg}\right.$ '), $\mathrm{E}=76.4 \mathrm{MeV} /$ nucleon; measured $\mathrm{E} \gamma, \mathrm{I} \gamma$, (particle) $\gamma$-coin following projectile Coulomb excitation. ${ }^{26,32,34} \mathrm{Mg}$ deduced transitions $\mathrm{B}(\mathrm{E} 2)$, deformation parameters. Comparison with previous work, model predictions. JOUR PRVCA 72054320

$$
\mathrm{A}=\mathbf{2 1 0}
$$

${ }^{210} \mathrm{~Pb} \quad 2005 \mathrm{SA} 52$ NUCLEAR REACTIONS ${ }^{208} \mathrm{~Pb}\left({ }^{6} \mathrm{He},{ }^{6} \mathrm{He}\right),\left({ }^{6} \mathrm{He}, \alpha\right), \mathrm{E}=14,16,17,18$, $22 \mathrm{MeV}$; measured $\sigma(\theta)$; deduced reaction mechanism features. JOUR JPGPE 31 S1953

${ }^{210} \mathrm{Bi}$ 2005BOZT NUCLEAR REACTIONS ${ }^{206} \mathrm{~Pb}(\mathrm{n}, \mathrm{X}),(\mathrm{n}, \gamma), \mathrm{E}=0-600 \mathrm{keV}$; measured total and capture $\sigma$; deduced resonance parameters. ${ }^{206} \mathrm{~Pb},{ }^{209} \mathrm{Bi}(\mathrm{n}, \gamma)$, $\mathrm{E}=$ thermal; measured $\sigma$. THESIS A Borella,Gent Univ 


\section{$\mathrm{A}=210$ (continued)}

${ }^{210} \mathrm{Ra}$

2005RE23

2005KU31

$$
{ }^{211} \mathrm{Ra}
$$

$2005 K \cup 31$

$\begin{array}{ll}{ }^{212} \mathrm{Po} & \\ { }^{212} \mathrm{Ra} & 2005 \mathrm{GA} 46\end{array}$

\section{$\mathrm{A}=\mathbf{2 1 2}$}

NUCLEAR REACTIONS ${ }^{208} \mathrm{~Pb},{ }^{209} \mathrm{Bi}\left({ }^{8} \mathrm{He}, 4 \mathrm{n}\right), \mathrm{E}=28 \mathrm{MeV}$; measured $\mathrm{E} \gamma, \mathrm{I} \gamma, \gamma \gamma$-coin. ${ }^{212} \mathrm{Po},{ }^{213} \mathrm{At}$ deduced levels, J, $\pi$. Exogam array. JOUR JPGPE 31 S1851 RADIOACTIVITY ${ }^{215,216,216 m, 217} \operatorname{Th}(\alpha)$ [from $\left.{ }^{170} \operatorname{Er}\left({ }^{50} \mathrm{Ti}, \mathrm{xn}\right)\right]$; measured $\mathrm{E} \alpha, \mathrm{I} \alpha, \mathrm{E} \gamma, \mathrm{I} \gamma, \alpha \gamma$-coin, $\mathrm{T}_{1 / 2} .{ }^{211,212,213} \mathrm{Ra}$ deduced levels, J, $\pi$, ICC. JOUR ZAANE 25397

\section{$\mathrm{A}=\mathbf{2 1 3}$}

${ }^{213}$ At $\quad 2005$ GA46

NUCLEAR REACTIONS ${ }^{208} \mathrm{~Pb},{ }^{209} \mathrm{Bi}\left({ }^{8} \mathrm{He}, 4 \mathrm{n}\right), \mathrm{E}=28 \mathrm{MeV}$; measured $\mathrm{E} \gamma, \mathrm{I} \gamma, \gamma \gamma$-coin. ${ }^{212} \mathrm{Po},{ }^{213}$ At deduced levels, J, $\pi$. Exogam array. JOUR JPGPE 31 S1851

${ }^{213} \mathrm{Ra} \quad 2005 \mathrm{KU} 31$

${ }^{213}$ Th 2005LE42 RADIOACTIVITY ${ }^{215,216,216 m, 217} \operatorname{Th}(\alpha)$ [from $\left.{ }^{170} \operatorname{Er}\left({ }^{50} \mathrm{Ti}, \mathrm{xn}\right)\right]$; measured $\mathrm{E} \alpha, \mathrm{I} \alpha, \mathrm{E} \gamma, \mathrm{I} \gamma, \alpha \gamma$-coin, $\mathrm{T}_{1 / 2} .{ }^{211,212,213} \mathrm{Ra}$ deduced levels, J, $\pi$, ICC. JOUR ZAANE 25397

RADIOACTIVITY ${ }^{217,218,218 m, 219} \mathrm{U}(\alpha)$; measured $\mathrm{E} \alpha, \mathrm{T}_{1 / 2}$. ${ }^{217,218,219} \mathrm{U}$ deduced ground state $\mathrm{J}, \pi .{ }^{218} \mathrm{U}$ deduced isomer $\mathrm{J}, \pi$. Implications for $\mathrm{Z}=92$ shell closure discussed. JOUR ZAANE 25 s01 183

\section{$\mathrm{A}=\mathbf{2 1 4}$}

${ }^{214} \mathrm{Th} \quad$ 2005LE42

RADIOACTIVITY ${ }^{217,218,218 m, 219} \mathrm{U}(\alpha)$; measured $\mathrm{E} \alpha, \mathrm{T}_{1 / 2}$. ${ }^{217,218,219} \mathrm{U}$ deduced ground state $\mathrm{J}, \pi \cdot{ }^{218} \mathrm{U}$ deduced isomer $\mathrm{J}, \pi$. Implications for $\mathrm{Z}=92$ shell closure discussed. JOUR ZAANE $25 \mathrm{~s} 01$ 183

\section{$\mathrm{A}=\mathbf{2 1 5}$}

${ }^{215} \mathrm{Th} \quad 2005 \mathrm{KU} 31$
RADIOACTIVITY ${ }^{215,216,216 m, 217} \operatorname{Th}(\alpha)$ [from $\left.{ }^{170} \operatorname{Er}\left({ }^{50} \mathrm{Ti}, \mathrm{xn}\right)\right]$; measured $\mathrm{E} \alpha, \mathrm{I} \alpha, \mathrm{E} \gamma, \mathrm{I} \gamma, \alpha \gamma$-coin, $\mathrm{T}_{1 / 2} .{ }^{211,212,213} \mathrm{Ra}$ deduced levels, J, $\pi$, ICC. JOUR ZAANE 25397 


\section{$\mathrm{A}=215$ (continued)}

2005LE42 RADIOACTIVITY ${ }^{217,218,218 m, 219} \mathrm{U}(\alpha)$; measured $\mathrm{E} \alpha, \mathrm{T}_{1 / 2}$. ${ }^{217,218,219} \mathrm{U}$ deduced ground state $\mathrm{J}, \pi .{ }^{218} \mathrm{U}$ deduced isomer $\mathrm{J}, \pi$. Implications for $\mathrm{Z}=92$ shell closure discussed. JOUR ZAANE 25 s01 183

\section{$\mathrm{A}=216$}

${ }^{216} \mathrm{Th} \quad 2005 \mathrm{KU} 31$

RADIOACTIVITY ${ }^{215,216,216 m, 217} \mathrm{Th}(\alpha)$ [from $\left.{ }^{170} \operatorname{Er}\left({ }^{50} \mathrm{Ti}, \mathrm{xn}\right)\right]$; measured $\mathrm{E} \alpha, \mathrm{I} \alpha, \mathrm{E} \gamma, \mathrm{I} \gamma, \alpha \gamma$-coin, $\mathrm{T}_{1 / 2} .{ }^{211,212,213} \mathrm{Ra}$ deduced levels, J, $\pi$, ICC. JOUR ZAANE 25397

\section{$\mathrm{A}=\mathbf{2 1 7}$}

${ }^{217} \mathrm{Th} \quad 2005 \mathrm{KU} 31$

RADIOACTIVITY ${ }^{215,216,216 m, 217} \operatorname{Th}(\alpha)$ [from $\left.{ }^{170} \operatorname{Er}\left({ }^{50} \mathrm{Ti}, \mathrm{xn}\right)\right]$; measured $\mathrm{E} \alpha, \mathrm{I} \alpha, \mathrm{E} \gamma, \mathrm{I} \gamma, \alpha \gamma$-coin, $\mathrm{T}_{1 / 2} .{ }^{211,212,213} \mathrm{Ra}$ deduced levels, J, $\pi$, ICC. JOUR ZAANE 25397

${ }^{217} \mathrm{U} \quad$ 2005LE42 NUCLEAR REACTIONS ${ }^{182} \mathrm{~W}\left({ }^{40} \mathrm{Ar}, \mathrm{xn}\right){ }^{217} \mathrm{U} /{ }^{218} \mathrm{U} /{ }^{218 m} \mathrm{U} /{ }^{219} \mathrm{U}$, $\mathrm{E}=186 \mathrm{MeV}$; measured $\mathrm{E} \alpha, \alpha \alpha-$, (recoil) $\alpha$-coin; deduced production $\sigma$. JOUR ZAANE 25 s01 183

2005LE42 RADIOACTIVITY ${ }^{217,218,218 m, 219} \mathrm{U}(\alpha)$; measured $\mathrm{E} \alpha, \mathrm{T}_{1 / 2}$. ${ }^{217,218,219} \mathrm{U}$ deduced ground state $\mathrm{J}, \pi \cdot{ }^{218} \mathrm{U}$ deduced isomer $\mathrm{J}, \pi$. Implications for $\mathrm{Z}=92$ shell closure discussed. JOUR ZAANE 25 s01 183

\section{$\mathrm{A}=\mathbf{2 1 8}$}

${ }^{218} \mathrm{U} \quad$ 2005LE42

NUCLEAR REACTIONS ${ }^{182} \mathrm{~W}\left({ }^{40} \mathrm{Ar}, \mathrm{xn}\right){ }^{217} \mathrm{U} /{ }^{218} \mathrm{U} /{ }^{218 m} \mathrm{U} /{ }^{219} \mathrm{U}$, $\mathrm{E}=186 \mathrm{MeV}$; measured $\mathrm{E} \alpha, \alpha \alpha-$, (recoil) $\alpha$-coin; deduced production $\sigma$. JOUR ZAANE 25 s01 183

2005LE42 RADIOACTIVITY ${ }^{217,218,218 m, 219} \mathrm{U}(\alpha)$; measured $\mathrm{E} \alpha, \mathrm{T}_{1 / 2}$. ${ }^{217,218,219} \mathrm{U}$ deduced ground state $\mathrm{J}, \pi \cdot{ }^{218} \mathrm{U}$ deduced isomer $\mathrm{J}, \pi$. Implications for $\mathrm{Z}=92$ shell closure discussed. JOUR ZAANE 25 s01 183

\section{$\mathrm{A}=\mathbf{2 1 9}$}

${ }^{219} \mathrm{U} \quad$ 2005LE42

NUCLEAR REACTIONS ${ }^{182} \mathrm{~W}\left({ }^{40} \mathrm{Ar}, \mathrm{xn}\right){ }^{217} \mathrm{U} /{ }^{218} \mathrm{U} /{ }^{218 m} \mathrm{U} /{ }^{219} \mathrm{U}$, $\mathrm{E}=186 \mathrm{MeV}$; measured $\mathrm{E} \alpha, \alpha \alpha$-, (recoil) $\alpha$-coin; deduced production $\sigma$. JOUR ZAANE 25 s01 183

2005LE42 RADIOACTIVITY ${ }^{217,218,218 m, 219} \mathrm{U}(\alpha)$; measured $\mathrm{E} \alpha, \mathrm{T}_{1 / 2}$. ${ }^{217,218,219} \mathrm{U}$ deduced ground state $\mathrm{J}, \pi \cdot{ }^{218} \mathrm{U}$ deduced isomer $\mathrm{J}, \pi$. Implications for $\mathrm{Z}=92$ shell closure discussed. JOUR ZAANE 25 s01 183 


$$
\mathrm{A}=\mathbf{2 2 0}
$$

No references found

$$
\mathrm{A}=221
$$

No references found

$$
\mathrm{A}=222
$$

${ }^{222} \mathrm{Rn} \quad 2004 \mathrm{KU} 35 \quad$ RADIOACTIVITY ${ }^{238} \mathrm{Pu},{ }^{226} \mathrm{Ra}(\alpha) ;{ }^{152} \mathrm{Eu}(\mathrm{EC}) ;$ measured low-energy electron spectra, angular distributions, (electron) $\alpha-$, (electron) $\gamma-$, (electron)(X-ray)-coin. JOUR BRSPE 681358

$$
\mathrm{A}=223
$$

No references found

$$
\mathrm{A}=224
$$

No references found

$$
\mathrm{A}=225
$$

No references found

$$
\mathrm{A}=\mathbf{2 2 6}
$$

${ }^{226} \mathrm{Ra} \quad 2004 \mathrm{KU} 35 \quad$ RADIOACTIVITY ${ }^{238} \mathrm{Pu},{ }^{226} \mathrm{Ra}(\alpha) ;{ }^{152} \mathrm{Eu}(\mathrm{EC}) ;$ measured low-energy electron spectra, angular distributions, (electron) $\alpha-$, (electron) $\gamma-$, (electron)(X-ray)-coin. JOUR BRSPE 681358

$$
\mathrm{A}=227
$$

No references found

$$
\mathrm{A}=228
$$

No references found 


\section{$\mathrm{A}=229$}

${ }^{229} \mathrm{Ra} \quad$ 2005HE26 ATOMIC MASSES ${ }^{229,230,231,232} \mathrm{Ra},{ }^{230} \mathrm{Fr}$; measured masses. Penning trap mass spectrometer. JOUR ZAANE 25 s01 17

\section{$\mathrm{A}=\mathbf{2 3 0}$}

${ }^{230} \mathrm{Fr} \quad 2005 \mathrm{HE} 26$

${ }^{230} \mathrm{Ra} \quad 2005 \mathrm{HE} 26$

.

(2005

${ }^{231} \mathrm{Ra} \quad 2005 \mathrm{HE} 26$

${ }^{232} \mathrm{Ra} \quad 2005 \mathrm{HE} 26$

ATOMIC MASSES $229,230,231,232 \mathrm{Ra},{ }^{230} \mathrm{Fr}$; measured masses. Penning trap mass spectrometer. JOUR ZAANE 25 s01 17

\section{$A=233$}

${ }^{233} \mathrm{Th}$ 2004HA64 NUCLEAR REACTIONS ${ }^{232} \mathrm{Th}(\mathrm{n}, \gamma), \mathrm{E}=0.05-2 \mathrm{MeV} ;{ }^{230} \mathrm{Th}$, ${ }^{231,233} \mathrm{~Pa}(\mathrm{n}, \mathrm{F}), \mathrm{E}=0.5-10 \mathrm{MeV}$; measured $\sigma$. Comparison with previous results. JOUR BJPHE 34814

\section{$\mathrm{A}=\mathbf{2 3 4}$}

${ }^{234} \mathrm{U} \quad 2004 \mathrm{KU} 35 \quad$ RADIOACTIVITY ${ }^{238} \mathrm{Pu},{ }^{226} \mathrm{Ra}(\alpha) ;{ }^{152} \mathrm{Eu}(\mathrm{EC})$; measured low-energy electron spectra, angular distributions, (electron) $\alpha-$, (electron) $\gamma^{-}$, (electron)(X-ray)-coin. JOUR BRSPE 681358

\section{$\mathrm{A}=\mathbf{2 3 5}$}

${ }^{235} \mathrm{Ac} \quad 2005 \mathrm{GEZW}$

ATOMIC MASSES ${ }^{235}$ Ac; measured mass, $\mathrm{T}_{1 / 2}$. 185,186,187,188,189,190,191,192,193,194,195,196 $\mathrm{Bi}$; measured masses, proton separation energies. ${ }^{207 m} \mathrm{Tl}$; measured $\mathrm{T}_{1 / 2}$. Stored beams, Schottky mass spectrometry. PREPRINT nucl-ex/0510009,10/4/2005 


\section{$\mathrm{A}=236$}

\begin{tabular}{|c|c|c|}
\hline \multirow[t]{2}{*}{${ }^{236} \mathrm{U}$} & $2005 \mathrm{CSZZ}$ & $\begin{array}{l}\text { NUCLEAR REACTIONS }{ }^{235} \mathrm{U}(\mathrm{d}, \mathrm{pF}), \mathrm{E}=13 \mathrm{MeV} \text {; measured Ep, } \\
\text { fission fragment angular correlations. }{ }^{236} \mathrm{U} \text { deduced hyperdeformed } \\
\text { resonances. REPT MLL } 2004 \text { Annual,P19, Csige } \\
\text { NUCLEAR REACTIONS }{ }^{232} \mathrm{Th},{ }^{238} \mathrm{U}(\mathrm{n}, \mathrm{F}), \mathrm{E}=21-95 \mathrm{MeV} ; \text { measured } \\
\text { fission fragments angular distributions, anisotropy. }{ }^{232} \mathrm{Th},{ }^{238} \mathrm{U}(\mathrm{n}, \mathrm{F}), \\
\mathrm{E}=0-95 \mathrm{MeV} ;{ }^{232} \mathrm{Th},{ }^{238} \mathrm{U}(\mathrm{n}, 2 \mathrm{n}),(\mathrm{n}, 3 \mathrm{n}),(\mathrm{n}, \mathrm{xnF}), \mathrm{E}=0-20 \mathrm{MeV} ; \\
\text { calculated } \sigma \text {, fission fragments angular anisotropy. }{ }^{238} \mathrm{U}(\mathrm{n}, \mathrm{pX}), \\
\mathrm{E}=25-65 \mathrm{MeV} \text {; calculated } \sigma . \text { Multichance fission, saddle-point } \\
\text { statistical model analysis. JOUR NUPAB } 76019\end{array}$ \\
\hline & \multicolumn{2}{|r|}{$\mathrm{A}=237$} \\
\hline${ }^{237} \mathrm{U}$ & 2005RY03 & $\begin{array}{l}\text { NUCLEAR REACTIONS }{ }^{232} \mathrm{Th},{ }^{238} \mathrm{U}(\mathrm{n}, \mathrm{F}), \mathrm{E}=21-95 \mathrm{MeV} \text {; measured } \\
\text { fission fragments angular distributions, anisotropy. }{ }^{232} \mathrm{Th},{ }^{238} \mathrm{U}(\mathrm{n}, \mathrm{F}), \\
\mathrm{E}=0-95 \mathrm{MeV} ;{ }^{232} \mathrm{Th},{ }^{238} \mathrm{U}(\mathrm{n}, 2 \mathrm{n}),(\mathrm{n}, 3 \mathrm{n}),(\mathrm{n}, \mathrm{xnF}), \mathrm{E}=0-20 \mathrm{MeV} ; \\
\text { calculated } \sigma \text {, fission fragments angular anisotropy. }{ }^{238} \mathrm{U}(\mathrm{n}, \mathrm{pX}), \\
\mathrm{E}=25-65 \mathrm{MeV} \text {; calculated } \sigma . \text { Multichance fission, saddle-point } \\
\text { statistical model analysis. JOUR NUPAB } 76019\end{array}$ \\
\hline${ }^{237} \mathrm{~Np}$ & 2005MA90 & $\begin{array}{l}\text { RADIOACTIVITY }{ }^{242} \operatorname{Am}\left(\beta^{-}\right),(\mathrm{EC})\left[\text { from }{ }^{241} \operatorname{Am}(\mathrm{n}, \gamma)\right] ; \text { measured } \\
\beta \gamma \text {-coin; deduced source activity. }{ }^{241} \operatorname{Am}(\alpha) ; \text { measured } \mathrm{E} \alpha \text {. JOUR } \\
\text { NIMAE } 553559\end{array}$ \\
\hline & 2005PA56 & $\begin{array}{l}\text { RADIOACTIVITY }{ }^{252} \mathrm{Cf}(\mathrm{SF}) \text {; measured neutron emission rates. } \\
{ }^{241} \mathrm{Am}(\alpha) \text {; measured neutron emission rates for Am-Be source. } \\
\text { Manganese sulphate bath system. JOUR KPSJA } 47603\end{array}$ \\
\hline
\end{tabular}

\section{$\mathrm{A}=238$}

${ }^{238} \mathrm{U} \quad 2005 \mathrm{YO} 12$

RADIOACTIVITY ${ }^{238} \mathrm{U}(\mathrm{SF})$; measured spontaneous fission decay constant. Solid-state nuclear track detectors. JOUR NIMAE 555386 NUCLEAR REACTIONS ${ }^{237} \mathrm{~Np}(\mathrm{n}, \gamma), \mathrm{E}=0.01-10 \mathrm{eV}$; measured $\sigma$. Comparison with previous results. JOUR NIMBE 241176

RADIOACTIVITY ${ }^{238} \mathrm{Pu},{ }^{226} \mathrm{Ra}(\alpha) ;{ }^{152} \mathrm{Eu}(\mathrm{EC})$; measured low-energy electron spectra, angular distributions, (electron) $\alpha-$, (electron) $\gamma-$, (electron)(X-ray)-coin. JOUR BRSPE 681358

$$
\mathrm{A}=\mathbf{2 3 9}
$$

No references found

$$
\mathrm{A}=\mathbf{2 4 0}
$$

${ }^{240} \mathrm{Pu} \quad$ 2005THZZ

NUCLEAR REACTIONS ${ }^{232} \mathrm{Th},{ }^{238} \mathrm{U}(\alpha, 2 \mathrm{n}), \mathrm{E}=20-27 \mathrm{MeV}$; measured prompt and delayed fission fragment yields; deduced excitation functions for isomeric and prompt fission. REPT MLL 2004 Annual,P17,Thirolf 


\section{$\mathrm{A}=241$}

${ }^{241} \mathrm{Am} \quad$ 2005Ma90 RADIOACTIVITY ${ }^{242} \mathrm{Am}\left(\beta^{-}\right)$, (EC) [from $\left.{ }^{241} \mathrm{Am}(\mathrm{n}, \gamma)\right]$; measured $\beta \gamma$-coin; deduced source activity. ${ }^{241} \operatorname{Am}(\alpha)$; measured E $\alpha$. JOUR NIMAE 553559

2005PA56 RADIOACTIVITY ${ }^{252} \mathrm{Cf}(\mathrm{SF})$; measured neutron emission rates. ${ }^{241} \operatorname{Am}(\alpha)$; measured neutron emission rates for Am-Be source. Manganese sulphate bath system. JOUR KPSJA 47603

\section{$\mathrm{A}=242$}

${ }^{242} \mathrm{Pu}$ 2005MA90 RADIOACTIVITY ${ }^{242} \mathrm{Am}\left(\beta^{-}\right)$, (EC) [from $\left.{ }^{241} \mathrm{Am}(\mathrm{n}, \gamma)\right]$; measured $\beta \gamma$-coin; deduced source activity. ${ }^{241} \mathrm{Am}(\alpha)$; measured $\mathrm{E} \alpha$. JOUR NIMAE 553559

${ }^{242} \mathrm{Am} \quad$ 2005MA90 RADIOACTIVITY ${ }^{242} \mathrm{Am}\left(\beta^{-}\right)$, (EC) [from $\left.{ }^{241} \mathrm{Am}(\mathrm{n}, \gamma)\right]$; measured $\beta \gamma$-coin; deduced source activity. ${ }^{241} \operatorname{Am}(\alpha)$; measured $\mathrm{E} \alpha$. JOUR NIMAE 553559

${ }^{242} \mathrm{Cm}$ 2005MA90 RADIOACTIVITY ${ }^{242} \mathrm{Am}\left(\beta^{-}\right),(\mathrm{EC})\left[\right.$ from $\left.{ }^{241} \mathrm{Am}(\mathrm{n}, \gamma)\right]$; measured $\beta \gamma$-coin; deduced source activity. ${ }^{241} \operatorname{Am}(\alpha)$; measured $\mathrm{E} \alpha$. JOUR NIMAE 553559

\section{$\mathrm{A}=243$}

${ }^{243} \mathrm{Es}$ 2005HE27 RADIOACTIVITY $247,249,251,253,255 \mathrm{Md}(\alpha)$ [from ${ }^{209} \mathrm{Bi}\left({ }^{40} \mathrm{Ar}, \mathrm{xn}\right)$, $\left({ }^{50} \mathrm{Ti}, \mathrm{xn}\right),{ }^{207,207} \mathrm{~Pb},{ }^{209} \mathrm{Bi}\left({ }^{48} \mathrm{Ca}, \mathrm{xn}\right)$ and subsequent decay]; measured $\mathrm{E} \alpha, \mathrm{E} \gamma, \alpha \gamma$-coin; deduced branching ratios, hindrance factors. $243,245,247,249,251$ Es deduced levels, J, $\pi$, configurations, deformation. Comparison with model predictions. JOUR ZAANE 26233

\section{$\mathrm{A}=244$}

${ }^{244} \mathrm{Cm} \quad$ 2004NA44 RADIOACTIVITY ${ }^{244} \mathrm{Cm}(\mathrm{SF})$; measured fission fragments isomeric yield ratios; deduced fragment angular momentum distributions. JOUR RAACA 921

\section{$\mathrm{A}=245$}

${ }^{245} \mathrm{Es} \quad 2005 \mathrm{HE} 27 \quad$ RADIOACTIVITY $247,249,251,253,255 \mathrm{Md}(\alpha)$ [from ${ }^{209} \mathrm{Bi}\left({ }^{40} \mathrm{Ar}, \mathrm{xn}\right)$, $\left({ }^{50} \mathrm{Ti}, \mathrm{xn}\right),{ }^{207,207} \mathrm{~Pb},{ }^{209} \mathrm{Bi}\left({ }^{48} \mathrm{Ca}, \mathrm{xn}\right)$ and subsequent decay]; measured $\mathrm{E} \alpha, \mathrm{E} \gamma, \alpha \gamma$-coin; deduced branching ratios, hindrance factors. $243,245,247,249,251$ Es deduced levels, J, $\pi$, configurations, deformation. Comparison with model predictions. JOUR ZAANE 26233

$$
\mathrm{A}=\mathbf{2 4 6}
$$

No references found 


\section{$\mathrm{A}=\mathbf{2 4 7}$}

\begin{tabular}{|c|c|c|}
\hline \multirow[t]{2}{*}{${ }^{247} \mathrm{Es}$} & 2005GR36 & $\begin{array}{l}\text { RADIOACTIVITY }{ }^{255} \mathrm{Lr},{ }^{251} \mathrm{Md}(\alpha) \text { from }{ }^{209} \mathrm{Bi}\left({ }^{48} \mathrm{Ca}, 2 \mathrm{n}\right) \text { and } \\
\text { subsequent decay]; measured } \mathrm{E} \alpha, \alpha \alpha \text {-coin; deduced excited state decay. }\end{array}$ \\
\hline & 2005HE27 & $\begin{array}{l}\text { RADIOACTIVITY }{ }^{247,249,251,253,255} \mathrm{Md}(\alpha) \text { [from }{ }^{209} \mathrm{Bi}\left({ }^{40} \mathrm{Ar}, \mathrm{xn}\right), \\
\left({ }^{50} \mathrm{Ti}, \mathrm{xn}\right),{ }^{207,207} \mathrm{~Pb},{ }^{209} \mathrm{Bi}\left({ }^{48} \mathrm{Ca}, \mathrm{xn}\right) \text { and subsequent decay]; measured } \\
\mathrm{E} \alpha, \mathrm{E} \gamma, \alpha \gamma \text {-coin; deduced branching ratios, hindrance factors. } \\
243,245,247,249,251 \mathrm{Es} \text { deduced levels, J, } \pi \text {, configurations, deformation. }\end{array}$ \\
\hline${ }^{247} \mathrm{Md}$ & 2005HE27 & $\begin{array}{l}\text { Comparison with model predictions. JOUR ZAANE } 26233 \\
\text { RADIOACTIVITY }{ }^{247,249,251,253,255} \mathrm{Md}(\alpha) \text { [from }{ }^{209} \mathrm{Bi}\left({ }^{40} \mathrm{Ar}, \mathrm{xn}\right), \\
\left({ }^{50} \mathrm{Ti}, \mathrm{xn}\right),{ }^{207,207} \mathrm{~Pb},{ }^{209} \mathrm{Bi}\left({ }^{48} \mathrm{Ca}, \mathrm{xn}\right) \text { and subsequent decay]; measured } \\
\mathrm{E} \alpha, \mathrm{E} \gamma, \alpha \gamma \text {-coin; deduced branching ratios, hindrance factors. } \\
{ }^{243,245,247,249,251} \text { Es deduced levels, J, } \pi \text {, configurations, deformation. }\end{array}$ \\
\hline & & Comparison with model predictions. JOUR ZAANE 26233 \\
\hline
\end{tabular}

\section{$\mathrm{A}=248$}

No references found

\section{$\mathrm{A}=\mathbf{2 4 9}$}

${ }^{249} \mathrm{Es} \quad 2005 \mathrm{HE} 27$

${ }^{249} \mathrm{Md} \quad 2005 \mathrm{HE} 27$
RADIOACTIVITY ${ }^{247,249,251,253,255} \mathrm{Md}(\alpha)$ [from ${ }^{209} \mathrm{Bi}\left({ }^{40} \mathrm{Ar}, \mathrm{xn}\right)$, $\left({ }^{50} \mathrm{Ti}, \mathrm{xn}\right),{ }^{207,207} \mathrm{~Pb},{ }^{209} \mathrm{Bi}\left({ }^{48} \mathrm{Ca}, \mathrm{xn}\right)$ and subsequent decay]; measured $\mathrm{E} \alpha, \mathrm{E} \gamma, \alpha \gamma$-coin; deduced branching ratios, hindrance factors. $243,245,247,249,251$ Es deduced levels, J, $\pi$, configurations, deformation. Comparison with model predictions. JOUR ZAANE 26233 RADIOACTIVITY ${ }^{247,249,251,253,255} \mathrm{Md}(\alpha)$ [from ${ }^{209} \mathrm{Bi}\left({ }^{40} \mathrm{Ar}, \mathrm{xn}\right)$, $\left({ }^{50} \mathrm{Ti}, \mathrm{xn}\right),{ }^{207,207} \mathrm{~Pb},{ }^{209} \mathrm{Bi}\left({ }^{48} \mathrm{Ca}, \mathrm{xn}\right)$ and subsequent decay]; measured $\mathrm{E} \alpha, \mathrm{E} \gamma, \alpha \gamma$-coin; deduced branching ratios, hindrance factors. 243,245,247,249,251 Es deduced levels, J, $\pi$, configurations, deformation. Comparison with model predictions. JOUR ZAANE 26233

$$
\mathrm{A}=\mathbf{2 5 0}
$$

No references found

\section{$\mathrm{A}=\mathbf{2 5 1}$}

$\begin{array}{ll}{ }^{251} \mathrm{Cf} & 2005 \mathrm{AHO9} \\ { }^{251} \mathrm{Es} & 2005 \mathrm{AHO9}\end{array}$

RADIOACTIVITY ${ }^{255} \mathrm{Fm}(\alpha)$; measured $\mathrm{E} \gamma, \mathrm{I} \gamma \cdot{ }^{251} \mathrm{Es}(\mathrm{EC}) ;$ measured $\mathrm{E} \gamma, \mathrm{I} \gamma, \mathrm{E}(\mathrm{ce}), \mathrm{I}(\mathrm{ce}) .{ }^{251} \mathrm{Cf}$ deduced levels, J, $\pi$, configurations, vibrational states. JOUR PRVCA 72054308

RADIOACTIVITY ${ }^{255} \mathrm{Fm}(\alpha)$; measured $\mathrm{E} \gamma, \mathrm{I} \gamma \cdot{ }^{251} \mathrm{Es}(\mathrm{EC})$; measured $\mathrm{E} \gamma, \mathrm{I} \gamma, \mathrm{E}(\mathrm{ce}), \mathrm{I}(\mathrm{ce}) .{ }^{251} \mathrm{Cf}$ deduced levels, J, $\pi$, configurations, vibrational states. JOUR PRVCA 72054308 


\section{$\mathrm{A}=251$ (continued)}

2005HE27 RADIOACTIVITY ${ }^{247,249,251,253,255} \mathrm{Md}(\alpha)$ [from ${ }^{209} \mathrm{Bi}\left({ }^{40} \mathrm{Ar}, \mathrm{xn}\right)$, $\left({ }^{50} \mathrm{Ti}, \mathrm{xn}\right),{ }^{207,207} \mathrm{~Pb},{ }^{209} \mathrm{Bi}\left({ }^{48} \mathrm{Ca}, \mathrm{xn}\right)$ and subsequent decay]; measured $\mathrm{E} \alpha, \mathrm{E} \gamma, \alpha \gamma$-coin; deduced branching ratios, hindrance factors. $243,245,247,249,251$ Es deduced levels, J, $\pi$, configurations, deformation. Comparison with model predictions. JOUR ZAANE 26233

${ }^{251} \mathrm{Md}$ 2005GR36 RADIOACTIVITY ${ }^{255} \mathrm{Lr},{ }^{251} \mathrm{Md}(\alpha)$ [from ${ }^{209} \mathrm{Bi}\left({ }^{48} \mathrm{Ca}, 2 \mathrm{n}\right)$ and subsequent decay]; measured $\mathrm{E} \alpha, \alpha \alpha$-coin; deduced excited state decay. JOUR ZAANE 25 s01 599

2005GR36 NUCLEAR REACTIONS ${ }^{205} \mathrm{Tl}\left({ }^{48} \mathrm{Ca}, 2 \mathrm{n}\right), \mathrm{E}=218 \mathrm{MeV}$; measured E $\gamma$, I $\gamma$, (recoil) $\gamma$-coin. Jurogam array. JOUR ZAANE 25 s01 599

2005HE27 RADIOACTIVITY ${ }^{247,249,251,253,255} \mathrm{Md}(\alpha)$ [from ${ }^{209} \mathrm{Bi}\left({ }^{40} \mathrm{Ar}\right.$, xn), $\left({ }^{50} \mathrm{Ti}, \mathrm{xn}\right),{ }^{207,207} \mathrm{~Pb},{ }^{209} \mathrm{Bi}\left({ }^{48} \mathrm{Ca}, \mathrm{xn}\right)$ and subsequent decay]; measured $\mathrm{E} \alpha, \mathrm{E} \gamma, \alpha \gamma$-coin; deduced branching ratios, hindrance factors. $243,245,247,249,251$ Es deduced levels, J, $\pi$, configurations, deformation. Comparison with model predictions. JOUR ZAANE 26233

\section{$\mathrm{A}=252$}

${ }^{252} \mathrm{Cf} \quad 2005 \mathrm{~F} 017$

RADIOACTIVITY ${ }^{252} \mathrm{Cf}(\mathrm{SF})$; measured $\mathrm{E} \gamma, \mathrm{I} \gamma, \gamma \gamma$-coin. ${ }^{98} \mathrm{Sr}$, ${ }^{102,104} \mathrm{Zr},{ }^{137} \mathrm{Xe},{ }^{143} \mathrm{Ba},{ }^{152} \mathrm{Ce}$ levels deduced $\mathrm{T}_{1 / 2}$. Gammasphere array, time-gated triple-coincidence method. JOUR ZAANE 25 s01 465

2005HW06 RADIOACTIVITY ${ }^{252} \mathrm{Cf}(\mathrm{SF})$; measured $\mathrm{E} \gamma$, I $\gamma, \gamma \gamma$-coin. ${ }^{95,97} \mathrm{Sr},{ }^{99} \mathrm{Zr}$, ${ }^{108} \mathrm{Tc},{ }^{133,134} \mathrm{Te},{ }^{137} \mathrm{Xe}$ levels deduced $\mathrm{T}_{1 / 2}$. Gammasphere array, time-gated triple-coincidence method. JOUR ZAANE 25 s01 463

2005J024 RADIOACTIVITY ${ }^{252} \mathrm{Cf}(\mathrm{SF})$; measured E $\gamma$, I $\gamma, \gamma \gamma$-coin. ${ }^{162,164} \mathrm{Gd}$ deduced levels, J, $\pi$. Gammasphere array, level systematics in neighboring nuclides discussed. JOUR ZAANE 25 s01 467

2005LU21 RADIOACTIVITY ${ }^{252} \mathrm{Cf}(\mathrm{SF})$; measured $\mathrm{E} \gamma, \mathrm{I} \gamma, \gamma \gamma$-coin. ${ }^{99,101} \mathrm{Y}$, ${ }^{101,105} \mathrm{Nb}$ deduced levels, J, $\pi$, configurations, rotational bands, shape transition features. Gammasphere array, triaxial-rotor-plus-quasiparticle calculations. JOUR JPGPE 311303

2005LU24 RADIOACTIVITY ${ }^{252} \mathrm{Cf}(\mathrm{SF})$; measured $\mathrm{E} \gamma, \mathrm{I} \gamma, \gamma \gamma$-coin. ${ }^{99,101} \mathrm{Y}$, ${ }^{101,105} \mathrm{Nb}$ deduced levels, J, $\pi$, configurations, deformation. Gammasphere array, triaxial-rotor-plus-particle calculations. JOUR ZAANE 25 s01 469

2005PA56 RADIOACTIVITY ${ }^{252} \mathrm{Cf}(\mathrm{SF})$; measured neutron emission rates. ${ }^{241} \operatorname{Am}(\alpha)$; measured neutron emission rates for Am-Be source. Manganese sulphate bath system. JOUR KPSJA 47603

2005SH49 RADIOACTIVITY ${ }^{252} \mathrm{Cf}(\mathrm{SF})$; measured Doppler-shifted E $\gamma, \mathrm{I} \gamma, \gamma \gamma$ (fragment) $\gamma$-coin. ${ }^{144} \mathrm{Ba}$ deduced transitions $\mathrm{T}_{1 / 2}, \mathrm{~B}(\mathrm{E} 2)$, transition dipole, quadrupole, and octupole moments for alternating-parity band. Gammasphere array, cluster-model analysis. JOUR ZAANE 25387

2005SM08 RADIOACTIVITY ${ }^{252} \mathrm{Cf}(\mathrm{SF})$; measured $\mathrm{E} \gamma, \mathrm{I} \gamma(\theta, \mathrm{H}, \mathrm{t}), \gamma \gamma$-coin. ${ }^{96,100,102} \mathrm{Zr},{ }^{102,104,106,108} \mathrm{Mo},{ }^{106,108,110,112} \mathrm{Ru},{ }^{110,114,116} \mathrm{Pd}$ levels deduced g factors, B(E2). Gammasphere array, time-integral perturbed angular correlation technique. Comparison with interacting boson model predictions. JOUR JPGPE 31 S1433 


\section{$\mathrm{A}=252$ (continued)}

$2005 Z$ Z36 RADIOACTIVITY ${ }^{252} \mathrm{Cf}(\mathrm{SF})$; measured E $\gamma, \mathrm{I} \gamma, \gamma \gamma$-coin. ${ }^{106} \mathrm{Mo}$ deduced high-spin levels, $\mathrm{J}, \pi$, chiral vibrational bands. Gammasphere array, tilted-axis cranking model analysis. JOUR ZAANE 25 s01 459

\section{$\mathrm{A}=253$}

${ }^{253} \mathrm{Md} \quad$ 2005HE27

RADIOACTIVITY ${ }^{247,249,251,253,255} \mathrm{Md}(\alpha)$ [from ${ }^{209} \mathrm{Bi}\left({ }^{40} \mathrm{Ar}, \mathrm{xn}\right)$, $\left({ }^{50} \mathrm{Ti}, \mathrm{xn}\right),{ }^{207,207} \mathrm{~Pb},{ }^{209} \mathrm{Bi}\left({ }^{48} \mathrm{Ca}, \mathrm{xn}\right)$ and subsequent decay]; measured $\mathrm{E} \alpha, \mathrm{E} \gamma, \alpha \gamma$-coin; deduced branching ratios, hindrance factors. $243,245,247,249,251$ Es deduced levels, J, $\pi$, configurations, deformation. Comparison with model predictions. JOUR ZAANE 26233

\section{$\mathrm{A}=254$}

${ }^{254} \mathrm{No} \quad 2005 \mathrm{EE} 01$

NUCLEAR REACTIONS ${ }^{208} \mathrm{~Pb}\left({ }^{48} \mathrm{Ca}, 2 \mathrm{n}\right)$, E not given; measured $\mathrm{E} \gamma$, $\mathrm{I} \gamma$, (recoil) $\gamma$-coin. ${ }^{254}$ No deduced rotational band levels, J, $\pi$. Jurogam array, recoil-decay tagging. JOUR ZAANE 25 s01 605

2005EE02 NUCLEAR REACTIONS ${ }^{208} \mathrm{~Pb}\left({ }^{48} \mathrm{Ca}, 2 \mathrm{n}\right), \mathrm{E}=219,221 \mathrm{MeV}$; measured $\mathrm{E} \gamma, \mathrm{I} \gamma, \gamma \gamma$-, (recoil) $\gamma$-coin. ${ }^{254}$ No deduced levels, J, $\pi$, rotational band, non-yrast state. Jurogam array, recoil-decay tagging. JOUR ZAANE 26227

\section{$\mathrm{A}=255$}

${ }^{255} \mathrm{Fm} \quad 2005 \mathrm{AHO9}$

RADIOACTIVITY ${ }^{255} \mathrm{Fm}(\alpha)$; measured $\mathrm{E} \gamma, \mathrm{I} \gamma \cdot{ }^{251} \mathrm{Es}(\mathrm{EC})$; measured $\mathrm{E} \gamma, \mathrm{I} \gamma, \mathrm{E}(\mathrm{ce}), \mathrm{I}(\mathrm{ce}) .{ }^{251} \mathrm{Cf}$ deduced levels, J, $\pi$, configurations, vibrational states. JOUR PRVCA 72054308

${ }^{255} \mathrm{Md}$ 2005HE27 RADIOACTIVITY ${ }^{247,249,251,253,255} \mathrm{Md}(\alpha)$ [from ${ }^{209} \mathrm{Bi}\left({ }^{40} \mathrm{Ar}, \mathrm{xn}\right)$, $\left({ }^{50} \mathrm{Ti}, \mathrm{xn}\right),{ }^{207,207} \mathrm{~Pb},{ }^{209} \mathrm{Bi}\left({ }^{48} \mathrm{Ca}, \mathrm{xn}\right)$ and subsequent decay]; measured $\mathrm{E} \alpha, \mathrm{E} \gamma, \alpha \gamma$-coin; deduced branching ratios, hindrance factors. $243,245,247,249,251$ Es deduced levels, J, $\pi$, configurations, deformation. Comparison with model predictions. JOUR ZAANE 26233

${ }^{255} \mathrm{Lr}$ 2005GR36 RADIOACTIVITY ${ }^{255} \mathrm{Lr},{ }^{251} \mathrm{Md}(\alpha)\left[\right.$ from ${ }^{209} \mathrm{Bi}\left({ }^{48} \mathrm{Ca}, 2 \mathrm{n}\right)$ and subsequent decay]; measured $\mathrm{E} \alpha, \alpha \alpha$-coin; deduced excited state decay. JOUR ZAANE 25 s01 599

$$
\mathrm{A}=\mathbf{2 5 6}
$$

No references found

$$
\mathrm{A}=257
$$

No references found 


$$
\mathrm{A}=\mathbf{2 5 8}
$$

No references found

$$
\mathrm{A}=\mathbf{2 5 9}
$$

No references found

$$
\mathrm{A}=\mathbf{2 6 0}
$$

No references found

$$
\mathrm{A}=\mathbf{2 6 1}
$$

No references found

$$
\mathrm{A}=\mathbf{2 6 2}
$$

No references found

$$
\mathrm{A}=\mathbf{2 6 3}
$$

No references found

$$
\mathrm{A}=\mathbf{2 6 4}
$$

No references found

$$
\mathrm{A}=265
$$

No references found

$$
\mathrm{A}=\mathbf{2 6 6}
$$

No references found

$$
\mathrm{A}=\mathbf{2 6 7}
$$

${ }^{267} \mathrm{Rf} \quad 20050 \mathrm{G03}$ RADIOACTIVITY ${ }^{294} 118,{ }^{290,291,292,293} 116,{ }^{287,288,289} 114,{ }^{285} 112$ ${ }^{275} \mathrm{Hs}(\alpha) ;{ }^{286} 114,{ }^{283} 112,{ }^{279} \mathrm{Ds},{ }^{271} \mathrm{Sg}(\alpha)$, (SF); ${ }^{282,284} 112,{ }^{281} \mathrm{Ds}$, ${ }^{267} \mathrm{Rf}(\mathrm{SF}) ;$ measured $\mathrm{E} \alpha, \mathrm{T}_{1 / 2}$, branching ratios. JOUR ZAANE $25 \mathrm{~s} 01$ 589 


\section{$\mathrm{A}=267$ (continued)}

${ }^{267} \mathrm{Db} \quad$ 20050G02 RADIOACTIVITY ${ }^{287,288} 115,{ }^{283,284} 113,{ }^{279,280} \mathrm{Rg},{ }^{275,276} \mathrm{Mt},{ }^{272} \mathrm{Bh}(\alpha)$ [from ${ }^{243} \mathrm{Am}\left({ }^{48} \mathrm{Ca}, \mathrm{xn}\right)$ and subsequent decay]; measured $\mathrm{E} \alpha, \mathrm{T}_{1 / 2}$; deduced $\mathrm{Q} \alpha .{ }^{267,268} \mathrm{Db}(\mathrm{SF}) ;$ measured $\mathrm{T}_{1 / 2}$. JOUR PRVCA 72034611

\section{$\mathrm{A}=268$}

${ }^{268} \mathrm{Db} \quad$ 20050G02 RADIOACTIVITY $287,288115,{ }^{283,284} 113,{ }^{279,280} \mathrm{Rg},{ }^{275,276} \mathrm{Mt},{ }^{272} \mathrm{Bh}(\alpha)$ [from ${ }^{243} \mathrm{Am}\left({ }^{48} \mathrm{Ca}, \mathrm{xn}\right)$ and subsequent decay]; measured $\mathrm{E} \alpha, \mathrm{T}_{1 / 2}$; deduced $\mathrm{Q} \alpha .{ }^{267,268} \mathrm{Db}(\mathrm{SF}) ;$ measured $\mathrm{T}_{1 / 2}$. JOUR PRVCA 72034611

$$
\mathrm{A}=\mathbf{2 6 9}
$$

No references found

$$
\mathrm{A}=\mathbf{2 7 0}
$$

No references found

$$
\mathrm{A}=271
$$

${ }^{271} \mathrm{Sg} \quad 20050 G 03 \quad$ RADIOACTIVITY ${ }^{294} 118,{ }^{290,291,292,293} 116,{ }^{287,288,289} 114,{ }^{285} 112$, ${ }^{275} \mathrm{Hs}(\alpha) ;{ }^{286} 114,{ }^{283} 112,{ }^{279} \mathrm{Ds},{ }^{271} \mathrm{Sg}(\alpha)$, (SF); ${ }^{282,284} 112,{ }^{281} \mathrm{Ds}$, ${ }^{267} \mathrm{Rf}(\mathrm{SF}) ;$ measured $\mathrm{E} \alpha, \mathrm{T}_{1 / 2}$, branching ratios. JOUR ZAANE $25 \mathrm{~s} 01$ 589

${ }^{271} \mathrm{Bh} \quad 20050 \mathrm{G} 02 \quad$ RADIOACTIVITY ${ }^{287,288} 115,{ }^{283,284} 113,{ }^{279,280} \mathrm{Rg},{ }^{275,276} \mathrm{Mt},{ }^{272} \mathrm{Bh}(\alpha)$ [from ${ }^{243} \mathrm{Am}\left({ }^{48} \mathrm{Ca}, \mathrm{xn}\right)$ and subsequent decay]; measured $\mathrm{E} \alpha, \mathrm{T}_{1 / 2}$; deduced $\mathrm{Q} \alpha .{ }^{267,268} \mathrm{Db}(\mathrm{SF}) ;$ measured $\mathrm{T}_{1 / 2}$. JOUR PRVCA 72034611

$$
\mathrm{A}=272
$$

${ }^{272} \mathrm{Bh}$ 20050G02 RADIOACTIVITY ${ }^{287,288} 115,{ }^{283,284} 113,{ }^{279,280} \mathrm{Rg},{ }^{275,276} \mathrm{Mt},{ }^{272} \mathrm{Bh}(\alpha)$ [from ${ }^{243} \mathrm{Am}\left({ }^{48} \mathrm{Ca}, \mathrm{xn}\right)$ and subsequent decay]; measured $\mathrm{E} \alpha, \mathrm{T}_{1 / 2}$; deduced $\mathrm{Q} \alpha .{ }^{267,268} \mathrm{Db}(\mathrm{SF}) ;$ measured $\mathrm{T}_{1 / 2}$. JOUR PRVCA 72034611

$$
\mathrm{A}=\mathbf{2 7 3}
$$

No references found

$$
\mathrm{A}=\mathbf{2 7 4}
$$

No references found 


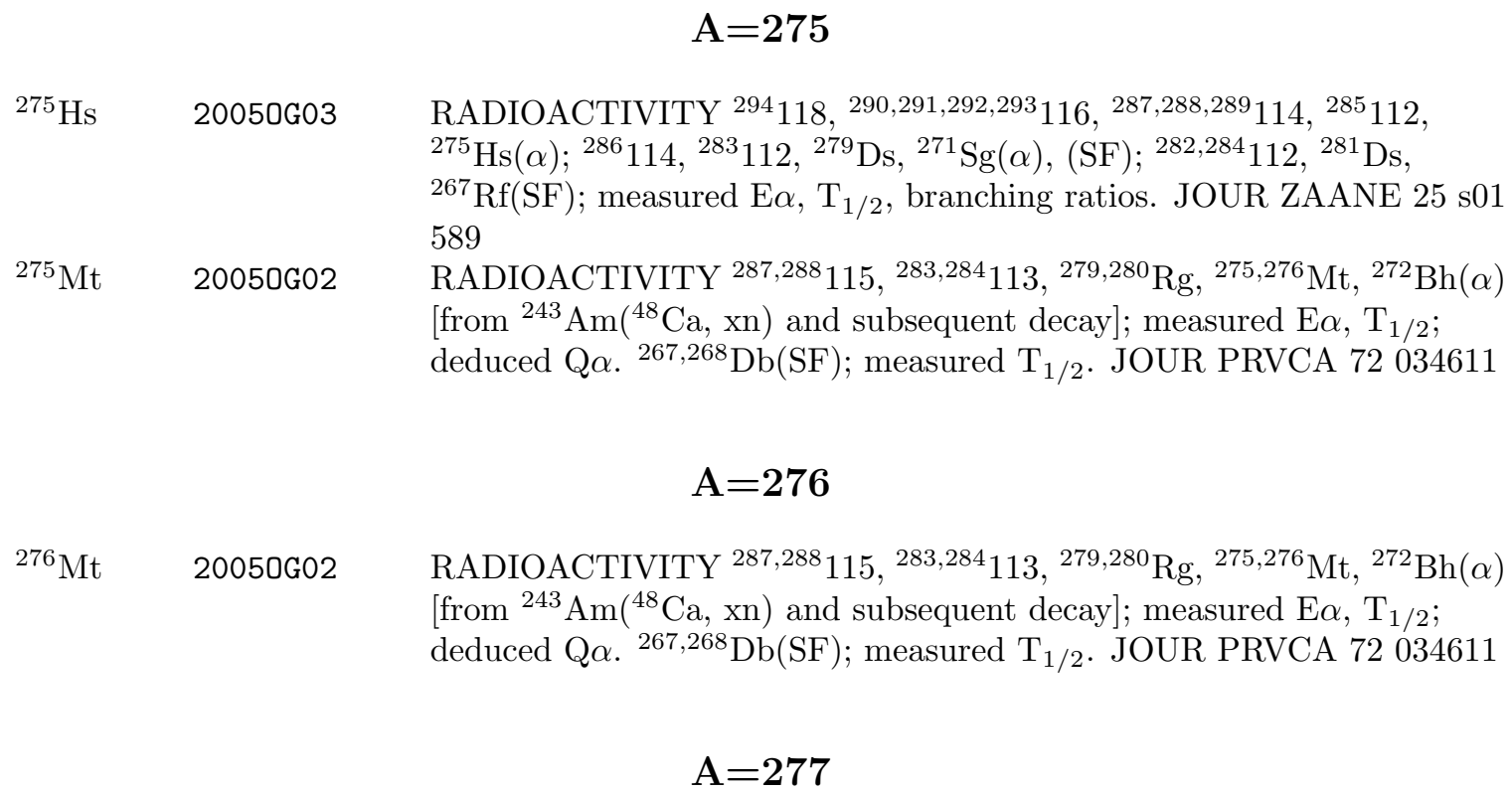

No references found

$$
\mathrm{A}=\mathbf{2 7 8}
$$

No references found

\section{$\mathrm{A}=\mathbf{2 7 9}$}

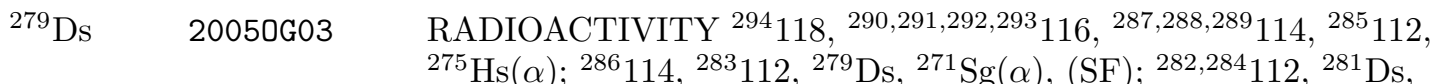
${ }^{267} \mathrm{Rf}(\mathrm{SF}) ;$ measured $\mathrm{E} \alpha, \mathrm{T}_{1 / 2}$, branching ratios. JOUR ZAANE $25 \mathrm{~s} 01$ 589

${ }^{279} \mathrm{Rg} \quad 20050 G 02 \quad$ RADIOACTIVITY ${ }^{287,288} 115,{ }^{283,284} 113,{ }^{279,280} \mathrm{Rg},{ }^{275,276} \mathrm{Mt},{ }^{272} \mathrm{Bh}(\alpha)$ [from ${ }^{243} \mathrm{Am}\left({ }^{48} \mathrm{Ca}, \mathrm{xn}\right)$ and subsequent decay]; measured $\mathrm{E} \alpha, \mathrm{T}_{1 / 2}$; deduced $\mathrm{Q} \alpha .{ }^{267,268} \mathrm{Db}(\mathrm{SF})$; measured $\mathrm{T}_{1 / 2}$. JOUR PRVCA 72034611

\section{$\mathrm{A}=\mathbf{2 8 0}$}

${ }^{280} \mathrm{Rg} \quad$ 20050G02 RADIOACTIVITY ${ }^{287,288} 115,{ }^{283,284} 113,{ }^{279,280} \mathrm{Rg},{ }^{275,276} \mathrm{Mt},{ }^{272} \mathrm{Bh}(\alpha)$ [from ${ }^{243} \mathrm{Am}\left({ }^{48} \mathrm{Ca}\right.$, xn $)$ and subsequent decay]; measured $\mathrm{E} \alpha, \mathrm{T}_{1 / 2}$; deduced $\mathrm{Q} \alpha .{ }^{267,268} \mathrm{Db}(\mathrm{SF}) ;$ measured $\mathrm{T}_{1 / 2}$. JOUR PRVCA 72034611 


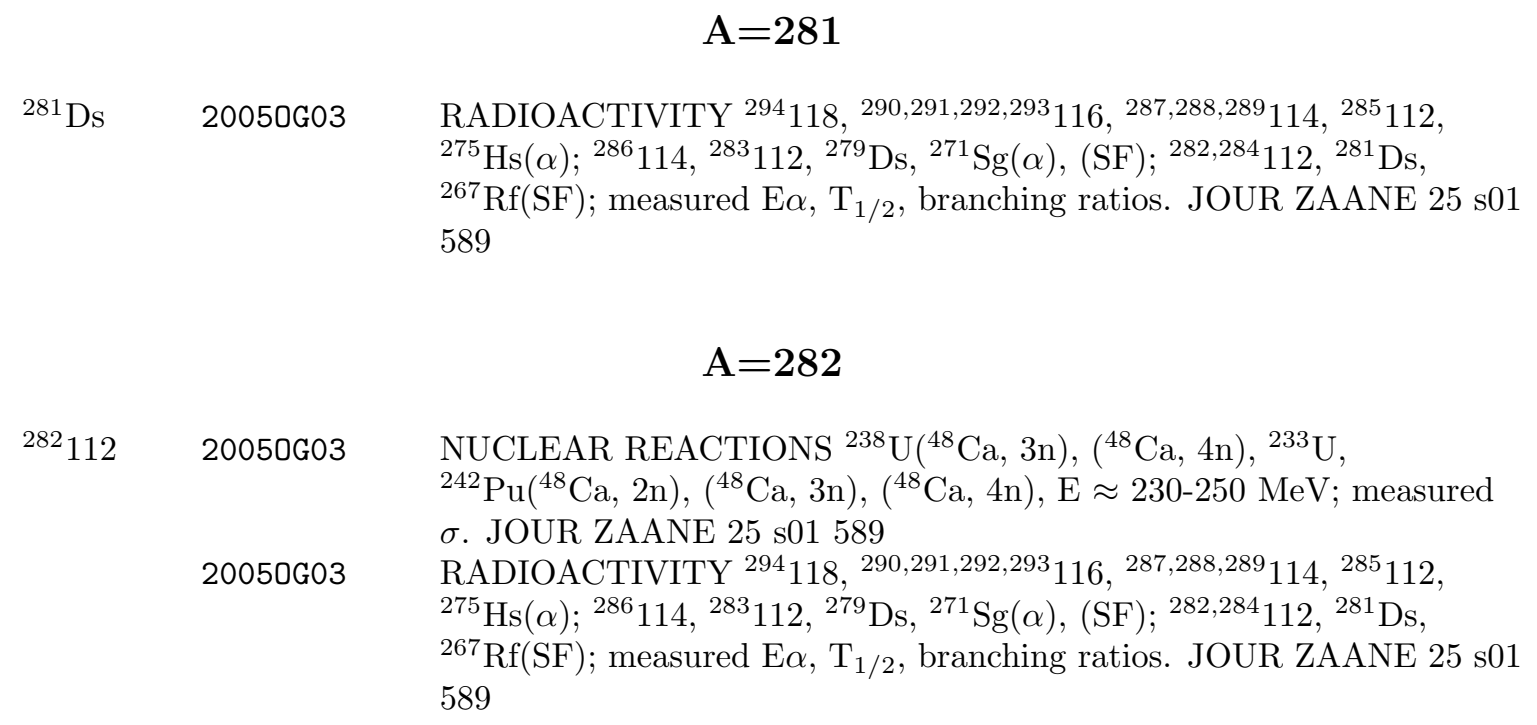

\section{$\mathrm{A}=282$}
${ }^{282} 112$ 20050G03 NUCLEAR REACTIONS ${ }^{238} \mathrm{U}\left({ }^{48} \mathrm{Ca}, 3 \mathrm{n}\right),\left({ }^{48} \mathrm{Ca}, 4 \mathrm{n}\right),{ }^{233} \mathrm{U}$, ${ }^{242} \mathrm{Pu}\left({ }^{48} \mathrm{Ca}, 2 \mathrm{n}\right),\left({ }^{48} \mathrm{Ca}, 3 \mathrm{n}\right),\left({ }^{48} \mathrm{Ca}, 4 \mathrm{n}\right), \mathrm{E} \approx 230-250 \mathrm{MeV}$; measured $\sigma$. JOUR ZAANE $25 \mathrm{~s} 01589$
20050G03 RADIOACTIVITY ${ }^{294} 118,{ }^{290,291,292,293} 116,{ }^{287,288,289} 114,{ }^{285} 112$, ${ }^{275} \mathrm{Hs}(\alpha) ;{ }^{286} 114,{ }^{283} 112,{ }^{279} \mathrm{Ds},{ }^{271} \mathrm{Sg}(\alpha)$, (SF); ${ }^{282,284} 112,{ }^{281} \mathrm{Ds}$, ${ }^{267} \mathrm{Rf}(\mathrm{SF})$; measured $\mathrm{E} \alpha, \mathrm{T}_{1 / 2}$, branching ratios. JOUR ZAANE $25 \mathrm{~s} 01$ 589

\section{$\mathrm{A}=283$}

${ }^{283} 112 \quad$ 2005HOZX

NUCLEAR REACTIONS ${ }^{238} \mathrm{U}\left({ }^{48} \mathrm{Ca}, \mathrm{xn}\right), \mathrm{E}=233,236,239 \mathrm{MeV}$; measured fission fragment spectra; deduced evidence for ${ }^{283} 112$. PREPRINT Hofmann $20050 G 03 \quad$ NUCLEAR REACTIONS ${ }^{238} \mathrm{U}\left({ }^{48} \mathrm{Ca}, 3 \mathrm{n}\right),\left({ }^{48} \mathrm{Ca}, 4 \mathrm{n}\right),{ }^{233} \mathrm{U}$, ${ }^{242} \mathrm{Pu}\left({ }^{48} \mathrm{Ca}, 2 \mathrm{n}\right),\left({ }^{48} \mathrm{Ca}, 3 \mathrm{n}\right),\left({ }^{48} \mathrm{Ca}, 4 \mathrm{n}\right), \mathrm{E} \approx 230-250 \mathrm{MeV}$; measured $\sigma$. JOUR ZAANE $25 \mathrm{~s} 01589$

20050G03 RADIOACTIVITY ${ }^{294} 118,{ }^{290,291,292,293} 116,{ }^{287,288,289} 114,{ }^{285} 112$, ${ }^{275} \mathrm{Hs}(\alpha) ;{ }^{286} 114,{ }^{283} 112,{ }^{279} \mathrm{Ds},{ }^{271} \mathrm{Sg}(\alpha)$, (SF); ${ }^{282,284} 112,{ }^{281} \mathrm{Ds}$, ${ }^{267} \mathrm{Rf}(\mathrm{SF}) ;$ measured $\mathrm{E} \alpha, \mathrm{T}_{1 / 2}$, branching ratios. JOUR ZAANE $25 \mathrm{~s} 01$ 589

${ }^{283} 113$ 20050G02 RADIOACTIVITY ${ }^{287,288} 115,{ }^{283,284} 113,{ }^{279,280} \mathrm{Rg},{ }^{275,276} \mathrm{Mt},{ }^{272} \mathrm{Bh}(\alpha)$ [from ${ }^{243} \mathrm{Am}\left({ }^{48} \mathrm{Ca}, \mathrm{xn}\right)$ and subsequent decay]; measured $\mathrm{E} \alpha, \mathrm{T}_{1 / 2}$; deduced $\mathrm{Q} \alpha .{ }^{267,268} \mathrm{Db}(\mathrm{SF}) ;$ measured $\mathrm{T}_{1 / 2}$. JOUR PRVCA 72034611

\section{$\mathrm{A}=\mathbf{2 8 4}$}

${ }^{284} 112$ 20050G03 RADIOACTIVITY ${ }^{294} 118,{ }^{290,291,292,293} 116,{ }^{287,288,289} 114,{ }^{285} 112$, ${ }^{275} \mathrm{Hs}(\alpha) ;{ }^{286} 114,{ }^{283} 112,{ }^{279} \mathrm{Ds},{ }^{271} \mathrm{Sg}(\alpha)$, (SF); ${ }^{282,284} 112,{ }^{281} \mathrm{Ds}$, ${ }^{267} \mathrm{Rf}(\mathrm{SF}) ;$ measured $\mathrm{E} \alpha, \mathrm{T}_{1 / 2}$, branching ratios. JOUR ZAANE $25 \mathrm{~s} 01$ 589

${ }^{284} 113$ 20050G02 RADIOACTIVITY ${ }^{287,288} 115,{ }^{283,284} 113,{ }^{279,280} \mathrm{Rg},{ }^{275,276} \mathrm{Mt},{ }^{272} \mathrm{Bh}(\alpha)$ [from ${ }^{243} \mathrm{Am}\left({ }^{48} \mathrm{Ca}, \mathrm{xn}\right)$ and subsequent decay]; measured $\mathrm{E} \alpha, \mathrm{T}_{1 / 2}$; deduced $\mathrm{Q} \alpha .{ }^{267,268} \mathrm{Db}(\mathrm{SF}) ;$ measured $\mathrm{T}_{1 / 2}$. JOUR PRVCA 72034611 


\section{$\mathrm{A}=285$}

${ }^{285} 112$ 20050G03 RADIOACTIVITY ${ }^{294} 118,{ }^{290,291,292,293} 116,{ }^{287,288,289} 114,{ }^{285} 112$, ${ }^{275} \mathrm{Hs}(\alpha) ;{ }^{286} 114,{ }^{283} 112,{ }^{279} \mathrm{Ds},{ }^{271} \mathrm{Sg}(\alpha)$, (SF); ${ }^{282,284} 112,{ }^{281} \mathrm{Ds}$, ${ }^{267} \mathrm{Rf}(\mathrm{SF}) ;$ measured $\mathrm{E} \alpha, \mathrm{T}_{1 / 2}$, branching ratios. JOUR ZAANE $25 \mathrm{~s} 01$ 589

\section{$\mathrm{A}=286$}

$280050 G 03$

NUCLEAR REACTIONS ${ }^{238} \mathrm{U}\left({ }^{48} \mathrm{Ca}, 3 \mathrm{n}\right),\left({ }^{48} \mathrm{Ca}, 4 \mathrm{n}\right),{ }^{233} \mathrm{U}$, ${ }^{242} \mathrm{Pu}\left({ }^{48} \mathrm{Ca}, 2 \mathrm{n}\right),\left({ }^{48} \mathrm{Ca}, 3 \mathrm{n}\right),\left({ }^{48} \mathrm{Ca}, 4 \mathrm{n}\right), \mathrm{E} \approx 230-250 \mathrm{MeV}$; measured $\sigma$. JOUR ZAANE $25 \mathrm{~s} 01589$

20050G03 RADIOACTIVITY ${ }^{294} 118,{ }^{290,291,292,293} 116,{ }^{287,288,289} 114,{ }^{285} 112$, ${ }^{275} \mathrm{Hs}(\alpha) ;{ }^{286} 114,{ }^{283} 112,{ }^{279} \mathrm{Ds},{ }^{271} \mathrm{Sg}(\alpha)$, (SF); ${ }^{282,284} 112,{ }^{281} \mathrm{Ds}$, ${ }^{267} \mathrm{Rf}(\mathrm{SF}) ;$ measured $\mathrm{E} \alpha, \mathrm{T}_{1 / 2}$, branching ratios. JOUR ZAANE $25 \mathrm{~s} 01$ 589

\section{$\mathrm{A}=287$}

${ }^{287} 114$ 20050G03 NUCLEAR REACTIONS ${ }^{238} \mathrm{U}\left({ }^{48} \mathrm{Ca}, 3 \mathrm{n}\right),\left({ }^{48} \mathrm{Ca}, 4 \mathrm{n}\right),{ }^{233} \mathrm{U}$, ${ }^{242} \mathrm{Pu}\left({ }^{48} \mathrm{Ca}, 2 \mathrm{n}\right),\left({ }^{48} \mathrm{Ca}, 3 \mathrm{n}\right),\left({ }^{48} \mathrm{Ca}, 4 \mathrm{n}\right), \mathrm{E} \approx 230-250 \mathrm{MeV}$; measured $\sigma$. JOUR ZAANE 25 s01 589

20050G03 RADIOACTIVITY ${ }^{294} 118,{ }^{290,291,292,293} 116,{ }^{287,288,289} 114,{ }^{285} 112$, ${ }^{275} \mathrm{Hs}(\alpha) ;{ }^{286} 114,{ }^{283} 112,{ }^{279} \mathrm{Ds},{ }^{271} \mathrm{Sg}(\alpha),(\mathrm{SF}) ;{ }^{282,284} 112,{ }^{281} \mathrm{Ds}$, ${ }^{267} \mathrm{Rf}(\mathrm{SF}) ;$ measured $\mathrm{E} \alpha, \mathrm{T}_{1 / 2}$, branching ratios. JOUR ZAANE $25 \mathrm{~s} 01$ 589

${ }^{287} 115$ 20050G02 NUCLEAR REACTIONS ${ }^{243} \mathrm{Am}\left({ }^{48} \mathrm{Ca}, 3 \mathrm{n}\right),\left({ }^{48} \mathrm{Ca}, 4 \mathrm{n}\right), \mathrm{E}=248,253$ $\mathrm{MeV}$; measured delayed $\mathrm{E} \alpha, \alpha \alpha$-coin; deduced $\sigma$. JOUR PRVCA 72 034611

20050G02 RADIOACTIVITY ${ }^{287,288} 115,{ }^{283,284} 113,{ }^{279,280} \mathrm{Rg},{ }^{275,276} \mathrm{Mt},{ }^{272} \mathrm{Bh}(\alpha)$ [from ${ }^{243} \mathrm{Am}\left({ }^{48} \mathrm{Ca}, \mathrm{xn}\right)$ and subsequent decay]; measured $\mathrm{E} \alpha, \mathrm{T}_{1 / 2}$; deduced $\mathrm{Q} \alpha .{ }^{267,268} \mathrm{Db}(\mathrm{SF}) ;$ measured $\mathrm{T}_{1 / 2}$. JOUR PRVCA 72034611

\section{$\mathrm{A}=288$}

${ }^{288} 114 \quad$ NUCLEAR REACTIONS ${ }^{238} \mathrm{U}\left({ }^{48} \mathrm{Ca}, 3 \mathrm{n}\right),\left({ }^{48} \mathrm{Ca}, 4 \mathrm{n}\right),{ }^{233} \mathrm{U}$, ${ }^{242} \mathrm{Pu}\left({ }^{48} \mathrm{Ca}, 2 \mathrm{n}\right),\left({ }^{48} \mathrm{Ca}, 3 \mathrm{n}\right),\left({ }^{48} \mathrm{Ca}, 4 \mathrm{n}\right), \mathrm{E} \approx 230-250 \mathrm{MeV}$; measured $\sigma$. JOUR ZAANE 25 s01 589

20050G03 RADIOACTIVITY ${ }^{294} 118,{ }^{290,291,292,293} 116,{ }^{287,288,289} 114,{ }^{285} 112$, ${ }^{275} \mathrm{Hs}(\alpha) ;{ }^{286} 114,{ }^{283} 112,{ }^{279} \mathrm{Ds},{ }^{271} \mathrm{Sg}(\alpha)$, (SF); ${ }^{282,284} 112,{ }^{281} \mathrm{Ds}$, ${ }^{267} \mathrm{Rf}(\mathrm{SF}) ;$ measured $\mathrm{E} \alpha, \mathrm{T}_{1 / 2}$, branching ratios. JOUR ZAANE $25 \mathrm{~s} 01$ 589

${ }^{288} 115$ 20050G02 NUCLEAR REACTIONS ${ }^{243} \mathrm{Am}\left({ }^{48} \mathrm{Ca}, 3 \mathrm{n}\right),\left({ }^{48} \mathrm{Ca}, 4 \mathrm{n}\right), \mathrm{E}=248,253$ $\mathrm{MeV}$; measured delayed $\mathrm{E} \alpha, \alpha \alpha$-coin; deduced $\sigma$. JOUR PRVCA 72 034611

20050G02 RADIOACTIVITY $287,288115,{ }^{283,284} 113,{ }^{279,280} \mathrm{Rg},{ }^{275,276} \mathrm{Mt},{ }^{272} \mathrm{Bh}(\alpha)$ [from ${ }^{243} \mathrm{Am}\left({ }^{48} \mathrm{Ca}, \mathrm{xn}\right)$ and subsequent decay]; measured $\mathrm{E} \alpha, \mathrm{T}_{1 / 2}$; deduced $\mathrm{Q} \alpha .{ }^{267,268} \mathrm{Db}(\mathrm{SF})$; measured $\mathrm{T}_{1 / 2}$. JOUR PRVCA 72034611 


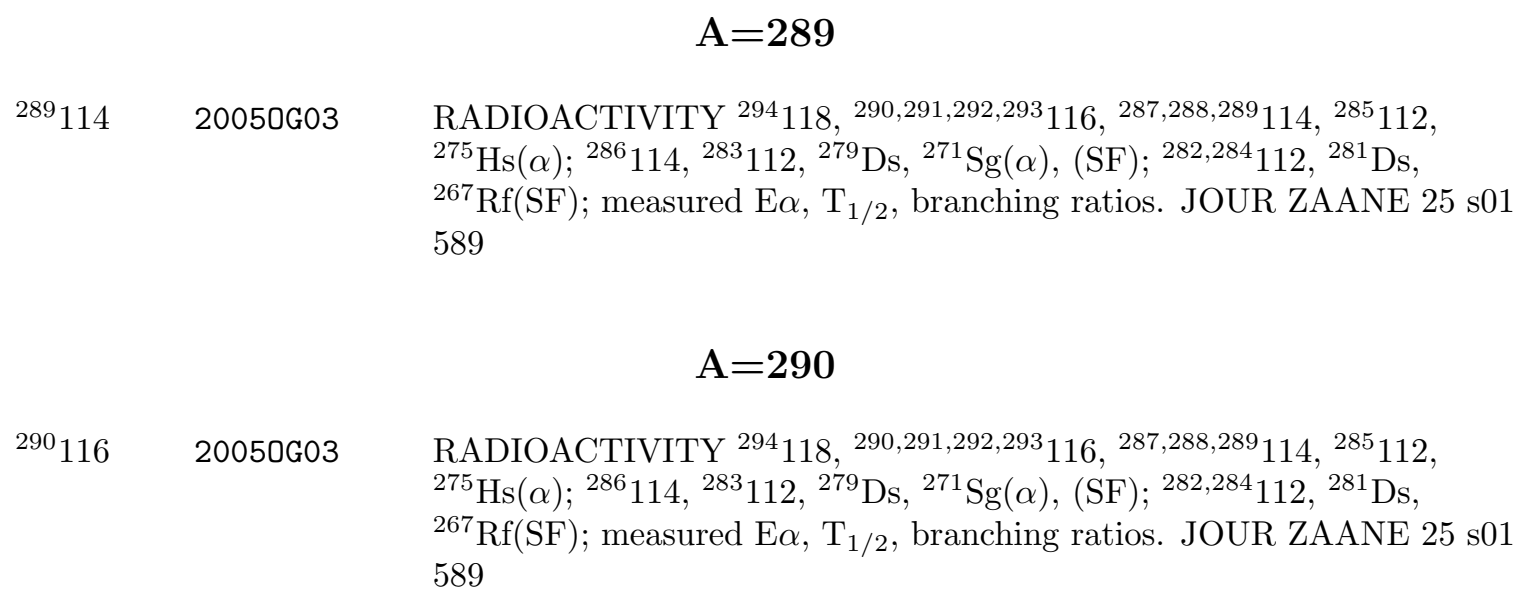

$\mathrm{A}=\mathbf{2 8 9}$

${ }^{289} 114$ 20050G03 RADIOACTIVITY ${ }^{294} 118,{ }^{290,291,292,293} 116,{ }^{287,288,289} 114,{ }^{285} 112$, ${ }^{275} \mathrm{Hs}(\alpha) ;{ }^{286} 114,{ }^{283} 112,{ }^{279} \mathrm{Ds},{ }^{271} \mathrm{Sg}(\alpha)$, (SF); ${ }^{282,284} 112,{ }^{281} \mathrm{Ds}$, ${ }^{267} \mathrm{Rf}(\mathrm{SF}) ;$ measured $\mathrm{E} \alpha, \mathrm{T}_{1 / 2}$, branching ratios. JOUR ZAANE $25 \mathrm{~s} 01$ 589

\section{$\mathrm{A}=\mathbf{2 9 0}$}

${ }^{290} 116 \quad 20050 G 03$

RADIOACTIVITY ${ }^{294} 118,{ }^{290,291,292,293} 116,{ }^{287,288,289} 114,{ }^{285} 112$, ${ }^{275} \mathrm{Hs}(\alpha) ;{ }^{286} 114,{ }^{283} 112,{ }^{279} \mathrm{Ds},{ }^{271} \mathrm{Sg}(\alpha)$, (SF); ${ }^{282,284} 112,{ }^{281} \mathrm{Ds}$, ${ }^{267} \mathrm{Rf}(\mathrm{SF})$; measured $\mathrm{E} \alpha, \mathrm{T}_{1 / 2}$, branching ratios. JOUR ZAANE $25 \mathrm{~s} 01$ 589

\section{$\mathrm{A}=\mathbf{2 9 1}$}

${ }^{291} 116$ 20050G03 RADIOACTIVITY ${ }^{294} 118,{ }^{290,291,292,293} 116,{ }^{287,288,289} 114,{ }^{285} 112$, ${ }^{275} \mathrm{Hs}(\alpha) ;{ }^{286} 114,{ }^{283} 112,{ }^{279} \mathrm{Ds},{ }^{271} \mathrm{Sg}(\alpha)$, (SF); ${ }^{282,284} 112,{ }^{281} \mathrm{Ds}$, ${ }^{267} \mathrm{Rf}(\mathrm{SF}) ;$ measured $\mathrm{E} \alpha, \mathrm{T}_{1 / 2}$, branching ratios. JOUR ZAANE $25 \mathrm{~s} 01$ 589

\section{$\mathrm{A}=292$}

${ }^{292} 116$ 20050G03 RADIOACTIVITY ${ }^{294} 118,{ }^{290,291,292,293} 116,{ }^{287,288,289} 114,{ }^{285} 112$, ${ }^{275} \mathrm{Hs}(\alpha) ;{ }^{286} 114,{ }^{283} 112,{ }^{279} \mathrm{Ds},{ }^{271} \mathrm{Sg}(\alpha)$, (SF); ${ }^{282,284} 112,{ }^{281} \mathrm{Ds}$, ${ }^{267} \mathrm{Rf}(\mathrm{SF}) ;$ measured $\mathrm{E} \alpha, \mathrm{T}_{1 / 2}$, branching ratios. JOUR ZAANE $25 \mathrm{~s} 01$ 589

\section{$\mathrm{A}=293$}

${ }^{293} 116$ 20050G03 RADIOACTIVITY ${ }^{294} 118,{ }^{290,291,292,293} 116,{ }^{287,288,289} 114,{ }^{285} 112$, ${ }^{275} \mathrm{Hs}(\alpha) ;{ }^{286} 114,{ }^{283} 112,{ }^{279} \mathrm{Ds},{ }^{271} \mathrm{Sg}(\alpha)$, (SF); ${ }^{282,284} 112,{ }^{281} \mathrm{Ds}$, ${ }^{267} \mathrm{Rf}(\mathrm{SF}) ;$ measured $\mathrm{E} \alpha, \mathrm{T}_{1 / 2}$, branching ratios. JOUR ZAANE $25 \mathrm{~s} 01$ 589

\section{$\mathrm{A}=294$}

${ }^{294} 118$ 20050G03 RADIOACTIVITY ${ }^{294} 118,{ }^{290,291,292,293} 116,{ }^{287,288,289} 114,{ }^{285} 112$, ${ }^{275} \mathrm{Hs}(\alpha) ;{ }^{286} 114,{ }^{283} 112,{ }^{279} \mathrm{Ds},{ }^{271} \mathrm{Sg}(\alpha)$, (SF); ${ }^{282,284} 112,{ }^{281} \mathrm{Ds}$, ${ }^{267} \mathrm{Rf}(\mathrm{SF})$; measured $\mathrm{E} \alpha, \mathrm{T}_{1 / 2}$, branching ratios. JOUR ZAANE $25 \mathrm{~s} 01$ 589 


\section{References}

2004AG09 Y.Agus, I.Celenk, A.Ozmen - Radiochim.Acta 92, 63 (2004)

Measurement of cross sections of threshold detectors with spectrum average technique

2004AL43 J.A.Alcantara-Nunez, J.R.B.Oliveira, E.W.Cybulska, N.H.Medina, M.N.Rao, R.V.Ribas, M.A.Rizzutto, W.A.Seale, F.Falla-Sotelo, K.T.Wiedemann, V.I.Dimitrov, S.Frauendorf - Braz.J.Phys. 34, 999 (2004)

Chiral Bands in ${ }^{105} \mathrm{Rh}$

2004AL44 J.A.Alcantara-Nunez, J.R.B.Oliveira, E.W.Cybulska, N.H.Medina, M.N.Rao, R.V.Ribas, M.A.Rizzutto, W.A.Seale, F.Falla-Sotelo - Braz.J.Phys. 34, 1005 (2004) Rotational and Vibrational bands in ${ }^{108} \mathrm{Pd}$

2004BE58 G.J.Beyer, S.K.Zeisler, D.W.Becker - Radiochim.Acta 92, 219 (2004)

The Auger-electron emitter ${ }^{165} \mathrm{Er}$ : excitation function of the ${ }^{165} \mathrm{Ho}(\mathrm{p}, \mathrm{n}){ }^{165} \mathrm{Er}$ process

2004 C029 S.Courtin, P.Baumann, Ph.Dessagne, F.Marechal, Ch.Miehe, F.Perrot, E.Poirier, M.Ramdhane, and the ISOLDE Collaboration - Braz.J.Phys. 34, 850 (2004)

Beta Decay and Structure of Exotic Nuclei in the Mass Regions N=Z, A 70 and Near the $\mathrm{N}=20$ Closed Shell

2004GA60 Yu.P.Gangrsky, V.I.Zhemenik, N.Yu.Maslova, G.V.Myshinsky, Kh.S.Norov, Yu.E.Penionzhkevich - Bull.Rus.Acad.Sci.Phys. 68, 1298 (2004)

Independent yields of $\mathrm{Kr}$ and Xe fragments from ${ }^{237} \mathrm{~Np}$ photofission

2004GE20 F.A.Genezini, C.B.Zamboni, M.T.F.da Cruz, J.Y.Zevallos-Chavez - Braz.J.Phys. 34, $722(2004)$

Angular Correlation Measurements in ${ }^{155} \mathrm{Eu}$ Nuclei

2004GL10 M.Glaser, D.B.Mackay, A.S.O.Ranicar, S.L.Waters, F.Brady, S.K.Luthra Radiochim.Acta 92, 951 (2004)

Improved targetry and production of iodine-124 for PET studies

2004 G059 S.K.Godovikov, S.M.Nikitin - Bull.Rus.Acad.Sci.Phys. 68, 1335 (2004)

Controlled radioactive decay of ${ }^{125 m}$ Te nuclei

2004GU21 V.Guimaraes - Braz.J.Phys. 34, 1012 (2004)

Nuclear Reaction Studies with Exotic Boron Beams

2004HA64 B.Haas - Braz.J.Phys. 34, 814 (2004)

Nuclear Data for New Fuel Cycles

2004HE35 A.Hermanne, S.Takacs, F.Tarkanyi, R.Bolbos - Radiochim.Acta 92, 215 (2004)

Cross section measurements of proton and deuteron induced formation of ${ }^{103} \mathrm{Ag}$ in natural palladium 
2004H025 J.Honzatko, I.Tomandl, A.M.Sukhovoi, V.A.Khitrov - Bull.Rus.Acad.Sci.Phys. 68, 1324 (2004)

Cascade $\gamma$-decay of compound states of ${ }^{71,74}$ Ge nuclei

2004KA68 S.Kastleiner, Yu.N.Shubin, F.M.Nortier, T.N.Van der Walt, S.M.Qaim -

Radiochim.Acta 92, 449 (2004)

Experimental studies and nuclear model calculations on (p, xn) and (p, pxn)

reactions on ${ }^{85} \mathrm{Rb}$ from their thresholds up to $100 \mathrm{MeV}$

2004KU33 J.Kuhnhenn, U.Herpers, R.Michel - Radiochim.Acta 92, 233 (2004)

New determination of the half-life of ${ }^{205} \mathrm{Bi}$

2004KU35 V.T.Kupryashkin, L.P.Sidorenko, A.I.Feoktistov, I.P.Shapovalova -

Bull.Rus.Acad.Sci.Phys. 68, 1358 (2004)

Angular distributions of near-zero-energy electrons emitted from surface of source in radioactive decay

2004KU36 R.Kuramoto, R.Lichtenthaler, A.Lepine-Szily, V.Guimaraes, G.F.Lima,

E.Benjamim, P.N.de Faria - Braz.J.Phys. 34, 933 (2004)

Spectroscopy of ${ }^{6} \mathrm{Li}$ Using the ${ }^{3} \mathrm{He}\left({ }^{7} \mathrm{Li}, \alpha\right){ }^{6} \mathrm{Li}$ Reaction

$2004 L 022$ Yu.E.Loginov, S.E.Malyutenkova - Bull.Rus.Acad.Sci.Phys. 68, 1292 (2004) $\gamma$-lines from ${ }^{183} \mathrm{~W}(\mathrm{n} \gamma){ }^{184} \mathrm{~W}$ reaction

2004MB08 G.V.Marti, A.J.Pacheco, J.E.Testoni, D.Abriola, O.A.Capurro, D.E.DiGregorio, J.O.Fernandez Niello, A.O.Macchiavelli, R.M.Clark, P.Fallon, A.Goergen, D.Ward, C.Y.Wu, A.Hayes, D.Cline, R.Teng - Braz.J.Phys. 34, 885 (2004)

Measurement of Inelastic-Scattering Cross Sections in the ${ }^{16} \mathrm{O}+{ }^{28} \mathrm{Si}$ System to Discriminate Regular and Chaotic Regimes

2004ME25 N.H.Medina, J.R.B.Oliveira, E.W.Cybulska, M.N.Rao, R.V.Ribas, M.A.Rizzutto, W.A.Seale, F.R.Espinoza-Quinones, D.Bazzacco, F.Brandolini, S.Lunardi, C.M.Petrache, Zs.Podolyak, C.Rossi-Alvarez, F.Soramel, C.A.Ur, M.A.Cardona, G.de Angelis, D.R.Napoli, P.Spolaore, A.Gadea, D.De Acuna, M.De Poli, E.Farnea, D.Foltescu, M.Ionescu-Bujor, A.Iordachescu - Braz.J.Phys. 34, 1002 (2004)

First Allowed Bandcrossing in Neutron Deficient Nucleus ${ }^{141} \mathrm{~Tb}$

2004M054 S.Mohammadi, Zs.Podolyak, G.de Angelis, M.Axiotis, D.Bazzacco, P.G.Bizzeti, F.Brandolini, R.Broda, D.Bucurescu, E.Farnea, W.Gelletly, A.Gadea, M.Ionescu-Bujor, A.Iordachescu, Th.Kroll, S.Longdown, S.Lunardi, N.Marginean, T.Martinez, N.H.Medina, B.Quintana, P.H.Regan, B.Rubio, C.A.Ur, J.J.Valiente Dobon, P.M.Walker, Y.H.Zhang - Braz.J.Phys. 34, 792 (2004)

High-Spin States Populated in Deep-Inelastic Reactions

2004NA44 H.Naik, R.J.Singh, R.H.Iyer - Radiochim.Acta 92, 1 (2004)

Fission fragment angular momentum in the spontaneous fission of ${ }^{244} \mathrm{Cm}$

2004R047 C.L.Rodrigues, M.R.D.Rodrigues, T.Borello-Lewin, L.B.Horodynski-Matsushigue, J.L.M.Duarte, G.M.Ukita, G.N.Hanninger - Braz.J.Phys. 34, 760 (2004) 
Coulomb-Nuclear Interference (CNI) Results of the Collective Quadrupolar Excitations in Odd and Even Ru Isotopes

2004R048 M.R.D.Rodrigues, C.L.Rodrigues, T.Borello-Lewin, L.B.Horodynski-Matsushigue, J.L.M.Duarte, G.M.Ukita - Braz.J.Phys. 34, 777 (2004)

Coulomb Nuclear Interference with Deuterons in even Palladium Isotopes

2004SC48 B.Scholten, S.Takacs, F.Tarkanyi, H.H.Coenen, S.M.Qaim - Radiochim.Acta 92, 203 (2004)

Excitation functions of deuteron induced nuclear reactions on enriched ${ }^{78} \mathrm{Kr}$ with particular relevance to the production of ${ }^{76} \mathrm{Br}$

2004 S036 F.A.Souza, A.Szanto de Toledo, M.G.Munhoz, J.Takahashi, N.Carlin, A.A.P.Suaide, M.M.de Moura, e.M.Szanto - Braz.J.Phys. 34, 907 (2004)

Study of the Effect of the Breakup on the Fusion Cross Section of the Systems ${ }^{6,7} \mathrm{Li}+{ }^{59} \mathrm{Co}$

2004SP06 I.Spahn, H.H.Coenen, S.M.Qaim - Radiochim.Acta 92, 183 (2004)

Enhanced production possibility for the therapeutic radionuclides ${ }^{64} \mathrm{Cu},{ }^{67} \mathrm{Cu}$ and

${ }^{89} \mathrm{Sr}$ via $(\mathrm{n}, \mathrm{p})$ reactions induced by fast spectral neutrons

2004 TA46 F.Tarkanyi, A.Hermanne, S.Takacs, Yu.N.Shubin, A.I.Dityuk - Radiochim.Acta 92, $223(2004)$

Cross sections for production of the therapeutic radioisotopes ${ }^{198} \mathrm{Au}$ and ${ }^{199} \mathrm{Au}$ in proton and deuteron induced reactions on ${ }^{198} \mathrm{Pt}$

2004TH15 M.Thoennessen - Acta Phys.Hung.N.S. 21, 379 (2004)

Pushing the Limits of Nuclear Stability

2004VA37 S.I.Vasiliev, Yu.G.Zdesenko, A.A.Klimenko, S.B.Osetrov, A.A.Smolnikov, V.I.Tretyak - Bull.Rus.Acad.Sci.Phys. 68, 1255 (2004)

Experimental limit on charge-nonconserving ${ }^{73} \mathrm{Ge} \beta$-decay probability

2004VE14 D.Verney, F.Ibrahim, O.Perru, O.Bajeat, C.Bourgeois, F.Clapier, E.Cottereau, C.Donzaud, S.Du, M.Ducourtieux, S.Essabaa, S.Gales, L.Gaudefroy, D.Guillemaud-Mueller, F.Hammache, F.Hosni, C.Lau, H.Lefort, F.Le Blanc, A.C.Mueller, N.Pauwels, J.C.Potier, F.Pougheon, J.Proust, B.Roussiere, J.Sauvage, O.Sorlin - Braz.J.Phys. 34, 979 (2004)

Study of the $\mathrm{N}=50$ Shell Closure Close to ${ }^{78} \mathrm{Ni}$

2004ZA15 G.S.Zahn, C.B.Zamboni, F.A.Genezini, M.P.Raele, M.T.F.da Cruz, J.Y.Zevallos-Chavez - Braz.J.Phys. 34, 719 (2004)

New Gamma Transitions in ${ }^{193}$ Ir From the Beta Decay of ${ }^{193}$ Os

2004ZH45 B.V.Zhuravlev, A.A.Lychagin, N.N.Titarenko, V.G.Demenkov, V.I.Trykova, A.Ya.Slekenichs - Bull.Rus.Acad.Sci.Phys. 68, 1319 (2004)

Level densities in ${ }^{90} \mathrm{Nb}$ and ${ }^{94} \mathrm{Nb}$ nuclei from neutron evaporation spectra

2005AD35 S.Adhikari, C.Samanta, C.Basu, S.Ray, A.Chatterjee, S.Kailas - Eur.Phys.J. A 25, Supplement 1, 299 (2005) 
Entrance channel dependence in compound nuclear reactions with loosely bound nuclei

2005AG15 U.Agvaanluvsan, E.Algin, J.A.Becker, M.Guttormsen, G.E.Mitchell, S.Siem, A.Schiller, A.Voinov - Nucl.Instrum.Methods Phys.Res. B241, 180 (2005) Investigation of the radiative strength function

2005AH07 J.Ahrens, S.Altieri, J.R.M.Annand, H.-J.Arends, R.Beck, A.Braghieri, N.d'Hose, H.Dutz, S.Goertz, P.Grabmayr, S.Hasegawa, E.Heid, H.Holvoet, L.Van Hoorebeke, N.Horikawa, T.Iwata, O.Jahn, P.Jennewein, R.Kondratiev, J.Krimmer, M.Lang, B.Lannoy, K.Livingston, J.C.McGeorge, W.Meyer, A.Panzeri, P.Pedroni, T.Pinelli, I.Preobrajenski, G.Reicherz, G.Rosner, M.Rost, T.Rostomyan, D.Ryckbosch, M.Schumacher, B.Seitz, G.Tamas, A.Thomas, R.van de Vyver, Th.Walcher, F.Zapadtka - Eur.Phys.J. A 26, 135 (2005)

Measurement of the G asymmetry for the $\gamma \mathrm{p} \rightarrow \mathrm{N} \pi$ channels in the $\Delta(1232)$ resonance region

2005AH09 I.Ahmad, J.P.Greene, E.F.Moore, F.G.Kondev, R.R.Chasman, C.E.Porter, L.K.Felker - Phys.Rev. C 72, 054308 (2005)

Energy levels of ${ }^{251} \mathrm{Cf}$ populated in the $\alpha$ decay of ${ }_{100}^{255} \mathrm{Fm}$ and EC decay of ${ }_{99}^{251} \mathrm{Es}$

2005AI06 A.Airapetian, and the HERMES Collaboration - Phys.Rev.Lett. 95, 242001 (2005) Measurement of the Tensor Structure Function $b_{1}$ of the Deuteron

2005AL37 G.D.Alkhazov, V.V.Astashin, A.G.Atamantchouk, W.Augustyniak, D.Bachelier, V.V.Baublis, M.Boivin, J.-L.Boyard, J.-F.Clavelin, P.Courtat, M.Denoit, R.Douet, C.Ellegaard, L.Farhi, C.Gaarde, R.Gacougnolle, V.Ya.Gerzenstein, V.L.Golovtsov, V.V.Golubev, T.Hennino, J.-C.Jourdain, A.V.Khanzadeev, B.G.Komkov, A.V.Kravtsov, L.G.Kudin, R.Kunne, J.Larsen, S.Lebon, L.V.Malinina, A.Maroni, J.-M.Martin, H.-P.Morsch, V.A.Mylnikov, E.M.Orischin, M.Osterlund, V.Poux, A.N.Prokofiev, P.Radvanyi, B.Ramstein, B.V.Razmyslovich, M.Roy-Stephan, V.M.Samsonov, R.Skowron, I.B.Smirnov, E.A.Strokovsky, I.I.Tkach, V.V.Vikhrov, S.S.Volkov, An.A.Vorobyov, A.A.Zhdanov, P.Zupranski - Nucl.Instrum.Methods Phys.Res. A551, 290 (2005)

SPES4- $\pi$ : installation for exclusive study of nuclear reactions

2005AL45 J.J.S.Alves, P.R.S.Gomes, J.Lubian, L.C.Chamon, R.M.Anjos, D.Pereira, E.S.Rossi, Jr., C.P.Silva, M.A.G.Alvarez, G.P.A.Nobre, L.R.Gasques - Braz.J.Phys. 35, 909 (2005)

Elastic, Inelastic Scatterings and Transfer Reactions for ${ }^{16,18} \mathrm{O}$ on ${ }^{58} \mathrm{Ni}$ Described by the Sao Paulo Potential

2005An24 E.Angeli, A.Tartari, M.Frignani, D.Mostacci, F.Rocchi, M.Sumini -

Appl.Radiat.Isot. 63, 545 (2005)

Preliminary results on the production of short-lived radioisotopes with a Plasma

Focus Device 
2005 AN26 K.Andgren, S.F.Ashley, P.H.Regan, E.A.McCutchan, N.V.Zamfir, L.Amon, R.B.Cakirli, R.F.Casten, R.M.Clark, G.Gurdal, K.L.Keyes, D.A.Meyer, M.N.Erduran, A.Papenberg, N.Pietralla, C.Plettner, G.Rainovski, R.V.Ribas, N.J.Thomas, J.Vinson, D.D.Warner, V.Werner, E.Williams - J.Phys.(London) G31, S1563 (2005)

$\mathrm{RDM}$ lifetime measurements in ${ }^{107} \mathrm{Cd}$

2005An30 C.Angulo, E.Casarejos, M.Couder, P.Demaret, P.Leleux, F.Vanderbist, A.Coc, J.Kiener, V.Tatischeff, T.Davinson, A.S.Murphy, N.L.Achouri, N.A.Orr,

D.Cortina-Gil, P.Figuera, B.R.Fulton, I.Mukha, E.Vangioni - Astrophys.J. 630, L105 (2005)

The ${ }^{7} \mathrm{Be}(\mathrm{d}, \mathrm{p}) 2 \alpha$ Cross Section at Big Bang Energies and the Primordial ${ }^{7} \mathrm{Li}$ Abundance

2005AR25 C.Arnaboldi, D.R.Artusa, F.T.Avignone III, M.Balata, I.Bandac, M.Barucci, J.W.Beeman, C.Brofferio, C.Bucci, S.Capelli, L.Carbone, S.Cebrian, O.Cremonesi, R.J.Creswick, A.de Waard, H.A.Farach, E.Fiorini, G.Frossati, E.Guardincerri, A.Giuliani, P.Gorla, E.E.Haller, R.J.McDonald, A.Morales, E.B.Norman, A.Nucciotti, E.Olivieri, M.Pallavicini, E.Palmieri, E.Pasca, M.Pavan, M.Pedretti, G.Pessina, S.Pirro, E.Previtali, L.Risegari, C.Rosenfeld, S.Sangiorgio, M.Sisti, A.R.Smith, L.Torres, G.Ventura - Phys.Rev.Lett. 95, 142501 (2005)

New Limit on the Neutrinoless $\beta \beta$ Decay of ${ }^{130} \mathrm{Te}$

2005 AR27 R.Arnold, C.Augier, J.Baker, A.Barabash, G.Broudin, V.Brudanin, A.J.Caffrey, E.Caurier, V.Egorov, K.Errahmane, A.I.Etienvre, J.L.Guyonnet, F.Hubert, Ph.Hubert, C.Jollet, S.Jullian, O.Kochetov, V.Kovalenko, S.Konovalov, D.Lalanne, F.Leccia, C.Longuemare, G.Lutter, Ch.Marquet, F.Mauger, F.Nowacki, H.Ohsumi, F.Piquemal, J.L.Reyss, R.Saakyan, X.Sarazin, L.Simard, F.Simkovic, Yu.Shitov, A.Smolnikov, L.Stekl, J.Suhonen, C.S.Sutton, G.Szklarz, J.Thomas, V.Timkin, V.Tretyak, V.Umatov, L.Vala, I.Vanushin, V.Vasilyev, V.Vorobel, Ts.Vylov Phys.Rev.Lett. 95, 182302 (2005)

First Results of the Search for Neutrinoless Double-Beta Decay with the NEMO 3 Detector

2005BA88 R.A.Bark, M.Lipoglavsek, S.M.Maliage, S.S.Ntshangase, A.Shevchenko J.Phys.(London) G31, S1747 (2005)

Aspects of nuclear physics research at iThemba LABS, South Africa

2005BA93 O.Bartholomy, and the CB-ELSA Collaboration - Phys.Rev.Lett. 94, 012003 (2005) Neutral-Pion Photoproduction off Protons in the Energy Range $0.3 \mathrm{GeV} \leq \mathrm{E} \gamma \leq 3$ $\mathrm{GeV}$

2005BA94 J.C.Batchelder, J.-C.Bilheux, C.R.Bingham, H.K.Carter, D.Fong, P.E.Garrett, R.Grzywacz, J.H.Hamilton, D.J.Hartley, J.K.Hwang, W.Krolas, W.D.Kulp, Y.Larochelle, A.Piechaczek, A.V.Ramayya, K.P.Rykaczewski, E.H.Spejewski, D.W.Stracener, M.N.Tantawy, J.A.Winger, J.L.Wood, E.F.Zganjar - Phys.Rev. C 72,044306 (2005)

New isomeric state in ${ }^{116} \mathrm{Ag}$ 
2005BA96 S.Barua, J.J.Das, A.Jhingan, N.Madhavan, T.Varughese, P.Sugathan, K.Kalita, S.Verma, B.Bhattacharjee, S.K.Datta, K.Boruah - Phys.Rev. C 72, 044602 (2005) Investigation of scattering between mirror nuclei ${ }^{7} \mathrm{Be}$ and ${ }^{7} \mathrm{Li}$

2005BB01 C.Bachelet, G.Audi, C.Gaulard, C.Guenaut, F.Herfurth, D.Lunney, M.De Saint Simon, C.Thibault - Eur.Phys.J. A 25, Supplement 1, 31 (2005)

Mass measurement of short-lived halo nuclides

2005BB02 J.C.Batchelder, M.Tantawy, C.R.Bingham, M.Danchev, D.J.Fong, T.N.Ginter, C.J.Gross, R.Grzywacz, K.Hagino, J.H.Hamilton, M.Karny, W.Krolas, C.Mazzocchi, A.Piechaczek, A.V.Ramayya, K.P.Rykaczewski, A.Stolz, J.A.Winger, C.-H.Yu, E.F.Zganjar - Eur.Phys.J. A 25, Supplement 1, 149 (2005)

Study of fine structure in the proton radioactivity of ${ }^{146} \mathrm{Tm}$

2005BB05 D.W.Bardayan, J.C.Blackmon, J.Gomez del Campo, R.L.Kozub, J.F.Liang, Z.Ma, D.Shapira, L.Sahin, M.S.Smith - Eur.Phys.J. A 25, Supplement 1, 643 (2005)

New ${ }^{19} \mathrm{Ne}$ resonance observed using an exotic ${ }^{18} \mathrm{~F}$ beam

2005BB06 L.Barron-Palos, E.Chavez, A.Huerta, M.E.Ortiz, G.Murillo, E.Aguilera, E.Martinez, E.Moreno, R.Policroniades, A.Varela - Eur.Phys.J. A 25, Supplement 1, 645 (2005)

${ }^{12} \mathrm{C}+{ }^{12} \mathrm{C}$ cross-section measurements at low energies

2005BE60 M.Belleguic, F.Azaiez, Zs.Dombradi, D.Sohler, M.J.Lopez-Jimenez, T.Otsuka, M.G.Saint-Laurent, O.Sorlin, M.Stanoiu, Y.Utsuno, Yu.-E.Penionzhkevich, N.L.Achouri, J.C.Angelique, C.Borcea, C.Bourgeois, J.M.Daugas, F.De Oliveira-Santos, Z.Dlouhy, C.Donzaud, J.Duprat, Z.Elekes, S.Grevy, D.Guillemaud-Mueller, S.Leenhardt, M.Lewitowicz, S.M.Lukyanov, W.Mittig, M.G.Porquet, F.Pougheon, P.Roussel-Chomaz, H.Savajols, Y.Sobolev, C.Stodel, J.Timar - Phys.Rev. C 72, 054316 (2005)

Search for neutron excitations across the $\mathrm{N}=20$ shell gap in ${ }^{25-29} \mathrm{Ne}$

2005BE61 N.Benczer-Koller, G.Kumbartzki, J.R.Cooper, T.J.Mertzimekis, M.J.Taylor, L.Bernstein, K.Hiles, P.Maier-Komor, M.A.McMahan, L.Phair, J.Powell, K.-H.Speidel, D.Wutte - Eur.Phys.J. A 25, Supplement 1, 203 (2005)

First $\mathrm{g}$-factor measurement using a radioactive ${ }^{76} \mathrm{Kr}$ beam

2005BEZS T.Behrens, R.Gernhauser, D.Habs, Th.Kroll, R.Krucken, R.Lutter, T.Morgan, M.Pasini, K.Rudolph, P.G.Thirolf, V.Bildstein, H.Scheit, J.Jolie, D.Martin, N.Warr, and the MINIBALL / REX-ISOLDE Collaboration - Maier-Leibnitz-Laboratorium 2004 Ann.Rept., p.14 (2005)

Coulomb Excitation of Neutron-rich Cd Isotopes

2005BI24 C.R.Bingham, M.N.Tantawy, J.C.Batchelder, M.Danchev, T.N.Ginter, C.J.Gross, D.J.Fong, R.Grzywacz, K.Hagino, J.H.Hamilton, M.Karny, W.Krolas, C.Mazzocchi, A.Piechaczek, A.V.Ramayya, K.Rykaczewski, A.Stolz, J.A.Winger, C.-H.Yu, E.F.Zganjar - Nucl.Instrum.Methods Phys.Res. B241, 185 (2005)

Nuclear structure studies at the proton drip line via proton radioactivity studies

2005BI25

J.Billowes - Eur.Phys.J. A 25, Supplement 1, 187 (2005)

Developments in laser spectroscopy at the Jyvaskyla IGISOL 
2005BL23 J.C.Blackmon, F.Carstoiu, L.Trache, D.W.Bardayan, C.R.Brune, C.A.Gagliardi, U.Greife, C.J.Gross, C.C.Jewett, R.L.Kozub, T.A.Lewis, J.F.Liang, B.H.Moazen, A.M.Mukhamedzhanov, C.D.Nesaraja, F.M.Nunes, P.D.Parker, L.Sahin, J.P.Scott, D.Shapira, M.S.Smith, J.S.Thomas, R.E.Tribble - Phys.Rev. C 72, 034606 (2005) Elastic scattering of the proton drip-line nucleus ${ }^{17} \mathrm{~F}$

2005Bl31 B.Blank, N.Adimi, A.Bey, G.Canchel, C.Dossat, A.Fleury, J.Giovinazzo, I.Matea, F.De Oliveira, I.Stefan, G.Georgiev, S.Grevy, J.C.Thomas, C.Borcea, D.Cortina, M.Caamano, M.Stanoiu, F.Aksouh - Eur.Phys.J. A 25, Supplement 1, 169 (2005) First observation of ${ }^{54} \mathrm{Zn}$ and its decay by two-proton emission

2005 B039 H.G.Bohlen, R.Kalpakchieva, W.von Oertzen, T.N.Massey, A.A.Ogloblin, G.de Angelis, Ch.Schulz, Tz.Kokalova, C.Wheldon - J.Phys.(London) G31, S1461 (2005) Structure studies of excited states of ${ }^{17} \mathrm{C}$ and ${ }^{16} \mathrm{C}$

2005B045 D.Borremans, D.L.Balabanski, K.Blaum, W.Geithner, S.Gheysen, P.Himpe, M.Kowalska, J.Lassen, P.Lievens, S.Mallion, R.Neugart, G.Neyens, N.Vermeulen, D.Yordanov - Phys.Rev. C 72, 044309 (2005); Erratum Phys.Rev. C 72, 059902 (2005)

New measurement and reevaluation of the nuclear magnetic and quadrupole moments of ${ }^{8} \mathrm{Li}$ and ${ }^{9} \mathrm{Li}$

2005B047 V.Bondarenko, J.Honzatko, I.Tomandl, T.von Egidy, H.-F.Wirth, A.M.Sukhovoj L.A.Malov, L.I.Simonova, P.Alexa, J.Berzins, R.Hertenberger, Y.Eisermann, G.Graw - Nucl.Phys. A762, 167 (2005)

Low-spin mixed particle-hole structures in ${ }^{185} \mathrm{~W}$

2005B049 P.Boutachkov, G.V.Rogachev, V.Z.Goldberg, A.Aprahamian, F.D.Becchetti, J.P.Bychowski, Y.Chen, G.Chubarian, P.A.DeYoung, J.J.Kolata, L.O.Lamm, G.F.Peaslee, M.Quinn, B.B.Skorodumov, A.Wohr - Eur.Phys.J. A 25, Supplement 1, $259(2005)$

Isobaric analog states of neutron-rich nuclei. Doppler shift as a measurement tool for resonance excitation functions

2005B0ZT A.Borella - Thesis, Gent Univ. Belgium (2005)

Determination of the neutron resonance parameters for ${ }^{206} \mathrm{~Pb}$ and of the thermal neutron capture cross section for ${ }^{206} \mathrm{~Pb}$ and ${ }^{209} \mathrm{Bi}$

2005BU37 M.J.Burns, R.Chapman, K.M.Spohr, J.Ollier, M.Labiche, X.Liang, E.Farnea, M.Axiotis, T.Martinez, D.R.Napoli, C.A.Ur, Th.Kroll - J.Phys.(London) G31, S1827 (2005)

High spin studies of the Er and Tm isotopes around $\mathrm{A}=166$

2005BUZZ D.Bucurescu, H.-F.Wirth, R.Hertenberger, G.Graw, D.A.Meyer, R.F.Casten, S.Heinze, J.Jolie - Maier-Leibnitz-Laboratorium 2004 Ann.Rept., p.16 (2005) Investigation of $0^{+}$Excitations in a Deformed Nucleus: ${ }^{168} \mathrm{Er}$

2005CA42 G.Cazzoli, C.Puzzarini, J.Gauss - Astrophys.J.Suppl.Ser. 159, 181 (2005) 
The rare isotopomers of $\mathrm{HCN}$ : $\mathrm{HC}^{15} \mathrm{~N}$ and $\mathrm{DC}^{15} \mathrm{~N}$ rotational spectrum and resolved nuclear hyperfine structures due to ${ }^{15} \mathrm{~N}$ and $\mathrm{D}$

2005CA43 M.P.Carpenter, F.G.Kondev, R.V.F.Janssens - J.Phys.(London) G31, S1599 (2005) Studies of neutron-deficient nuclei near the $\mathrm{Z}=82$ shell closure via cold fusion reactions

2005CA44 W.N.Catford, C.N.Timis, R.C.Lemmon, M.Labiche, N.A.Orr, L.Caballero, R.Chapman, M.Freer, M.Chartier, H.Savajols, M.Rejmund, N.Amzal, N.I.Ashwood, T.D.Baldwin, M.Burns, N.Curtis, G.de France, W.Gelletly, X.Liang, S.D.Pain, V.P.E.Pucknell, B.Rubio, O.Sorlin, K.Spohr, Ch.Theisen, D.D.Warner J.Phys.(London) G31, S1655 (2005)

Nucleon transfer via $(\mathrm{d}, \mathrm{p})$ using TIARA with a ${ }^{24}$ Ne radioactive beam

2005CA50 W.N.Catford, R.C.Lemmon, M.Labiche, C.N.Timis, N.A.Orr, L.Caballero, R.Chapman, M.Chartier, M.Rejmund, H.Savajols, and the TIARA Collaboration Eur.Phys.J. A 25, Supplement 1, 245 (2005)

First experiments on transfer with radioactive beams using the TIARA array

2005CH60 M.Chartier, M.B.Gomez Hornillos, W.Mittig, A.Lepine-Szily, L.Caballero Ontanaya, C.E.Demonchy, G.Georgiev, N.A.Orr, G.Politi, M.Rousseau, P.Roussel-Chomaz, A.C.C.Villari - J.Phys.(London) G31, S1771 (2005)

Direct mass measurement of $\mathrm{N} \sim \mathrm{Z}$ nuclei with $\mathrm{A}=64-80$ using the CSS2 cyclotron

2005CH62 Y.Cheng, B.Xia, Y.-N.Liu, Q.-X.Jin - Chin.Phys.Lett. 22, 2530 (2005)

Rhodium Mossbauer Effect Generated by Bremsstrahlung Excitation

2005CH65 A.Chakraborty, Krishichayan, S.S.Ghugre, R.Goswami, S.Mukhopadhyay, N.S.Pattabiraman, S.Ray, A.K.Sinha, S.Sarkar, P.V.Madhusudhana Rao, U.Garg, S.K.Basu, M.B.Chatterjee, M.S.Sarkar, L.Chaturvedi, A.Dhal, R.K.Sinha, I.M.Govil, R.K.Bhowmik, A.Jhingan, N.Madhavan, S.Muralithar, S.Nath, R.P.Singh, P.Sugathan - Phys.Rev. C 72, 054309 (2005)

Spectroscopy of ${ }^{90} \mathrm{Nb}$ at high spin

2005CH66 J.A.Church, C.M.Campbell, D.-C.Dinca, J.Enders, A.Gade, T.Glasmacher, Z.Hu, R.V.F.Janssens, W.F.Mueller, H.Olliver, B.C.Perry, L.A.Riley, K.L.Yurkewicz Phys.Rev. C 72, 054320 (2005)

Measurement of E2 transition strengths in ${ }^{32,34} \mathrm{Mg}$

2005CH67 R.S.Chakrawarthy, P.M.Walker, M.B.Smith, A.N.Andreyev, S.F.Ashley, G.C.Ball, J.A.Becker, J.J.Daoud, P.E.Garrett, G.Hackman, G.A.Jones, Y.Litvinov, A.C.Morton, C.J.Pearson, C.E.Svensson, S.J.Williams, E.F.Zganjar - Eur.Phys.J. A 25, Supplement 1, 125 (2005)

Discovery of a new $2.3 \mathrm{~s}$ isomer in neutron-rich ${ }^{174} \mathrm{Tm}$

2005CI07 J.A.Cizewski, K.L.Jones, S.D.Pain, J.S.Thomas, C.Baktash, D.W.Bardayan, J.C.Blackmon, C.Gross, J.F.Liang, D.Shapira, M.S.Smith, R.L.Kozub, B.H.Moazen, C.D.Nesaraja, H.K.Carter, M.S.Johnson, R.P.Fitzgerald, D.W.Visser, U.Greife, R.J.Livesay, W.Catford, Z.Ma - Nucl.Instrum.Methods Phys.Res. B241, 200 (2005) Neutron transfer reactions with neutron-rich radioactive ion beams 
2005CL07 R.M.Clark, L.W.Phair, M.Descovich, M.Cromaz, M.A.Deleplanque, P.Fallon, I.Y.Lee, A.O.Macchiavelli, M.A.McMahan, L.G.Moretto, E.Rodriguez-Vieitez, S.Sinha, F.S.Stephens, D.Ward, M.Wiedeking, L.A.Bernstein, J.T.Burke, J.A.Church - Phys.Rev. C 72, 054605 (2005)

Population of nuclei via ${ }^{7} \mathrm{Li}$-induced binary reactions

2005CL08 J.A.Clark, R.C.Barber, B.Blank, C.Boudreau, F.Buchinger, J.E.Crawford, J.P.Greene, S.Gulick, J.C.Hardy, A.A.Hecht, A.Heinz, J.K.P.Lee, A.F.Levand, B.F.Lundgren, R.B.Moore, G.Savard, N.D.Scielzo, D.Seweryniak, K.S.Sharma, G.D.Sprouse, W.Trimble, J.Vaz, J.C.Wang, Y.Wang, B.J.Zabransky, Z.Zhou Eur.Phys.J. A 25, Supplement 1, 629 (2005)

Investigating the rp-process with the Canadian Penning trap mass spectrometer

2005C022 A.Costin, N.Pietralla, T.Koike, C.Vaman, T.Ahn, G.Rainovski - Phys.Rev. C 72, 054305 (2005)

Subnanosecond lifetime measurement for the $\mathrm{T}=0,3_{1}^{+}$state of odd-odd $\mathrm{N}=\mathrm{Z}{ }^{58} \mathrm{Cu}$

2005C024 D.Cortina-Gil, J.Fernandez-Vazquez, T.Aumann, T.Baumann, J.Benlliure, M.J.G.Borge, L.V.Chulkov, U.Datta Pramanik, C.Forssen, L.M.Fraile, H.Geissel, J.Gerl, F.Hammache, K.Itahashi, R.Janik, B.Jonson, S.Mandal, K.Markenroth, M.Meister, M.Mocko, G.Munzenberg, T.Ohtsubo, A.Ozawa, Y.Prezado, V.Pribora, K.Riisager, H.Scheit, R.Schneider, G.Schrieder, H.Simon, B.Sitar, A.Stolz, P.Strmen, K.Summerer, I.Szarka, H.Weick - Eur.Phys.J. A 25, Supplement 1, 343 (2005) One-neutron knockout of ${ }^{23} \mathrm{O}$

2005C025 L.Corradi, A.M.Stefanini, S.Szilner, S.Beghini, B.R.Behera, E.Farnea, A.Gadea, E.Fioretto, F.Haas, A.Latina, N.Marginean, G.Montagnoli, G.Pollarolo, F.Scarlassara, M.Trotta, C.Ur, and the PRISMA-CLARA Collaboration Eur.Phys.J. A 25, Supplement 1, 427 (2005)

Multinucleon transfer reactions studied with the heavy-ion magnetic spectrometer PRISMA

2005CSZZ L.Csige, M.Csatlos, A.Krasznahorkay, P.G.Thirolf, T.Faestermann, Z.Gacsi, J.Gulyas, D.Habs, R.Hertenberger, M.Hunyadi, H.J.Maier, T.Morgan, O.Schaile, W.Schwerdtfeger, H.-F.Wirth - Maier-Leibnitz-Laboratorium 2004 Ann.Rept., p.19 (2005)

Angular Distribution of Fission Fragments from the ${ }^{236} \mathrm{U}(\mathrm{d}, \mathrm{pf})$ Reaction

2005CU05 D.M.Cullen, R.Glover, L.K.Pattison, P.M.Walker, S.Frauendorf, D.Almehed J.Phys.(London) G31, S1709 (2005)

Nuclear-tidal waves in the osmium nuclei

2005CU06 N.Curtis, N.I.Ashwood, W.N.Catford, N.M.Clarke, M.Freer, D.Mahboub, C.J.Metelko, S.D.Pain, N.Soic, D.C.Weisser - Phys.Rev. C 72, 044320 (2005) $\alpha+\mathrm{Li}$ and $\mathrm{H}+\mathrm{Be}$ decay of ${ }^{10,11,12} \mathrm{~B}$

2005CU07 X.Z.Cui, L.H.Zhu, X.G.Wu, Z.M.Wang, C.Y.He, Y.Liu, G.S.Li, S.X.Wen, Z.L.Zhang, R.Meng, R.G.Ma, P.Luo, Y.Zheng, M.M.Ndontchueng, J.D.Huo, C.X.Yang - Phys.Rev. C 72, 044322 (2005) 
High-spin states and shell structure of the odd-odd nucleus ${ }^{90} \mathrm{Nb}$

2005DA38 U.Datta Pramanik, P.Adrich, T.Aumann, K.Boretzky, D.Cortina, Th.W.Elze, H.Emling, H.Geissel, M.Hellstrom, K.L.Jones, L.H.Khiem, J.V.Kratz, R.Kulessa, Y.Leifels, G.Munzenberg, C.Nociforo, R.Palit, H.Scheit, H.Simon, K.Summerer, S.Typel, W.Walus, H.Weick - J.Phys.(London) G31, S1583 (2005)

Coulomb breakup of psd-shell neutron-rich nuclei

2005DA40Ｄ.Das, V.Natarajan - Europhys.Lett. 72, 740 (2005)

Hyperfine spectroscopy on the $6 \mathrm{P}_{3 / 2}$ state of ${ }^{133} \mathrm{Cs}$ using coherent control

2005DA41 J.J.Das, P.Sugathan, N.Madhavan, P.V.Madhusudhana Rao, A.Jhingan, T.Varughese, S.Barua, S.Nath, A.K.Sinha, B.Kumar, J.Zacharias Nucl.Instrum.Methods Phys.Res. B241, 953 (2005)

Production of light radioactive ion beams (RIB) using inverse kinematics

2005DA42 M.Danchev, J.Pavan, N.J.Stone, A.E.Stuchbery, C.Baktash, J.Beene, N.Benczer-Koller, C.R.Bingham, J.Dupak, A.Galindo-Uribarri, C.J.Gross, G.Kumbartzki, D.C.Radford, J.R.Stone, C.L.Timlin, C.-H.Yu, N.V.Zamfir Nucl.Instrum.Methods Phys.Res. B241, 971 (2005)

g-Factor measurements of first $2^{+}$states of heavy Te isotopes based on nuclear spin deorientation for nuclei recoiling in vacuum

2005DA43 U.Datta Pramanik, T.Aumann, K.Boretzky, D.Cortina, Th.W.Elze, H.Emling, H.Geissel, M.Hellstrom, K.L.Jones, L.H.Khiem, J.V.Kratz, R.Kulessa, Y.Leifels, G.Munzenberg, C.Nociforo, R.Palit, H.Scheit, H.Simon, K.Summerer, S.Typel, W.Walus, H.Weick - Eur.Phys.J. A 25, Supplement 1, 339 (2005)

Studies of light neutron-rich nuclei near the drip line

2005DE48 A.Dewald, O.Moller, B.Saha, K.Jessen, A.Fitzler, B.Melon, T.Pissulla, S.Heinze, J.Jolie, K.O.Zell, P.von Brentano, P.Petkov, S.Harissopulos, G.De Angelis,

T.Martinez, D.R.Napoli, N.Marginean, M.Axiotis, C.Rusu, D.Tonev, A.Gadea,

Y.H.Zhang, D.Bazzacco, S.Lunardi, C.A.Ur, R.Menegazzo, E.Farnea -

J.Phys.(London) G31, S1427 (2005)

Test of the critical point symmetry $\mathrm{X}(5)$ in the mass $\mathrm{A}=180$ region

2005DE54 G.Della Mea, A.Patelli, S.Restello, V.Rigato, A.Vomiero - Nucl.Instrum.Methods Phys.Res. B240, 803 (2005)

${ }^{14} \mathrm{~N}(\alpha, \mathrm{p}){ }^{17} \mathrm{O}$ nuclear reaction cross-section at $4.9-6.1 \mathrm{MeV}$

2005DEZT H.Denz, P.Amaudruz, J.T.Brack, J.Breitschopf, P.Camerini, J.L.Clark, H.Clement, L.Felawka, E.Fragiacomo, E.F.Gibson, N.Grion, G.J.Hofman, B.Jamieson, E.L.Mathie, R.Meier, G.Moloney, D.Ottewell, O.Patarakin, J.D.Patterson, M.M.Pavan, S.Piano, K.Raywood, R.A.Ristinen, R.Rui, M.E.Sevior, G.R.Smith, J.Stahov, R.Tacik, G.J.Wagner, F.von Wrochem, D.M.Yeomans nucl-ex/0512006,12/3/2005 (2005)

$\pi^{ \pm} \mathrm{p}$ differential cross sections at low energies 
2005DI16 C.Aa.Diget, F.C.Barker, M.G.Borge, J.Cederkall, V.N.Fedosseev, L.M.Fraile, B.R.Fulton, H.O.U.Fynbo, H.B.Jeppesen, B.Jonson, U.Koster, M.Meister, T.Nilsson, G.Nyman, Y.Prezado, K.Riisager, S.Rinta-Antila, O.Tengblad, M.Turrion, K.Wilhelmsen, J.Aysto - Nucl.Phys. A760, 3 (2005)

Properties of the ${ }^{12} \mathrm{C} 10 \mathrm{MeV}$ state determined through $\beta$-decay

2005D020 C.Dossat, A.Bey, B.Blank, G.Canchel, A.Fleury, J.Giovinazzo, I.Matea, F.de Oliveira Santos, G.Georgiev, S.Grevy, I.Stefan, J.C.Thomas, N.Adimi, C.Borcea, D.Cortina Gil, M.Caamano, M.Stanoiu, F.Aksouh, B.A.Brown, L.V.Grigorenko Phys.Rev. C 72, 054315 (2005)

Two-proton radioactivity studies with ${ }^{45} \mathrm{Fe}$ and ${ }^{48} \mathrm{Ni}$

2005DRZW G.D.Dracoulis, G.J.Lane, T.M.Peatey, A.P.Byrne, A.M.Baxter, P.M.Davidson, A.N.Wilson, T.Kibedi, F.R.Xu - ANU-P/1662 (2005)

E3 strength of the $11^{-}$to $8^{+}$isomeric decays in ${ }^{194} \mathrm{~Pb}$ and ${ }^{196} \mathrm{~Pb}$ and oblate deformation

2005 DU23 Ch.E.Dullmann, C.M.Folden III, K.E.Gregorich, D.C.Hoffman, D.Leitner, G.K.Pang, R.Sudowe, P.M.Zielinski, H.Nitsche - Nucl.Instrum.Methods Phys.Res. A551, 528 (2005)

Heavy-ion-induced production and physical preseparation of short-lived isotopes for chemistry experiments

2005EE01 S.Eeckhaudt, N.Amzal, J.E.Bastin, E.Bouchez, P.A.Butler, A.Chatillon, K.Eskola, J.Gerl, T.Grahn, A.Gorgen, P.T.Greenlees, R.-D.Herzberg, F.P.Hessberger, A.Hurstel, P.J.C.Ikin, G.D.Jones, P.Jones, R.Julin, S.Juutinen, H.Kettunen, T.L.Khoo, W.Korten, P.Kuusiniemi, Y.Le Coz, M.Leino, A.-P.Leppanen, P.Nieminen, J.Pakarinen, J.Perkowski, A.Pritchard, P.Reiter, P.Rahkila, C.Scholey, Ch.Theisen, J.Uusitalo, K.Van de Vel, J.Wilson, H.J.Wollersheim - Eur.Phys.J. A 25, Supplement 1, 605 (2005)

In-beam gamma-ray spectroscopy of ${ }^{254} \mathrm{No}$

2005EE02 S.Eeckhaudt, P.T.Greenlees, N.Amzal, J.E.Bastin, E.Bouchez, P.A.Butler, A.Chatillon, K.Eskola, J.Gerl, T.Grahn, A.Gorgen, R.-D.Herzberg, F.P.Hessberger, A.Hurstel, P.J.C.Ikin, G.D.Jones, P.Jones, R.Julin, S.Juutinen, H.Kettunen, T.L.Khoo, W.Korten, P.Kuusiniemi, Y.Le Coz, M.Leino, A.-P.Leppanen, P.Nieminen, J.Pakarinen, J.Perkowski, A.Pritchard, P.Reiter, P.Rahkila, C.Scholey, Ch.Theisen, J.Uusitalo, K.Van de Vel, J.Wilson - Eur.Phys.J. A 26, 227 (2005)

Evidence for non-yrast states in ${ }^{254} \mathrm{No}$

2005EK01 J.Ekman, L.-L.Andersson, C.Fahlander, E.K.Johansson, R.du Rietz, D.Rudolph Eur.Phys.J. A 25, Supplement 1, 363 (2005)

News on mirror nuclei in the sd and fp shells

2005EL10 H.M.El-Masri, P.M.Walker, G.D.Dracoulis, T.Kibedi, A.P.Byrne, A.M.Bruce, J.N.Orce, A.Emmanouilidis, D.M.Cullen, C.Wheldon, F.R.Xu - Phys.Rev. C 72, $054306(2005)$

High-K states in the odd-odd nuclide ${ }^{180} \mathrm{Re}$ 
2005ERZZ T.Eronen, V.Elomaa, U.Hager, J.Hakala, A.Jokinen, I.Moore, H.Penttila, S.Rahaman, S.Rinta-Antila, A.Saastamoinen, T.Sonoda, J.Aysto, A.Bey , B.Blank, G.Canchel, C.Dossat, J.Giovinazzo, I.Matea, N.Adimi - nucl-ex/0512010,12/12/2005 (2005)

Q-value of the superallowed $\beta$ decay of ${ }^{62} \mathrm{Ga}$

2005FE11 J.O.Fernandez-Niello, A.Priller, A.Arazi, D.Djokic, R.Golser, W.Kutschera, P.Steier, C.Vockenhuber, A.Wallner - Nucl.Instrum.Methods Phys.Res. B240, 495 (2005) A study of the tandem-terminal-stripper reaction ${ }^{1} \mathrm{H}\left({ }^{12} \mathrm{C}, \gamma\right){ }^{13} \mathrm{~N}$ with accelerator mass spectrometry

2005F014 B.Fornal, S.Zhu, R.V.F.Janssens, M.Honma, R.Broda, B.A.Brown, M.P.Carpenter, S.J.Freeman, N.Hammond, F.G.Kondev, W.Krolas, T.Lauritsen, S.N.Liddick, C.J.Lister, S.Lunardi, P.F.Mantica, N.Marginean, T.Mizusaki, E.F.Moore, T.Otsuka, T.Pawlat, D.Seweryniak, B.E.Tomlin, C.A.Ur, I.Wiedenhover, J.Wrzesinski - Phys.Rev. C 72, 044315 (2005)

Yrast structure of neutron-rich ${ }^{53} \mathrm{Ti}$

2005F017 D.Fong, J.K.Hwang, A.V.Ramayya, J.H.Hamilton, C.J.Beyer, K.Li, P.M.Gore, E.F.Jones, Y.X.Luo, J.O.Rasmussen, S.J.Zhu, S.C.Wu, I.Y.Lee, P.Fallon, M.A.Stoyer, S.J.Asztalos, T.N.Ginter, J.D.Cole, G.M.Ter-Akopian, A.Daniel, R.Donangelo - Eur.Phys.J. A 25, Supplement 1, 465 (2005)

Investigations of short half-life states from $\mathrm{SF}$ of ${ }^{252} \mathrm{Cf}$

2005FR29 S.J.Freeman, R.V.F.Janssens, A.N.Deacon, F.Xu, I.J.Calderin, M.P.Carpenter, P.Chowdhury, S.M.Fischer, N.J.Hammond, M.Honma, T.Lauritsen, C.J.Lister, T.L.Khoo, G.Mukherjee, D.Seweryniak, J.F.Smith, S.L.Tabor, B.J.Varley, S.Zhu J.Phys.(London) G31, S1465 (2005)

Structure of the neutron-rich Cr isotopes

2005Fu16 Y.Fujita, T.Adachi, P.von Brentano, G.P.A.Berg, C.Fransen, D.De Frenne, H.Fujita, K.Fujita, K.Hatanaka, E.Jacobs, K.Nakanishi, A.Negret, N.Pietralla, L.Popescu, B.Rubio, Y.Sakemi, Y.Shimbara, Y.Shimizu, Y.Tameshige, A.Tamii, M.Yosoi, K.O.Zell - Phys.Rev.Lett. 95, 212501 (2005)

Gamow-Teller Strengths in Proton-Rich Exotic Nuclei Deduced in the Combined Analysis of Mirror Transitions

2005GA44 A.Gadea, N.Marginean, L.Corradi, S.M.Lenzi, C.A.Ur, E.Farnea, G.de Angelis, E.Fioretto, D.R.Napoli, A.M.Stefanini, S.Szilner, M.Axiotis, B.R.Behera, A.Latina, C.Rusu, W.Zhimin, D.Bazzacco, S.Beghini, S.Lunardi, G.Montagnoli, R.Menegazzo, F.Scarlassara, F.Della Vedova, M.Nespolo, A.Bracco, F.Camera, S.Leoni, B.Million, M.Pignanelli, G.Pollarolo, M.Trotta, P.G.Bizzeti, A.M.Bizzeti-Sona, D.Curien, P.Medina, M.Chambit, R.Chapman, X.Liang, S.J.Freeman, A.Smith, B.J.Varley, V.Pucknell, R.Lemmon - J.Phys.(London) G31, S1443 (2005)

The CLARA-PRISMA setup installed at LNL: first results 
2005GA45 L.Gaudefroy, O.Sorlin, D.Beaumel, Y.Blumenfeld, Z.Dombradi, S.Fortier, S.Franchoo, M.Gelin, J.Gibelin, S.Grevy, F.Hammache, F.Ibrahim, K.Kemper, K.L.Kratz, S.M.Lukyanov, C.Monrozeau, L.Nalpas, F.Nowacki, A.N.Ostrowski, Yu.-E.Penionzhkevich, E.Pollacco, P.Roussel-Chomaz, E.Rich, J.A.Scarpaci, M.G.St Laurent, D.Sohler, M.Stanoiu, E.Tryggestad, D.Verney - J.Phys.(London) G31, S1623 (2005)

Study of ${ }^{45}$ Ar through $(d, p)$ reaction at SPIRAL

2005GA46 A.B.Garnsworthy, N.J.Thompson, Zs.Podolyak, P.M.Walker, S.J.Williams, G.D.Dracoulis, G.de France, G.J.Lane, K.Andgren, A.M.Bruce, A.P.Byrne, W.N.Catford, B.Cederwall, G.A.Jones, B.McGuirk, S.Mandal, E.S.Paul, V.Pucknell, N.Redon, B.Rosse, R.J.Senior, G.Sletten - J.Phys.(London) G31, S1851 (2005) Spectroscopy of ${ }^{212} \mathrm{Po}$ and ${ }^{213} \mathrm{At}$ using a ${ }^{8} \mathrm{He}$ radioactive beam and EXOGAM

2005GA47 P.E.Garrett, W.D.Kulp, J.L.Wood, D.Bandyopadhyay, S.Christen, S.Choudry, A .Dewald, A.Fitzler, C .Fransen, K.Jessen, J.Jolie, A.Kloezer, P.Kudejova, A.Kumar, S.R.Lesher, A.Linnemann, A.Lisetskiy, D.Martin, M.Masur, M.T.McEllistrem, O.Moller, M.Mynk, J.N.Orce, P.Pejovic, T.Pissulla, J.M.Regis, A.Schiller, D.Tonev, S.W.Yates - J.Phys.(London) G31, S1855 (2005)

Octupole and hexadecapole bands in ${ }^{152} \mathrm{Sm}$

2005GA50 Yu.P.Gangrsky, V.I.Zhemenik, G.V.Mishinsky, Yu.E.Penionzhkevich - Yad.Fiz. 68, 1475 (2005); Phys.Atomic Nuclei 68, 1417 (2005)

Independent Yields of $\mathrm{Kr}$ and Xe Fragments in the Photofission of ${ }^{237} \mathrm{~Np}$ and ${ }^{243} \mathrm{Am}$ Odd Nuclei

2005GA54 A.Gade, D.Bazin, B.A.Brown, C.M.Campbell, J.A.Church, D.-C.Dinca, J.Enders, T.Glasmacher, P.G.Hansen, Z.Hu, K.W.Kemper, W.F.Mueller, H.Olliver, B.C.Perry, L.A.Riley, B.T.Roeder, B.M.Sherrill, J.R.Terry, J.A.Tostevin, K.L.Yurkewicz -

Eur.Phys.J. A 25, Supplement 1, 251 (2005)

Spectroscopic factors in exotic nuclei from nucleon-knockout reactions

2005GA56 A.Gadea - Eur.Phys.J. A 25, Supplement 1, 421 (2005)

First results of the CLARA-PRISMA setup installed at LNL

2005GAZT C.Gaulard, G.Audi, C.Bachelet, D.Lunney, M.de Saint Simon, C.Thibault, N.Vieira - nucl-ex/0511007,11/2/2005 (2005)

Accurate mass measurements of ${ }^{26} \mathrm{Ne},{ }^{26-30} \mathrm{Na},{ }^{29-33} \mathrm{Mg}$ performed with the MISTRAL spectrometer

2005GAZU Ju.M.Gavriljuk, A.M.Gangapshev, V.V.Kuzminov, S.I.Panasenko, S.S.Ratkevich nucl-ex/0510071,10/26/2005 (2005)

Results of a search for $2 \beta$-decay of ${ }^{136} \mathrm{Xe}$ with high-pressure copper proportional counters in Baksan Neutrino Observatory

2005GAZV Ju.M.Gavriljuk, V.N.Gavrin, A.M.Gangapshev, V.V.Kazalov, V.V.Kuzminov, N.Ya.Osetrova, I.I.Pul'nikov, A.V.Ryabukhin, A.N.Shubin, G.M.Skorynin, S.I.Panasenko, S.S.Ratkevich - nucl-ex/0510070,10/26/2005 (2005)

Next stage of search for $2 \mathrm{~K}(2 \nu)$-capture of ${ }^{78} \mathrm{Kr}$ 
2005GE09 G.Georgiev, I.Matea, D.L.Balabanski, J.M.Daugas, F.de Oliveira Santos, S.Franchoo, F.Ibrahim, F.Le Blanc, M.Lewitowicz, G.Lo Bianco, S.Lukyanov, V.Meot, P.Morel, G.Neyens, Yu.E.Penionzhkevich, A.Saltarelli, O.Sorlin, M.Stanoiu, M.Tarisien, N.Vermeulen, D.Verney , D.Yordanov - J.Phys.(London) G31, S1439 (2005) g-factor measurement of the $9 / 2^{+}$isomeric state in ${ }^{65} \mathrm{Ni}$

2005GE10 H.Geissel, Yu.A.Litvinov - J.Phys.(London) G31, S1779 (2005)

Precision experiments with relativistic exotic nuclei at GSI

2005GEZW H.Geissel, Yu.A.Litvinov, B.Pfeiffer, F.Attallah, G.Audi, K.Beckert, P.Beller, F.Bosch, D.Boutin, T.J.Burvenich, L.Chen, T.Faestermann, M.Falch, B.Franzke, M.Hausmann, E.Kaza, T.Kerscher, P.Kienle, O.Klepper, R.Knobel, C.Kozhuharov, K.-L.Kratz, S.A.Litvinov, K.E.G.Lobner, L.Maier, M.Matos, F.Montes, G.Munzenberg, C.Nociforo, F.Nolden, Yu.N.Novikov, T.Ohtsubo, A.Ostrowski, Z.Patyk, W.Plass, M.Portillo, T.Radon, H.Schatz, C.Scheidenberger, J.Stadlmann, M.Steck, B.Sun, K.Takahashi, G.Vorobjev, H.Weick, M.Winkler, H.Wollnik, T.Yamaguchi - nucl-ex/0510009,10/4/2005 (2005)

Present and Future Experiments with Stored Exotic Nuclei at Relativistic Energies

2005GI15 J.Giovinazzo - J.Phys.(London) G31, S1509 (2005)

The two-proton radioactivity in the $\mathrm{A} \sim 50$ mass region

2005GI17 A.Giannatiempo, A.Perego, P.Sona, A.Nannini, H.Mach, B.Fogelberg, M.J.G.Borge, O.Tengblad, L.M.Fraile, A.J.Aas, K.Gulda - Phys.Rev. C 72, 044308 (2005)

Spectroscopy and lifetime measurements of states in ${ }^{76} \mathrm{Kr}$ populated in ${ }^{76} \mathrm{Rb}$ decay

2005GI18 L.Giot, P.Roussel-Chomaz, N.Alamanos, F.Auger, M.-D.Cortina-Gil, Ch.E.Demonchy, J.Fernandez, A.Gillibert, C.Jouanne, V.Lapoux, R.S.Mackintosh, W.Mittig, L.Nalpas, A.Pakou, S.Pita, E.C.Pollacco, A.Rodin, K.Rusek, H.Savajols, J.L.Sida, F.Skaza, S.Stepantsov, G.Ter-Akopian, I.Thompson, R.Wolski -

Eur.Phys.J. A 25, Supplement 1, 267 (2005)

Study of the ground-state wave function of ${ }^{6} \mathrm{He}$ via the ${ }^{6} \mathrm{He}(\mathrm{p}, \mathrm{t}) \alpha$ transfer reaction

2005GL09 K.A.Gladnishki, D.L.Balabanski, P.Petkov, A.Dewald, D.Tonev, M.Axiotis, A.Fitzler, M.Danchev, S.Harissopulos, S.Lalkovski, N.Marginean, T.Martinez, O.Moeller, G.Neyens, A.Spyrou, E.A.Stefanova, C.Ur - J.Phys.(London) G31, S1559 (2005)

Lifetime measurements in the Yrast magnetic band in ${ }^{193} \mathrm{~Pb}$

2005G034 T.Gomi, T.Motobayashi, Y.Ando, N.Aoi, H.Baba, K.Demichi, Z.Elekes, N.Fukuda, Zs.Fulop, U.Futakami, H.Hasegawa, Y.Higurashi, K.Ieki, N.Imai, M.Ishihara, K.Ishikawa, N.Iwasa, H.Iwasaki, S.Kanno, Y.Kondo, T.Kubo, S.Kubono, M.Kunibu, K.Kurita, Y.U.Matsuyama, S.Michimasa, T.Minemura, M.Miura, H.Murakami, T.Nakamura, M.Notani, S.Ota, A.Saito, H.Sakurai, M.Serata, S.Shimoura, T.Sugimoto, E.Takeshita, S.Takeuchi, Y.Togano, K.Ue, K.Yamada, Y.Yanagisawa, K.Yoneda, A.Yoshida - J.Phys.(London) G31, S1517 (2005)

Coulomb dissociation experiment for explosive hydrogen burning: study of the ${ }^{22} \mathrm{Mg}(\mathrm{p}, \gamma){ }^{23} \mathrm{Al}$ reaction 
2005 G036 M.B.Gomez Hornillos, M.Chartier, W.Mittig, B.Blank, F.Chautard, C.E.Demonchy, A.Gillibert, B.Jacquot, B.Jurado, N.Lecesne, A.Lepine-Szily, N.A.Orr, P.Roussel-Chomaz, H.Savajols, A.C.C.Villari - J.Phys.(London) G31, S1869 (2005) Mass measurements with the CIME cyclotron at GANIL

2005G043 A.Gorgen, E.Clement, A.Chatillon, A.Dewald, W.Korten, Y.Le Coz, N.Marginean, B.Melon, R.Menegazzo, O.Moller, Ch.Theisen, D.Tonev, C.A.Ur, K.O.Zell -

Eur.Phys.J. A 26, 153 (2005)

Lifetime measurement in ${ }^{74} \mathrm{Kr}$ and ${ }^{76} \mathrm{Kr}$

2005GR25 C.J.Gross - J.Phys.(London) G31, S1639 (2005)

Recent results at HRIBF using neutron-rich radioactive ion beams

2005GR26 I.Grabowska-Bold - Eur.Phys.J. C 44, S1 (2005)

Measurement of deeply virtual Compton scattering using the ZEUS detector at HERA

2005GR28 N.Grion, M.Bregant, P.Camerini, E.Fragiacomo, S.Piano, R.Rui, E.F.Gibson, G.Hofman, E.L.Mathie, R.Meier, M.E.Sevior, G.R.Smith, R.Tacik, for the CHAOS Collaboration - Nucl.Phys. A763, 80 (2005)

The $\pi \rightarrow \pi \pi$ process in nuclei and the restoration of chiral symmetry

2005GR30 S.Grevy, F.Negoita, I.Stefan, N.L.Achouri, J.C.Angelique, B.Bastin, R.Borcea, A.Buta, J.M.Daugas, F.De Oliveira, O.Giarmana, C.Jollet, B.Laurent, M.Lazar, E.Lienard, F.Marechal, J.Mrazek, D.Pantelica, Y.Penionzhkevich, S.Pietri, O.Sorlin, M.Stanoiu, C.Stodel, M.G.St-Laurent - Eur.Phys.J. A 25, Supplement 1, 111 (2005) Observation of the $0_{2}^{+}$state in ${ }^{44} \mathrm{~S}$

2005GR32 R.Grzywacz, M.Karny, K.P.Rykaczewski, J.C.Batchelder, C.R.Bingham, D.Fong, C.J.Gross, W.Krolas, C.Mazzocchi, A.Piechaczek, M.N.Tantawy, J.A.Winger, E.F.Zganjar - Eur.Phys.J. A 25, Supplement 1, 145 (2005)

Discovery of the new proton emitter ${ }^{144} \mathrm{Tm}$

2005GR35 T.Grahn, A.Dewald, O.Moller, C.W.Beausang, S.Eeckhaudt, P.T.Greenlees, J.Jolie, P.Jones, R.Julin, S.Juutinen, H.Kettunen, T.Kroll, R.Krucken, M.Leino, A.-P.Leppanen, P.Maierbeck, D.A.Meyer, P.Nieminen, M.Nyman, J.Pakarinen, P.Petkov, P.Rahkila, B.Saha, C.Scholey, J.Uusitalo - Eur.Phys.J. A 25, Supplement 1, 441 (2005)

RDDS lifetime measurement with JUROGAM + RITU

2005GR36 P.T.Greenlees, N.Amzal, J.E.Bastin, E.Bouchez, P.A.Butler, A.Chatillon, O.Dorvaux, S.Eeckhaudt, K.Eskola, B.Gall, J.Gerl, T.Grahn, A.Gorgen, N.J.Hammond, K.Hauschild, R.-D.Herzberg, F.-P.Hessberger, R.D.Humphreys, A.Hurstel, D.G.Jenkins, G.D.Jones, P.Jones, R.Julin, S.Juutinen, H.Kankaanpaa, A.Keenan, H.Kettunen, F.Khalfallah, T.L.Khoo, W.Korten, P.Kuusiniemi, Y.Le Coz, M.Leino, A.-P.Leppanen, M.Muikku, P.Nieminen, J.Pakarinen, P.Rahkila, P.Reiter, M.Rousseau, C.Scholey, Ch.Theisen, J.Uusitalo, J.Wilson, H.-J.Wollersheim - Eur.Phys.J. A 25, Supplement 1, 599 (2005)

In-beam and decay spectroscopy of transfermium elements 
2005GU25 F.Q.Guo, J.Powell, D.W.Lee, D.Leitner, M.A.McMahan, D.M.Moltz, J.P.O'Neil, K.Perajarvi, L.Phair, C.A.Ramsey, X.J.Xu, J.Cerny - Phys.Rev. C 72, 034312 (2005)

Reexamination of the energy levels of ${ }^{15} \mathrm{~F}$ by ${ }^{14} \mathrm{O}+{ }^{1} \mathrm{H}$ elastic resonance scattering

2005GU27 C.Guenaut, G.Audi, D.Beck, K.Blaum, G.Bollen, P.Delahaye, F.Herfurth, A.Kellerbauer, H.-J.Kluge, D.Lunney, S.Schwarz, L.Schweikhard, C.Yazidjian J.Phys.(London) G31, S1765 (2005)

Mass measurements of ${ }^{56-57} \mathrm{Cr}$ and the question of shell reincarnation at $\mathrm{N}=32$

2005GU28 G.Gurdal, H.Amro, C.W.Beausang, D.S.Brenner, M.P.Carpenter, R.F.Casten, C.Engelhardt, G.B.Hagemann, C.R.Hansen, D.J.Hartley, B.Herskind, H.Hubel, T.L.Khoo, T.Lauritsen, W.C.Ma, D.A.Meyer, E.F.Moore, A.Neusser, P.Bringel, D.G.Roux, G.Sletten, R.B.Yadav, Y.Zhang - J.Phys.(London) G31, S1873 (2005) Quadrupole moment measurements of TSD1 and TSD2 bands in ${ }^{167} \mathrm{Lu}$

2005 GU29 B.Guo, Z.H.Li, W.P.Liu, X.X.Bai, G.Lian, S.Q.Yan, B.X.Wang, S.Zeng, J.Su, Y.Lu - Nucl.Phys. A761, 162 (2005)

The ${ }^{8} \mathrm{Li}(\mathrm{d}, \mathrm{p}){ }^{9} \mathrm{Li}$ reaction and astrophysical ${ }^{8} \mathrm{~B}(\mathrm{p}, \gamma){ }^{9} \mathrm{C}$ reaction rate

2005GU32 P.Guazzoni, L.Zetta, B.F.Bayman, A.Covello, A.Gargano, G.Graw, R.Hertenberger, H.-F.Wirth, M.Jaskola - Phys.Rev. C 72, 044604 (2005)

${ }^{120} \mathrm{Sn}$ homologous levels via the ${ }^{123} \mathrm{Sb}(\mathrm{p}(\mathrm{pol}), \alpha){ }^{120} \mathrm{Sn}$ reaction: Experimental evidence and microscopic calculations

2005GU37 C.Guenaut, G.Audi, D.Beck, K.Blaum, G.Bollen, P.Delahaye, F.Herfurth, A.Kellerbauer, H.-J.Kluge, D.Lunney, S.Schwarz, L.Schweikhard, C.Yazidjian Eur.Phys.J. A 25, Supplement 1, 35 (2005)

Extending the mass "backbone" to short-lived nuclides with ISOLTRAP

2005GUZW P.Guazzoni, L.Zetta, F.Della Vedova, S.M.Lenzi, A.Vitturi, G.Graw, R.Hertenberger, H.-F.Wirth, M.Jaskola - Maier-Leibnitz-Laboratorium 2004 Ann.Rept., p.7 (2005)

The ${ }^{48} \mathrm{Sc}(\mathrm{p}(\mathrm{pol}), \alpha)^{42} \mathrm{Ca}$ Reaction and the Corresponding Homologous States

2005GUZX P.Guazzoni, S.Russo, M.Sassi, L.Zetta, F.Della Vedova, S.M.Lenzi, A.Vitturi, G.Graw, R.Hertenberger, H.-F.Wirth, M.Jaskola - Maier-Leibnitz-Laboratorium 2004 Ann.Rept., p.6 (2005) The ${ }^{44} \mathrm{Ca}(\mathrm{p}(\mathrm{pol}), \alpha)^{41} \mathrm{~K}$ Reaction

2005HA56 S.Harissopulos, A.Lagoyannis, A.Spyrou, Ch.Zarkadas, S.Galanopoulos, G.Perdikakis, H.-W.Becker, C.Rolfs, F.Strieder, R.Kunz, M.Fey, J.W.Hammer, A.Dewald, K.-O.Zell, P.von Brentano, R.Julin, P.Demetriou - J.Phys.(London) G31, S1417 (2005)

Proton and alpha-particle capture reactions at sub-Coulomb energies relevant to the p process 
2005HA57 B.Hadinia, B.Cederwall, J.Blomqvist, E.Ganioglu, P.T.Greenlees, K.Andgren, I.G.Darby, S.Eeckhaudt, E.Ideguchi, P.M.Jones, D.T.Joss, R.Julin, S.Juutinen, S.Ketelhut, K.Lagergren, A.-P.Leppanen, M.Leino, M.Nyman, J.Pakarinen, E.S.Paul, M.Petri, P.Rahkila, M.Sandzelius, J.Saren, C.Scholey, J.Uusitalo, R.Wadsworth, R.Wyss - Phys.Rev. C 72, 041303 (2005)

First identification of excited states in ${ }^{106} \mathrm{Te}$ and evidence for isoscalar-enhanced vibrational collectivity

2005HA60 T.Hayakawa, T.Shizuma, T.Kajino, S.Chiba, N.Shinohara, T.Nakagawa, T.Arima Astrophys.J. 628, 533 (2005)

New s-Process Path and Its Implications for a ${ }^{187}$ Re$^{187}$ Os

Nucleo-Cosmochronometer

2005HA64 M.Hatano, H.Sakai, T.Wakui, T.Uesaka, N.Aoi, Y.Ichikawa, T.Ikeda, K.Itoh, H.Iwasaki, T.Kawabata, H.Kuboki, Y.Maeda, N.Matsui, T.Ohnishi, T.K.Onishi, T.Saito, N.Sakamoto, M.Sasano, Y.Satou, K.Sekiguchi, K.Suda, A.Tamii, Y.Yanagisawa, K.Yako - Eur.Phys.J. A 25, Supplement 1, 255 (2005)

First experiment of ${ }^{6} \mathrm{He}$ with a polarized proton target

2005HE24 M.He, S.Jiang, Y.Nagashima, Y.Yang, T.Takahashi, K.Liu, K.Sasa, K.Dong, S.Wu, T.Matsuhiro, H.Tosaki, X.Ren, R.Seki, K.Sueki - Nucl.Instrum.Methods Phys.Res. B240, $612(2005)$

Measurement of the cross-section of ${ }^{14} \mathrm{~N}\left({ }^{16} \mathrm{O}, \alpha\right){ }^{26} \mathrm{Al}$ with AMS

2005HE26 F.Herfurth, G.Audi, D.Beck, K.Blaum, G.Bollen, P.Delahaye, S.George, C.Guenaut, A.Herlert, A.Kellerbauer, H.-J.Kluge, D.Lunney, M.Mukherjee, S.Rahaman, S.Schwarz, L.Schweikhard, C.Weber, C.Yazidjian - Eur.Phys.J. A 25, Supplement 1, 17 (2005)

Recent high-precision mass measurements with the Penning trap spectrometer ISOLTRAP

2005HE27 F.P.Hessberger, S.Antalic, B.Streicher, S.Hofmann, D.Ackermann, B.Kindler, I.Kojouharov, P.Kuusiniemi, M.Leino, B.Lommel, R.Mann, K.Nishio, S.Saro, B.Sulignano - Eur.Phys.J. A 26, 233 (2005)

Energy systematics of low-lying Nilsson levels in odd-mass einsteinium isotopes

2005HOZW M.J.Hornish, L.De Braeckeleer, A.S.Barabash, V.I.Umatov nucl-ex/0512030,12/20/2005 (2005)

Double Beta Decay of ${ }^{100}$ Mo to Excited Final States

2005HOZX S.Hofmann, D.Ackermann, S.Antalic, H.G.Burkhard, R.Dressler, F.P.Hessberger, B.Kindler, I.Kojouharov, P.Kuusiniemi, M.Leino, B.Lommel, R.Mann, G.Munzenberg, K.Nishio, A.G.Popeko, S.Saro, H.J.Schott, B.Streicher, B.Sulignano, J.Uusitalo, A.V.Yeremin - Priv.Comm. (2005)

Synthesis of SHE at ship

2005 HW06 J.K.Hwang, A.V.Ramayya, J.H.Hamilton, D.Fong, C.J.Beyer, K.Li, P.M.Gore, E.F.Jones, Y.X.Luo, J.O.Rasmussen, S.J.Zhu, S.C.Wu, I.Y.Lee, M.A.Stoyer, J.D.Cole, G.M.Ter-Akopian, A.Daniel, R.Donangelo - Eur.Phys.J. A 25, Supplement $1,463(2005)$ 
Half-life measurement of excited states in neutron-rich nuclei

2005HY04 B.Hyland, D.Melconian, G.C.Ball, J.R.Leslie, C.E.Svensson, P.Bricault, E.Cunningham, M.Dombsky, G.F.Grinyer, G.Hackman, K.Koopmans, F.Sarazin, M.A.Schumaker, H.C.Scraggs, M.B.Smith, P.M.Walker - J.Phys.(London) G31, S1885 (2005)

Precision half-life measurement of ${ }^{62} \mathrm{Ga}$

2005 ID03 E.Ideguchi, M.Niikura, C.Ishida, T.Fukuchi, H.Baba, N.Hokoiwa, H.Iwasaki, T.Koike, T.Komatsubara, T.Kubo, M.Kurokawa, S.Michimasa, K.Miyakawa, K.Morimoto, T.Ohnishi, S.Ota, A.Ozawa, S.Shimoura, T.Suda, M.Tamaki, I.Tanihata, Y.Wakabayashi, K.Yoshida, B.Cederwall - Eur.Phys.J. A 25, Supplement 1, 429 (2005)

Study of high-spin states in the ${ }^{48} \mathrm{Ca}$ region by using secondary fusion reactions

2005IL03 C.Iliadis, A.Champagne, J.Jose - J.Phys.(London) G31, S1785 (2005)

Explosive hydrogen burning of ${ }^{23} \mathrm{Na}$ in classical novae

2005IM02 G.Imbriani, H.Costantini, A.Formicola, A.Vomiero, C.Angulo, D.Bemmerer, R.Bonetti, C.Broggini, F.Confortola, P.Corvisiero, J.Cruz, P.Descouvemont, Z.Fulop, G.Gervino, A.Guglielmetti, C.Gustavino, Gy.Gyurky, A.P.Jesus, M.Junker, J.N.Klug, A.Lemut, R.Menegazzo, P.Prati, V.Roca, C.Rolfs, M.Romano, C.Rossi-Alvarez, F.Schumann, D.Schurmann, E.Somorjai, O.Straniero, F.Strieder, F.Terrasi, H.P.Trautvetter - Eur.Phys.J. A 25, 455 (2005)

S-factor of ${ }^{14} \mathrm{~N}(\mathrm{p}, \gamma){ }^{15} \mathrm{O}$ at astrophysical energies

20051002 M.Ionescu-Bujor, A.Iordachescu, D.Bucurescu, N.Marginean - Phys.Rev. C 72, $044313(2005)$

New short-lived isomers in ${ }^{84} \mathrm{Y}$

2005IW03 H.Iwasaki, N.Aoi, S.Takeuchi, S.Ota, H.Sakurai, M.Tamaki, T.K.Onishi, E.Takeshita, H.J.Ong, N.Iwasa, H.Baba, Z.Elekes, T.Fukuchi, Y.Ichikawa, M.Ishihara, S.Kanno, R.Kanungo, S.Kawai, T.Kubo, K.Kurita, S.Michimasa, M.Niikura, A.Saito, Y.Satou, S.Shimoura, H.Suzuki, M.K.Suzuki, Y.Togano, Y.Yanagisawa, T.Motobayashi - Eur.Phys.J. A 25, Supplement 1, 415 (2005) Intermediate-energy Coulomb excitation of the neutron-rich Ge isotopes around $\mathrm{N}=$ 50

2005JA17 Th.Jagemann, J.Jochum, F.V.Feilitzsch - Nucl.Instrum.Methods Phys.Res. A551, $245(2005)$

Neutron scattering facility for the measurement of nuclear recoil quenching factors

2005JE07 D.G.Jenkins, C.J.Lister, M.P.Carpenter, P.Chowdhury, N.J.Hammond, R.V.F.Janssens, T.L.Khoo, T.Lauritsen, D.Seweryniak, T.Davinson, P.J.Woods, A.Jokinen, H.Penttila - Phys.Rev. C 72, 031303 (2005)

Mirror energy differences in the $\mathrm{A}=31$ mirror nuclei, ${ }^{31} \mathrm{~S}$ and ${ }^{31} \mathrm{P}$, and their significance in electromagnetic spin-orbit splitting 
2005J018 D.T.Joss, J.Simpson, D.E.Appelbe, K.Lagergren, C.J.Barton, B.Cederwall, S.Eeckhaudt, T.Grahn, P.M.Jones, R.Julin, S.Juutinen, B.Hadinia, H.Kettunen, M.Leino, A.-P.Leppanen, P.Nieminen, R.D.Page, J.Pakarinen, E.S.Paul, J.Perkowski, P.Rahkila, M.A.Riley, C.Scholey, J.Uusitalo, K.Van de Vel, D.D.Warner, D.R.Wiseman - J.Phys.(London) G31, S1715 (2005)

Yrast structures in the light Pt isotopes ${ }^{169-173} \mathrm{Pt}$

2005J019 G.A.Jones, Zs.Podolyak, P.M.Walker, P.H.Regan, G.de Angelis, M.Axiotis, D.Bazzacco, P.G.Bizzeti, F.Brandolini, R.Broda, D.Bucurescu, E.Farnea, W.Gelletly, A.Gadea, M.Ionescu-Bujor, A.Iordachescu, Th.Kroll, S.D.Langdown, S.Lunardi, N.Marginean, T.Martinez, N.H.Medina, B.Quintana, B.Rubio, C.A.Ur, J.J.Valiente-Dobon, S.J.Williams, Y.H.Zhang - J.Phys.(London) G31, S1891 (2005) Population of yrast states in ${ }^{191}$ Os using deep-inelastic reactions

2005J020 P.Joshi, S.Finnigan, D.B.Fossan, T.Koike, E.S.Paul, G.Rainovski, K.Starosta, C.Vaman, R.Wadsworth - J.Phys.(London) G31, S1895 (2005)

Evidence for a new region of chirality around $\mathrm{A} \sim 104$

2005J021 K.Joo, for the CLAS Collaboration - Phys.Rev. C 72, 058202 (2005)

Measurement of the polarized structure function $\sigma_{L T^{\prime}}$ for pion electroproduction in the Roper-resonance region

2005J022 A.Jokinen, T.Eronen, U.Hager, J.Hakala, S.Kopecky, A.Nieminen, S.Rinta-Antila, J.Aysto - Eur.Phys.J. A 25, Supplement 1, 27 (2005)

Ion manipulation and precision measurements at JYFLTRAP

2005J023 K.L.Jones, C.Baktash, D.W.Bardayan, J.C.Blackmon, W.N.Catford, J.A.Cizewski, R.P.Fitzgerald, U.Greife, M.S.Johnson, R.L.Kozub, R.J.Livesay, Z.Ma, C.D.Nesaraja, D.Shapira, M.S.Smith, J.S.Thomas, D.Visser - Eur.Phys.J. A 25, Supplement 1, 283 (2005)

Developing techniques to study $\mathrm{A} \sim 132$ nuclei with $(\mathrm{d}, \mathrm{p})$ reactions in inverse kinematics

2005J024 E.F.Jones, J.H.Hamilton, P.M.Gore, A.V.Ramayya, J.K.Hwang, A.P.deLima Eur.Phys.J. A 25, Supplement 1, 467 (2005)

Identification of levels in ${ }^{162,164} \mathrm{Gd}$ and decrease in moment of inertia between $\mathrm{N}=$ 98-100

2005JU12 D.S.Judson, A.M.Bruce, M.J.Taylor, G.D.Dracoulis, T.Kibedi, A.P.Byrne, K.H.Maier, P.Nieminen, J.N.Orce - J.Phys.(London) G31, S1899 (2005)

Microsecond isomers in ${ }^{125} \mathrm{Sb}$

2005KA39 A.Kankainen, G.K.Vorobjev, S.A.Eliseev, W.Huang, J.Huikari, A.Jokinen, A.Nieminen, Yu.N.Novikov, H.Penttila, A.V.Popov, S.Rinta-Antila, H.Schatz, D.M.Seliverstov, Yu.P.Suslov, J.Aysto - Eur.Phys.J. A 25, 355 (2005) Isomers of astrophysical interest in neutron-deficient nuclei at masses $\mathrm{A}=81,85$ and 86 
2005KA45 T.Kautzsch, A.Wohr, W.B.Walters, K.-L.Kratz, B.Pfeiffer, M.Hannawald, J.Shergur, O.Arndt, S.Hennrich, S.Falahat, T.Griesel, O.Keller, A.Aprahamian, B.A.Brown, P.F.Mantica, M.A.Stoyer, H.L.Ravn, for the ISOLDE IS333 and Rochester CHICO / Gammasphere Collaborations - Eur.Phys.J. A 25, Supplement 1,117 (2005)

Structure of neutron-rich even-even ${ }^{124,126} \mathrm{Cd}$

2005KA46 A.Kankainen, S.A.Eliseev, T.Eronen, S.P.Fox, U.Hager, J.Hakala, W.Huang, J.Huikari, D.Jenkins, A.Jokinen, S.Kopecky, I.Moore, A.Nieminen, Yu.N.Novikov, H.Penttila, A.V.Popov, S.Rinta-Antila, H.Schatz, D.M.Seliverstov, G.K.Vorobjev, Y.Wang, J.Aysto, and the IS403 Collaboration - Eur.Phys.J. A 25, Supplement 1, $129(2005)$

Beta-delayed gamma and proton spectroscopy near the $\mathrm{Z}=\mathrm{N}$ line

2005KA47 M.Karny, L.Batist, A.Banu, F.Becker, A.Blazhev, K.Burkard, W.Bruchle, J.Doring, T.Faestermann, M.Gorska, H.Grawe, Z.Janas, A.Jungclaus, M.Kavatsyuk, O.Kavatsyuk, R.Kirchner, M.La Commara, S.Mandal, C.Mazzocchi, K.Miernik, I.Mukha, S.Muralithar, C.Plettner, A.Plochocki, E.Roeckl, M.Romoli, K.Rykaczewski, M.Schadel, K.Schmidt, R.Schwengner, J.Zylicz - Eur.Phys.J. A 25, Supplement 1, 135 (2005)

Beta-decay studies near ${ }^{100} \mathrm{Sn}$

2005KA48 M.Kavatsyuk, O.Kavatsyuk, L.Batist, A.Banu, F.Becker, A.Blazhev, W.Bruchle, K.Burkard, J.Doring, T.Faestermann, M.Gorska, H.Grawe, Z.Janas, A.Jungclaus, M.Karny, R.Kirchner, M.La Commara, S.Mandal, C.Mazzocchi, I.Mukha, S.Muralithar, C.Plettner, A.Plochocki, E.Roeckl, M.Romoli, M.Schadel, R.Schwengner, J.Zylicz - Eur.Phys.J. A 25, Supplement 1, 139 (2005) Beta-decay spectroscopy of ${ }^{103,105} \mathrm{Sn}$

2005KA51 R.Kanungo, M.Chiba, B.Abu-Ibrahim, S.Adhikari, D.Q.Fang, N.Iwasa, K.Kimura, K.Maeda, S.Nishimura, T.Ohnishi, A.Ozawa, C.Samanta, T.Suda, T.Suzuki, Q.Wang, C.Wu, Y.Yamaguchi, K.Yamada, A.Yoshida, T.Zheng, I.Tanihata Eur.Phys.J. A 25, Supplement 1, 327 (2005)

Observation of a two-proton halo in ${ }^{17} \mathrm{Ne}$

2005KA52 S.A.Karamian - Yad.Fiz. 68, 1827 (2005); Phys.Atomic Nuclei 68, 1765 (2005) Comparative Analysis of the ${ }^{178 m 2} \mathrm{Hf}$ Yield at Reactions with Different Projectiles

2005KAZU T.Kawabata, H.Akimune, H.Fujita, Y.Fujita, M.Fujiwara, K.Hara, K.Hatanaka, M.Itoh, Y.Kanada-En'yo, S.Kishi, K.Nakanishi, H.Sakaguchi, Y.Shimbara, A.Tamii, S.Terashima, M.Uchida, T.Wakasa, Y.Yasuda, H.P.Yoshida, M.Yosoi nucl-ex/0512040,12/25/2005 (2005)

Indication of dilute $2 \alpha+\mathrm{t}$ cluster structure in ${ }^{11} \mathrm{~B}$

2005KE07 K.C.Kelley, N.E.Hertel, E.J.Pitcher, M.Devlin, S.G.Mashnik - Nucl.Phys. A760, 225 (2005)

${ }^{148} \mathrm{Gd}$ production cross section measurements for $600-$ and $800-\mathrm{MeV}$ protons on tantalum, tungsten, and gold 
2005KE08 K.L.Keyes, A.Papenberg, R.Chapman, J.Ollier, X.Liang, M.J.Burns, M.Labiche, K.M.Spohr, N.Amzal, C.Beck, P.Bednarczyk, F.Haas, G.Duchene, P.Papka, B.Gebauer, T.Kokalova, S.Thummerer, W.von Oertzen, C.Wheldon J.Phys.(London) G31, S1903 (2005)

Spectroscopy of $\mathrm{Ne}, \mathrm{Na}$ and $\mathrm{Mg}$ isotopes approaching the Island of Inversion

2005KE10

2005KE11

2005KI17

2005KI19

2005KI20

2005KI21
H.Kettunen, T.Enqvist, K.Eskola, T.Grahn, P.T.Greenlees, K.Helariutta, P.Jones, R.Julin, S.Juutinen, H.Kankaanpaa, A.Keenan, H.Koivisto, P.Kuusiniemi, M.Leino, A.-P.Leppanen, M.Muikku, P.Nieminen, J.Pakarinen, P.Rahkila, J.Uusitalo -

Eur.Phys.J. A 25, Supplement 1, 181 (2005)

Decay studies of neutron-deficient odd-mass At and Bi isotopes

K.L.Keyes, A.Papenberg, R.Chapman, J.Ollier, X.Liang, M.J.Burns, M.Labiche, K.-M.Spohr, N.Amzal, C.Beck, P.Bednarczyk, F.Haas, G.Duchene, P.Papka, B.Gebauer, T.Kokalova, S.Thummerer, W.von Oertzen, C.Wheldon - Eur.Phys.J. A 25, Supplement 1, 431 (2005)

Spectroscopy of Ne and Na isotopes: Preliminary results from a EUROBALL + Binary Reaction Spectrometer experiment

R.U.Khafizov, N.Severijns, O.Zimmer, H.-F.Wirth, D.Rich, S.V.Tolokonnikov, V.A.Solovei, M.R.Kolhidashvili - nucl-ex/0512001,12/1/2005 (2005)

Discovery of the neutron radiative decay

T.Kii, T.Shima, T.Baba, Y.Nagai - Nucl.Instrum.Methods Phys.Res. A552, 329 (2005)

A time projection chamber for the study of nuclear photodisintegration

St.Kistryn, E.Stephan, A.Biegun, K.Bodek, A.Deltuva, E.Epelbaum, K.Ermisch, W.Glockle, J.Golak, N.Kalantar-Nayestanaki, H.Kamada, M.Kis, B.Klos, A.Kozela, J.Kuros-Zolnierczuk, M.Mahjour-Shafiei, U.-G.Meissner, A.Micherdzinska, A.Nogga, P.U.Sauer, R.Skibinski, R.Sworst, H.Witala, J.Zejma, W.Zipper - Phys.Rev. C 72, 044006 (2005)

Systematic study of three-nucleon force effects in the cross section of the deuteron-proton breakup at $130 \mathrm{MeV}$

E.R.Kinney, J.L.Matthews, P.A.M.Gram, D.W.MacArthur, E.Piasetzky, G.A.Rebka, Jr., D.A.Roberts - Phys.Rev. C 72, 044608 (2005)

Inclusive pion double charge exchange in ${ }^{4} \mathrm{He}$ at intermediate energies

O.A.Kiselev, F.Aksouh, A.Bleile, O.V.Bochkarev, L.V.Chulkov, D.Cortina-Gil, A.V.Dobrovolsky, P.Egelhof, H.Geissel, M.Hellstrom, N.B.Isaev, B.G.Komkov, M.Matos, F.V.Moroz, G.Munzenberg, M.Mutterer, V.A.Mylnikov, S.R.Neumaier, V.N.Pribora, D.M.Seliverstov, L.O.Sergueev, A.Shrivastava, K.Summerer, H.Weick, M.Winkler, V.I.Yatsoura - Eur.Phys.J. A 25, Supplement 1, 215 (2005)

Investigation of nuclear matter distribution of the neutron-rich He isotopes by proton elastic scattering at intermediate energies 
2005KN02 H.H.Knudsen, H.O.U.Fynbo, M.J.G.Borge, R.Boutami, P.Dendooven, C.Aa.Diget, T.Eronen, S.Fox, L.M.Fraile, B.Fulton, J.Huikary, H.B.Jeppesen, A.S.Jokinen, B.Jonson, A.Kankainen, I.Moore, A.Nieminen, G.Nyman, H.Penttila, K.Riisager, S.Rinta-Antila, O.Tengblad, Y.Wang, K.Wilhelmsen, J.Aysto - Phys.Rev. C 72, $044312(2005)$

$\beta$-decay of ${ }^{13} \mathrm{O}$

2005K032 C.Kohstall, D.Belic, P.von Brentano, C.Fransen, A.Gade, R.-D.Herzberg, J.Jolie, U.Kneissl, A.Linnemann, A.Nord, N.Pietralla, H.H.Pitz, M.Scheck, F.Stedile, V.Werner, S.W.Yates - Phys.Rev. C 72, 034302 (2005)

Low-lying dipole excitations in vibrational nuclei: The Cd isotopic chain studied in photon scattering experiments

2005K040 A.Korgul, H.Mach, B.A.Brown, A.Covello, A.Gargano, B.Fogelberg, R.Schuber, W.Kurcewicz, E.Werner-Malento, R.Orlandi, M.Sawicka - Eur.Phys.J. A 25, Supplement 1, $123(2005)$

On the structure of the anomalously low-lying $5 / 2^{+}$state of ${ }^{135} \mathrm{Sb}$

2005K041 M.Kowalska, D.Yordanov, K.Blaum, D.Borremans, P.Himpe, P.Lievens, S.Mallion, R.Neugart, G.Neyens, N.Vermeulen - Eur.Phys.J. A 25, Supplement 1, 193 (2005)

Laser and $\beta$-NMR spectroscopy on neutron-rich magnesium isotopes

2005K043 U.Koster, O.Arndt, U.C.Bergmann, R.Catherall, J.Cederkall, I.Dillmann, M.Dubois, F.Durantel, L.Fraile, S.Franchoo, G.Gaubert, L.Gaudefroy, O.Hallmann, C.Huet-Equilbec, B.Jacquot, P.Jardin, K.L.Kratz, N.Lecesne, R.Leroy, A.Lopez, L.Maunoury, J.Y.Pacquet, B.Pfeiffer, M.G.Saint-Laurent, C.Stodel, A.C.C.Villari, L.Weissman - Eur.Phys.J. A 25, Supplement 1, 729 (2005)

ISOL beams of neutron-rich oxygen isotopes

2005KR14 K.Kramer, D.S.Armstrong, T.D.Averett, W.Bertozzi, S.Binet, C.Butuceanu, A.Camsonne, G.D.Cates, J.-P.Chen, S.Choi, E.Chudakov, F.Cusanno, A.Deur, P.Djawotho, D.Dutta, J.M.Finn, H.Gao, F.Garibaldi, O.Gayou, R.Gilman, A.Glamazdin, V.Gorbenko, K.A.Griffioen, J.-O.Hansen, D.W.Higinbotham, W.Hinton, T.Horn, C.W.de Jager, X.Jiang, W.Korsch, J.LeRose, D.Lhuillier, N.Liyanage, D.J.Margaziotis, K.McCormick, Z.-E.Meziani, R.Michaels, B.Milbrath, B.Moffit, S.Nanda, C.F.Perdrisat, R.Pomatsalyuk, V.Punjabi, B.Reitz, J.Roche, R.Roche, M.Roedelbronn, N.Savvinov, J.Secrest, J.Singh, S.Sirca, K.Slifer, P.Solvignon, D.J.Steiner, R.Suleiman, V.Sulkosky, A.Tobias, A.Vacheret, Y.Xiao, X.Zheng, J.Zhou, L.Zhu, X.Zhu, P.A.Zolnierczuk - Phys.Rev.Lett. 95, 142002 (2005) $\mathrm{Q}^{2}$ Dependence of the Neutron Spin Structure Function $g_{2}^{n}$ at Low $\mathrm{Q}^{2}$

2005KR15 A.Krishna, K.Pandey, A.Wasan, V.Natarajan - Europhys.Lett. 72, 221 (2005) High-resolution hyperfine spectroscopy of excited states using electromagnetically induced transparency

2005KU28 A.Kumar, J.N.Orce, S.R.Lesher, C.J.McKay, M.T.McEllistrem, S.W.Yates Phys.Rev. C 72, 034313 (2005)

Heterogeneous vibrations in ${ }^{112} \mathrm{Sn}$ 
2005KU31 P.Kuusiniemi, F.P.Hessberger, D.Ackermann, S.Hofmann, B.Sulignano, I.Kojouharov, R.Mann - Eur.Phys.J. A 25, 397 (2005)

Decay studies of ${ }^{215-217}$ Th using ER- $\gamma-\alpha-\gamma$ coincidences

2005KU34 R.Kumar, A.Kumar, S.K.Chamoli, K.Singh, M.Sharma, D.Mehta, N.Singh, S.S.Ghugre, N.S.Pattabiraman, L.Chaturvedi, P.K.Joshi, H.C.Jain, Z.Naik, C.R.Praharaj, I.M.Govil - Phys.Rev. C 72, 044319 (2005)

Polarization measurement and $\gamma$-ray spectroscopy of ${ }^{122} \mathrm{Cs}$

2005KU36 S.Kumar, S.V.Kumar, G.L.N.Reddy, V.Kain, J.V.Ramana, V.S.Raju -

Nucl.Instrum.Methods Phys.Res. B240, 704 (2005)

Depth profiling of nitrogen using $429 \mathrm{keV}$ and $897 \mathrm{keV}$ resonances in the ${ }^{15} \mathrm{~N}(\mathrm{p}$, $\alpha \gamma)^{12} \mathrm{C}$ reaction

2005KU37 A.Kumar, J.N.Orce, S.R.Lesher, C.J.McKay, M.T.McEllistrem, S.W.Yates Eur.Phys.J. A 25, Supplement 1, 443 (2005)

Lifetime measurements and low-lying structure in ${ }^{112} \mathrm{Sn}$

2005LA28 M.Labiche, C.N.Timis, R.C.Lemmon, W.N.Catford, R.Chapman, N.Amzal, N.I.Ashwood, T.D.Baldwin, M.Burns, L.Caballero, M.Chartier, N.Curtis, G.de France, W.Gelletly, X.Liang, M.Freer, N.A.Orr, S.D.Pain, V.P.E.Pucknell, M.Rejmund, B.Rubio, H.Savajols, O.Sorlin, K.Spohr, Ch.Theisen, D.D.Warner J.Phys.(London) G31, S1691 (2005)

Study of transfer reactions in inverse kinematics with the TIARA array

2005LA29 S.Lakshmi, H.C.Jain, P.K.Joshi, I.Mazumdar, R.Palit, A.K.Jain, S.S.Malik Nucl.Phys. A761, 1 (2005)

High spin structure of ${ }^{136} \mathrm{Ce}$

2005LA30 V.P.Ladygin, L.S.Azhgirey, S.V.Afanasiev, V.V.Arkhipov, V.K.Bondarev, Yu.T.Borzunov, G.Filipov, L.B.Golovanov, A.Yu.Isupov, V.I.Ivanov, A.A.Kartamyshev, V.A.Kashirin, A.N.Khrenov, V.I.Kolesnikov, V.A.Kuznetsov, A.G.Litvinenko, S.G.Reznikov, P.A.Rukoyatkin, A.Yu.Semenov, I.A.Semenova, G.D.Stoletov, A.P.Tzvinev, N.P.Yudin, V.N.Zhmyrov, L.S.Zolin - Phys.Lett. B 629, $60(2005)$

Tensor analyzing power $\mathrm{A}_{y y}$ in deuteron inclusive breakup on hydrogen and carbon at $9 \mathrm{GeV} / \mathrm{c}$ and large proton transverse momenta

2005LA32 K.Lagergren, M.A.Riley, J.Simpson, D.E.Appelbe, P.Bednarczyk, D.B.Campbell, C.Chandler, P.T.W.Choy, D.Curien, D.T.Joss, E.S.Paul - Phys.Rev. C 72, 057303 (2005)

Observation of the second proton alignment in ${ }^{160} \mathrm{Tm}$

2005LE34 F.Le Blanc, L.Cabaret, E.Cottereau, J.E.Crawford, S.Essabaa, J.Genevey, R.Horn, G.Huber, J.Lassen, J.K.P.Lee, G.Le Scornet, J.Lettry, J.Obert, J.Oms, A.Ouchrif, J.Pinard, H.Ravn, B.Roussiere, J.Sauvage, D.Verney - Phys.Rev. C 72, 034305 (2005)

Charge-radius change and nuclear moments in the heavy tin isotopes from laser spectroscopy: Charge radius of ${ }^{132} \mathrm{Sn}$ 
2005LE35 S.Leoni, G.Benzoni, A.Bracco, N.Blasi, F.Camera, C.Grassi, P.Mason, B.Million, A.Paleni, M.Pignanelli, E.Vigezzi, O.Wieland, M.Matsuo, T.Dossing, B.Herskind, G.B.Hagemann, J.Wilson, A.Maj, M.Kmiecik, G.Lo Bianco, C.M.Petrache, M.Castoldi, A.Zucchiatti, G.de Angelis, D.R.Napoli, P.Bednarczyk, D.Curien Phys.Rev. C 72, 034307 (2005)

Damping mechanisms and order-to-chaos transition in the warm rotating ${ }^{163} \mathrm{Er}$ nucleus

2005LE38 J.Leske, K.-H.Speidel, S.Schielke, J.Gerber, P.Maier-Komor, T.Engeland, M.Hjorth-Jensen - Phys.Rev. C 72, 044301 (2005)

Dominant $\left(\mathrm{g}_{9 / 2}\right)^{2}$ neutron configuration in the $4_{1}^{+}$state of ${ }^{68} \mathrm{Zn}$ based on new $\mathrm{g}$ factor measurements

2005LE42 A.-P.Leppanen, J.Uusitalo, S.Eeckhaudt, T.Enqvist, K.Eskola, T.Grahn, F.P.Hessberger, P.T.Greenlees, P.Jones, R.Julin, S.Juutinen, H.Kettunen, P.Kuusiniemi, M.Leino, P.Nieminen, J.Pakarinen, J.Perkowski, P.Rahkila, C.Scholey, G.Sletten - Eur.Phys.J. A 25, Supplement 1, 183 (2005)

Alpha-decay study of ${ }^{218} \mathrm{U}$; a search for the sub-shell closure at $\mathrm{Z}=92$

2005LH01 G.Lhersonneau, S.Brant - Phys.Rev. C 72, 034308 (2005)

Levels in ${ }^{99} \mathrm{Zr}$ observed in the decay of ${ }^{99} \mathrm{Y}$

2005LI47 Z.Liu, P.J.Woods, K.Schmidt, H.Mahmud, P.S.L.Munro, A.Blazhev, J.Doring, H.Grawe, M.Hellstrom, R.Kirchner, Z.K.Li, C.Mazzocchi, I.Mukha, C.Plettner, E.Roeckl, M.La Commara - Phys.Rev. C 72, 047301 (2005)

Reinvestigation of direct proton decay of ${ }^{105} \mathrm{Sb}$

2005LI53 S.N.Liddick, P.F.Mantica, R.Broda, B.A.Brown, M.P.Carpenter, A.D.Davies, B.Fornal, M.Horoi, R.V.F.Janssens, A.C.Morton, W.F.Mueller, J.Pavan, H.Schatz, A.Stolz, S.L.Tabor, B.E.Tomlin, M.Wiedeking - Phys.Rev. C 72, 054321 (2005) $\beta$-decay of odd-A ${ }^{57} \mathrm{Ti}$ and ${ }^{59} \mathrm{~V}$

2005LU21 Y.X.Luo, J.O.Rasmussen, I.Stefanescu, A.Gelberg, J.H.Hamilton, A.V.Ramayya, J.K.Hwang, S.J.Zhu, P.M.Gore, D.Fong, E.F.Jones, S.C.Wu, I.Y.Lee, T.N.Ginter, W.C.Ma, G.M.Ter-Akopian, A.V.Daniel, M.A.Stoyer, R.Donangelo J.Phys.(London) G31, 1303 (2005)

Shape trends and triaxiality in neutron-rich odd-mass $\mathrm{Y}$ and $\mathrm{Nb}$ isotopes

2005LU24 Y.X.Luo, J.O.Rasmussen, J.H.Hamilton, A.V.Ramayya, A.Gelberg, I.Stefanescu, J.K.Hwang, S.J.Zhu, P.M.Gore, D.Fong, E.F.Jones, S.C.Wu, I.Y.Lee, T.N.Ginter, W.C.Ma, G.M.Ter-Akopian, A.V.Daniel, M.A.Stoyer, R.Donangelo - Eur.Phys.J. A 25, Supplement 1, 469 (2005)

Shape transitions and triaxiality in neutron-rich odd-mass $\mathrm{Y}$ and $\mathrm{Nb}$ isotopes

2005MA81 H.Mach, P.M.Walker, R.Julin, M.Leino, S.Juutinen, M.Stanoiu, Zs.Podolyak, R.Wood, A.M.Bruce, T.Back, J.A.Cameron, B.Cederwall, J.Ekman, B.Fogelberg, P.T.Greenlees, M.Hellstrom, P.Jones, W.Klamra, K.Lagergren, A.-P.Leppanen, P.Nieminen, R.Orlandi, J.Pakarinen, P.Rahkila, D.Rudolph, G.Simpson, J.Uusitalo, C.Wheldon - J.Phys.(London) G31, S1421 (2005) 
Application of ultra-fast timing techniques to the study of exotic and weakly produced nuclei

2005MA84 P.Mason, G.Benzoni, A.Bracco, F.Camera, B.Million, O.Wieland, S.Leoni, A.K.Singh, A.Al-Khatib, H.Hubel, P.Bringel, A.Burger, A.Neusser, G.Schonwasser, G.B.Hagemann, C.R.Hansen, B.Herskind, G.Sletten, A.Algora, Zs.Dombradi, J.Gal, G.Kalinka, J.Molnar, B.M.Nyako, D.Sohler, J.Timar, L.Zolnai, M.Kmiecik, A.Maj, J.Styczen, K.Zuber, F.Azaiez, K.Hauschild, A.Korichi, A.Lopez-Martens, J.Roccaz, S.Siem, F.Hannachi, J.N.Scheurer, P.Bednarczyk, Th.Byrski, D.Curien, O.Dorvaux, G.Duchene, B.Gall, F.Khalfallah, I.Piqueras, J.Robin, K.Juhasz, S.B.Patel, A.O.Evans, G.Rainovski, C.M.Petrache, D.Petrache, G.La Rana, R.Moro, G.De Angelis, P.Fallon, I.-Y.Lee, J.C.Lisle, B.Cederwall, K.Lagergren, R.M.Lieder, E.Podsvirova, W.Gast, H.Jager, N.Redon, A.Gorgen - J.Phys.(London) G31, S1729 (2005)

Octupole signatures in ${ }^{124,125} \mathrm{Ba}$

2005MA86 F.Marechal, D.L.Balabanski, D.Borremans, J.-M.Daugas, F.de Oliveira Santos, P.Dessagne, G.Georgiev, J.Giovinazzo, S.Grevy, P.Himpe, C.Jollet, I.Matea, G.Neyens, F.Perrot, E.Poirier, O.Roig, M.Stanoiu, C.Stodel, J.-C.Thomas, K.Turzo, D.Yordanov, E.Caurier, F.Nowacki, A.Poves - Phys.Rev. C 72, 044314 (2005) $\beta$ decay of ${ }^{31} \mathrm{Mg}$ : Extending the "island of inversion"

2005MA90 N.L.Maidana, M.N.Takeda, M.S.Dias, M.F.Koskinas, V.R.Vanin Nucl.Instrum.Methods Phys.Res. A553, 559 (2005)

Absolute measurement of ${ }^{242 g} \mathrm{Am}$ sources activities in the ${ }^{241} \mathrm{Am}(\mathrm{n}, \gamma)$ cross-section determination-Improvement by simulation

2005MA92 N.W.Makau, T.E.Derry - Nucl.Instrum.Methods Phys.Res. A555, 31 (2005) A new way of depositing thin targets of monolayer thickness for nuclear reaction analysis: A case of the $550 \mathrm{keV}{ }^{13} \mathrm{C}(\mathrm{p}, \gamma){ }^{14} \mathrm{~N}$ resonant nuclear reaction

2005MA93 P.F.Mantica, S.N.Liddick, B.E.Tomlin - Nucl.Instrum.Methods Phys.Res. B241, 195 (2005)

Beta-decay studies of neutron-rich nuclei produced using projectile fragmentation at the NSCL

2005MA95 C.Mazzocchi, R.Grzywacz, J.C.Batchelder, C.R.Bingham, D.Fong, J.H.Hamilton, J.K.Hwang, M.Karny, W.Krolas, S.N.Liddick, A.C.Morton, P.F.Mantica, W.F.Mueller, K.P.Rykaczewski, M.Steiner, A.Stolz, J.A.Winger - Eur.Phys.J. A 25, Supplement 1, 93 (2005)

Beta-delayed $\gamma$ and neutron emission near the double shell closure at ${ }^{78} \mathrm{Ni}$

2005 MA96 H.Mach, L.M.Fraile, O.Tengblad, R.Boutami, C.Jollet, W.A.Plociennik, D.T.Yordanov, M.Stanoiu, M.J.G.Borge, P.A.Butler, J.Cederkall, Ph.Dessagne, B.Fogelberg, H.Fynbo, P.Hoff, A.Jokinen, A.Korgul, U.Koster, W.Kurcewicz, F.Marechal, T.Motobayashi, J.Mrazek, G.Neyens, T.Nilsson, S.Pedersen, A.Poves, B.Rubio, E.Ruchowska, and the ISOLDE Collaboration - Eur.Phys.J. A 25, Supplement 1, 105 (2005)

New structure information on ${ }^{30} \mathrm{Mg},{ }^{31} \mathrm{Mg}$ and ${ }^{32} \mathrm{Mg}$ 
2005MAZL M.Mahgoub, A.Bergmaier, D.Bucurescu, T.Behrens, G.Dollinger, T.Faestermann, R.Gernhauser, R.Hertenberger, Th.Kroll, R.Krucken, P.Maierbeck, F.Nebel, M.Schlarb, H.-F.Wirth - Maier-Leibnitz-Laboratorium 2004 Ann.Rept., p.9 (2005) One and two Neutron Transfer Reactions in Normal and Inverse Kinematics

2005MAZM P.Maierbeck, T.Behrens, A.Bergmaier, M.Bohmer, T.Faestermann, R.Gernhauser, R.Hertenberger, Th.Kroll, R.Krucken, R.Lutter, M.Mahgoub, L.Maier,

S.Nimmrichter, B.Sailer, M.Schlarb, H.-F.Wirth, T.Nilsson, M.Pantea, A.Richter, G.Schrieder, S.Volz, N.Braun, B.Bruyneel, T.Striepling, P.Reiter, A.Wiens, N.Warr, and the MINIBALL Collaboration - Maier-Leibnitz-Laboratorium 2004 Ann.Rept., p.8 (2005)

Study of ${ }^{49} \mathrm{Ca}$ in One-Neutron Transfer Reactions

2005MC12 P.McEwan, M.Freer, N.Ashwood, N.Curtis, D.Price, V.Ziman, H.G.Bohlen, Tz.Kokalova, Ch.Schulz, R.Torabi, W.von Oertzen, C.Wheldon, T.N.Massey, R.Kalpakchieva, W.Catford - J.Phys.(London) G31, S1921 (2005)

Investigation into the structure of ${ }^{14} \mathrm{C}$ using recoil co-incidence techniques

2005MC13 C.J.McKay, J.N.Orce, S.R.Lesher, D.Bandyopadhyay, M.T.McEllistrem, C.Fransen, J.Jolie, A.Linnemann, N.Pietralla, V.Werner, S.W.Yates - Eur.Phys.J. A 25,

Supplement 1, 377 (2005); Eur.Phys.J. A 25, Supplement 1, 773 (2005)

Identification of mixed-symmetry states in odd-A ${ }^{93} \mathrm{Nb}$

2005ME19 D.A.Meyer, G.Graw, R.Hertenberger, H.-F.Wirth, R.F.Casten, P.von Brentano, D.Bucurescu, S.Heinze, J.L.Jerke, J.Jolie, R.Krucken, M.Mahgoub, P.Pejovic, O.Moller, D.Mucher, C.Scholl - J.Phys.(London) G31, S1399 (2005)

Systematic exploration of $0^{+}$states in structurally diverse nuclei

2005MI28 T.Mitsui, R.Masuda, S.Kitao, M.Seto - J.Phys.Soc.Jpn. 74, 3122 (2005)

Nuclear Resonant Scattering of Synchrotron Radiation by ${ }^{158} \mathrm{Gd}$

2005MI32 S.Michimasa, S.Shimoura, H.Iwasaki, M.Tamaki, S.Ota, N.Aoi, H.Baba, N.Iwasa, S.Kanno, S.Kubono, K.Kurita, M.Kurokawa, T.Minemura, T.Motobayashi, M.Notani, H.J.Ong, A.Saito, H.Sakurai, S.Takeuchi, E.Takeshita, Y.Yanagisawa, A.Yoshida - Eur.Phys.J. A 25, Supplement 1, 367 (2005)

Study of single-particle states in ${ }^{23} \mathrm{~F}$ using proton transfer reaction

2005MIZT S.Michimasa, S.Shimoura, H.Iwasaki, M.Tamaki, S.Ota, N.Aoi, H.Baba, N.Iwasa, S.Kanno, S.Kubono, K.Kurita, M.Kurokawa, T.Minemura, T.Motobayashi, M.Notani, H.J.Ong, A.Saito, H.Sakurai, E.Takeshita, S.Takeuchi, Y.Yanagisawa, A.Yoshida - CNS-REP-67 (2005)

Proton Single-Particle States in the Neutron-rich ${ }^{23}$ F Nucleus

2005M033 O.Moller, P.Petkov, B.Melon, A.Dewald, A.Fitzler, J.Jolie, D.Tonev, S.Christen, B.Saha, K.O.Zell, M.Heidemann - Phys.Rev. C 72, 034306 (2005)

Lifetimes of the first excited $2^{+}$states in ${ }^{176,178,180} \mathrm{Os}$

2005NA36 S.Nakayama, T.Yamagata, H.Akimune, Y.Arimoto, H.Daito, H.Ejiri, H.Fujimura, Y.Fujita, M.Fujiwara, K.Fushimi, M.B.Greenfield, H.Kohri, N.Koori, K.Takahisa, T.Takeuchi, M.Tanaka, K.Yonehara, H.P.Yoshida - Phys.Rev. C 72, 041001 (2005) 
M1 cross section for the photodisintegration of deuterium using the ${ }^{2} \mathrm{H}\left({ }^{7} \mathrm{Li},{ }^{7} \mathrm{Be}\right)$ reaction

2005NA37 S.Naguleswaran, R.S.Chakrawarthy, U.Garg, K.L.Lamkin, G.Smith, J.C.Walpe, A.Galindo-Uribarri, V.P.Janzen, D.C.Radford, R.Kaczarowski, D.B.Fossan, D.R.Lafosse, P.Vaska, Ch.Droste, T.Morek, S.Pilotte, J.DeGraaf, T.Drake, R.Wyss Phys.Rev. C 72, 044304 (2005)

Magnetic and intruder rotational bands in ${ }^{113} \mathrm{In}$

2005NA43 B.S.Nara Singh, M.Hass, G.Goldring, D.Ackermann, J.Gerl, F.P.Hessberger, S.Hofmann, I.Kojouharov, P.Kuusiniemi, H.Schaffner, B.Sulignano, B.A.Brown Eur.Phys.J. A 25, Supplement 1, 703 (2005)

Parity non-conservation in the $\gamma$-decay of polarized $17 / 2^{-}$isomers in ${ }^{93} \mathrm{Tc}$

2005NC01 N.J.Ncapayi, S.M.Mullins, M.Benatar, E.Gueorgueiva, J.J.Lawrie, G.K.Mabala, S.Mukherjee, S.H.T.Murray, K.P.Mutshena, R.T.Newman, J.F.Sharpey-Schafer, F.D.Smit, P.Vymers - Eur.Phys.J. A 26, 265 (2005)

Characterization of quasiparticle states at and beyond stability in ytterbium isotopes: Spectroscopy of ${ }^{175} \mathrm{Yb},{ }^{176} \mathrm{Yb}$ and ${ }^{177} \mathrm{Yb}$

2005NE14 A.Negret, T.Adachi, C.Baumer, A.M.van den Berg, G.P.A.Berg, P.von Brentano, D.Frekers, D.De Frenne, K.Fujita, Y.Fujita, E.-W.Grewe, P.Haefner, K.Hatanaka, M.Hunyadi, E.Jacobs, A.Korff, K.Nakanishi, L.Popescu, S.Rakers, Y.Sakemi, Y.Shimbara, Y.Shimizu, Y.Tameshige, A.Tamii, M.Uchida, H.J.Wortche, M.Yosoi J.Phys.(London) G31, S1931 (2005)

High resolution study of the Gamow-Teller strength distribution starting from the ground state of ${ }^{14} \mathrm{~N}$ in the $\beta^{-}$and $\beta^{+}$directions

2005NI20 B.Nilsson, J.-O.Adler, B.-E.Andersson, J.R.M.Annand, I.Akkurt, M.J.Boland, G.I.Crawford, K.G.Fissum, K.Hansen, P.D.Harty, D.G.Ireland, L.Isaksson, M.Karlsson, M.Lundin, J.C.McGeorge, G.J.Miller, H.Ruijter, A.Sandell, B.Schroder, D.A.Sims, D.Watts - Phys.Lett. B 626, 65 (2005)

Near-threshold measurement of the ${ }^{4} \mathrm{He}(\gamma, \mathrm{n})$ reaction

2005N013 T.Noro, T.Yonemura, S.Asaji, N.S.Chant, K.Fujita, Y.Hagihara, K.Hatanaka, G.C.Hillhouse, T.Ishida, M.Itoh, S.Kishi, M.Nakamura, Y.Nagasue, H.Sakaguchi, Y.Sakemi, Y.Shimizu, H.Takeda, Y.Tamesige, S.Terashima, M.Uchida, T.Wakasa, Y.Yasuda, H.P.Yoshida, M.Yosoi - Phys.Rev. C 72, 041602 (2005)

Analyzing powers for exclusive $1 \mathrm{~s}_{1 / 2}$ proton knockout from light nuclei

2005N015 W.Nortershauser, B.A.Bushaw, A.Dax, G.W.F.Drake, G.Ewald, S.Gotte, R.Kirchner, H.-J.Kluge, Th.Kuhl, R.Sanchez, A.Wojtaszek, Z.-C.Yan, C.Zimmermann - Eur.Phys.J. A 25, Supplement 1, 199 (2005)

Measurement of the nuclear charge radii of ${ }^{8,9} \mathrm{Li}$ : The last step towards the determination of the charge radius of ${ }^{11} \mathrm{Li}$

20050B04 S.Oberstedt, A.Oberstedt, D.Rochman, F.Gonnenwein, I.Tsekhanovich, J.Becker, A.Sartz, H.Bax, F.-J.Hambsch, S.Raman - Nucl.Phys. A761, 173 (2005)

Light charged particle emission in the reaction ${ }^{251} \mathrm{Cf}\left(\mathrm{n}_{t h}, \mathrm{f}\right)$ 
20050G02 Yu.Ts.Oganessian, V.K.Utyonkov, S.N.Dmitriev, Yu.V.Lobanov, M.G.Itkis, A.N.Polyakov, Yu.S.Tsyganov, A.N.Mezentsev, A.V.Yeremin, A.A.Voinov, E.A.Sokol, G.G.Gulbekian, S.L.Bogomolov, S.Iliev, V.G.Subbotin, A.M.Sukhov, G.V.Buklanov, S.V.Shishkin, V.I.Chepigin, G.K.Vostokin, N.V.Aksenov, M.Hussonnois, K.Subotic, V.I.Zagrebaev, K.J.Moody, J.B.Patin, J.F.Wild, M.A.Stoyer, N.J.Stoyer, D.A.Shaughnessy, J.M.Kenneally, P.A.Wilk,

R.W.Lougheed, H.W.Gaggeler, D.Schumann, H.Bruchertseifer, R.Eichler Phys.Rev. C 72, 034611 (2005)

Synthesis of elements 115 and 113 in the reaction ${ }^{243} \mathrm{Am}+{ }^{48} \mathrm{Ca}$

20050 G03 Yu.Ts.Oganessian, V.K.Utyonkov, Yu.V.Lobanov, F.Sh.Abdullin, A.N.Polyakov, I.V.Shirokovsky, Yu.S.Tsyganov, G.G.Gulbekian, S.L.Bogomolov, B.N.Gikal, A.N.Mezentsev, S.Iliev, V.G.Subbotin, A.M.Sukhov, A.A.Voinov, G.V.Buklanov, K.Subotic, V.I.Zagrebaev, M.G.Itkis, J.B.Patin, K.J.Moody, J.F.Wild, M.A.Stoyer, N.J.Stoyer, D.A.Shaughnessy, J.M.Kenneally, P.A.Wilk, R.W.Lougheed -

Eur.Phys.J. A 25, Supplement 1, 589 (2005)

New elements from Dubna

20050L04 J.Ollier, A.Hodsdon, R.Chapman, X.Liang, M.Burns, K.Keyes, M.Labiche, A.Papenberg, K.-M.Spohr, M.Davison, G.de Angelis, M.Axiotis, B.Behera, L.Corradi, A.Gadea, A.Latina, T.Kroll, N.Marginean, T.Martinez, D.R.Napoli, A.Stefanini, M.Trotta, S.Beghini, D.Bazzacco, E.Farnea, S.Lunardi, G.Montagnoli, F.Scarlassara, A.Deacon, A.G.Smith, J.F.Smith, D.Curien, F.Haas, D.Verney, A.Jungclaus, F.Azaiez, F.Ibrahim, M.Stanoiu, Zs.Dombradi - J.Phys.(London) G31, S1935 (2005)

Intruder configurations in neutron-rich $\mathrm{P}$ and $\mathrm{S}$ isotopes

20050N04 H.J.Ong, N.Imai, N.Aoi, H.Sakurai, Zs.Dombradi, A.Saito, Z.Elekes, H.Baba, K.Demichi, Zs.Fulop, J.Gibelin, T.Gomi, H.Hasegawa, M.Ishihara, H.Iwasaki, S.Kanno, S.Kawai, T.Kubo, K.Kurita, Y.U.Matsuyama, S.Michimasa, T.Minemura, T.Motobayashi, M.Notani, S.Ota, H.K.Sakai, S.Shimoura, E.Takeshita, S.Takeuchi, M.Tamaki, Y.Togano, K.Yamada, Y.Yanagisawa, K.Yoneda - Eur.Phys.J. A 25, Supplement 1, 347 (2005)

Inelastic proton scattering on ${ }^{16} \mathrm{C}$

20050R02 J.N.Orce, T.Kibedi, G.D.Dracoulis, R.Julin, S.W.Yates - J.Phys.(London) G31, S1705 (2005)

Conversion-electron study of $0^{+}$excitations in ${ }^{208} \mathrm{~Pb}$

2005PA56 H.Park, K.-O.Choi, J.-M.Lee, K.B.Lee, M.S.Hahn, M.Kralik - J.Korean Phys.Soc. 47, $603(2005)$

Absolute Measurement of the Neutron Emission Rate with a Manganese Sulphate Bath System

2005PA68 S.D.Pain, W.N.Catford, N.A.Orr, J.C.Angelique, N.I.Ashwood, V.Bouchat, N.M.Clarke, N.Curtis, M.Freer, B.R.Fulton, F.Hanappe, M.Labiche, J.L.Lecouey, R.C.Lemmon, D.Mahboub, A.Ninane, G.Normand, N.Soic, L.Stuttge, C.N.Timis, J.A.Tostevin, J.S.Winfield, V.Ziman - Eur.Phys.J. A 25, Supplement 1, 349 (2005) Experimental evidence of a $\nu\left(1 \mathrm{~d}_{5 / 2}\right)^{2}$ component to the ${ }^{12}$ Be ground state 
2005PA69 J.Pakarinen, I.Darby, S.Eeckhaudt, T.Enqvist, T.Grahn, P.Greenlees, F.Johnston-Theasby, P.Jones, R.Julin, S.Juutinen, H.Kettunen, M.Leino, A.-P.Leppanen, P.Nieminen, M.Nyman, R.Page, P.Raddon, P.Rahkila, C.Scholey, J.Uusitalo, R.Wadsworth - Eur.Phys.J. A 25, Supplement 1, 449 (2005)

Probing the three shapes in ${ }^{186} \mathrm{~Pb}$ using in-beam $\gamma$-ray spectroscopy

2005PazV S.D.Pain, W.N.Catford, N.A.Orr, J.C.Angelique, N.I.Ashwood, V.Bouchat, N.M.Clarke, N.Curtis, M.Freer, B.R.Fulton, F.Hanappe, M.Labiche, J.L.Lecouey, R.C.Lemmon, D.Mahboub, A.Ninane, G.Normand, N.Soic, L.Stuttge, C.N.Timis, J.A.Tostevin, J.S.Winfield, V.Ziman - nucl-ex/0510048,10/16/2005 (2005) Structure of ${ }^{12} \mathrm{Be}$ : intruder d-wave strength at $\mathrm{N}=8$

2005PE18 R.Peusquens, R.S.Chakrawarthy, A.Dewald, P.Petkov, P.von Brentano, R.Krucken, S.Kasemann, H.Tiesler, K.O.Zell, S.Lunardi, D.Bazzacco, F.Brandolini, N.H.Medina, P.Pavan, C.M.Petrache, C.Rossi-Alvarez, G.de Angelis, G.Maron, M.de Poli, D.R.Napoli - Phys.Rev. C 72, 031304 (2005)

Decay out of the highly deformed band in ${ }^{133} \mathrm{Nd}$

2005PE23 K.Perajarvi, J.Cerny, U.Hager, J.Hakala, J.Huikari, A.Jokinen, P.Karvonen, J.Kurpeta, D.Lee, I.Moore, H.Penttila, A.Popov, J.Aysto - Eur.Phys.J. A 25, Supplement 1, 749 (2005)

Production of beams of neutron-rich nuclei between $\mathrm{Ca}$ and $\mathrm{Ni}$ using the ion-guide technique

2005P017 L.Popescu, T.Adachi, G.P.A.Berg, P.von Brentano, D.De Frenne, K.Fujita, Y.Fujita, K.Hatanaka, E.Jacobs, A.Negret, K.Nakanishi, Y.Sakemi, Y.Shimbara, Y.Shimizu, Y.Tameshige, A.Tamii, M.Uchida, M.Yosoi - J.Phys.(London) G31, S1945 (2005) Gamow-Teller transitions in the ${ }^{64} \mathrm{Ni}\left({ }^{3} \mathrm{He}, \mathrm{t}\right){ }^{64} \mathrm{Cu}$ reaction

2005PR20

P.Prati, D.Bemmerer, R.Bonetti, C.Broggini, F.Confortola, P.Corvisiero, H.Costantini, J.Cruz, A.Formicola, Z.Fulop, G.Gervino, A.Guglielmetti, C.Gustavino, G.Gyurky, G.Imbriani, A.P.Jesus, M.Junker, A.Lemut, R.Menegazzo, V.Roca, C.Rolfs, M.Romano, C.Rossi Alvarez, F.Schumann, E.Somorjai, O.Straniero, F.Strieder, F.Terrasi, H.P.Trautvetter - J.Phys.(London) G31, S1537 (2005)

Recent results from the LUNA facility at Gran Sasso

2005PYZZ

I.Pysmenetska, S.Walter, J.Enders, H.von Garrel, O.Karg, U.Kneissl, C.Kohstall, P.von Neumann-Cosel, H.H.Pitz, V.Yu.Ponomarev, M.Scheck, F.Stedile, S.Volz nucl-ex/0512013,12/8/2005 (2005)

Two-phonon $1^{-}$state in ${ }^{112} \mathrm{Sn}$ observed in resonant photon scattering

2005RA26 B.Rakvin, N.Maltar-Strmecki - Chem.Phys.Lett. 415, 161 (2005) Study of the first stable L-alanine paramagnetic center by 2D-HYSCORE spectroscopy: Detection of ${ }^{14} \mathrm{~N}$ hyperfine and quadrupole splitting 
2005RA27 F.Raiola, B.Burchard, Z.Fulop, G.Gyurky, S.Zeng, J.Cruz, A.Di Leva, B.Limata, M.Fonseca, H.Luis, M.Aliotta, H.W.Becker, C.Broggini, A.D'Onofrio, L.Gialanella, G.Imbriani, A.P.Jesus, M.Junker, J.P.Ribeiro, V.Roca, C.Rolfs, M.Romano, E.Somorjai, F.Strieder, F.Terrasi, for the LUNA Collaboration - J.Phys.(London) G31, 1141 (2005)

Electron screening in $\mathrm{d}(\mathrm{d}, \mathrm{p}) \mathrm{t}$ for deuterated metals: temperature effects

2005RA29 D.R.Rao, K.V.Sai, M.Sainath, K.Venkataramaniah - Eur.Phys.J. A 26, 41 (2005)

Precision electron-gamma spectroscopic measurements in ${ }^{75} \mathrm{As}$

2005RA30 M.A.Rahman, M.S.Chowdhury - Phys.Rev. C 72, 054303 (2005)

Level structure of ${ }^{95} \mathrm{Nb}$

2005RA32 D.C.Radford, C.Baktash, C.J.Barton, J.Batchelder, J.R.Beene, C.R.Bingham, M.A.Caprio, M.Danchev, B.Fuentes, A.Galindo-Uribarri, J.Gomez del Campo, C.J.Gross, M.L.Halbert, D.J.Hartley, P.Hausladen, J.K.Hwang, W.Krolas, Y.Larochelle, J.F.Liang, P.E.Mueller, E.Padilla, J.Pavan, A.Piechaczek, D.Shapira, D.W.Stracener, R.L.Varner, A.Woehr, C.-H.Yu, N.V.Zamfir - Eur.Phys.J. A 25, Supplement 1, 383 (2005)

Coulomb excitation and transfer reactions with neutron-rich radioactive beams

2005RA33 M.P.Raele, C.B.Zamboni, G.S.Zahn, F.A.Genezini - Braz.J.Phys. 35, 839 (2005) Decay of ${ }^{155} \mathrm{Sm}$

2005RE23 J.J.Ressler, C.W.Beausang, R.F.Casten, N.V.Zamfir, H.Ai, H.Amro, M.Babilon, R.B.Cakirli, J.A.Caggiano, G.Gurdal, A.Heinz, R.O.Hughes, S.D.Langdown, E.A.McCutchan, D.A.Meyer, C.Plettner, J.Qian, P.H.Regan, M.J.S.Sciacchitano, N.J.Thomas, E.Williams, A.Yamamoto - J.Phys.(London) G31, S1605 (2005) Isomers and seniority in the trans- $\mathrm{Pb}$ nuclei

2005RE25 R.Reifarth, E.-I.Esch, A.Alpizar-Vicente, E.M.Bond, T.A.Bredeweg, S.E.Glover, U.Greife, R.Hatarik, R.C.Haight, A.Kronenberg, J.M.O'Donnell, R.S.Rundberg, J.M.Schwantes, J.L.Ullmann, D.J.Vieira, J.B.Wilhelmy, J.M.Wouters Nucl.Instrum.Methods Phys.Res. B241, 176 (2005) $(\mathrm{n}, \gamma)$ measurements on radioactive isotopes with DANCE

2005RI16 M.A.Riley, M.K.Djongolov, A.O.Evans, D.J.Hartley, R.V.F.Janssens, E.S.Paul, J.Simpson, A.A.Aguilar, D.E.Appelbe, C.R.Bingham, D.B.Campbell, M.P.Carpenter, P.Chowdhury, P.T.W.Choy, R.M.Clark, M.Cromaz, D.M.Cullen, M.Danchev, G.D.Dracoulis, P.Fallon, A.Gorgen, G.B.Hagemann, D.T.Joss, J.Goon, R.A.Kaye, T.L.Khoo, F.G.Kondev, R.W.Laird, K.Lagergren, T.Lauritsen, A.O.Macchiavelli, B.McClain, E.F.Moore, G.Mukherjee, E.Ngijoi-Yogo, P.J.Nolan, H.I.Park, A.Pipidis, L.L.Riedinger, G.Sletten, S.K.Tandel, P.M.Walker, D.Ward, I.Ragnarsson, F.Saric, J.Zhang - J.Phys.(London) G31, S1735 (2005)

Beyond band termination in ${ }^{157} \mathrm{Er}$ and the search for wobbling excitations in strongly deformed ${ }^{174} \mathrm{Hf}$ 
2005RI17 S.Rigby, D.M.Cullen, D.T.Scholes, C.Scholey, P.Rahkila, S.Eeckhaudt, T.Grahn, P.Greenlees, P.M.Jones, R.Julin, S.Juutinen, H.Kettunen, M.Leino, A.Leppanen, P.Nieminen, M.Nyman, J.Pakarinen, J.Uusitalo - J.Phys.(London) G31, S1949 (2005)

Mass-140 isomers near the proton dripline

2005RI19 S.Rinta-Antila, Y.Wang, P.Dendooven, J.Huikari, A.Jokinen, A.Kankainen, V.S.Kolhinen, G.Lhersonneau, A.Nieminen, S.Nummela, H.Penttila, K.Perajarvi, J.Szerypo, J.C.Wang, J.Aysto, and the ISOLDE Collaboration - Eur.Phys.J. A 25, Supplement 1, 119 (2005)

Structure of doubly-even cadmium nuclei studied by $\beta^{-}$decay

2005R035 S.D.Rosner, D.Masterman, T.J.Scholl, R.A.Holt - Can.J.Phys. 83, 841 (2005) Measurement of hyperfine structure and isotope shifts in Nd II

2005R037 G.V.Rogachev, A.A.Aprahamian, F.D.Becchetti, P.Boutachkov, Y.Chen, G.Chubarian, P.A.DeYoung, A.Fomichev, V.Z.Goldberg, M.S.Golovkov, J.J.Kolata, Yu.Ts.Oganessian, G.F.Peaslee, M.Quinn, A.Rodin, B.B.Skorodumov, R.S.Slepnev, G.Ter-Akopian, W.H.Trzaska, A.Wohr, R.Wolski - Nucl.Instrum.Methods Phys.Res. B241, 977 (2005)

Isobaric analog states as a tool for spectroscopy of exotic nuclei

2005R039 D.Rodriguez, V.S.Kolhinen, G.Audi, J.Aysto, D.Beck, K.Blaum, G.Bollen, F.Herfurth, A.Jokinen, A.Kellerbauer, H.-J.Kluge, M.Oinonen, H.Schatz, E.Sauvan, S.Schwarz - Eur.Phys.J. A 25, Supplement 1, 41 (2005)

Mass measurement on the rp-process waiting point ${ }^{72} \mathrm{Kr}$

2005R040 A.P.Robinson, C.N.Davids, D.Seweryniak, P.J.Woods, B.Blank, M.P.Carpenter, T.Davinson, S.J.Freeman, N.Hammond, N.Hoteling, R.V.F.Janssens, T.L.Khoo, Z.Liu, G.Mukherjee, C.Scholey, J.Shergur, S.Sinha, A.A.Sonzogni, W.B.Walters, A.Woehr - Eur.Phys.J. A 25, Supplement 1, 155 (2005)

Recoil decay tagging study of ${ }^{146} \mathrm{Tm}$

2005R042 M.Romoli, M.Mazzocco, E.Vardaci, M.Di Pietro, A.De Francesco, R.Bonetti, A.De Rosa, T.Glodariu, A.Guglielmetti, G.Inglima, M.La Commara, B.Martin, V.Masone, P.Parascandolo, D.Pierroutsakou, M.Sandoli, P.Scopel, C.Signorini, F.Soramel, L.Stroe, J.Greene, A.Heinz, D.Henderson, C.L.Jiang, E.F.Moore, R.C.Pardo, K.E.Rehm, A.Wuosmaa, J.F.Liang - Eur.Phys.J. A 25, Supplement 1, 289 (2005) The EXODET apparatus: Features and first experimental results

2005RU18 A.A.Rudchik, A.T.Rudchik, G.M.Kozeratska, O.A.Ponkratenko, E.I.Koshchy, A.Budzanowski, B.Czech, S.Kliczewski, R.Siudak, I.Skwirczynska, A.Szczurek, S.Yu.Mezhevych, K.W.Kemper, J.Choinski, T.Czosnyka, L.Glowacka - Phys.Rev. C $72,034608(2005)$

${ }^{7} \mathrm{Li}+{ }^{11} \mathrm{~B}$ elastic and inelastic scattering in a coupled-reaction-channels approach

2005RUZZ G.Rusev, E.Grosse, M.Erhard, A.Junghans, K.Kosev, K.-D.Schilling, R.Schwengner, A.Wagner - nucl-ex/0512027,12/20/2005 (2005)

Pygmy dipole strength close to particle-separation energies - the case of the Mo isotopes 
2005RY03 I.V.Ryzhov, M.S.Onegin, G.A.Tutin, J.Blomgren, N.Olsson, A.V.Prokofiev, P.-U.Renberg - Nucl.Phys. A760, 19 (2005)

Influence of multichance fission on fragment angular anisotropy in the ${ }^{232} \mathrm{Th}(\mathrm{n}, \mathrm{f})$ and ${ }^{238} \mathrm{U}(\mathrm{n}, \mathrm{f})$ reactions at intermediate energies

2005SA52 A.M.Sanchez-Benitez, D.Escrig, M.A.G.Alvarez, M.V.Andres, C.Angulo, M.J.G.Borge, J.Cabrera, S.Cherubini, J.M.Espino, P.Figuera, M.Freer,

J.E.Garcia-Ramos, J.Gomez-Camacho, M.Gulino, O.R.Kakuee, I.Martel, C.Metelco, A.M.Moro, J.Rahighi, K.Rusek, D.Smirnov, O.Tengblad, P.Van Duppen, V.Ziman J.Phys.(London) G31, S1953 (2005)

Scattering of ${ }^{6} \mathrm{He}$ at energies around the Coulomb barrier

2005SC20 A.Schiller, T.Baumann, J.Dietrich, S.Kaiser, W.Peters, M.Thoennessen - Phys.Rev. C 72, $037601(2005)$

Search for particle-bound ${ }^{26} \mathrm{O}$ and ${ }^{28} \mathrm{~F}$ in p-stripping reactions

2005SC22 C.Scholey, M.Sandzelius, S.Eeckhaudt, T.Grahn, P.T.Greenlees, P.Jones, R.Julin, S.Juutinen, M.Leino, A.-P.Leppanen, P.Nieminen, M.Nyman, J.Perkowski, J.Pakarinen, P.Rahkila, P.M.Rahkila, J.Uusitalo, K.Van de Vel, B.Cederwall, B.Hadinia, K.Lagergren, D.T.Joss, D.E.Appelbe, C.J.Barton, J.Simpson, D.D.Warner, I.G.Darby, R.D.Page, E.S.Paul, D.Wiseman - J.Phys.(London) G31, S1719 (2005)

In-beam and decay spectroscopy of very neutron deficient iridium nuclei

2005SC26 P.Schury, G.Bollen, D.A.Davies, A.Doemer, D.Lawton, D.J.Morrissey, J.Ottarson, A.Prinke, R.Ringle, T.Sun, S.Schwarz, L.Weissman - Eur.Phys.J. A 25, Supplement $1,51(2005)$

Precision experiments with rare isotopes with LEBIT at MSU

2005SC27 H.Scheit, O.Niedermaier, V.Bildstein, H.Boie, J.Fitting, R.von Hahn, F.Kock, M.Lauer, U.K.Pal, H.Podlech, R.Repnow, D.Schwalm, C.Alvarez, F.Ames, G.Bollen, S.Emhofer, D.Habs, O.Kester, R.Lutter, K.Rudolph, M.Pasini, P.G.Thirolf, B.H.Wolf, J.Eberth, G.Gersch, H.Hess, P.Reiter, O.Thelen, N.Warr, D.Weisshaar, F.Aksouh, P.Van den Bergh, P.Van Duppen, M.Huyse, O.Ivanov, P.Mayet, J.Van de Walle, J.Aysto, P.A.Butler, J.Cederkall, P.Delahaye, H.O.U.Fynbo, L.M.Fraile, O.Forstner, S.Franchoo, U.Koster, T.Nilsson, M.Oinonen, T.Sieber, F.Wenander, M.Pantea, A.Richter, G.Schrieder, H.Simon, T.Behrens, R.Gernhauser, T.Kroll, R.Krucken, M.Munch, T.Davinson, J.Gerl, G.Huber, A.Hurst, J.Iwanicki, B.Jonson, P.Lieb, L.Liljeby, A.Schempp, A.Scherillo, P.Schmidt, G.Walter - Eur.Phys.J. A 25, Supplement 1, 397 (2005)

Coulomb excitation of neutron-rich beams at REX-ISOLDE

2005SC28 H.Schatz, P.T.Hosmer, A.Aprahamian, O.Arndt, R.R.C.Clement, A.Estrade, K.-L.Kratz, S.N.Liddick, P.F.Mantica, W.F.Mueller, F.Montes, A.C.Morton, M.Ouellette, E.Pellegrini, B.Pfeiffer, P.Reeder, P.Santi, M.Steiner, A.Stolz, B.E.Tomlin, W.B.Walters, A.Wohr - Eur.Phys.J. A 25, Supplement 1, 639 (2005) The half-life of the doubly-magic r-process nucleus ${ }^{78} \mathrm{Ni}$ 
2005 Sc29 D.Schurmann, A.Di Leva, L.Gialanella, D.Rogalla, F.Strieder, N.De Cesare, A.D'Onofrio, G.Imbriani, R.Kunz, C.Lubritto, A.Ordine, V.Roca, C.Rolfs, M.Romano, F.Schumann, F.Terrasi, H.-P.Trautvetter - Eur.Phys.J. A 26, 301 (2005) First direct measurement of the total cross-section of ${ }^{12} \mathrm{C}(\alpha, \gamma){ }^{16} \mathrm{O}$

2005SCZT D.Schurmann, A.Di Leva, L.Gialanella, D.Rogalla, F.Strieder, N.De Cesare, A.D'Onofrio, G.Imbriani, R.Kunz, C.Lubritto, A.Ordine, V.Roca, C.Rolfs, M.Romano, F.Schumann, F.Terrasi, H.-P.Trautvetter - nucl-ex/0511050,11/29/2005 (2005)

First direct measurement of the total cross section of ${ }^{12} \mathrm{C}(\alpha, \gamma){ }^{16} \mathrm{O}$

2005SCZV W.Schwerdtfeger, B.Bruyneel, T.Faestermann, R.Gernhauser, D.Habs, Th.Kroll, R.Krucken, M.Lauer, R.Lutter, H.J.Maier, T.Morgan, M.Munch, O.Niedermaier, P.Reiter, O.Schaile, H.Scheit, P.G.Thirolf, W.von Oertzen, N.Warr, D.Weisshaar, H.Wolter - Maier-Leibnitz-Laboratorium 2004 Ann.Rept., p.4 (2005)

Towards Two-Neutron Transfer around the Island of Inversion

2005SE22 K.Sekiguchi, H.Sakai, H.Witala, W.Glockle, J.Golak, K.Hatanaka, M.Hatano, K.Itoh, H.Kamada, H.Kuboki, Y.Maeda, A.Nogga, H.Okamura, T.Saito, N.Sakamoto, Y.Sakemi, M.Sasano, Y.Shimizu, K.Suda, A.Tamii, T.Uesaka, T.Wakasa, K.Yako - Phys.Rev.Lett. 95, 162301 (2005)

Resolving the Discrepancy of $135 \mathrm{MeV}$ pd Elastic Scattering Cross Section and Relativistic Effects

2005SE23 M.Segawa, A.Tomyo, Y.Nagai, Y.Temma, T.Masaki, T.Shima, J.Nishiyama, T.Ohsaki, M.Igashira - J.Phys.Soc.Jpn. 74, 2981 (2005)

New Approach for Measuring the $(\mathrm{n}, \gamma)$ Cross Section of a Nucleus by a Few keV Neutron

2005SE26 D.Seweryniak, C.N.Davids, A.Robinson, P.J.Woods, B.Blank, M.P.Carpenter, T.Davinson, S.J.Freeman, N.Hammond, N.Hoteling, R.V.F.Janssens, T.L.Khoo, Z.Liu, G.Mukherjee, J.Shergur, S.Sinha, A.A.Sonzogni, W.B.Walters, A.Woehr Eur.Phys.J. A 25, Supplement 1, 159 (2005)

Particle-core coupling in the transitional proton emitters ${ }^{145,146,147} \mathrm{Tm}$

2005SEZV K.Sekiguchi, H.Sakai, H.Witala, W.Gloeckle, J.Golak, K.Hatanaka, M.Hatano, K.Itoh, H.Kamada, H.Kuboki, Y.Maeda, A.Nogga, H.Okamura, T.Saito, N.Sakamoto, Y.Sakemi, M.Sasano, Y.Shimizu, K.Suda, A.Tamii, T.Uesaka, T.Wakasa, K.Yako - nucl-ex/0510005,10/3/2005 (2005)

Resolving the Discrepancy of $135 \mathrm{MeV}$ pd Elastic Scattering Cross Sections and Relativistic Effects

2005 SH46 S.Shimoura - J.Phys.(London) G31, S1759 (2005)

Single particle states in exotic nuclei via nucleon transfer reactions at 30-60 A MeV

2005SH49 T.M.Shneidman, R.V.Jolos, R.Krucken, A.Aprahamian, D.Cline, J.R.Cooper, M.Cromaz, R.M.Clark, C.Hutter, A.O.Macchiavelli, W.Scheid, M.A.Stoyer, C.Y.Wu - Eur.Phys.J. A 25, 387 (2005)

E2 transitions between positive- and negative-parity states of the ground-state alternating-parity bands 
2005SH51 T.Shima, S.Naito, Y.Nagai, T.Baba, K.Tamura, T.Takahashi, T.Kii, H.Ohgaki, H.Toyokawa - Phys.Rev. C 72, 044004 (2005)

Simultaneous measurement of the photodisintegration of ${ }^{4} \mathrm{He}$ in the giant dipole resonance region

2005 SH52 K.S.Sharma, J.Vaz, R.C.Barber, F.Buchinger, J.A.Clark, J.E.Crawford, H.Fukutani, J.P.Greene, S.Gulick, A.Heinz, J.K.P.Lee, G.Savard, Z.Zhou, J.C.Wang -

Eur.Phys.J. A 25, Supplement 1, 45 (2005)

Atomic mass ratios for some stable isotopes of platinum relative to ${ }^{197} \mathrm{Au}$

2005SH53 J.Shergur, N.Hoteling, A.Wohr, W.B.Walters, O.Arndt, B.A.Brown, C.N.Davids, D.J.Dean, K.-L.Kratz, B.Pfeiffer, D.Seweryniak, and the ISOLDE Collaboration Eur.Phys.J. A 25, Supplement 1, 121 (2005)

New level information on $\mathrm{Z}=51$ isotopes, ${ }^{111} \mathrm{Sb}_{60}$ and ${ }^{134,135} \mathrm{Sb}_{83,84}$

2005SHZS A.Shrivastava, A.Navin, N.Keeley, K.Mahata, K.Ramachandran, V.Nanal, V.V.Parkar, A.Chatterjee, S.Kailas - nucl-ex/0512032,12/21/2005 (2005)

Evidence for transfer followed by breakup in ${ }^{7} \mathrm{Li}+{ }^{65} \mathrm{Cu}$

2005 SI28 M.A.G.Silveira, N.H.Medina, J.A.Alcantara-Nunez, E.W.Cybulska, H.Dias, J.R.B.Oliveira, M.N.Rao, R.V.Ribas, W.A.Seale, K.T.Wiedemann, B.A.Brown, M.Honma, T.Mizusaki, T.Otsuka - J.Phys.(London) G31, S1577 (2005)

Nuclear structure of the odd-odd nucleus ${ }^{58} \mathrm{Co}$

2005SI31 K.Singh, Z.Naik, R.Kumar, J.Goswamy, D.Mehta, N.Singh, C.R.Praharaj, E.S.Paul, K.P.Singh, R.P.Singh, S.Muralithar, N.Madhavan, J.J.Das, S.Nath, A.Jhingan, P.Sugathan, R.K.Bhowmik - Eur.Phys.J. A 25, 345 (2005)

Rotational structures in ${ }^{123} \mathrm{Cs}$

2005 SI32 J.M.Sisterson, F.D.Brooks, A.Buffler, M.S.Allie, D.T.L.Jones, M.B.Chadwick Nucl.Instrum.Methods Phys.Res. B240, 617 (2005)

Cross-section measurements for neutron-induced reactions in copper at neutron energies of 70.7 and $110.8 \mathrm{MeV}$

2005SI34 G.Sikler, G.Audi, D.Beck, K.Blaum, G.Bollen, F.Herfurth, A.Kellerbauer, H.-J.Kluge, D.Lunney, M.Oinonen, C.Scheidenberger, S.Schwarz, J.Szerypo, C.Weber - Nucl.Phys. A763, 45 (2005); Erratum Nucl.Phys. A768, 160 (2006) Mass measurements on neutron-deficient $\mathrm{Sr}$ and neutron-rich $\mathrm{Sn}$ isotopes with the ISOLTRAP mass spectrometer

2005SI37 M.A.G.Silveira, N.H.Medina, J.R.B.Oliveira, J.A.Alcantara-Nunez, E.W.Cybulska, H.Dias, M.N.Rao, R.V.Ribas, W.A.Seale, K.T.Wiedemann - Braz.J.Phys. 35, 821 (2005) In-Beam Gamma Ray Spectroscopy of ${ }^{58} \mathrm{Co}$

2005SM08 A.G.Smith, R.Orlandi, D.Patel, G.S.Simpson, R.M.Wall, J.F.Smith, O.J.Onakanmi, I.Ahmad, J.P.Greene, M.P.Carpenter, T.Lauritsen, C.J.Lister, R.V.F.Janssens, F.G.Kondev, D.Seweryniak, B.J.P.Gall, O.Dorvaux, B.Roux - J.Phys.(London) G31, S1433 (2005) 
The magnetic properties of collective states in $\mathrm{A} \sim 100$ fission fragments

2005 S013 N.Soic, M.Freer, L.Donadille, N.M.Clarke, P.J.Leask, W.N.Catford, K.L.Jones, D.Mahboub, B.R.Fulton, B.J.Greenhalgh, D.L.Watson, D.C.Weisser -

J.Phys.(London) G31, S1701 (2005)

Three-centre cluster structure in ${ }^{11} \mathrm{C}$ and ${ }^{11} \mathrm{~B}$

2005 S014 F.A.Souza, R.Liguori Neto, M.M.de Moura, M.G.Munhoz, A.A.P.Suaide, E.M.Szanto, J.Takahashi, A.Szanto de Toledo, N.Carlin - Braz.J.Phys. 35, 888 (2005)

Direct Measurement of the Breakup Process

2005SPZY K.-H.Speidel, S.Schielke, J.Leske, C.C.Dey, J.Gerber, P.Maier-Komor, S.J.Q.Robinson, Y.Y.Sharon, L.Zamick - Maier-Leibnitz-Laboratorium 2004 Ann.Rept., p.5 (2005)

First Measurements of g Factors of the $2_{1}^{+}$States in ${ }^{36,38} \mathrm{Ar}$ and their Shell Model Interpretation

2005ST29 A.Stolz, T.Baumann, N.H.Frank, T.N.Ginter, G.W.Hitt, E.Kwan, M.Mocko, W.Peters, A.Schiller, C.S.Sumithrarachchi, M.Thoennessen - Phys.Lett. B 627, 32 (2005)

First observation of ${ }^{60} \mathrm{Ge}$ and ${ }^{64} \mathrm{Se}$

2005ST30 M.F.M.Steenbakkers, M.F.van Batenburg, Th.S.Bauer, H.P.Blok, T.Botto, G.E.Dodge, D.L.Groep, P.Heimberg, W.H.A.Hesselink, D.W.Higinbotham, E.Jans, Y.Jun, T.J.Ketel, L.Lapikas, D.J.J.de Lange, B.E.Norum, I.Passchier, R.Starink, L.Todor, H.de Vries - Phys.Rev.Lett. 95, 172501 (2005)

Quasifree $\pi^{0}$ and $\pi^{-}$Electroproduction on ${ }^{4} \mathrm{He}$ in the $\Delta$-Resonance Region

2005ST33 N.J.Stone, A.E.Stuchbery, M.Danchev, J.Pavan, C.L.Timlin, C.Baktash, C.Barton, J.R.Beene, N.Benczer-Koller, C.R.Bingham, J.Dupak, A.Galindo-Uribarri, C.J.Gross, G.Kumbartzki, D.C.Radford, J.R.Stone, N.V.Zamfir - Eur.Phys.J. A 25, Supplement 1, 205 (2005)

First nuclear moment measurement with radioactive beams by recoil-in-vacuum method: g-factor of the $2_{1}^{+}$state in ${ }^{132} \mathrm{Te}$

2005ST34 A.Stolz, T.Baumann, N.H.Frank, T.N.Ginter, G.W.Hitt, E.Kwan, M.Mocko, W.Peters, A.Schiller, C.S.Sumithrarachchi, M.Thoennessen - Eur.Phys.J. A 25, Supplement 1, 335 (2005)

Discovery of ${ }^{60} \mathrm{Ge}$ and ${ }^{64} \mathrm{Se}$

2005SU25 T.Sumikama, T.Iwakoshi, T.Nagatomo, M.Ogura, Y.Nakashima, H.Fujiwara, K.Matsuta, T.Minamisono, M.Mihara, M.Fukuda, K.Minamisono, T.Yamaguchi Eur.Phys.J. A 25, Supplement 1, 709 (2005)

Alignment correlation term in mass A $=8$ system and G-parity irregular term

2005SUZU A.C.Sunde, R.Chankova, M.Guttormsen, F.Ingebretsen, S.Messelt, J.Rekstad, S.Siem, N.U.H.Syed, S.W.Odegard, T.Lonnroth, A.Schiller, A.Voinov nucl-ex/0511054,11/30/2005 (2005)

Microcanonical entropies and radiative strength functions of ${ }^{50,51} \mathrm{~V}$ 
2005Sz04 F.Szelecsenyi, G.F.Steyn, Z.Kovacs, C.Vermeulen, N.P.van der Meulen, S.G.Dolley, T.N.van der Walt, K.Suzuki, K.Mukai - Nucl.Instrum.Methods Phys.Res. B240, 625 (2005)

Investigation of the ${ }^{66} \mathrm{Zn}(\mathrm{p}, 2 \mathrm{pn}){ }^{64} \mathrm{Cu}$ and ${ }^{68} \mathrm{Zn}(\mathrm{p}, \mathrm{x}){ }^{64} \mathrm{Cu}$ nuclear processes up to $100 \mathrm{MeV}$ : Production of ${ }^{64} \mathrm{Cu}$

2005TA26 F.Tarkanyi, B.Kiraly, F.Ditroi, S.Takacs, J.Csikai, A.Hermanne, M.S.Uddin, M.Hagiwara, M.Baba, T.Ido, Yu.N.Shubin, A.K.Dityuk - Nucl.Instrum.Methods Phys.Res. B239, 293 (2005)

Activation cross sections of proton induced nuclear reactions on iridium

2005TA27 M.J.Taylor, G.Hammond, M.A.Bentley, F.Becker, J.Grebosz, A.Banu, C.J.Barton, T.Beck, P.Bednarczyk, A.Bracco, A.M.Bruce, L.C.Bullock, A .Burger, F.Camera, C.Chandler, P.Doornenbal, J.Gerl, H.Geissel, M.Gorska, M.Hellstrom, D.S.Judson, I.Kojouharov, N.Kurz, R.Lozeva, A.Maj, S.Mandal, B.McGuirk, S.Muralithar, E.S.Paul, Z.Podolyak, W.Prokopowicz, D.Rudolph, N.Saito, T.R.Saito, H.Schaffner, J.Simpson, D.D.Warner, H.Weick, C.Wheldon, M.Winkler, H.J.Wollersheim J.Phys.(London) G31, S1527 (2005)

Spectroscopy of nuclei approaching the proton drip-line using a secondary-fragmentation technique with the RISING detector array

2005 TA28 W.P.Tan, J.Gorres, J.Daly, M.Couder, A.Couture, H.Y.Lee, E.Stech, E.Strandberg, C.Ugalde, M.Wiescher - Phys.Rev. C 72, 041302 (2005)

Lifetime of the astrophysically important $4.03-\mathrm{MeV}$ state in ${ }^{19} \mathrm{Ne}$

2005TA31 M.N.Tantawy, C.R.Bingham, C.Mazzocchi, R.Grzywacz, W.Krolas, K.P.Rykaczewski, J.C.Batchelder, C.J.Gross, D.Fong, J.H.Hamilton, D.J.Hartley, J.K.Hwang, Y.Larochelle, A.Piechaczek, A.V.Ramayya, D.Shapira, J.A.Winger, C.-H.Yu, E.F.Zganjar - Eur.Phys.J. A 25, Supplement 1, 151 (2005)

Study of the $\mathrm{N}=77$ odd-Z isotones near the proton-drip line

2005 TA32 M.Takechi, M.Fukuda, M.Mihara, T.Chinda, T.Matsumasa, H.Matsubara, Y.Nakashima, K.Matsuta, T.Minamisono, R.Koyama, W.Shinosaki, M.Takahashi, A.Takizawa, T.Ohtsubo, T.Suzuki, T.Izumikawa, S.Momota, K.Tanaka, T.Suda, M.Sasaki, S.Sato, A.Kitagawa - Eur.Phys.J. A 25, Supplement 1, 217 (2005) Reaction cross-sections for stable nuclei and nucleon density distribution of proton drip-line nucleus ${ }^{8} \mathrm{~B}$

$2005 T A 33$ K.Tanaka, M.Fukuda, M.Mihara, M.Takechi, T.Chinda, T.Sumikama, S.Kudo, K.Matsuta, T.Minamisono, T.Suzuki, T.Ohtubo, T.Izumikawa, S.Momota, T.Yamaguchi, T.Onishi, A.Ozawa, I.Tanihata, T.Zheng - Eur.Phys.J. A 25, Supplement 1, 221 (2005)

Nucleon density distribution of proton drip-line nucleus ${ }^{17} \mathrm{Ne}$

2005 TE04 S.A.Telezhnikov, C.Granja, H.T.Hiep, J.Honzatko, M.Kralik, M.-E.Montero-Cabrera, S.Pospisil - Nucl.Phys. A763, 31 (2005)

Primary gamma transitions in ${ }^{173,174} \mathrm{Yb}$ in neutron capture at isolated resonances 
2005TE05 G.M.Ter-Akopian, A.S.Fomichev, M.S.Golovkov, L.V.Grigorenko, S.A.Krupko, Yu.Ts.Oganessian, A.M.Rodin, S.I.Sidorchuk, R.S.Slepnev, S.V.Stepantsov, R.Wolski, A.A.Korsheninnikov, E.Yu.Nikolskii, P.Roussel-Chomaz, W.Mittig, R.Palit, V.Bouchat, V.Kinnard, T.Materna, F.Hanappe, O.Dorvaux, L.Stuttge, C.Angulo, V.Lapoux, R.Raabe, L.Nalpas, A.A.Yukhimchuk, V.V.Perevozchikov, Yu.I.Vinogradov, S.K.Grishechkin, S.V.Zlatoustovskii - Eur.Phys.J. A 25,

Supplement 1, 315 (2005)

New insights into the resonance states of ${ }^{5} \mathrm{H}$ and ${ }^{5} \mathrm{He}$

2005 TH12 J.S.Thomas, D.W.Bardayan, J.C.Blackmon, J.A.Cizewski, R.P.Fitzgerald, U.Greife, C.J.Gross, M.S.Johnson, K.L.Jones, R.L.Kozub, J.F.Liang, R.J.Livesay, Z.Ma, B.H.Moazen, C.D.Nesaraja, D.Shapira, M.S.Smith, D.W.Visser - Eur.Phys.J. A 25, Supplement 1, 371 (2005)

Single-neutron excitations in neutron-rich $\mathrm{N}=51$ nuclei

2005THzZ P.G.Thirolf, D.Habs, R.Hertenberger, H.J.Maier, T.Morgan, O.Schaile, J.Schreiber, W.Schwerdtfeger, J.Szerypo - Maier-Leibnitz-Laboratorium 2004 Ann.Rept., p.17 (2005)

Search for a new Picosecond Fission Isomer in ${ }^{234} \mathrm{U}$

2005 T11 C.Timis, J.C.Angelique, A.Buta, N.L.Achouri, D.Baiborodin, P.Baumann, C.Borcea, S.Courtin, P.Dessagne, Z.Dlouhy, J.M.Daugas, S.Grevy, D.Guillemaud-Mueller, A.Knipper, F.R.Lecolley, J.L.Lecouey, M.Lewitowicz, E.Lienard, S.M.Lukyanov, F.M.Marques, C.Miehe, J.Mrazek, F.Negoita, F.Nowacki, F.de Oliveira, N.A.Orr, J.Peter, S.Pietri, Yu.E.Penionzhkevich, E.Poirier, M.Stanoiu, G.Walter - J.Phys.(London) G31, S1965 (2005) Spectroscopy around $\mathrm{N}=20$ shell closure: $\beta$-n decay study of ${ }^{35} \mathrm{Al}$

2005T014 A.Tomyo, Y.Temma, M.Segawa, Y.Nagai, H.Makii, T.Shima, T.Ohsaki, M.Igashira Astrophys.J. 623, L153 (2005)

${ }^{62} \mathrm{Ni}(\mathrm{n}, \gamma){ }^{63} \mathrm{Ni}$ reaction and overproduction of $\mathrm{Ni}$ isotopes

2005 T015 B.S.Tomar, K.S.Babu, K.Sudarshan, R.Tripathi, A.Goswami - Pramana 64, 1 (2005) Angular momentum transfer in incomplete fusion

2005 T022 D.Tonev, G.de Angelis, P.Petkov, A.Dewald, A.Gadea, P.Pejovic, D.L.Balabanski, P.Bednarczyk, F.Camera, A.Fitzler, O.Moller, N.Marginean, A.Paleni, C.Petrache, K.O.Zell, Y.H.Zhang - Eur.Phys.J. A 25, Supplement 1, 447 (2005)

Check for chirality in real nuclei

2005TR13 V.Tripathi, S.L.Tabor, P.F.Mantica, C.R.Hoffman, M.Wiedeking, A.D.Davies, S.N.Liddick, W.F.Mueller, A.Stolz, B.E.Tomlin, A.Volya - Eur.Phys.J. A 25, Supplement 1, 101 (2005)

Voyage to the "Island of Inversion": ${ }^{29} \mathrm{Na}$

2005TU09 A.Tumino, C.Spitaleri, C.Bonomo, S.Cherubini, P.Figuera, M.Gulino, M.La Cognata, L.Lamia, A.Musumarra, M.G.Pellegriti, R.G.Pizzone, A.Rinollo, S.Romano - Eur.Phys.J. A 25, Supplement 1, 649 (2005)

Quasi-free ${ }^{6} \mathrm{Li}(\mathrm{n}, \alpha)^{3} \mathrm{H}$ reaction at low energy from ${ }^{2} \mathrm{H}$ break-up 
2005UU03 J.Uusitalo, S.Eeckhaudt, T.Enqvist, K.Eskola, T.Grahn, P.T.Greenlees, P.Jones, R.Julin, S.Juutinen, H.Kettunen, P.Kuusiniemi, M.Leino, A.-P.Leppanen, P.Nieminen, M.Nyman, J.Pakarinen, P.Rahkila, C.Scholey - Eur.Phys.J. A 25, Supplement 1, 179 (2005)

Alpha-decay studies using the JYFL gas-filled recoil separator RITU

2005VA30 J.J.Valiente-Dobon, T.Steinhardt, C.E.Svensson, A.V.Afanasjev, I.Ragnarsson, C.Andreoiu, R.A.E.Austin, M.P.Carpenter, D.Dashdorj, G.de Angelis, F.Donau, J.Eberth, E.Farnea, S.J.Freeman, A.Gadea, P.E.Garrett, A.Gorgen, G.F.Grinyer, B.Hyland, D.Jenkins, F.Johnston-Theasby, P.Joshi, A.Jungclaus, K.P.Lieb, A.O.Macchiavelli, E.F.Moore, G.Mukherjee, D.R.Napoli, A.A.Phillips, C.Plettner, W.Reviol, D.Sarantites, H.Schnare, M.A.Schumaker, R.Schwengner, D.Seweryniak, M.B.Smith, I.Stefanescu, O.Thelen, R.Wadsworth - Phys.Rev.Lett. 95, 232501 (2005)

Evidence for Nontermination of Rotational Bands in ${ }^{74} \mathrm{Kr}$

2005VA31 R.L.Varner, J.R.Beene, C.Baktash, A.Galindo-Uribarri, C.J.Gross, J.Gomez del Campo, M.L.Halbert, P.A.Hausladen, Y.Larochelle, J.F.Liang, J.Mas, P.E.Mueller, E.Padilla-Rodal, D.C.Radford, D.Shapira, D.W.Stracener, J.-P.Urrego-Blanco, C.-H.Yu - Eur.Phys.J. A 25, Supplement 1, 391 (2005)

Coulomb excitation measurements of transition strengths in the isotopes ${ }^{132,134} \mathrm{Sn}$

2005VE07 Ts.Venkova, W.Gast, R.M.Lieder, D.Bazzacco, G.de Angelis, E.O.Lieder, A.A.Pasternak, R.Menegazzo, S.Lunardi, C.Rossi Alvarez, C.Ur, T.Martinez, M.Axiotis, D.Napoli, W.Urban, T.Rzaca-Urban, S.Frauendorf - Eur.Phys.J. A 26, 19 (2005)

Suppression of band crossing in the neutron-rich nuclei ${ }^{172,173} \mathrm{Yb}$ due to the absence of a static pair field

2005VE08 V.A.Vesna, Yu.M.Gledenov, V.V.Nesvizhevsky, A.K.Petukhov, P.V.Sedyshev, T.Soldner, O.Zimmer, E.V.Shulgina - Pisma Zh.Eksp.Teor.Fiz. 82, 519 (2005); JETP Lett. 82, 463 (2005)

Discovery of a P-Odd Effect in Triton Emission from the reaction ${ }^{6} \operatorname{Li}(\mathrm{n}, \alpha)^{3} \mathrm{H}$

2005WA29 J.Wang, X.Wang, T.Su - Phys.Rev. C 72, 037604 (2005)

Measurements of the ${ }^{71} \mathrm{Ga}(\mathrm{n}, 2 \mathrm{n})^{70} \mathrm{Ga}$ cross section in the neutron energy range of 13.5-14.7 MeV

2005WA31 A.Wagner, R.Beyer, M.Erhard, F.Donau, E.Grosse, A.Hartmann, A.R.Junghans, L.Kaubler, K.Kosev, S.Mallion, C.Nair, N.Nankov, G.Rusev, K.D.Schilling, W.Schulze, R.Schwengner - J.Phys.(London) G31, S1969 (2005)

The new bremsstrahlung facility at the superconducting electron accelerator ELBE

2005WA34 T.J.Wasowicz, R.Drozdowski, J.Kwela - Eur.Phys.J. D 36, 249 (2005)

Hyperfine structure and isotope shift study in a singly ionized lead

2005WE11 C.Weber, G.Audi, D.Beck, K.Blaum, G.Bollen, F.Herfurth, A.Kellerbauer, H.-J.Kluge, D.Lunney, S.Schwarz - Phys.Lett. A 347, 81 (2005)

Weighting excited nuclear states with a Penning trap mass spectrometer 
2005WH04 C.Wheldon, G.D.Dracoulis, A.N.Wilson, P.M.Davidson, A.P.Byrne, D.M.Cullen, L.K.Pattison, S.V.Rigby, D.T.Scholes, G.Sletten, R.Wood - Nucl.Phys. A763, 1 (2005)

Gamma-ray spectroscopy of the doubly-odd nuclide ${ }^{184} \mathrm{Re}$

2005WI19 O.Wieland, S.Barlini, V.L.Kravchuk, A.Bracco, F.Camera, F.Gramegna, A.Maj, G.Benzoni, N.Blasi, S.Brambilla, M.Brekiesz, M.Bruno, G.Casini, M.Chiari, E.Geraci, A.Giussani, M.Kmiecik, S.Leoni, A.Lanchais, P.Mastinu, B.Million, A.Moroni, A.Nannini, A.Ordine, G.Vannini, L.Vannucci - J.Phys.(London) G31, S1973 (2005)

The $\gamma$-decay of the GDR in highly excited Ce nuclei

2005WI20 S.J.Williams, P.M.Jenneson, P.E.Garrett, P.H.Regan, C.Andreoiu, G.C.Ball, R.S.Chakrawarthy, J.J.Daoud, G.F.Grinyer, G.Hackman, B.Hyland, N.A.Mlwilo, A.C.Morton, C.J.Pearson, J.J.Ressler, M.A.Schumaker, M.B.Smith, C.E.Svensson, J.J.Valiente Dobon - J.Phys.(London) G31, S1979 (2005)

Testing the integration of $\mathrm{BaF}_{2}$ detectors into the $8 \pi$ array: fast-timing measurements at TRIUMF

2005WI21 A.N.Wilson, A.K.Singh, H.Hubel, P.M.Davidson, A.Gorgen, D.Rossbach, A.Korichi, A.Astier, F.Azaiez, D.Bazzacco, C.Bourgeois, N.Buforn, A.P.Byrne, G.D.Dracoulis, F.Hannachi, K.Hauschild, W.Korten, T.Kroll, G.J.Lane, A.Lopez-Martens, N.Redon, P.Reiter, C.Rossi-Alvarez, G.Schonwasser, O.Stezowski, P.G.Thirolf Phys.Rev.Lett. 95, 182501 (2005)

Excitation Energies of Superdeformed States in ${ }^{196} \mathrm{~Pb}$ : Towards a Systematic Study of the Second Well in $\mathrm{Pb}$ Isotopes

2005WI23 K.T.Wiedemann, N.H.Medina, J.R.B.Oliveira, J.A.Alcantara-Nunez, W.A.Seale, R.V.Ribas, E.W.Cybulska, M.A.G.Silveira - Braz.J.Phys. 35, 898 (2005)

Yield Ratios in Particle- $\gamma$ Coincidence as a Spectroscopic Tool

2005WIZY A.N.Wilson, A.K.Singh, H.Hubel, P.M.Davidson, A.Gorgen, D.Rossbach, A.Korichi, A.Astier, F.Azaiez, D.Bazzacco, C.Bourgeois, N.Buforn, A.P.Byrne, G.D.Dracoulis, F.Hannachi, K.Hauschild, W.Korten, T.Kroll, G.J.Lane, A.Lopez-Martens, N.Redon, P.Reiter, C.Rossi-Alvarez, G.Schonwasser, O.Stezowski, P.G.Thirolf ANU-P/1667 (2005)

Excitation energies of superdeformed states in ${ }^{196} \mathrm{~Pb}$ : towards a systematic study of the second well in $\mathrm{Pb}$ isotopes

2005WRZZ A.Wronska, V.Hejny, C.Wilkin, S.Dymov, C.Hanhart, V.Komarov, H.R.Koch, A.Kulikov, A.Magiera, T.Mersmann, S.Mikirtytchiants, H.Ohm, D.Prasuhn, R.Schleichert, H.J.Stein, H.Stroher - nucl-ex/0510056,10/20/2005 (2005)

Near threshold $\eta$ meson production in the $\mathrm{dd} \rightarrow{ }^{4} \mathrm{He} \eta$ reaction

$2005 X 106$ C.Y.Xie, X.H.Zhou, Y.H.Zhang, Y.X.Guo, X.G.Lei, Y.Zheng, M.L.Liu, L.T.Song, H.L.Wang, W.T.Guo, H.P.Yu, L.H.Zhu, X.G.Wu, F.R.Xu - Phys.Rev. C 72, 044302 (2005)

Low-spin signature inversion in the $\pi \mathrm{h}_{9 / 2}(\mathrm{x}) \nu \mathrm{i}_{13 / 2}$ oblate band of ${ }^{190} \mathrm{Tl}$ 
$2005 Y$ K26 K.Yamada, T.Motobayashi, N.Aoi, H.Baba, K.Demichi, Z.Elekes, J.Gibelin, T.Gomi, H.Hasegawa, N.Imai, H.Iwasaki, S.Kanno, T.Kubo, K.Kurita, Y.U.Matsuyama, S.Michimasa, T.Minemura, M.Notani, T.K.Onishi, H.J.Ong, S.Ota, A.Ozawa, A.Saito, H.Sakurai, S.Shimoura, E.Takeshita, S.Takeuchi, M.Tamaki, Y.Togano, Y.Yanagisawa, K.Yoneda, I.Tanihata - Eur.Phys.J. A 25, Supplement 1, 409 (2005)

Reduced transition probabilities for the first $2^{+}$excited state in ${ }^{46} \mathrm{Cr},{ }^{50} \mathrm{Fe}$, and ${ }^{54} \mathrm{Ni}$

2005YAZW S.W.Yates - Priv.Comm. (2005)

2005 YE05 Y.L.Ye, D.Y.Pang, G.L.Zhang, D.X.Jiang, T.Zheng, Z.H.Li, X.Q.Li, Q.J.Wang, Y.C.Ge, C.E.Wu, J.Wang, Z.Q.Chen, Y.Ai, A.Ozawa, Y.Yamaguchi, D.Q.Fang, I.Tanihata - J.Phys.(London) G31, S1647 (2005)

Study of the halo nucleus ${ }^{6} \mathrm{He}$ through the direct nuclear reactions

2005 Y012 T.Yoshioka, T.Tsuruta, H.Iwano, T.Danhara - Nucl.Instrum.Methods Phys.Res. A555, 386 (2005); Erratum Nucl.Instrum.Methods Phys.Res. A557, 689 (2006) Spontaneous fission decay constant of ${ }^{238} \mathrm{U}$ determined by SSNTD method using CR-39 and DAP plates

2005 YU07 C.-H.Yu, C.Baktash, J.C.Batchelder, J.R.Beene, C.Bingham, M.Danchev, A.Galindo-Uribarri, C.J.Gross, P.A.Hausladen, W.Krolas, J.F.Liang, E.Padilla, J.Pavan, D.C.Radford - Eur.Phys.J. A 25, Supplement 1, 395 (2005)

Coulomb excitation of odd-A neutron-rich radioactive beams

2005ZA14 N.V.Zamfir, R.O.Hughes, R.F.Casten, D.C.Radford, C.J.Barton, C.Baktash, M.A.Caprio, A.Galindo-Uribarri, C.J.Gross, P.A.Hausladen, E.A.McCutchan, J.J.Ressler, D.Shapira, D.W.Stracener, C.-H.Yu - Eur.Phys.J. A 25, Supplement 1, $389(2005)$

${ }^{132} \mathrm{Te}$ and single-particle density-dependent pairing

$2005 Z$ G15 G.S.Zahn, C.B.Zamboni, F.A.Genezini, M.P.Raele, J.Y.Zevallos-Chavez, M.T.F.da Cruz - Braz.J.Phys. 35, 843 (2005)

Investigation of Excited Levels in ${ }^{193}$ Ir From the Beta Decay of ${ }^{193}$ Os

2005ZEZZ R.G.T.Zegers, H.Akimune, Sam M.Austin, D.Bazin, A.M.van den Berg, G.P.A.Berg, B.A.Brown, A.L.Cole, I.Daito, Y.Fujita, M.Fujiwara, S.Gales, M.N.Harakeh, H.Hashimoto, R.Hayami, G.W.Hitt, M.E.Howard, M.Itoh, J.Janecke, T.Kawabata, K.Kawase, M.Kinoshita, T.Nakamura, K.Nakanishi, S.Nakayama, S.Okamura, W.A.Richter, D.A.Roberts, B.M.Sherrill, Y.Shimbara, M.Steiner, M.Uchida, H.Ueno, T.Yamagata, M.Yosoi - nucl-ex/0512025,12/20/2005 (2005)

The $\left(\mathrm{t},{ }^{3} \mathrm{He}\right)$ and $\left({ }^{3} \mathrm{He}, \mathrm{t}\right)$ reactions as probes of Gamow-Teller strength

2005ZH30 Y.H.Zhang, Y.D.Fang, G.de Angelis, N.Marginean, A.Gadea, D.R.Napoli, M.Axiotis, C.Rusu, T.Martinez, H.L.Wang, X.H.Zhou, W.T.Guo, M.L.Liu, Y.X.Guo, X.G.Lei, M.Oshima, Y.Toh, F.R.Xu - J.Phys.(London) G31, S1545 (2005) High-spin states of an odd-odd ${ }^{184} \mathrm{Au}$ nucleus 
$2005 Z$ Z31 X.H.Zhou, C.Y.Xie, Y.H.Zhang, Y.X.Guo, X.G.Lei, Y.Zheng, M.L.Liu, L.T.Song, H.L.Wang, W.T.Guo - J.Phys.(London) G31, S1985 (2005)

First observation of low-spin signature inversion in the $\pi \mathrm{h}_{9 / 2}(\mathrm{X}) \nu \mathrm{i}_{13 / 2}$ oblate band

2005ZH32 Y.-H.Zhang, S.Guo, X.-H.Zhou, L.Ma, M.Oshima, Y.Toh, M.Koizumi, A.Osa, A.Kimura, Y.Hatsukawa, M.Sugawara, H.Kusakari - Chin.Phys.Lett. 22, 2788 (2005)

Observation of High-j Two-Quasiparticle Bands in Odd-Odd ${ }^{174}$ Re

$2005 Z$ S36 S.J.Zhu, J.H.Hamilton, A.V.Ramayya, P.M.Gore, J.O.Rasmussen, V.Dimitrov, S.Frauendorf, R.Q.Xu, J.K.Hwang, D.Fong, L.M.Yang, K.Li, Y.J.Chen, X.Q.Zhang, E.F.Jones, Y.X.Luo, I.Y.Lee, W.C.Ma, J.D.Cole, M.W.Drigert, M.Stoyer, G.M.Ter-Akopian, A.V.Daniel - Eur.Phys.J. A 25, Supplement 1, 459 (2005) Soft chiral vibrations in ${ }^{106} \mathrm{Mo}$

2006B001 Ch.Bobin, J.Bouchard - Appl.Radiat.Isot. 64, 124 (2006)

A $4 \pi(\mathrm{LS}) \beta-\gamma$ coincidence system using a TDCR apparatus in the $\beta$ channel

2006DI01

F.Ditroi, F.Tarkanyi, J.Csikai, M.S.Uddin, M.Hagiwara, M.Baba, Yu.N.Shubin, S.F.Kovalev - Nucl.Instrum.Methods Phys.Res. B243, 20 (2006)

Excitation functions of long lived products in deuteron induced nuclear reactions on platinum up to $40 \mathrm{MeV}$

2006DR01 Ch.Droste, B.Czajkowska, Z.Marcinkowska, R.M.Lieder, T.Morek, T.Rzaca-Urban, W.Gast - Nucl.Instrum.Methods Phys.Res. A556, 182 (2006)

Polarisation sensitivity of the CLUSTER detector used in EUROBALL array

2006HA01 K.F.Hassan, S.M.Qaim, Z.A.Saleh, H.H.Coenen - Appl.Radiat.Isot. 64, 101 (2006) Alpha-particle induced reactions on ${ }^{n a t} \mathrm{Sb}$ and ${ }^{121} \mathrm{Sb}$ with particular reference to the production of the medically interesting radionuclide ${ }^{124} \mathrm{I}$

2006LE01 P.Leleux, P.Lipnik, S.wa Kitwanga, J.Vanhorenbeeck - Nucl.Instrum.Methods Phys.Res. A556, 397 (2006)

About the neutron yield from the $\mathrm{p}+{ }^{13} \mathrm{C}$ interaction at $30 \mathrm{MeV}$ 